\title{
Deoxofluorination of (Hetero)aromatic Acids
}

Serhii Trofymchuk, ${ }^{1}$ Maksym Bugera, ${ }^{1,2}$ Anton A. Klipkov, ${ }^{1,2}$ Bohdan Razhyk, ${ }^{1}$ Sergey Semenov, ${ }^{1}$ Karen Tarasenko, ${ }^{2,3}$ Viktoriia S. Starova, ${ }^{4}$ Olga A. Zaporozhets, ${ }^{4}$ Oksana Y. Tananaiko, ${ }^{4}$ Anatoliy N. Alekseenko, ${ }^{5}$ Yurii Pustovit, ${ }^{5}$ Oleksandr Kiriakov, ${ }^{1}$ Igor I. Gerus, ${ }^{3}$ Andrei A. Tolmachev ${ }^{1,4}$ Pavel K. Mykhailiuk ${ }^{1,4 *}$

\footnotetext{
${ }^{1}$ Enamine Ltd.; Chervonotkatska 78, 02094 Kyiv (Ukraine), www.enamine.net; www.mykhailiukchem.org, pavel.mykhailiuk@gmail.com

${ }^{2}$ V.P. Kukhar Institute of Bioorganic Chemistry and Petrochemistry NAS of Ukraine; Murmanskaya 1, 02094 Kyiv (Ukraine)

${ }^{3}$ UkrOrgSyntez Ltd. (UORSY), 29 Schorsa Str., 01133 Kyiv (Ukraine)

${ }^{4}$ Taras Shevchenko National University of Kyiv; Chemistry Department; Volodymyrska 64, 01601 Kyiv (Ukraine).

${ }^{5}$ Institute of Organic Chemistry NAS of Ukraine, Murmanskaya 5, 02094 Kyiv (Ukraine)
} 


\section{Table of contents}

Compound 1a

Compound 2a

Compound $3 \mathrm{a}^{*}$. .11

Compound $4 \mathrm{a}^{*}$. 14

Compound 5a* 17

Compound $6 \mathrm{a}$

Compound $7 \mathrm{a}$ 22

Compound 8a .25

Compound 9a* .28

Compound 10a 31

Compound 11a .34

Compound 12a .37

Compound 13a 40

Compound 14a. .43

Compound 15a. 46

Compound 16a* 49

Compound 17a. .52

Compound 18a. .55

Compound 19a .58

Compound 20a. 61

Compound 21a. 64

Compound 22a. 67

Compound 23a. .70

Compound 24a* .73

Compound 25a* .76

Compound 26a* .79

Compound 27a 82

Compound 28a*... 85

Compound 29a. .88

Compound 30a. .91

Compound 31a. .94

Compound 32a. .97

Compound 33a* 100

Compound 34a. 103 


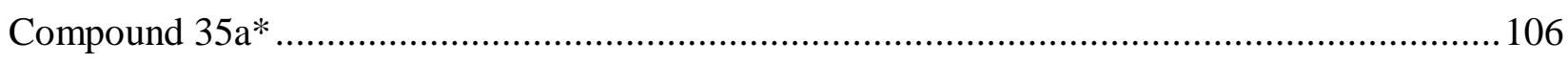

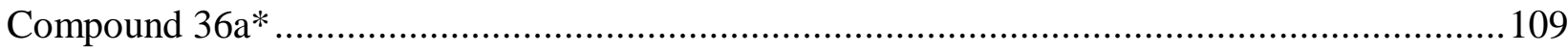

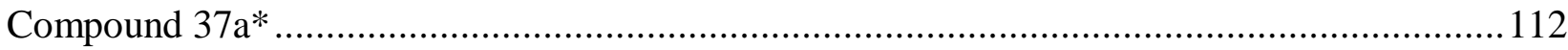

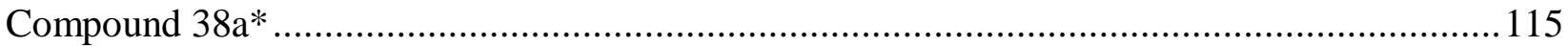

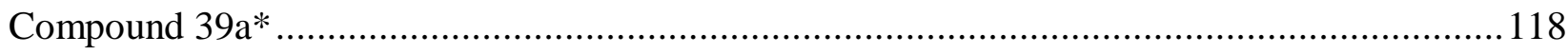

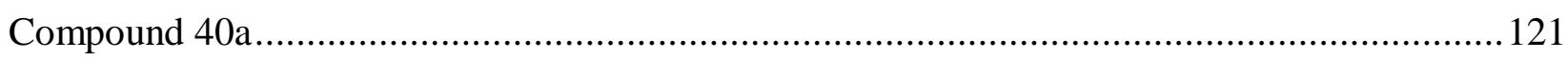

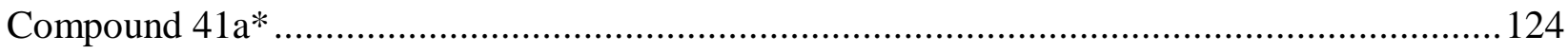

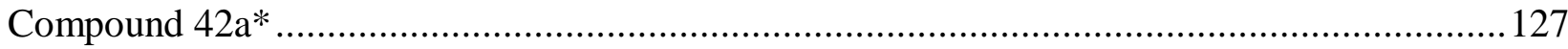

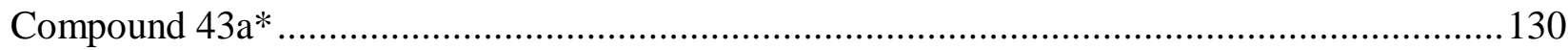

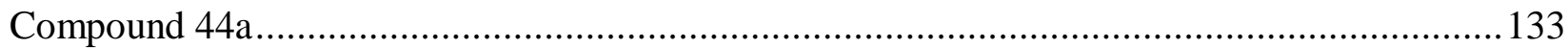

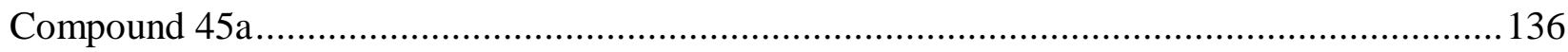

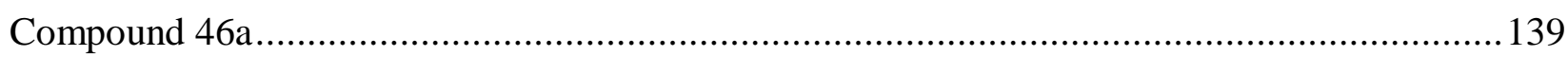

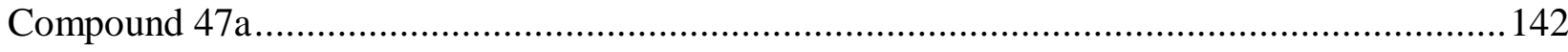

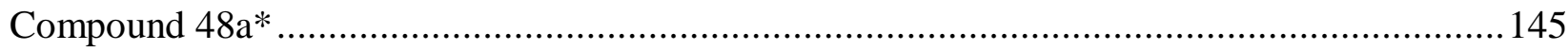

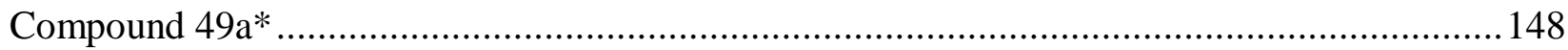

Compound 50a

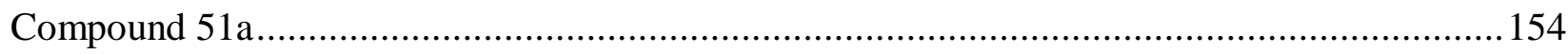

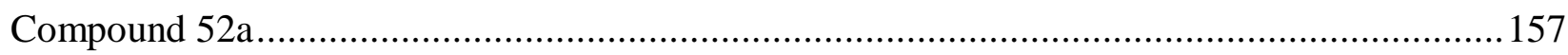

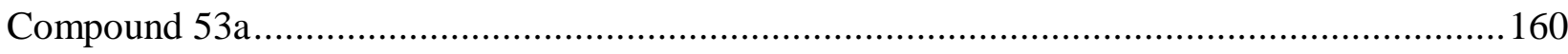

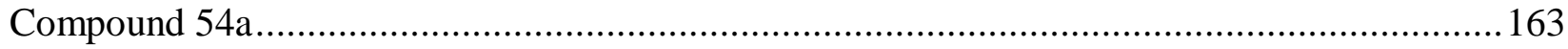

Compound 55a

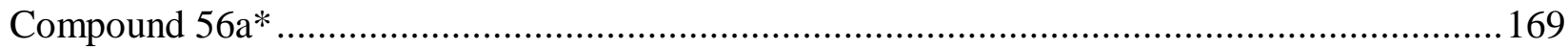

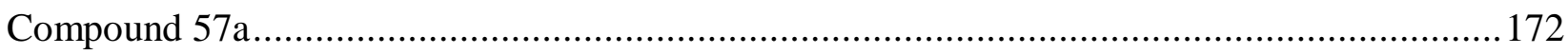

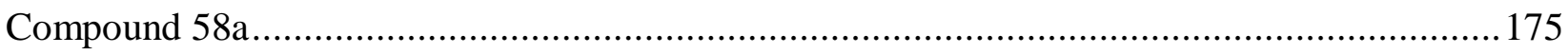

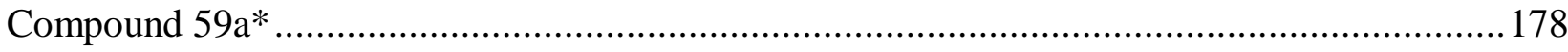

Compound 60a

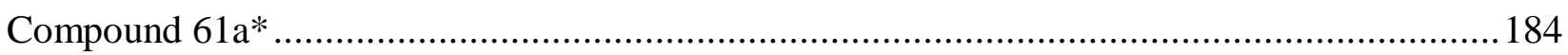

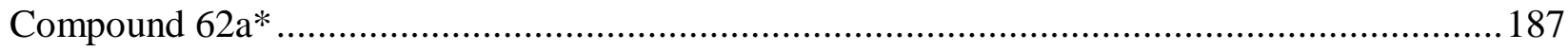

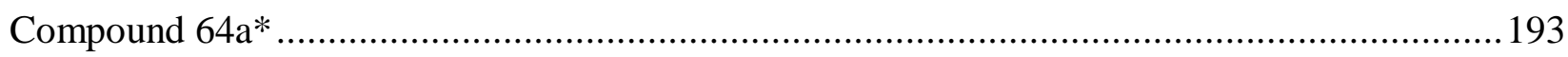

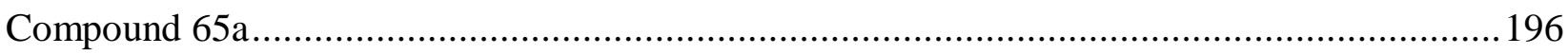

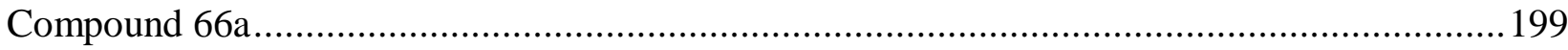

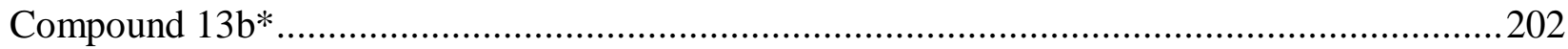

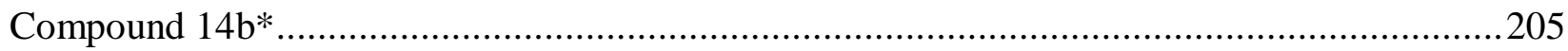

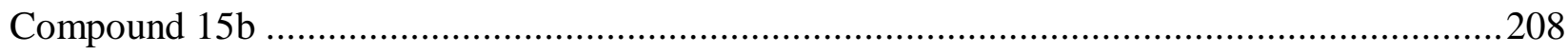

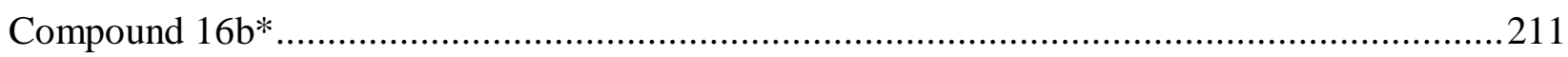




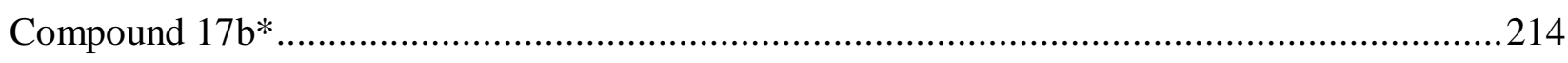

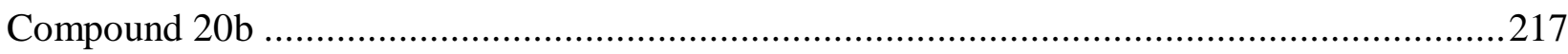

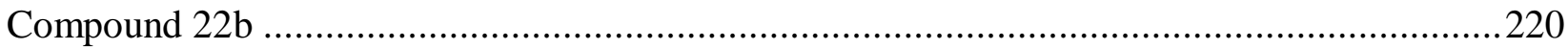

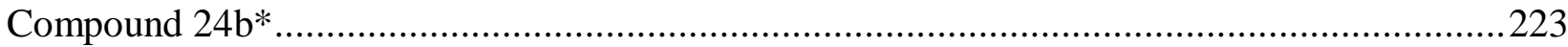

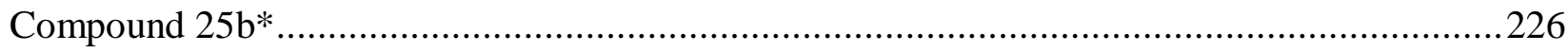

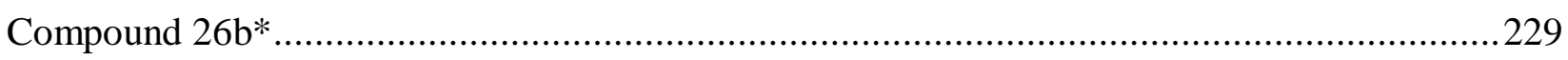

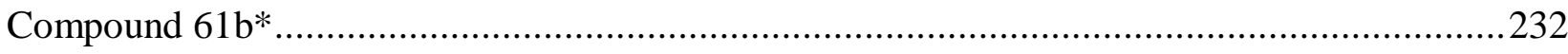

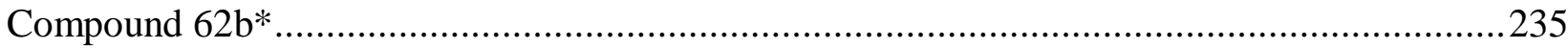

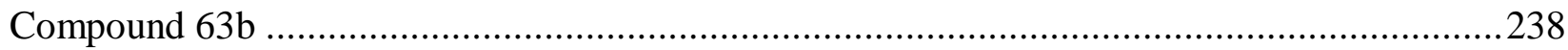

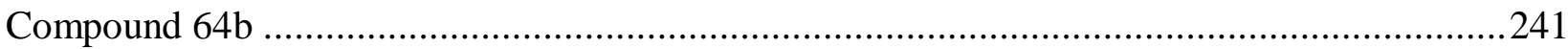

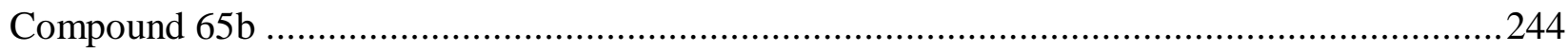

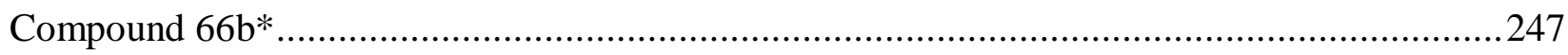

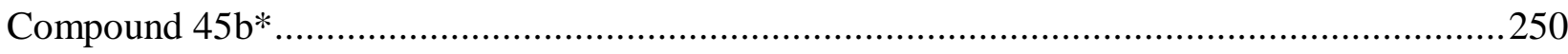

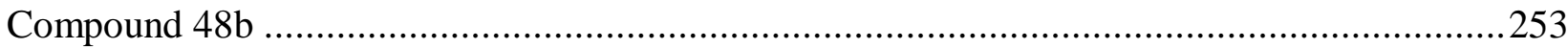

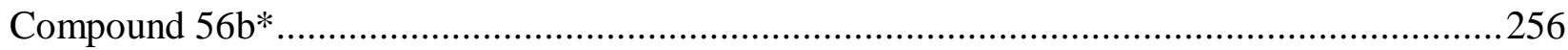

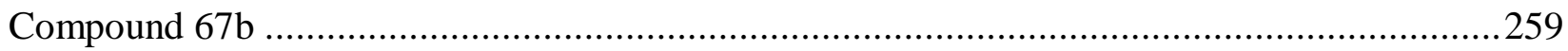

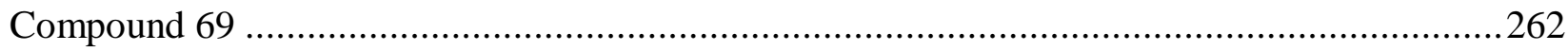

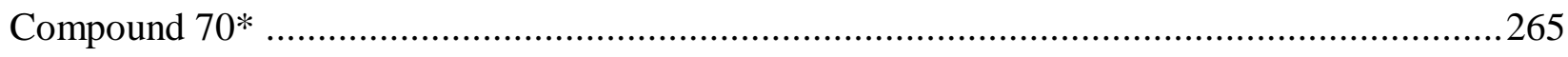

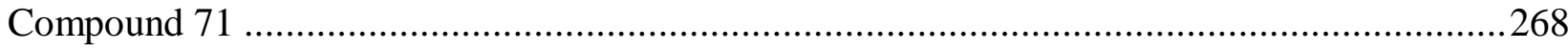

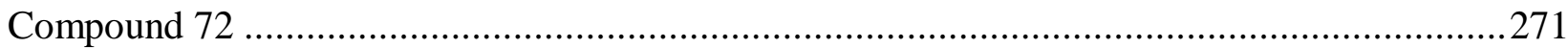

Determination $p K_{a}$ and $p I$ for $\mathrm{CF}_{3}$-homo-GABA isomers by potentiometric titration $\mathrm{pK}_{\mathrm{a}}$ Determination of homo-GABA and its trifluoromethyl derivatives .....................................274

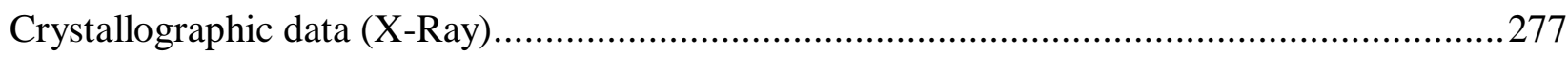

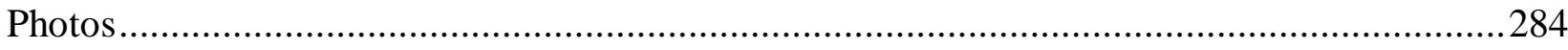


Compound $1 \mathrm{a}^{*}$

${ }^{1} \mathrm{H}$ NMR (400 MHz, $\left.\mathrm{CDCl}_{3}\right)$

i.

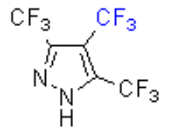
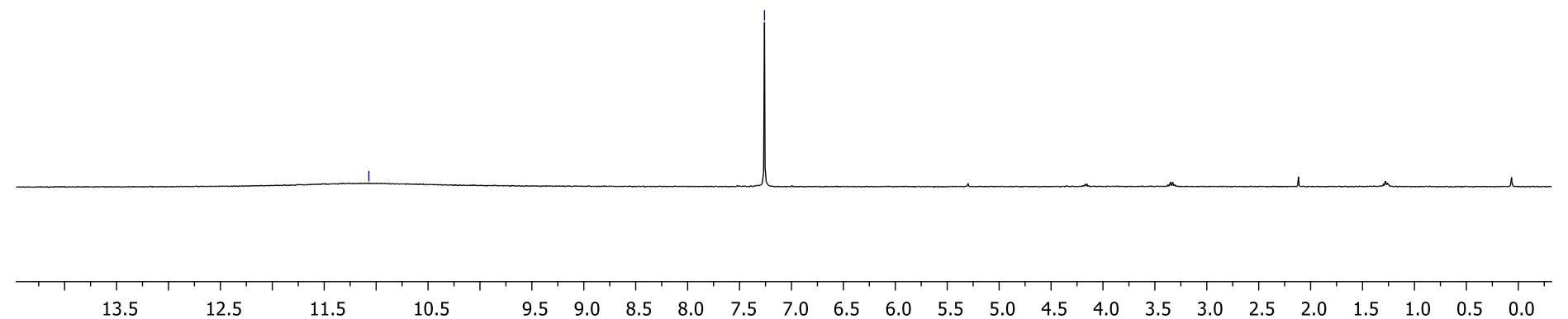

Compound 1a is described in: J. Org. Chem. 2012, 77, 1, 47-56. 


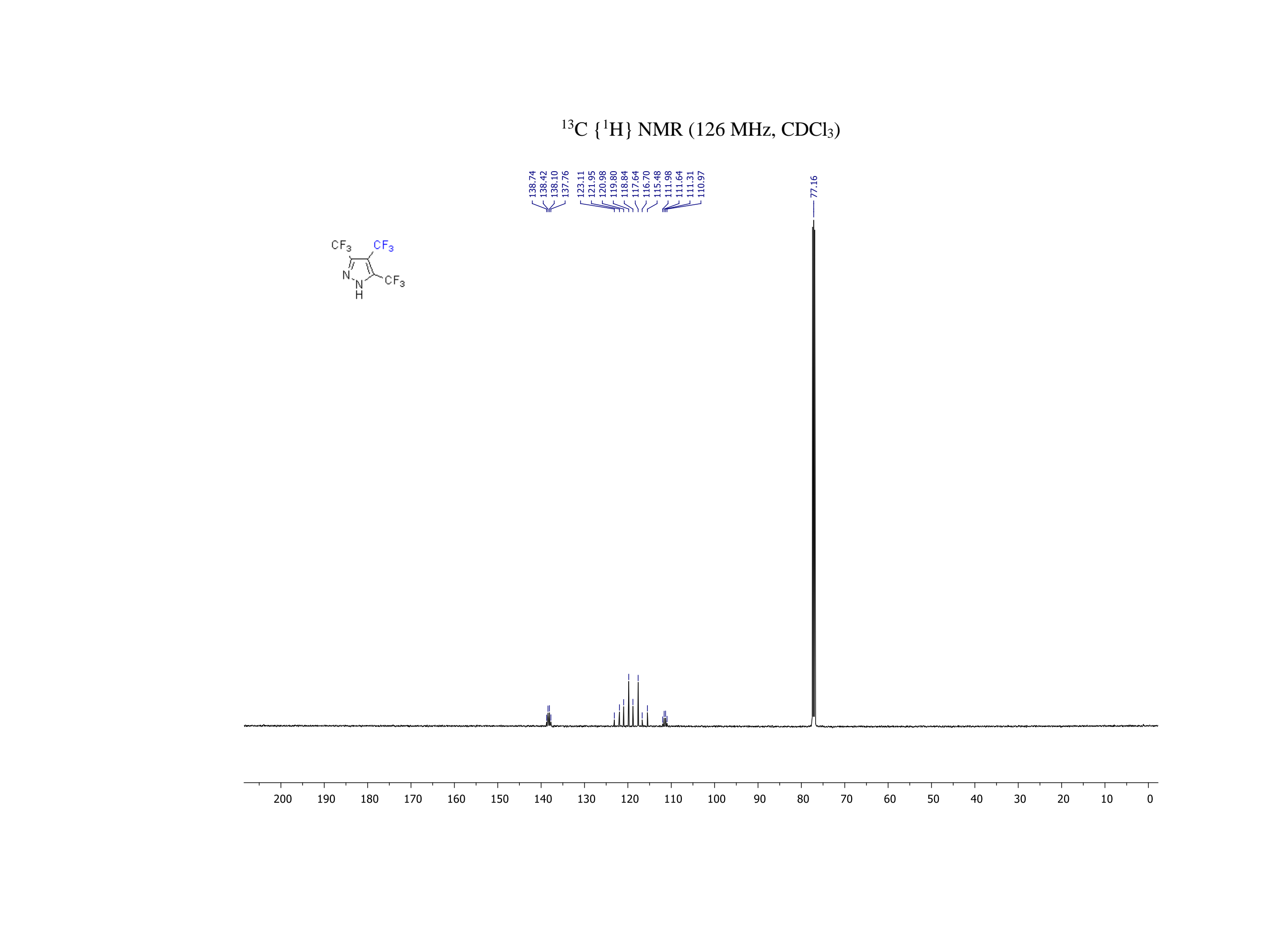


${ }^{19} \mathrm{~F}$ NMR (376 MHz, $\mathrm{CDCl}_{3}$ )

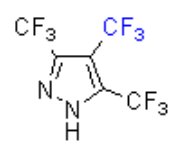

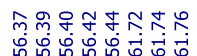

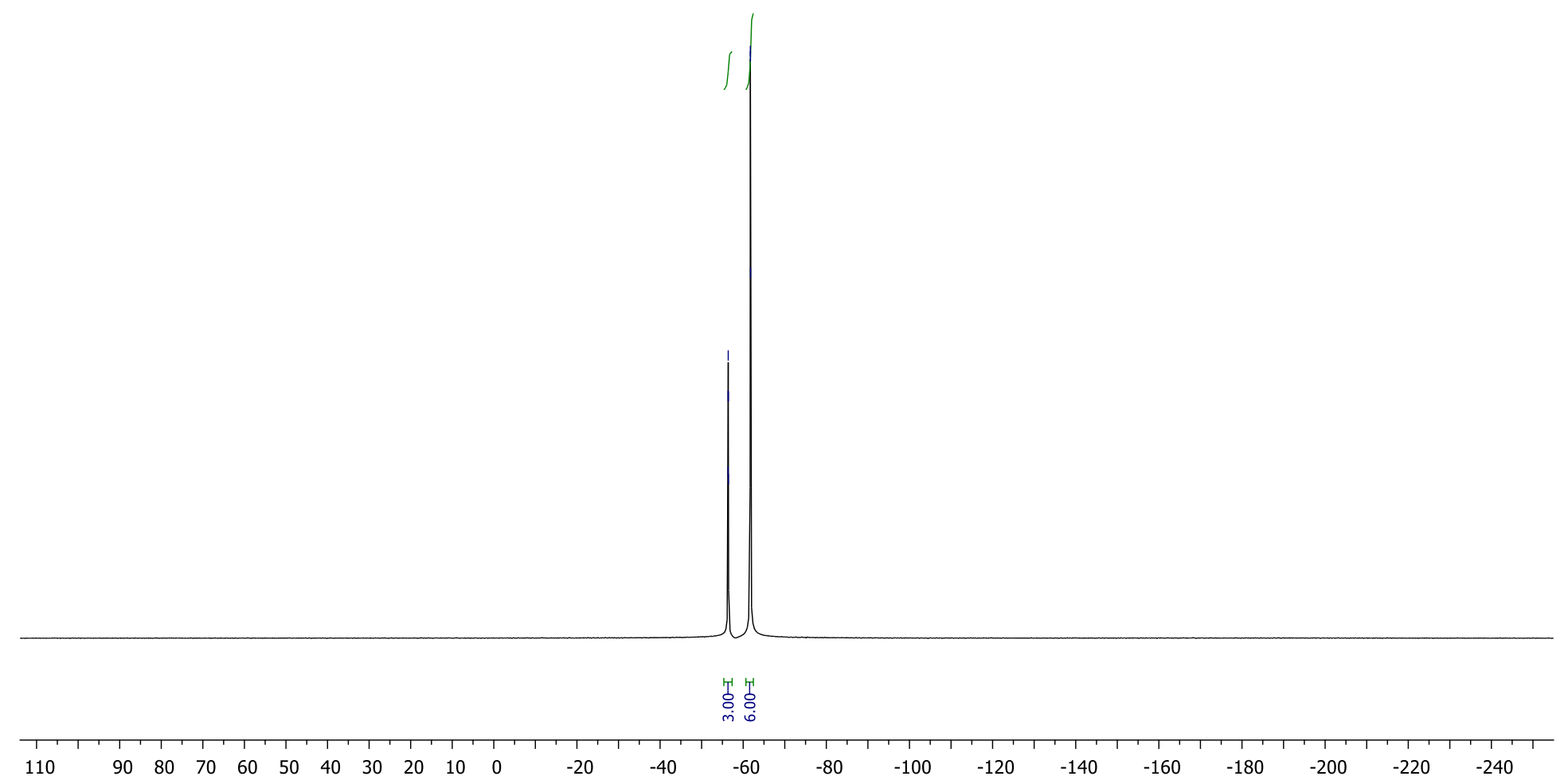


Compound 2a

${ }^{1} \mathrm{H}$ NMR $\left(400 \mathrm{MHz}, \mathrm{CDCl}_{3}\right)$

$\mathrm{Br}_{\mathrm{Br}}^{\mathrm{CF}}$

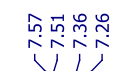

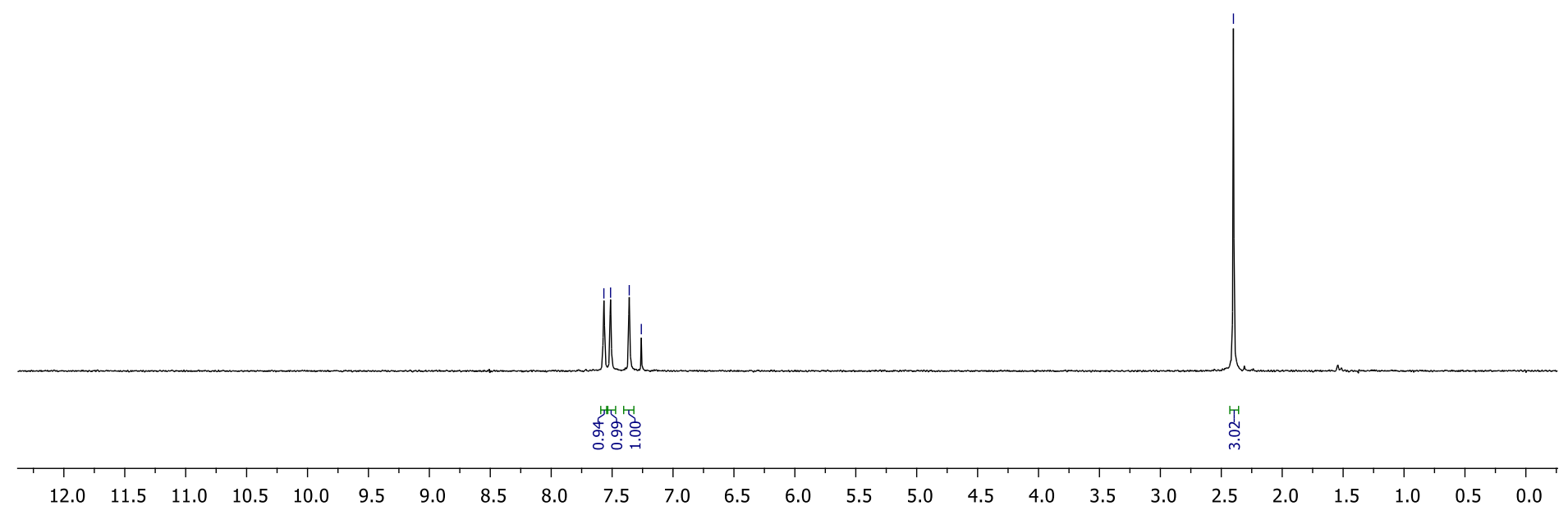


${ }^{13} \mathrm{C}\left\{{ }^{1} \mathrm{H}\right\}$ NMR $\left(126 \mathrm{MHz}, \mathrm{CDCl}_{3}\right)$

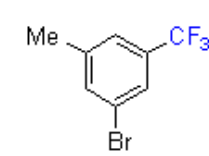

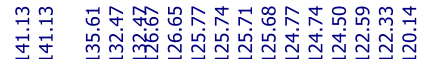

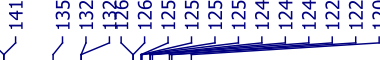

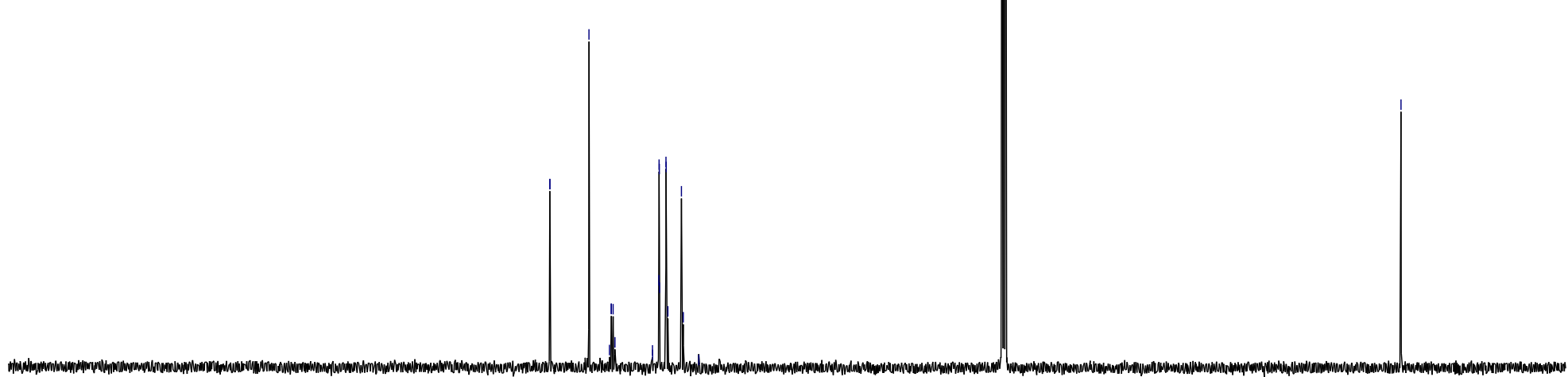


${ }^{19} \mathrm{~F}$ NMR (376 MHz, $\left.\mathrm{CDCl}_{3}\right)$

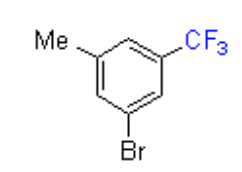

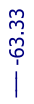

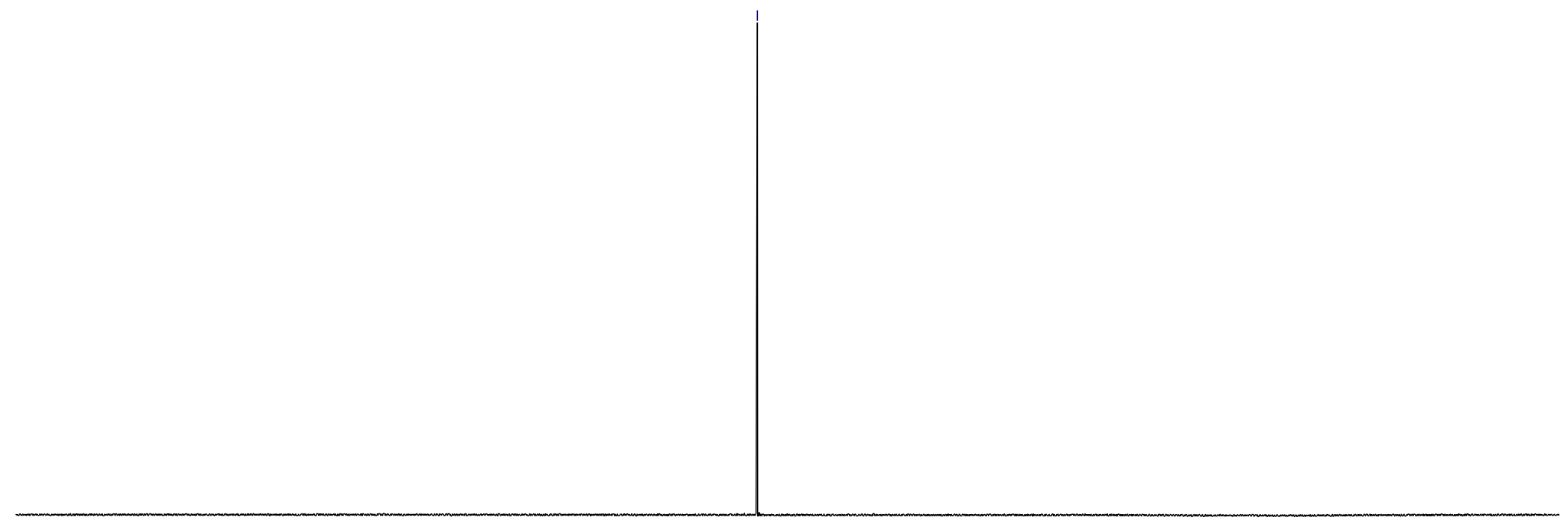


Compound $3 \mathrm{a}^{*}$

${ }^{1} \mathrm{H}$ NMR (400 MHz, $\mathrm{CDCl}_{3}$ )
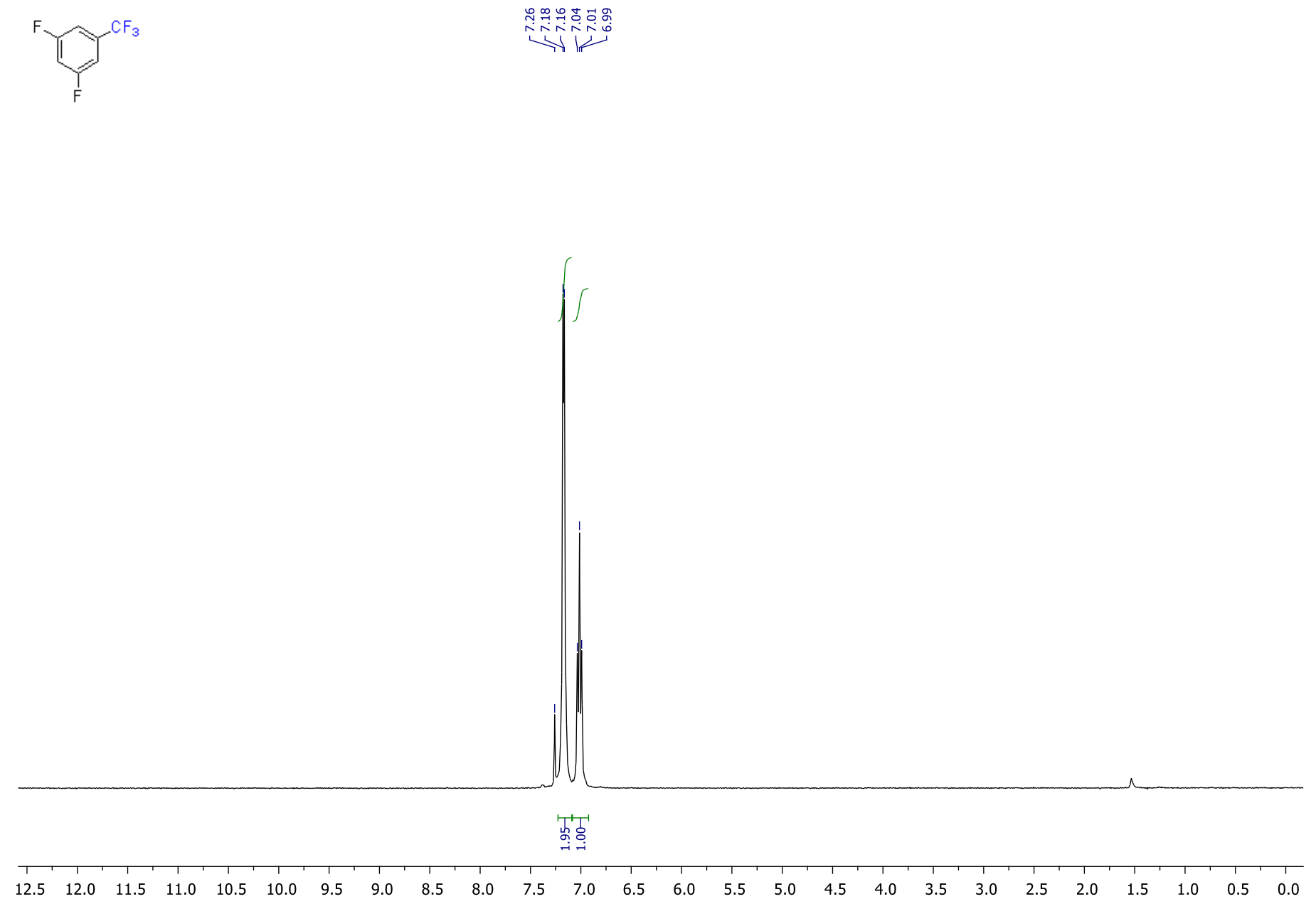

Compound 3a is also described in: Angew. Chem. Int. Ed. 2012, 51, 31, $7767-7770$. 
${ }^{13} \mathrm{C}\left\{{ }^{1} \mathrm{H}\right\}$ NMR $\left(126 \mathrm{MHz}, \mathrm{CDCl}_{3}\right)$
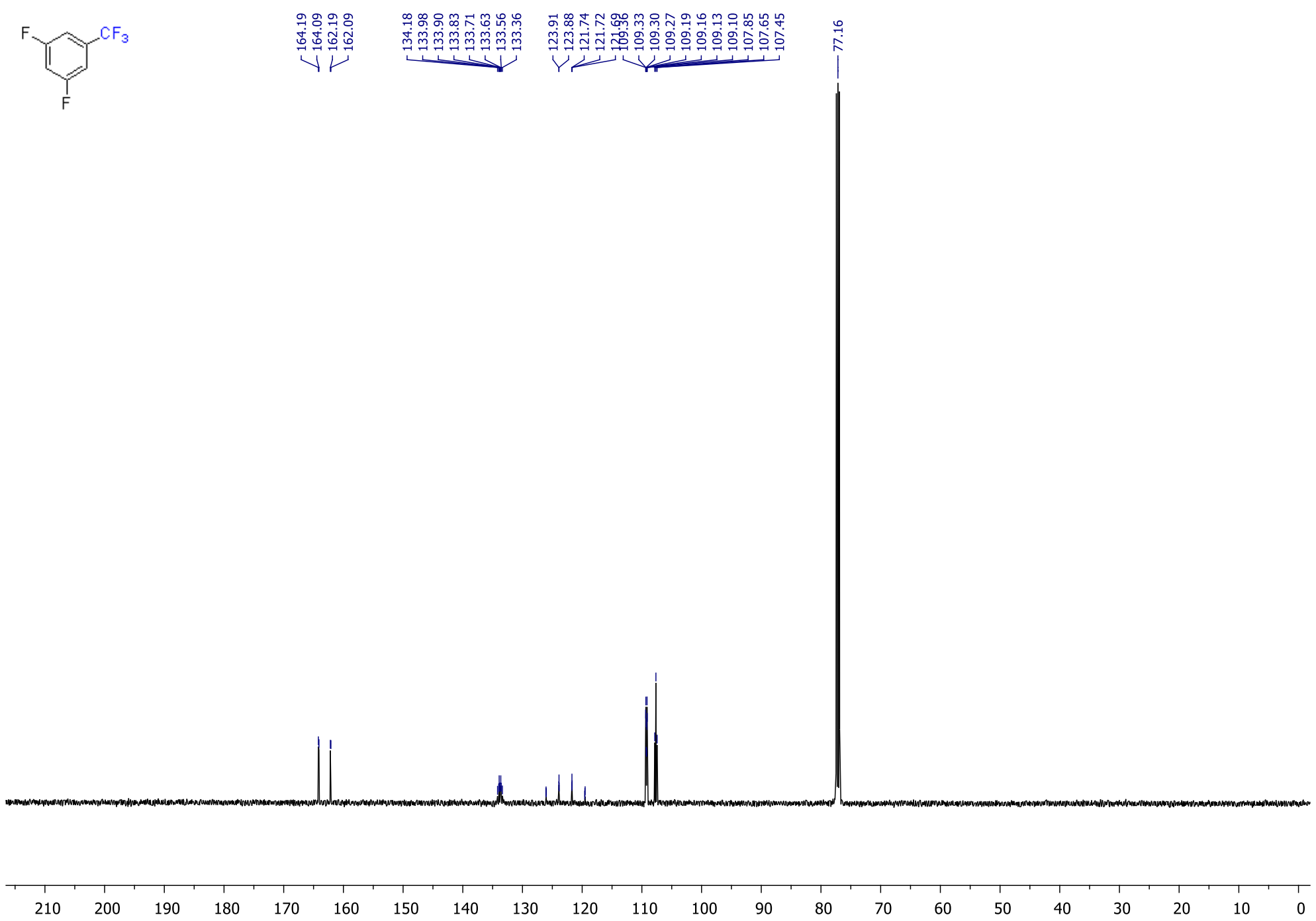
${ }^{19} \mathrm{~F}$ NMR (376 MHz, $\mathrm{CDCl}_{3}$ )
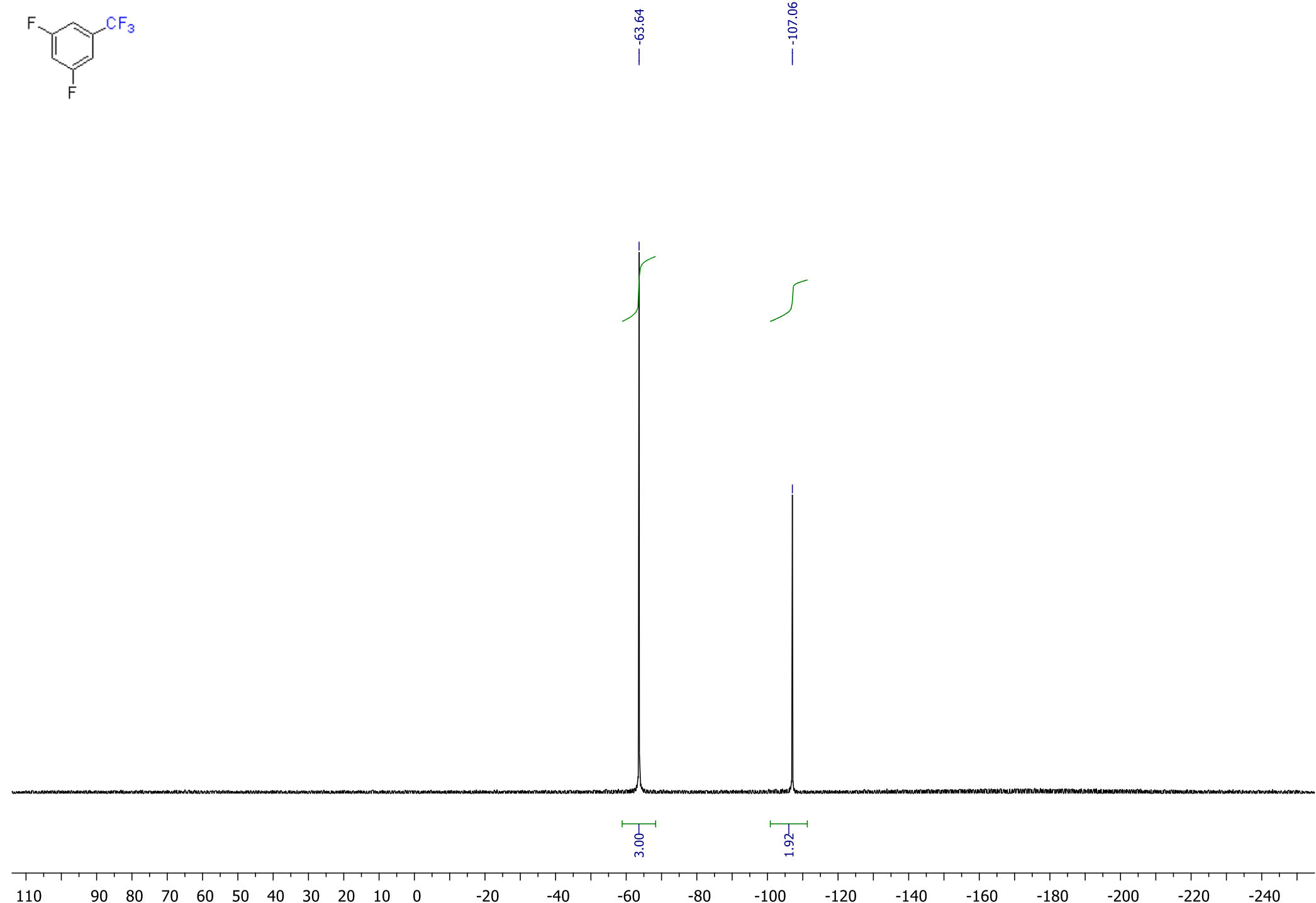
Compound $4 a^{*}$

${ }^{1} \mathrm{H} \mathrm{NMR}\left(400 \mathrm{MHz}, \mathrm{CDCl}_{3}\right)$

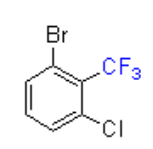

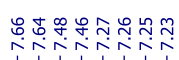

iniving
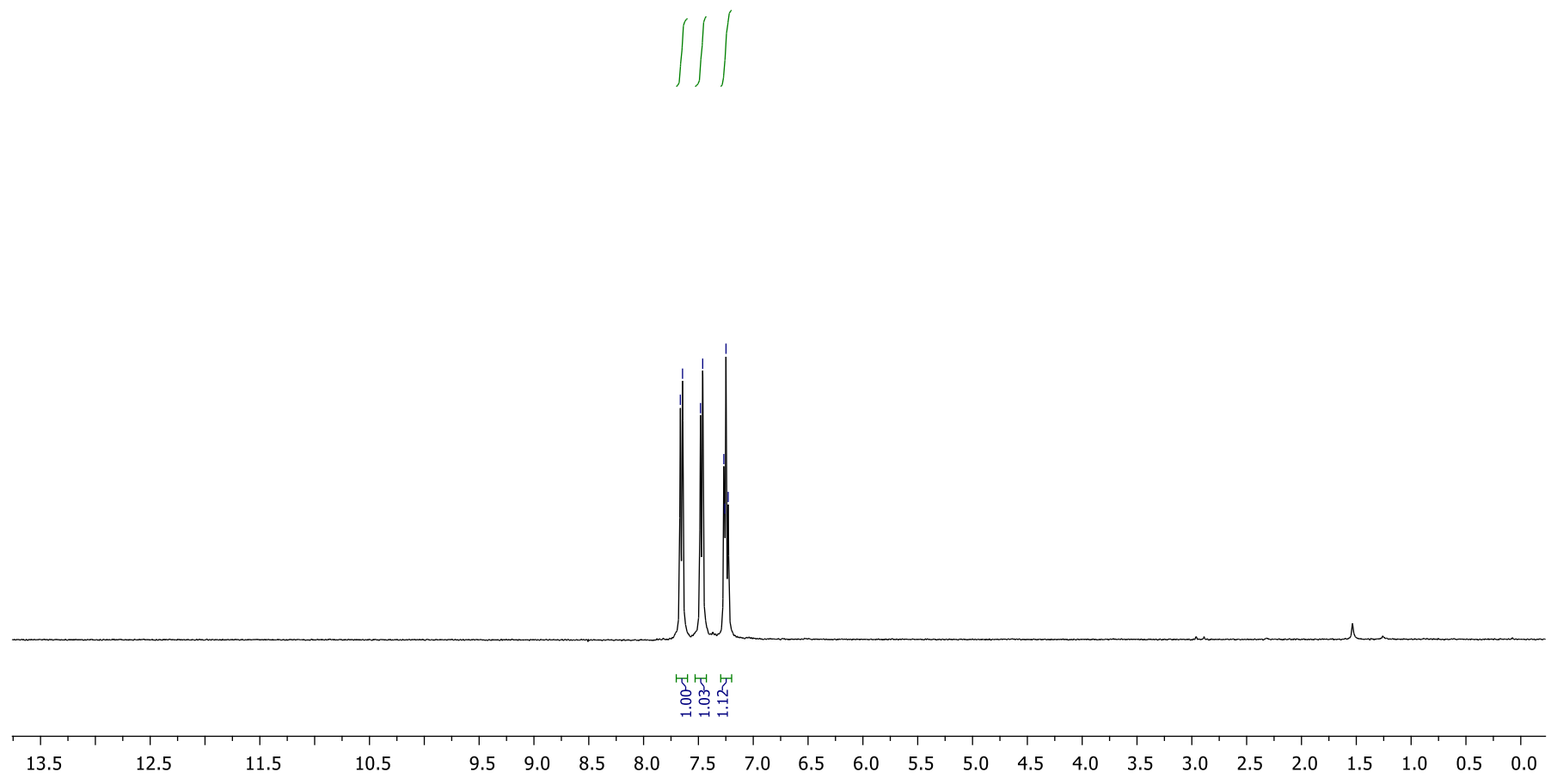

Compound 4a is also described in: a) Angew. Chem. Int. Ed. 2012, 51, 31, 7767 - 7770; b) ACS Med. Chem. Lett. 2017, 8, 1, 90-95; c) Angew. Chem. Int. Ed. 2018, 57, 46, 15081-15085; d) J. Am. Chem. Soc. 2019, 141, 4, 1788-1796. 
${ }^{13} \mathrm{C}\left\{{ }^{1} \mathrm{H}\right\}$ NMR $\left(126 \mathrm{MHz}, \mathrm{CDCl}_{3}\right)$
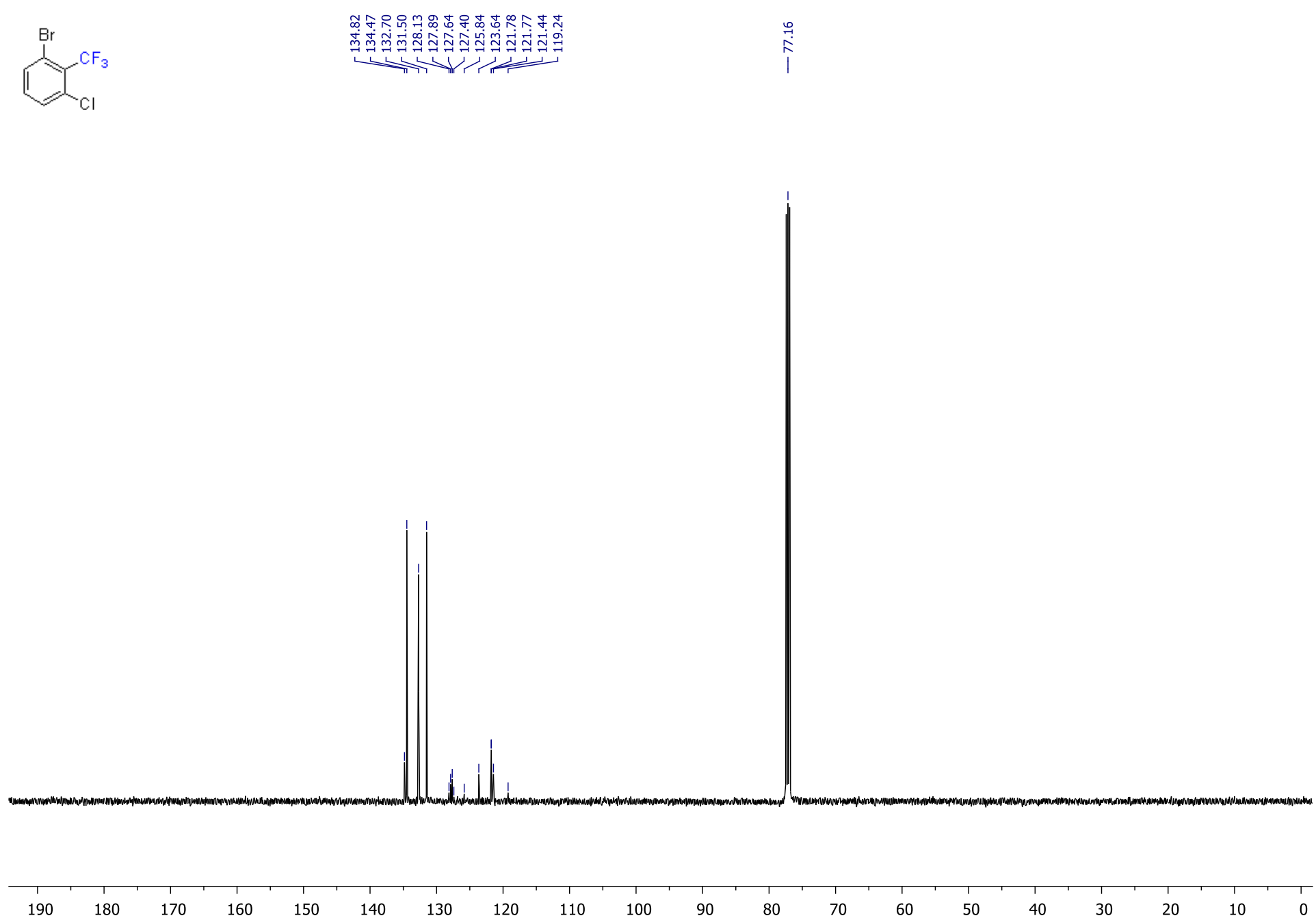
${ }^{19} \mathrm{~F}$ NMR (376 MHz, $\mathrm{CDCl}_{3}$ )

$\overbrace{\mathrm{Cl}}^{\mathrm{Br}}$

ֻิ

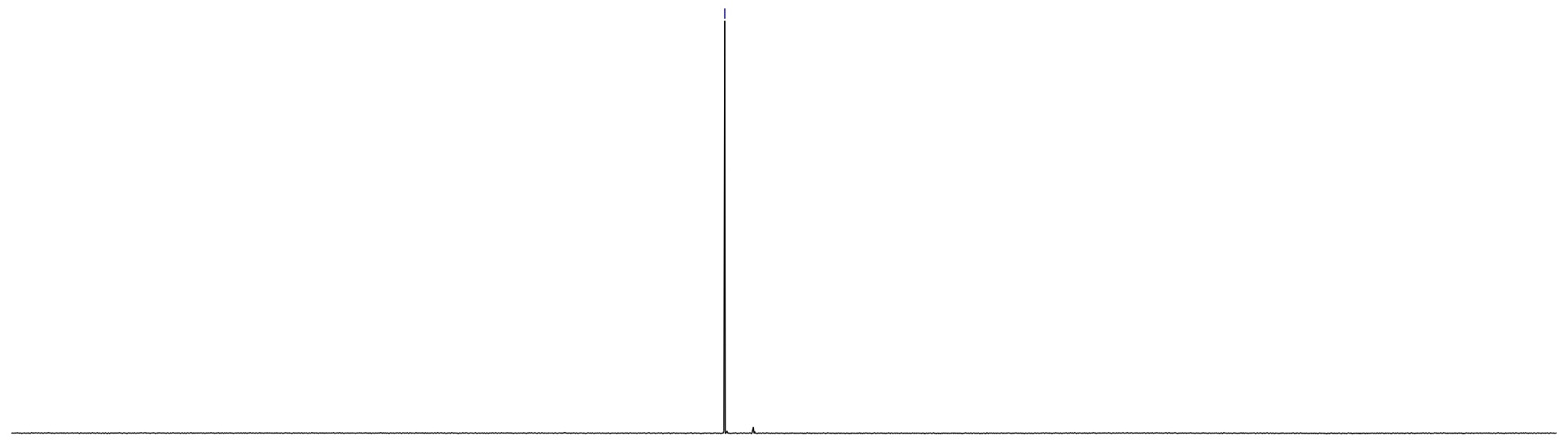

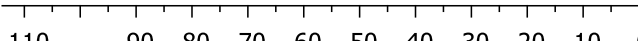

$\begin{array}{llllllllll}90 & 80 & 70 & 60 & 50 & 40 & 30 & 20 & 10 & 0\end{array}$ 
Compound 5a*

${ }^{1} \mathrm{H} \mathrm{NMR}\left(400 \mathrm{MHz}, \mathrm{CDCl}_{3}\right)$

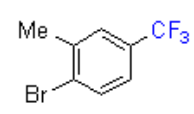

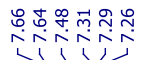

111

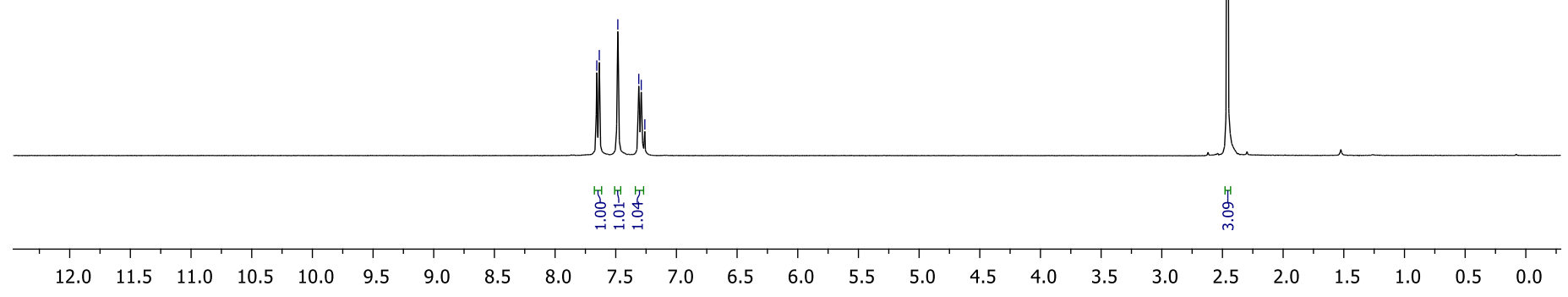

Compound 5a is also described in: a) ACS Med. Chem. Lett. 2017, 8, 1, 90-95; b) Angew. Chem. Int. Ed. 2018, 57, 46, 15081-15085; c) Angew. Chem. Int. Ed. 2018, 54, 34, 11040-11044; d) J. Am. Chem. Soc. 2019, 141, 4, 1788-1796. 
${ }^{13} \mathrm{C}\left\{{ }^{1} \mathrm{H}\right\}$ NMR $\left(126 \mathrm{MHz}, \mathrm{CDCl}_{3}\right)$

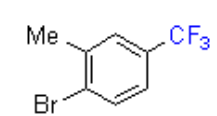

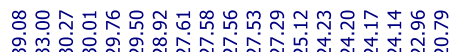

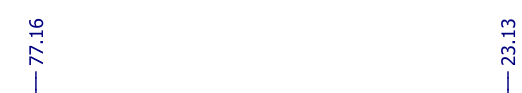

mam

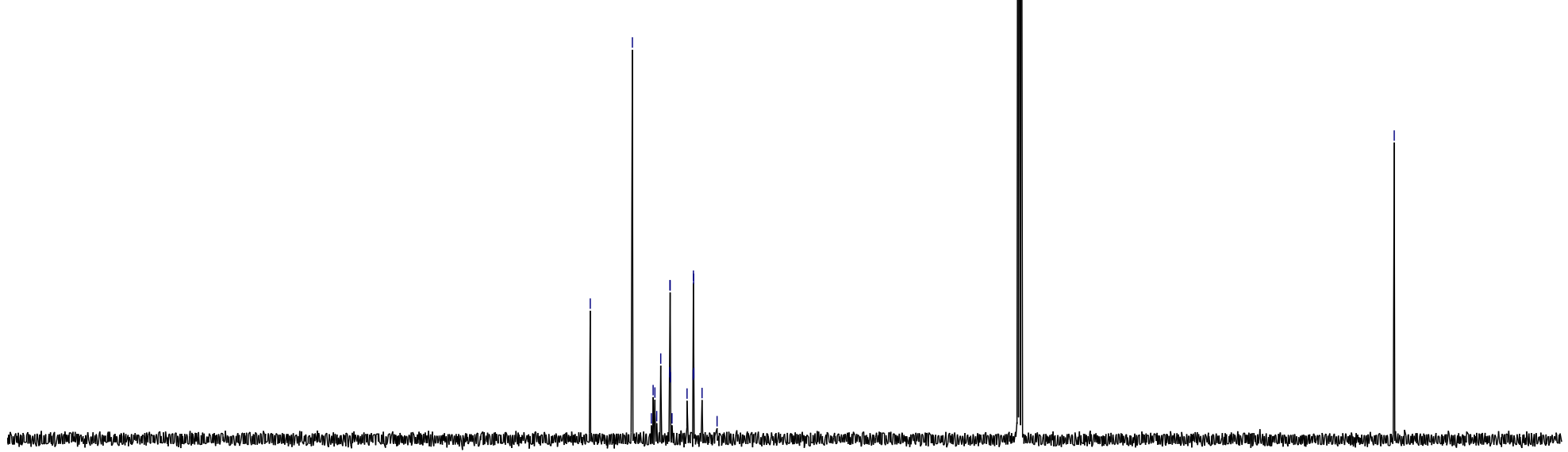

$\begin{array}{lllllllllllllllllllllll}220 & 210 & 200 & 190 & 180 & 170 & 160 & 150 & 140 & 130 & 120 & 110 & 100 & 90 & 80 & 70 & 60 & 50 & 40 & 30 & 20 & 10 & 0\end{array}$ 
Compound 6a

${ }^{1} \mathrm{H}$ NMR (400 MHz, $\mathrm{CDCl}_{3}$ )

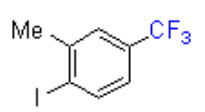

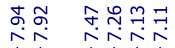

र iniv

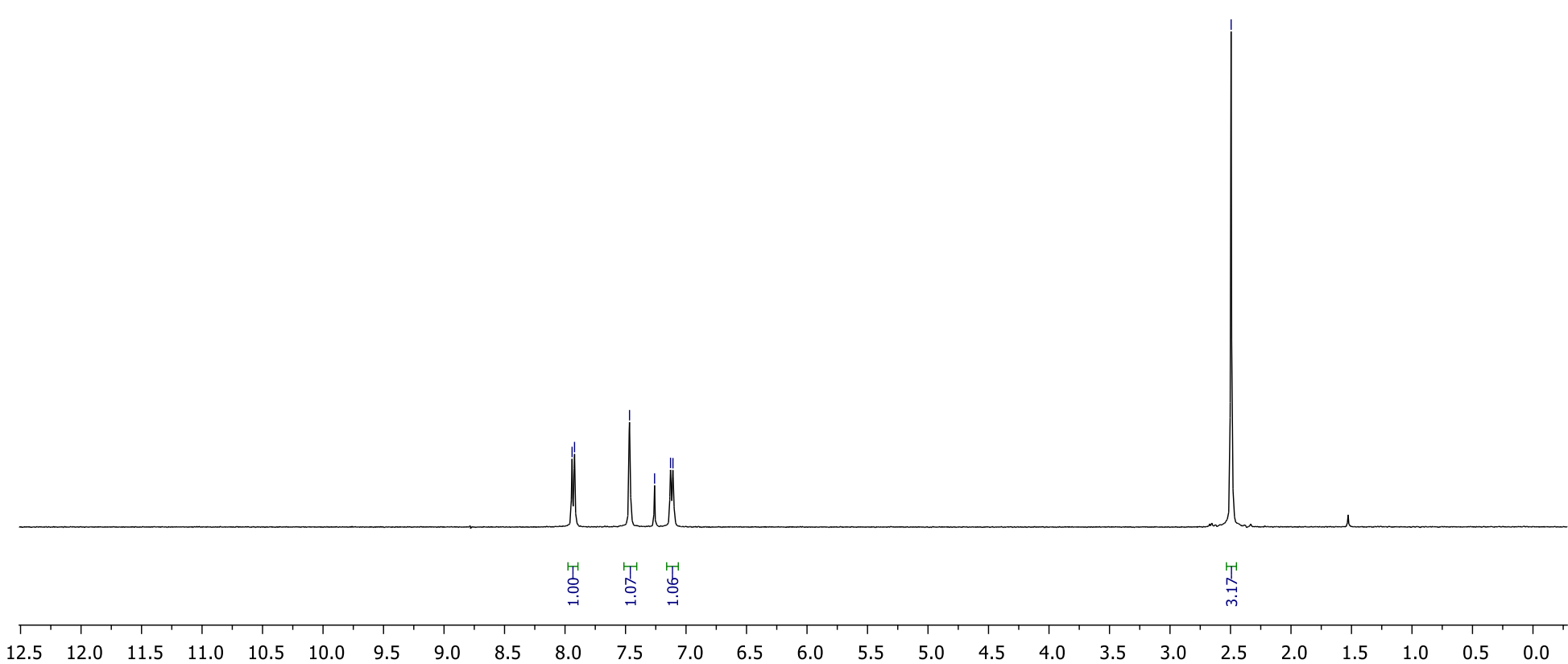


${ }^{13} \mathrm{C}\left\{{ }^{1} \mathrm{H}\right\}$ NMR $\left(126 \mathrm{MHz}, \mathrm{CDCl}_{3}\right)$

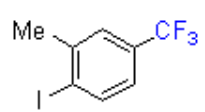

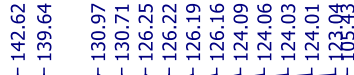

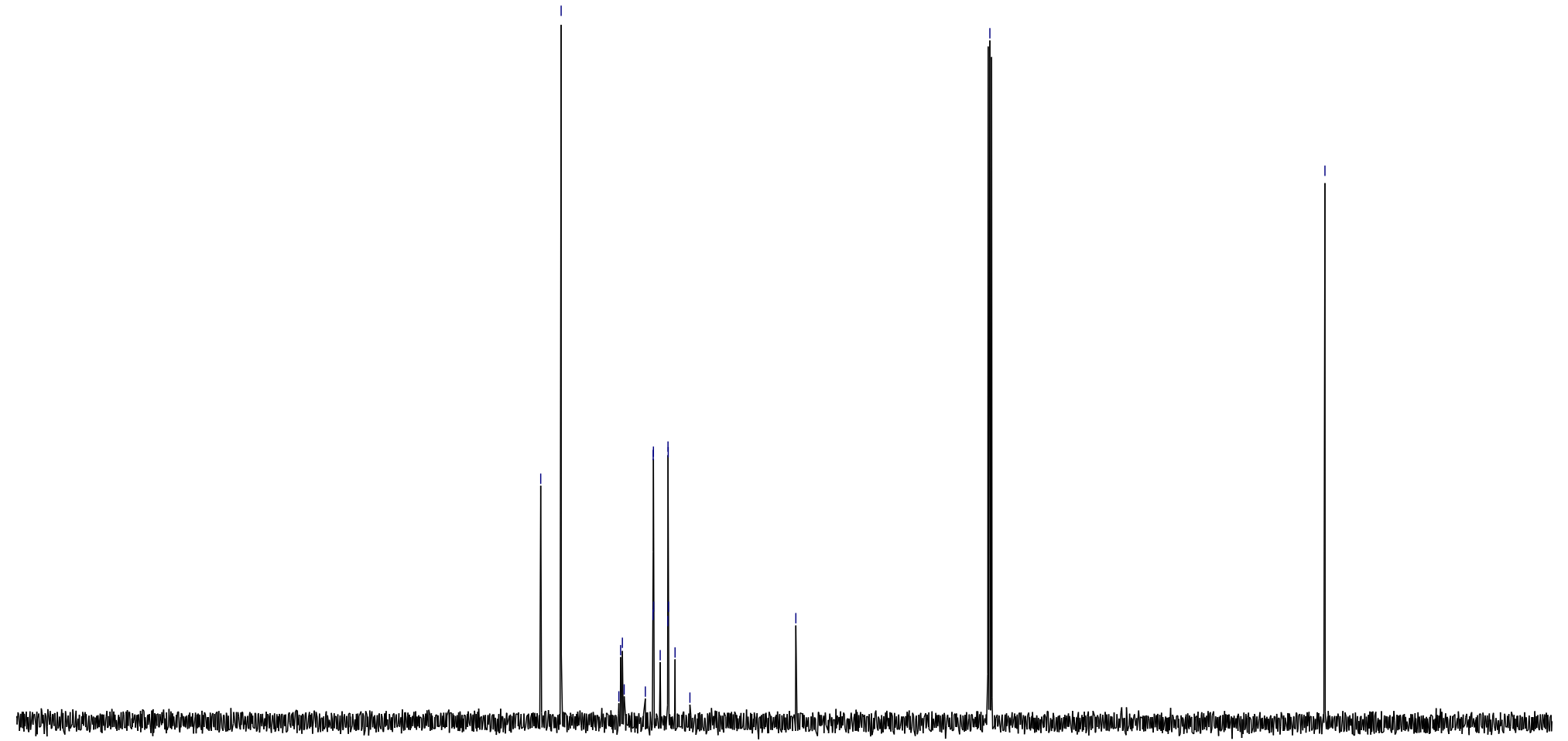


${ }^{19} \mathrm{~F}$ NMR (376 MHz, $\left.\mathrm{CDCl}_{3}\right)$
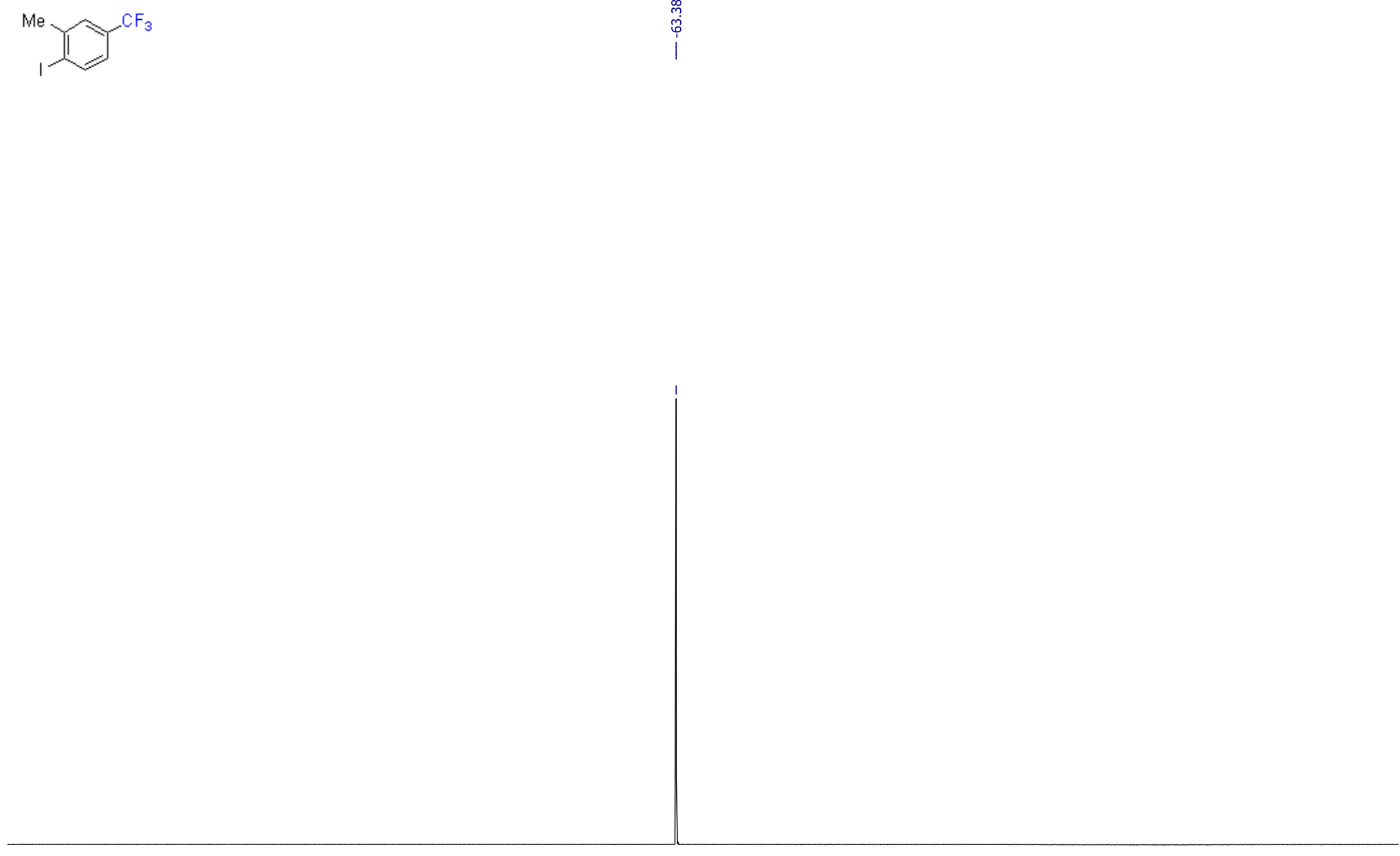
Compound 7a

${ }^{1} \mathrm{H}$ NMR (400 MHz, $\mathrm{CDCl}_{3}$ )

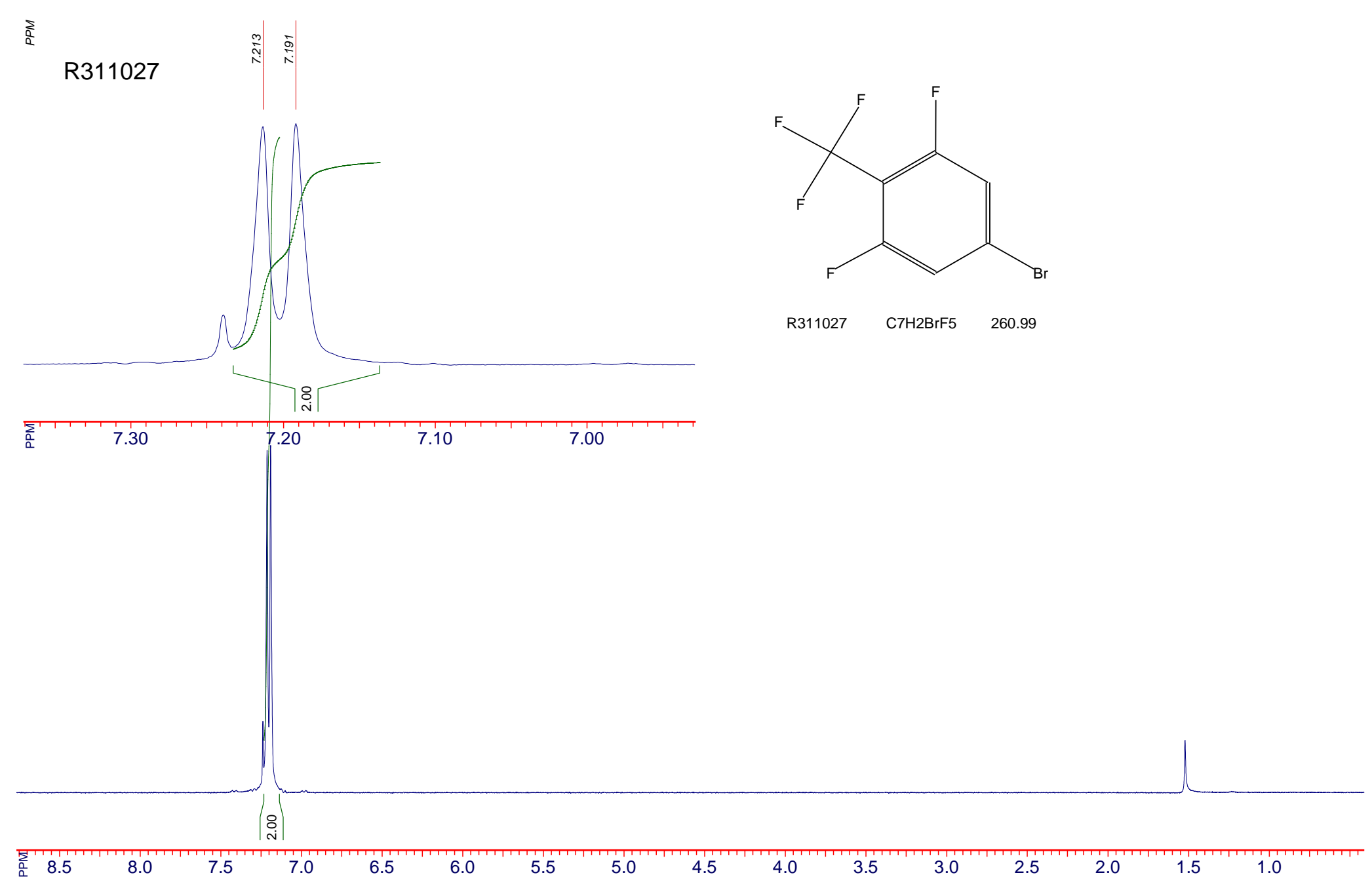


${ }^{13} \mathrm{C}\left\{{ }^{1} \mathrm{H}\right\}$ NMR $\left(126 \mathrm{MHz}, \mathrm{CDCl}_{3}\right)$

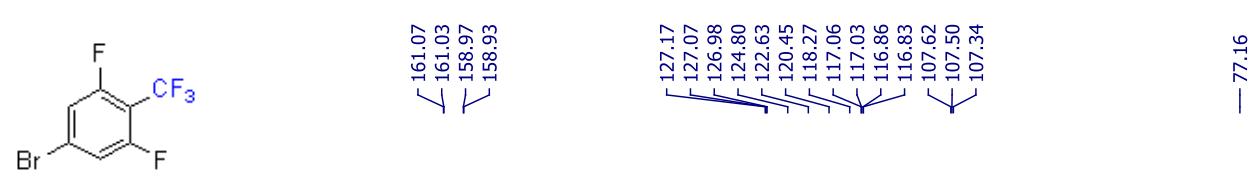

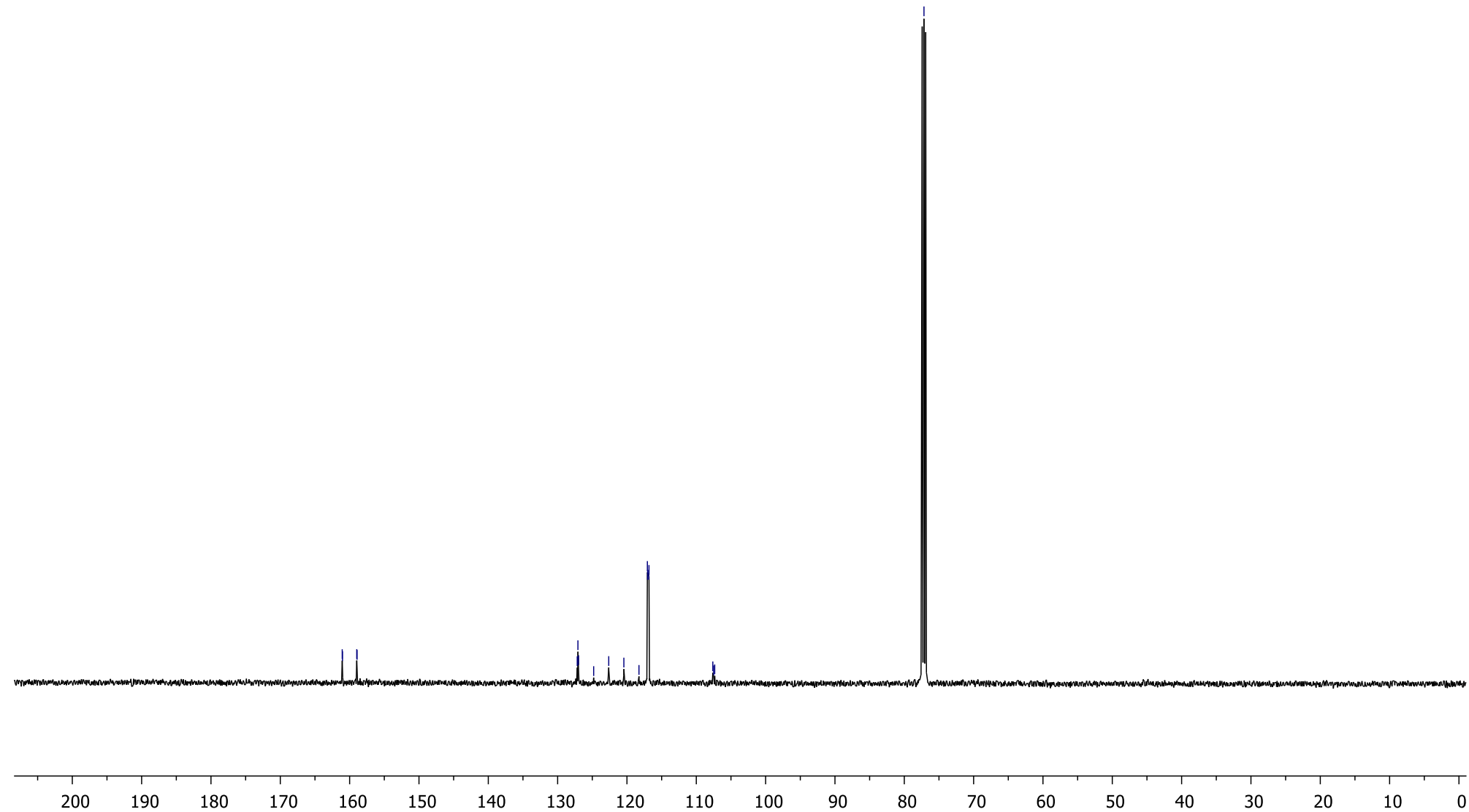


$\left.{ }^{19} \mathrm{~F} \mathrm{NMR} \mathrm{(376} \mathrm{MHz,} \mathrm{CDCl}_{3}\right)$

$\overbrace{\mathrm{F}}^{\mathrm{CF}_{3}}$

لَ

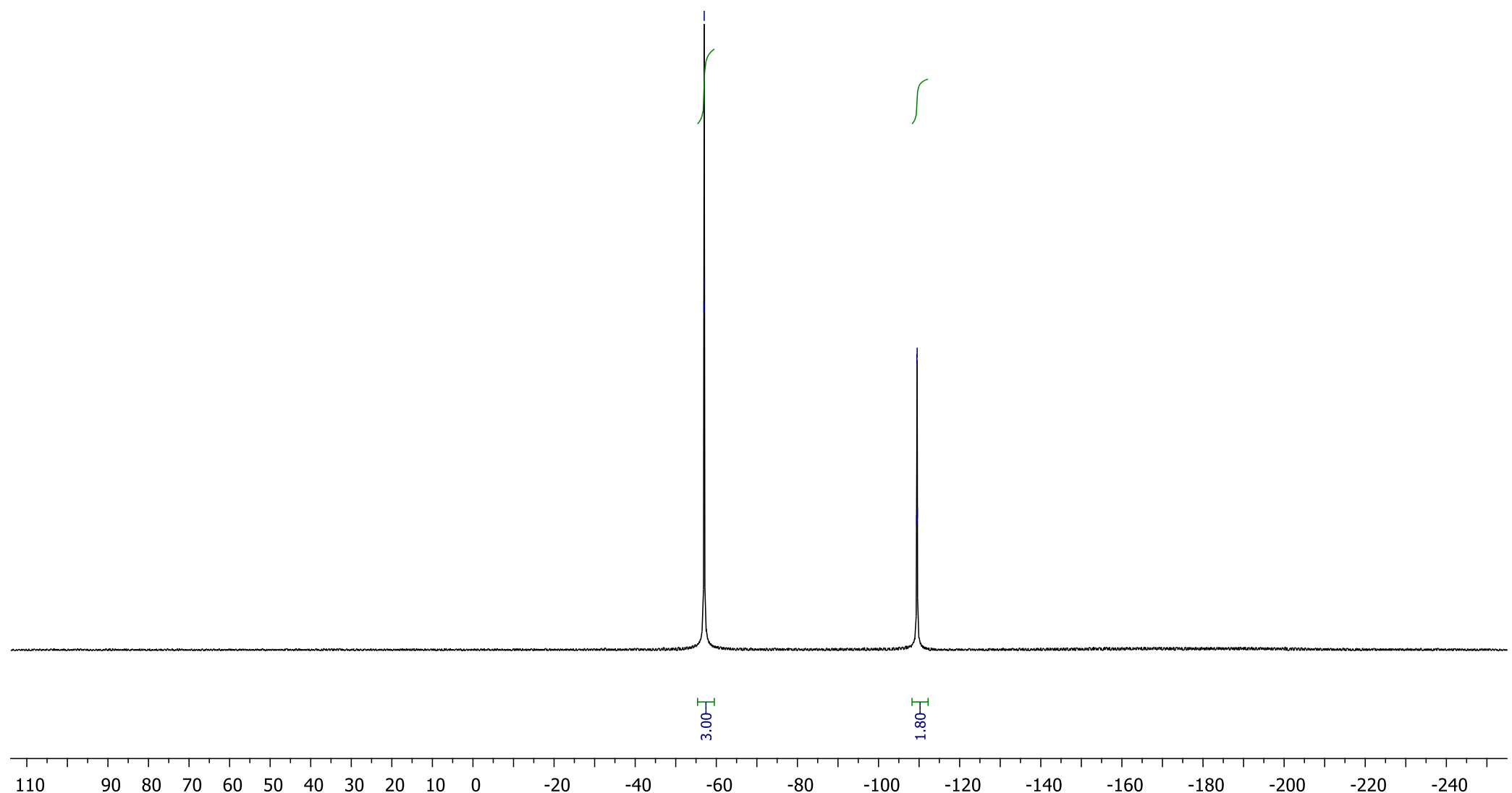


Compound 8a

${ }^{1} \mathrm{H}$ NMR $\left(400 \mathrm{MHz}, \mathrm{CDCl}_{3}\right)$

$\mathrm{CF}_{3}$

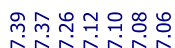

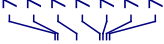
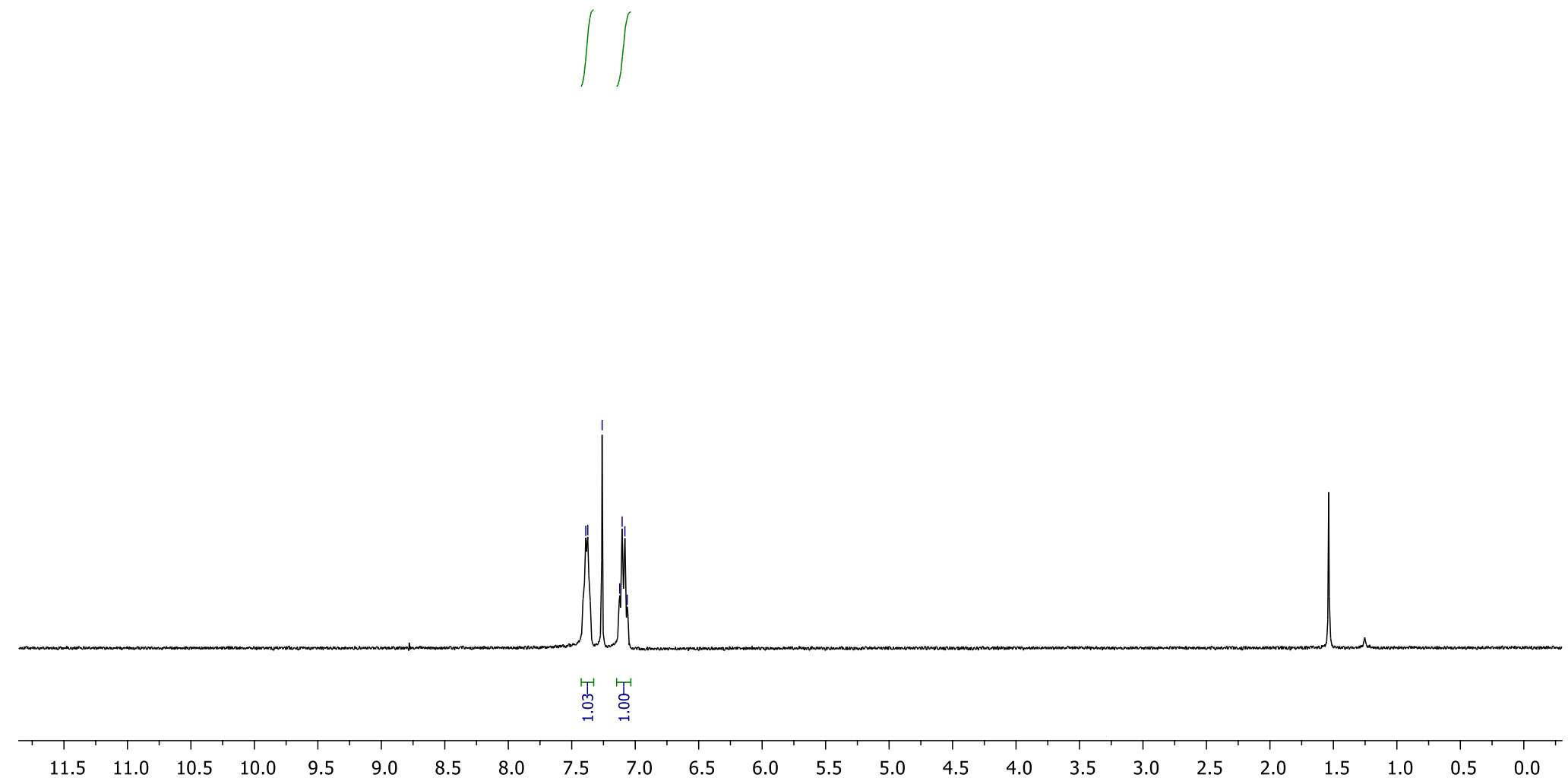
${ }^{13} \mathrm{C}\left\{{ }^{1} \mathrm{H}\right\}$ NMR $\left(151 \mathrm{MHz}, \mathrm{CDCl}_{3}\right)$

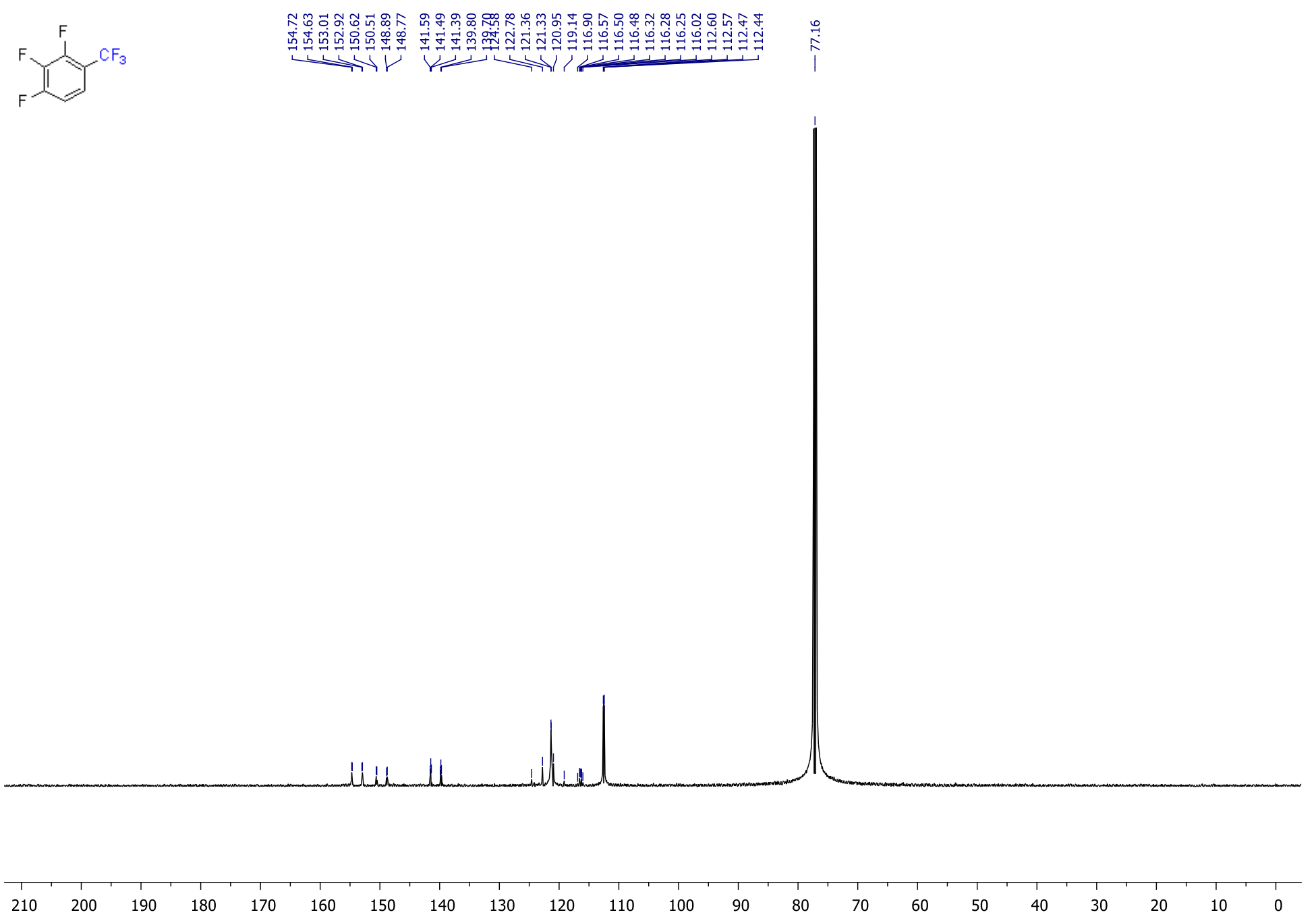


${ }^{19} \mathrm{~F}$ NMR (376 MHz, $\left.\mathrm{CDCl}_{3}\right)$

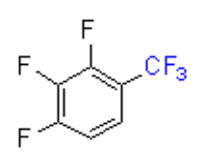

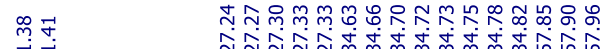

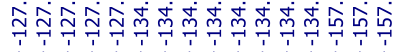

P

$\int$
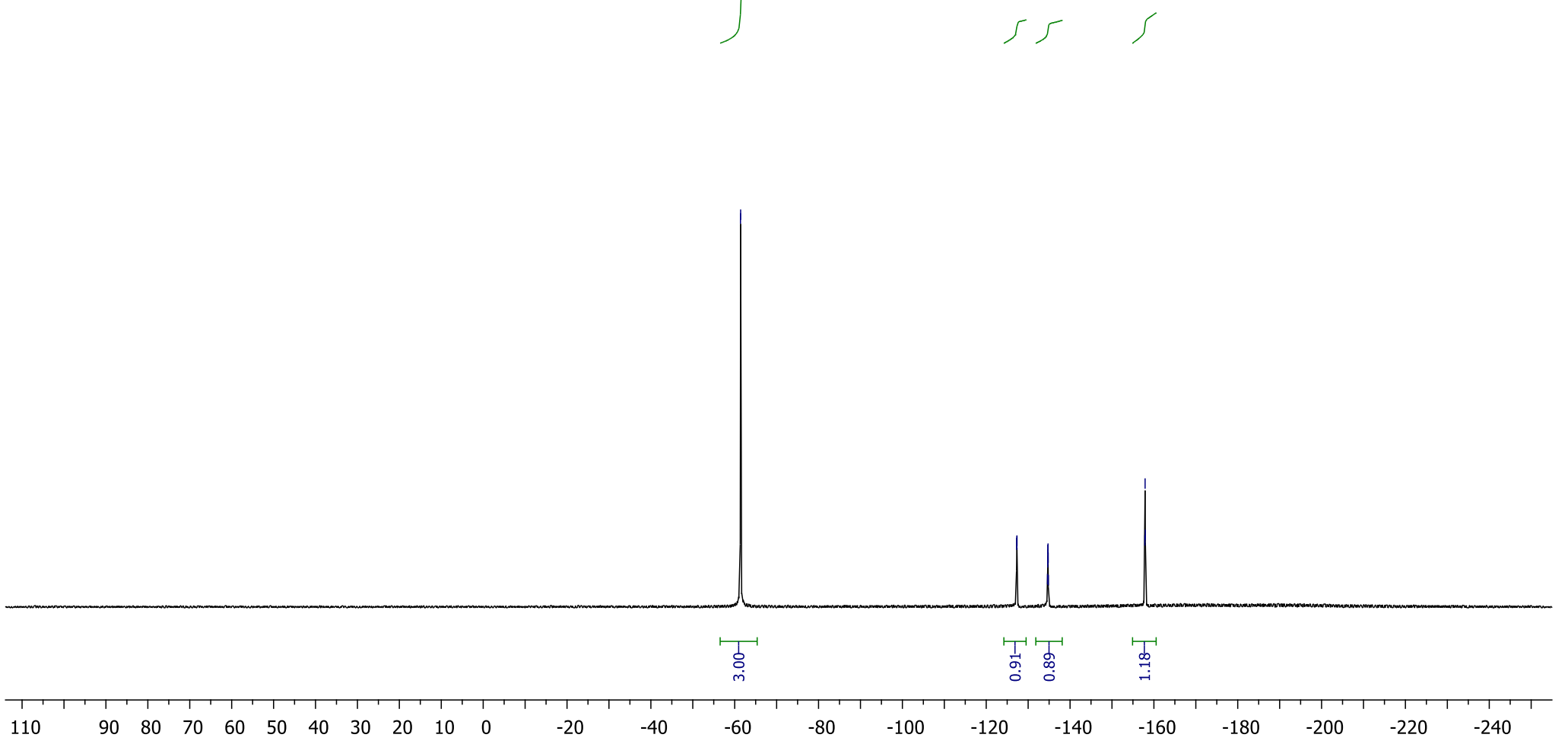
Compound $9 \mathrm{a}^{*}$

${ }^{1} \mathrm{H} \mathrm{NMR}\left(400 \mathrm{MHz}, \mathrm{CDCl}_{3}\right)$

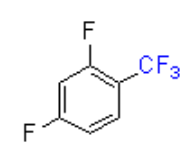

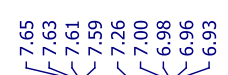

तो
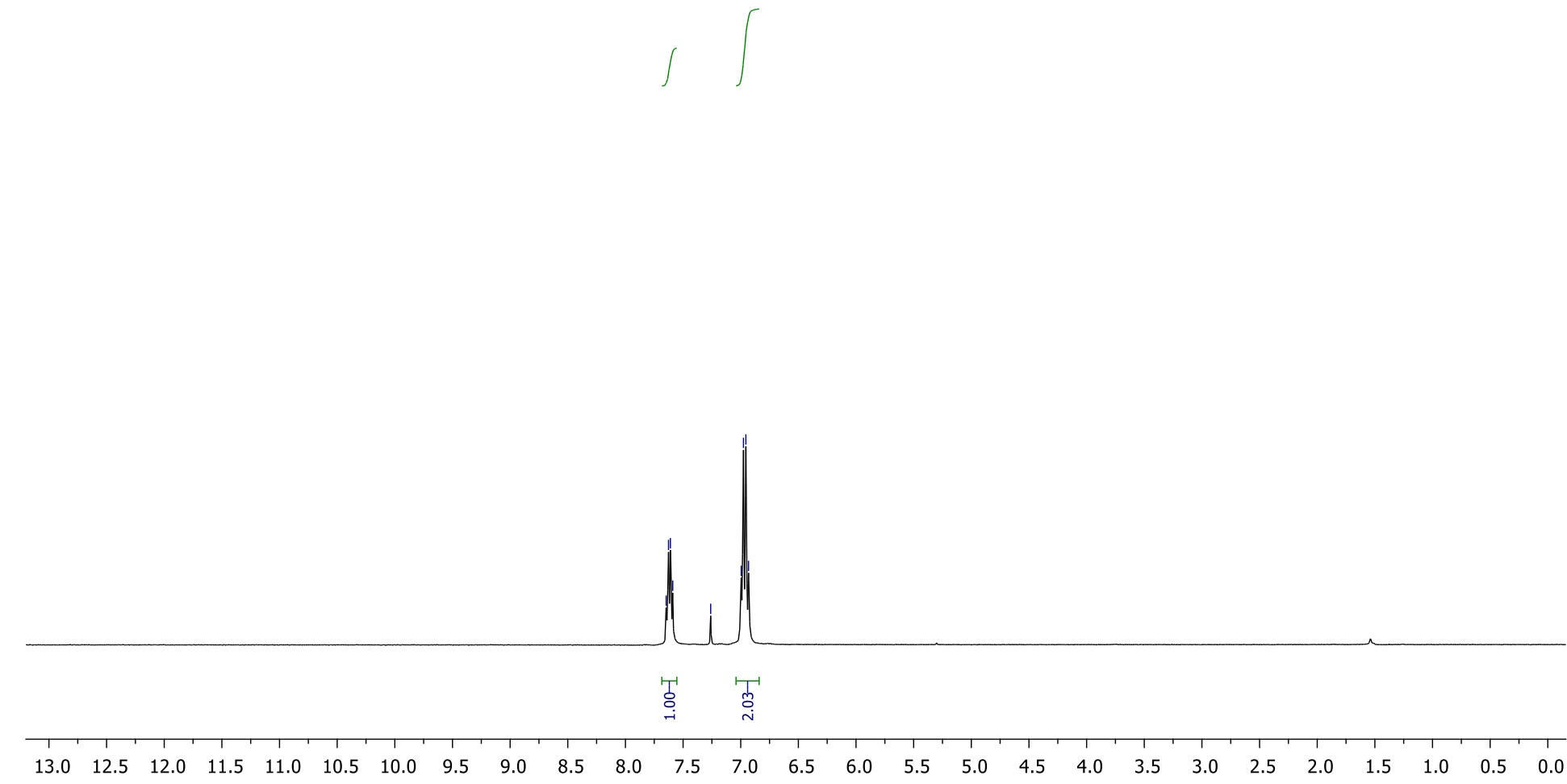

Compound 9a is also described in: Angew. Chem. Int. Ed. 2012, 51, 31, $7767-7770$. 
${ }^{13} \mathrm{C}\left\{{ }^{1} \mathrm{H}\right\}$ NMR $\left(126 \mathrm{MHz}, \mathrm{CDCl}_{3}\right)$
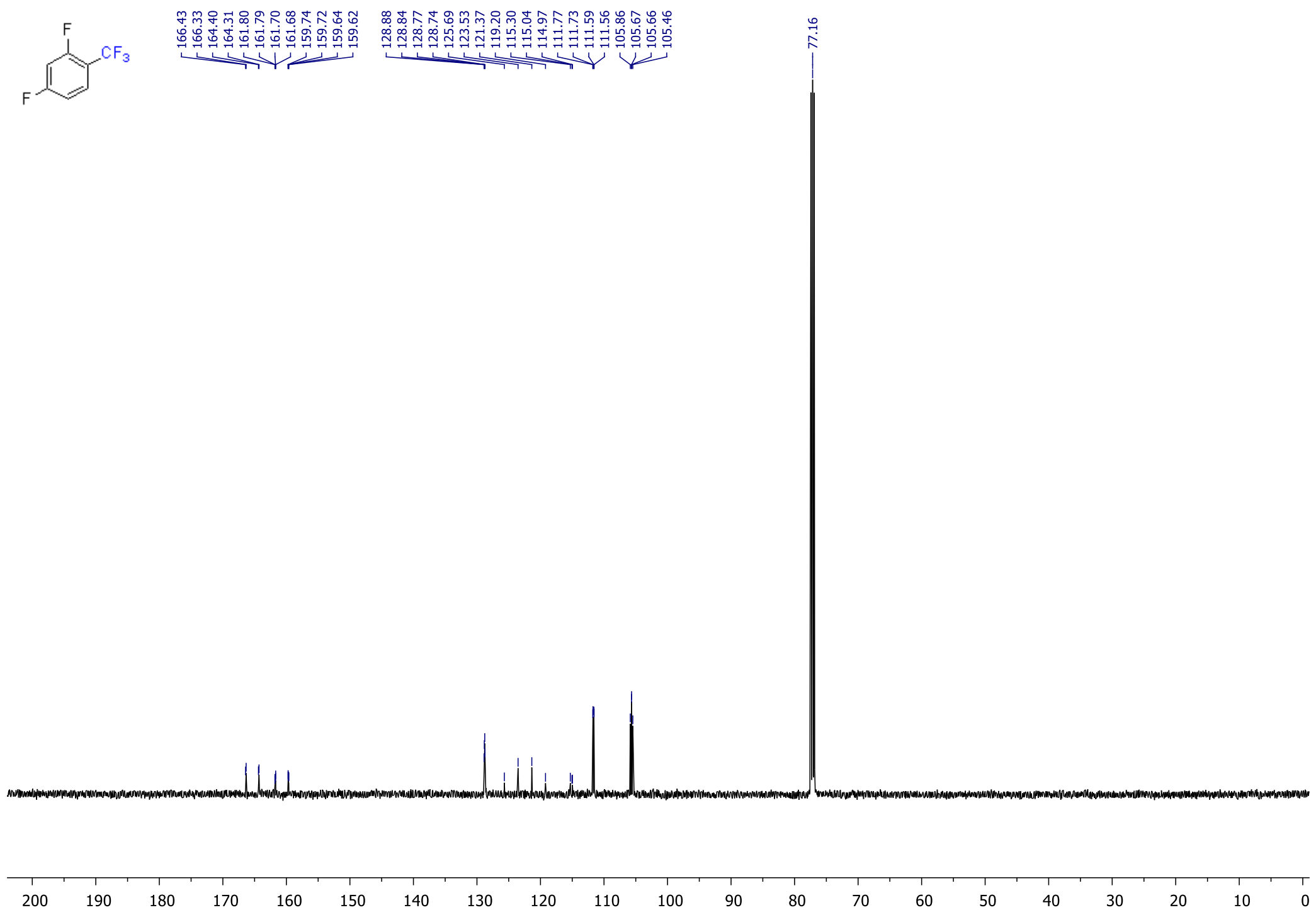
${ }^{19} \mathrm{~F}$ NMR (376 MHz, $\mathrm{CDCl}_{3}$ )
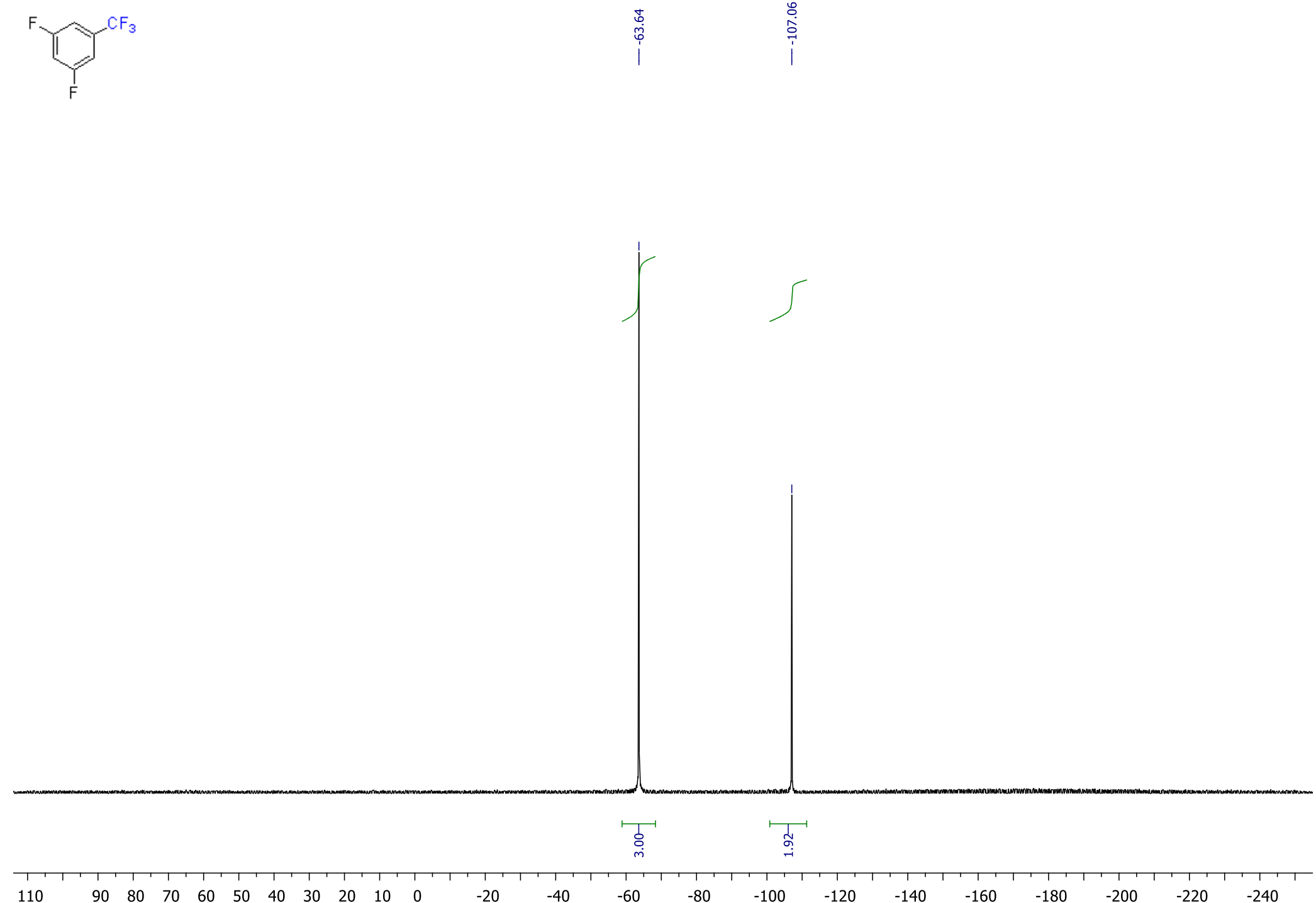
Compound 10a

${ }^{1} \mathrm{H}$ NMR (400 MHz, $\mathrm{CDCl}_{3}$ )

CFe

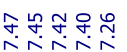

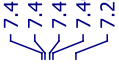

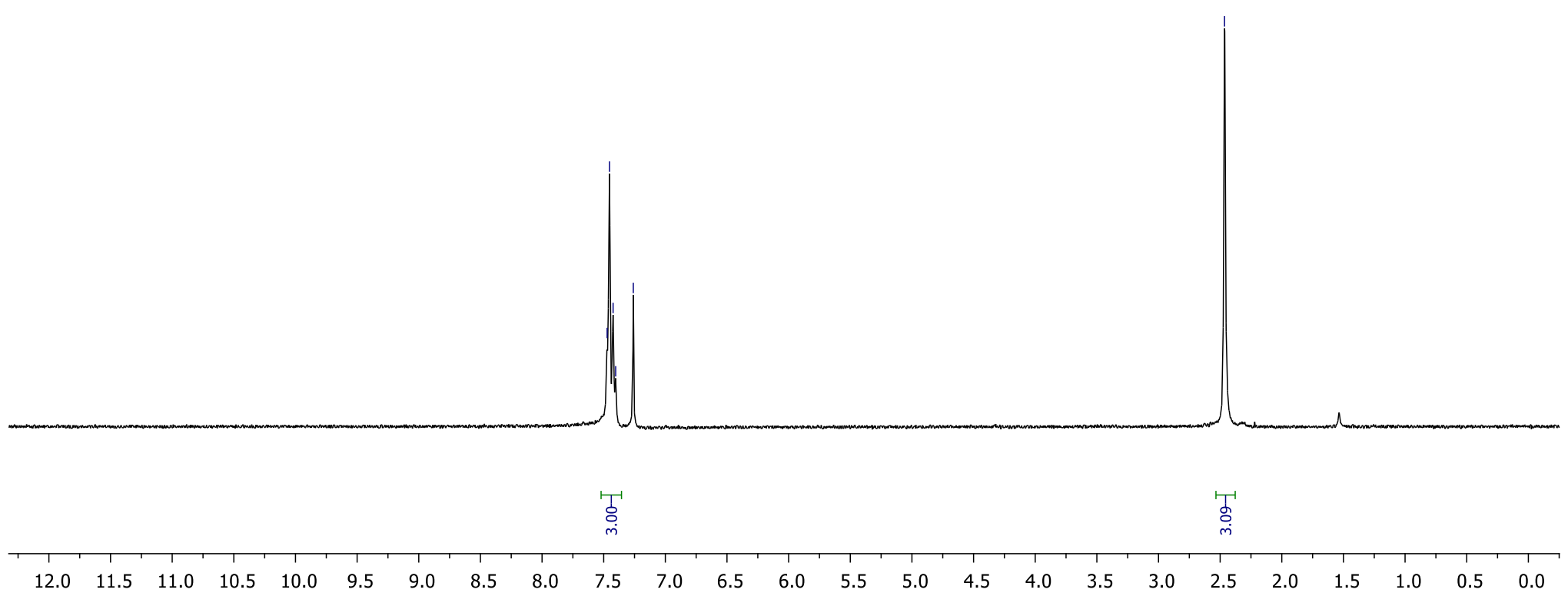


${ }^{13} \mathrm{C}\left\{{ }^{1} \mathrm{H}\right\}$ NMR $\left(126 \mathrm{MHz}, \mathrm{CDCl}_{3}\right)$

$\overbrace{}^{\mathrm{MeF}}$

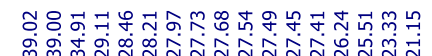

说

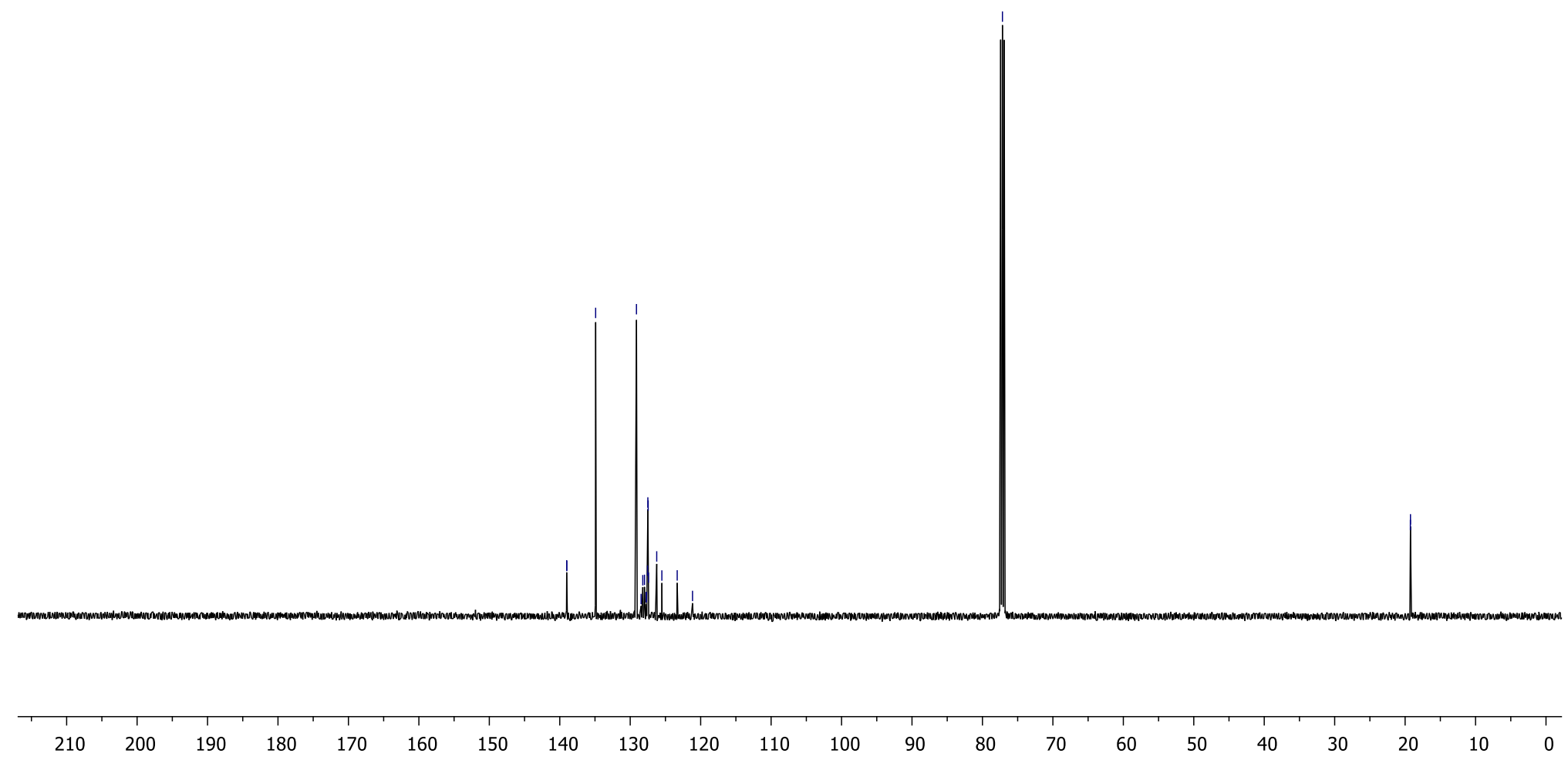


${ }^{19} \mathrm{~F}$ NMR (376 MHz, $\left.\mathrm{CDCl}_{3}\right)$

(Fe

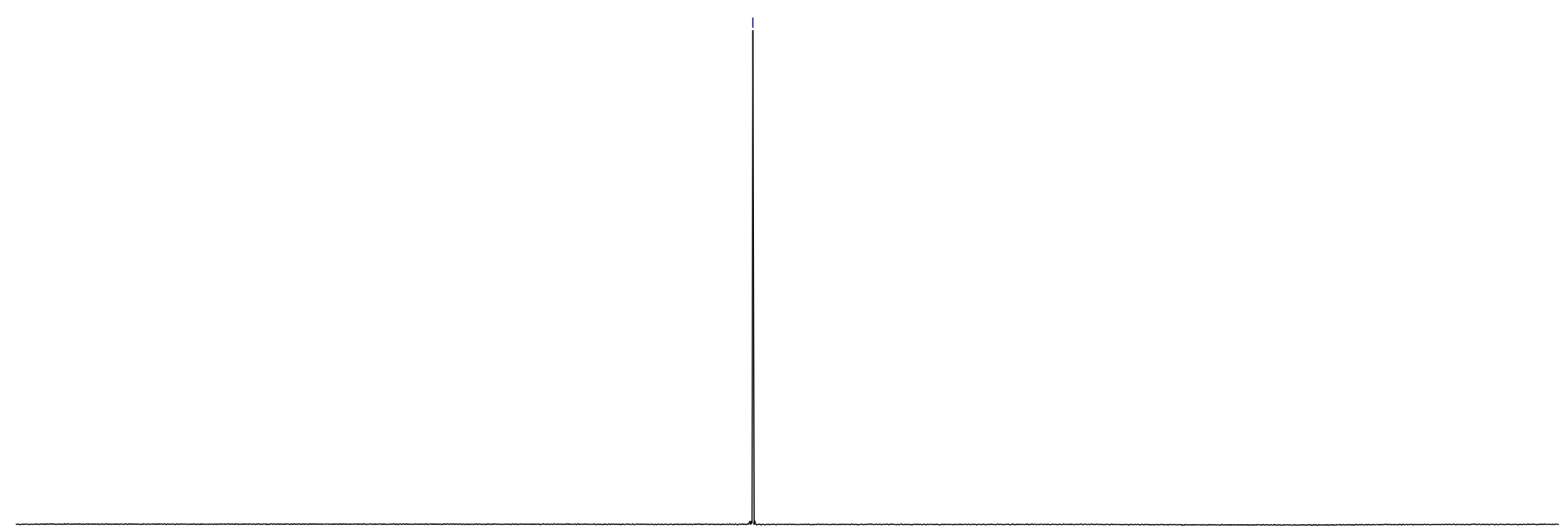

$\begin{array}{lllllllllll}110 & 90 & 80 & 70 & 60 & 50 & 40 & 30 & 20 & 10 & 0\end{array}$

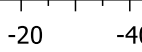

$-40 \quad-60$ $-80$ 
Compound 11a

${ }^{1} \mathrm{H}$ NMR (400 MHz, $\mathrm{CDCl}_{3}$ )

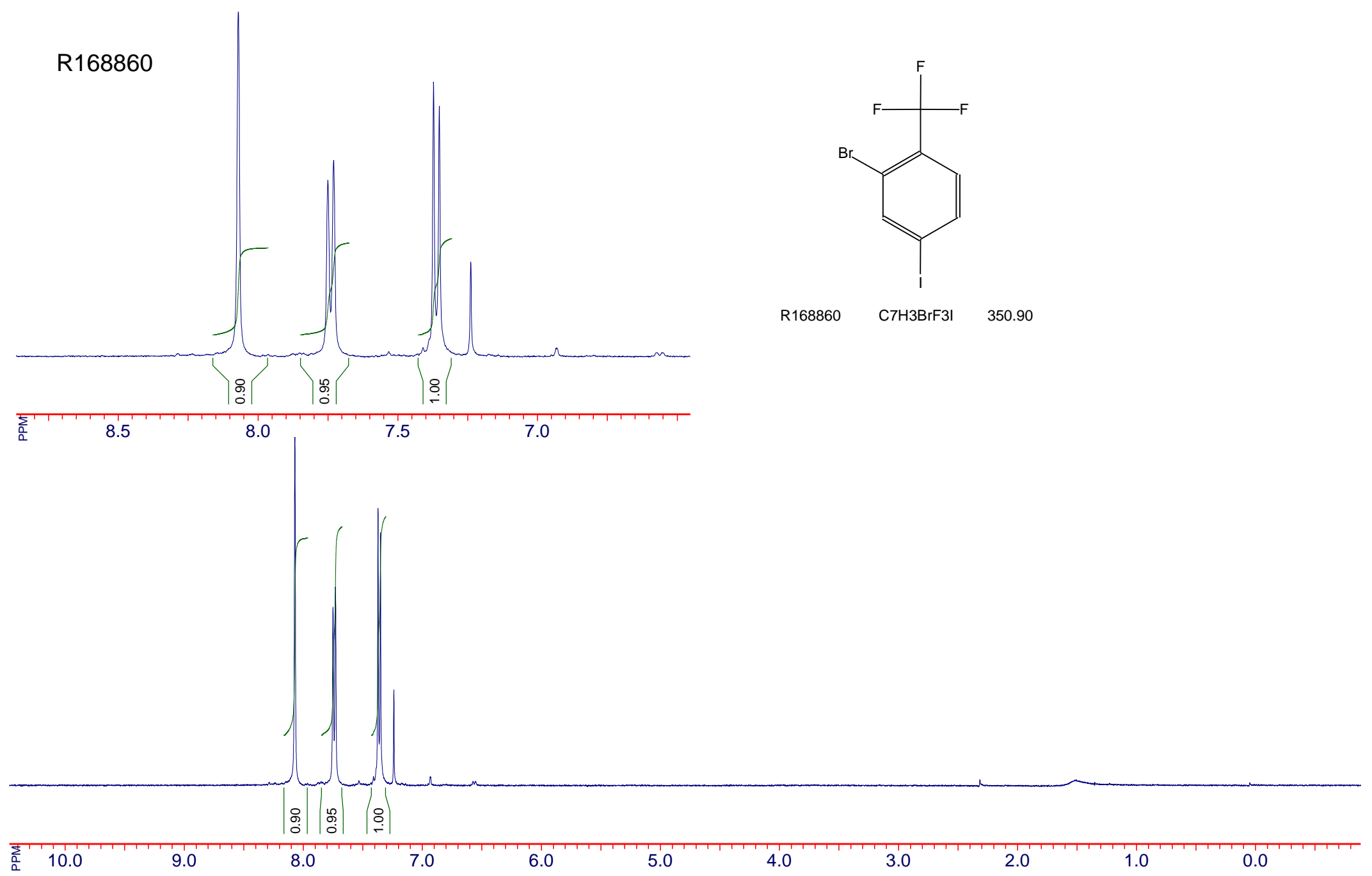


${ }^{13} \mathrm{C}\left\{{ }^{1} \mathrm{H}\right\}$ NMR $\left(126 \mathrm{MHz}, \mathrm{CDCl}_{3}\right)$

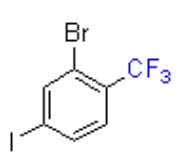

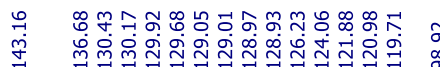

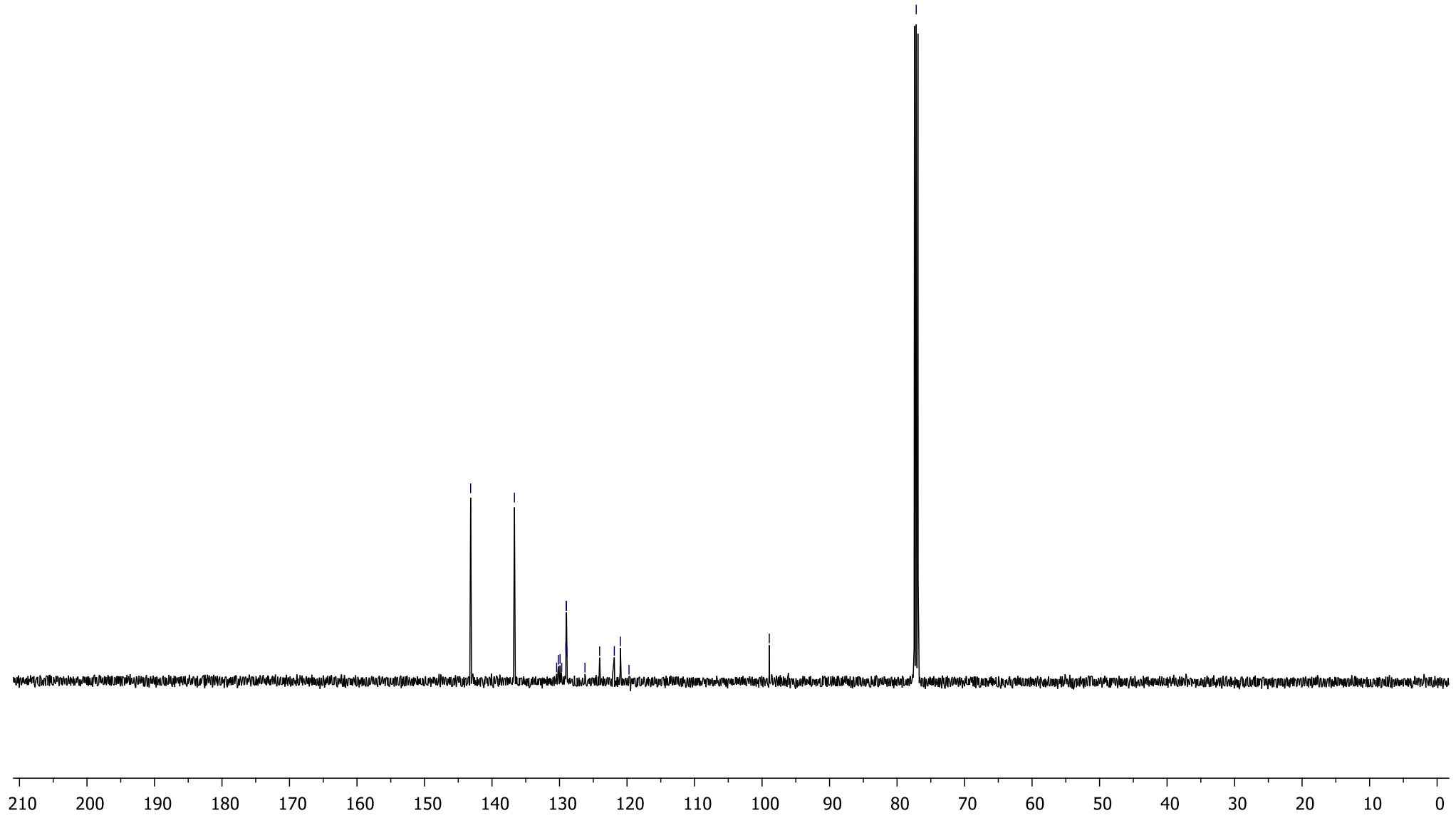


${ }^{19} \mathrm{~F}$ NMR $\left(376 \mathrm{MHz}, \mathrm{CDCl}_{3}\right)$

$\overbrace{1}^{\mathrm{Br}} \mathrm{CF}_{3}$

$\underset{\substack{F \\ \dot{b}}}{i}$

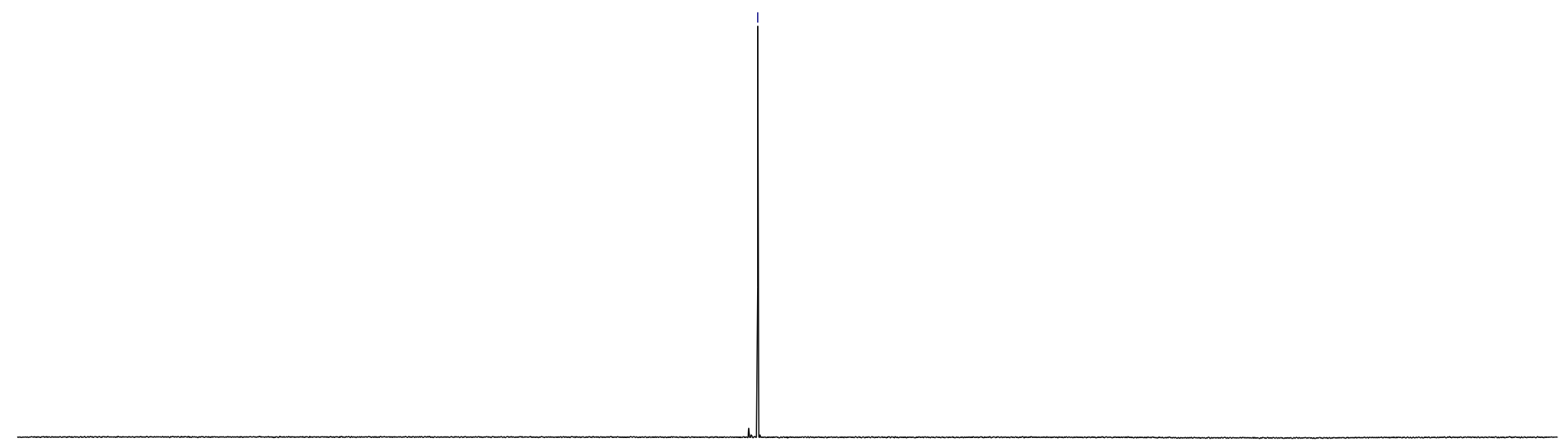

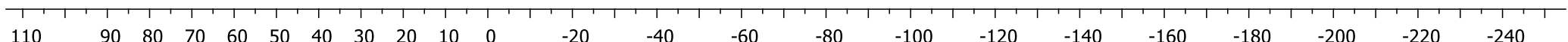


Compound 12a

${ }^{1} \mathrm{H}$ NMR (400 MHz, $\mathrm{CDCl}_{3}$ )

$\overbrace{}^{c 1}$

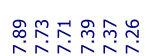

IN

$\iint$

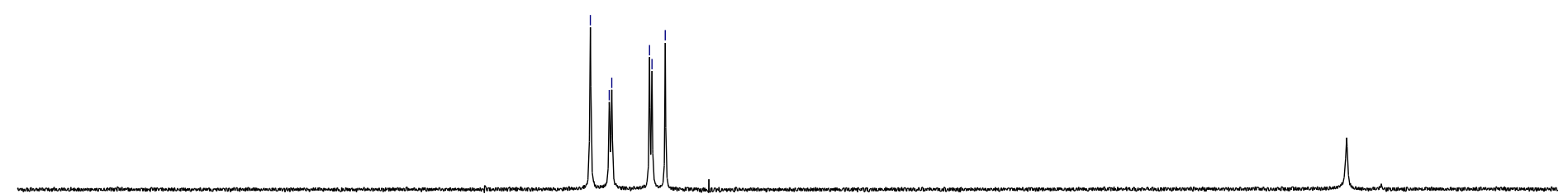

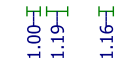

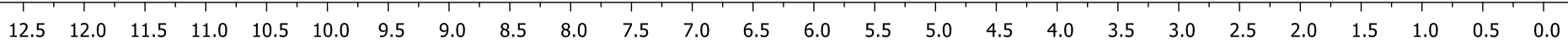


${ }^{13} \mathrm{C}\left\{{ }^{1} \mathrm{H}\right\}$ NMR $\left(126 \mathrm{MHz}, \mathrm{CDCl}_{3}\right)$
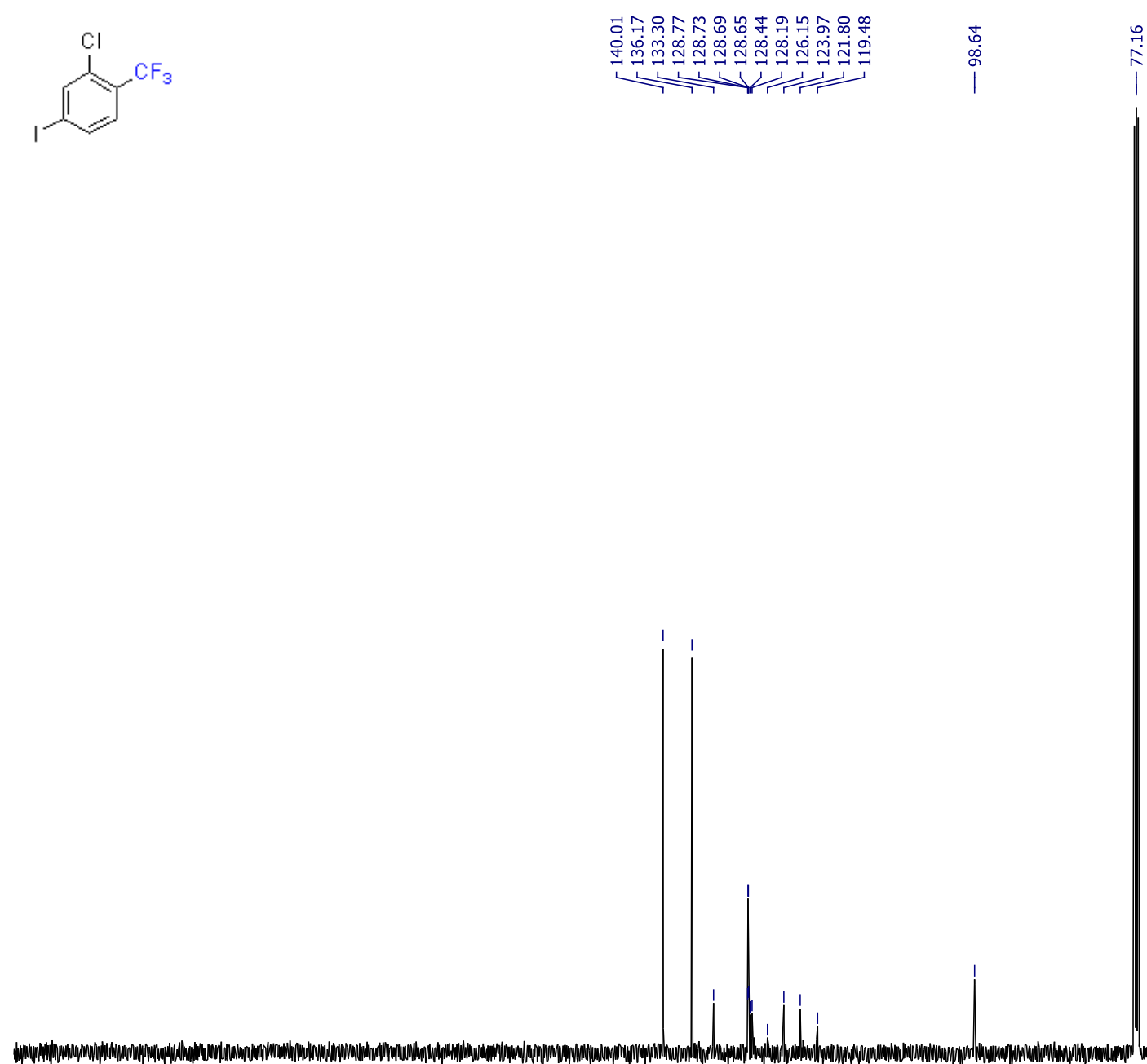

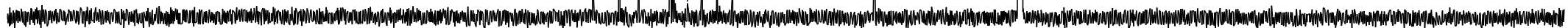

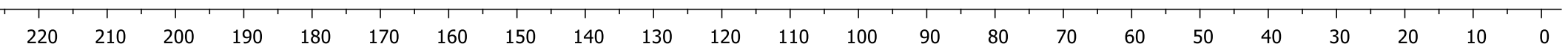


${ }^{19} \mathrm{~F}$ NMR $\left(376 \mathrm{MHz}, \mathrm{CDCl}_{3}\right)$

$\overbrace{}^{C l}$

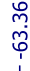

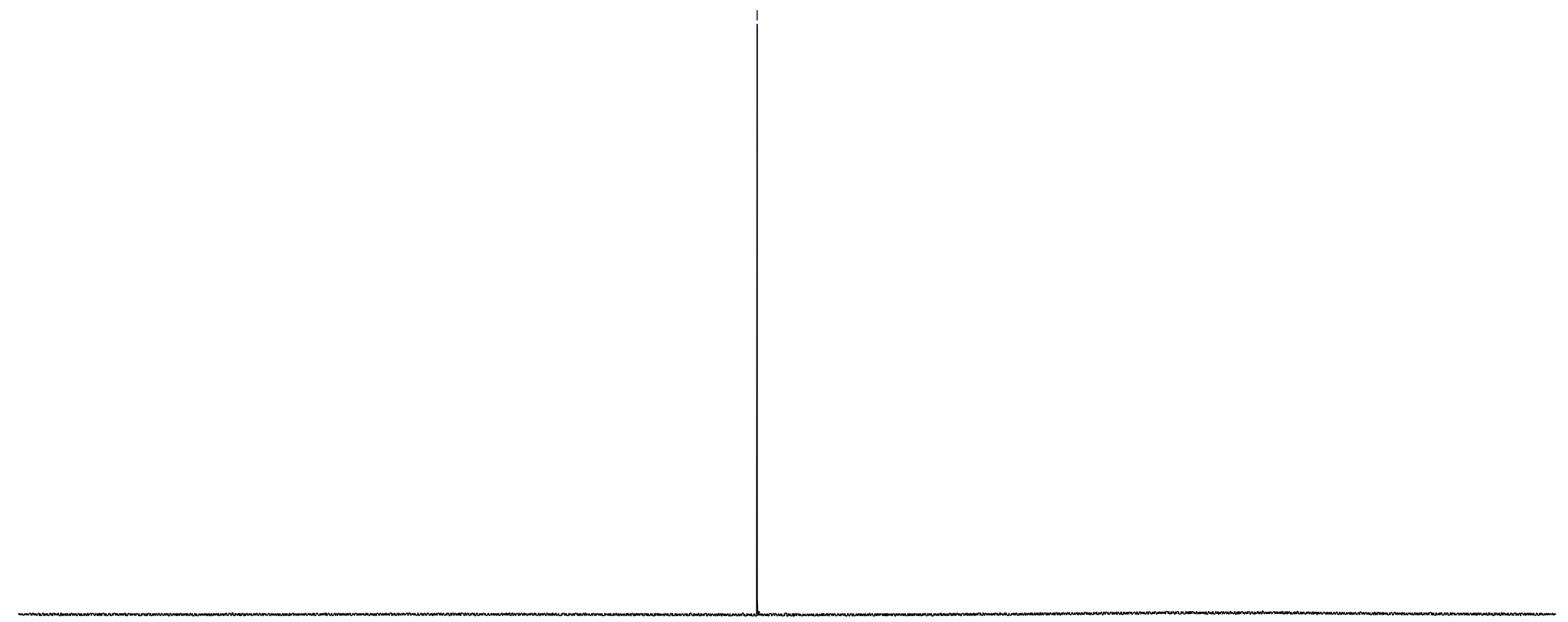

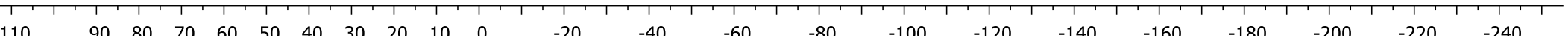


Compound 13a

${ }^{1} \mathrm{H}$ NMR (400 MHz, $\mathrm{CDCl}_{3}$ )

$\mathrm{CF}^{\mathrm{NO}_{3}}$

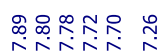

迕们

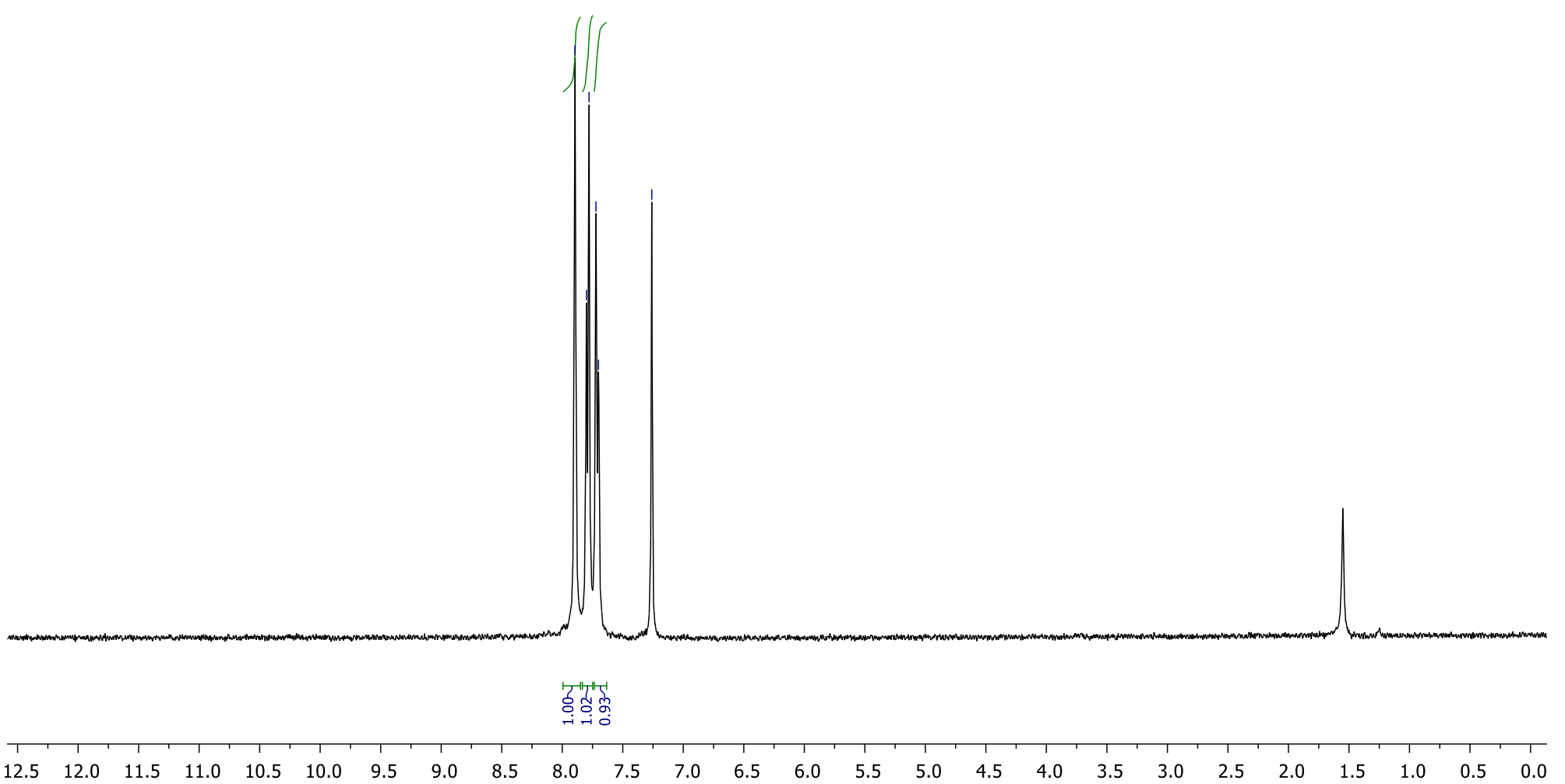


${ }^{13} \mathrm{C}\left\{{ }^{1} \mathrm{H}\right\}$ NMR (101 MHz, DMSO-d 6 )

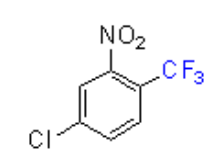

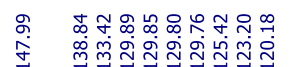

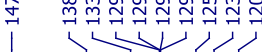

||||$_{n}^{\mid} \mid$

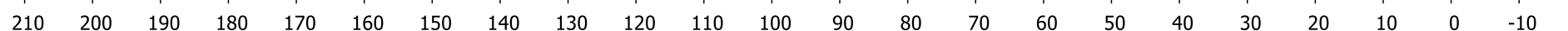


${ }^{19}$ F NMR (376 MHz, DMSO-d6)

$\mathrm{CF}_{3}$

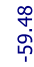

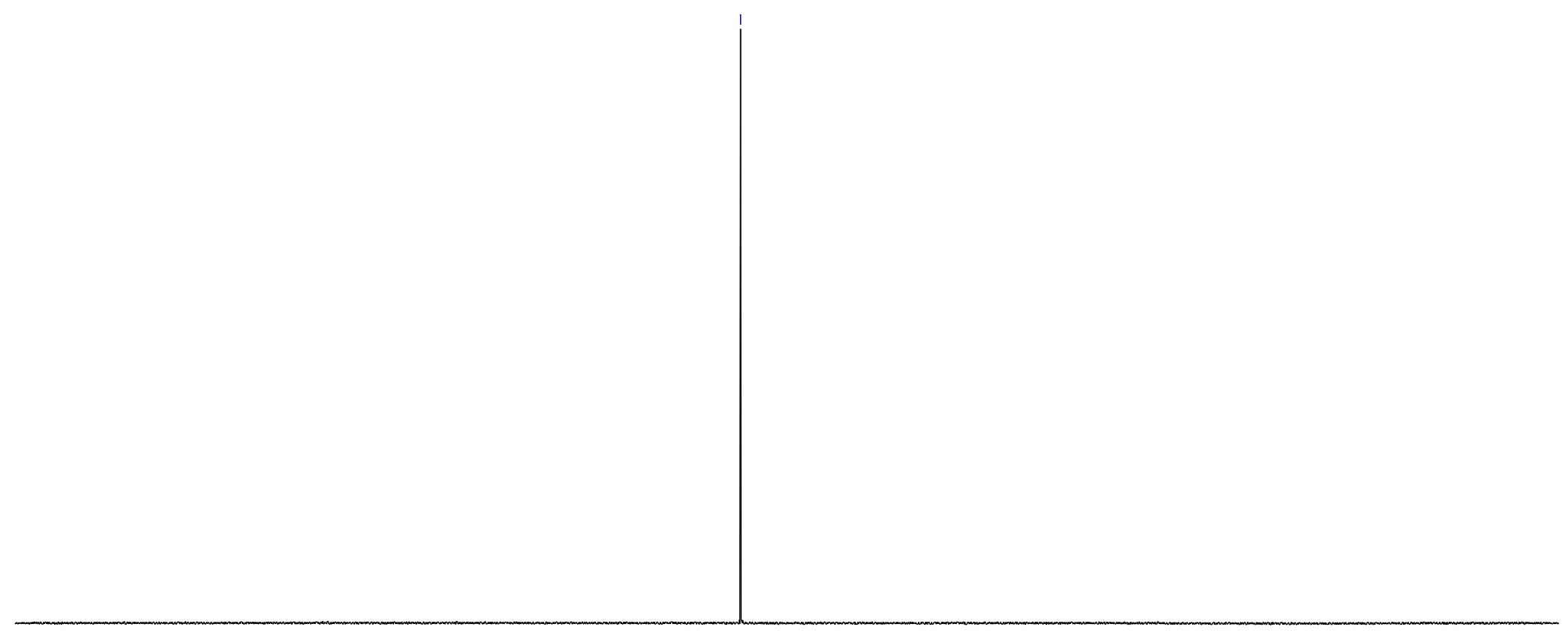

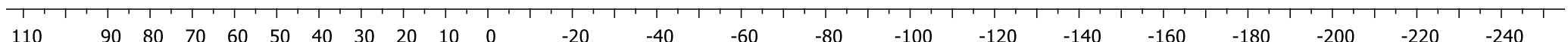


Compound 14a

${ }^{1} \mathrm{H}$ NMR $\left(400 \mathrm{MHz}, \mathrm{CDCl}_{3}\right)$

ind

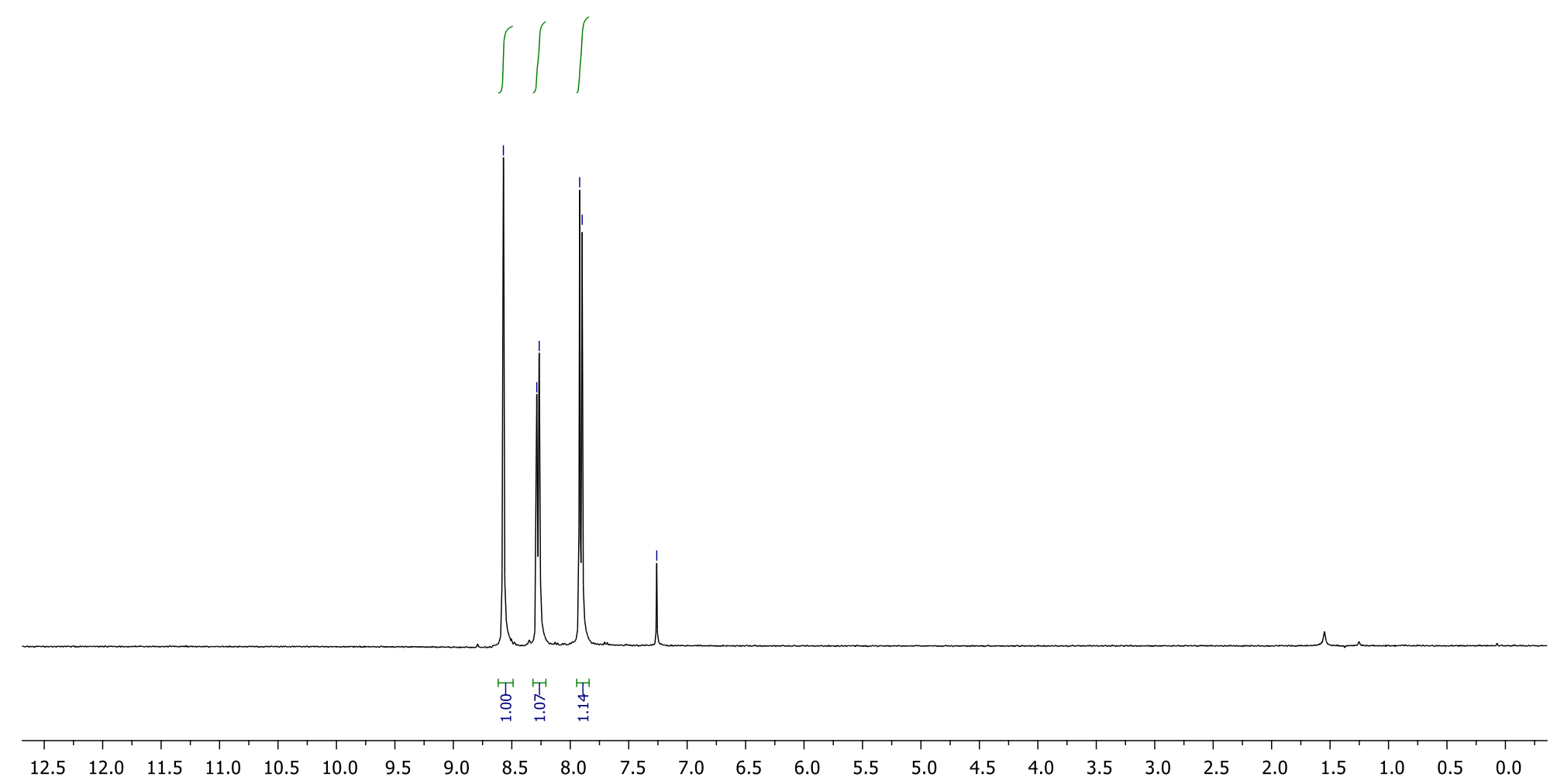


${ }^{13} \mathrm{C}\left\{{ }^{1} \mathrm{H}\right\}$ NMR $\left(126 \mathrm{MHz}, \mathrm{CDCl}_{3}\right)$

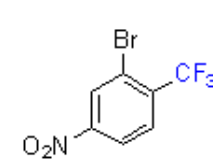

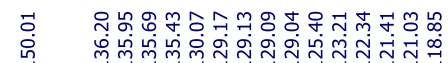

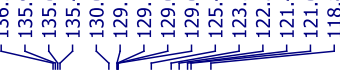

$\mathrm{O}_{2} \mathrm{~N}$

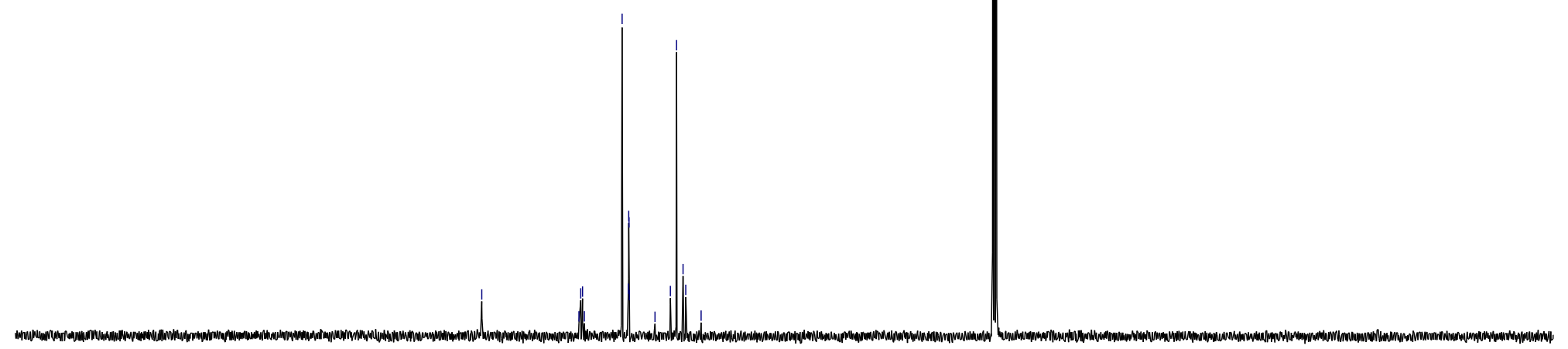

$\begin{array}{lllll}210 \quad 200 \quad 190 \quad 180 & 170\end{array}$ 
${ }^{19} \mathrm{~F}$ NMR $\left(376 \mathrm{MHz}, \mathrm{CDCl}_{3}\right)$

$\mathrm{O}_{2 \mathrm{~N}}^{\mathrm{Br} \mathrm{F}_{3}}$

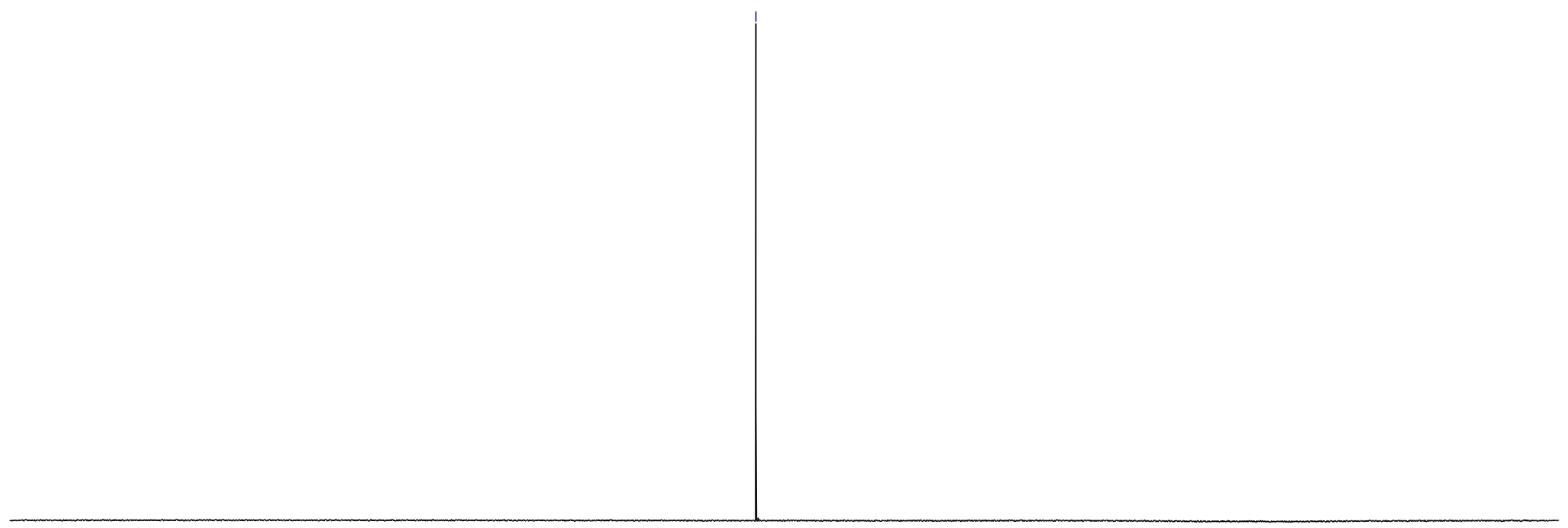

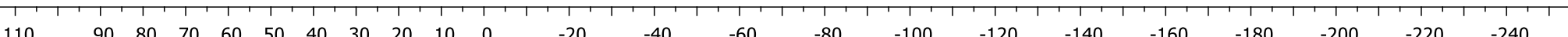


Compound 15a

${ }^{1} \mathrm{H}$ NMR (400 MHz, $\mathrm{CDCl}_{3}$ )

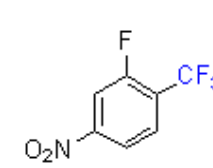

|

पर

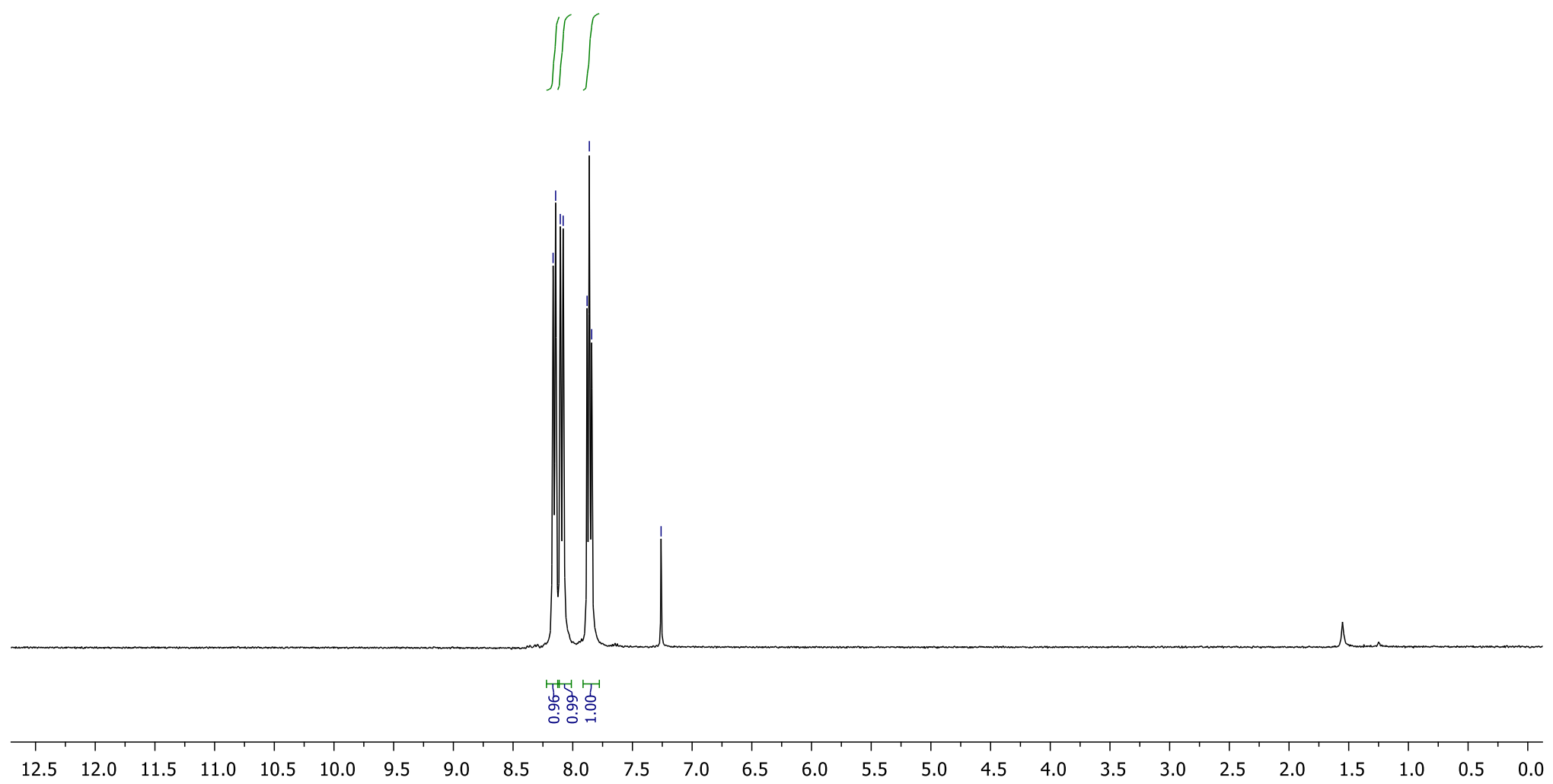




$$
I
$$


${ }^{19} \mathrm{~F}$ NMR (376 MHz, $\mathrm{CDCl}_{3}$ )
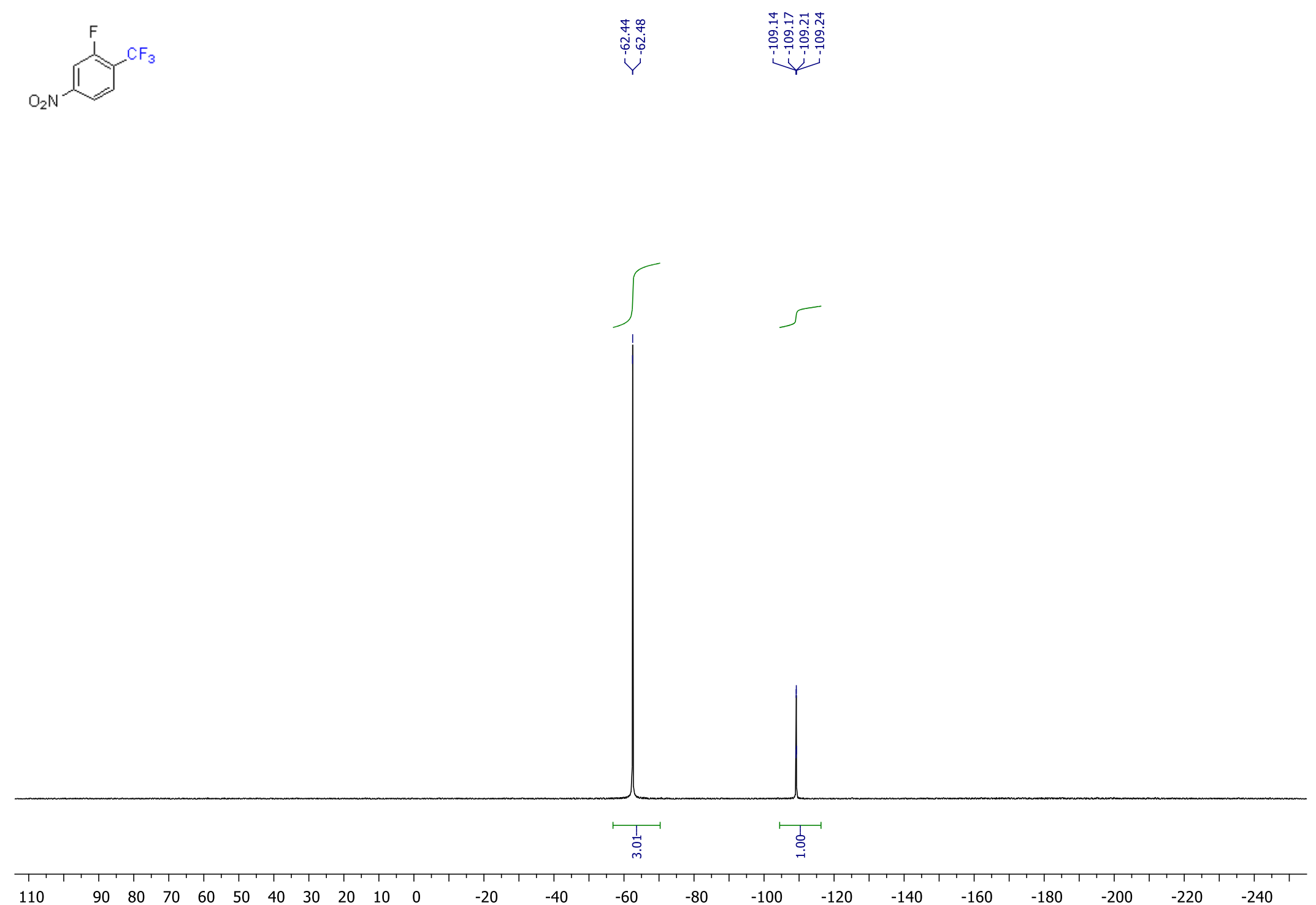
Compound 16a*

${ }^{1} \mathrm{H} \mathrm{NMR}\left(400 \mathrm{MHz}, \mathrm{CDCl}_{3}\right)$

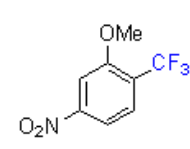

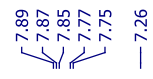

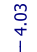

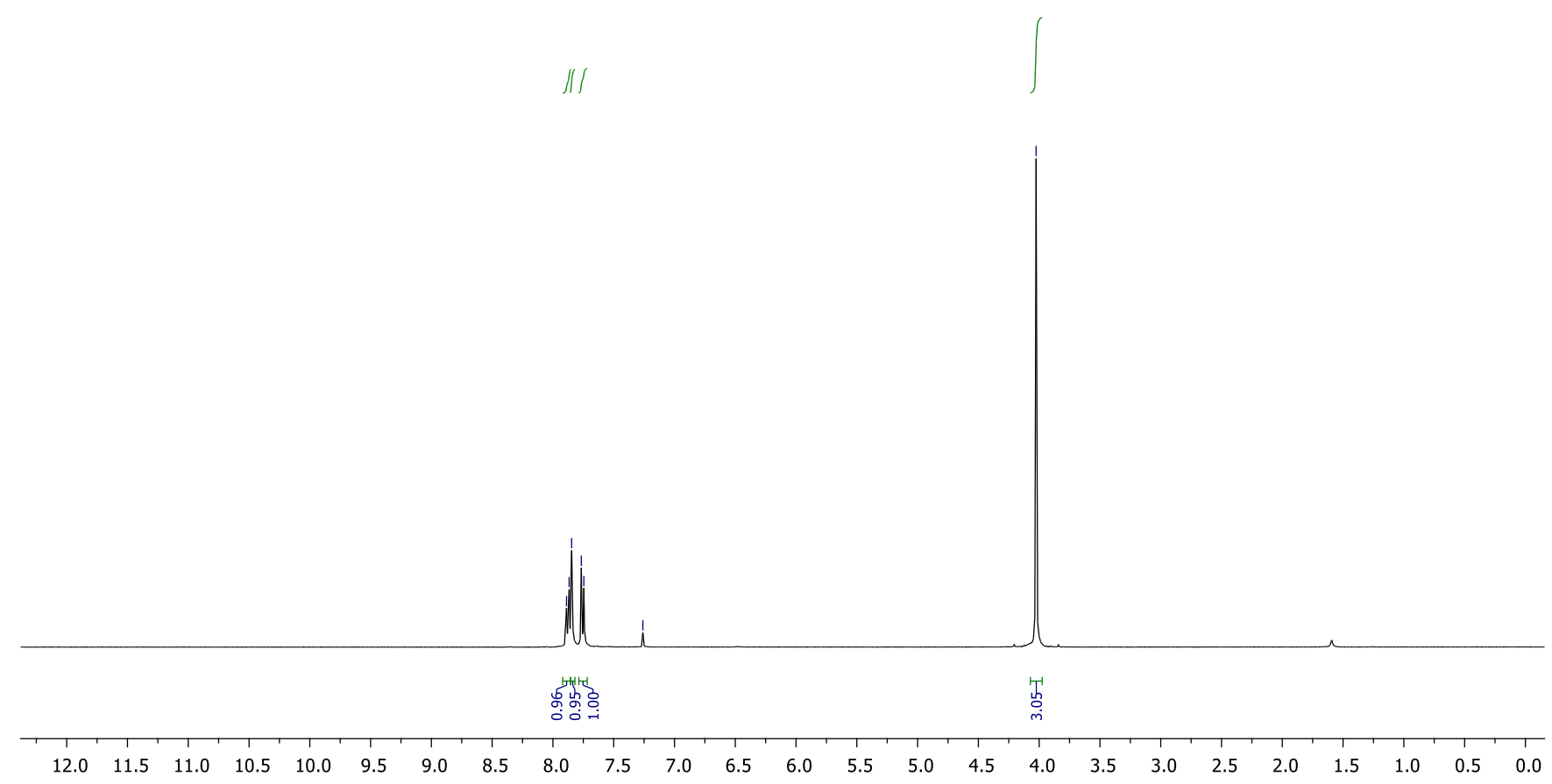

Compound 16a is also described in: a) Chem. Commun. 2014, 50, 10237-10240; b) J. Med. Chem. 2007, 50, 18, 4351-4373; c) Synthesis 2014, 46, 16, 21432148; d) J. Am. Chem. Soc. 2013, 135, 28, 10330-10333; e) J. Med. Chem. 2014, 57, 5, 1914-1931. 
${ }^{13} \mathrm{C}\left\{{ }^{1} \mathrm{H}\right\}$ NMR $\left(126 \mathrm{MHz}, \mathrm{CDCl}_{3}\right)$
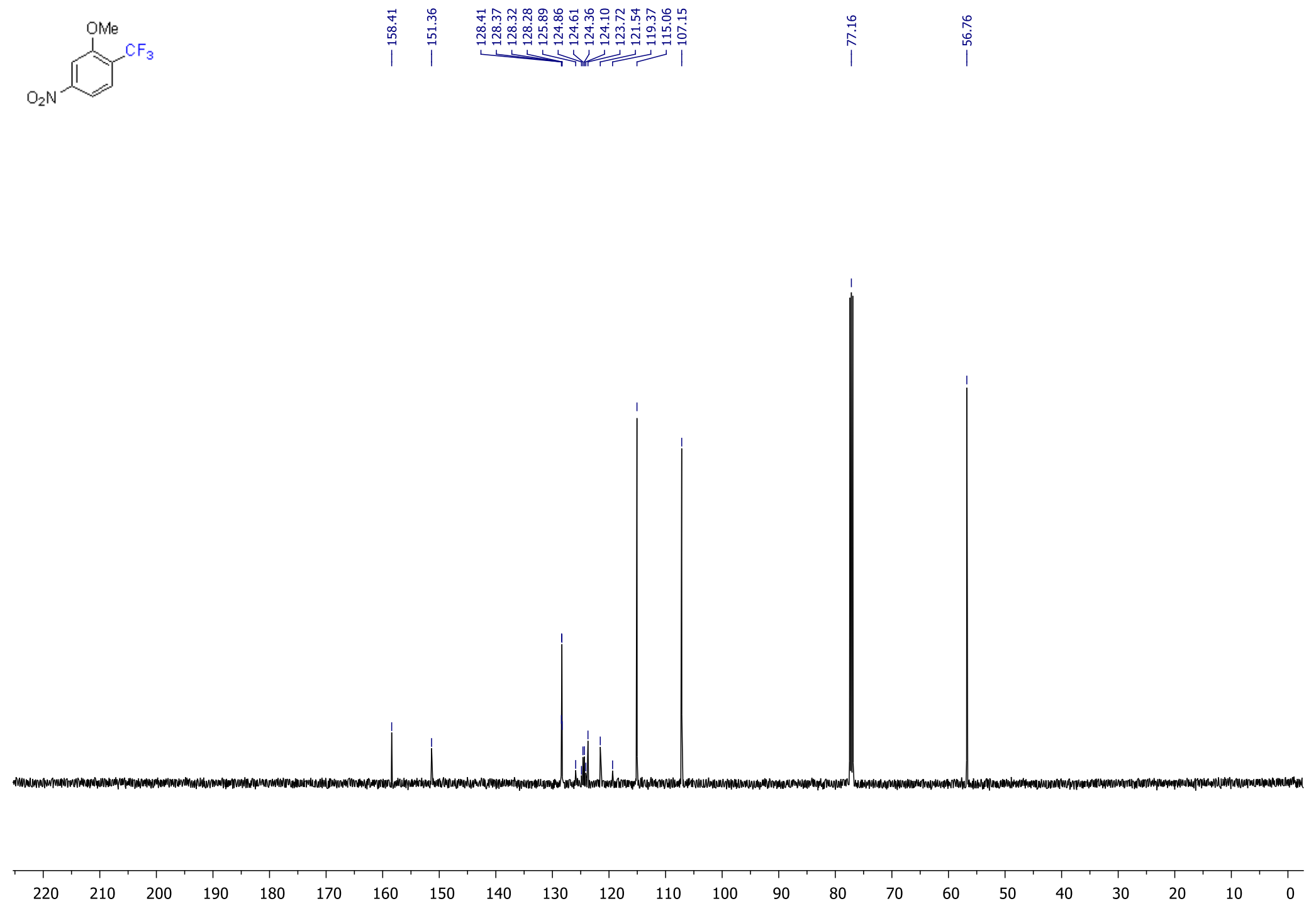
${ }^{19} \mathrm{~F}$ NMR (376 MHz, $\mathrm{CDCl}_{3}$ )

(N)

$\stackrel{\substack{0 \\ 0}}{\substack{i \\ i}}$
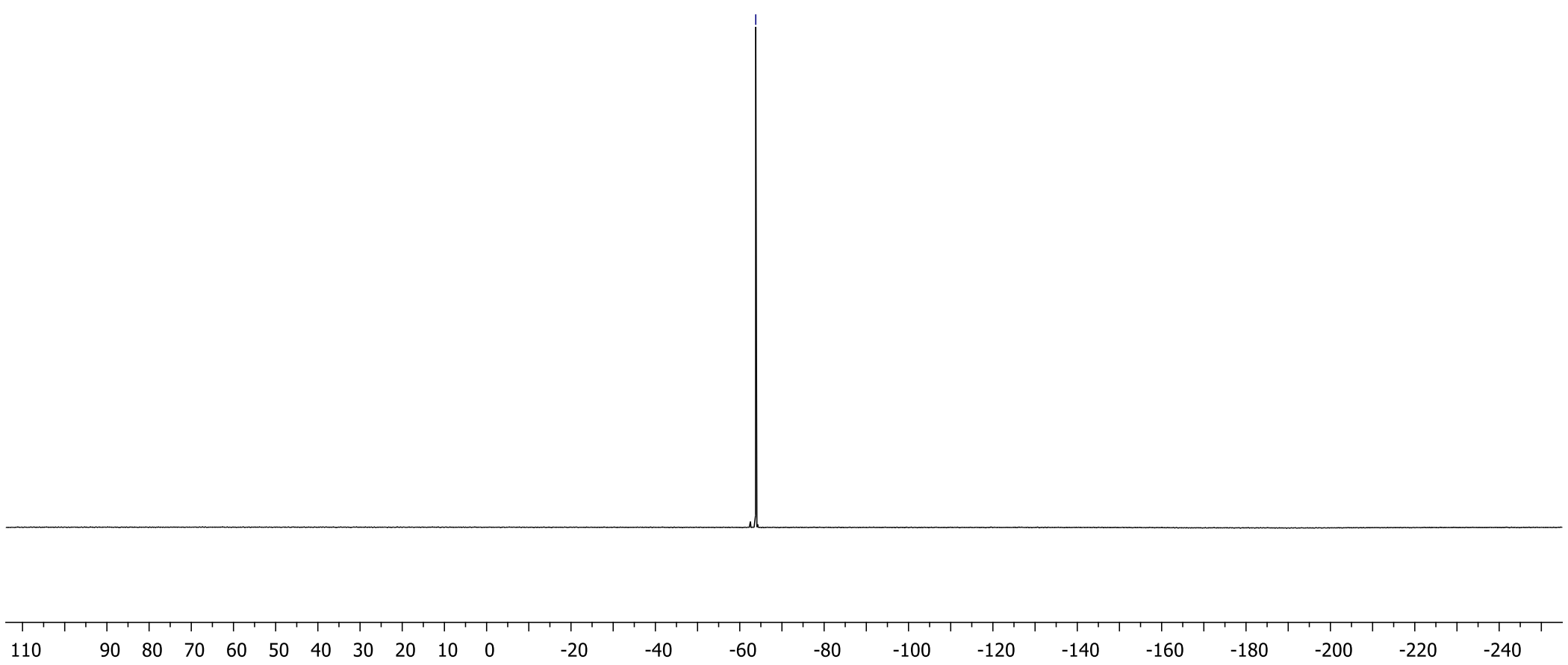
Compound 17a

${ }^{1} \mathrm{H}$ NMR (400 MHz, $\mathrm{CDCl}_{3}$ )

$\overbrace{}^{\mathrm{NO}_{2}}$

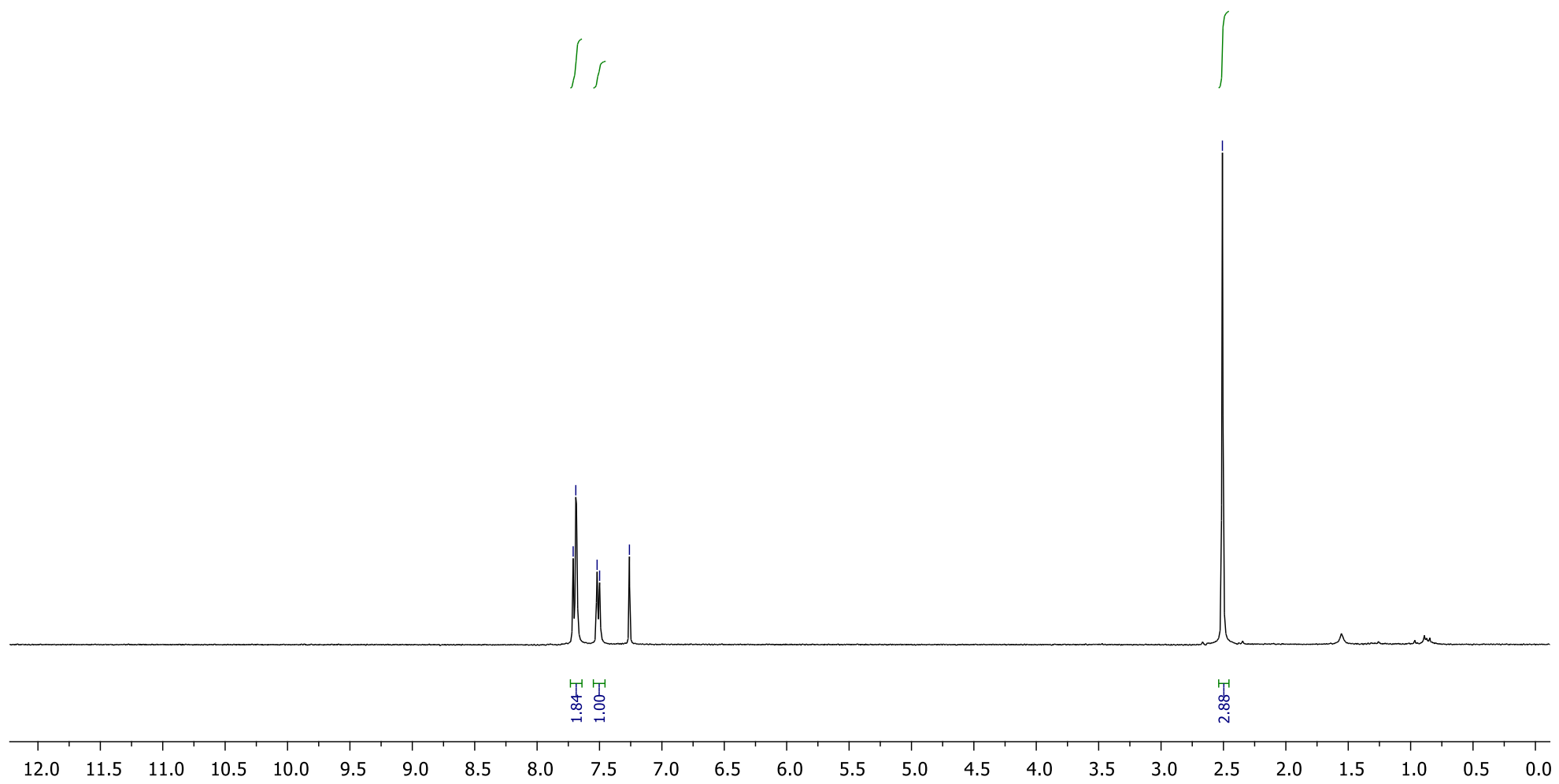


${ }^{13} \mathrm{C}\left\{{ }^{1} \mathrm{H}\right\}$ NMR $\left(126 \mathrm{MHz}, \mathrm{CDCl}_{3}\right)$

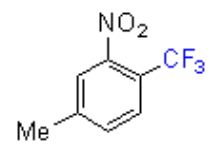

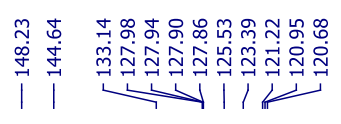

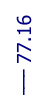

$\stackrel{\sim}{\sim}$

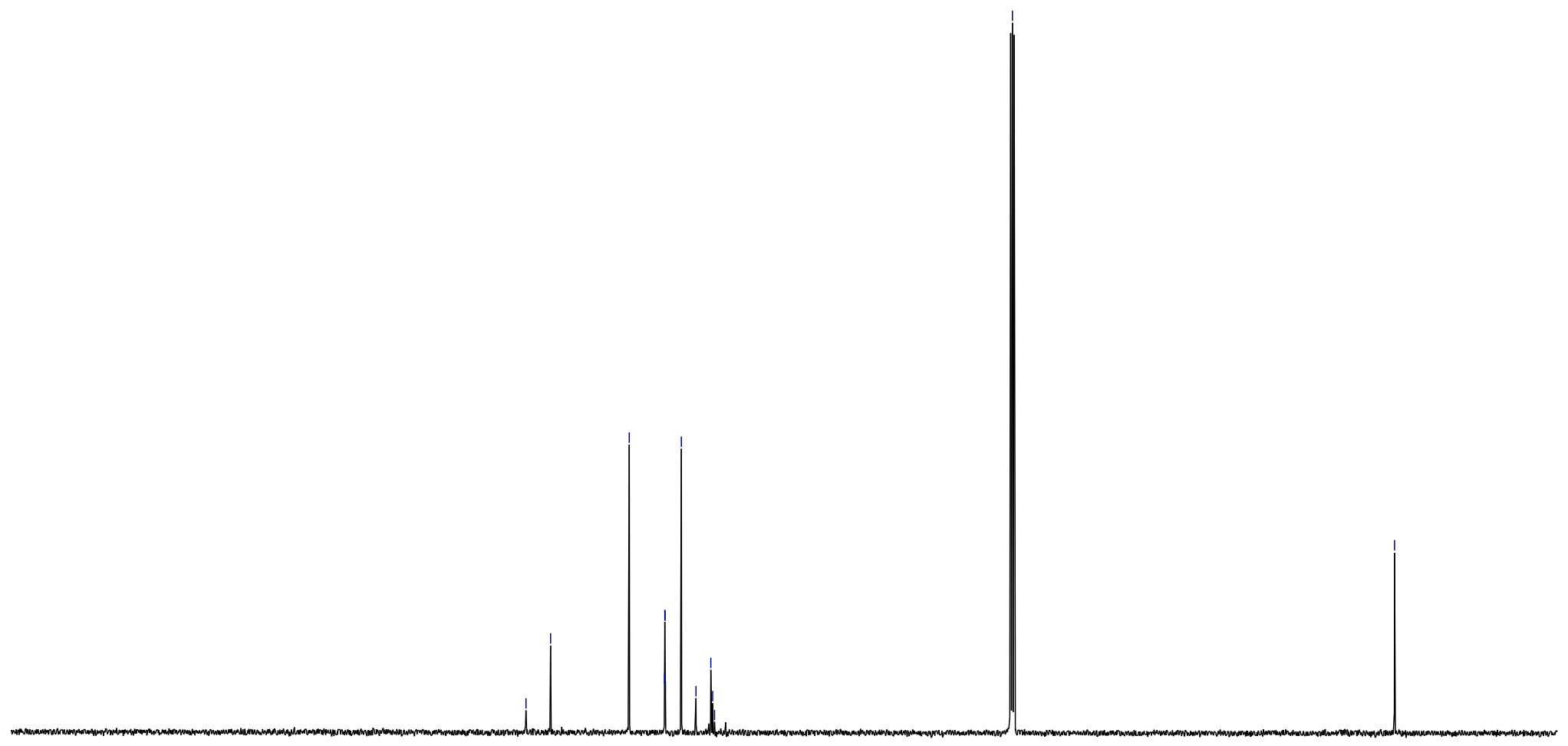

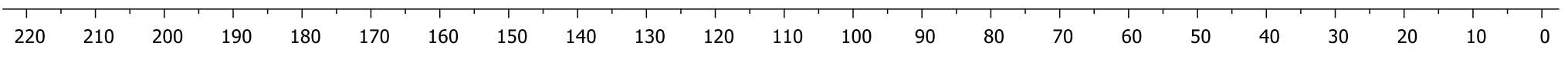

S53 
${ }^{19} \mathrm{~F}$ NMR (376 MHz, $\mathrm{CDCl}_{3}$ )

${ }^{\mathrm{NO}_{2}} \mathrm{CF}_{3}$

$\stackrel{\substack{0 \\ \varphi}}{i}$

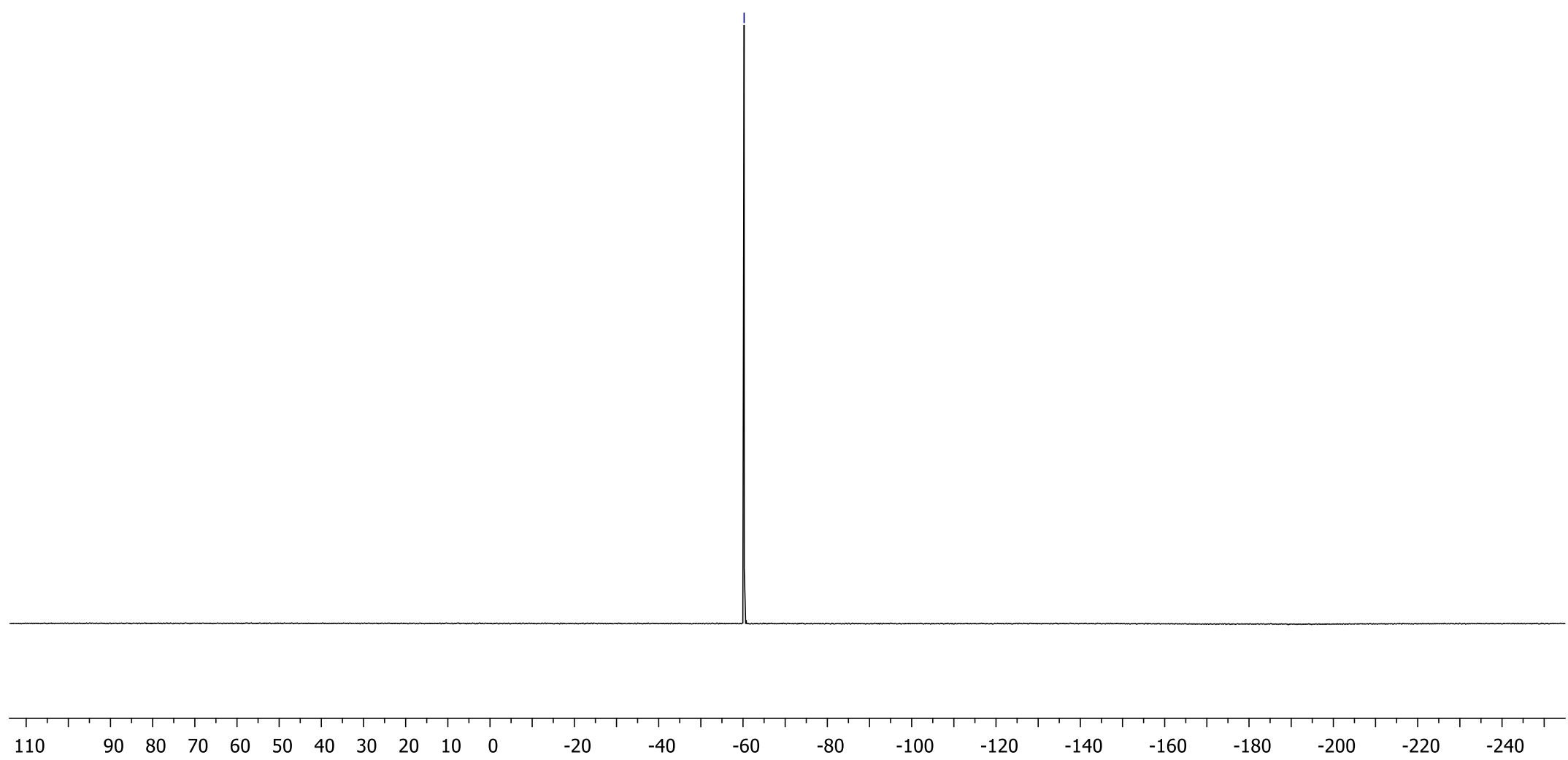


Compound 18a

${ }^{1} \mathrm{H}$ NMR $\left(400 \mathrm{MHz}, \mathrm{CDCl}_{3}\right)$
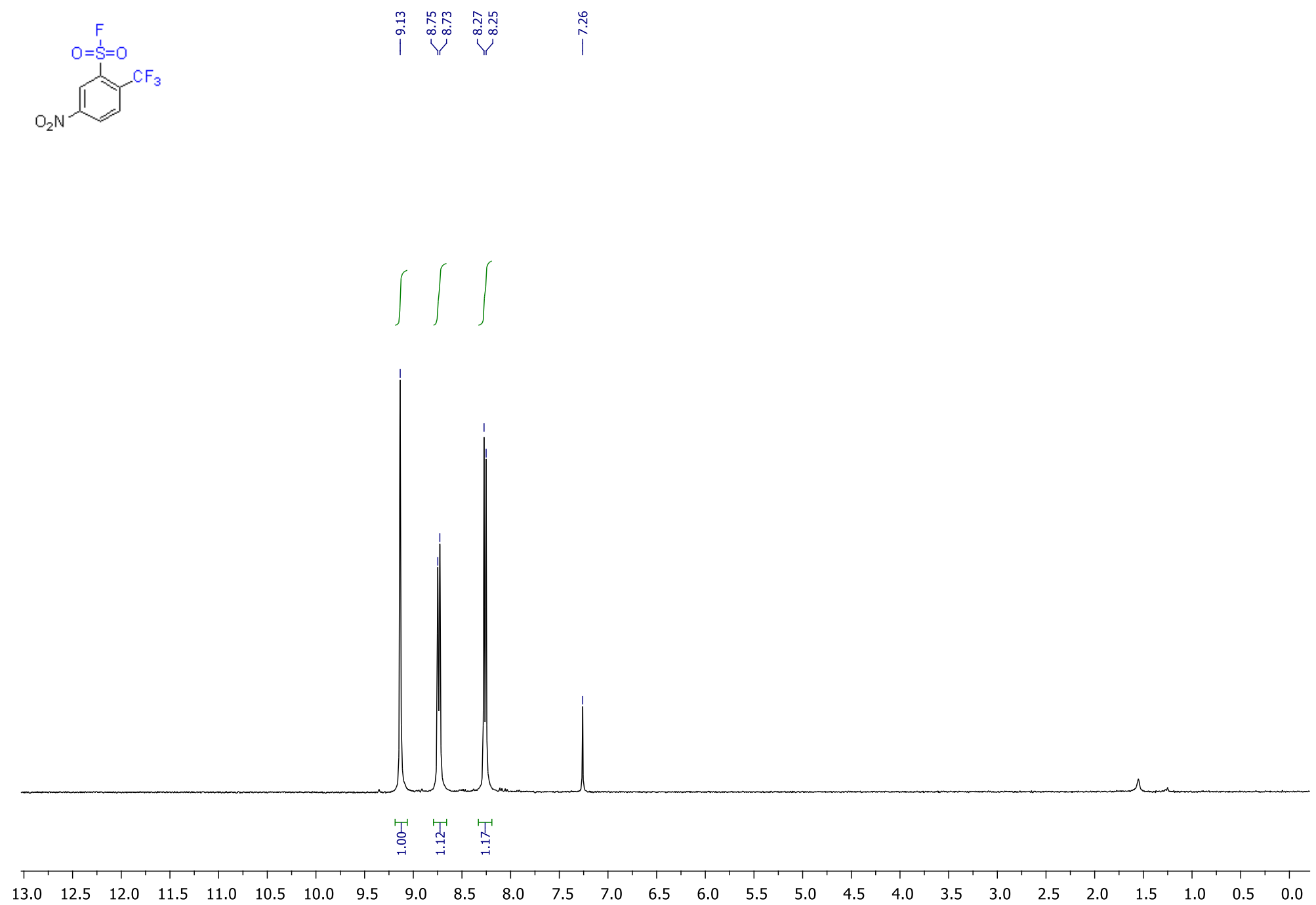
${ }^{13} \mathrm{C}\left\{{ }^{1} \mathrm{H}\right\}$ NMR $\left(126 \mathrm{MHz}, \mathrm{CDCl}_{3}\right)$

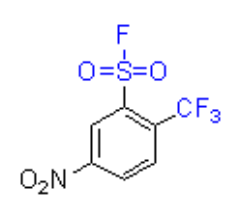

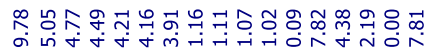

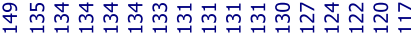

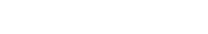

1

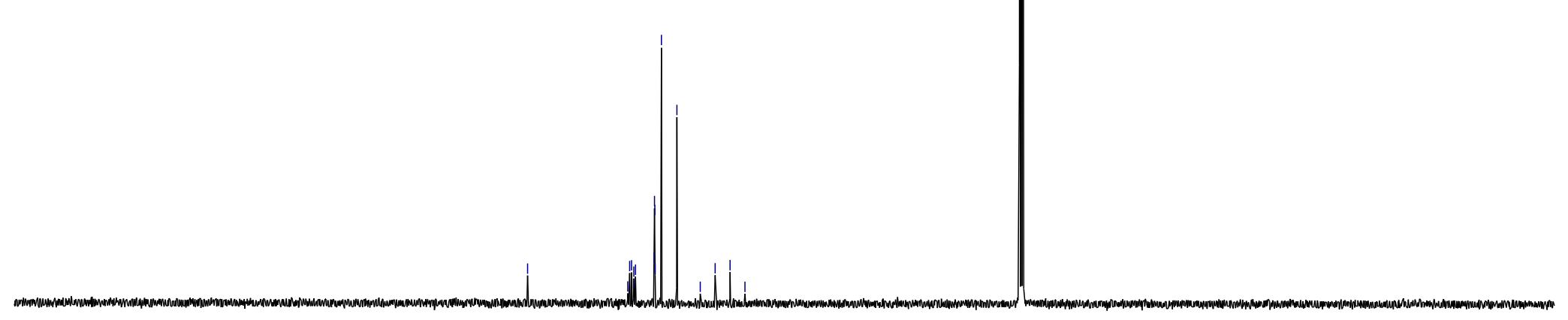


${ }^{19} \mathrm{~F}$ NMR (376 MHz, $\mathrm{CDCl}_{3}$ )

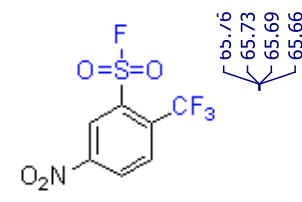

誉席

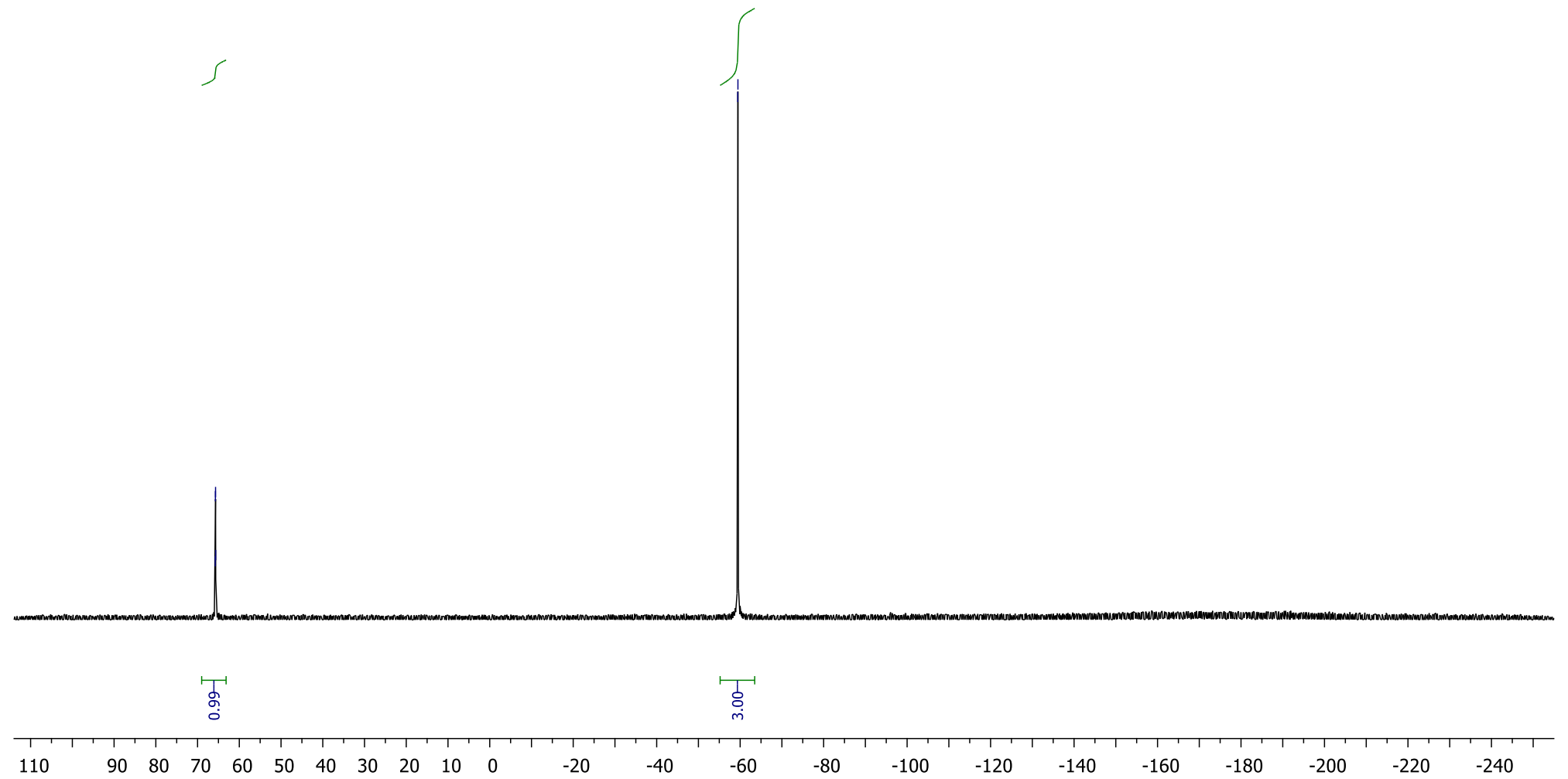


Compound 19a

${ }^{1} \mathrm{H}$ NMR (400 MHz, $\mathrm{CDCl}_{3}$ )

管

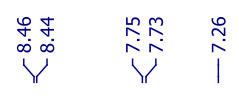
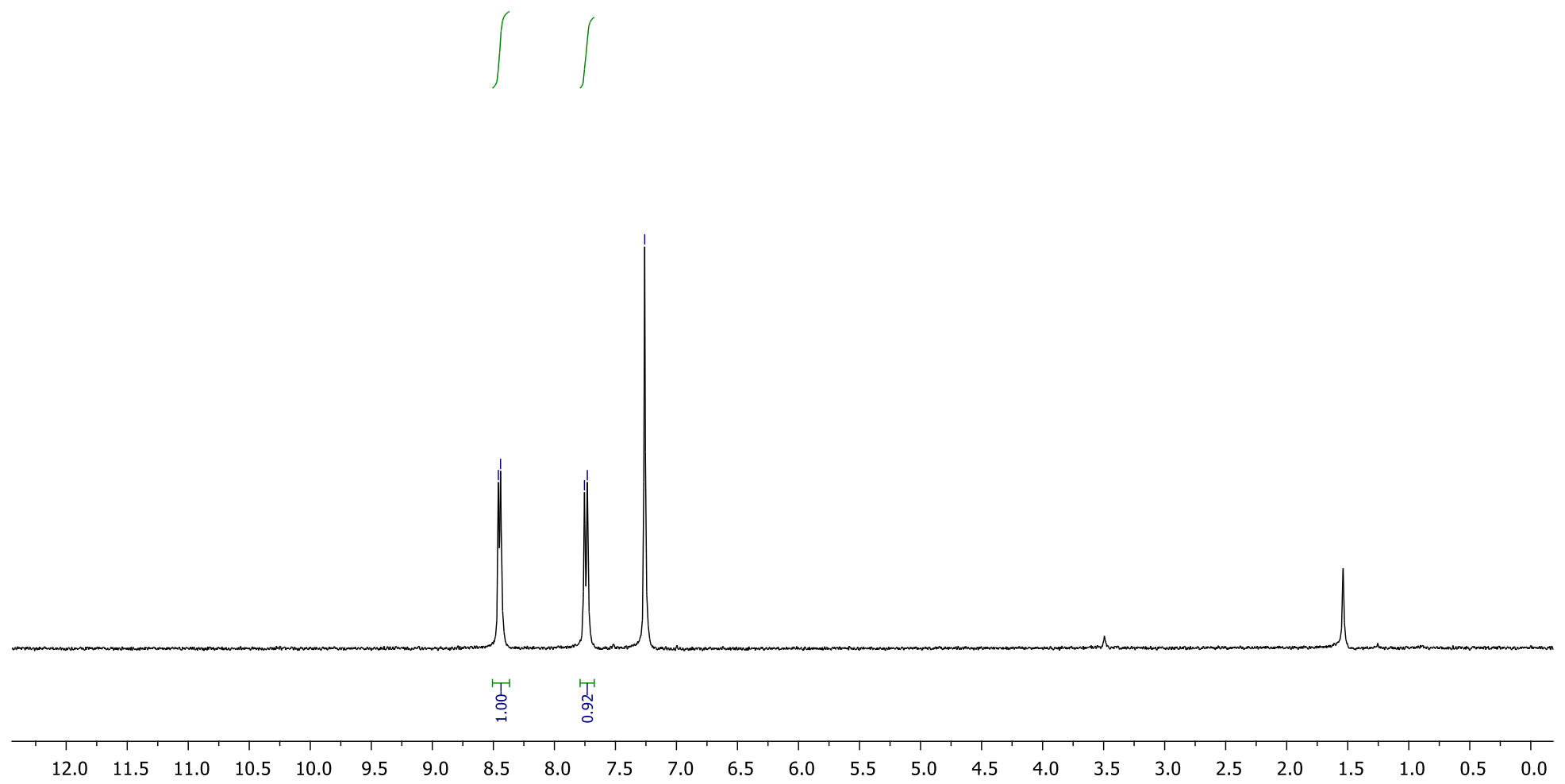


$$
=1
$$


${ }^{19} \mathrm{~F}$ NMR (376 MHz, $\mathrm{CDCl}_{3}$ )
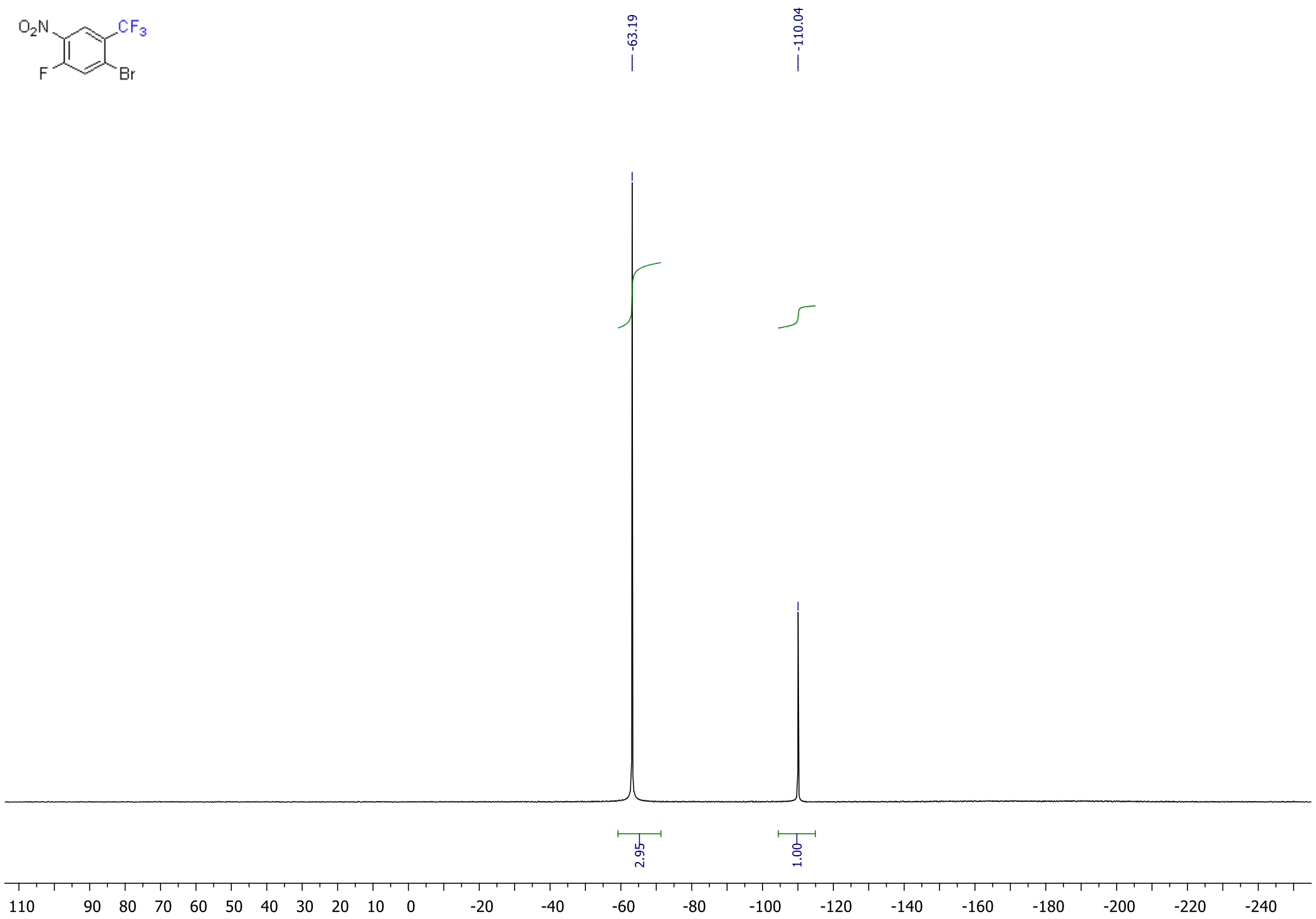
Compound 20a

${ }^{1} \mathrm{H}$ NMR (400 MHz, $\mathrm{CDCl}_{3}$ )

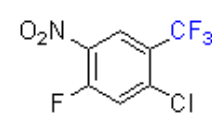

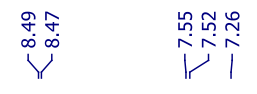

$\iint$

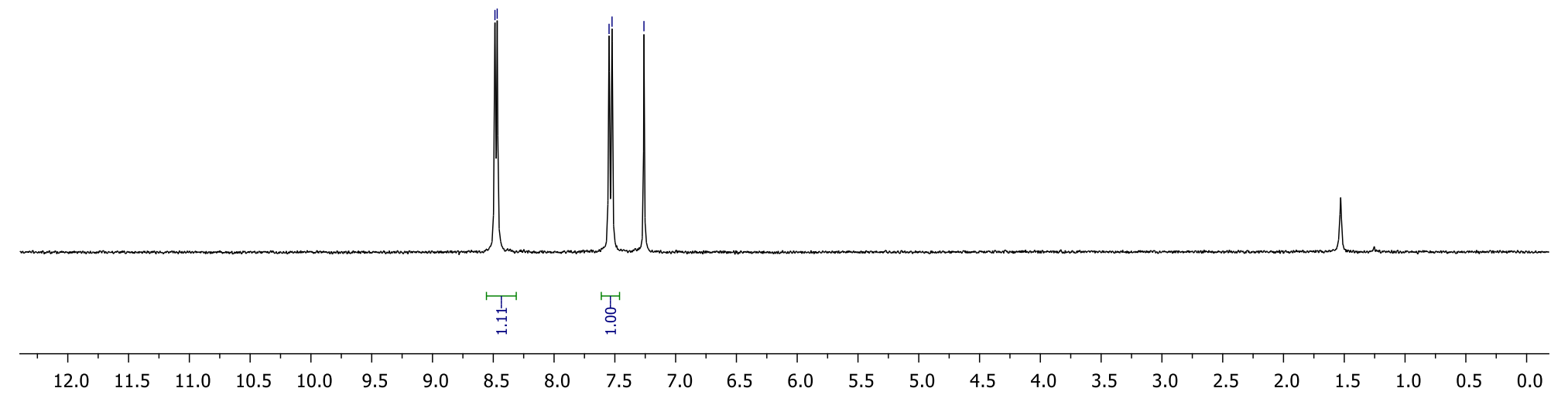


${ }^{13} \mathrm{C}\left\{{ }^{1} \mathrm{H}\right\}$ NMR $\left(151 \mathrm{MHz}, \mathrm{CDCl}_{3}\right)$

ICl

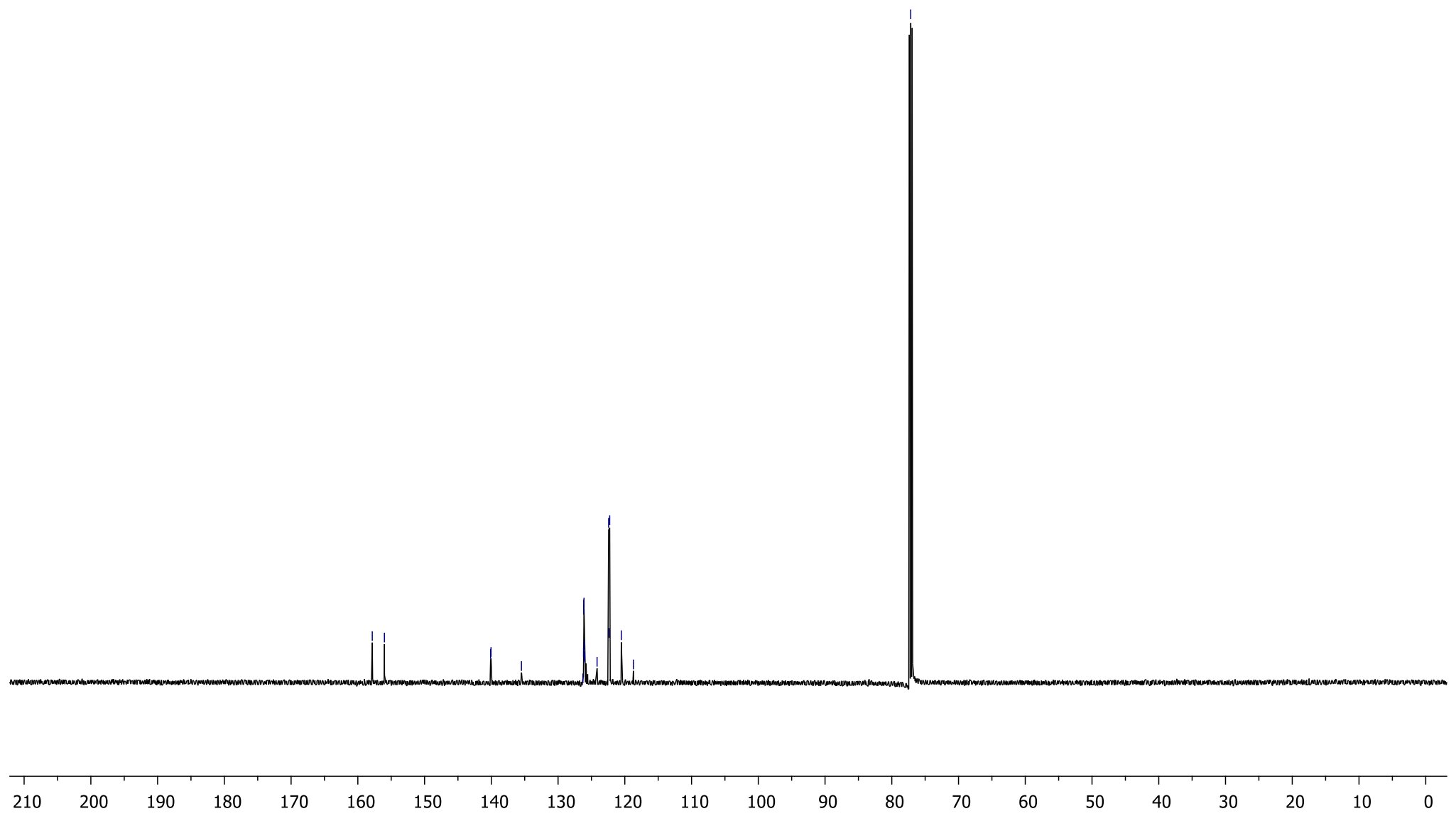


${ }^{19} \mathrm{~F}$ NMR (376 MHz, $\mathrm{CDCl}_{3}$ )
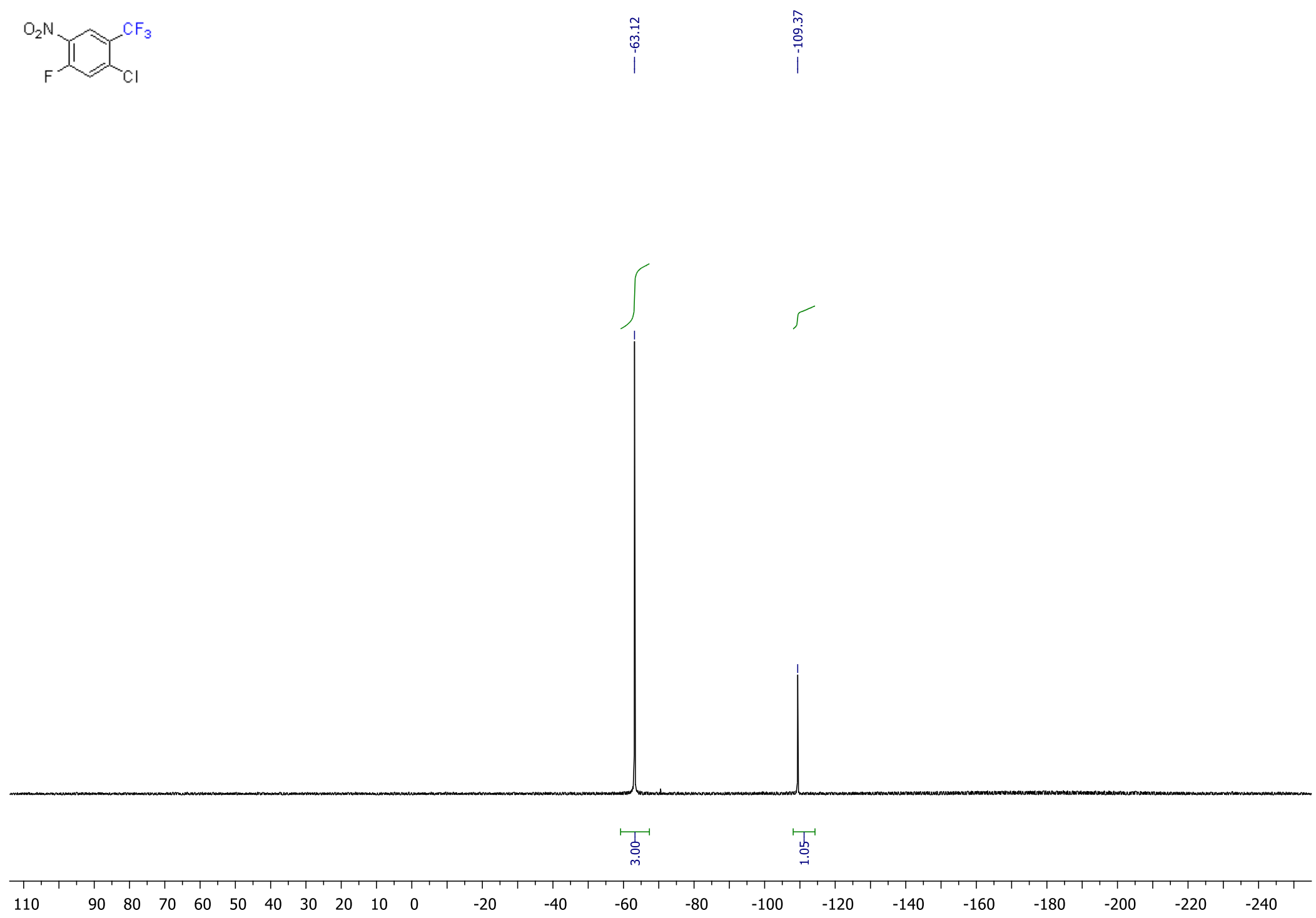
Compound 21a

${ }^{1} \mathrm{H}$ NMR (500 MHz, $\mathrm{CDCl}_{3}$ )
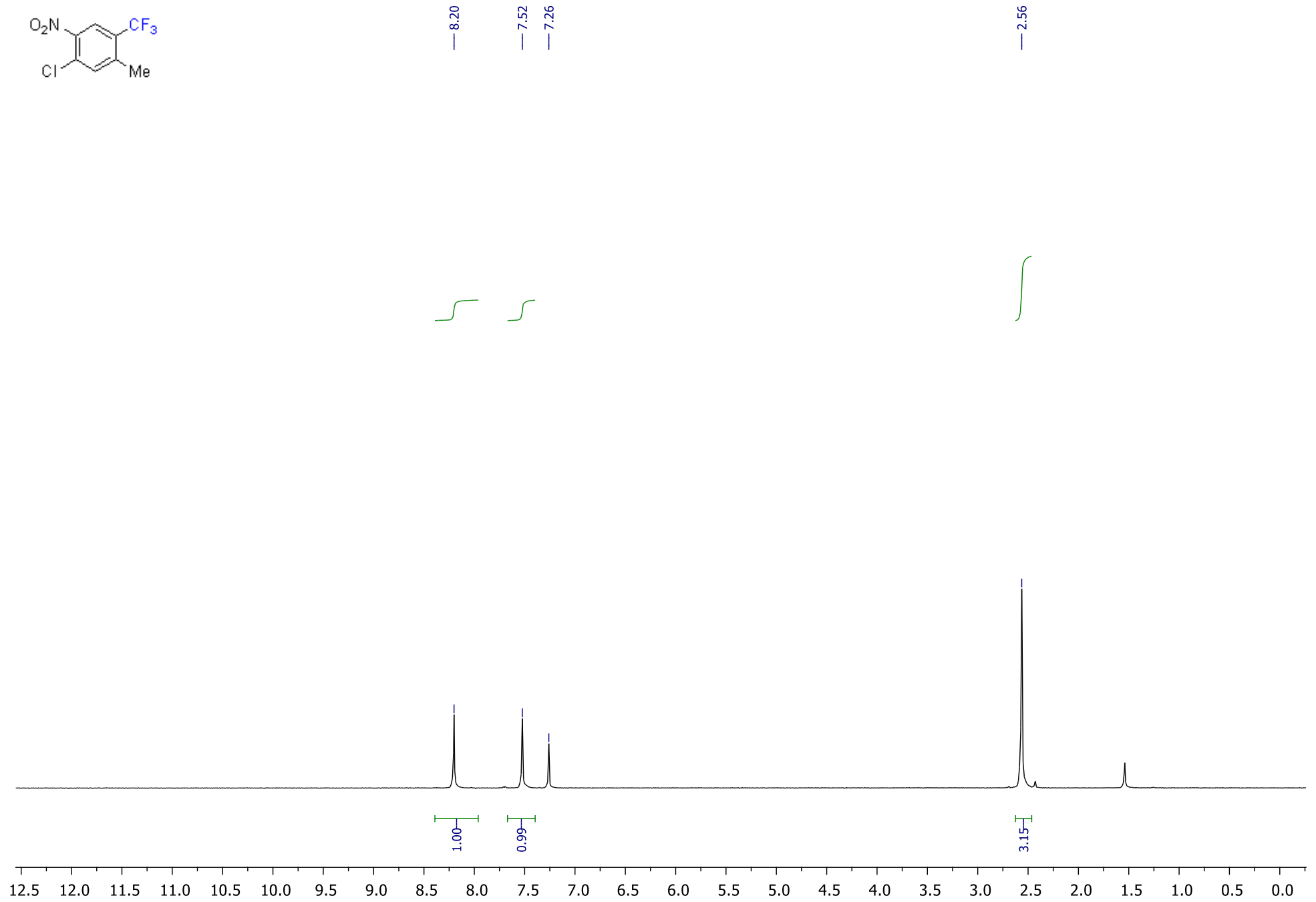
${ }^{13} \mathrm{C}\left\{{ }^{1} \mathrm{H}\right\}$ NMR $\left(151 \mathrm{MHz}, \mathrm{CDCl}_{3}\right)$

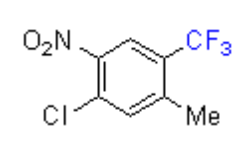

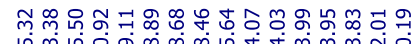

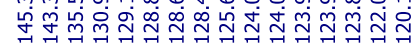

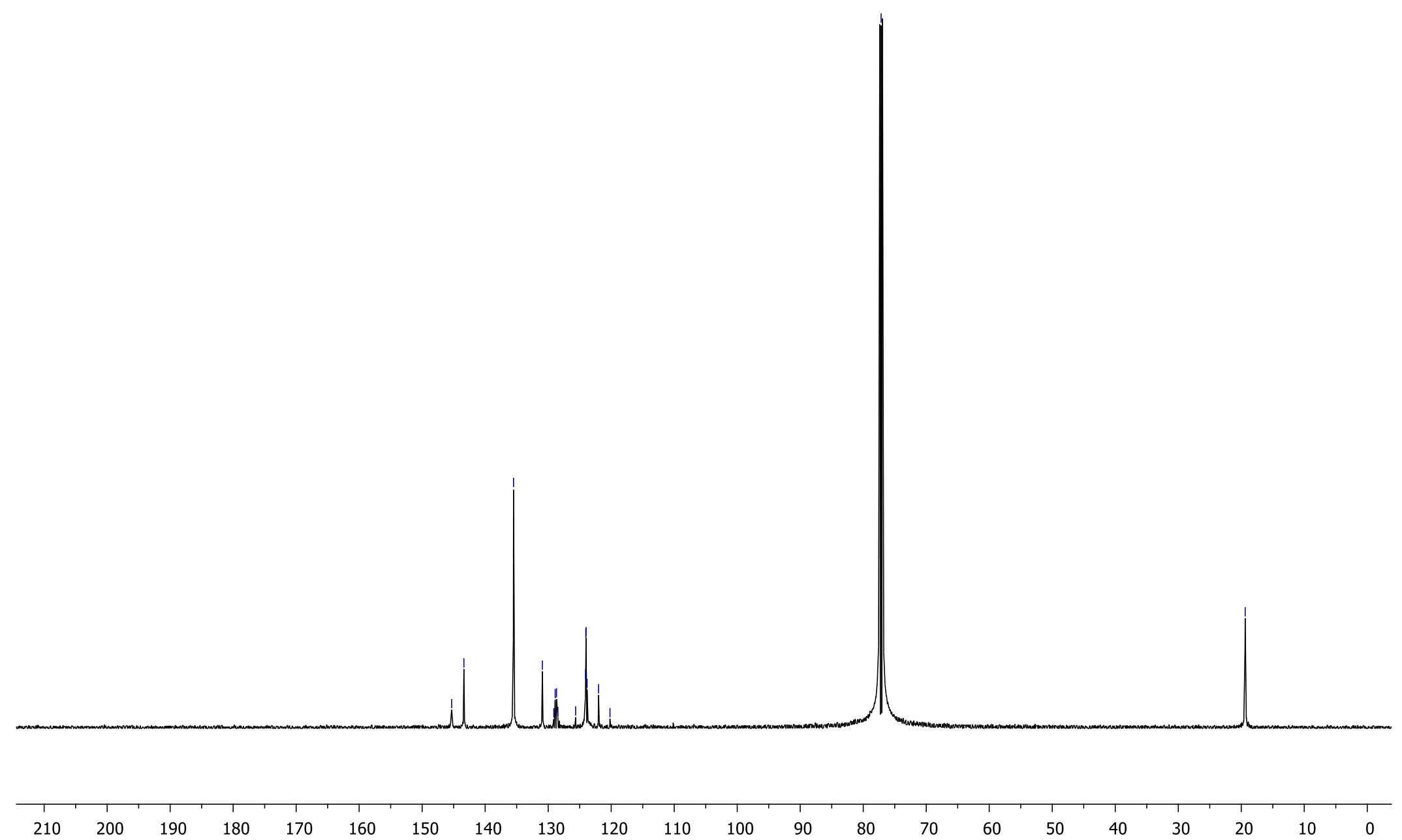


${ }^{19} \mathrm{~F}$ NMR $\left(376 \mathrm{MHz}, \mathrm{CDCl}_{3}\right)$

的

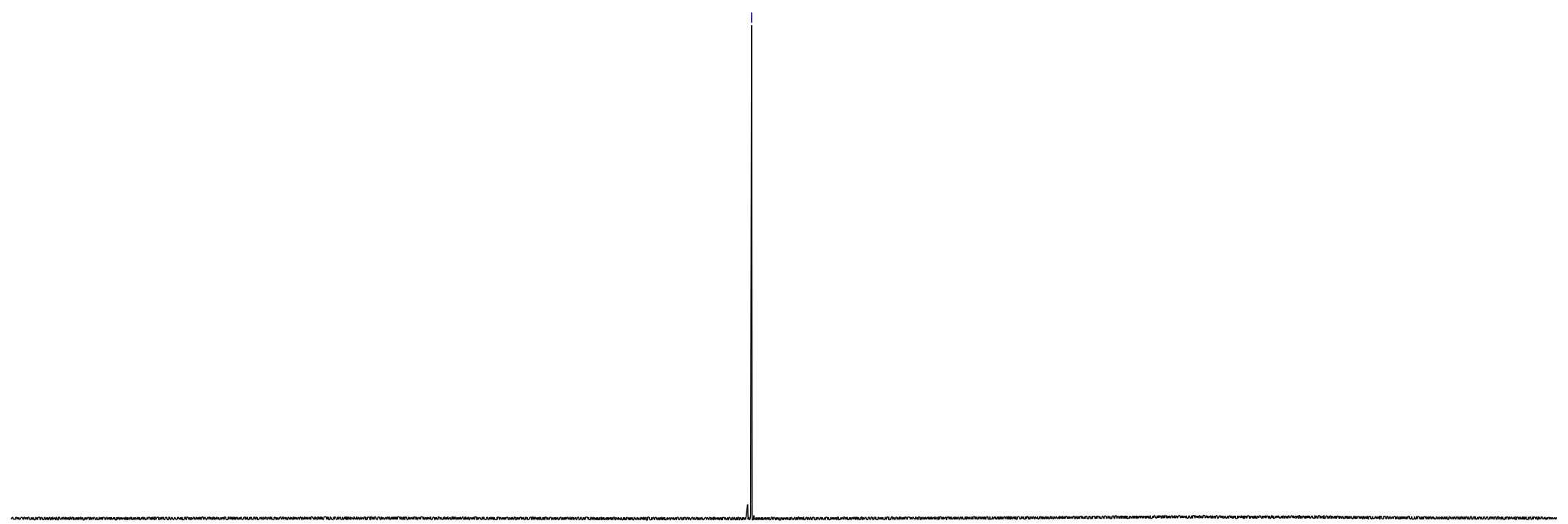

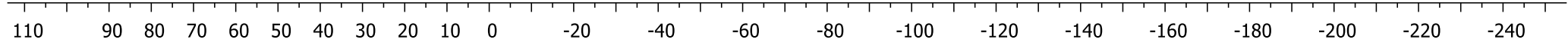


Compound 22a

${ }^{1} \mathrm{H}$ NMR (400 MHz, $\mathrm{CDCl}_{3}$ )

${ }_{\mathrm{Me}}^{\mathrm{O}_{2} \mathrm{~N}} \mathrm{C}_{\mathrm{F}}^{\mathrm{CF}_{3}}$

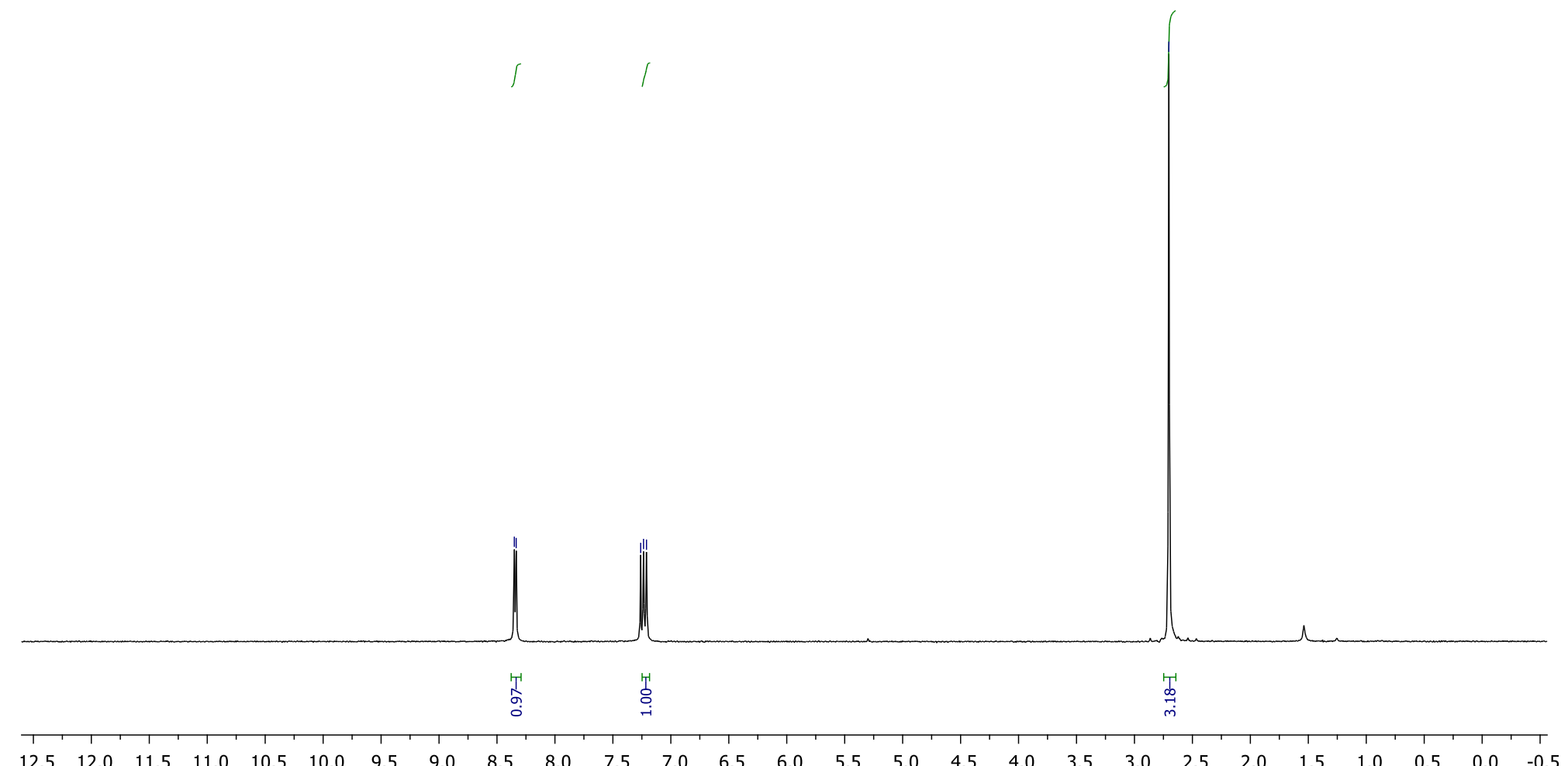


${ }^{13} \mathrm{C}\left\{{ }^{1} \mathrm{H}\right\}$ NMR $\left(126 \mathrm{MHz}, \mathrm{CDCl}_{3}\right)$

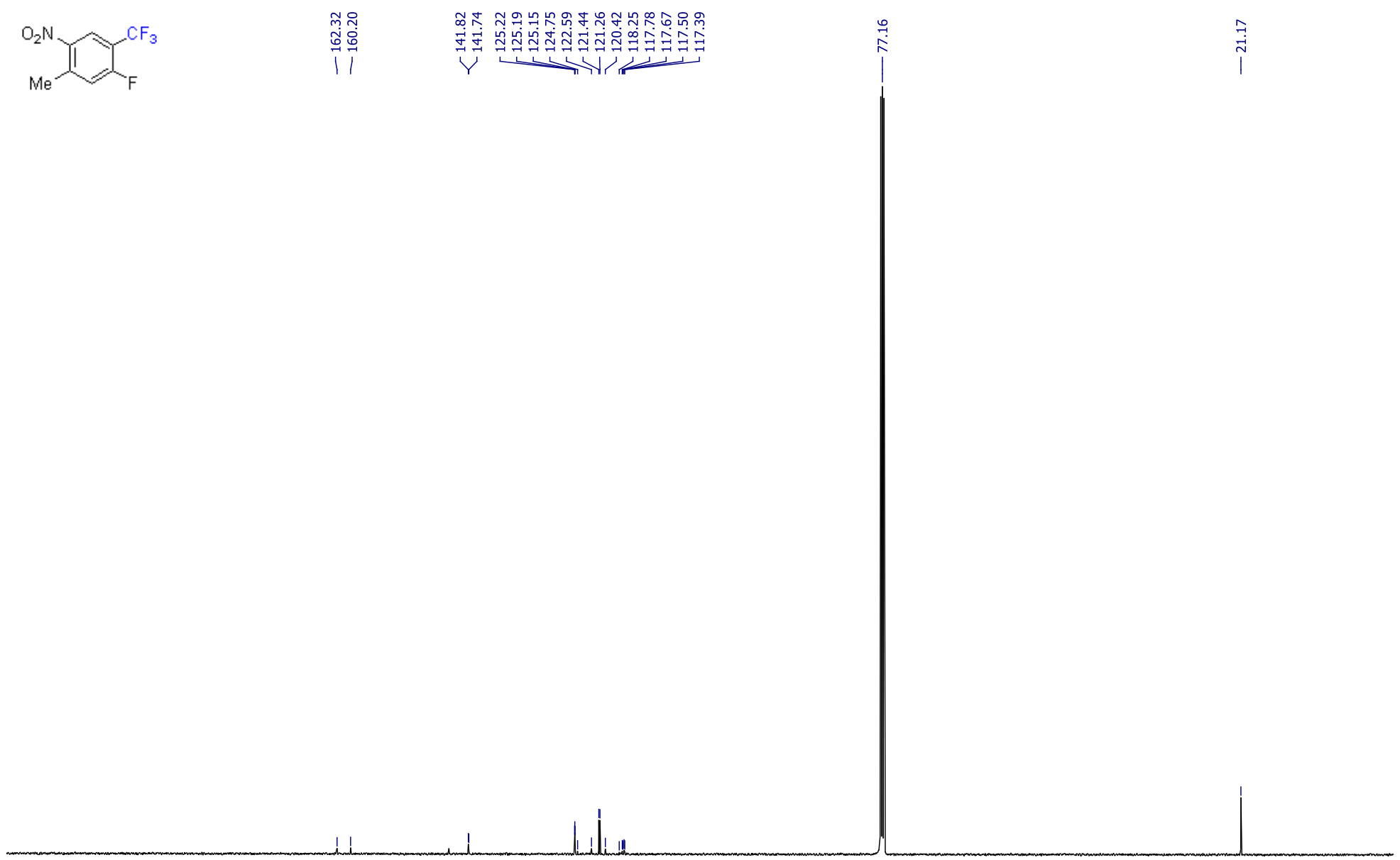

$\begin{array}{llllllllllllllllllll}210 & 200 & 190 & 180 & 170 & 160 & 150 & 140 & 130 & 120 & 110 & 100 & 90 & 80 & 70 & 60 & 50 & 40 & 30 & 20\end{array}$ 
$\left.{ }^{19} \mathrm{~F} \mathrm{NMR} \mathrm{(376} \mathrm{MHz,} \mathrm{CDCl}_{3}\right)$
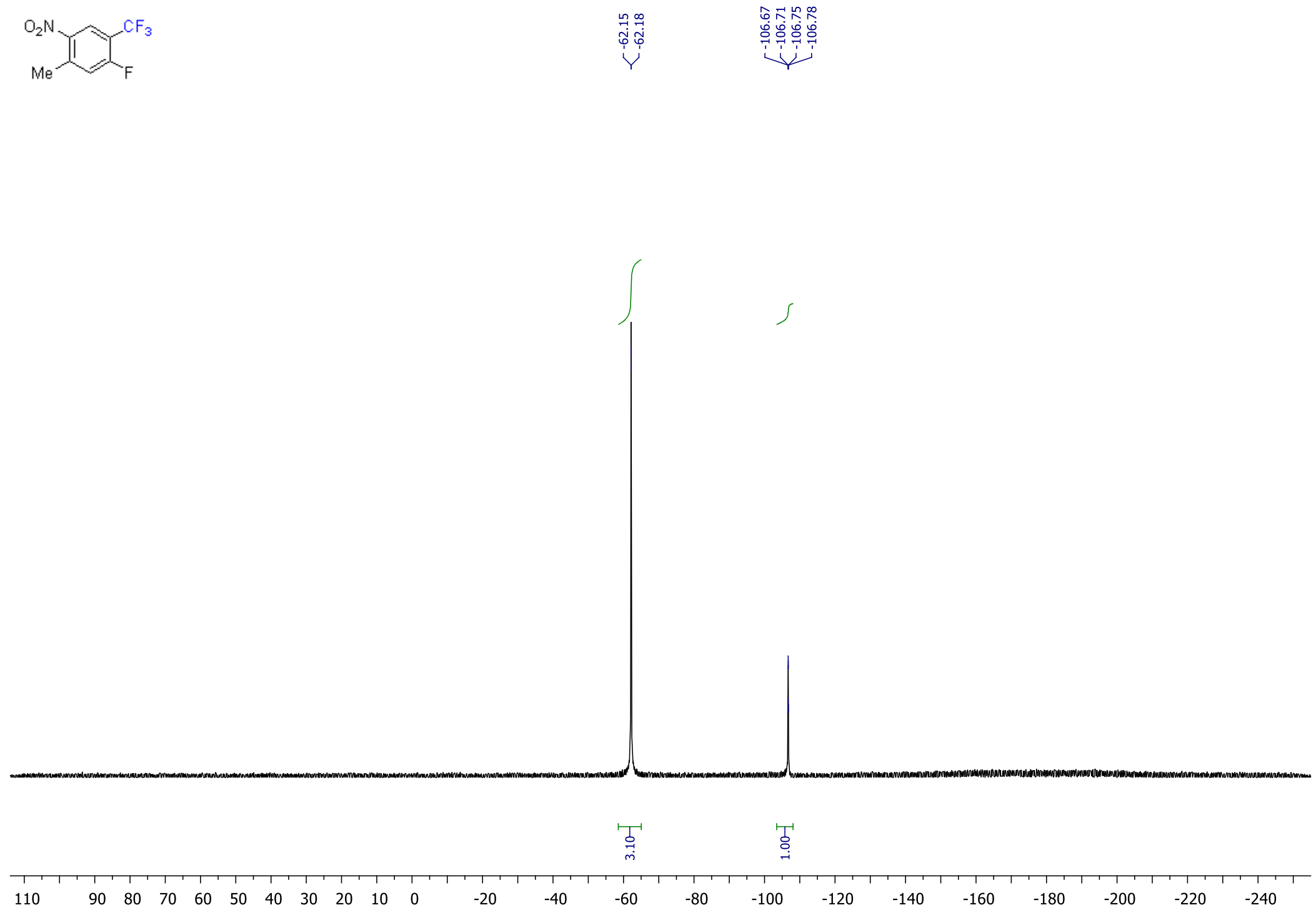
Compound 23a

${ }^{1} \mathrm{H}$ NMR (400 MHz, $\mathrm{CDCl}_{3}$ )

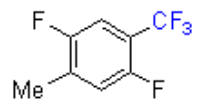

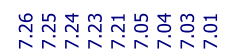

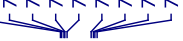

1

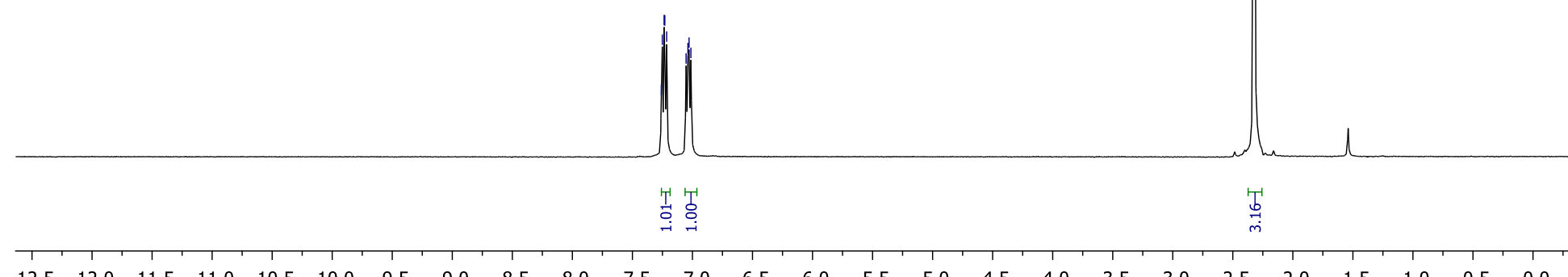

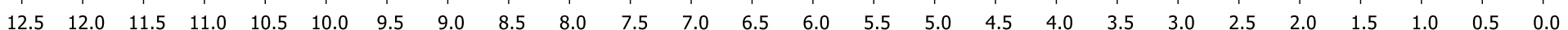


${ }^{13} \mathrm{C}\left\{{ }^{1} \mathrm{H}\right\}$ NMR $\left(151 \mathrm{MHz}, \mathrm{CDCl}_{3}\right)$

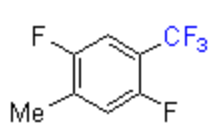

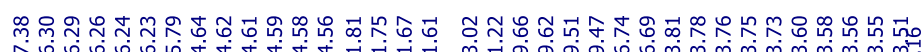

ஸी

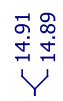

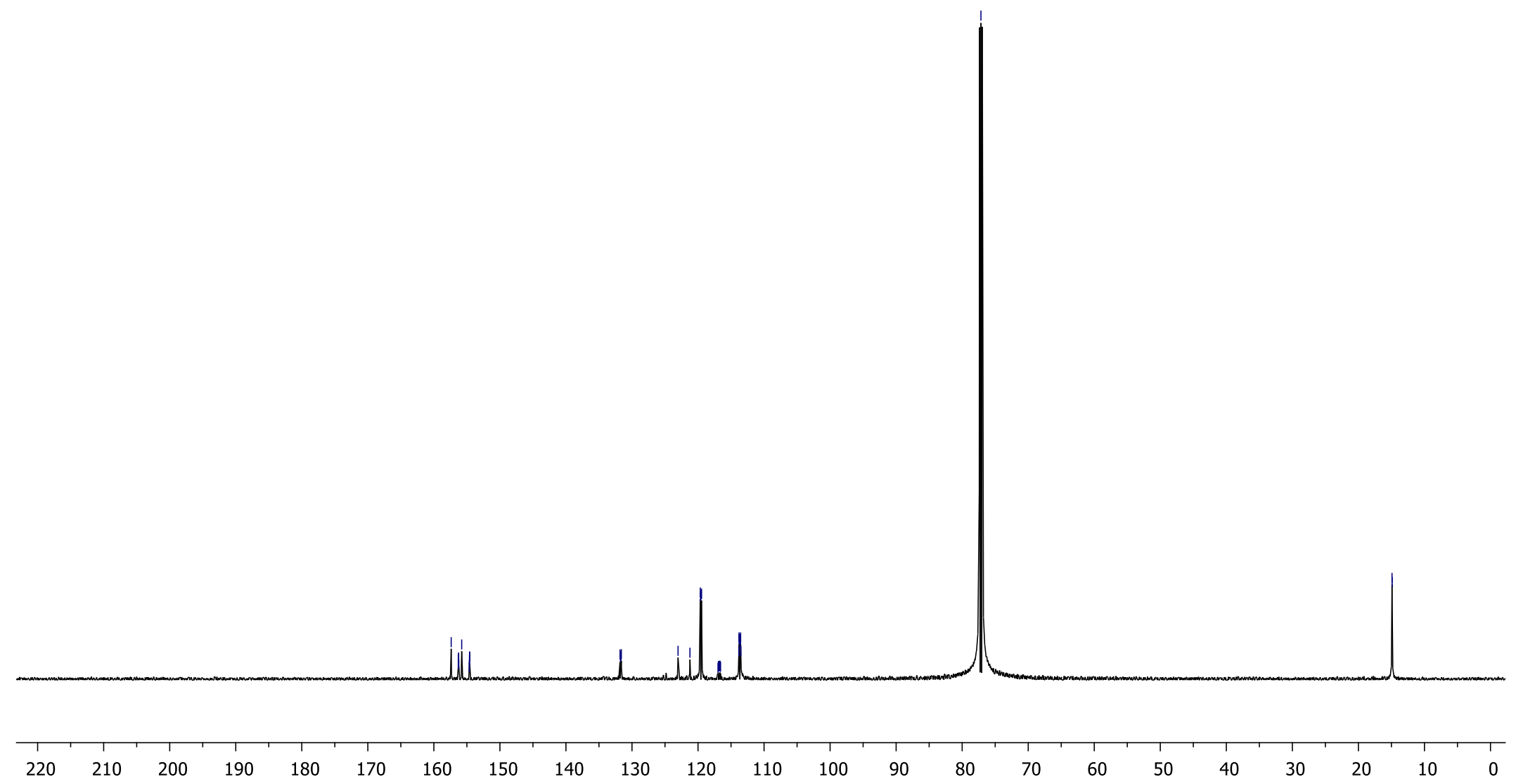


${ }^{19} \mathrm{~F}$ NMR (376 MHz, $\mathrm{CDCl}_{3}$ )
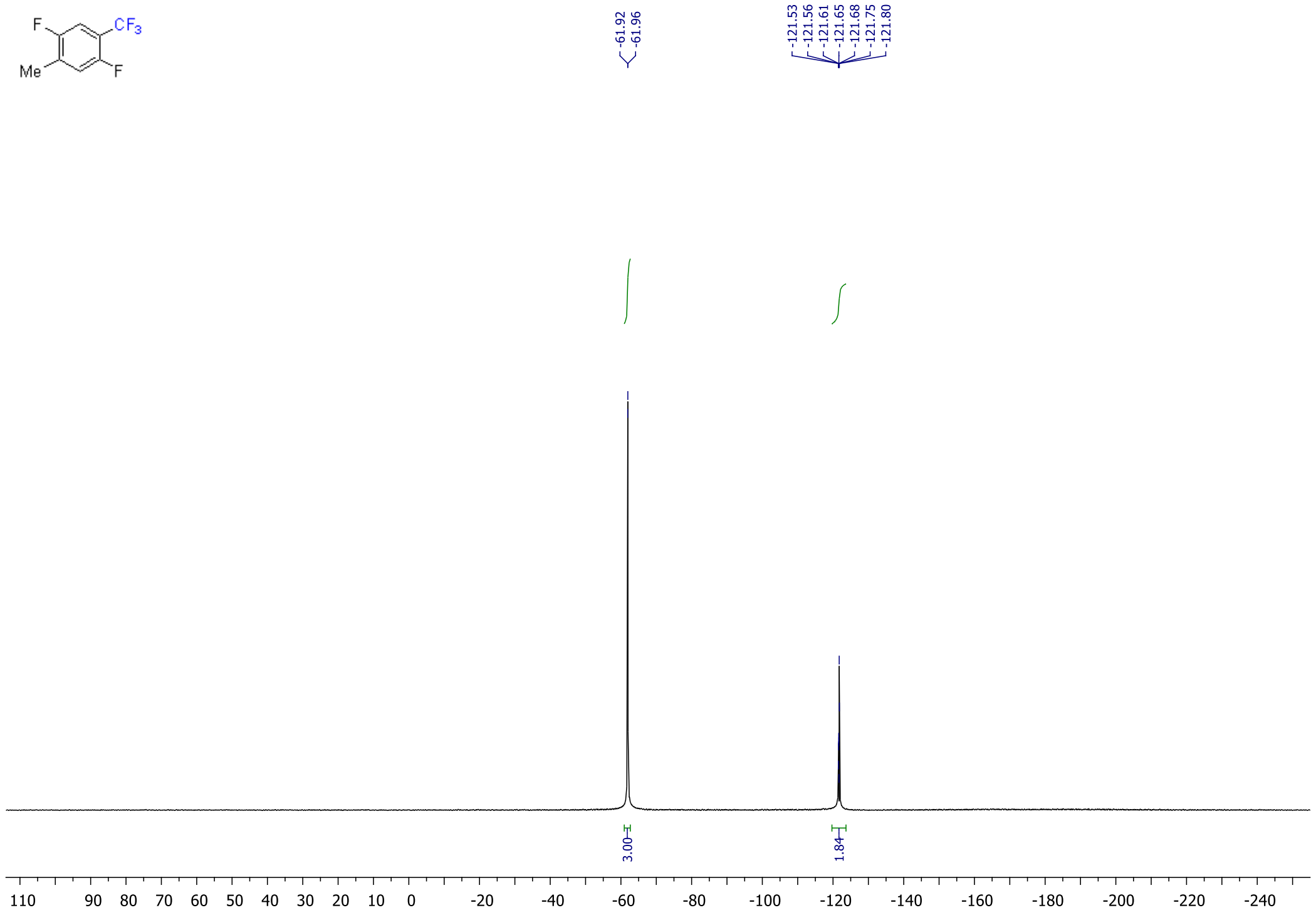
Compound $24 \mathrm{a}^{*}$

${ }^{1} \mathrm{H} \mathrm{NMR}\left(400 \mathrm{MHz}, \mathrm{CDCl}_{3}\right)$

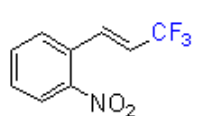

Y.
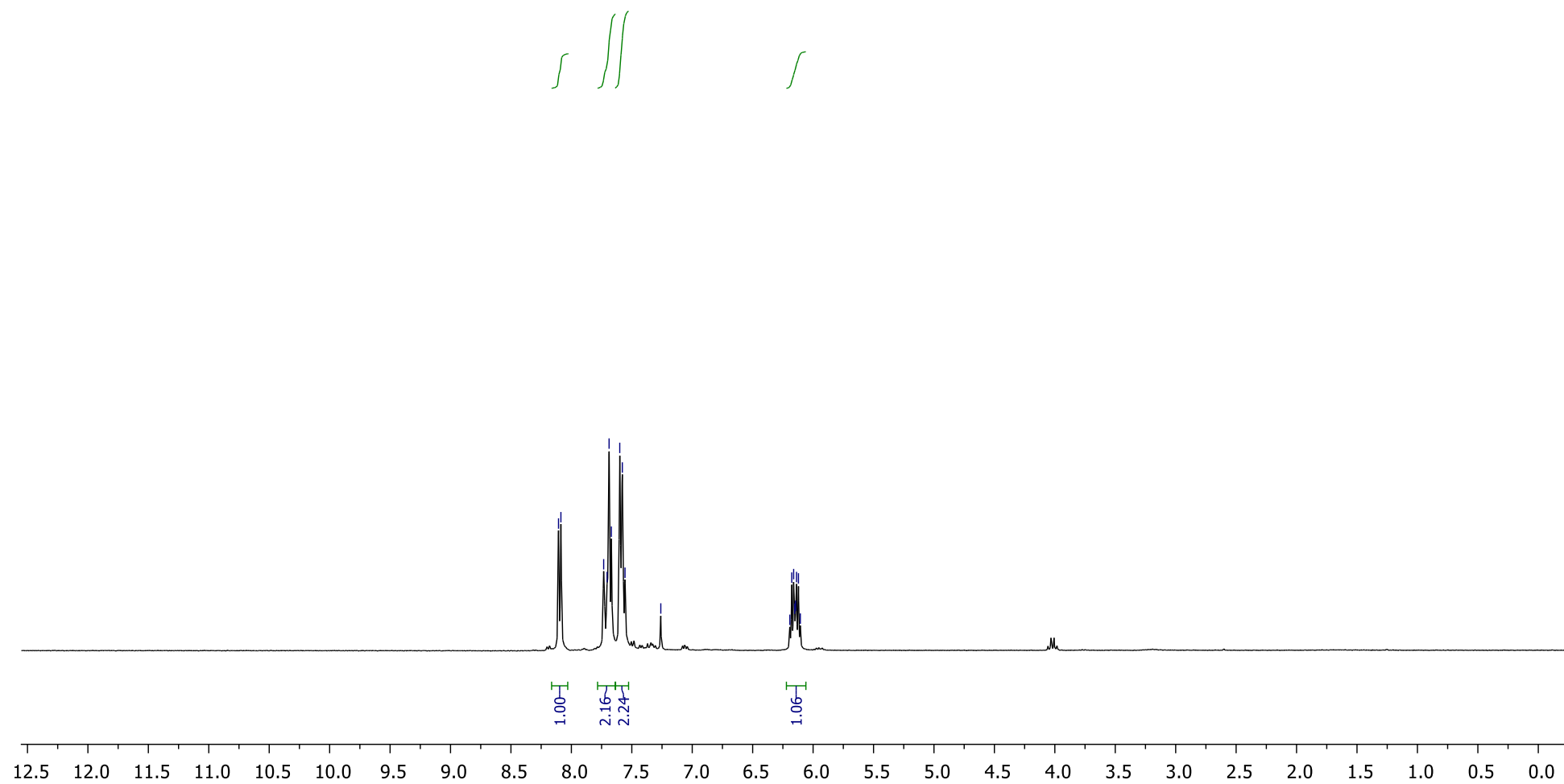

Compound 24a is also described in: a) Org. Lett. 2015, 17, 5, 1252-1255; b) Chem. Commun. 2014, 50, 2308-2310. 
${ }^{13} \mathrm{C}\left\{{ }^{1} \mathrm{H}\right\}$ NMR (101 MHz, $\left.\mathrm{CDCl}_{3}\right)$

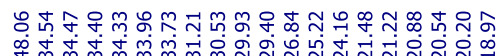

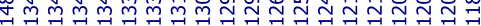

$\stackrel{\substack{i \\ i}}{a}$

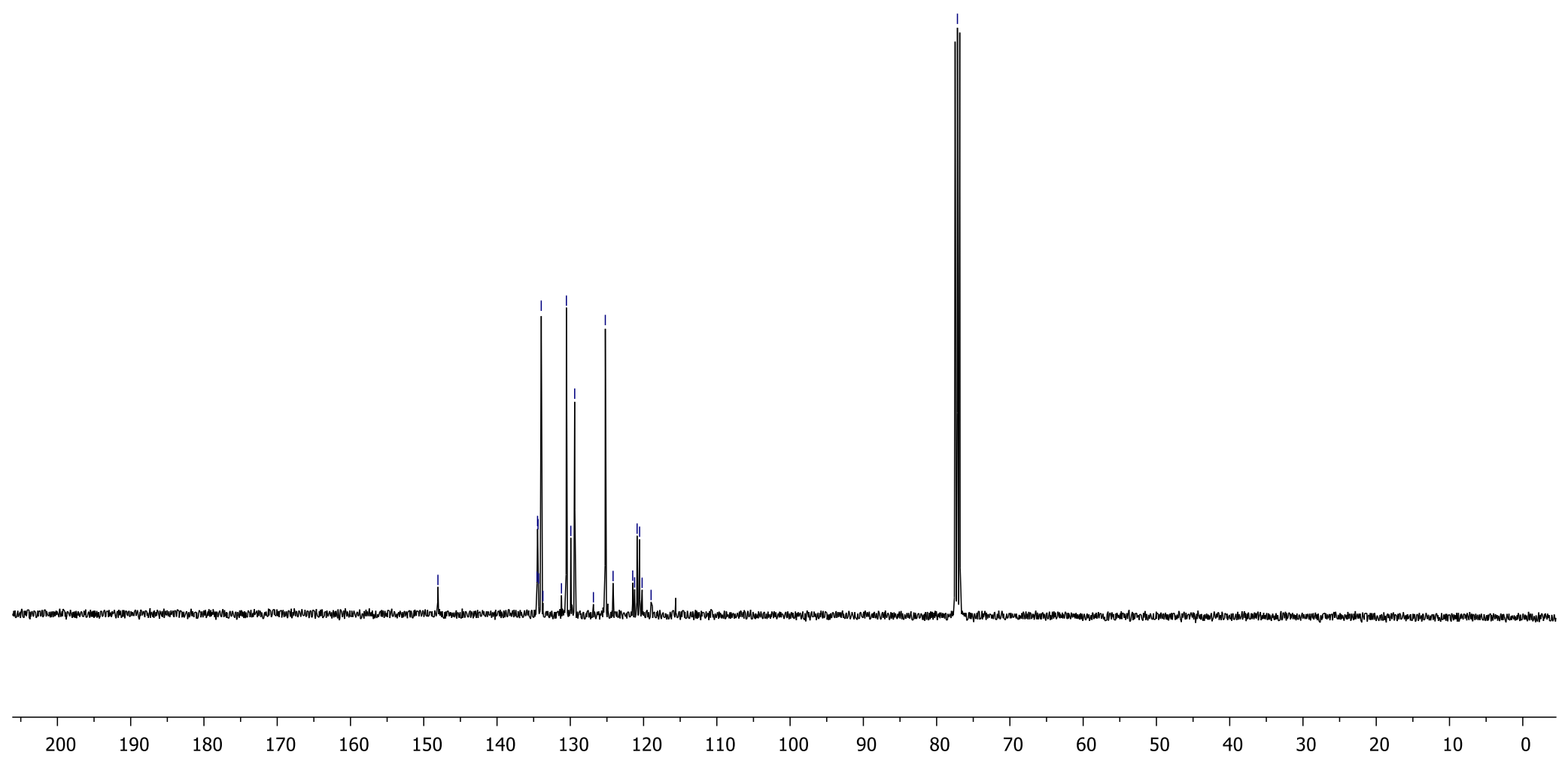


${ }^{19} \mathrm{~F}$ NMR $\left(376 \mathrm{MHz}, \mathrm{CDCl}_{3}\right)$

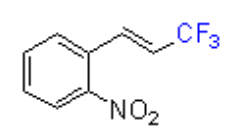

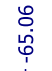

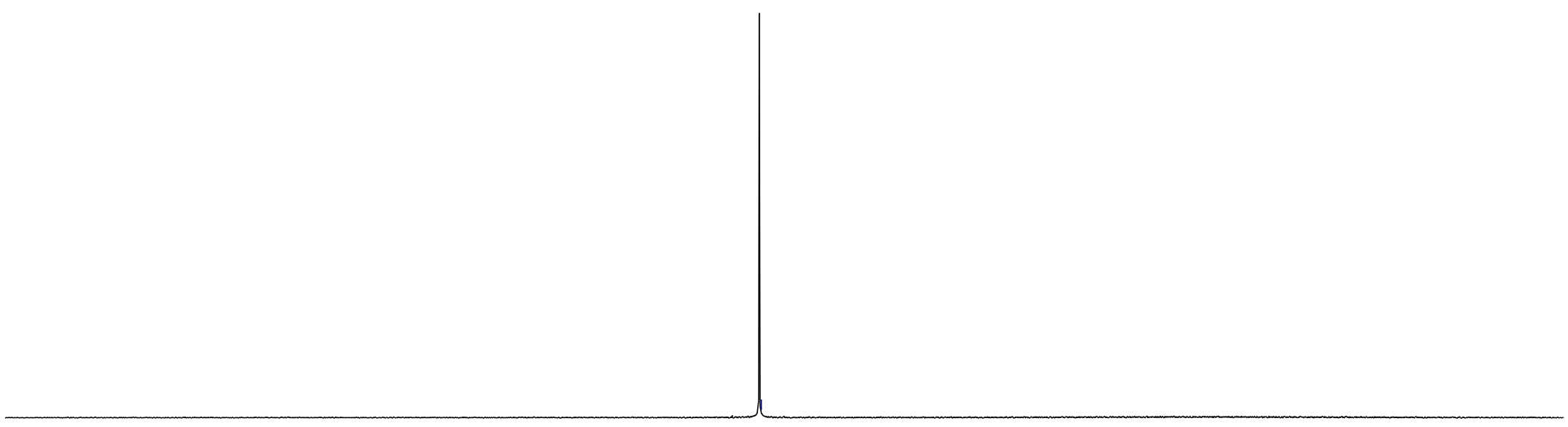

$\begin{array}{llllllllllllllllllllllllllllllllll} & 110 & 90 & 80 & 70 & 60 & 50 & 40 & 30 & 20 & 10 & 0 & -20 & -40 & -60 & -80 & -100 & -120 & -140 & -160 & -180 & -200 & -220 & -240 & \end{array}$ 

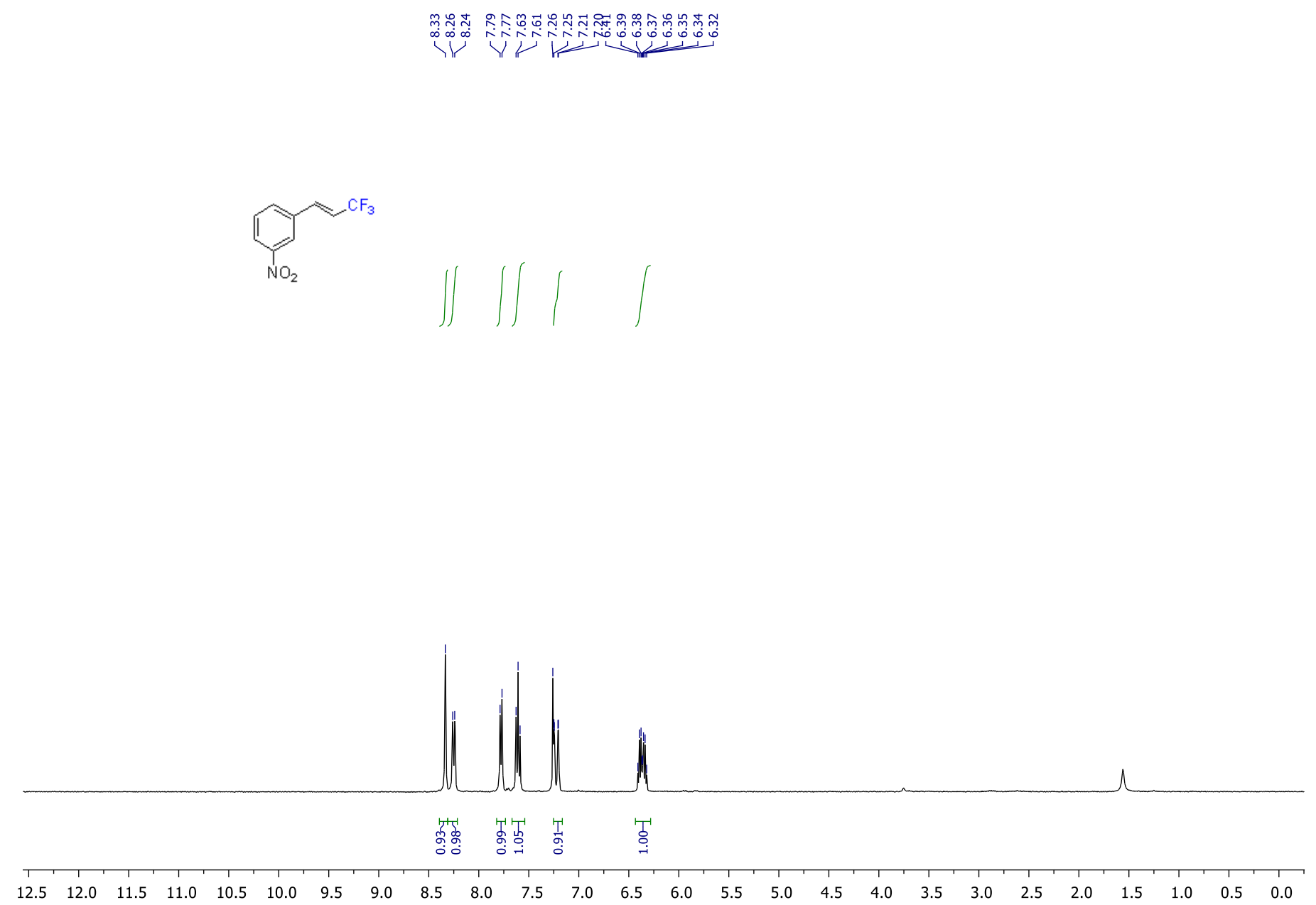

Compound 25a is also described in: a) J. Org. Chem. 2019, 84, 9, 5980-5986; b) Eur. J. Org. Chem. 2013, 35, 7996-8003; c) Eur. J. Org. Chem.2013, 24, 5247-5250. 
$\left.{ }^{19} \mathrm{~F} \mathrm{NMR} \mathrm{(376} \mathrm{MHz,} \mathrm{CDCl}_{3}\right)$

R1588643_F19\{H\} $19 \mathrm{~F}-\{1 \mathrm{H}\}$

mºn
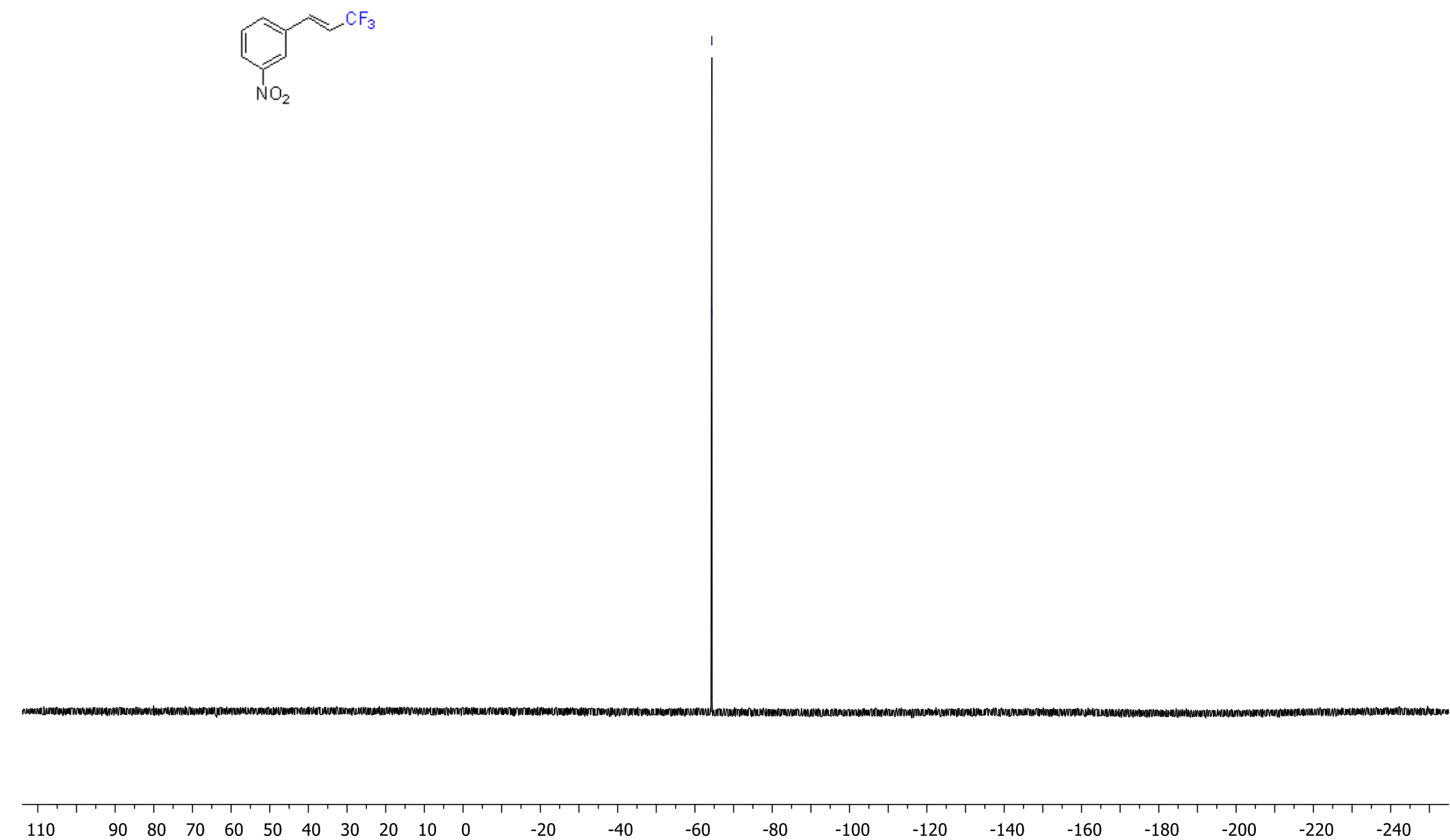
${ }^{13} \mathrm{C}\left\{{ }^{1} \mathrm{H}\right\}$ NMR $\left(126 \mathrm{MHz}, \mathrm{CDCl}_{3}\right)$

R1588643_C1:

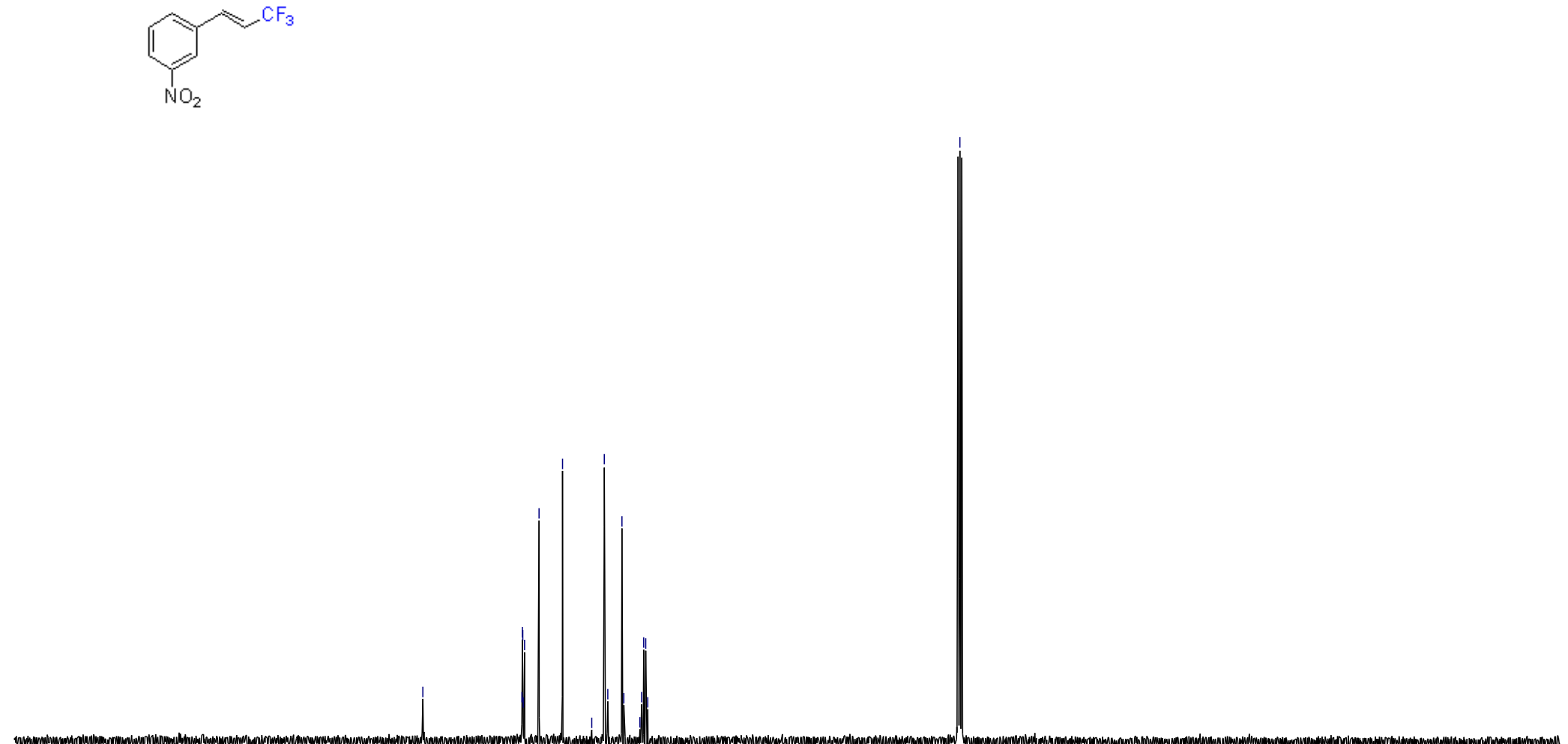



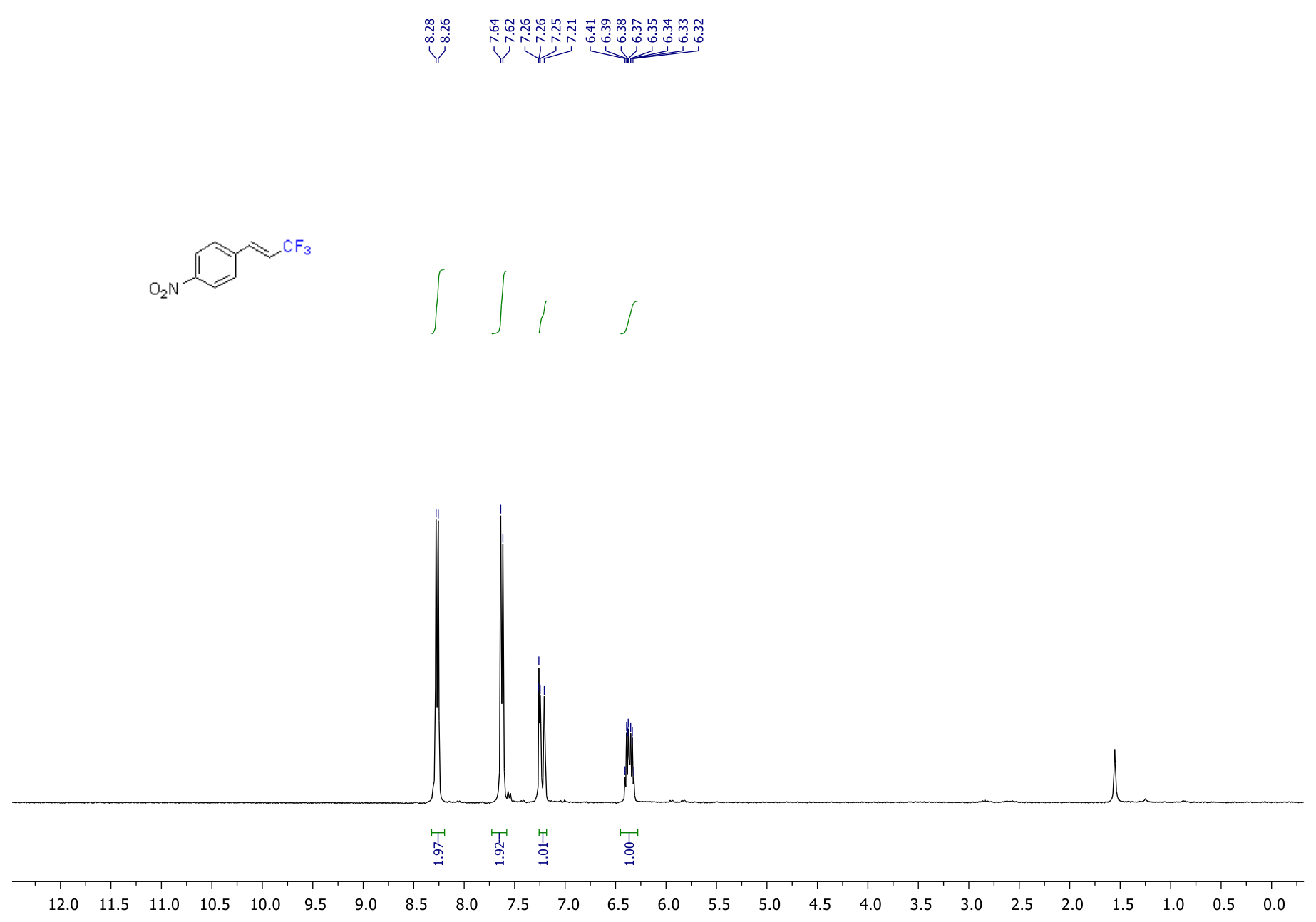

Compound 26a is also described in: a) J. Org. Chem. 2019, 84, 9, 5980-5986; b) Eur. J. Org. Chem. 2013, 35, 7996-8003; c) Beilstein J. Org. Chem. 2013, 9, 2635-2640; d) Synthesis 1981, 5, 365-366; e) J. Fluorine Chem. 2015, 171, 169-173. 
${ }^{19} \mathrm{~F}$ NMR $\left(376 \mathrm{MHz}, \mathrm{CDCl}_{3}\right)$

R1588633_F19\{H\}

$19 \mathrm{~F}-\{1 \mathrm{H}\}$

$\underset{\substack{0 \\+}}{i}$

O $\mathrm{O}_{2} \mathrm{~N} \mathrm{CF}_{3}$

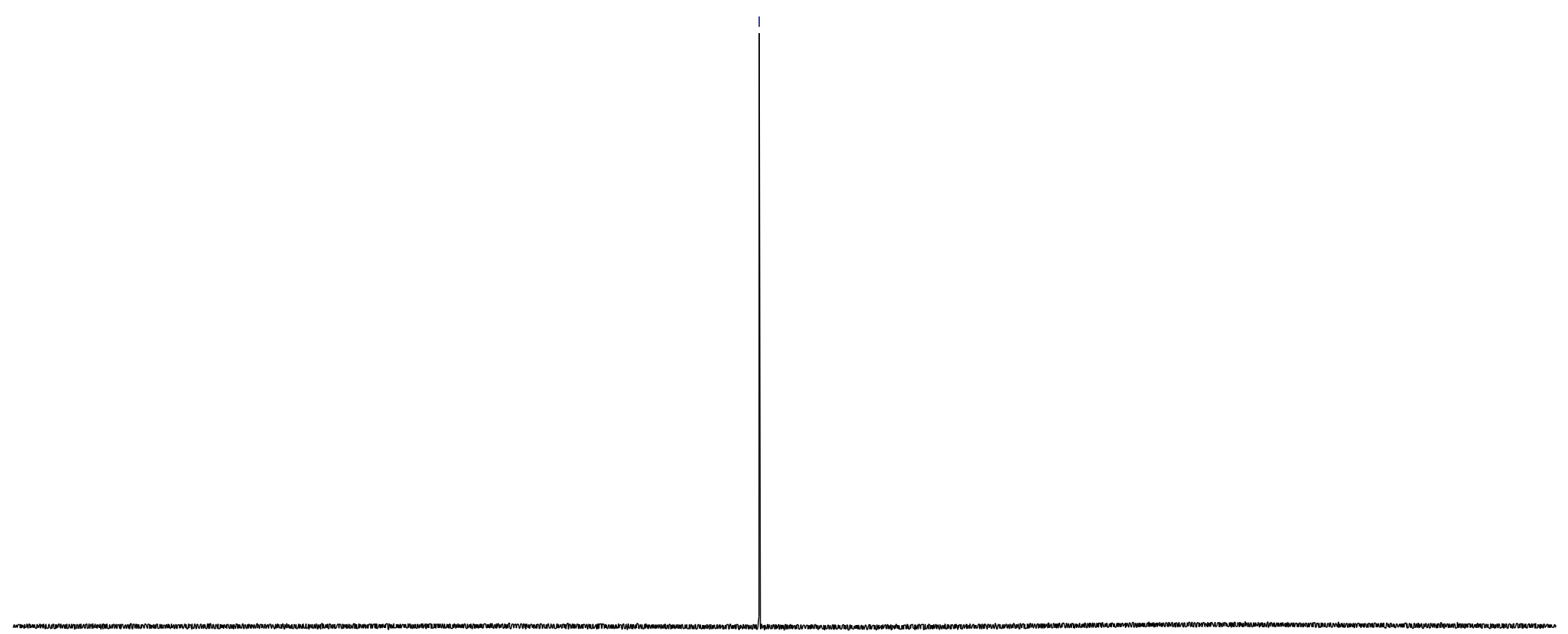

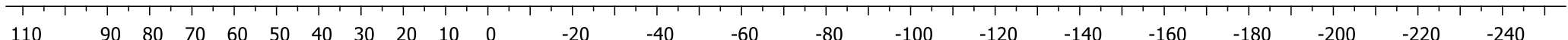


${ }^{13} \mathrm{C}\left\{{ }^{1} \mathrm{H}\right\} \mathrm{NMR}\left(126 \mathrm{MHz}, \mathrm{CDCl}_{3}\right)$

R1588633_C1:

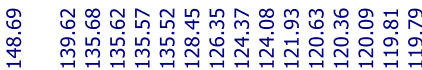

还证

$\stackrel{0}{\stackrel{2}{*}}$

$\mathrm{O}_{2} \mathrm{~N}$

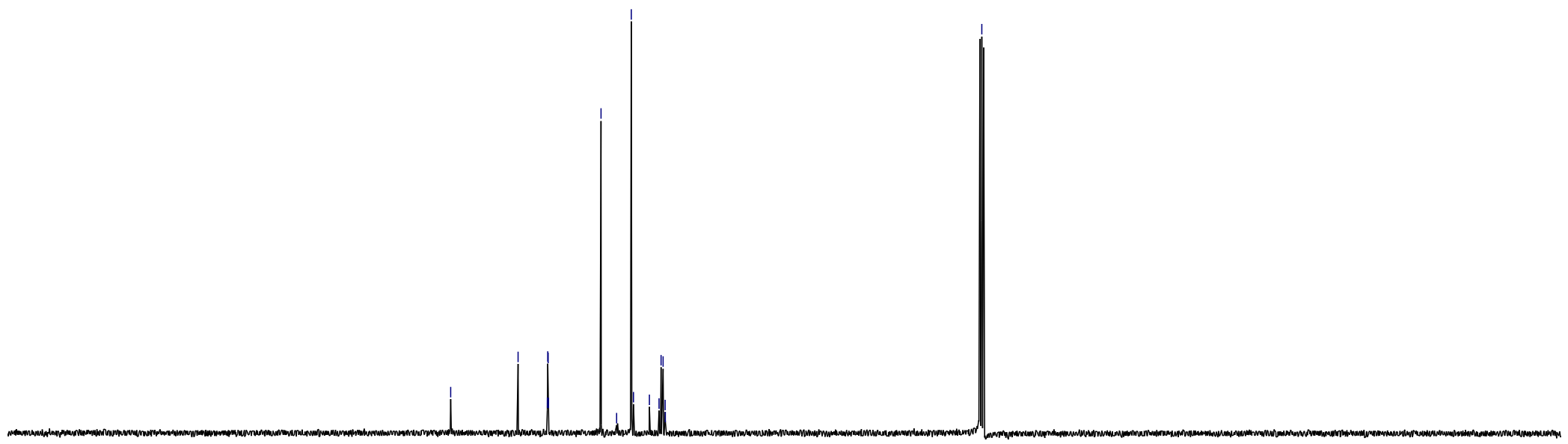

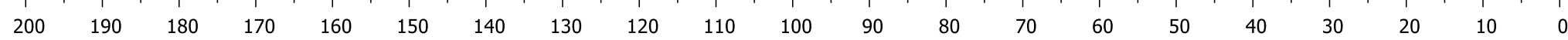


Compound 27a

${ }^{1} \mathrm{H} \mathrm{NMR}\left(400 \mathrm{MHz}, \mathrm{CDCl}_{3}\right)$

$\prod_{\mathrm{S}} \mathrm{CF}_{\mathrm{C}_{3}}$

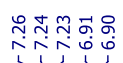
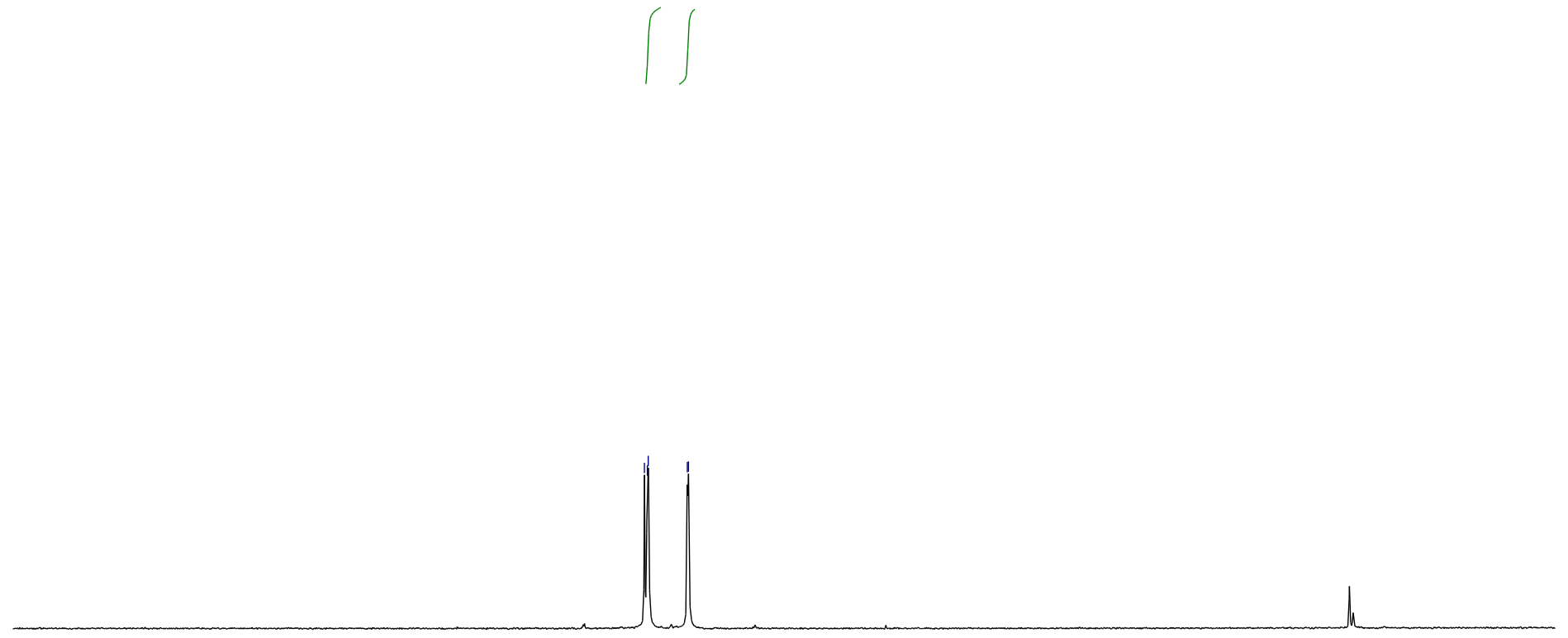

管畋

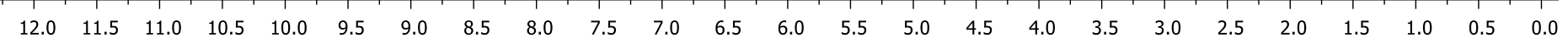


${ }^{13} \mathrm{C}\left\{{ }^{1} \mathrm{H}\right\} \mathrm{NMR}\left(126 \mathrm{MHz}, \mathrm{CDCl}_{3}\right)$

$\prod_{S} \prod_{\mathrm{CF}_{3}}$

Nㅜㄹ

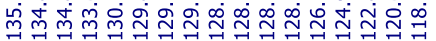

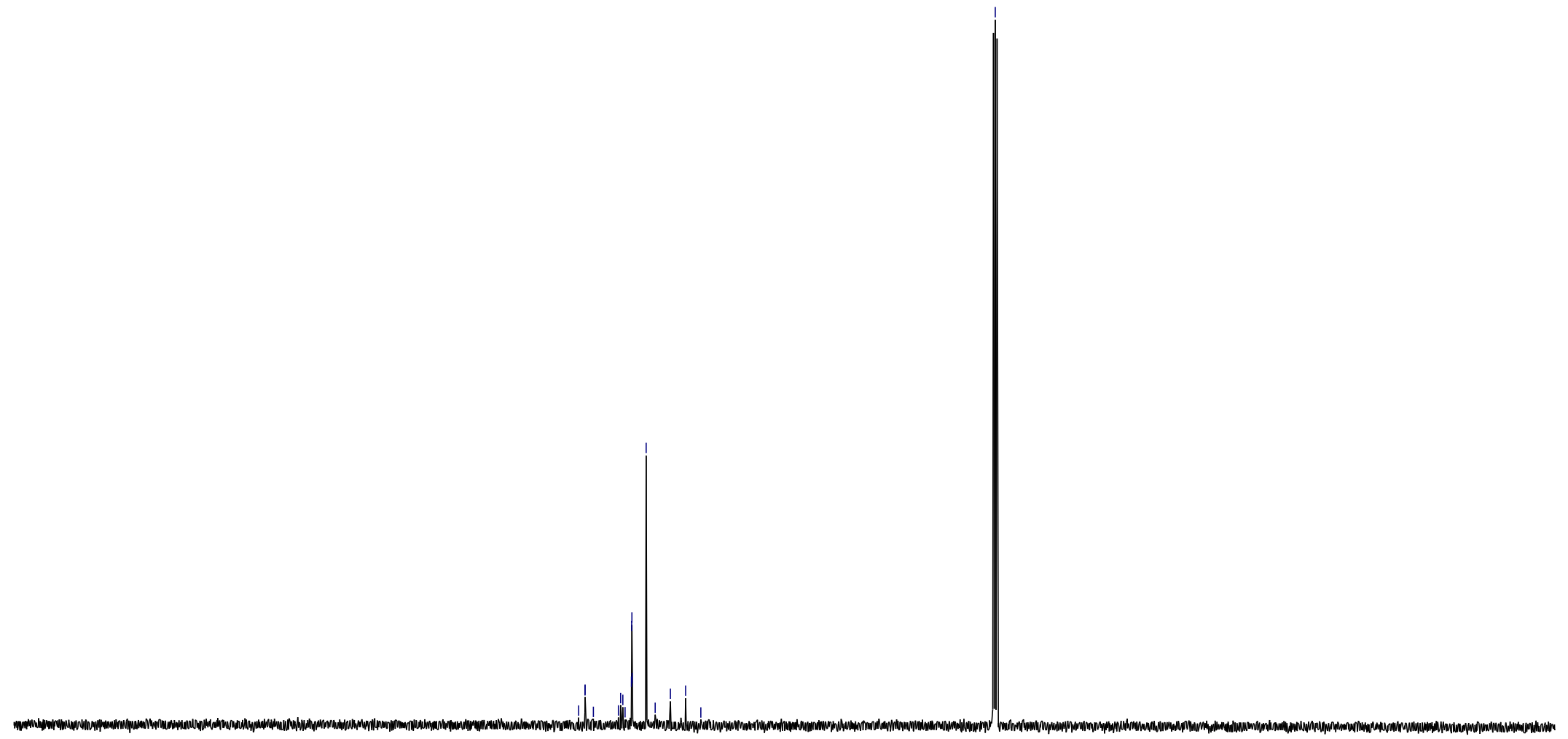

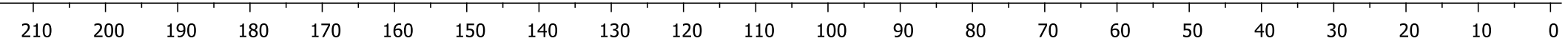


${ }^{19} \mathrm{~F}$ NMR (376 MHz, $\mathrm{CDCl}_{3}$ )

${ }_{\mathrm{C}} \mathrm{MS}_{\mathrm{S}}$

商

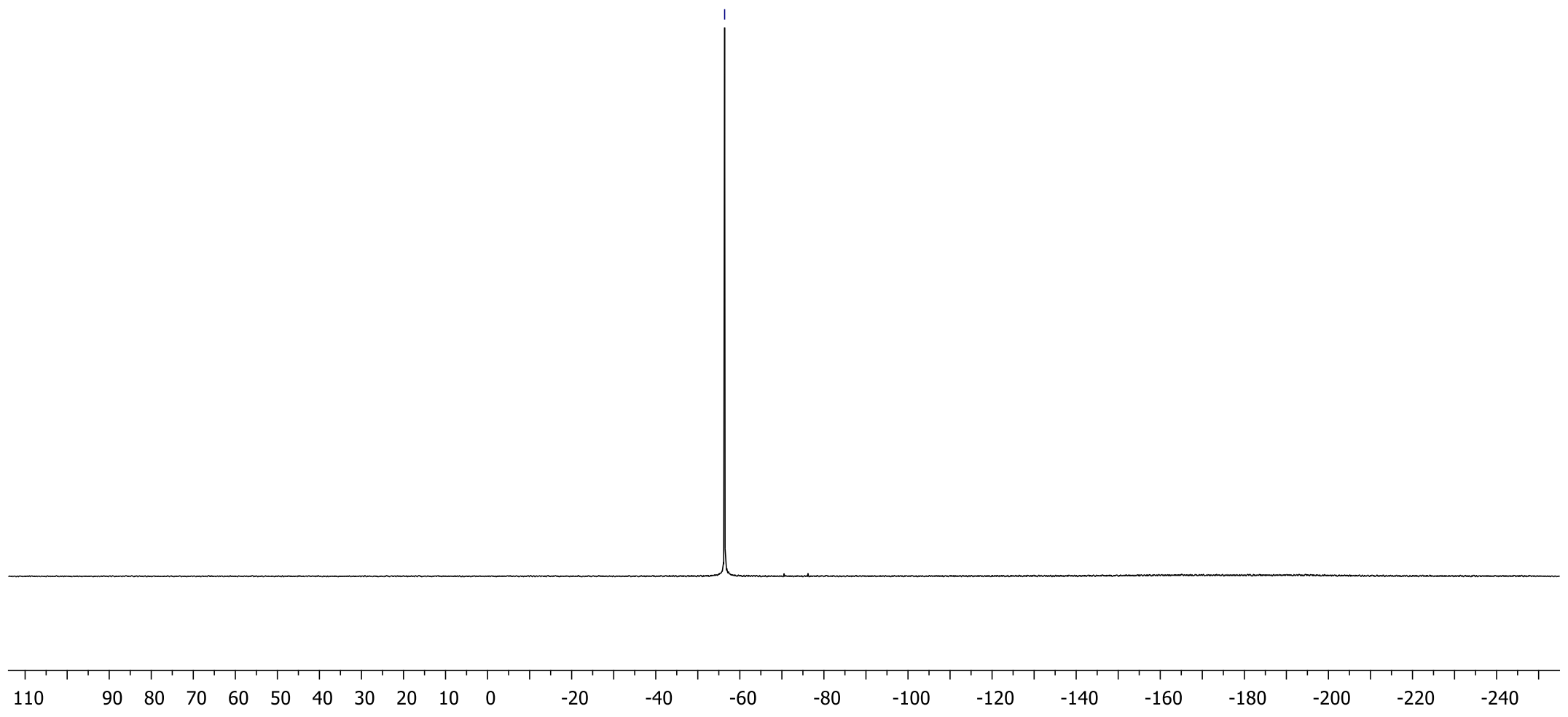


Compound 28a*

${ }^{1} \mathrm{H} \mathrm{NMR}\left(400 \mathrm{MHz}, \mathrm{CDCl}_{3}\right)$
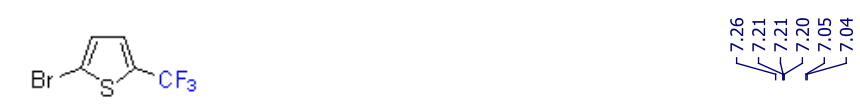

inin
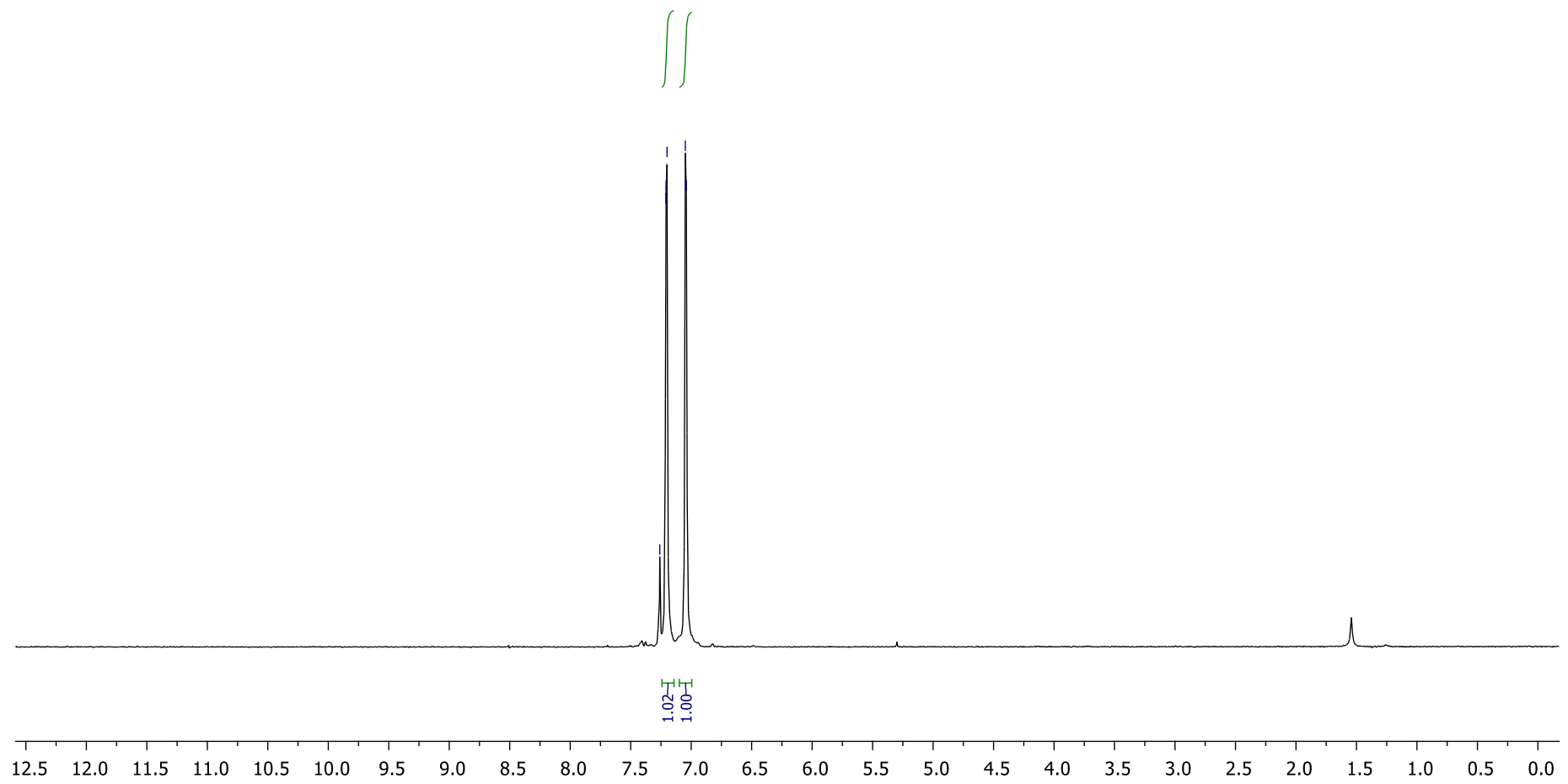

Compound 28a is also described in: J. Fluorine Chem. 2010, 131, 98-105. 


$$
=1
$$


${ }^{19} \mathrm{~F}$ NMR $\left(376 \mathrm{MHz}, \mathrm{CDCl}_{3}\right)$

$\mathrm{Br}_{\mathrm{S}} \prod_{\mathrm{CF}_{3}}$

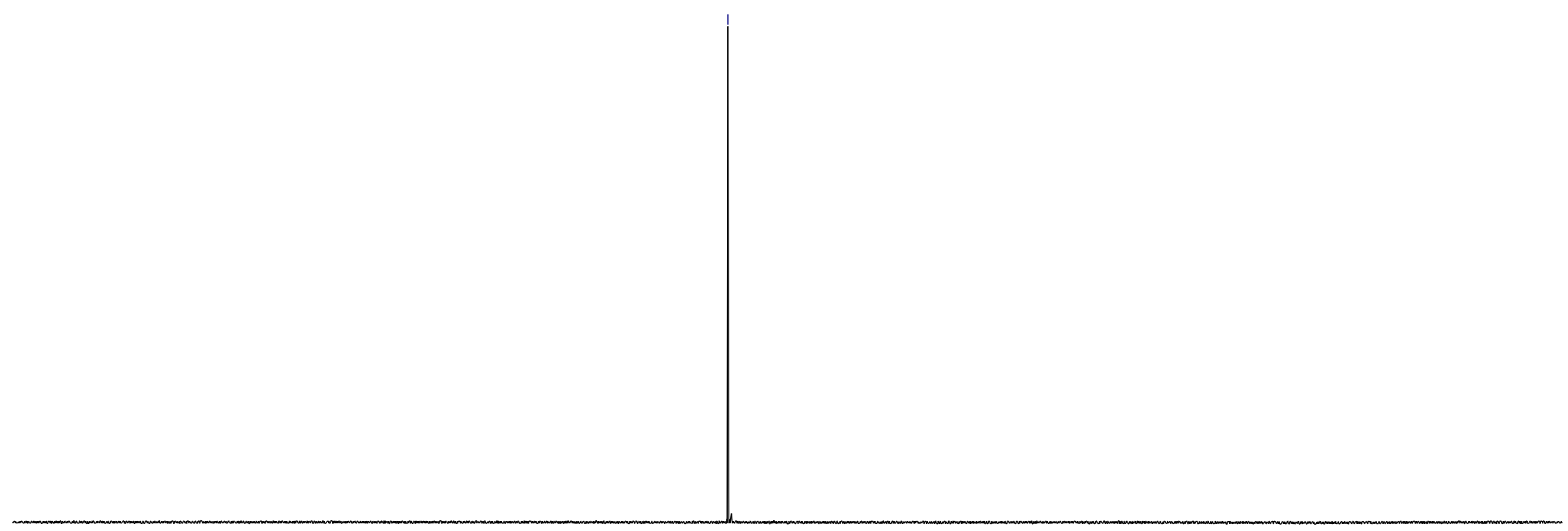

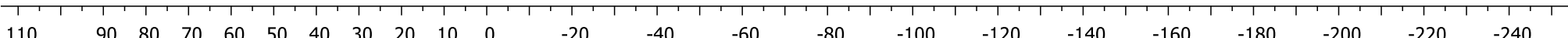


Compound 29a

${ }^{1} \mathrm{H}$ NMR (400 MHz, $\mathrm{CDCl}_{3}$ )

$\prod_{\mathrm{S}}^{\mathrm{Br}}$

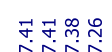

Vil

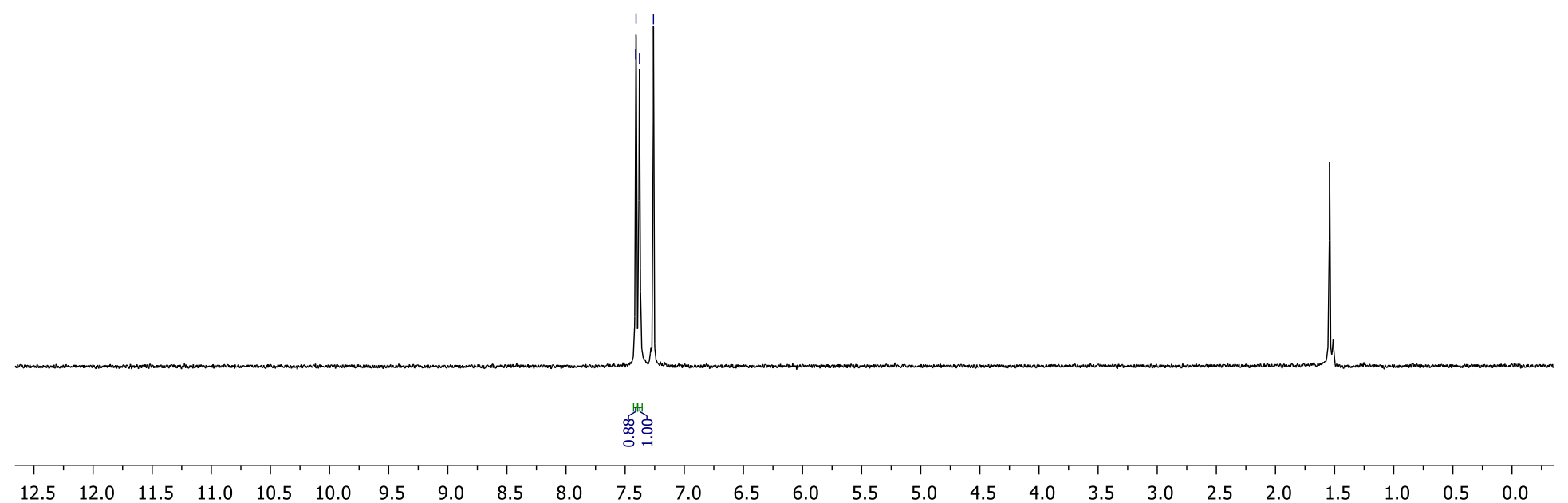


${ }^{13} \mathrm{C}\left\{{ }^{1} \mathrm{H}\right\}$ NMR $\left(126 \mathrm{MHz}, \mathrm{CDCl}_{3}\right)$

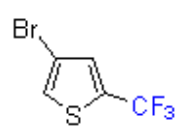

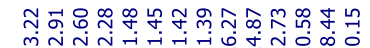

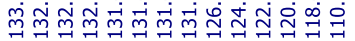

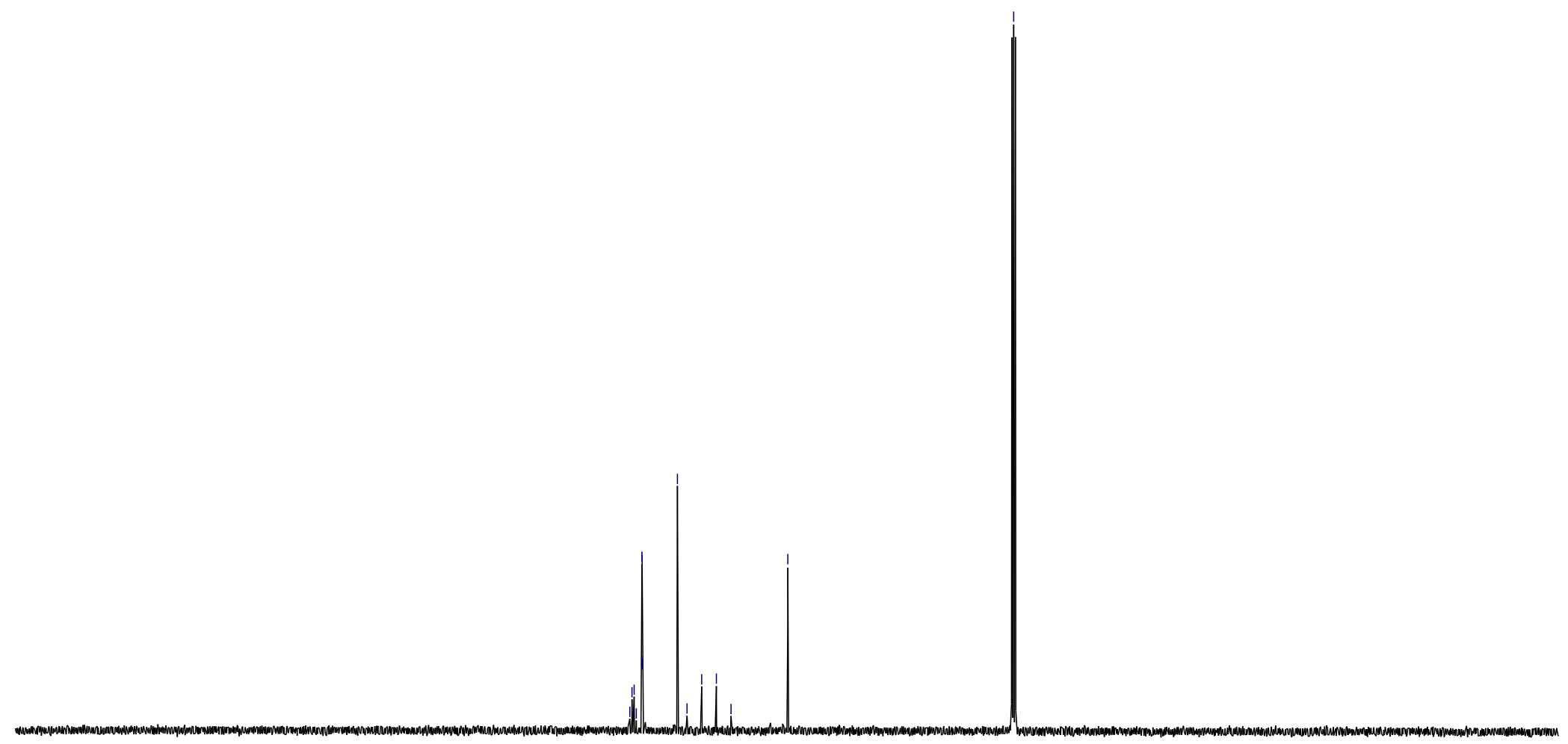


${ }^{19} \mathrm{~F}$ NMR (376 MHz, $\mathrm{CDCl}_{3}$ )

$\sum_{S} l_{C F_{3}}$

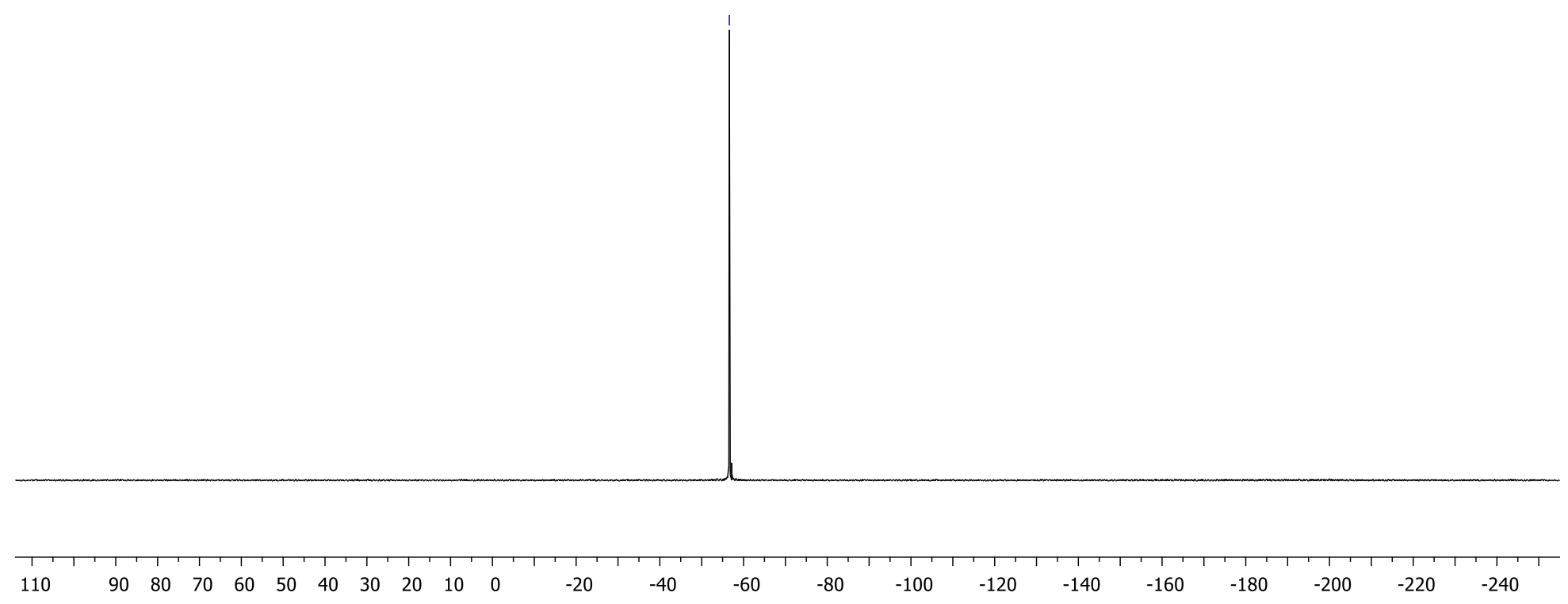


Compound 30a

${ }^{1} \mathrm{H}$ NMR $\left(400 \mathrm{MHz}, \mathrm{CDCl}_{3}\right)$

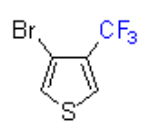

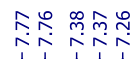
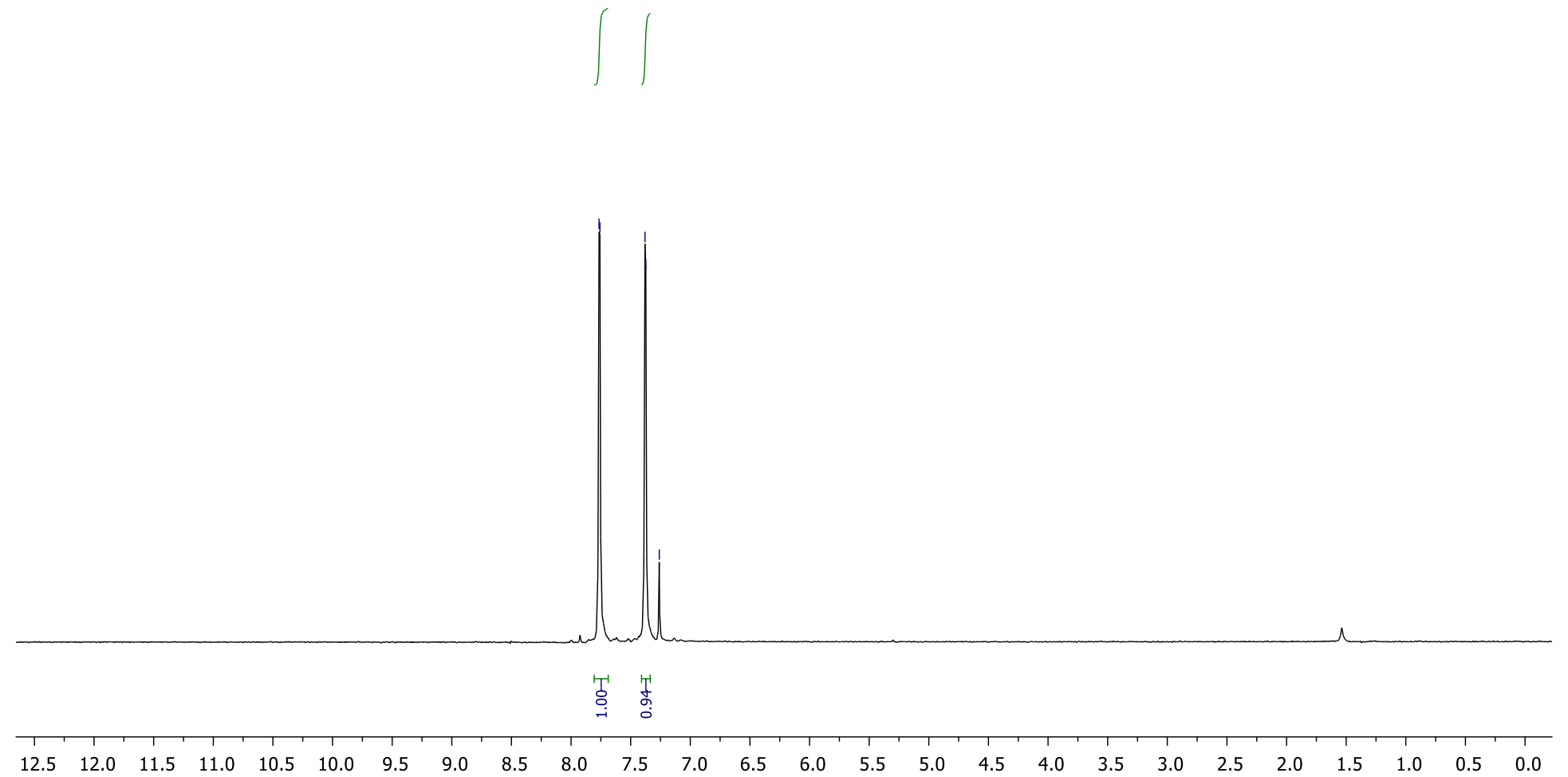


$$
\text { i }
$$


${ }^{19} \mathrm{~F}$ NMR $\left(376 \mathrm{MHz}, \mathrm{CDCl}_{3}\right)$

${ }_{5}^{\mathrm{Br}}$

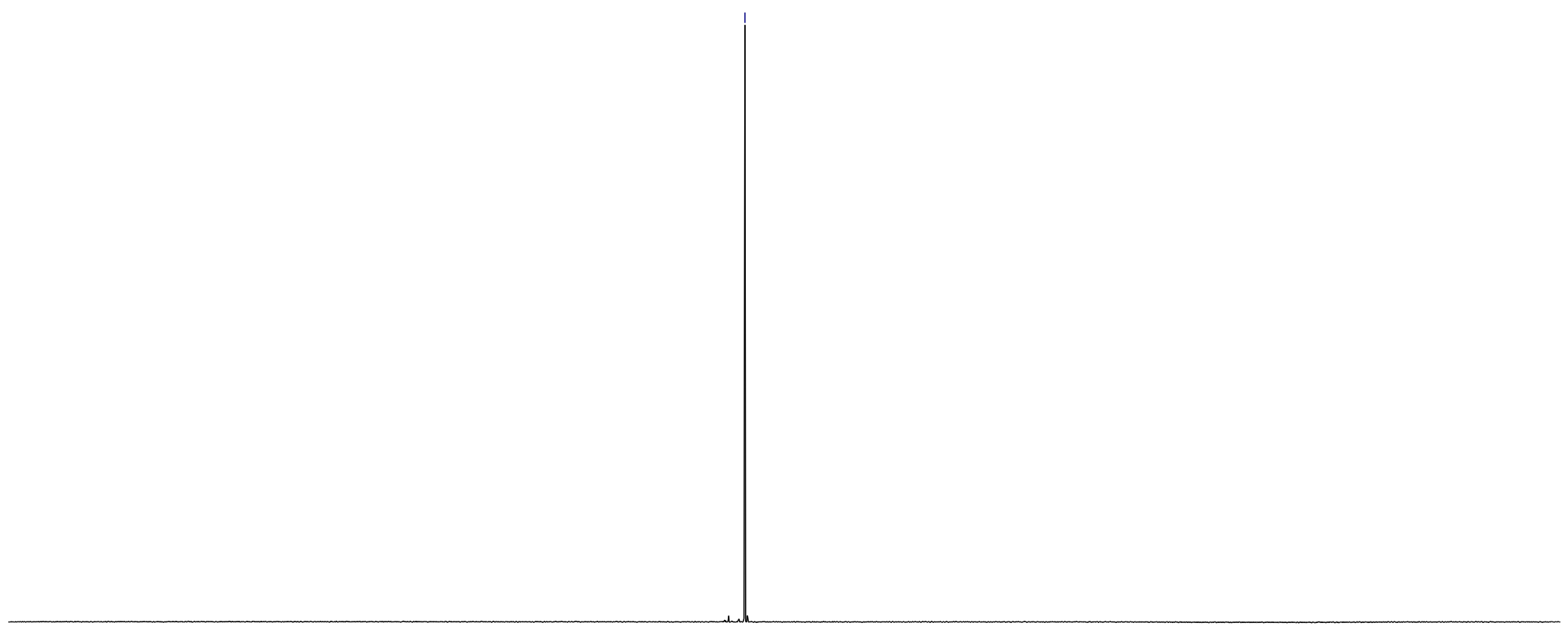


Compound 31a

${ }^{1} \mathrm{H}$ NMR (400 MHz, $\mathrm{CDCl}_{3}$ )

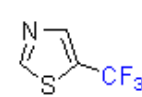

$\stackrel{\substack{\infty \\ \infty}}{\substack{\infty \\ \infty}}$

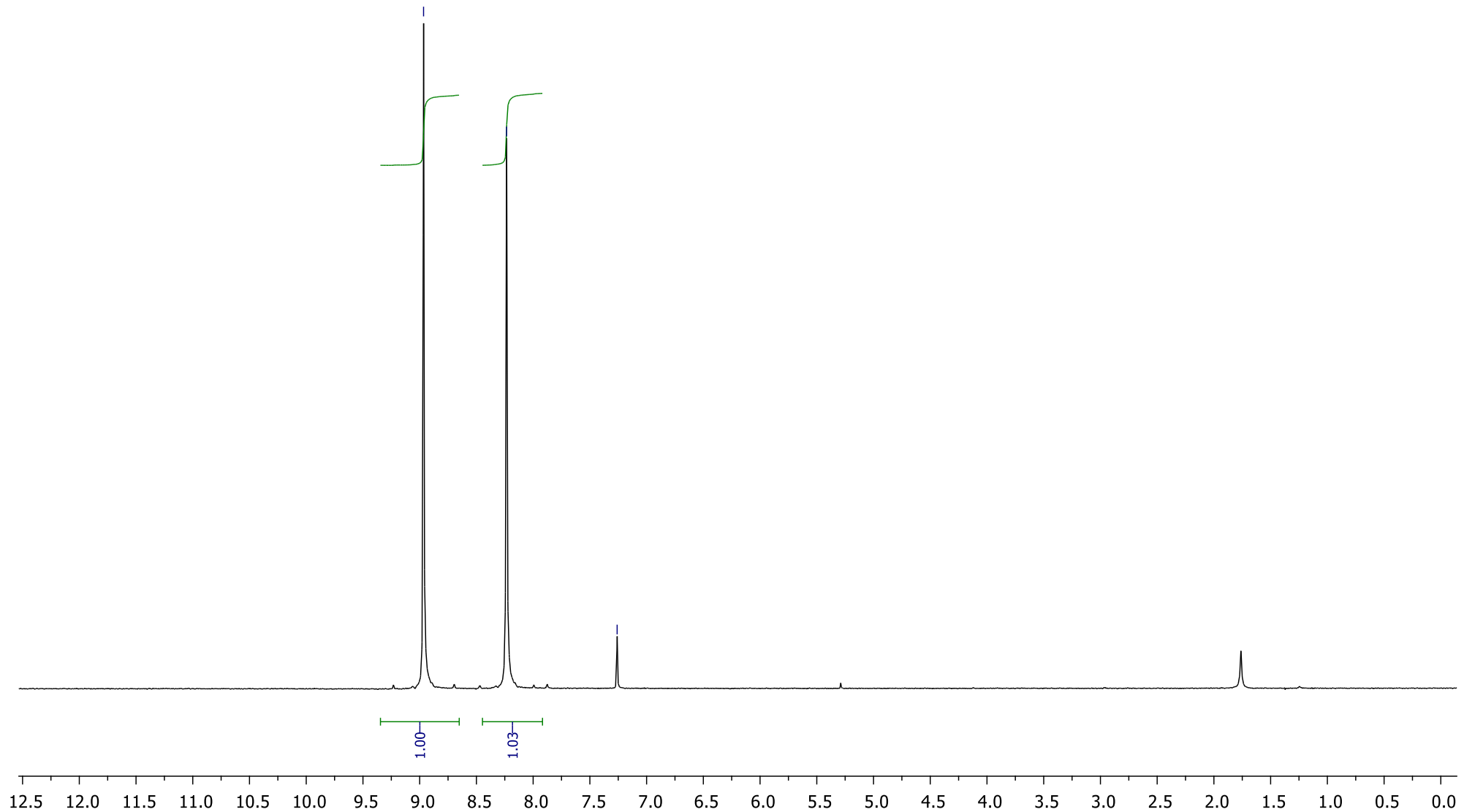


${ }^{13} \mathrm{C}\left\{{ }^{1} \mathrm{H}\right\}$ NMR $\left(101 \mathrm{MHz}, \mathrm{CDCl}_{3}\right)$

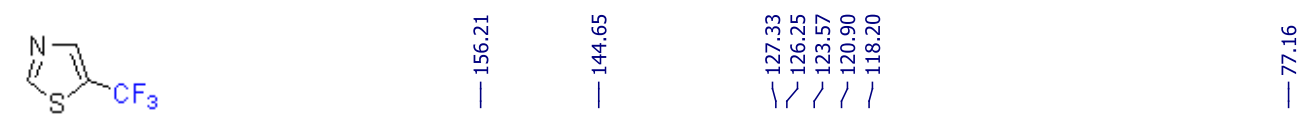

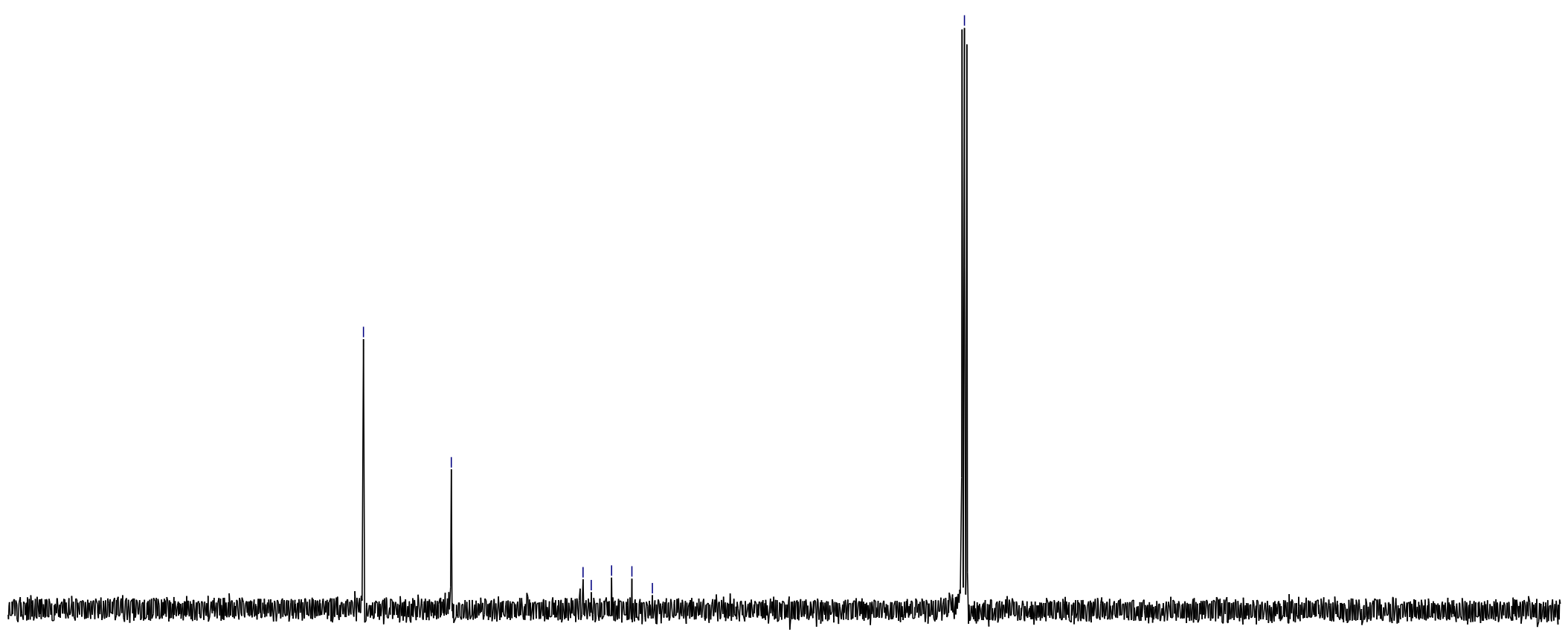

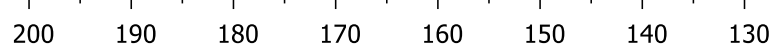


${ }^{19} \mathrm{~F}$ NMR (376 MHz, $\left.\mathrm{CDCl}_{3}\right)$

${ }_{S}^{\mathrm{N}} \mathrm{CF}_{3}$

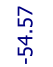

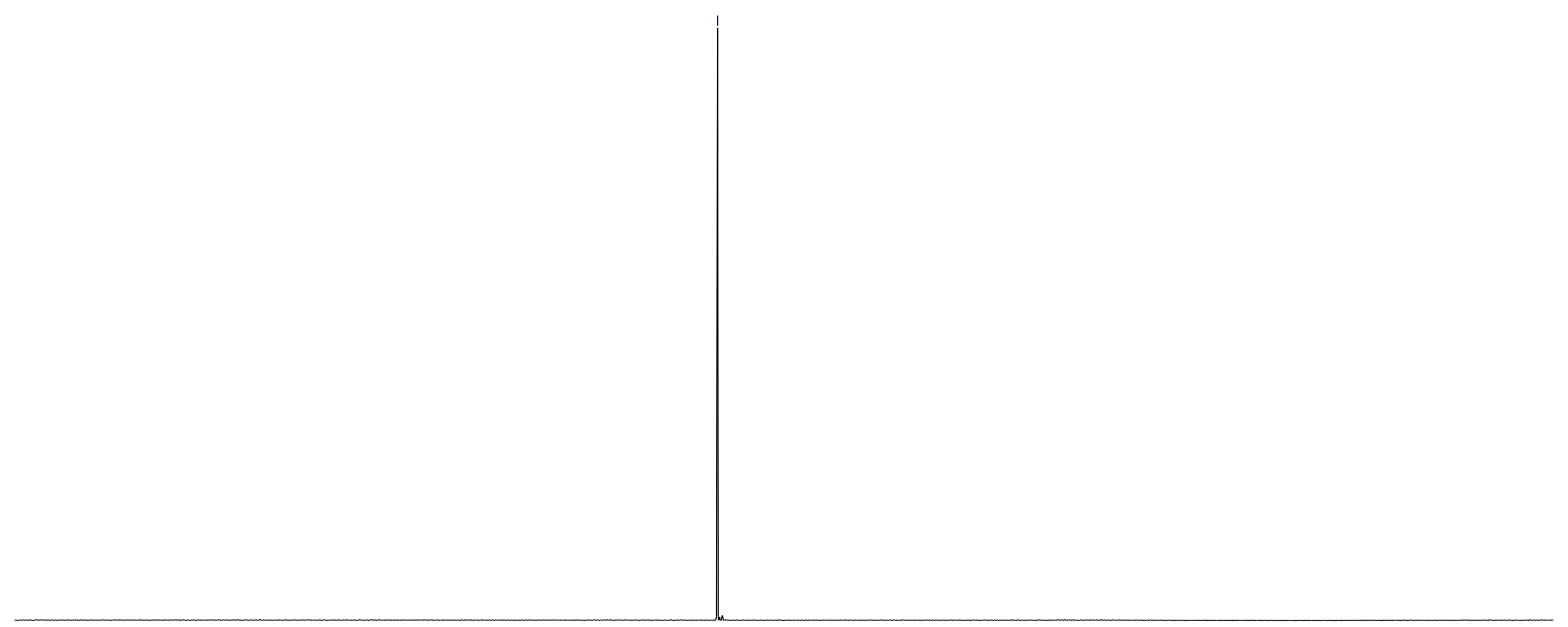

$\begin{array}{llllllllll}110 & 90 & 80 & 70 & 60 & 50 & 40 & 30 & 20 & 10\end{array}$

$-20$

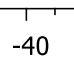

$-60$ 
Compound 32a

${ }^{1} \mathrm{H}$ NMR (400 MHz, $\mathrm{CDCl}_{3}$ )

${ }_{\mathrm{Br}} \stackrel{\mathrm{N}}{4} \mathrm{~h}_{\mathrm{CF}_{3}}$

$\stackrel{8}{i} \stackrel{\stackrel{2}{i}}{i}$

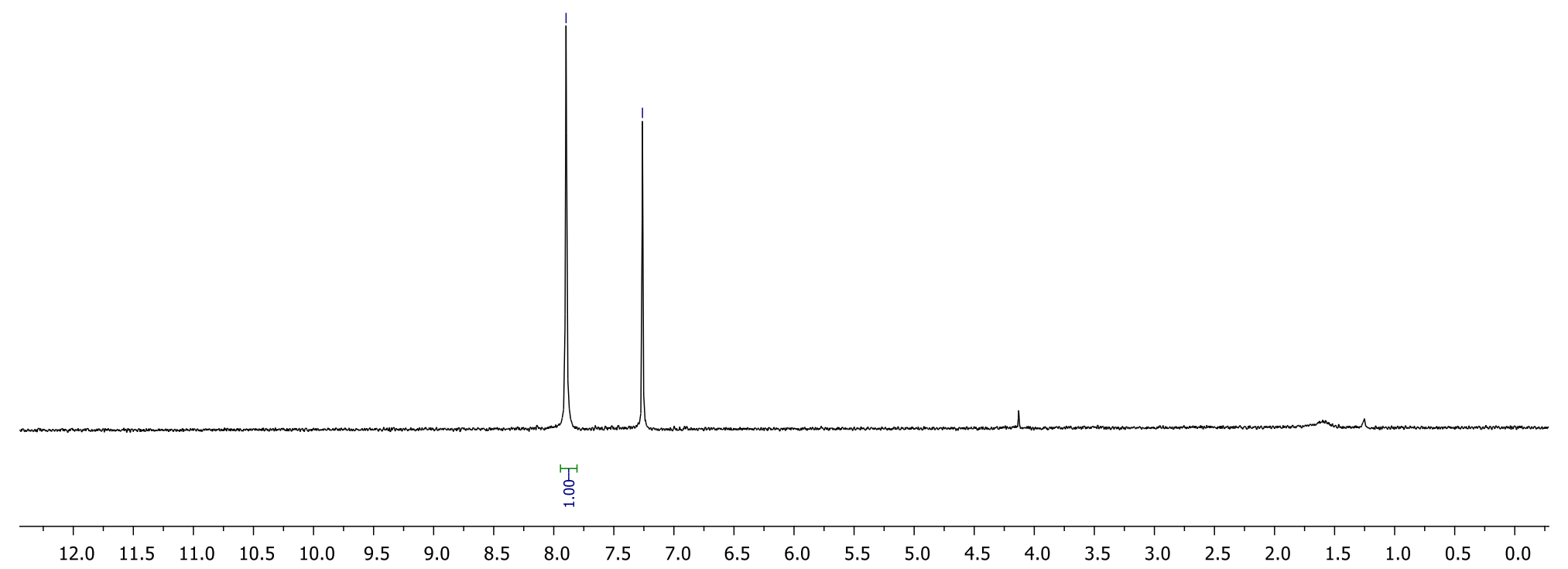


${ }^{13} \mathrm{C}\left\{{ }^{1} \mathrm{H}\right\}$ NMR (126 MHz, $\left.\mathrm{CDCl}_{3}\right)$
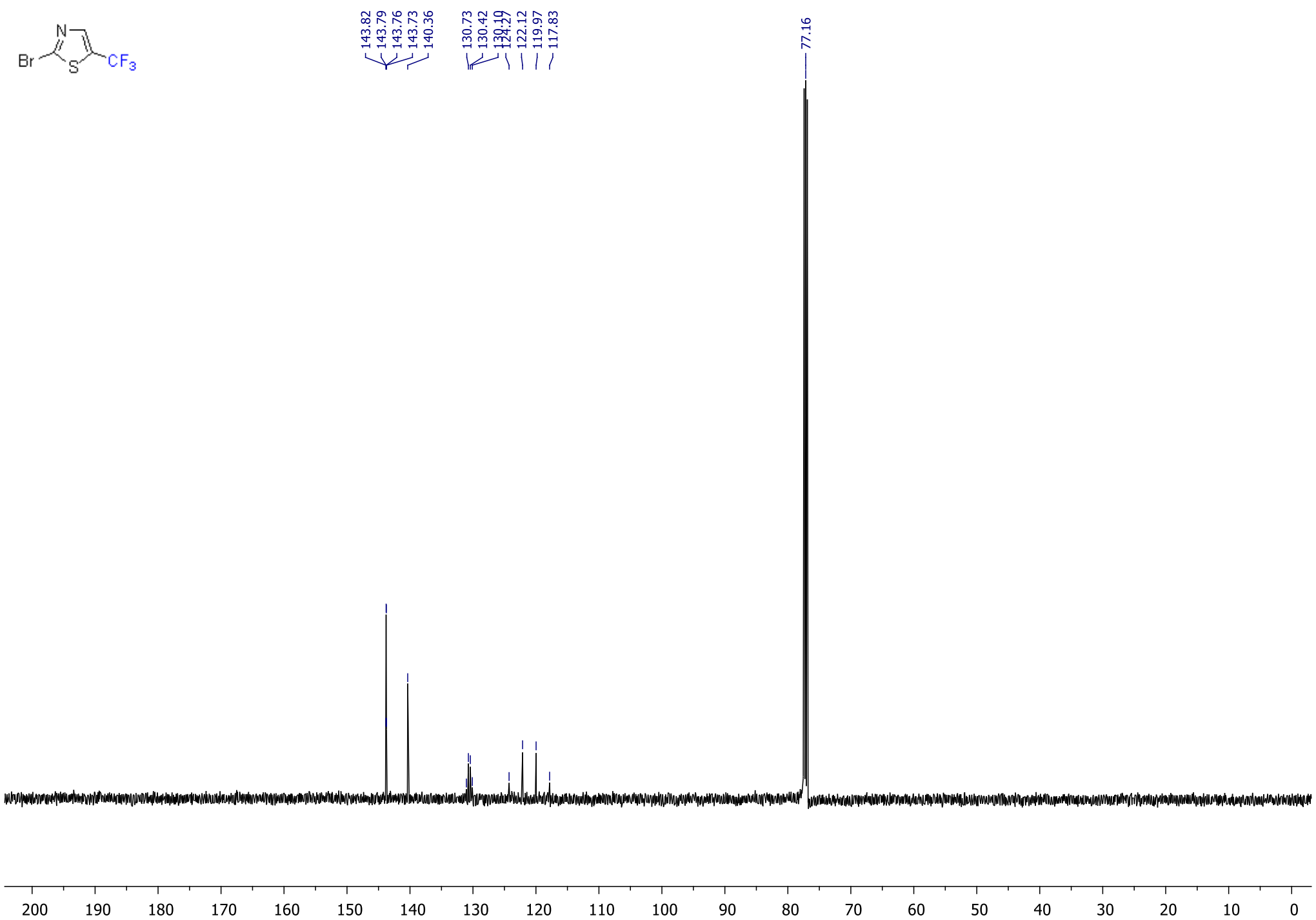
${ }^{19} \mathrm{~F}$ NMR (376 MHz, $\mathrm{CDCl}_{3}$ )

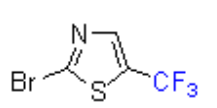

旋

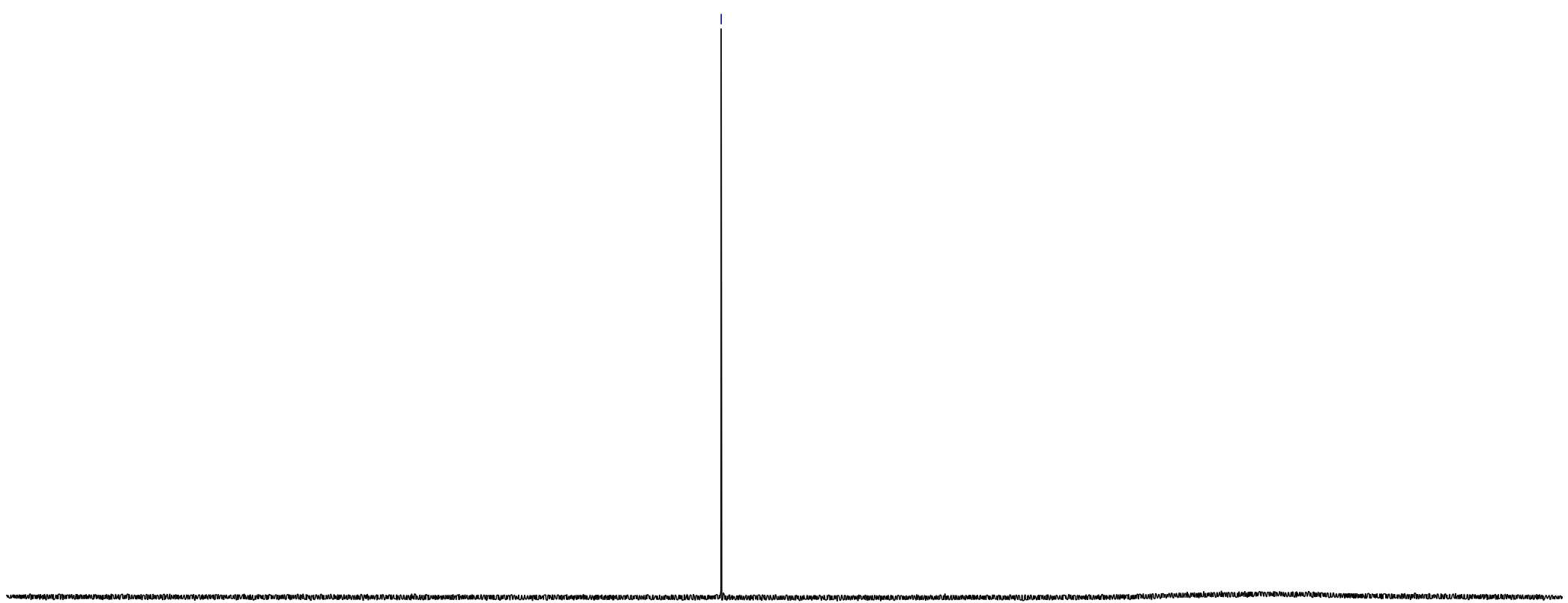


Compound 33a*

${ }^{1} \mathrm{H} \mathrm{NMR}\left(400 \mathrm{MHz}, \mathrm{CDCl}_{3}\right)$

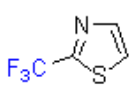

$\stackrel{i}{\operatorname{lol}} \stackrel{\substack{0 \\ 0}}{i}$
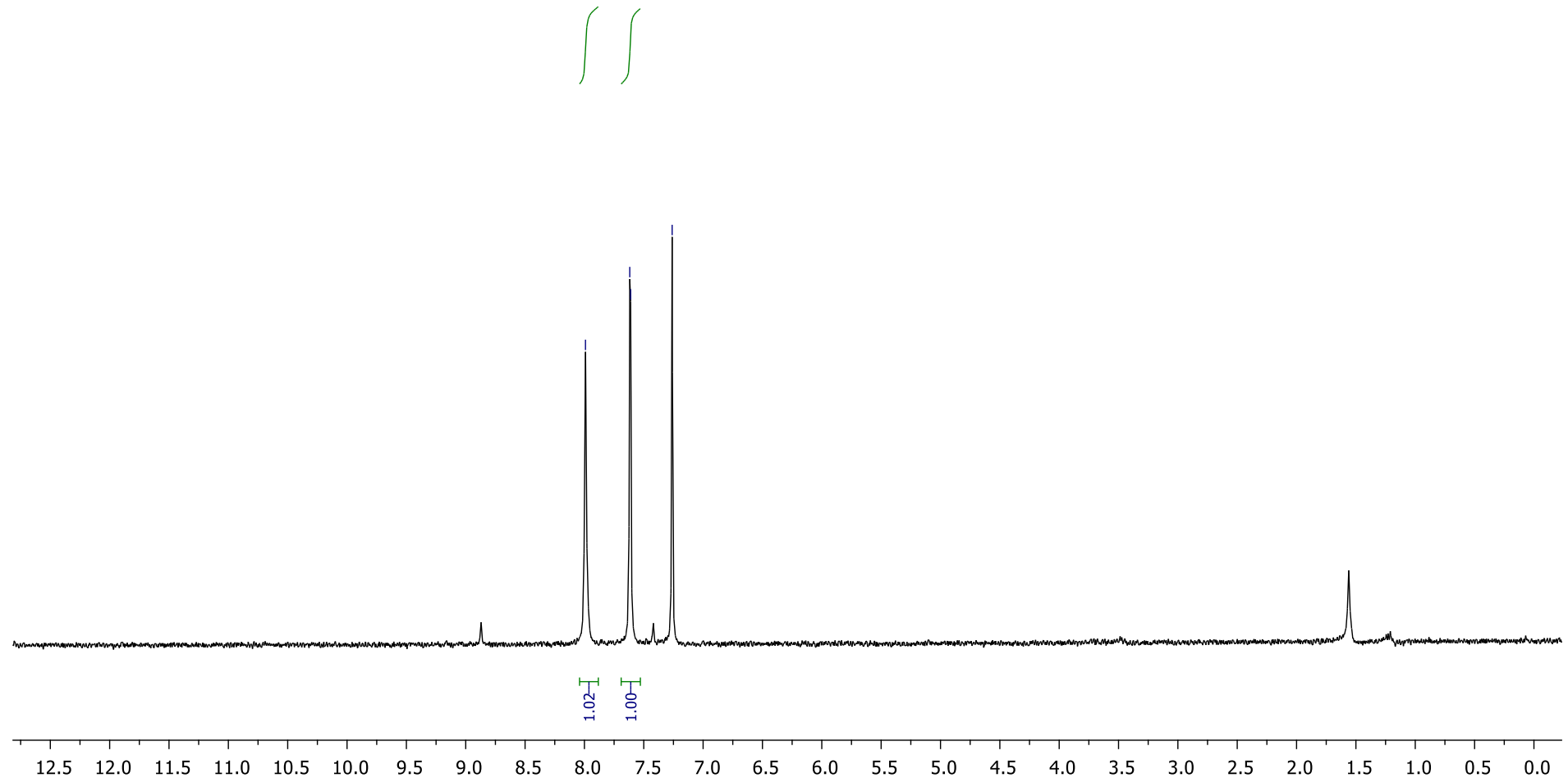

Compound 33a is also described in: J. Org. Chem. 2013, 78, 22, 11126-11146. 
${ }^{13} \mathrm{C}\left\{{ }^{1} \mathrm{H}\right\}$ NMR $\left(126 \mathrm{MHz}, \mathrm{CDCl}_{3}\right)$

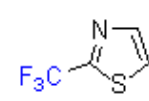

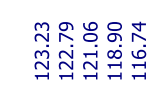

पil
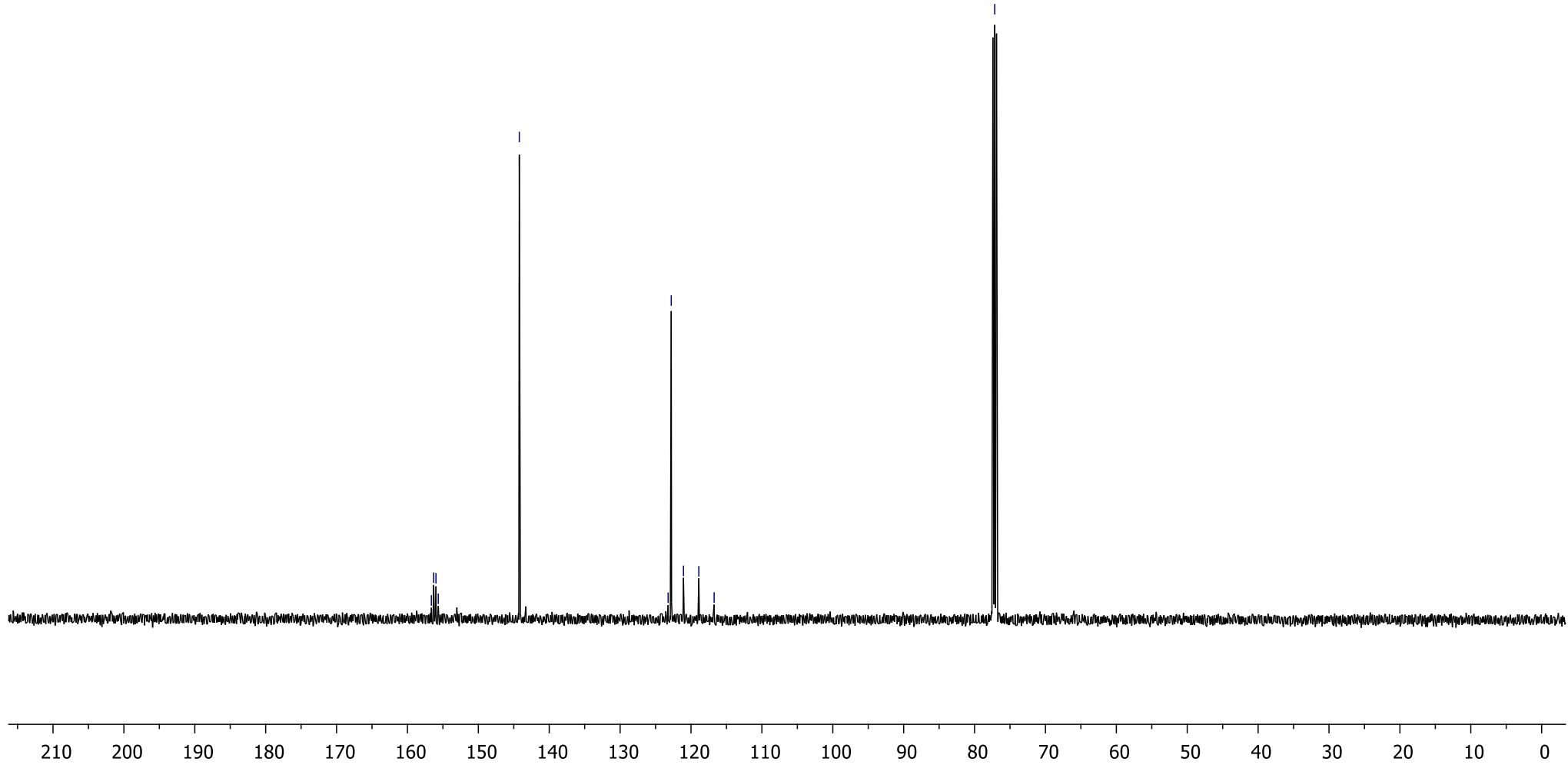
${ }^{19} \mathrm{~F}$ NMR $\left(376 \mathrm{MHz}, \mathrm{CDCl}_{3}\right)$

$\mathrm{F}_{3} \mathrm{C}-\sum^{\mathrm{N}}$

$\underset{\substack{i \\ i}}{\stackrel{0}{i}}$

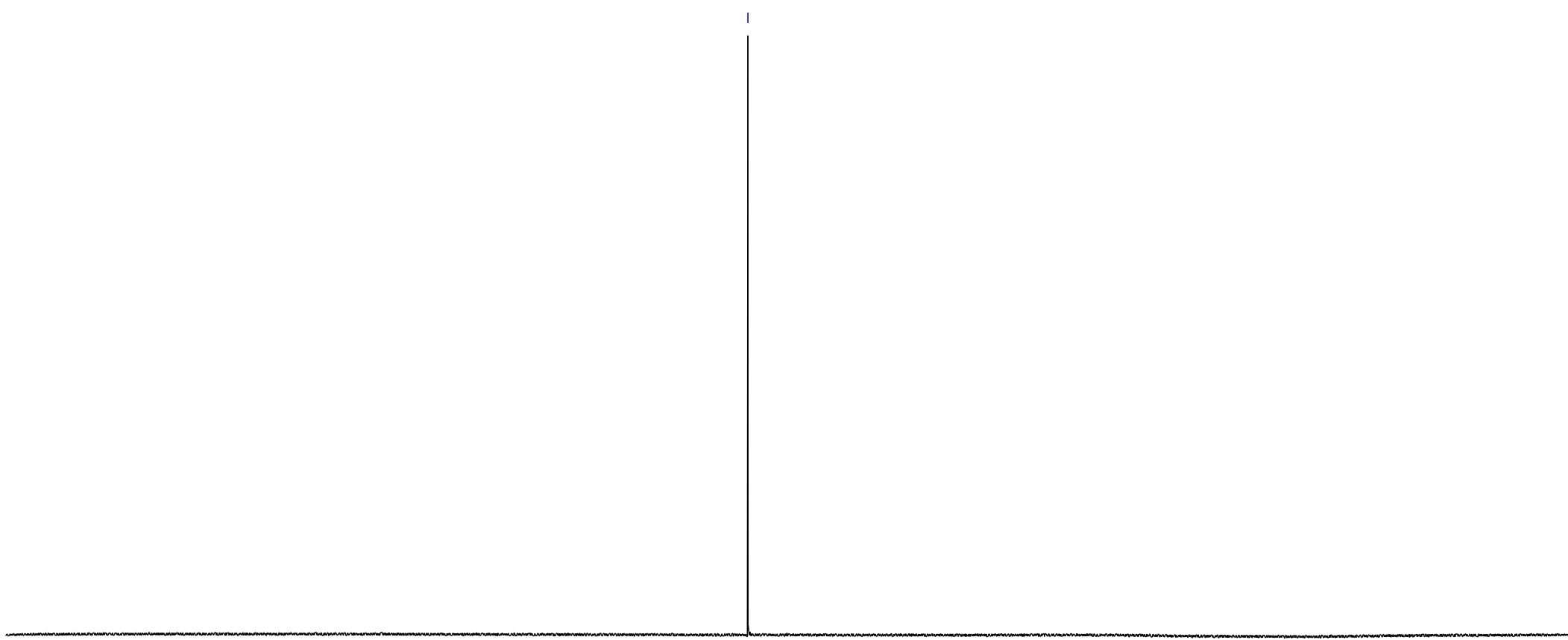

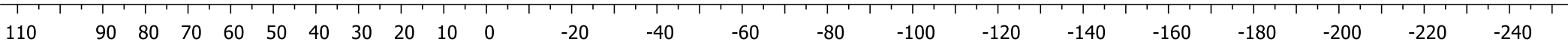


Compound 34a

${ }^{1} \mathrm{H}$ NMR (400 MHz, $\mathrm{CDCl}_{3}$ )

${ }_{{ }_{3} C} \stackrel{N}{N} \prod_{B r}$

$\stackrel{\infty}{i} \stackrel{\substack{i \\ i}}{i}$

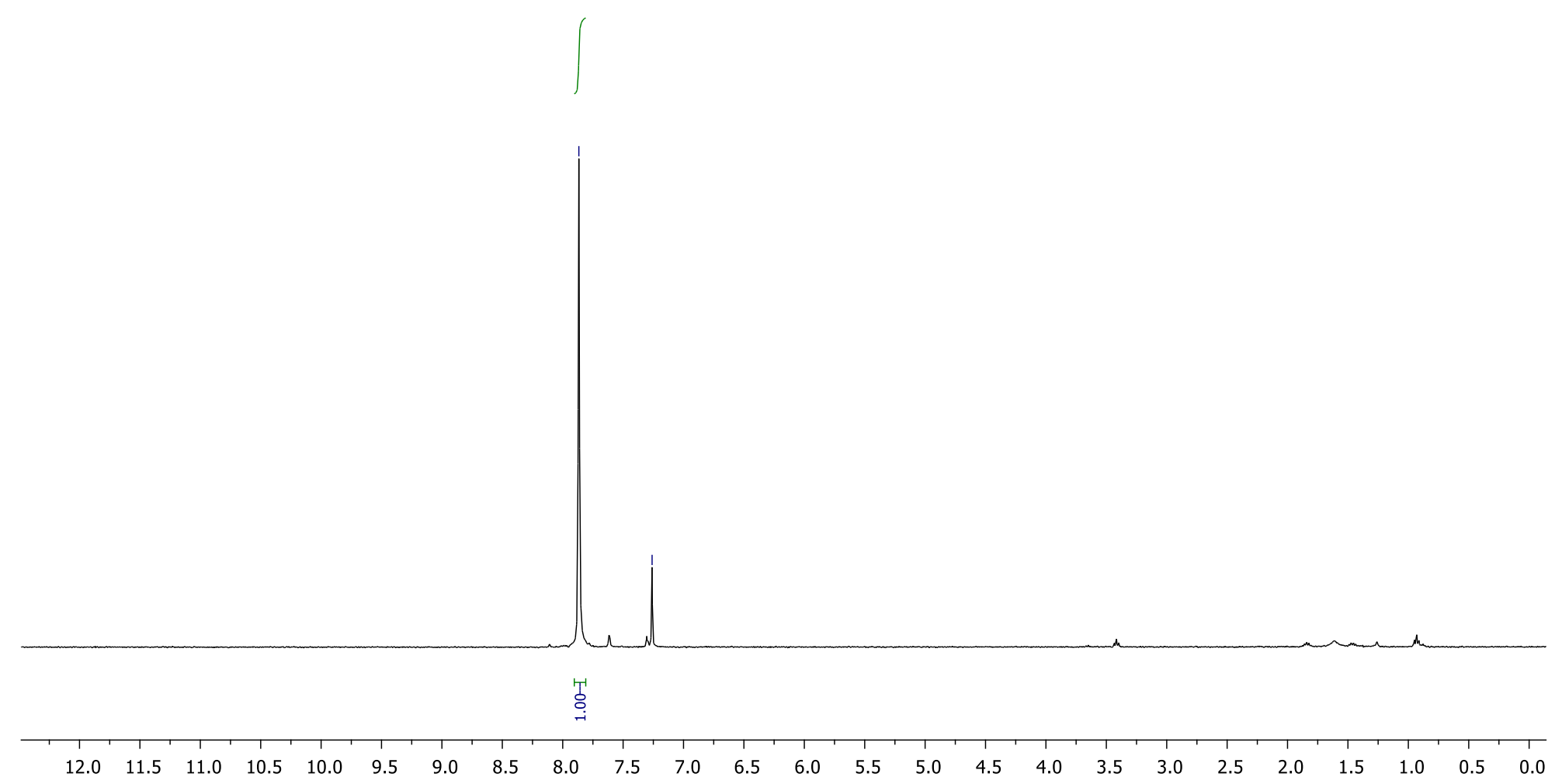


${ }^{13} \mathrm{C}\left\{{ }^{1} \mathrm{H}\right\}$ NMR (126 MHz, $\left.\mathrm{CDCl}_{3}\right)$

$\mathrm{F}_{3} \stackrel{\mathrm{C}}{\mathrm{N}} \prod_{\mathrm{Br}}$

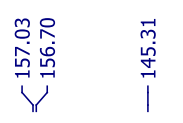

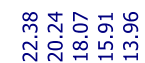

$\stackrel{\substack{i \\ i}}{i}$

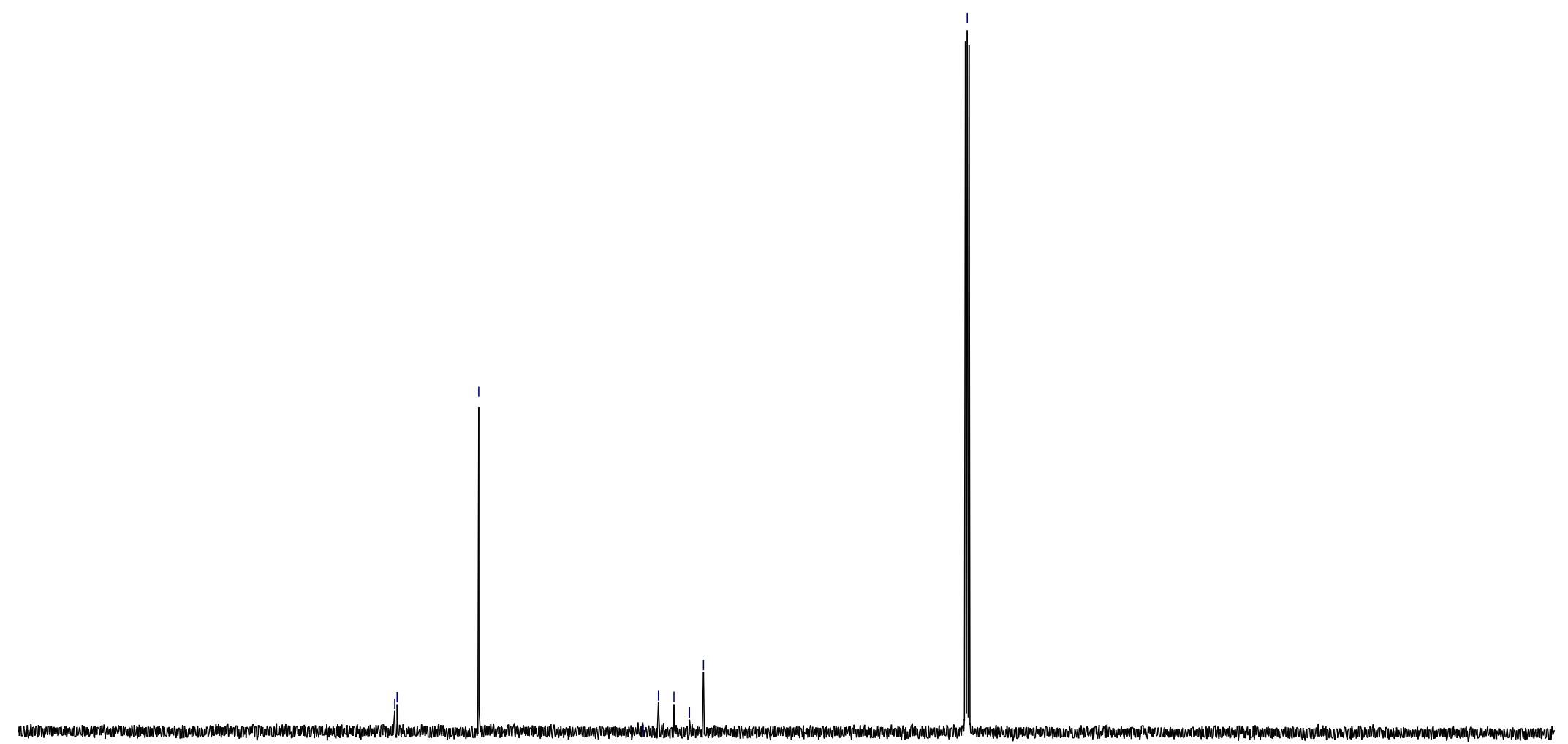


${ }^{19} \mathrm{~F}$ NMR (376 MHz, $\mathrm{CDCl}_{3}$ )

${ }_{\mathrm{F}_{3}} \mathrm{C} \stackrel{\mathrm{N}}{\not \prod_{\mathrm{Br}}}$
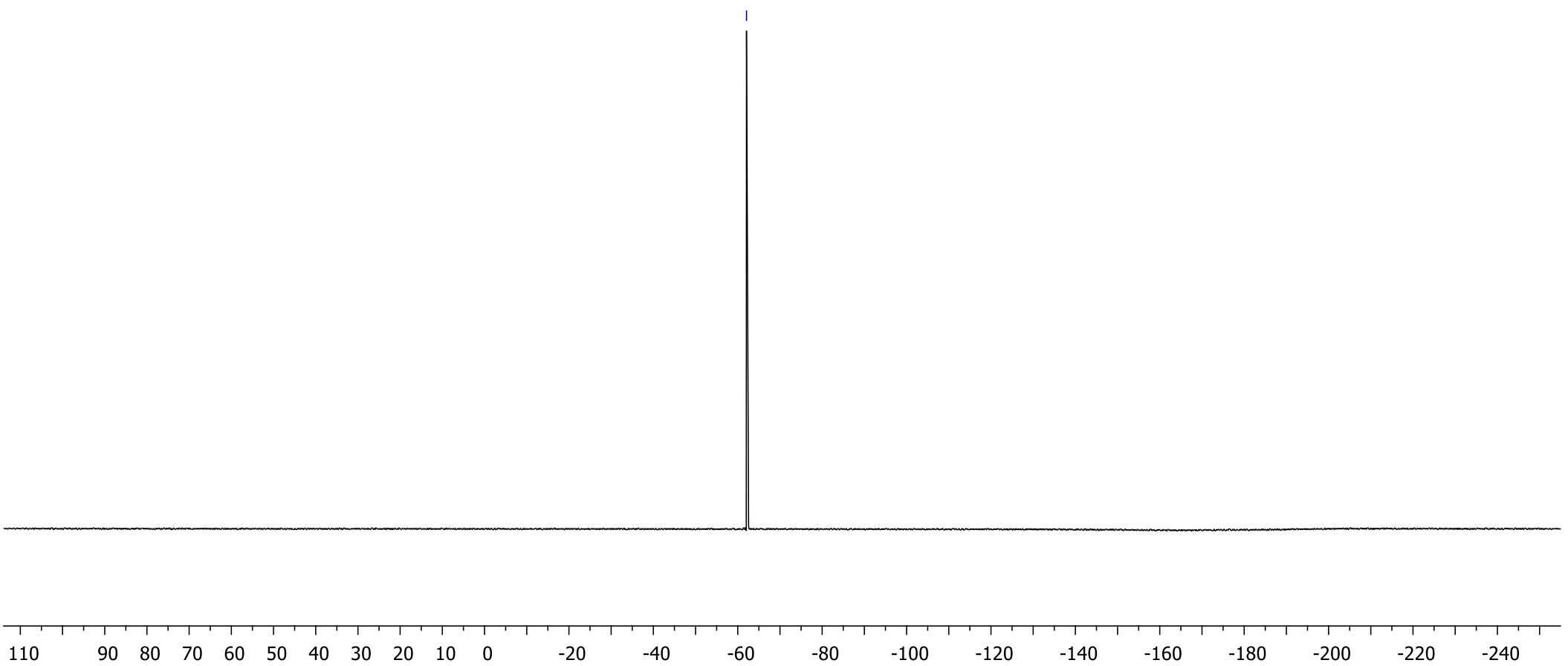
Compound 35a*

${ }^{1} \mathrm{H}$ NMR $\left(400 \mathrm{MHz}, \mathrm{CDCl}_{3}\right)$
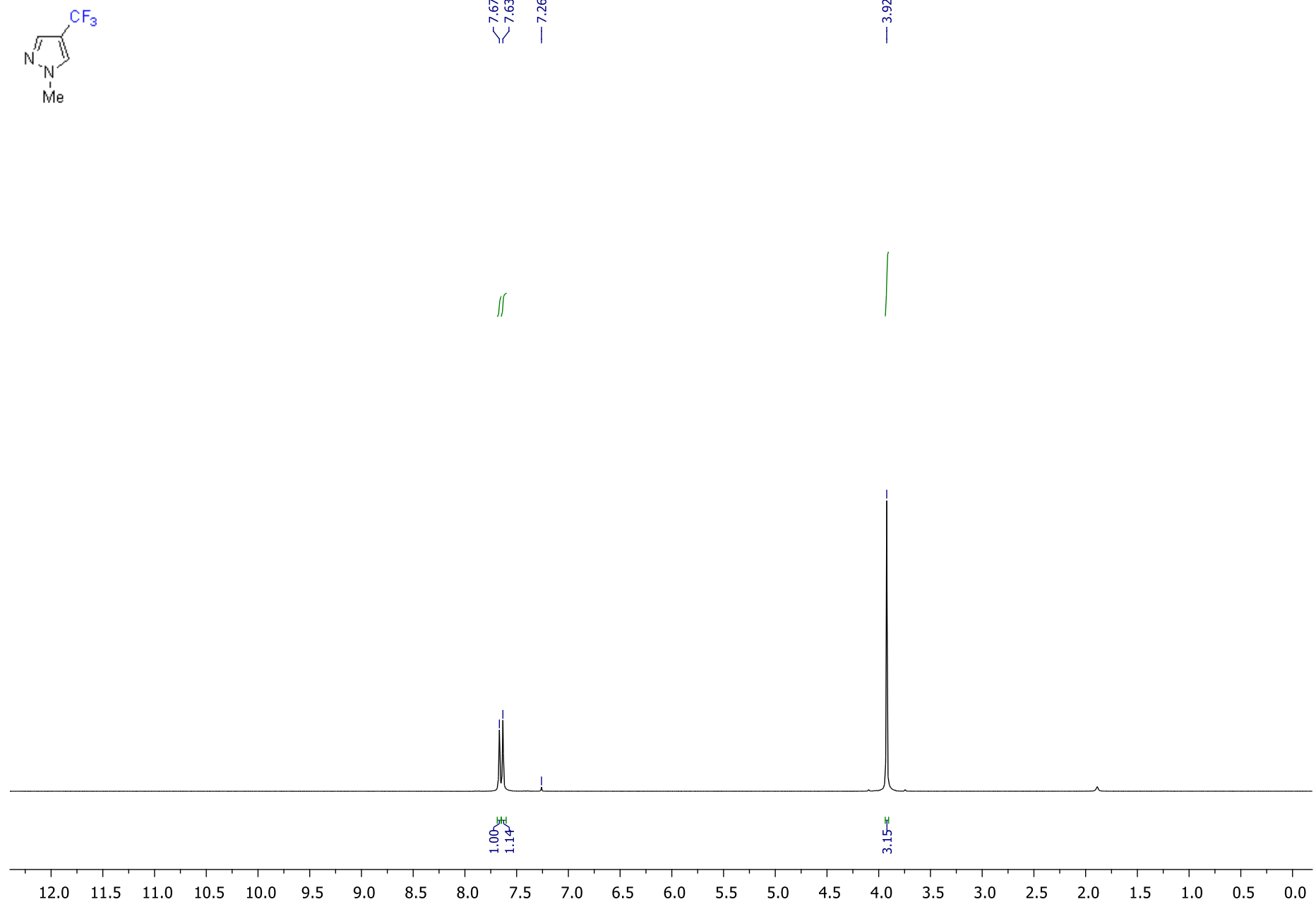

Compound 35a is also described in: a) Tetrahedron Lett. 1996, 37, 11, 1829-1832; b) J. Org. Chem. 2013, 78, 24, 12837-12843; c) Org. Lett. 2015, 17, 9, 2086-2089. 
${ }^{13} \mathrm{C}\left\{{ }^{1} \mathrm{H}\right\}$ NMR $\left(101 \mathrm{MHz}, \mathrm{CDCl}_{3}\right)$

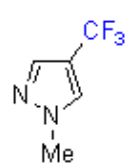

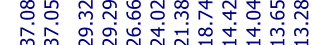

प vil

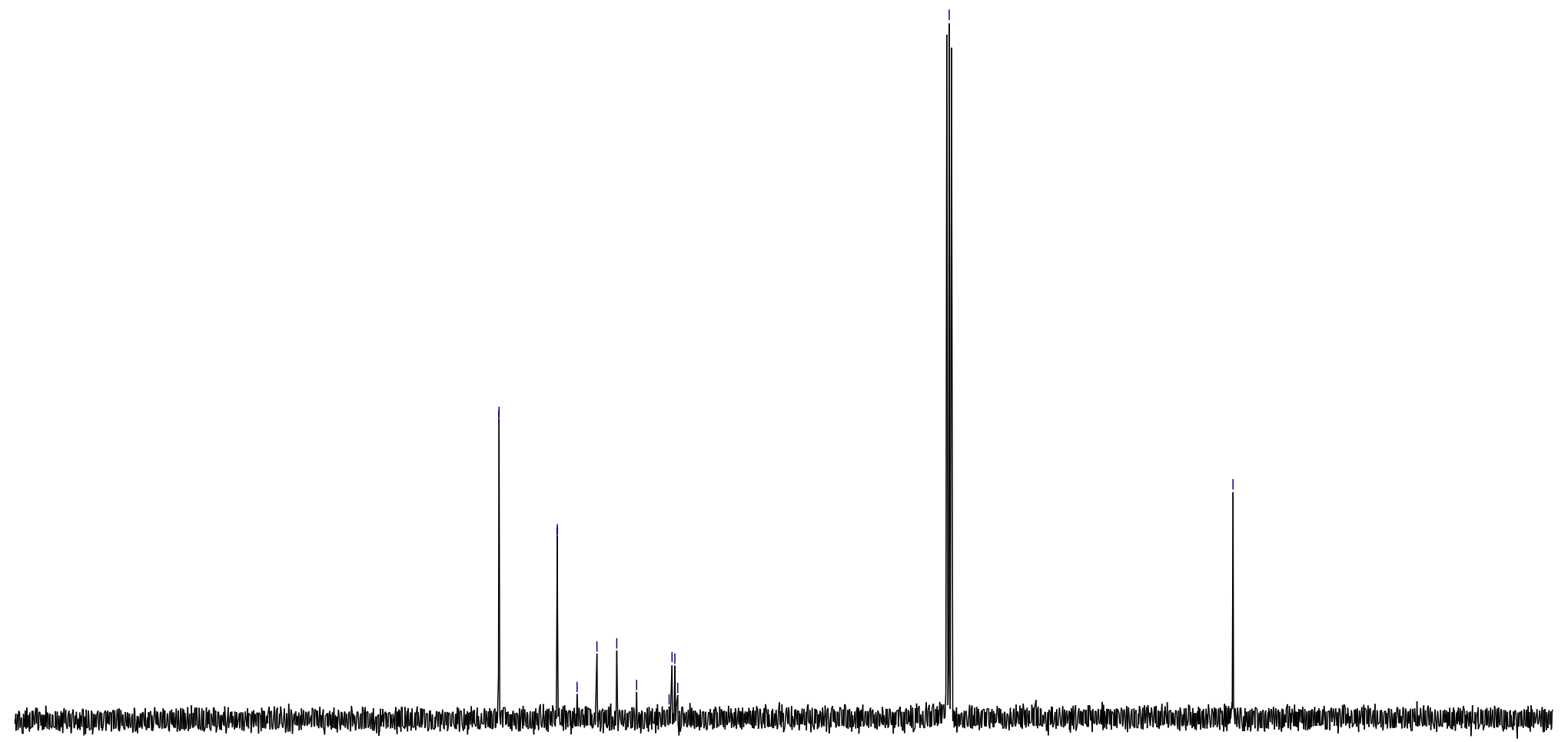


${ }^{19} \mathrm{~F}$ NMR $\left(376 \mathrm{MHz}, \mathrm{CDCl}_{3}\right)$

$\underbrace{\mathrm{CF}_{3}}_{\substack{n \\ \text { Me }}}$

$\stackrel{\substack{\infty \\ \dot{\varphi}}}{i}$

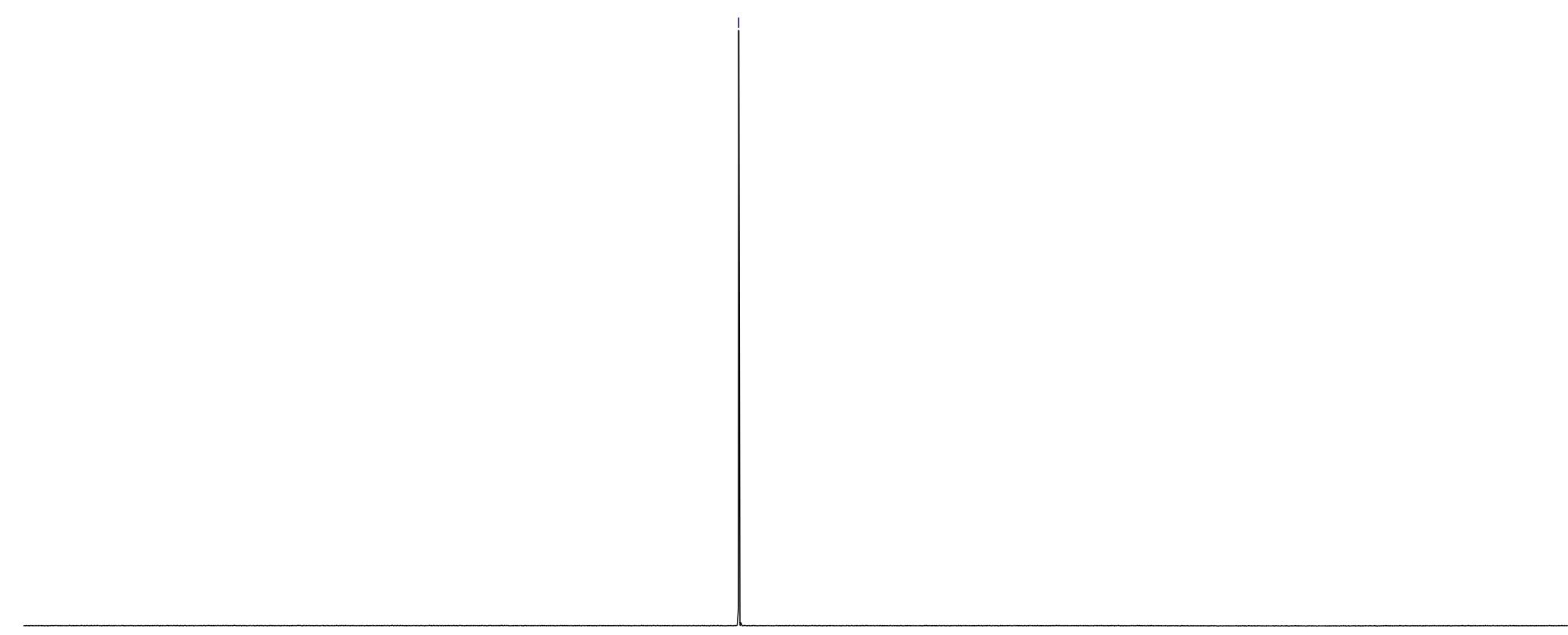

$110 \quad \begin{array}{llllllllllllllllllllllll} & 90 & 70 & 60 & 50 & 40 & 30 & 20 & 10 & 0 & -20 & -40 & -60 & -80 & -100 & -120 & -140 & -160 & -180 & -200 & -220 & -240 & \end{array}$ 

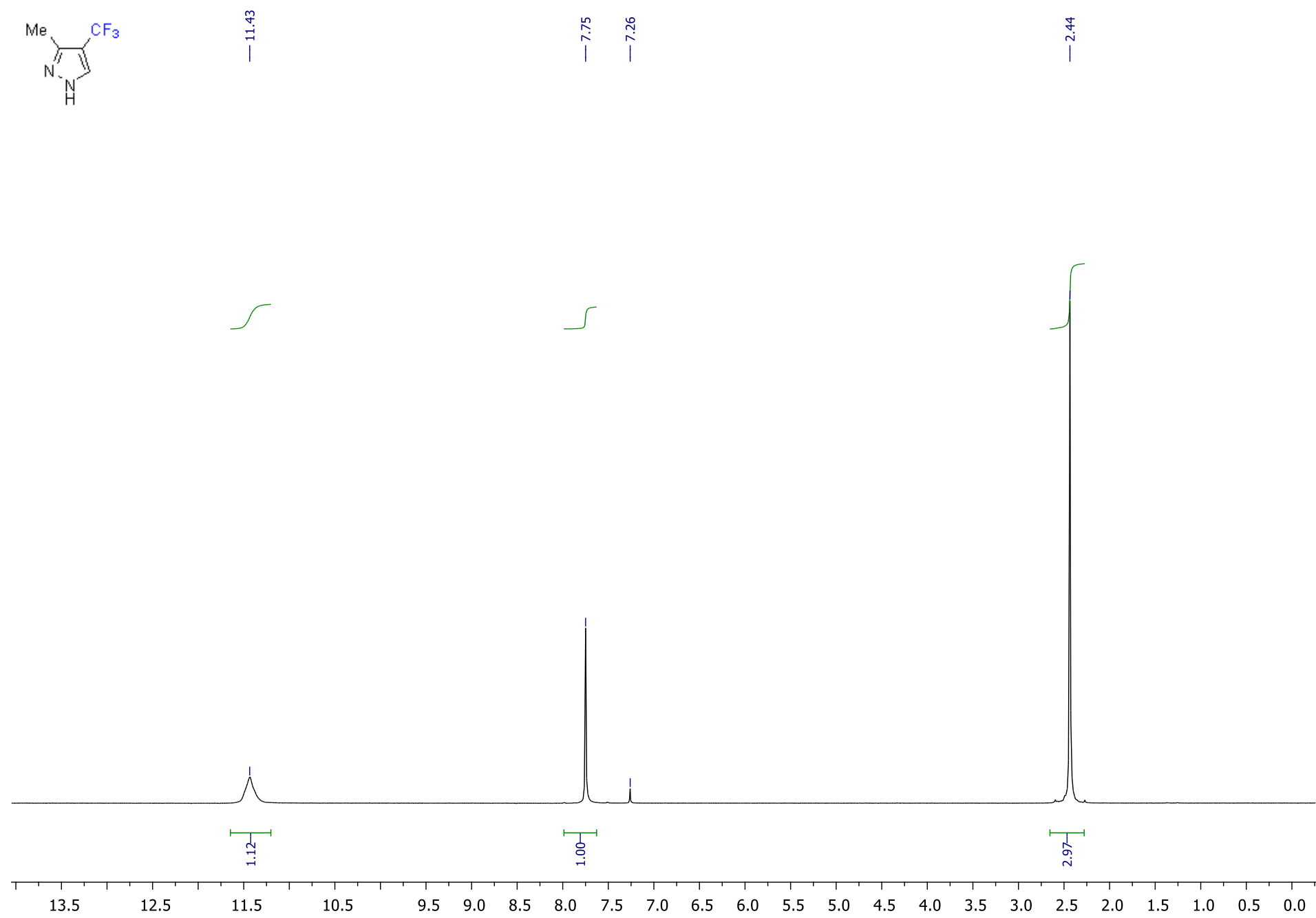

Compound 36a is also described in: J. Fluorine Chem. 2010, 131, 98-105. 


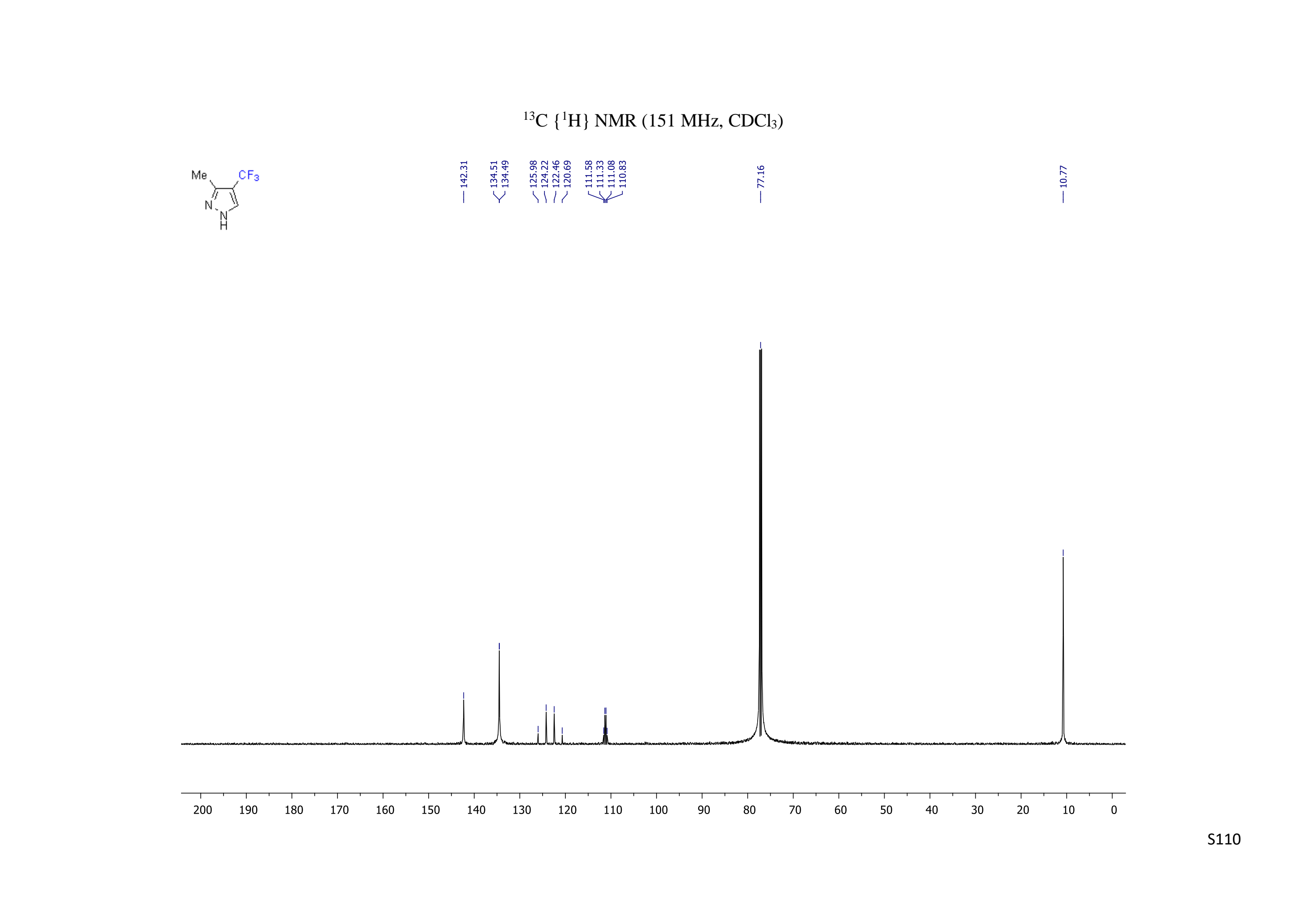


${ }^{19} \mathrm{~F}$ NMR $\left(376 \mathrm{MHz}, \mathrm{CDCl}_{3}\right)$

Me

$\stackrel{\stackrel{m}{?}}{\stackrel{m}{?}}$

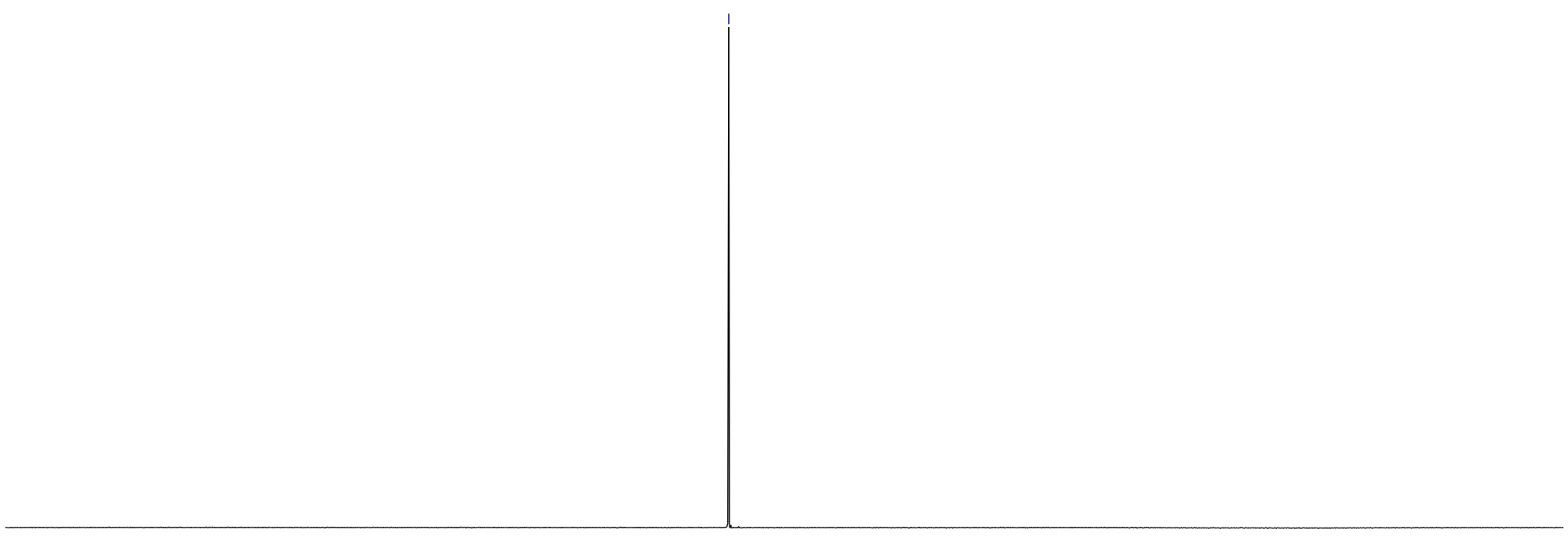

$110 \quad$\begin{tabular}{rlllllllllllllllllllllllllllllll}
\hline 0 & 80 & 70 & 60 & 50 & 40 & 30 & 20 & 10 & 0 & -20 & -40 & -60 & -80 & -100 & -120 & -140 & -160 & -180 & -200 & -220 & -240
\end{tabular} 
Compound $37 \mathrm{a}^{*}$

${ }^{1} \mathrm{H}$ NMR $\left(400 \mathrm{MHz}, \mathrm{CDCl}_{3}\right)$

$$
{ }_{3} C{ }_{\mathrm{H}}^{\mathrm{Br}}
$$

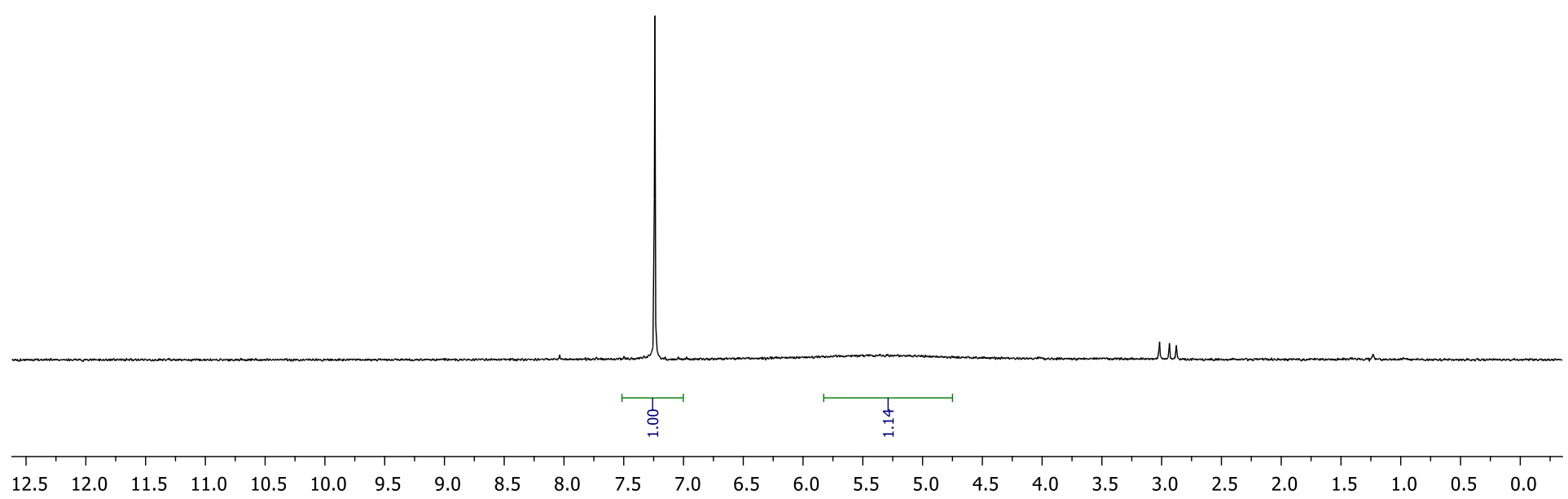

Compound 37a is also described in: J. Org. Chem. 1998, 63, 25, 9448-9454. 


$$
I
$$


${ }^{19} \mathrm{~F}$ NMR (376 MHz, $\mathrm{CDCl}_{3}$ )

等

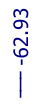

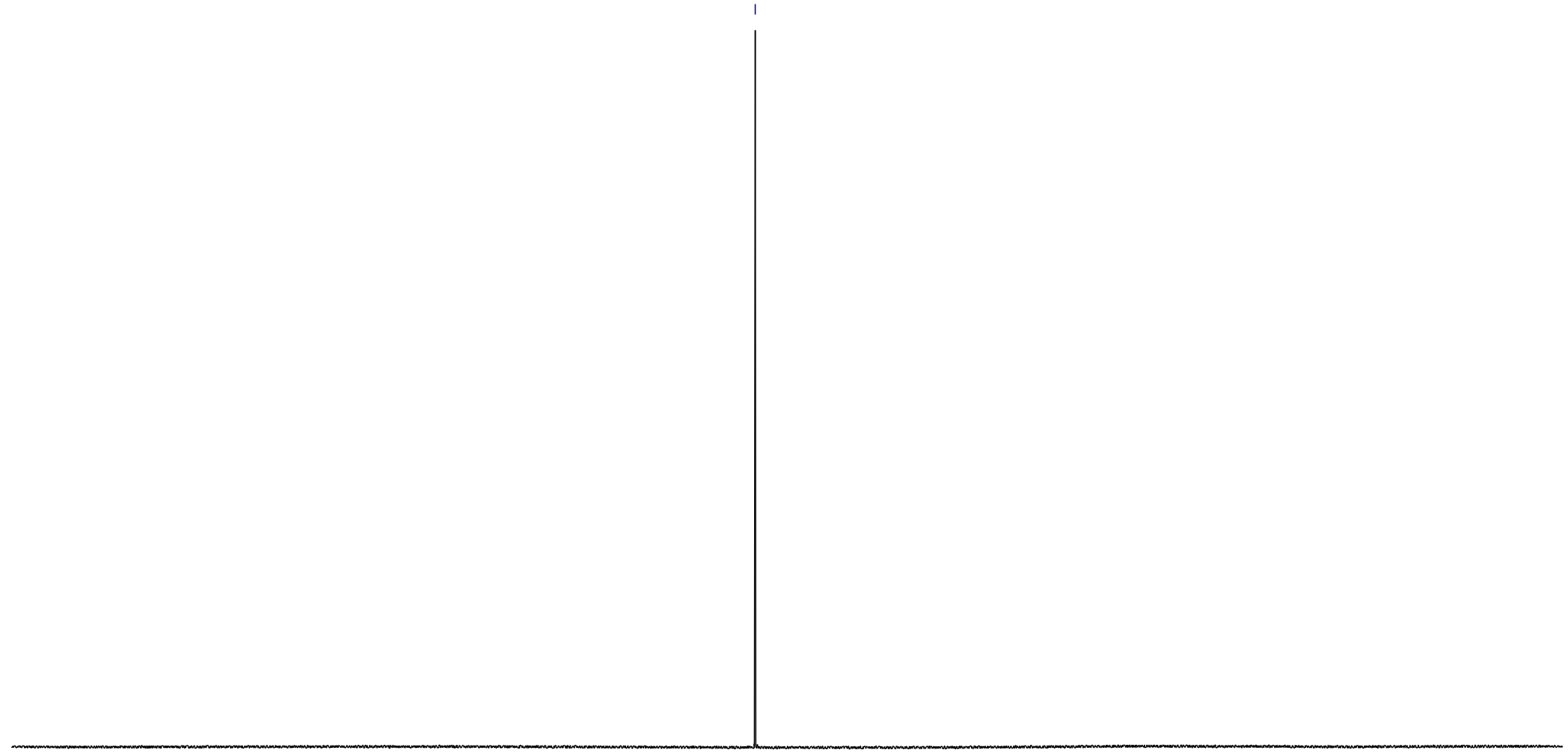


Compound 38a*

${ }^{1} \mathrm{H}$ NMR (400 MHz, DMSO-d 6 )

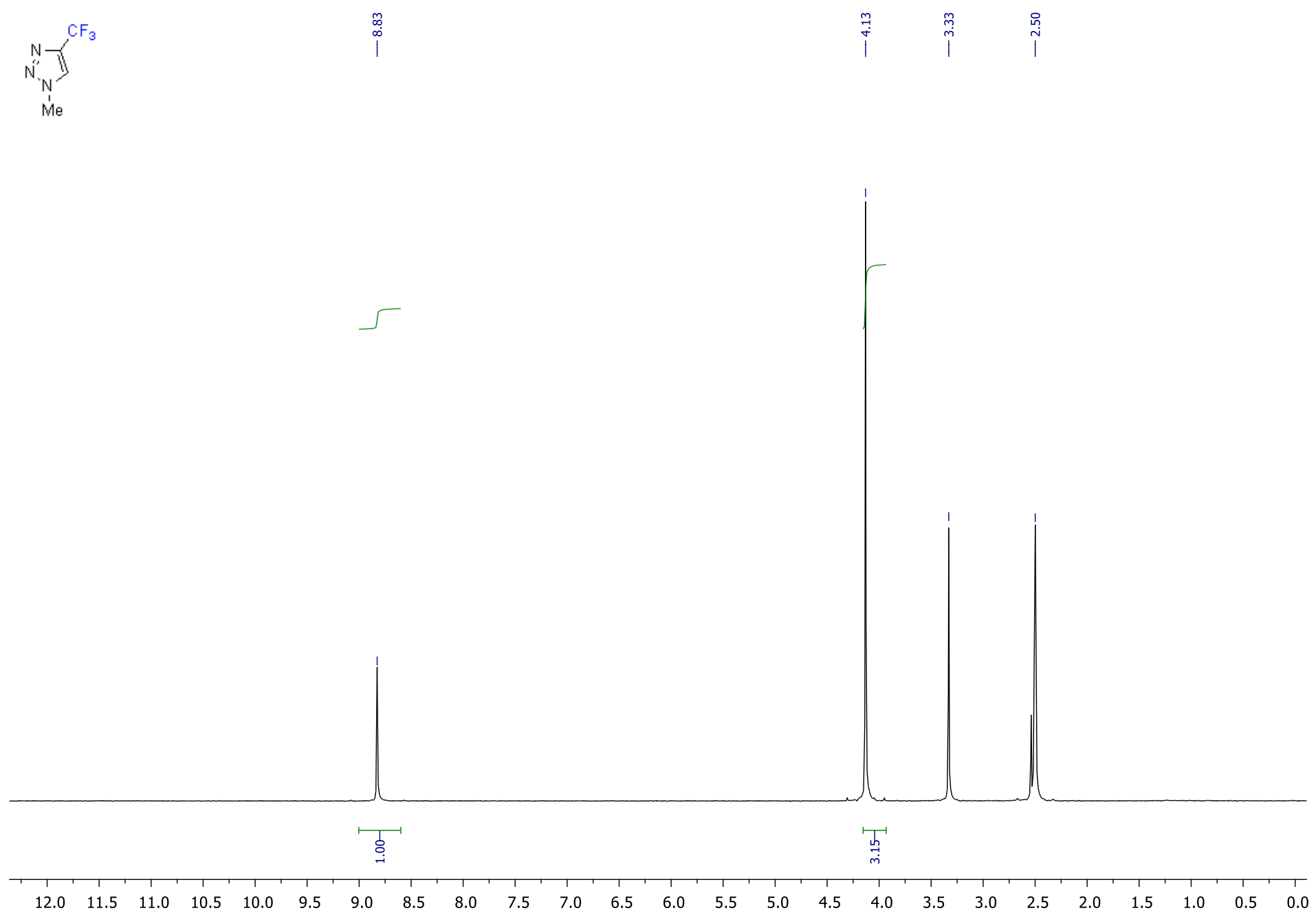

Compound 38a is also described in: J. Org. Chem. USSR, 1989, 25, 8.1, $1456-1461$. 
${ }^{13} \mathrm{C}\left\{{ }^{1} \mathrm{H}\right\}$ NMR (101 MHz, DMSO-d 6 )

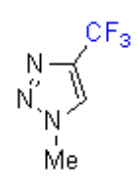$$
\text { 중ํำ }
$$

舟品:

lit 1/11

minumum

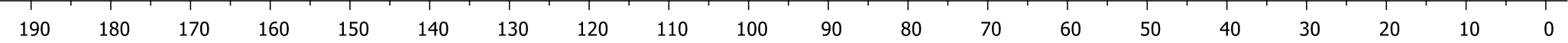


${ }^{19}$ F NMR (376 MHz, DMSO-d 6 )

$\underbrace{\mathrm{NF}_{3}}_{\mathrm{Me}}$

$\stackrel{\substack{0 \\ i}}{1}$

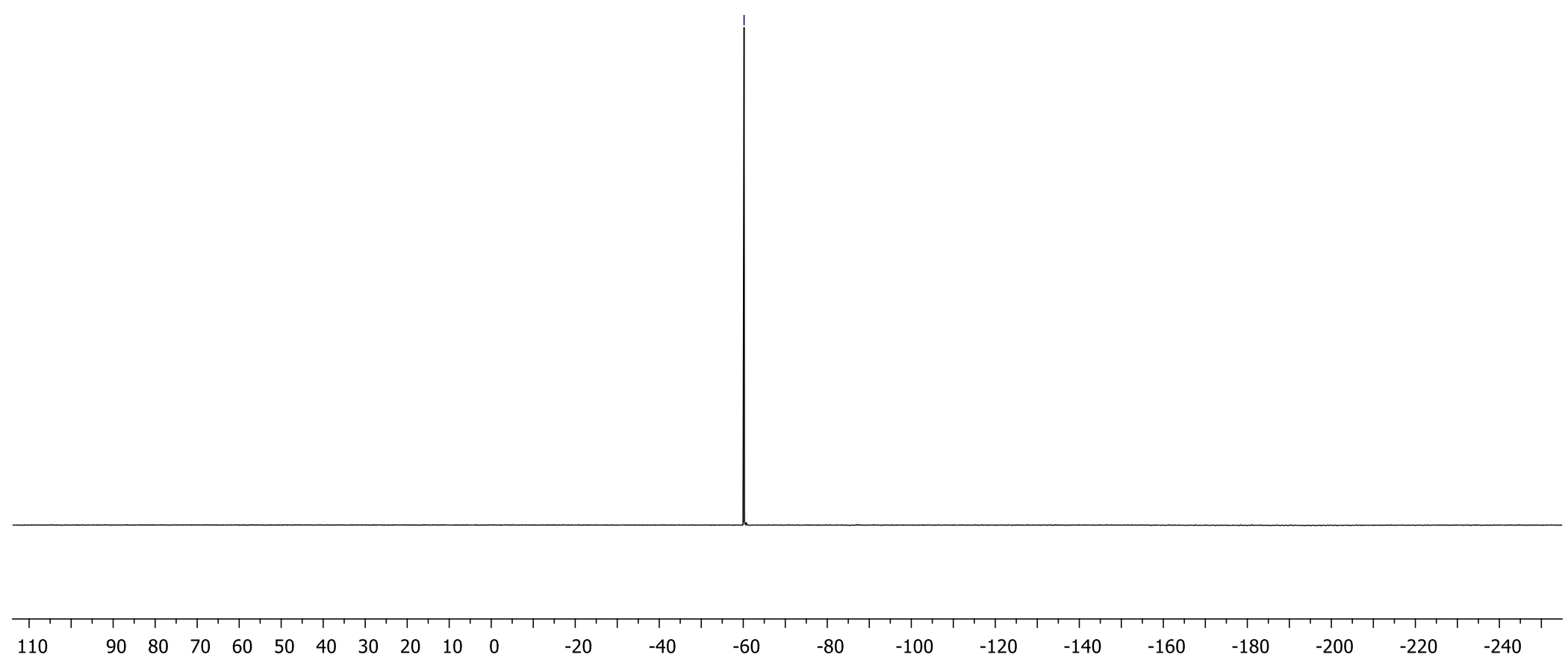


Compound 39a*

${ }^{1} \mathrm{H} \mathrm{NMR}\left(400 \mathrm{MHz}, \mathrm{CDCl}_{3}\right)$
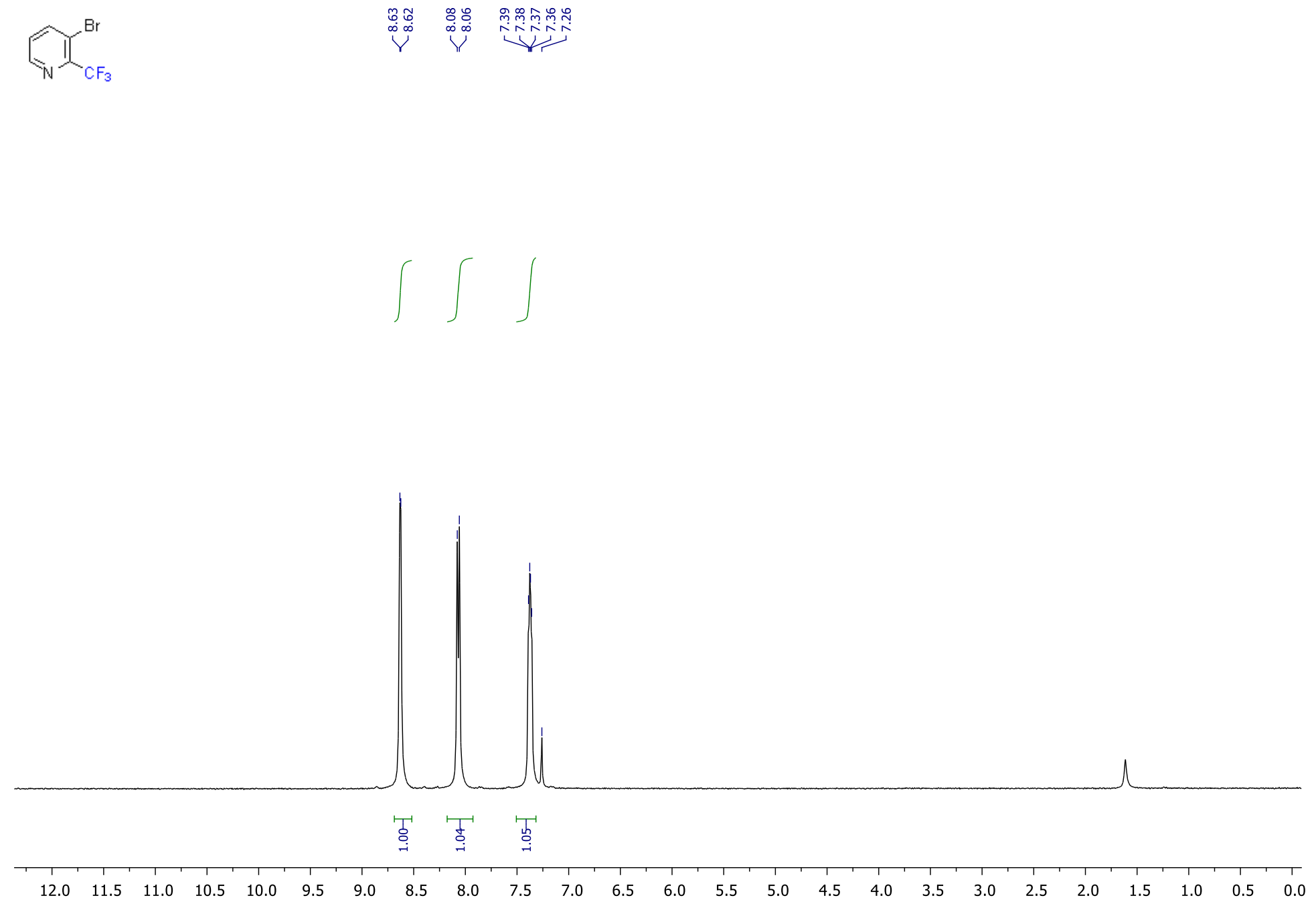

Compound 39a is also described in: Eur. J. Org. Chem. 2003, 8, 1559-1568. 
${ }^{13} \mathrm{C}\left\{{ }^{1} \mathrm{H}\right\}$ NMR $\left(151 \mathrm{MHz}, \mathrm{CDCl}_{3}\right)$

$\overbrace{\mathrm{N} F_{3}}^{\mathrm{Br}}$

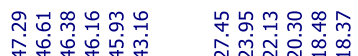

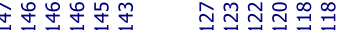

W यो

$\stackrel{0}{i}$

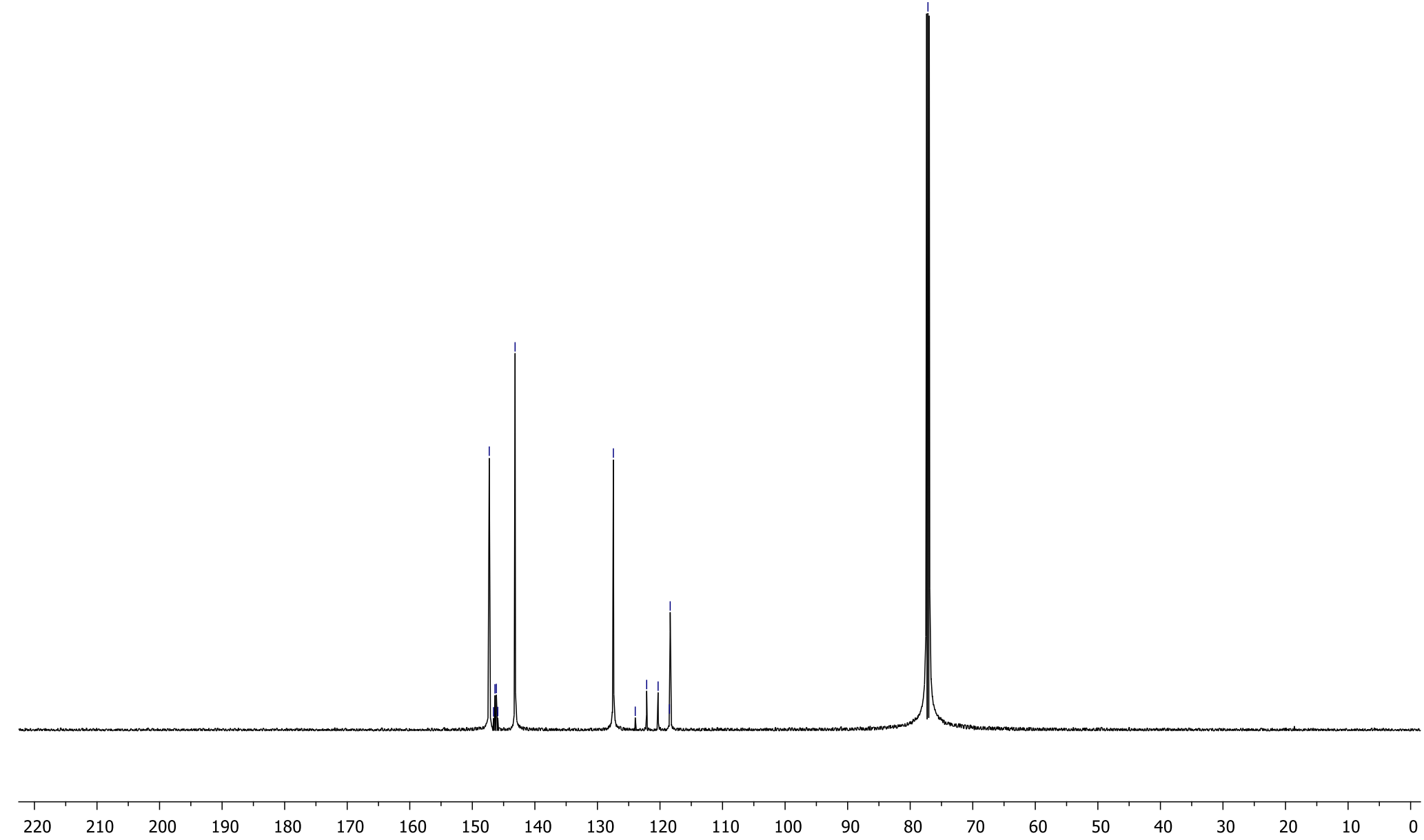


${ }^{19} \mathrm{~F}$ NMR $\left(376 \mathrm{MHz}, \mathrm{CDCl}_{3}\right)$

$\mathrm{Sich}_{\mathrm{CF}_{3}}^{\mathrm{Br}}$

$\stackrel{\infty}{\stackrel{\infty}{0}}$

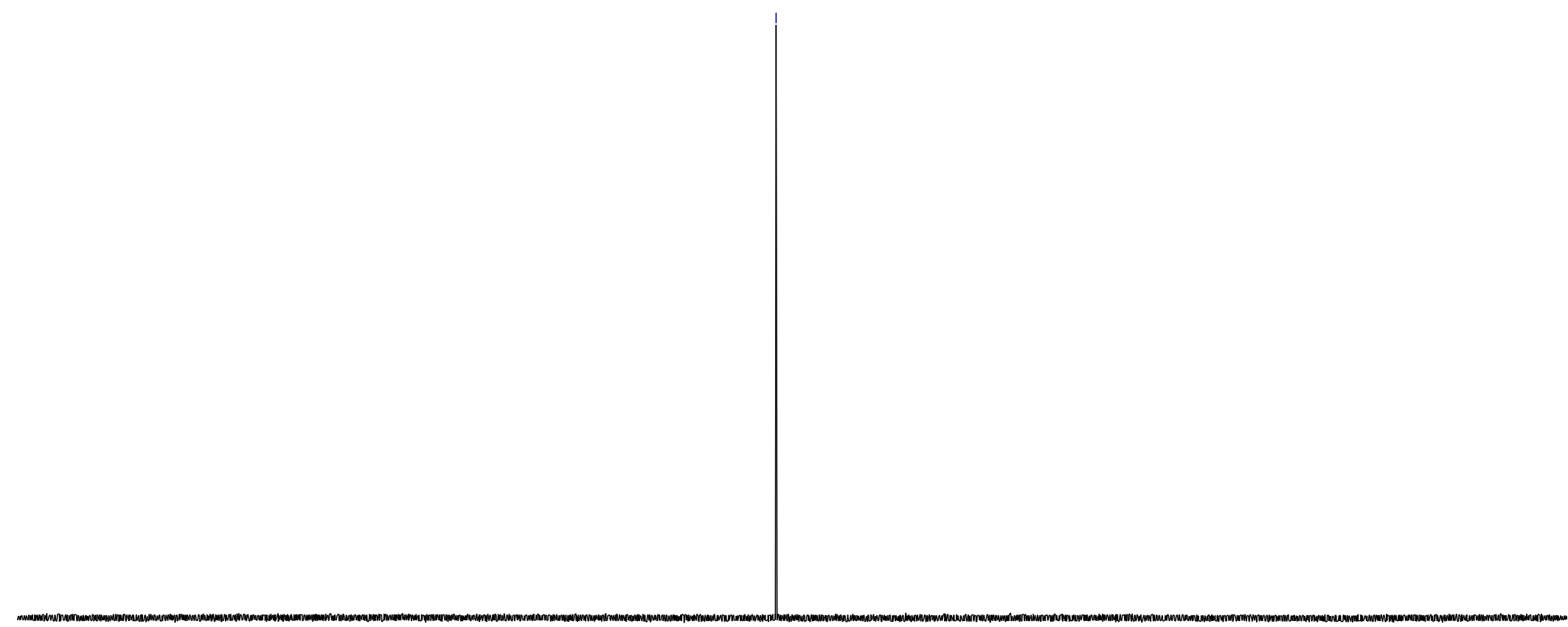

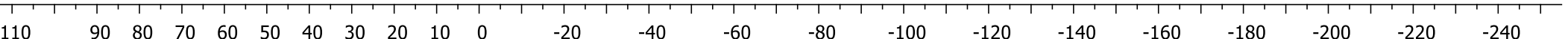


Compound 40a

${ }^{1} \mathrm{H}$ NMR $\left(400 \mathrm{MHz}, \mathrm{CDCl}_{3}\right)$

$\overbrace{\mathrm{N}}^{\mathrm{F}}$

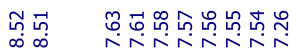
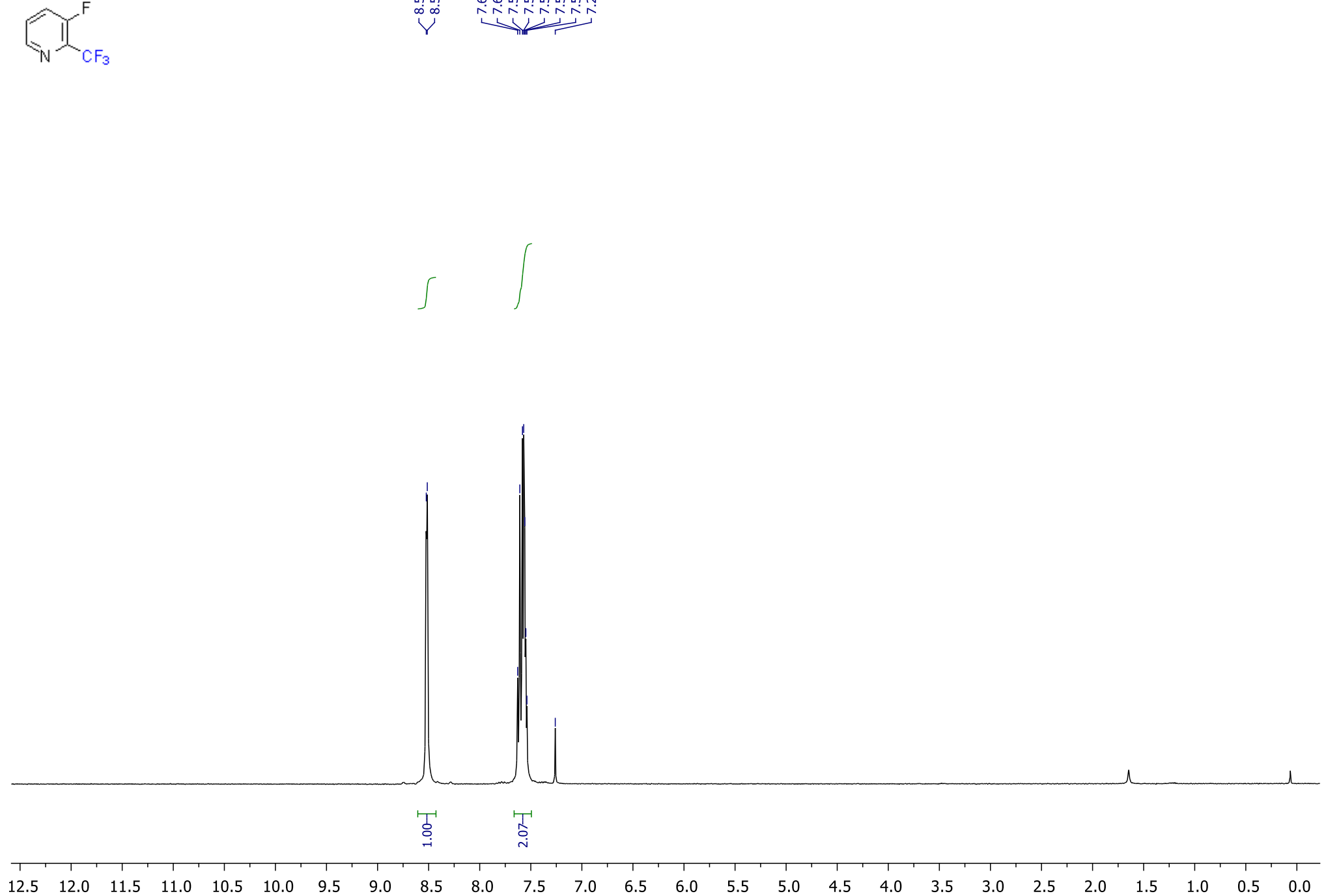
${ }^{13} \mathrm{C}\left\{{ }^{1} \mathrm{H}\right\}$ NMR $\left(126 \mathrm{MHz}, \mathrm{CDCl}_{3}\right)$

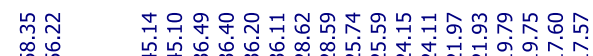

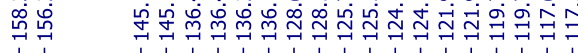

$\overbrace{\mathrm{N}}^{\mathrm{F}}$

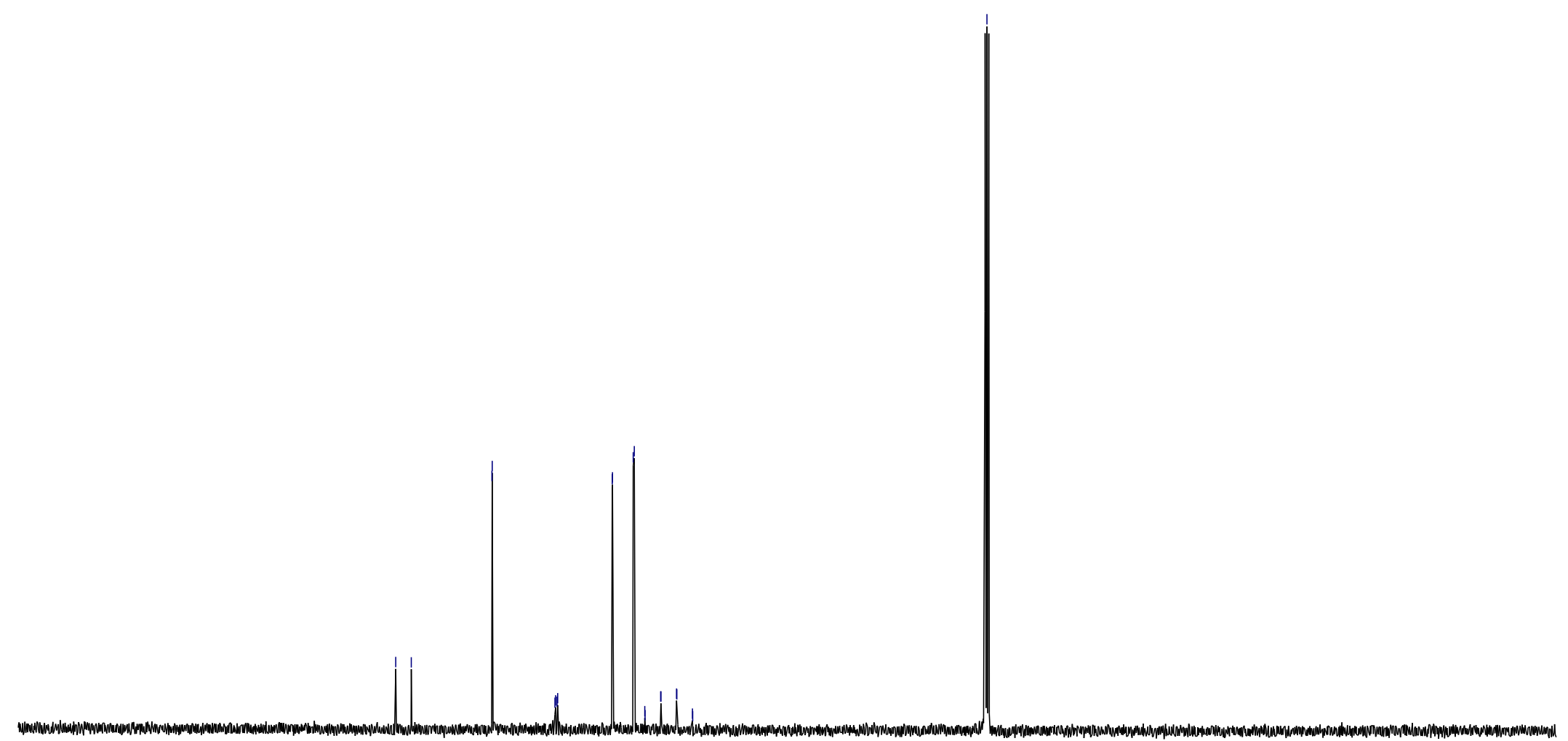


${ }^{19} \mathrm{~F}$ NMR (376 MHz, $\mathrm{CDCl}_{3}$ )

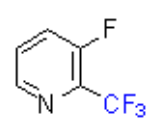

0
0

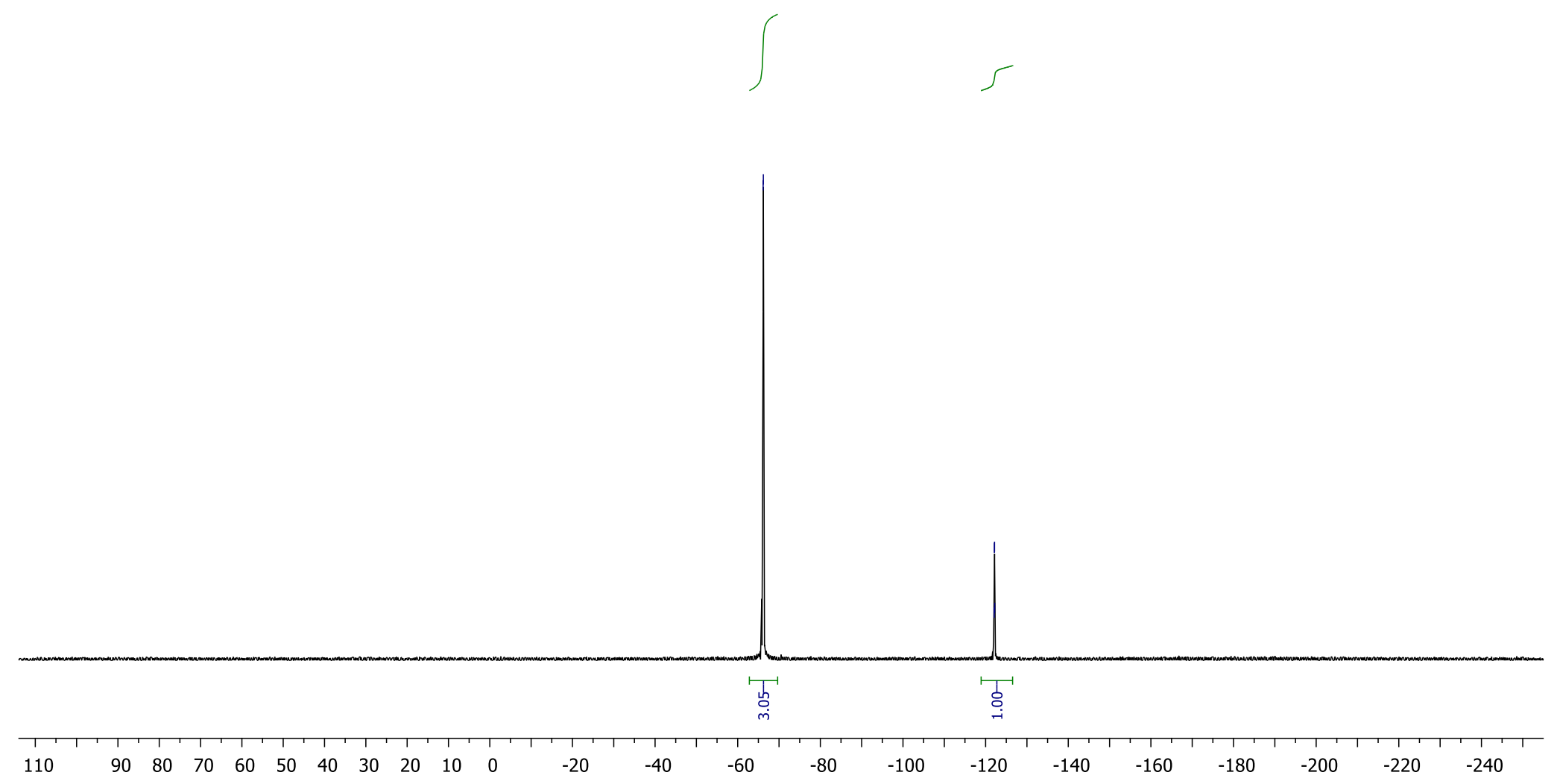


Compound $41 \mathrm{a}^{*}$

${ }^{1} \mathrm{H}$ NMR $\left(400 \mathrm{MHz}, \mathrm{CDCl}_{3}\right)$
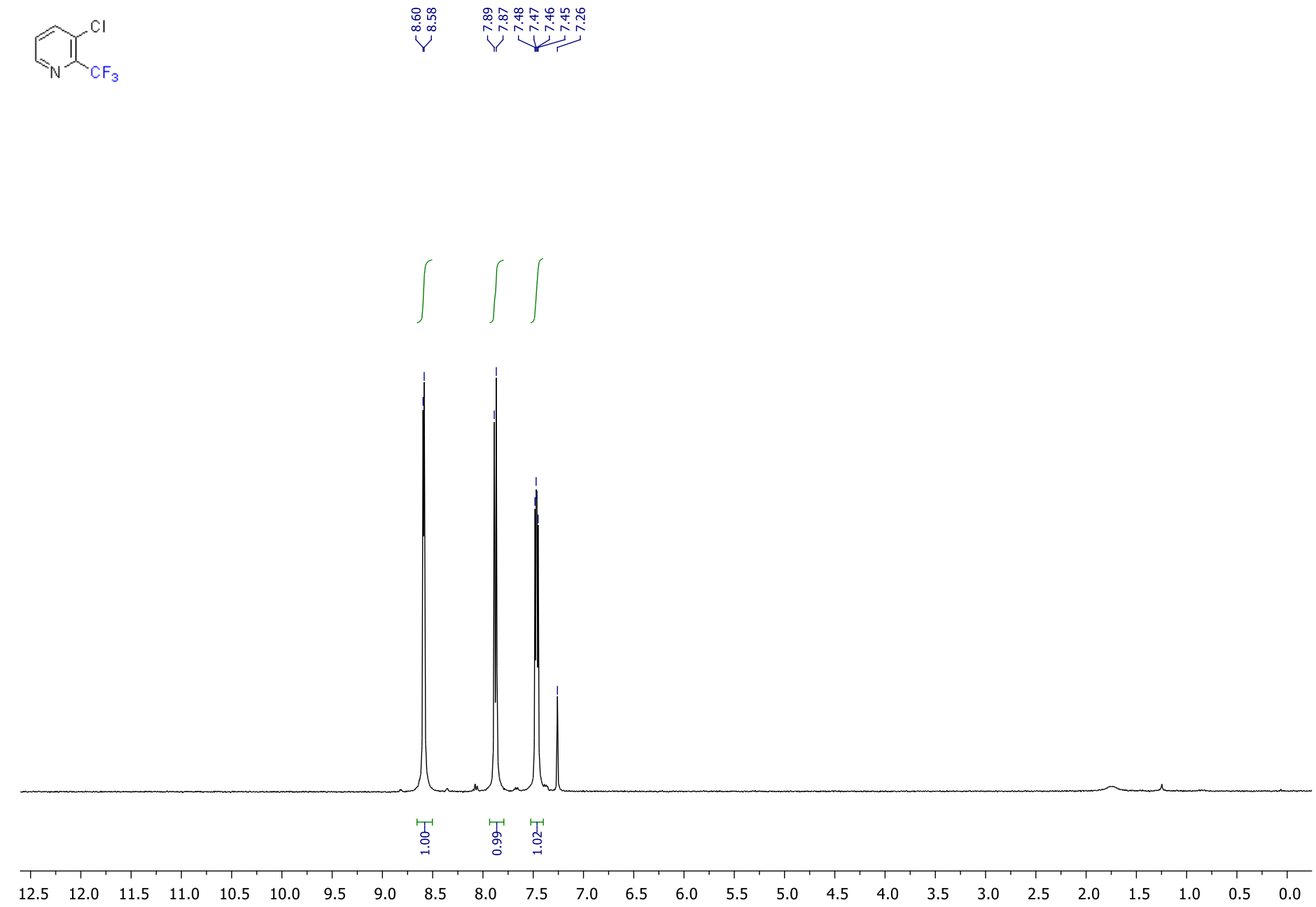

Compound 41a is also described in: a) Synthesis, 2004, 10, 1619 - 1624; b) Chem. Eur. J. 2016, 22, 6, 2075 - 2084; c) RSC Adv. 2016, 6, $75465-75469$. 


$$
\text { II }
$$


${ }^{19} \mathrm{~F}$ NMR (376 MHz, $\mathrm{CDCl}_{3}$ )

$\mathrm{C}_{\mathrm{CF}_{3}}^{\mathrm{Cl}}$

ف̊.

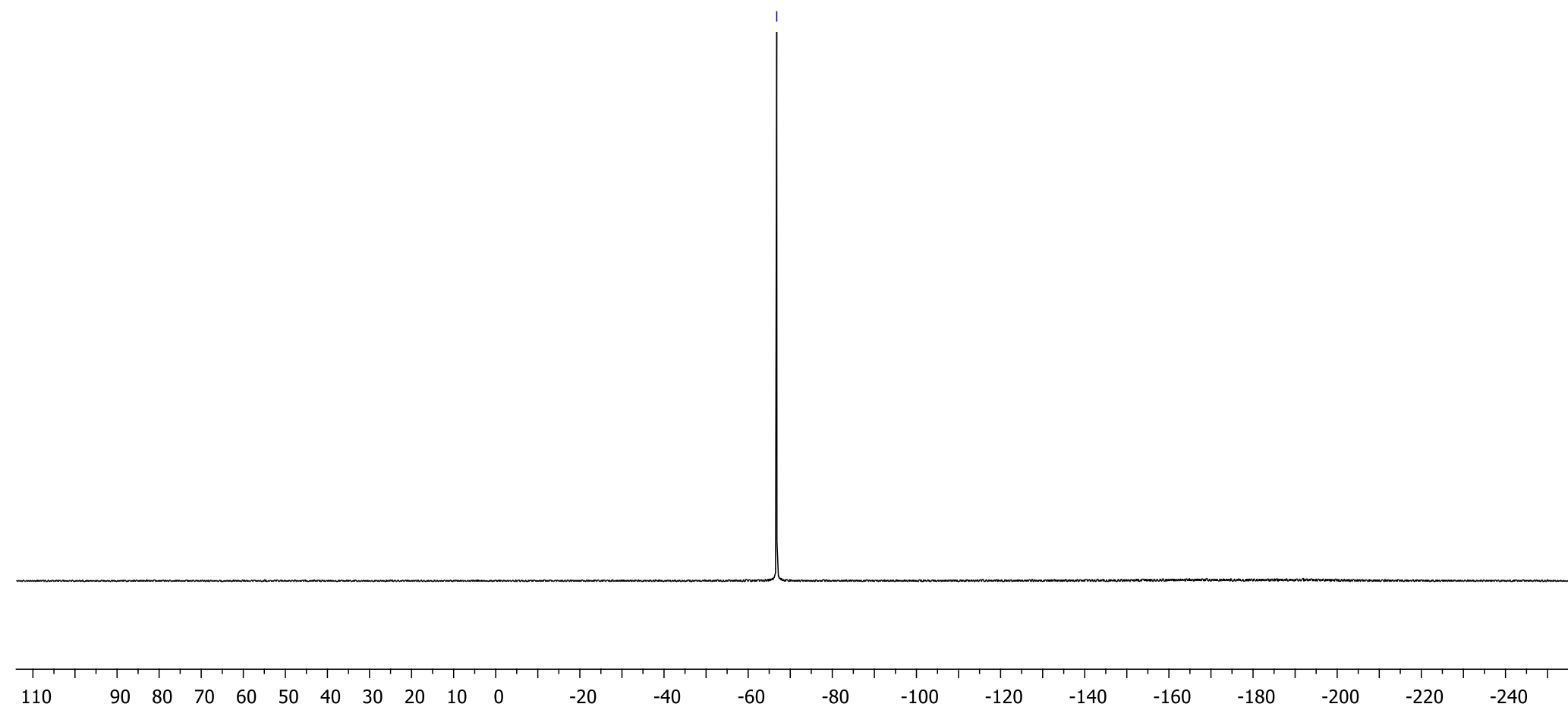


Compound $42 \mathrm{a}^{*}$
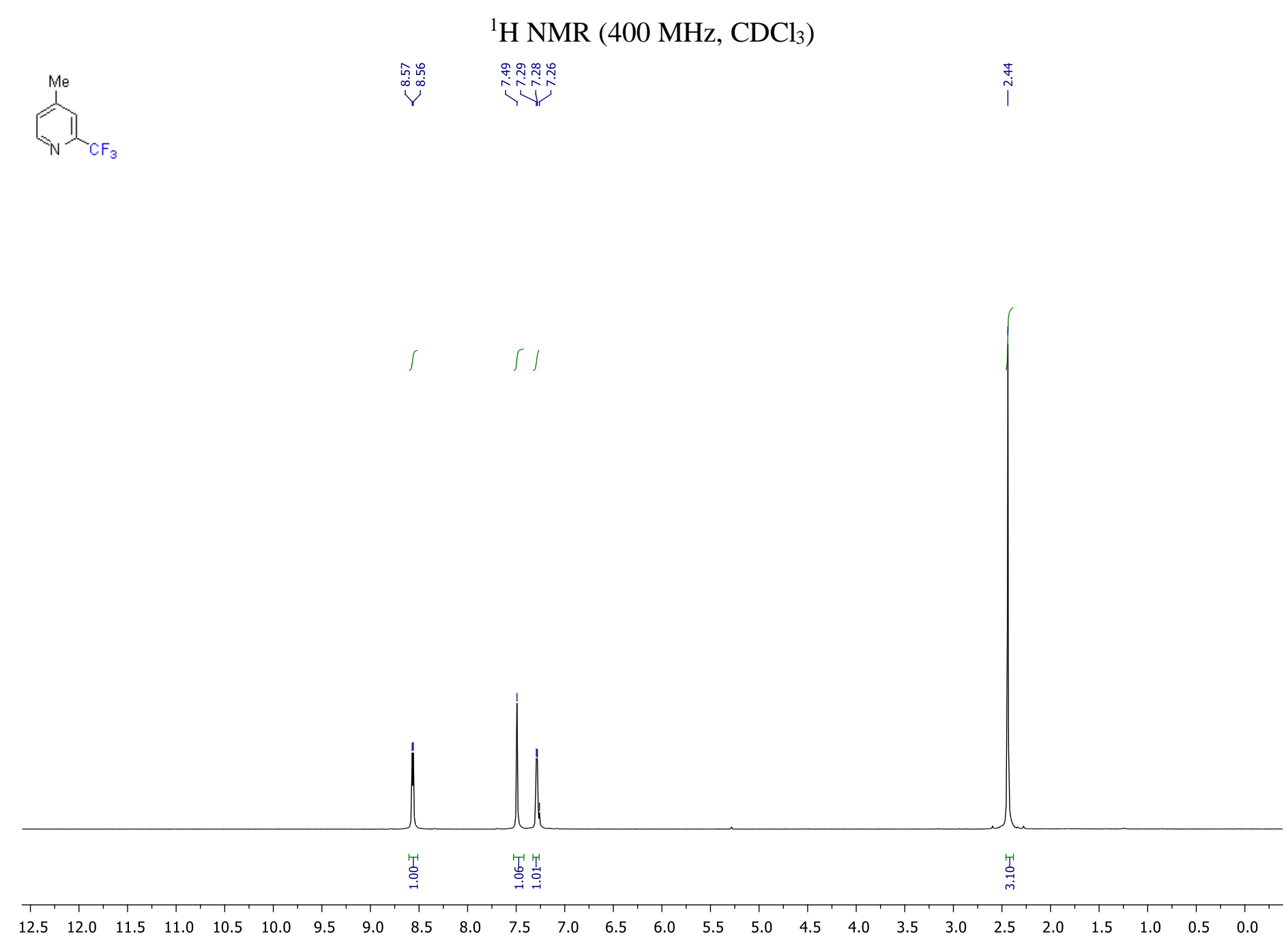

Compound 42a is also described in: a) J. Org. Chem. 2013, 78, 22, 11126-11146; b) ARKIVOC, 2012, 4, 118 - 126; c) J. Org. Chem. 1965, 30, 4, 12491250. 
${ }^{13} \mathrm{C}\left\{{ }^{1} \mathrm{H}\right\}$ NMR $\left(126 \mathrm{MHz}, \mathrm{CDCl}_{3}\right)$
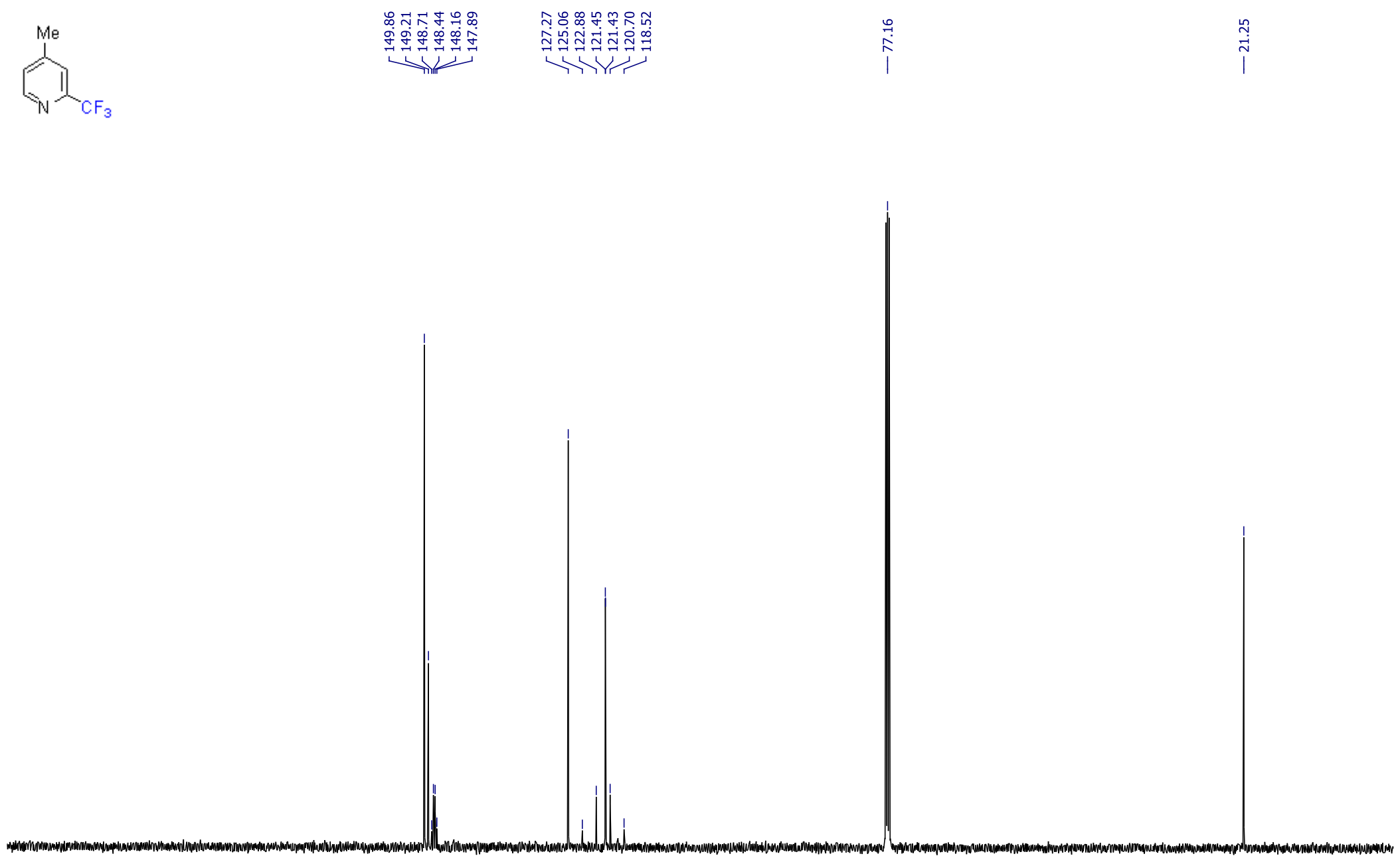

$210 \quad 200$ 
${ }^{19} \mathrm{~F}$ NMR (376 MHz, $\mathrm{CDCl}_{3}$ )

$\mathrm{N}_{\mathrm{CF}_{3}}^{\mathrm{Me}}$

象

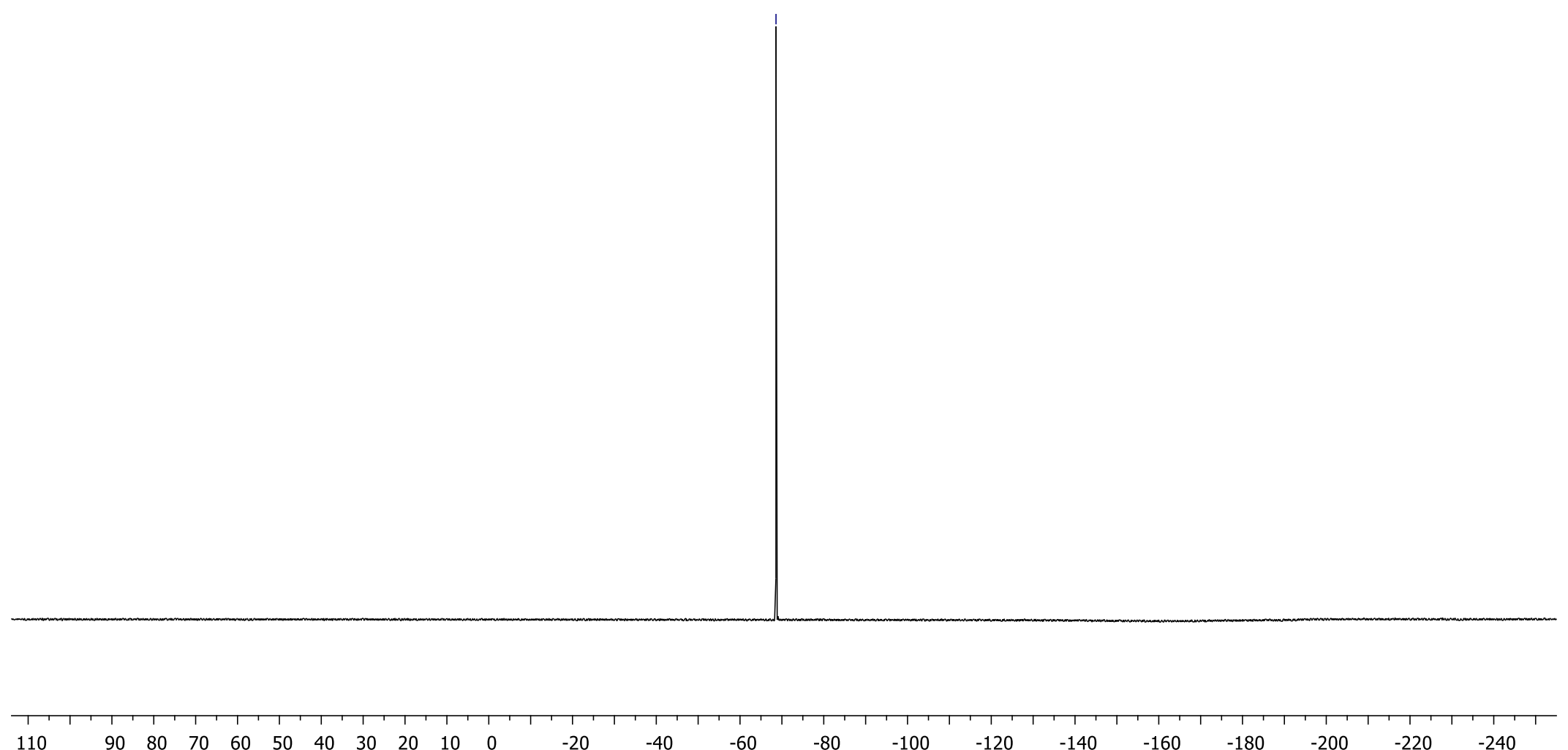


Compound $43 \mathrm{a}^{*}$

${ }^{1} \mathrm{H} \mathrm{NMR}\left(400 \mathrm{MHz}, \mathrm{CDCl}_{3}\right)$
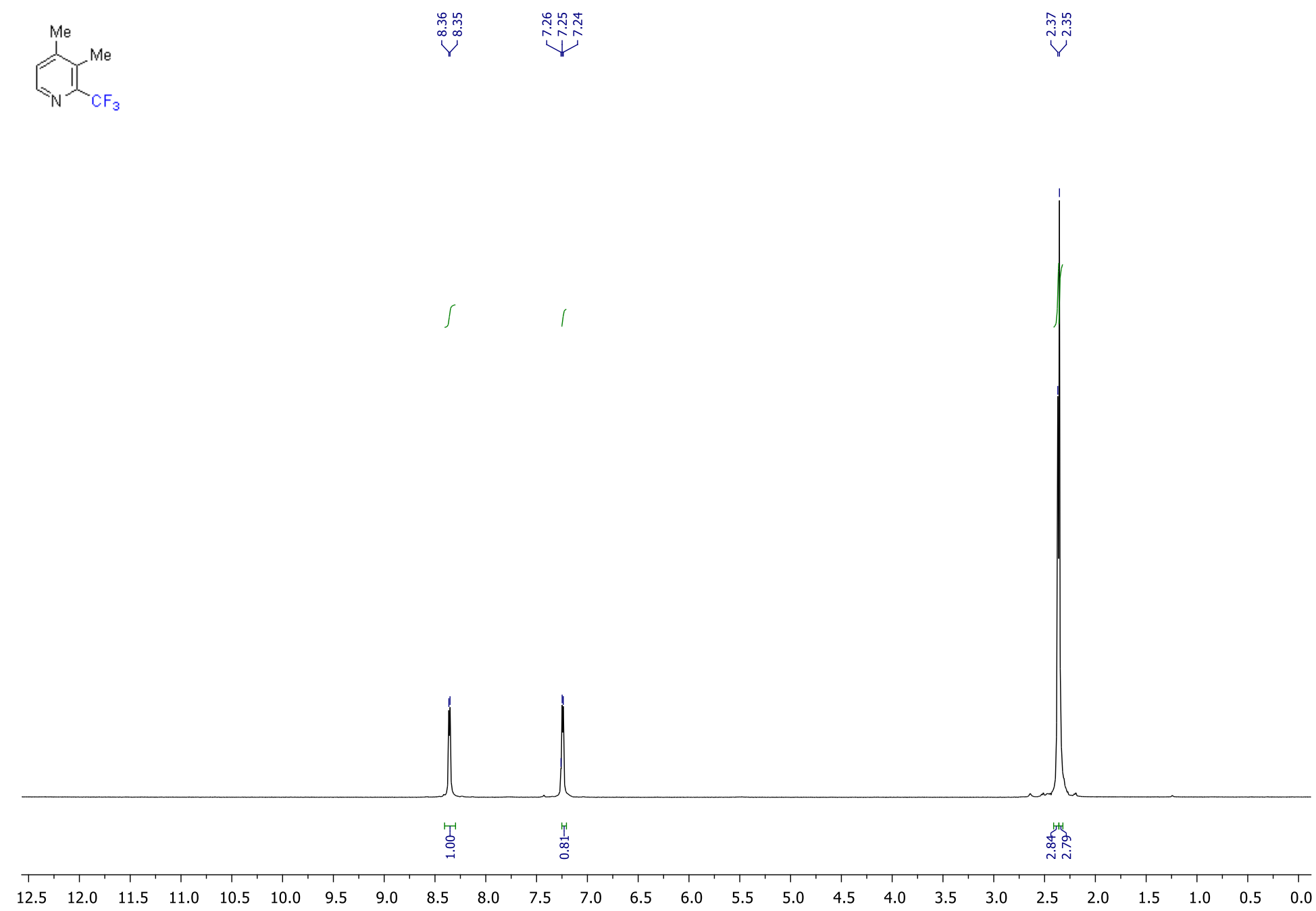

Compound 43a is also described in: J. Am. Chem. Soc. 2013, 135, 32, 12122-12134. 
${ }^{13} \mathrm{C}\left\{{ }^{1} \mathrm{H}\right\}$ NMR $\left(126 \mathrm{MHz}, \mathrm{CDCl}_{3}\right)$

$\mathrm{N}_{\mathrm{CF}_{3}}^{\mathrm{Me}}$

mmㅎำ

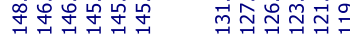

造证 行

$\stackrel{0}{i}$

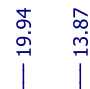

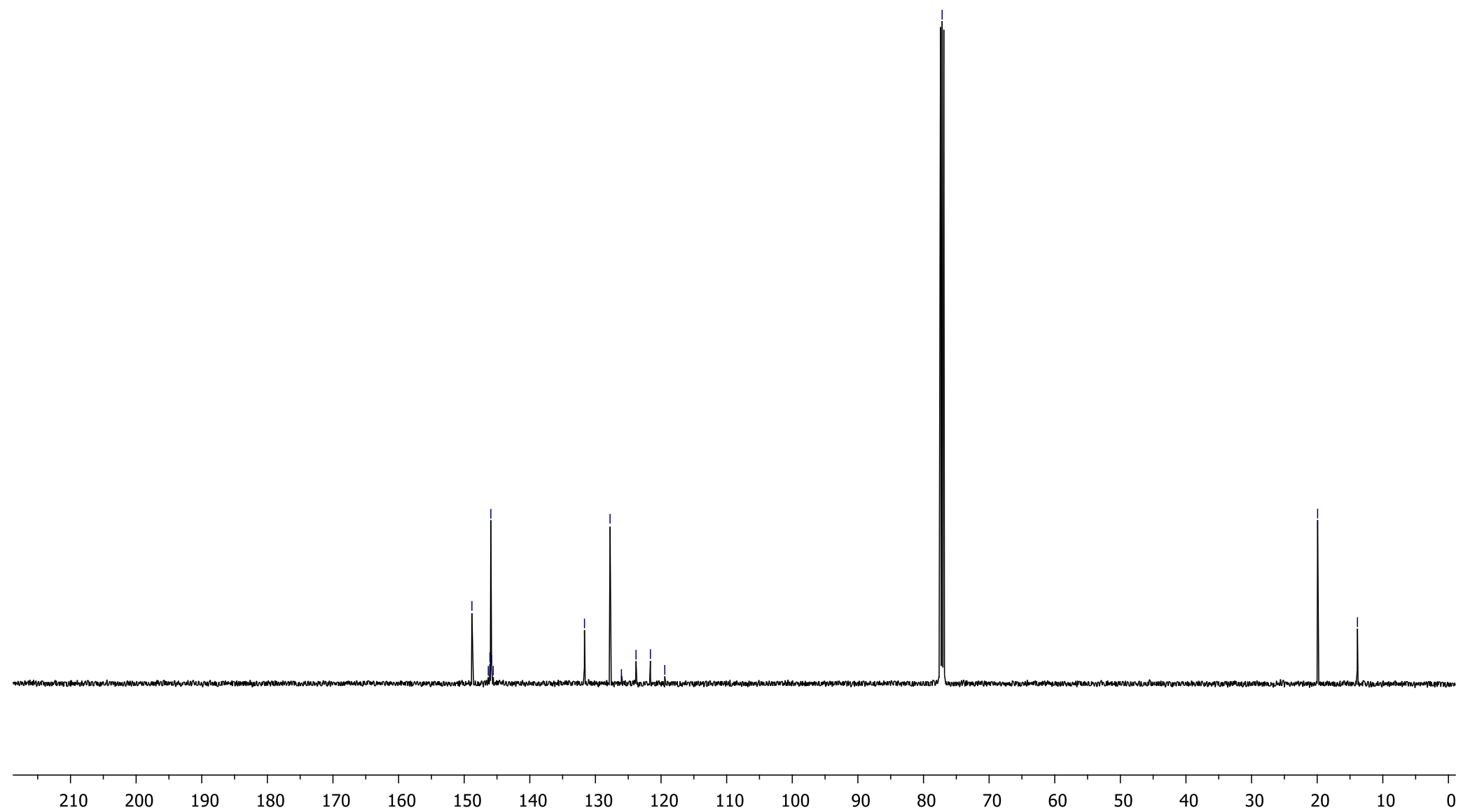


${ }^{19} \mathrm{~F}$ NMR $\left(376 \mathrm{MHz}, \mathrm{CDCl}_{3}\right)$

$\overbrace{N}^{\mathrm{Me}}$

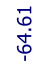

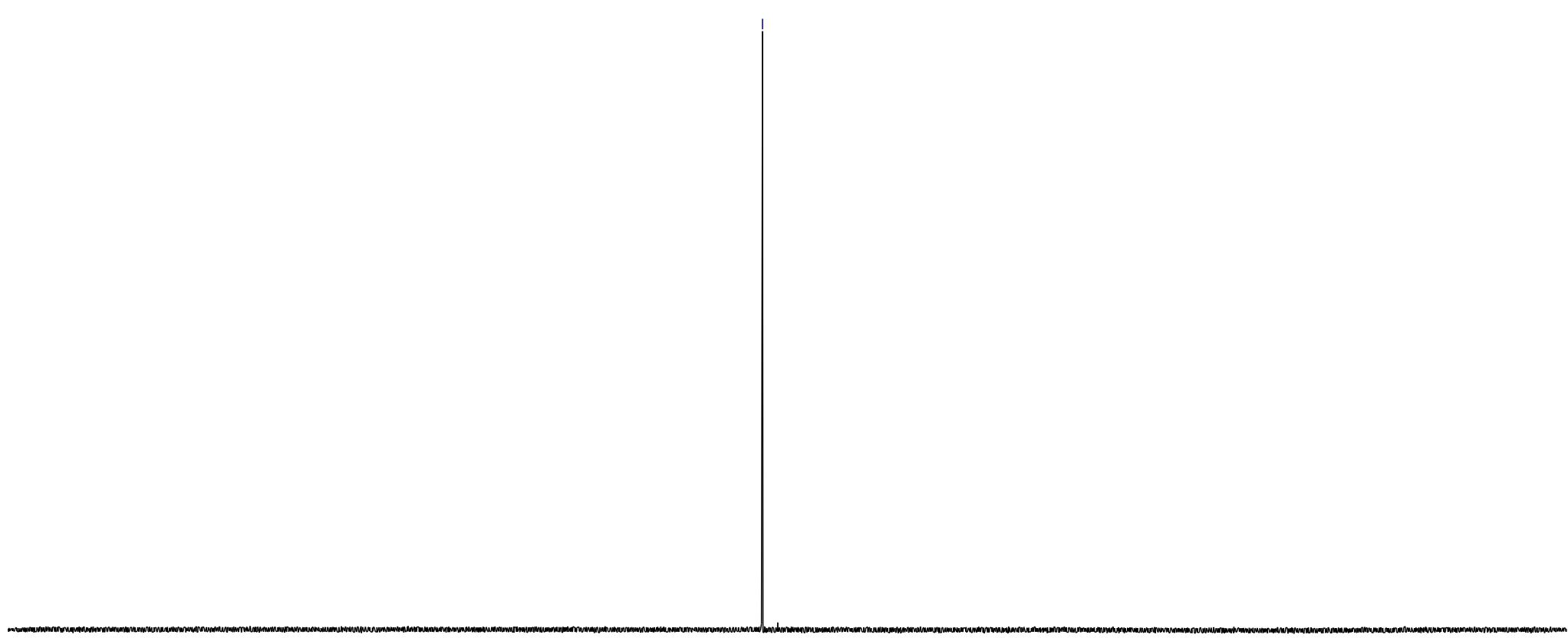

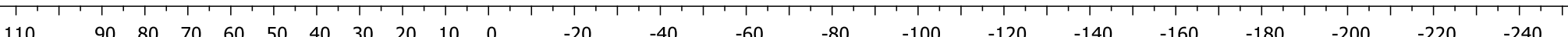


Compound 44a

${ }^{1} \mathrm{H}$ NMR (400 MHz, $\mathrm{CDCl}_{3}$ )

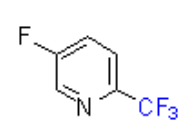

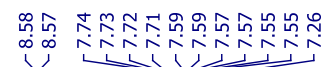
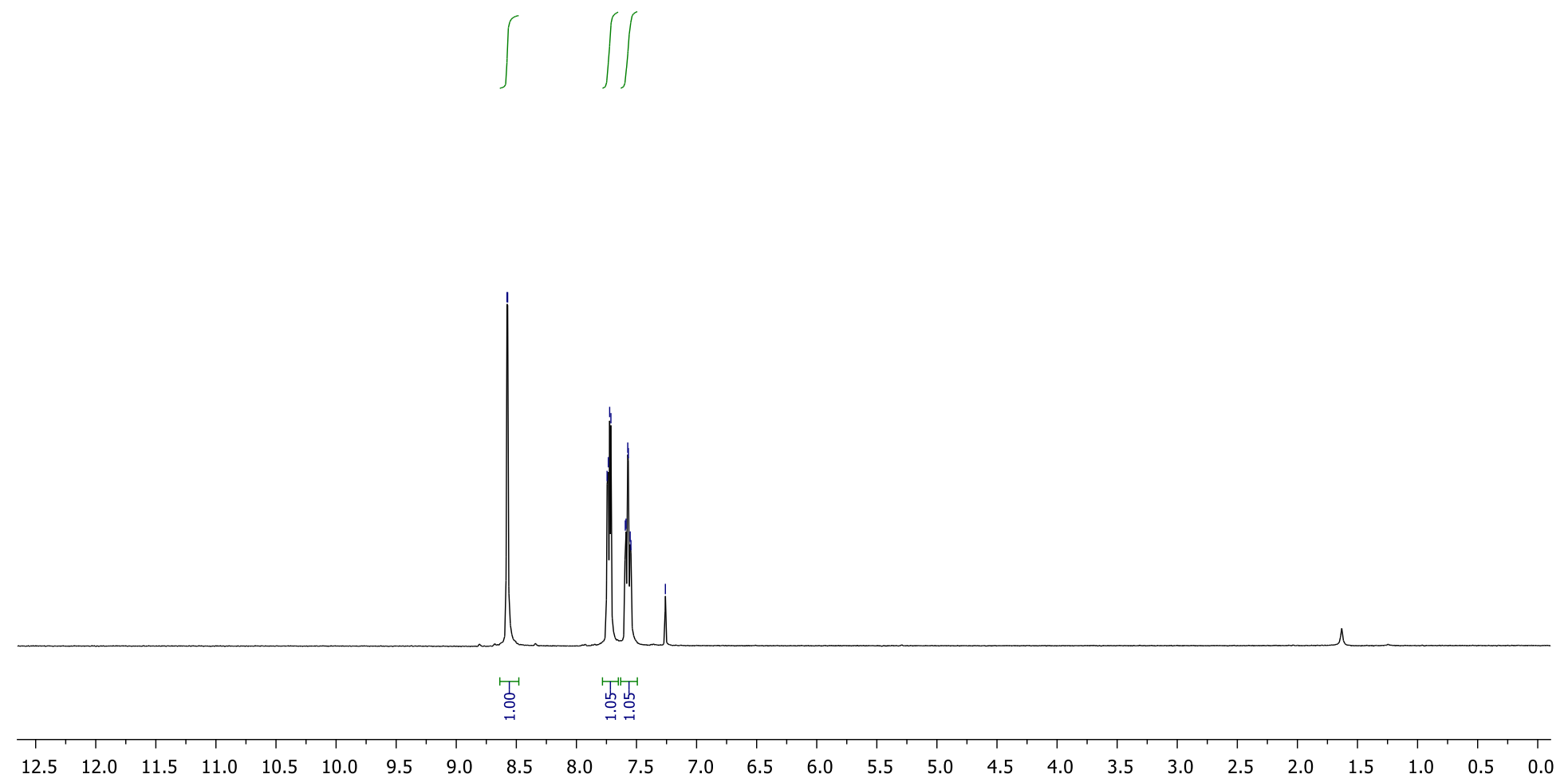
${ }^{13} \mathrm{C}\left\{{ }^{1} \mathrm{H}\right\}$ NMR $\left(126 \mathrm{MHz}, \mathrm{CDCl}_{3}\right)$

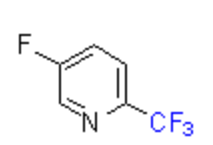

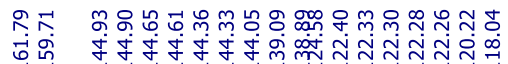

11

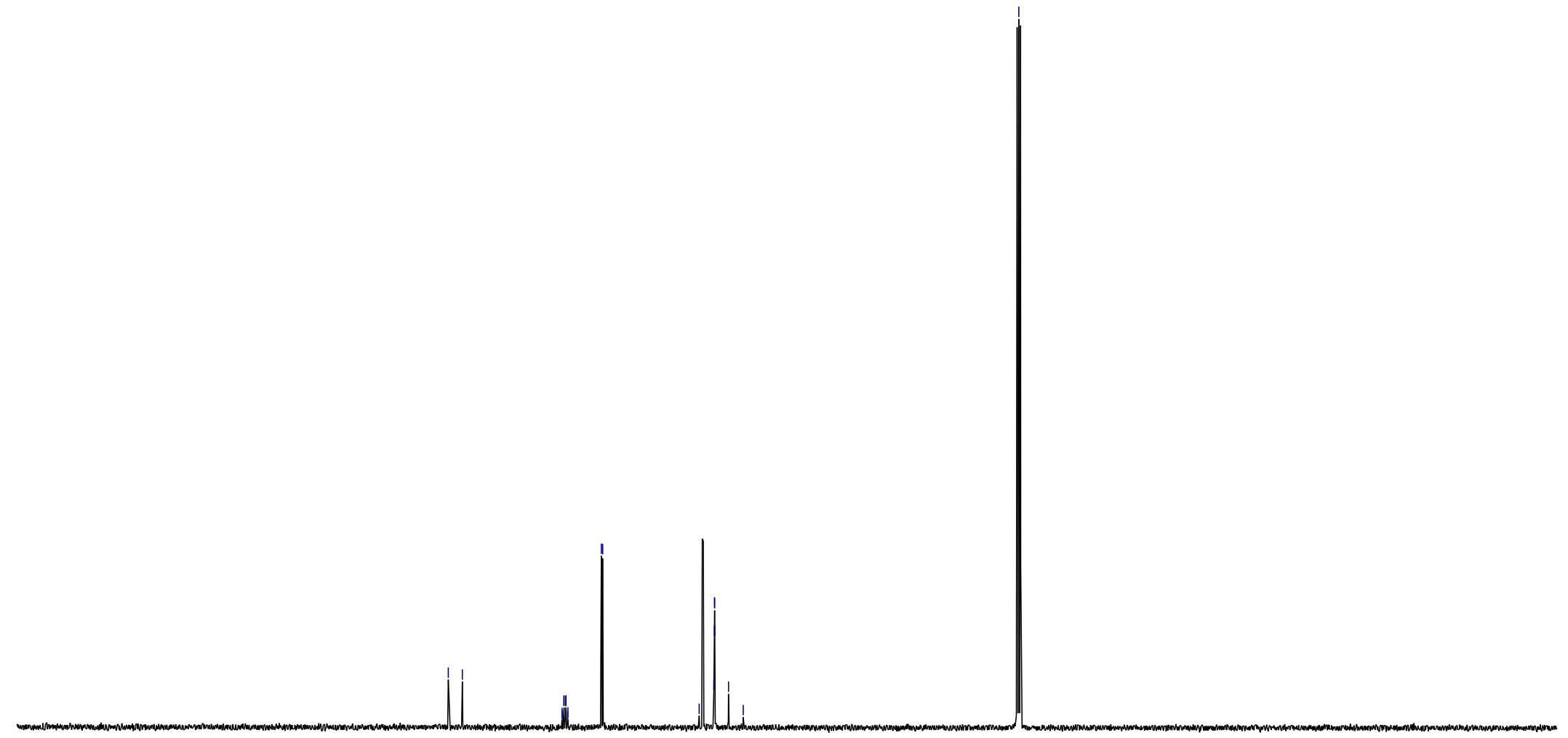

$220 \quad 210 \quad 200$

$190 \quad 180$

$\begin{array}{lllll}80 & 170 & 160 & 150 & 1\end{array}$

$40 \quad 130$

$\begin{array}{lll}120 & 110 \quad 100\end{array}$

80

70

$60 \quad 50$

$50 \quad 40$

30

$20 \quad 10 \quad 0$ 
${ }^{19} \mathrm{~F}$ NMR (376 MHz, $\mathrm{CDCl}_{3}$ )

Cl
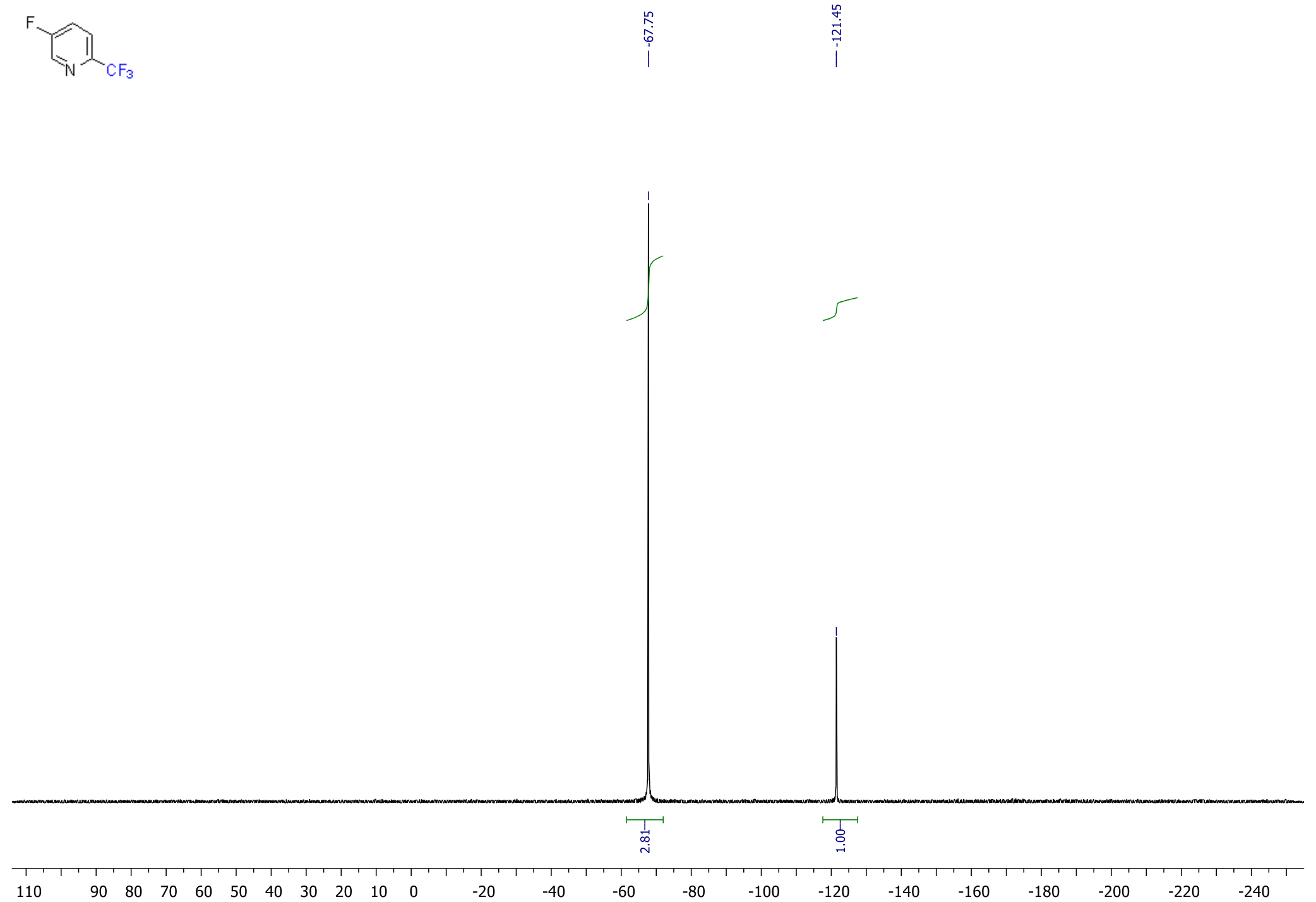
Compound 45a

${ }^{1} \mathrm{H}$ NMR (400 MHz, $\mathrm{CDCl}_{3}$ )

م

|

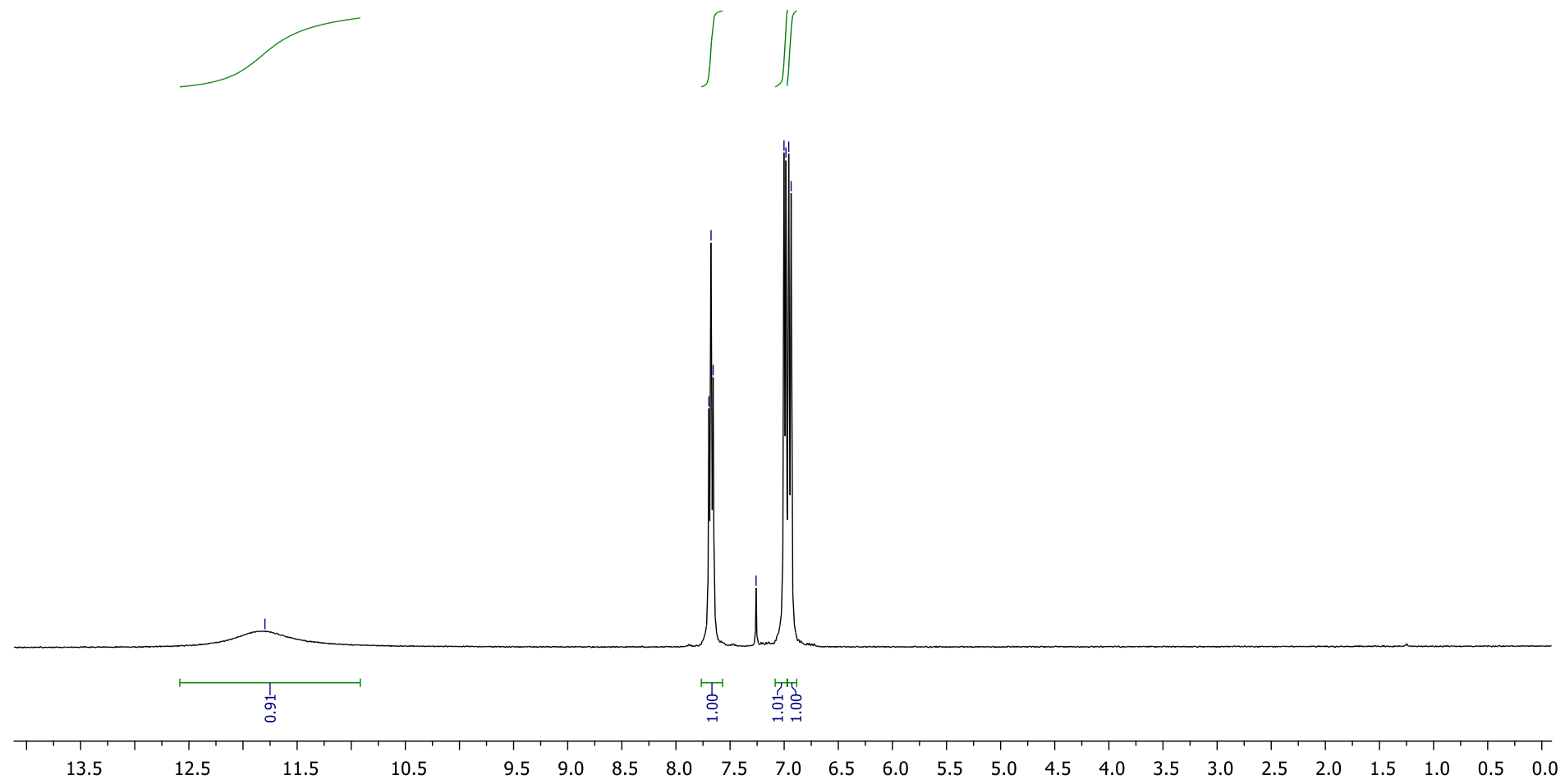


${ }^{13} \mathrm{C}\left\{{ }^{1} \mathrm{H}\right\}$ NMR $\left(151 \mathrm{MHz}, \mathrm{CDCl}_{3}\right)$
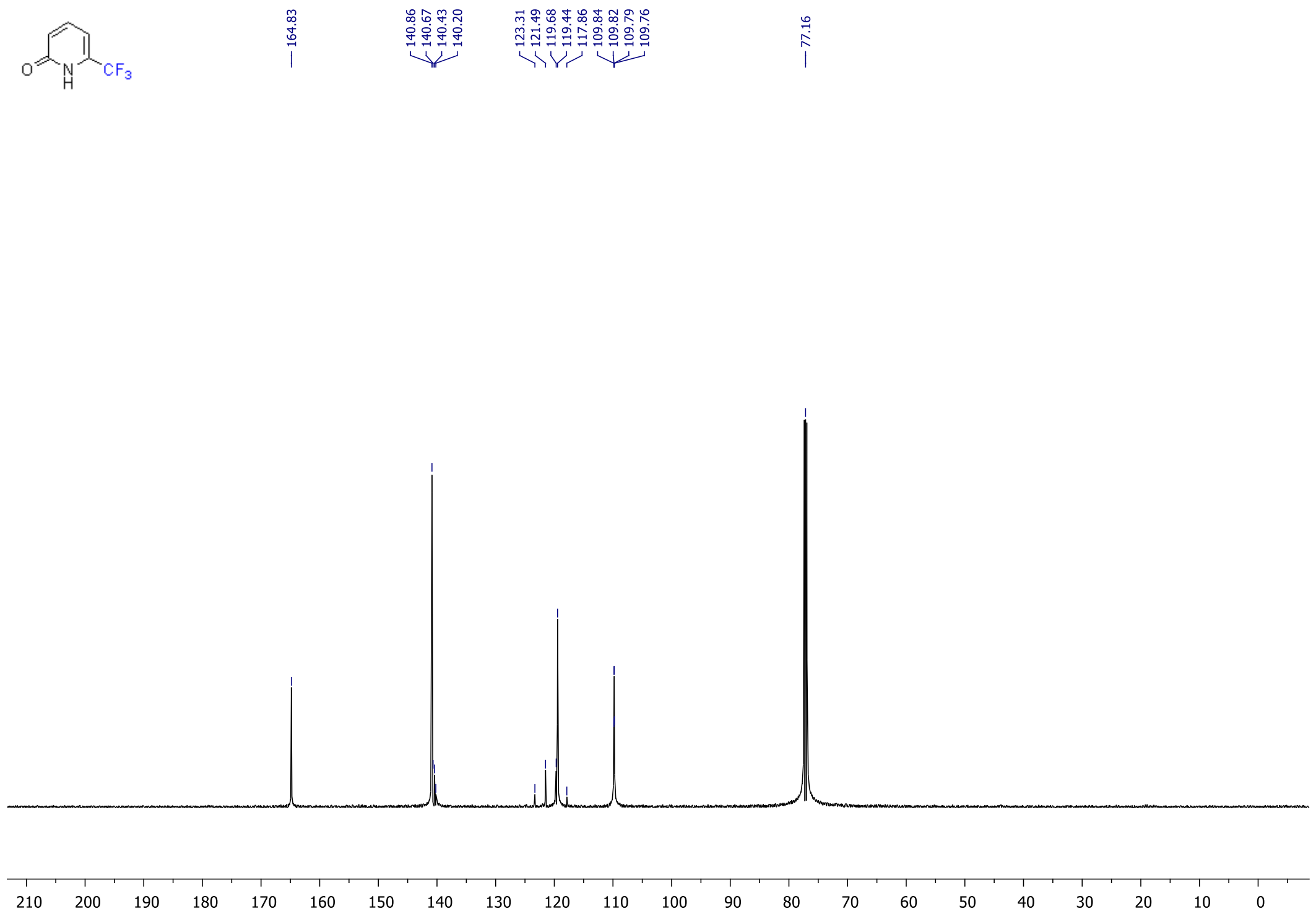

S137 
${ }^{19} \mathrm{~F}$ NMR (376 MHz, $\mathrm{CDCl}_{3}$ )

$\mathrm{C}_{\mathrm{H}} \mathrm{CH}_{\mathrm{CF}_{3}}$

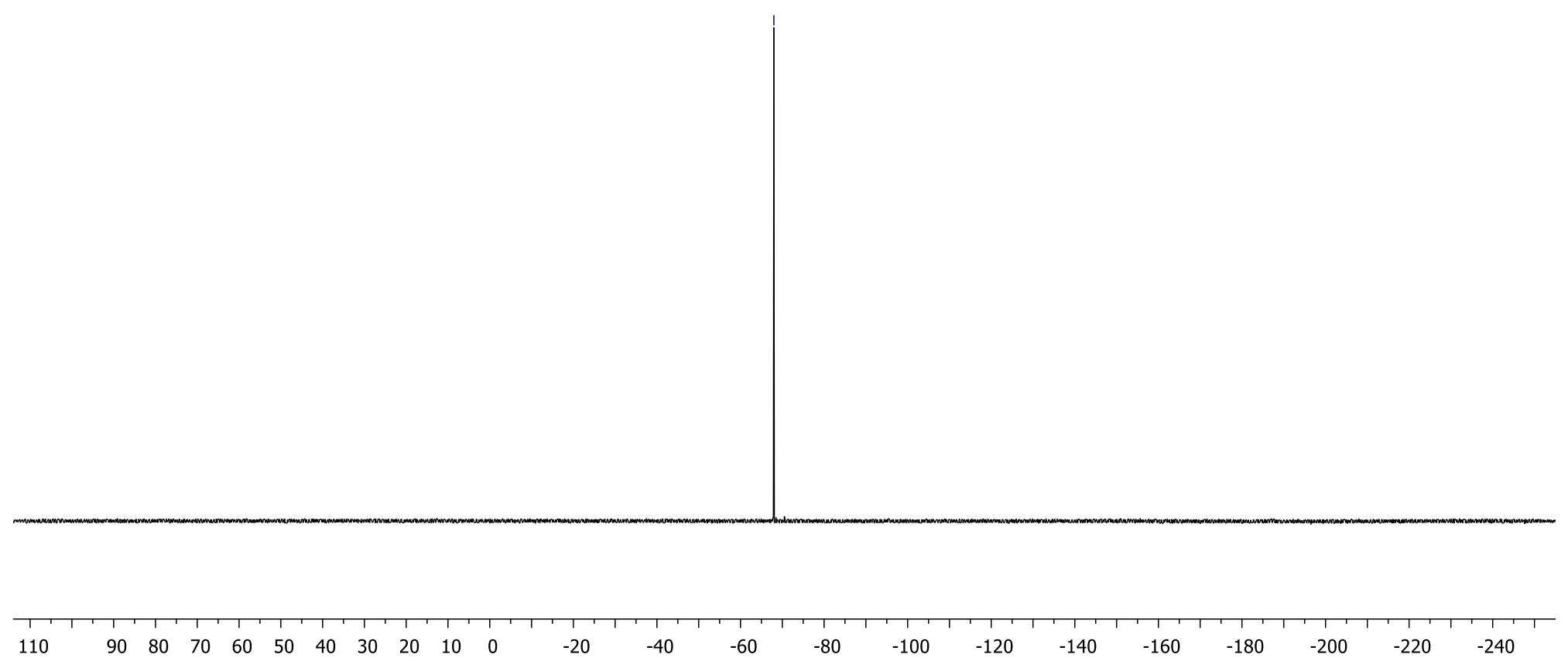


Compound 46a

${ }^{1} \mathrm{H}$ NMR $\left(400 \mathrm{MHz}, \mathrm{CDCl}_{3}\right)$

$\overbrace{\mathrm{N}}^{\mathrm{Br}} \mathrm{CF}_{3}$

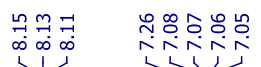

사

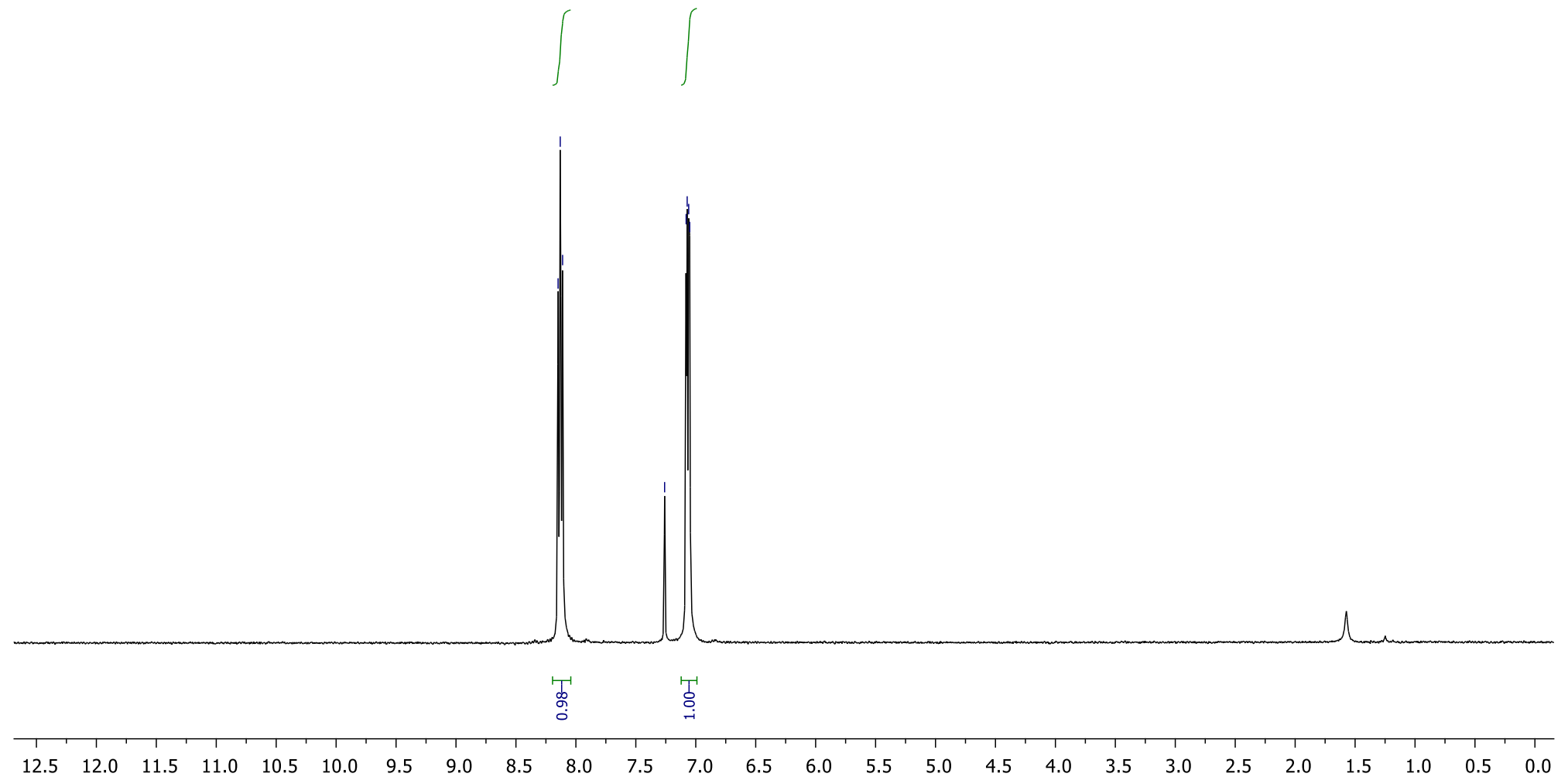


${ }^{13} \mathrm{C}\left\{{ }^{1} \mathrm{H}\right\}$ NMR $\left(151 \mathrm{MHz}, \mathrm{CDCl}_{3}\right)$

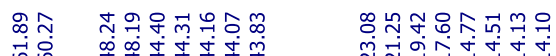

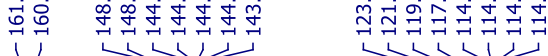

$\overbrace{\mathrm{N}}^{\mathrm{Br}}$

11 r

列证

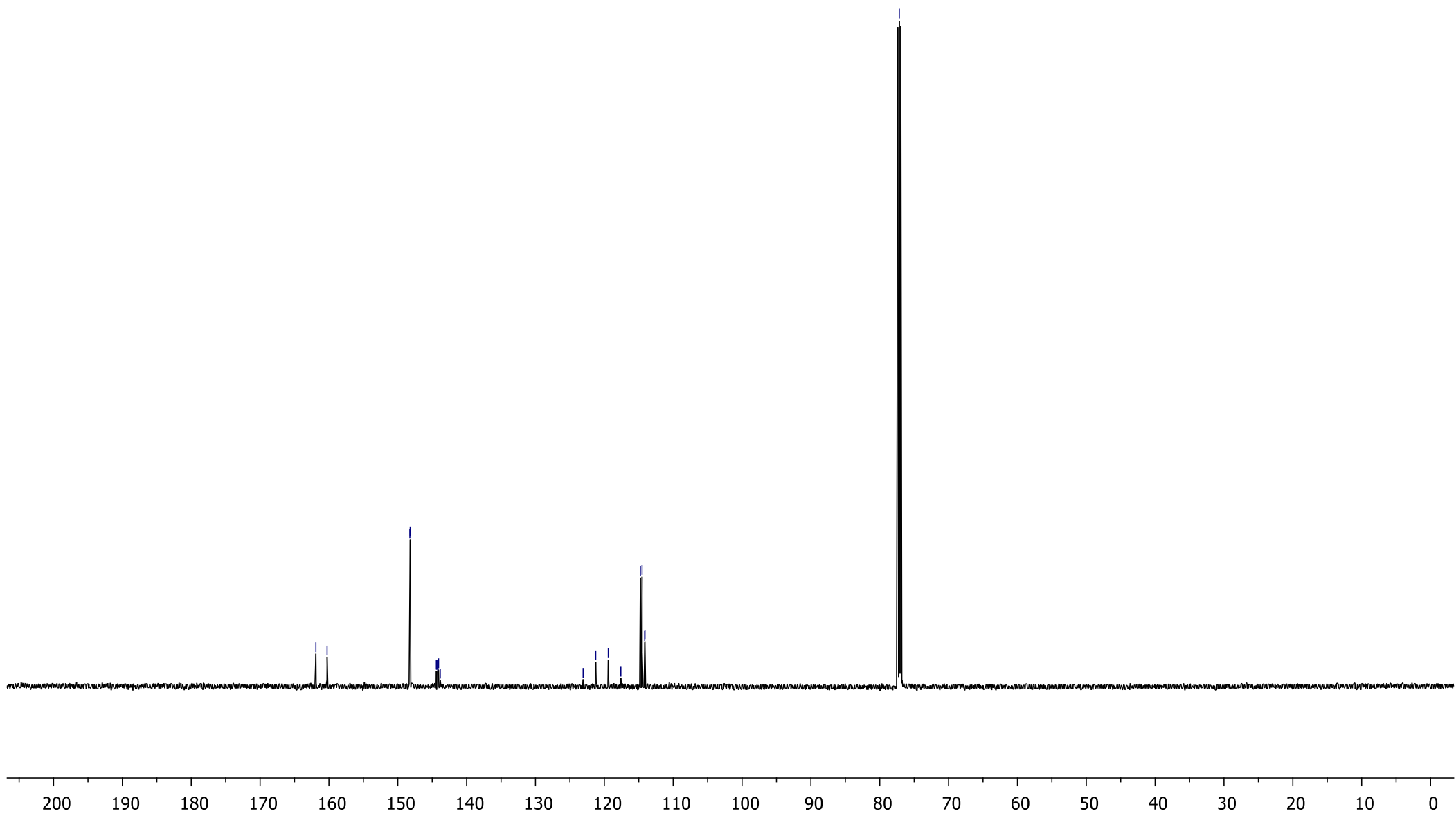


${ }^{19} \mathrm{~F}$ NMR (376 MHz, $\mathrm{CDCl}_{3}$ )

$\overbrace{\mathrm{N}}^{\mathrm{Br}}$

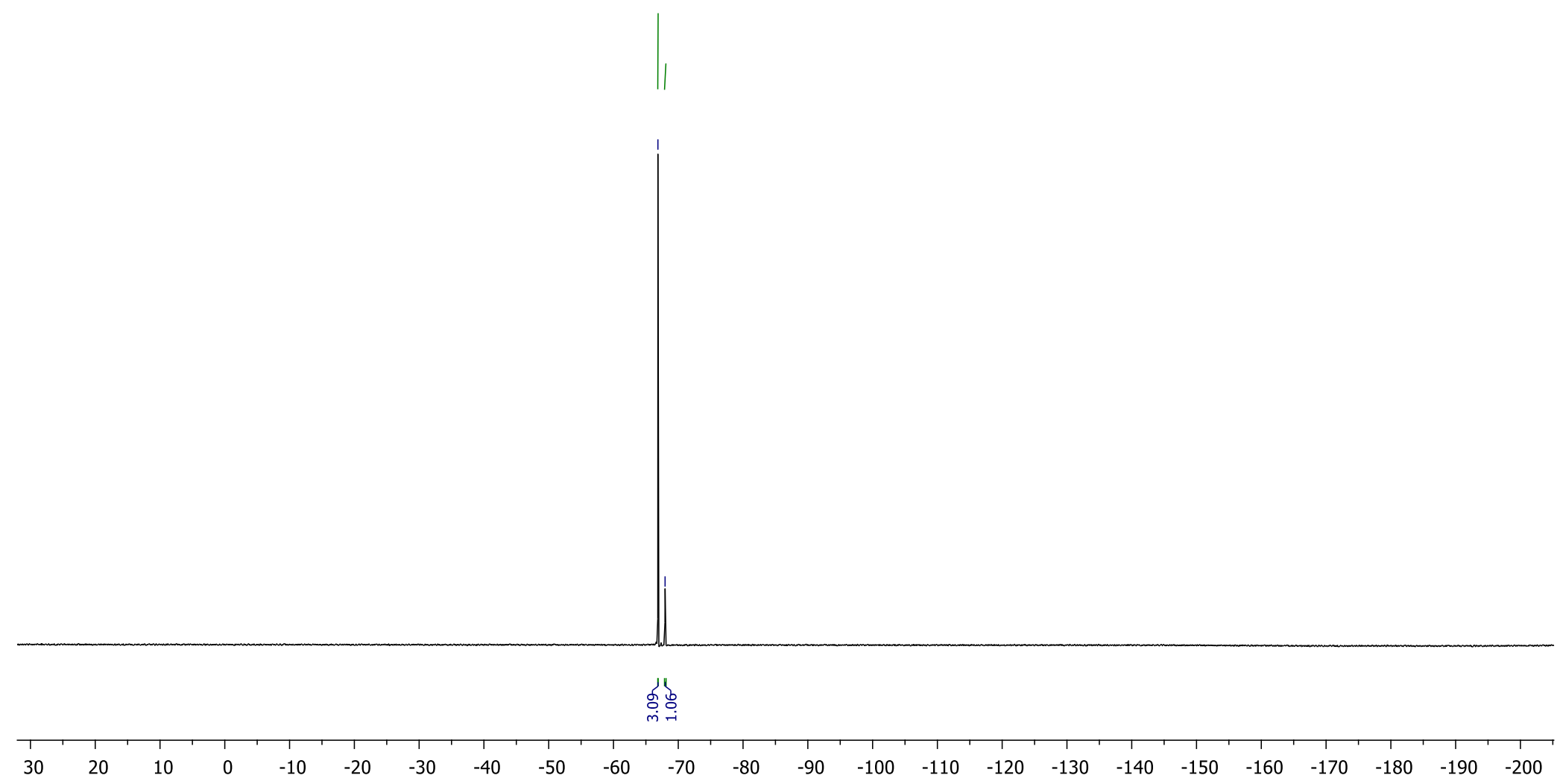


Compound 47a

${ }^{1} \mathrm{H}$ NMR (400 MHz, $\mathrm{CDCl}_{3}$ )

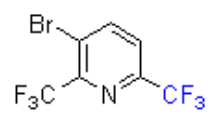

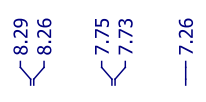

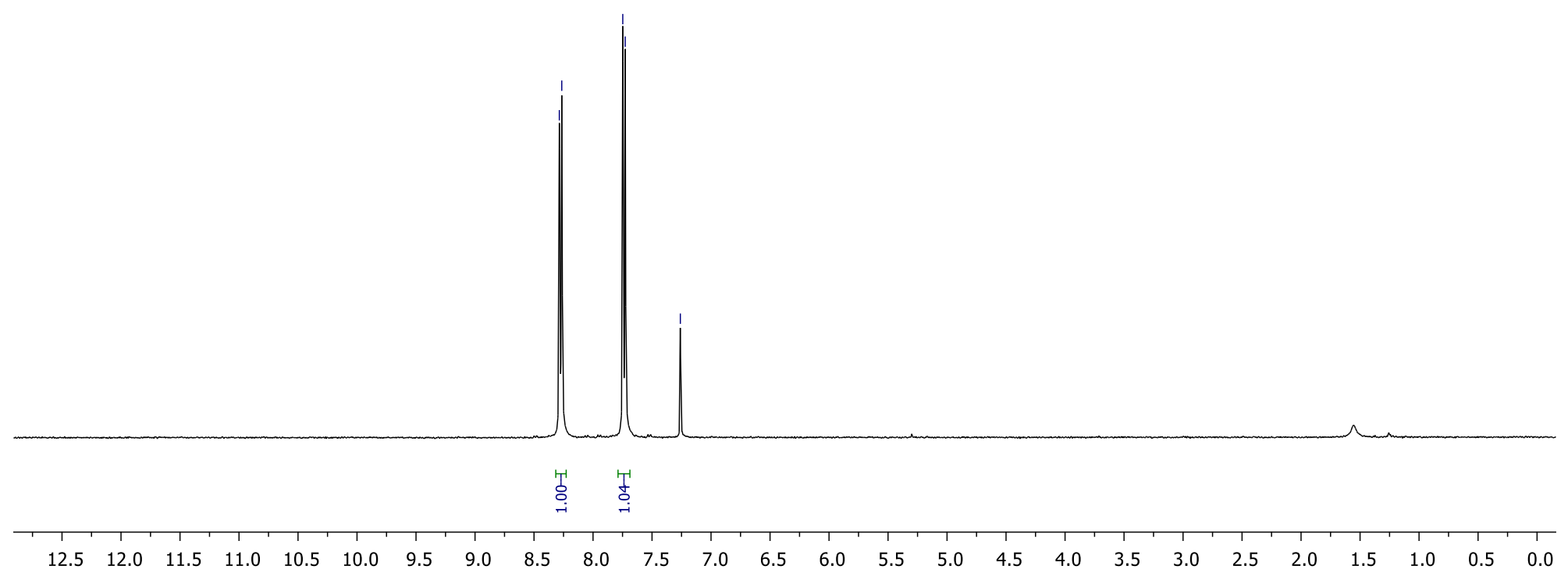

S142 
${ }^{13} \mathrm{C}\left\{{ }^{1} \mathrm{H}\right\}$ NMR $\left(151 \mathrm{MHz}, \mathrm{CDCl}_{3}\right)$

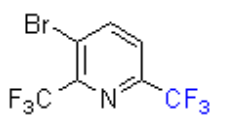

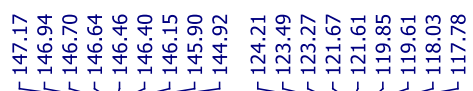

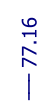
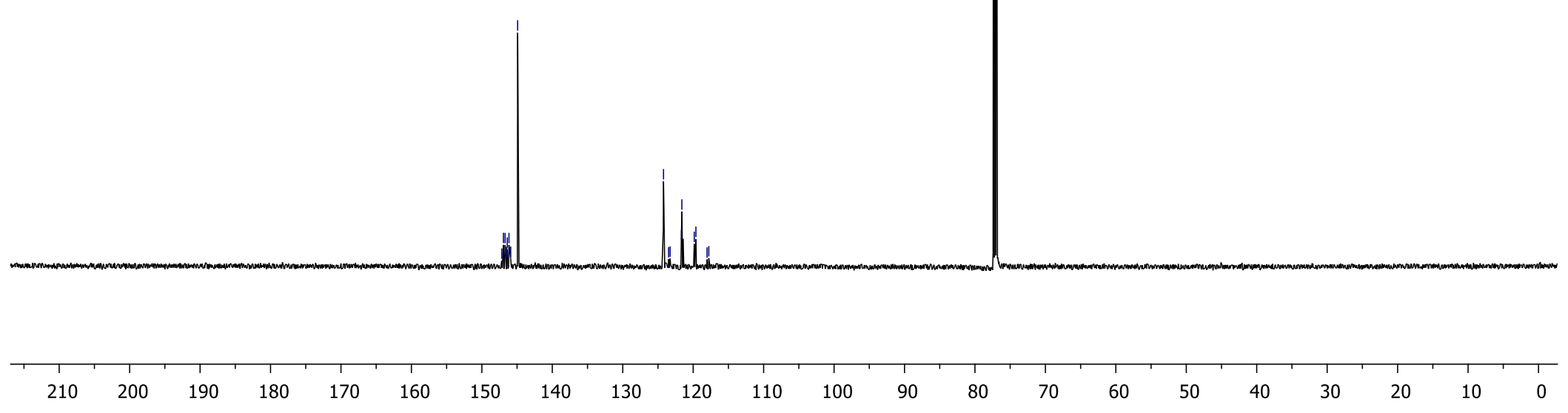

S143 
${ }^{19} \mathrm{~F}$ NMR (376 MHz, $\mathrm{CDCl}_{3}$ )
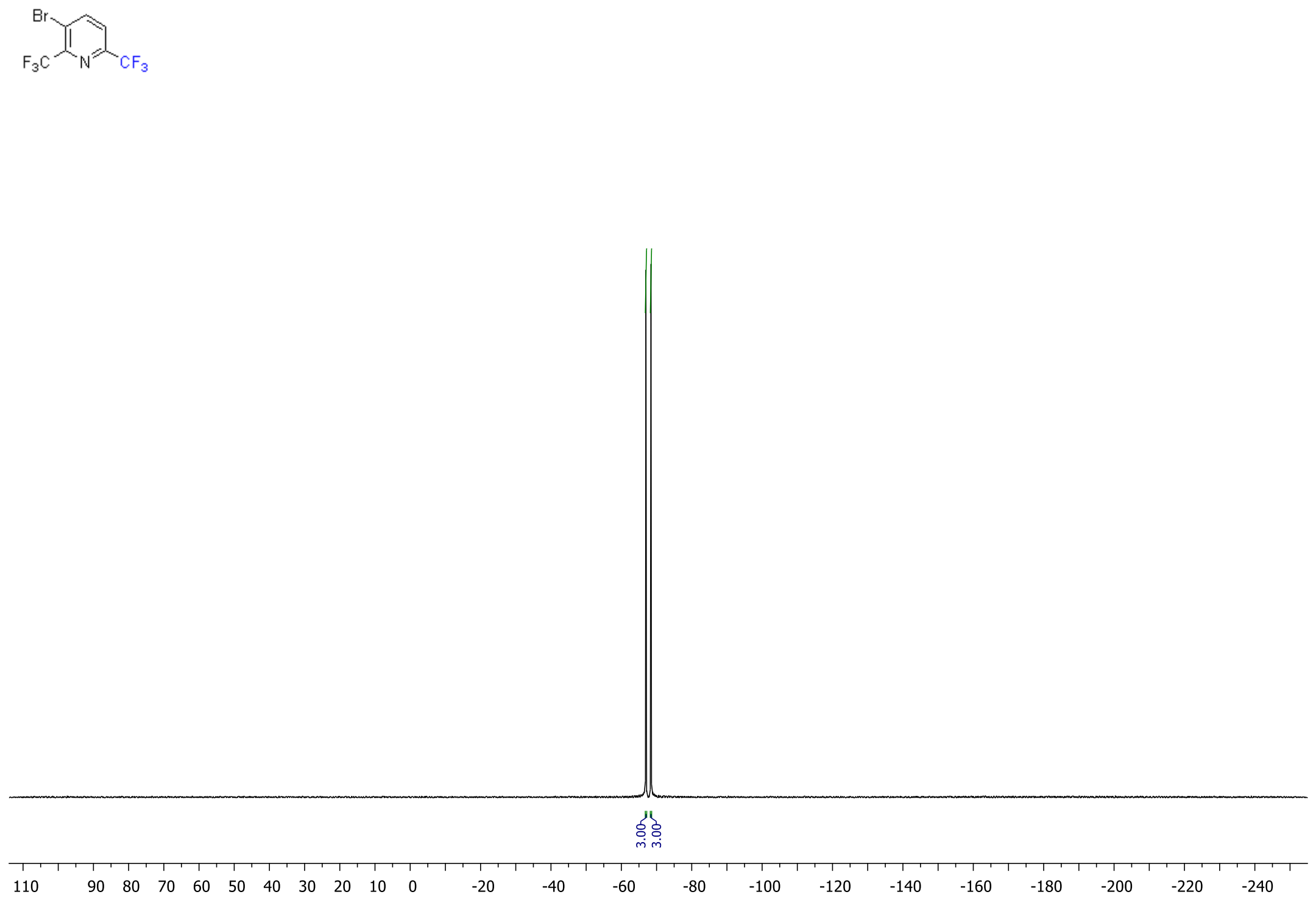
Compound $48 \mathrm{a}^{*}$

${ }^{1} \mathrm{H}$ NMR $\left(400 \mathrm{MHz}, \mathrm{CDCl}_{3}\right)$
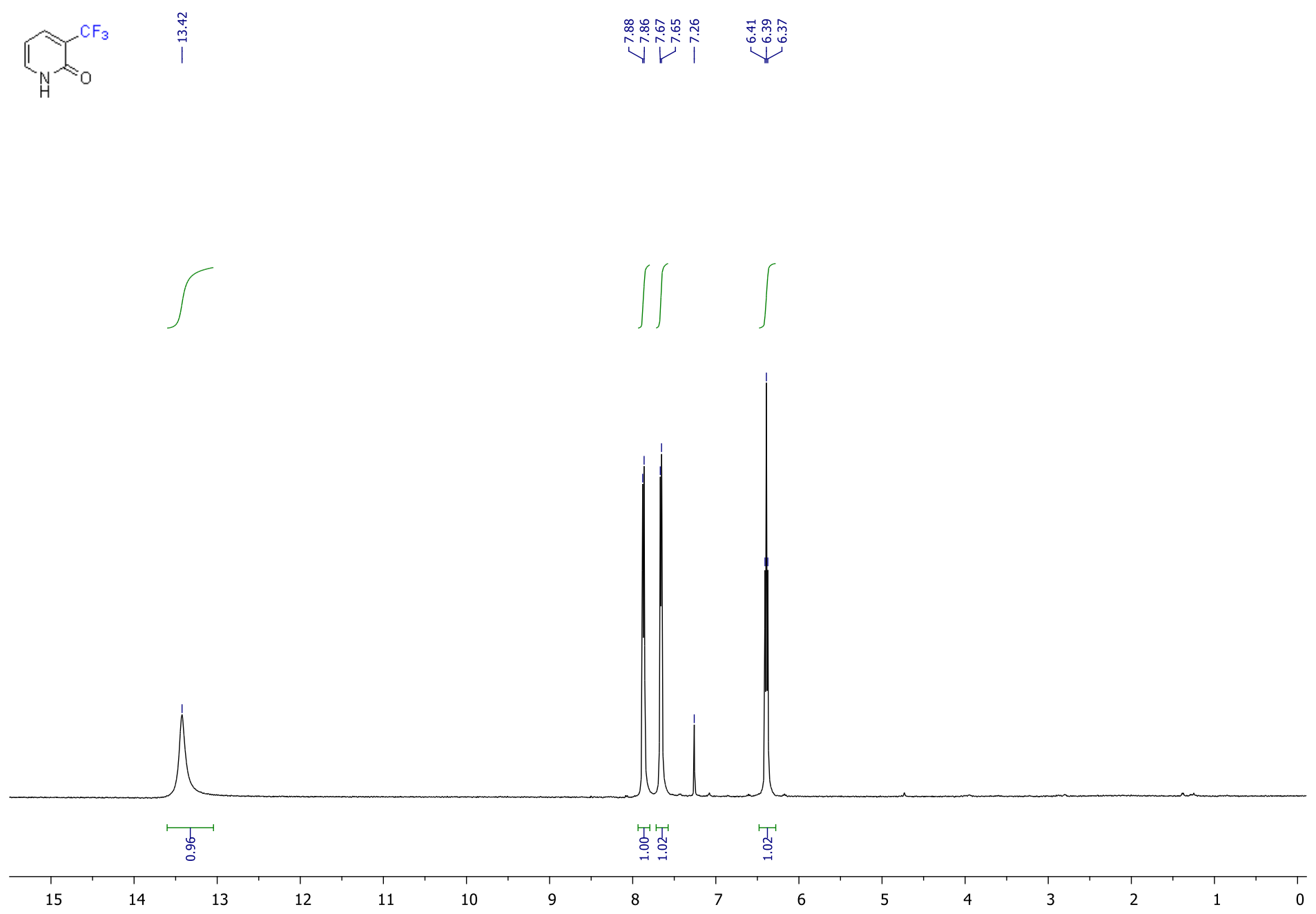

Compound 48a is also described in: a) J. Fluorine Chem. 2010, 131, 98-105; b) Synthetic Commun. 2006, 36, 9,1235 - 1245. 
${ }^{13} \mathrm{C}\left\{{ }^{1} \mathrm{H}\right\}$ NMR $\left(151 \mathrm{MHz}, \mathrm{CDCl}_{3}\right)$

$\overbrace{\mathrm{H}}^{\mathrm{N}_{\mathrm{O}}} \mathrm{CF}_{3}$

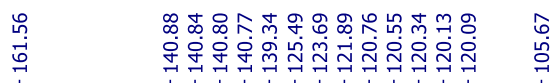

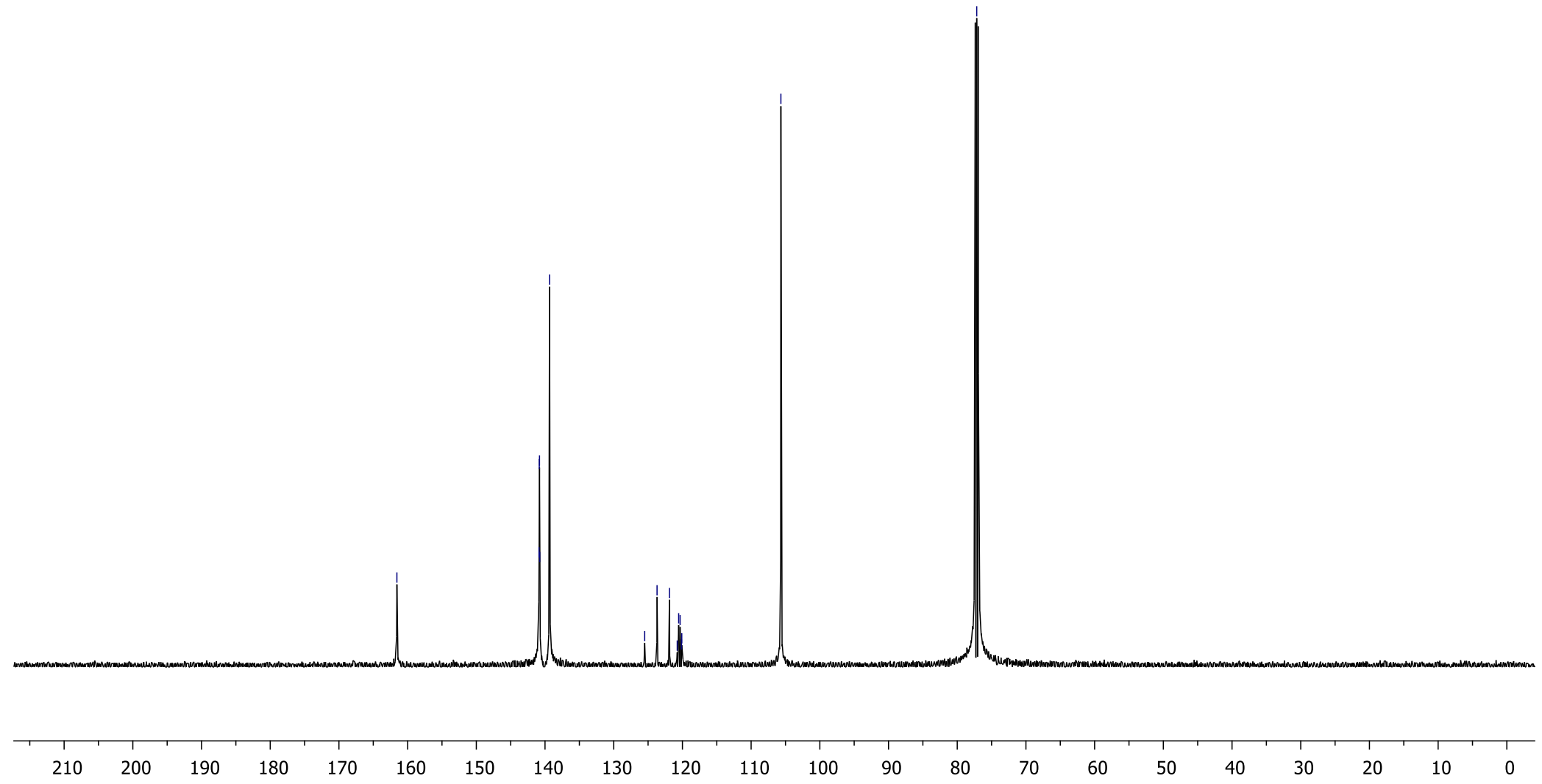


${ }^{19} \mathrm{~F}$ NMR (376 MHz, $\mathrm{CDCl}_{3}$ )

$\overbrace{\mathrm{H}}^{\mathrm{CF}_{3}}$

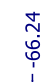

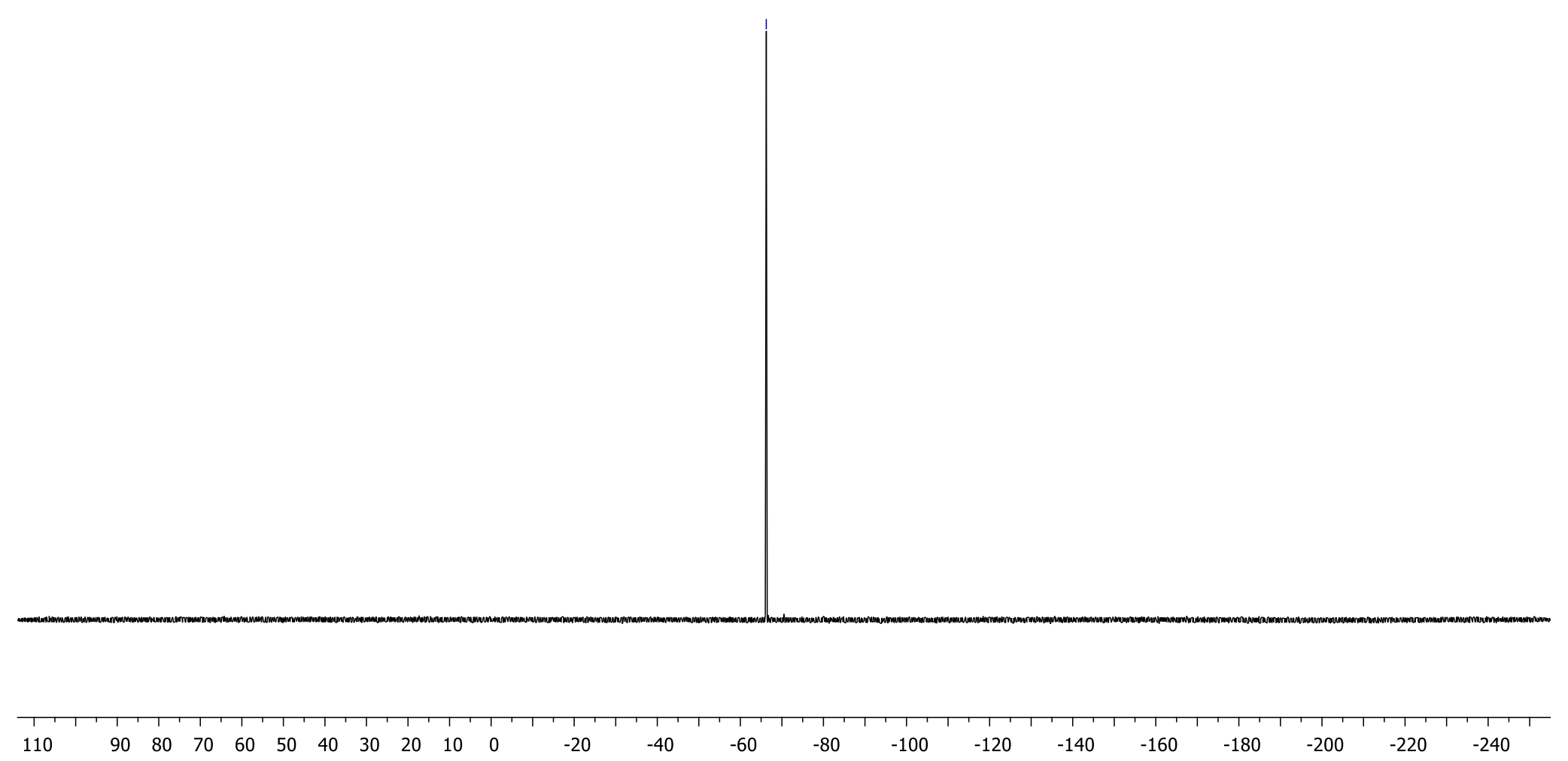


Compound $49 \mathrm{a}^{*}$

${ }^{1} \mathrm{H}$ NMR (400 MHz, DMSO-d $)$

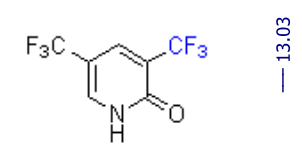

$\underset{\substack{0 \\ \infty}}{\substack{\infty \\ 11}}$

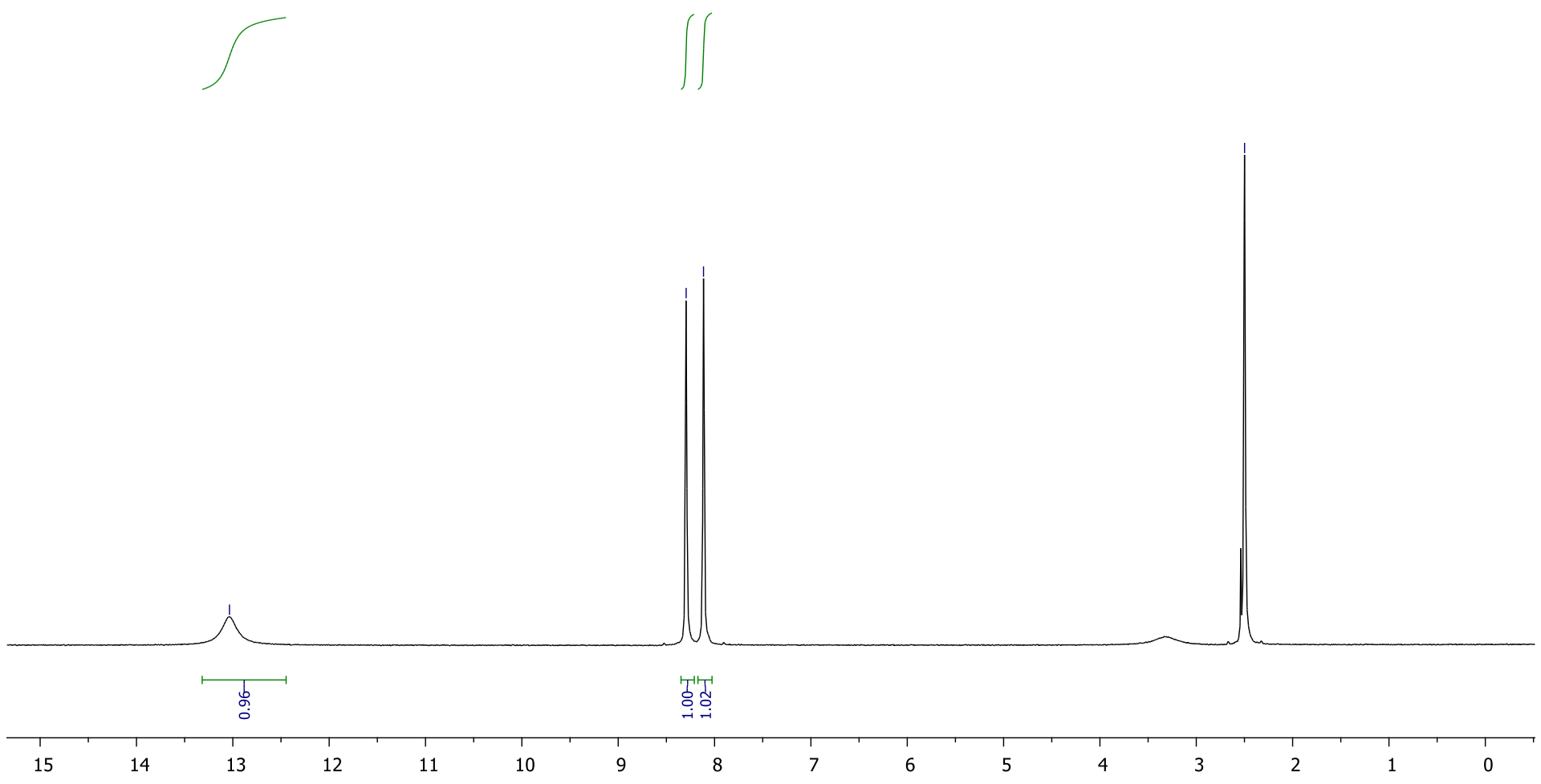

Compound 49a is also described in: a) Angew. Chem. Int. Ed. 2017, 56, 18, 5125-5129; b) J. Org. Chem. 1988, 53, 19, 4582-4585. 
${ }^{13} \mathrm{C}\left\{{ }^{1} \mathrm{H}\right\}$ NMR (126 MHz, DMSO-d 6 )

$\mathrm{N}_{\mathrm{H}}^{\mathrm{CF}_{3}}$

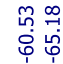

i
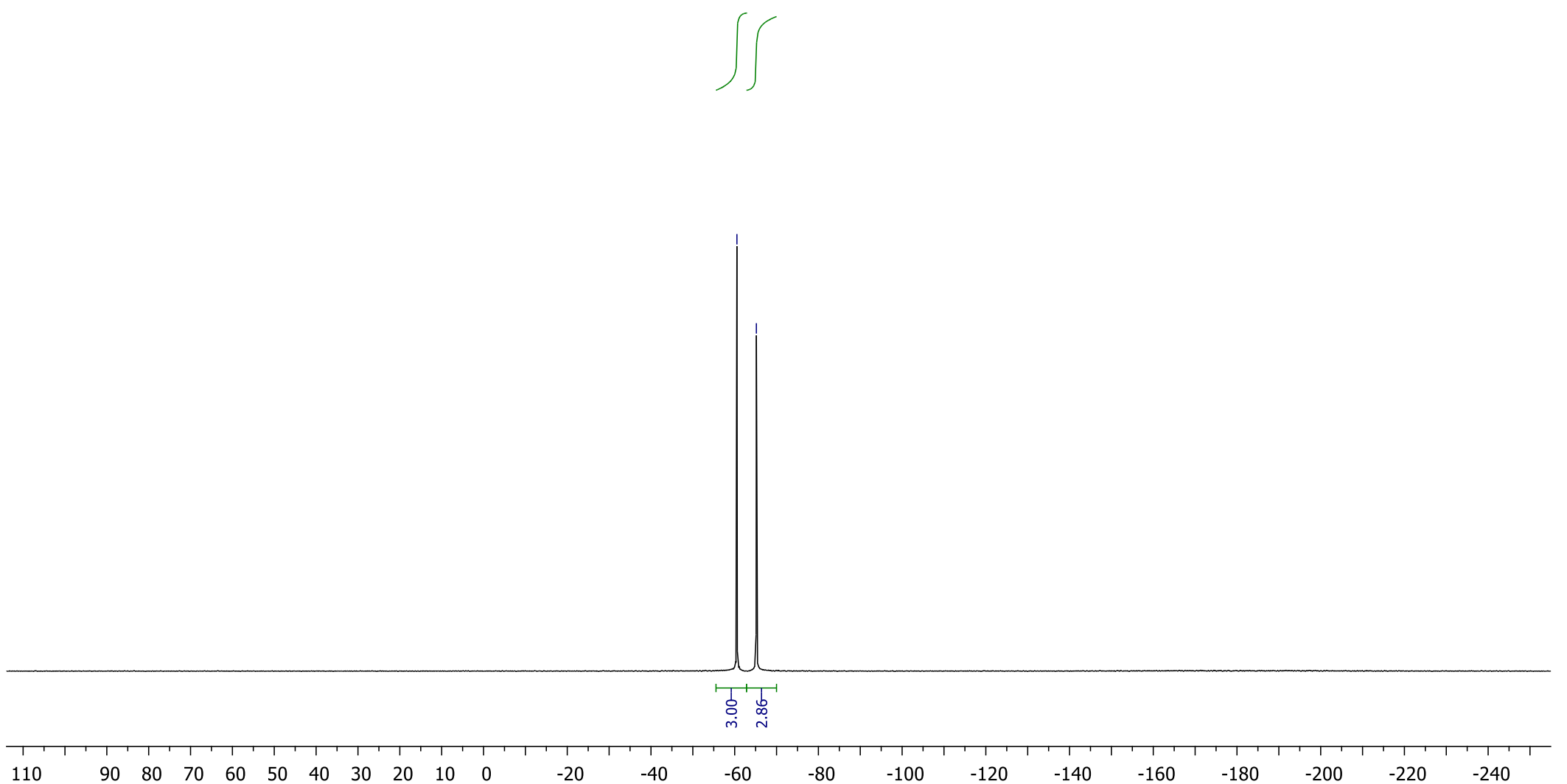
${ }^{19}$ F NMR (376 MHz, DMSO-d 6 )
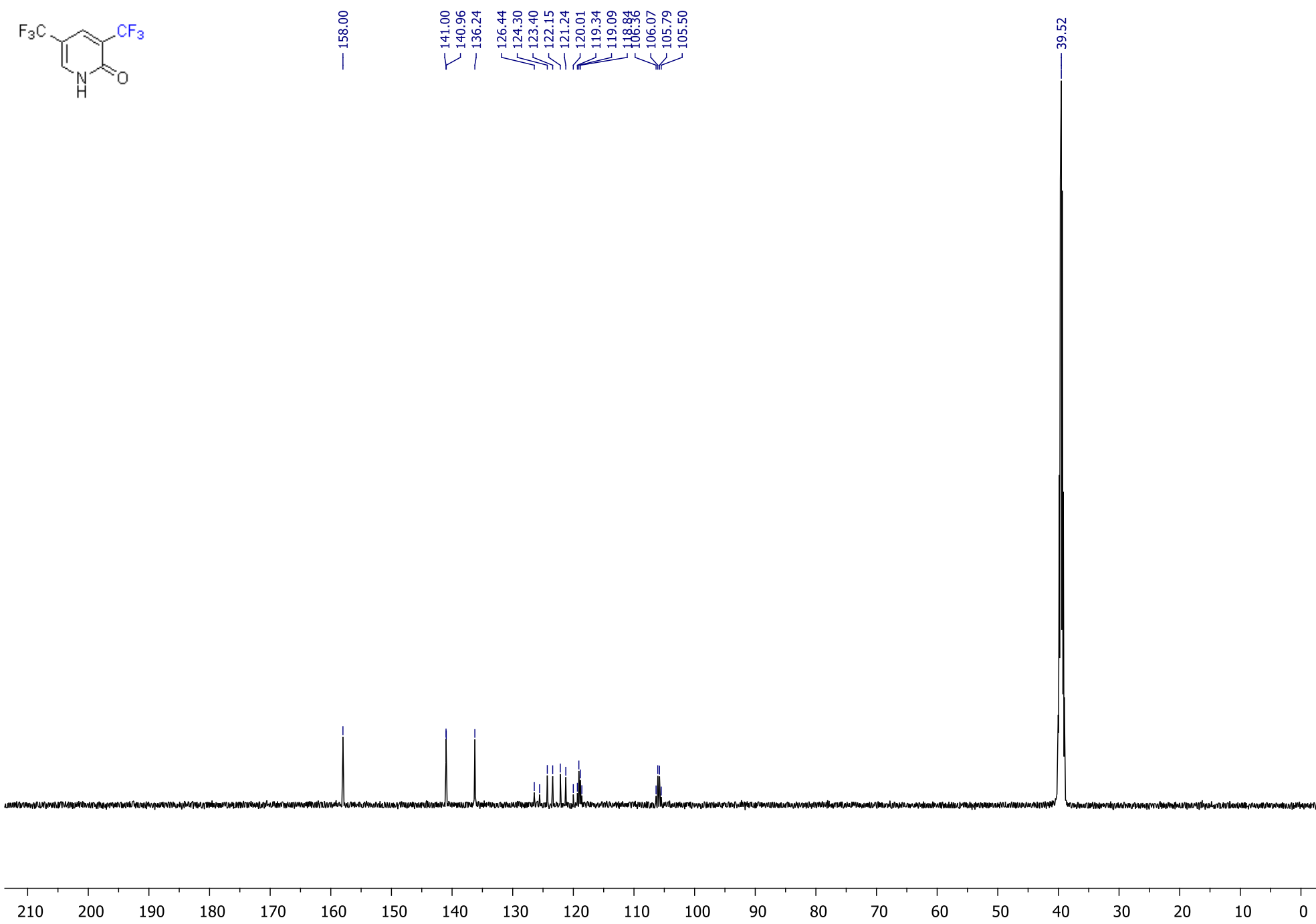
Compound 50a*

${ }^{1} \mathrm{H}$ NMR (400 MHz, DMSO-d 6 )

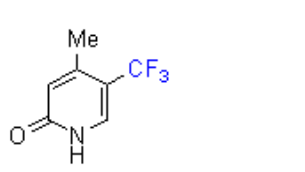

i

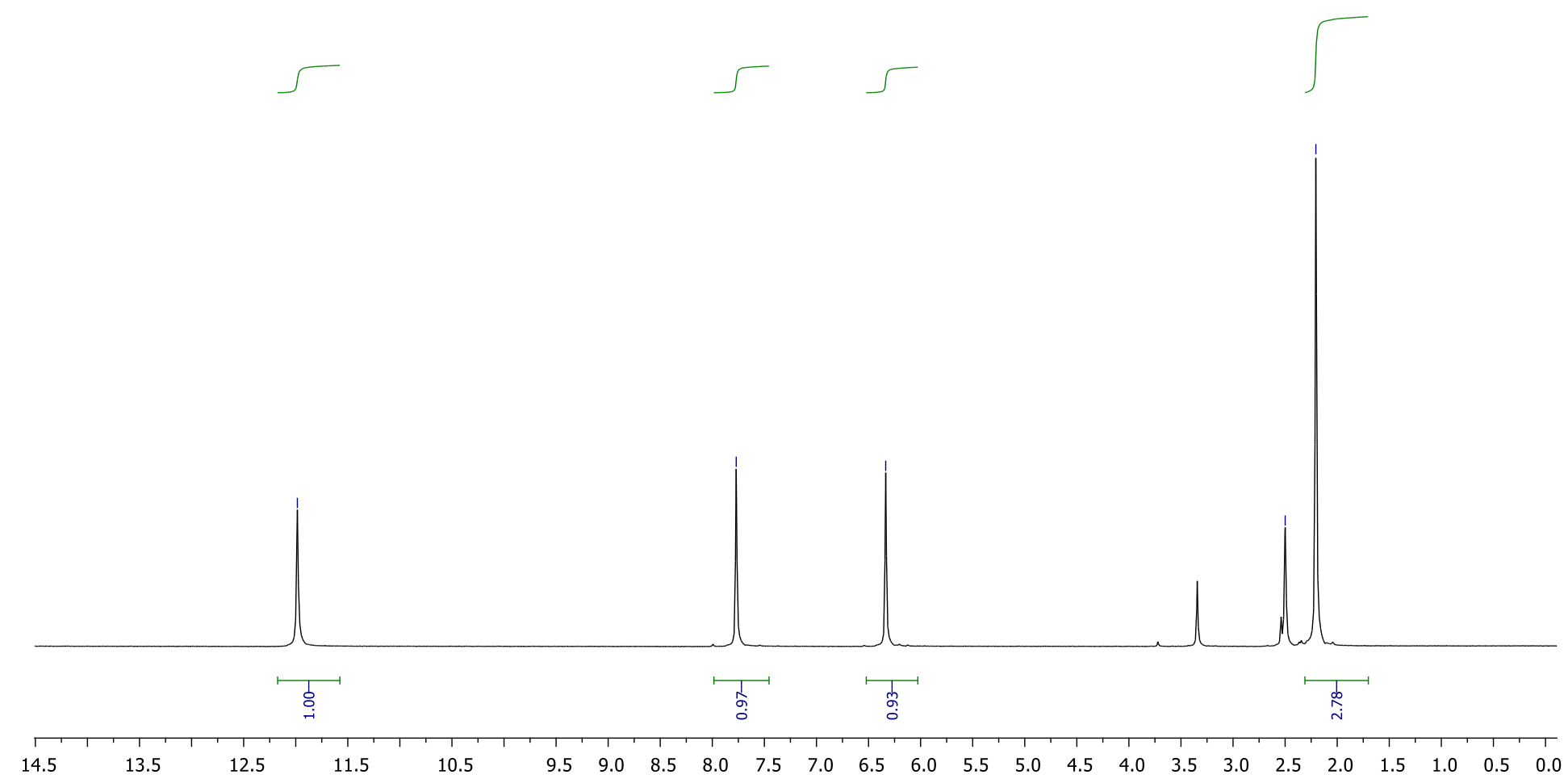

Compound 50a is also described in: J. Organomet. Chem. 2017, 843, 15, 14-19. 
${ }^{13} \mathrm{C}\left\{{ }^{1} \mathrm{H}\right\}$ NMR (151 MHz, DMSO-d 6 )

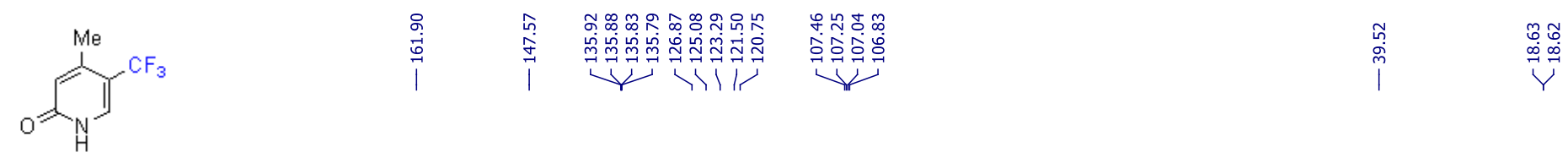

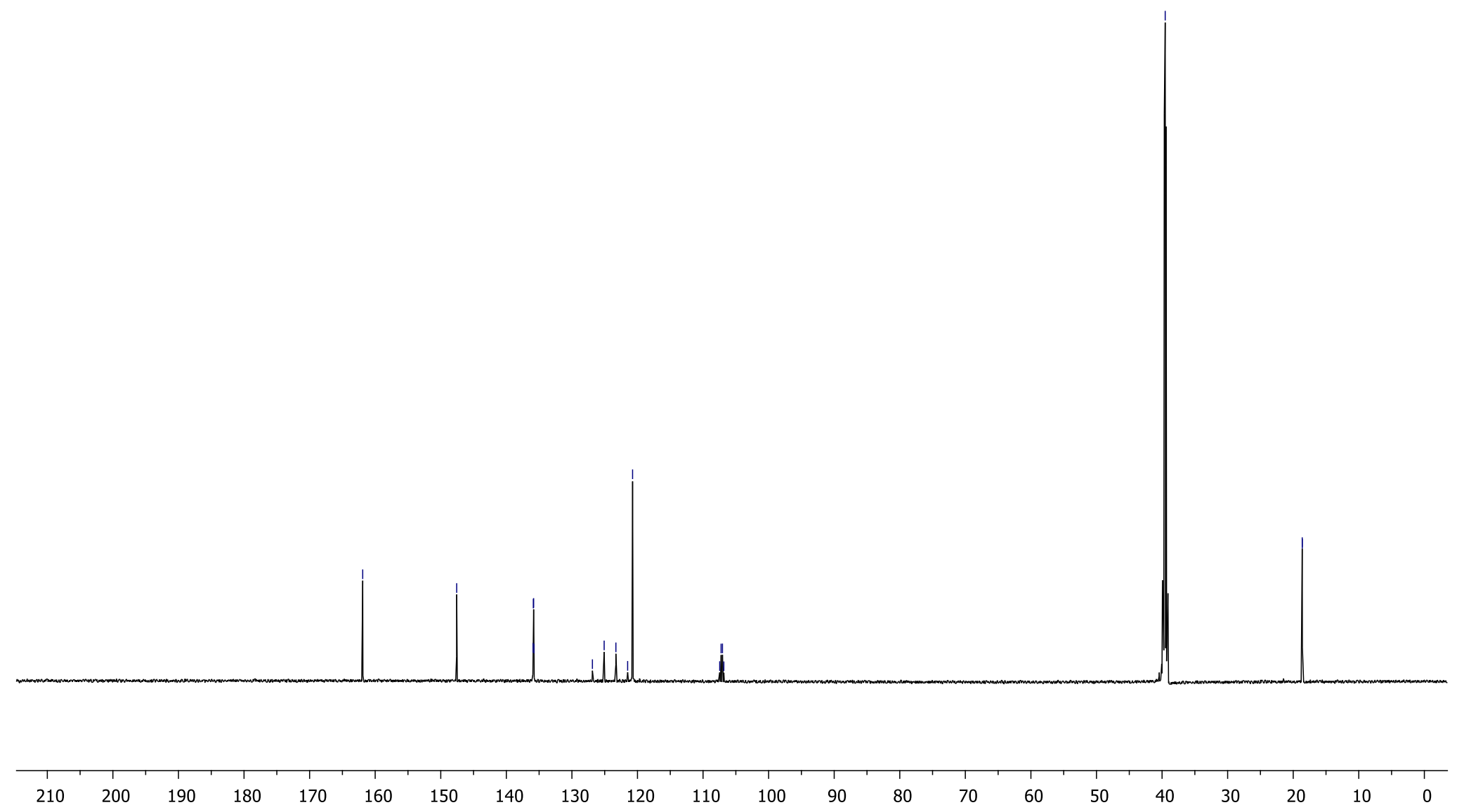


${ }^{19}$ F NMR (376 MHz, DMSO-d 6 )

(Ne)

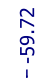

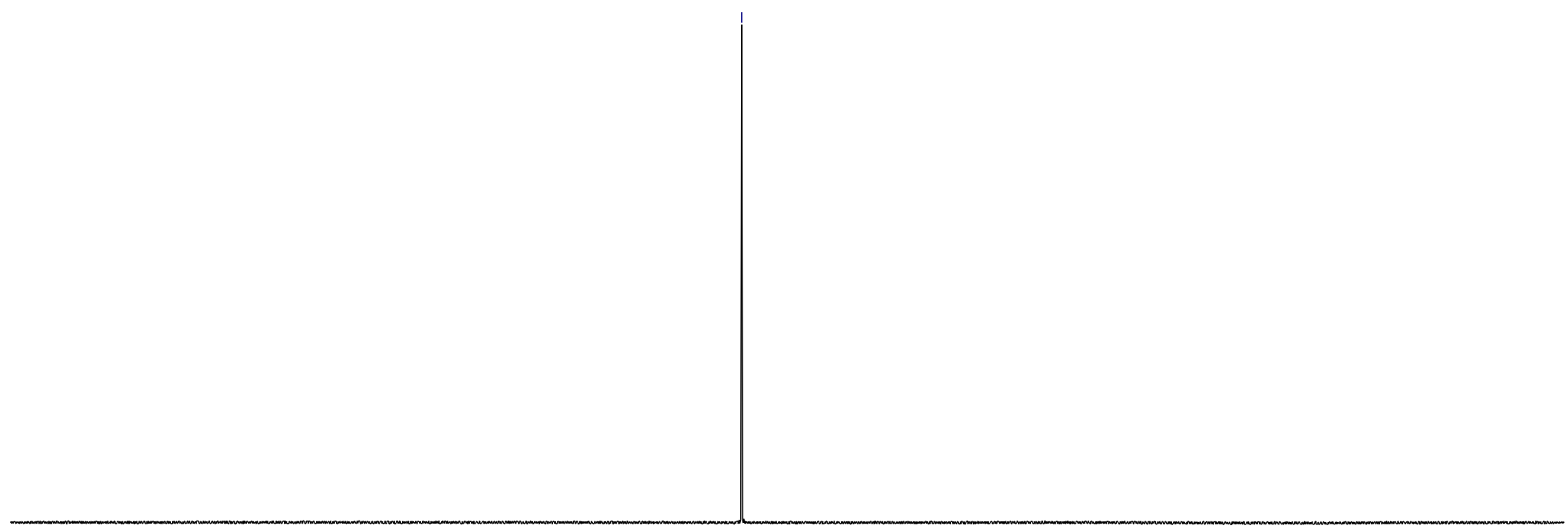

$\begin{array}{lllllllll}90 & 80 & 70 & 60 & 50 & 40 & 30 & 20 & 10\end{array}$

$-20$

$-40 \quad-60$

$-80 \quad-100$

$-120$

T1 T

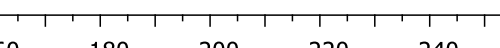


Compound 51a

${ }^{1} \mathrm{H}$ NMR (400 MHz, $\mathrm{CDCl}_{3}$ )

$\int_{N}^{C F_{3}}$

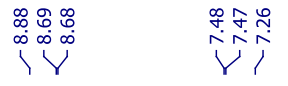
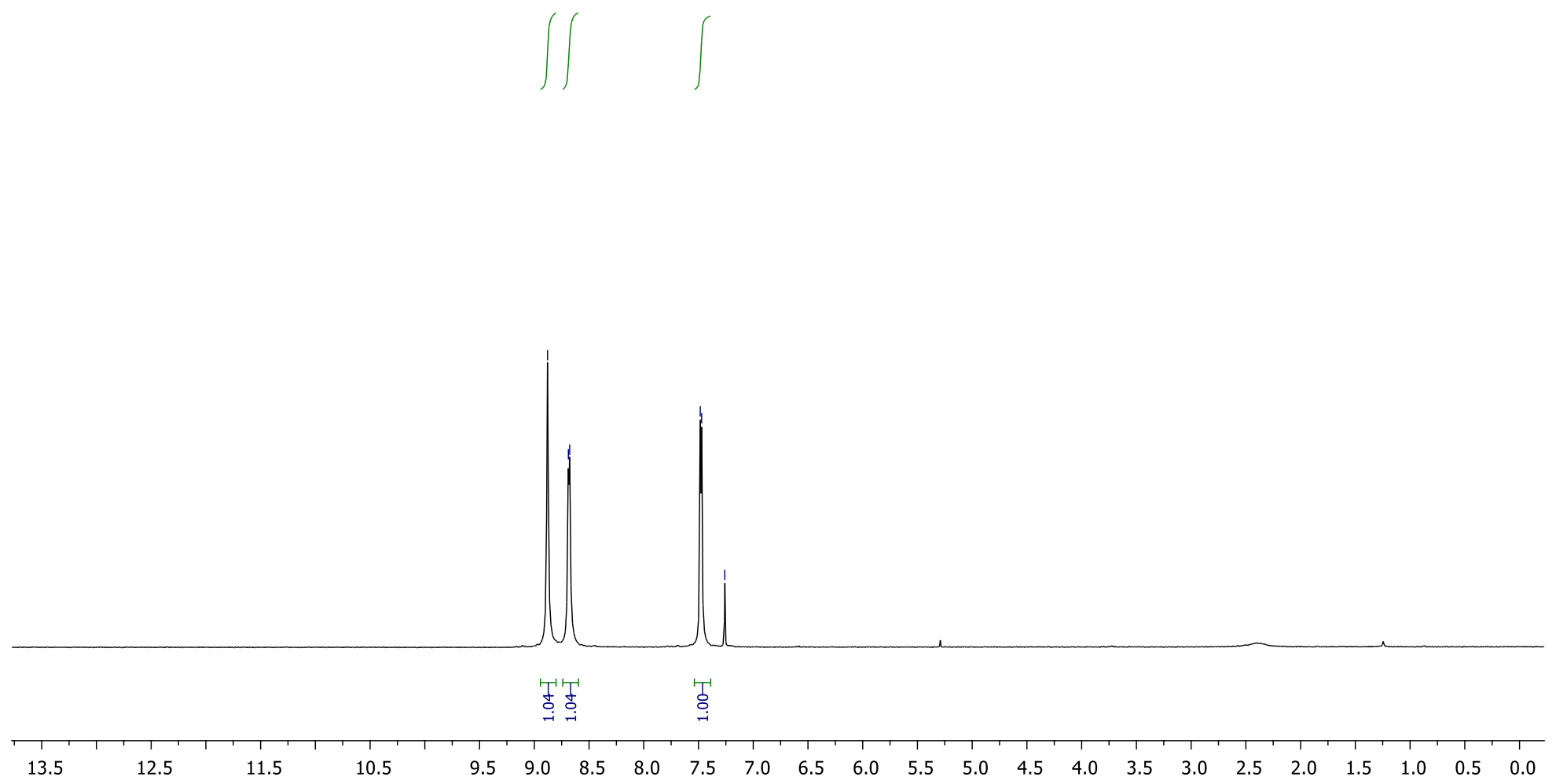
${ }^{13} \mathrm{C}\left\{{ }^{1} \mathrm{H}\right\}$ NMR $\left(126 \mathrm{MHz}, \mathrm{CDCl}_{3}\right)$

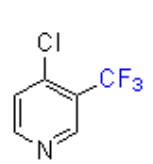

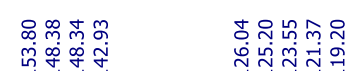

저궉ㄱ

$\stackrel{2}{\stackrel{2}{i}}$

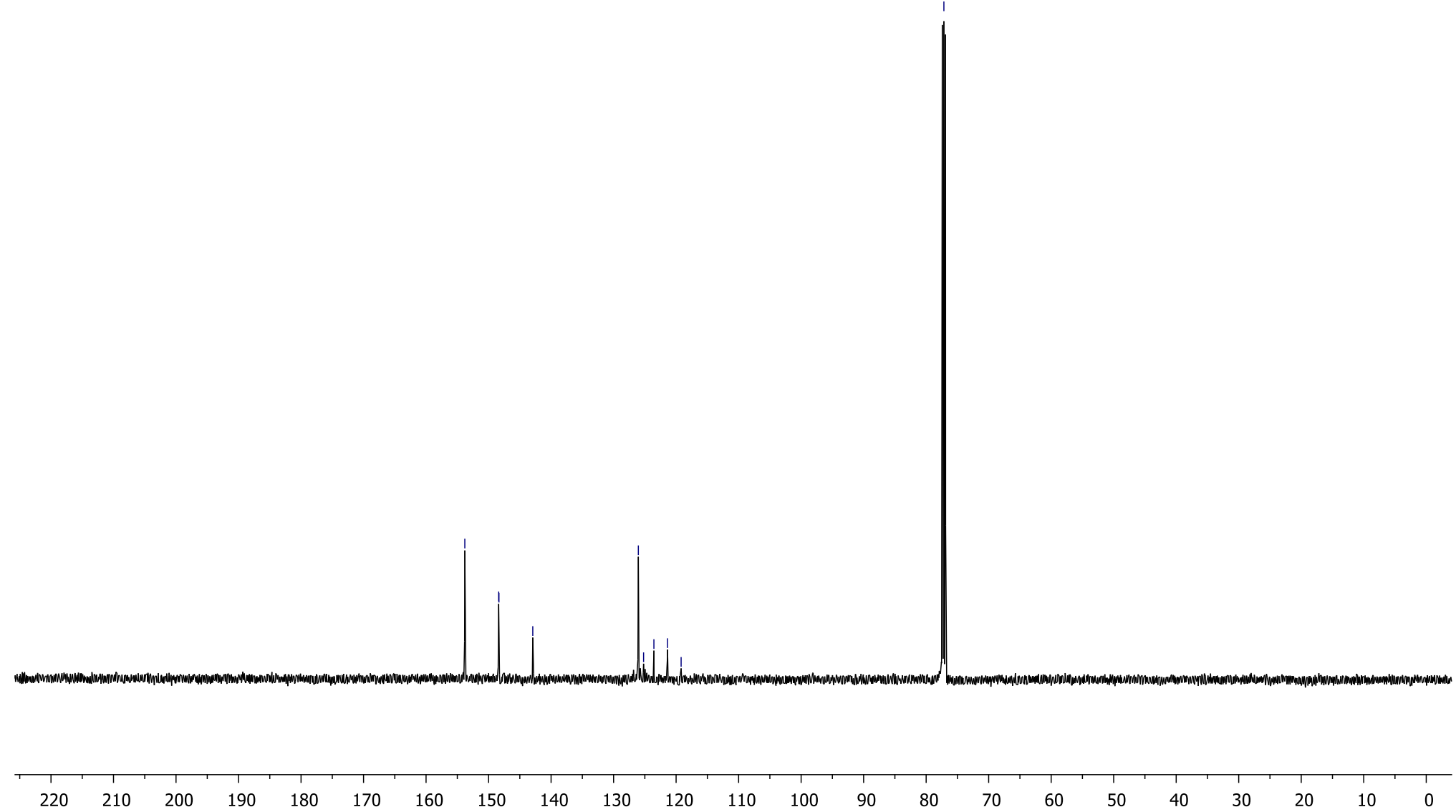


${ }^{19} \mathrm{~F}$ NMR (376 MHz, $\left.\mathrm{CDCl}_{3}\right)$

$\int_{N}^{C l} C_{3}^{F_{3}}$

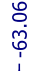

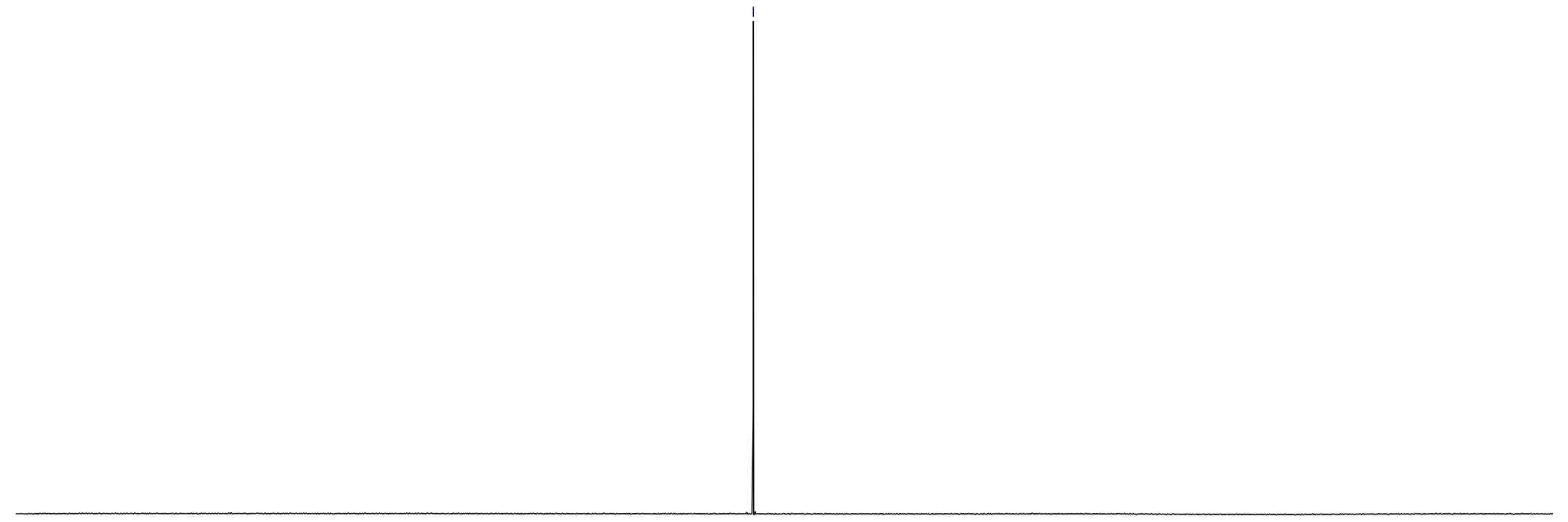

$\begin{array}{lllllllllll}110 & 90 & 80 & 70 & 60 & 50 & 40 & 30 & 20 & 10 & 0\end{array}$ 
Compound 52a

${ }^{1} \mathrm{H}$ NMR (400 MHz, $\mathrm{CDCl}_{3}$ )

$\mathrm{Cl}_{\mathrm{N}}^{\mathrm{CF}_{3}}$

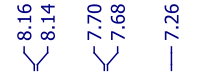
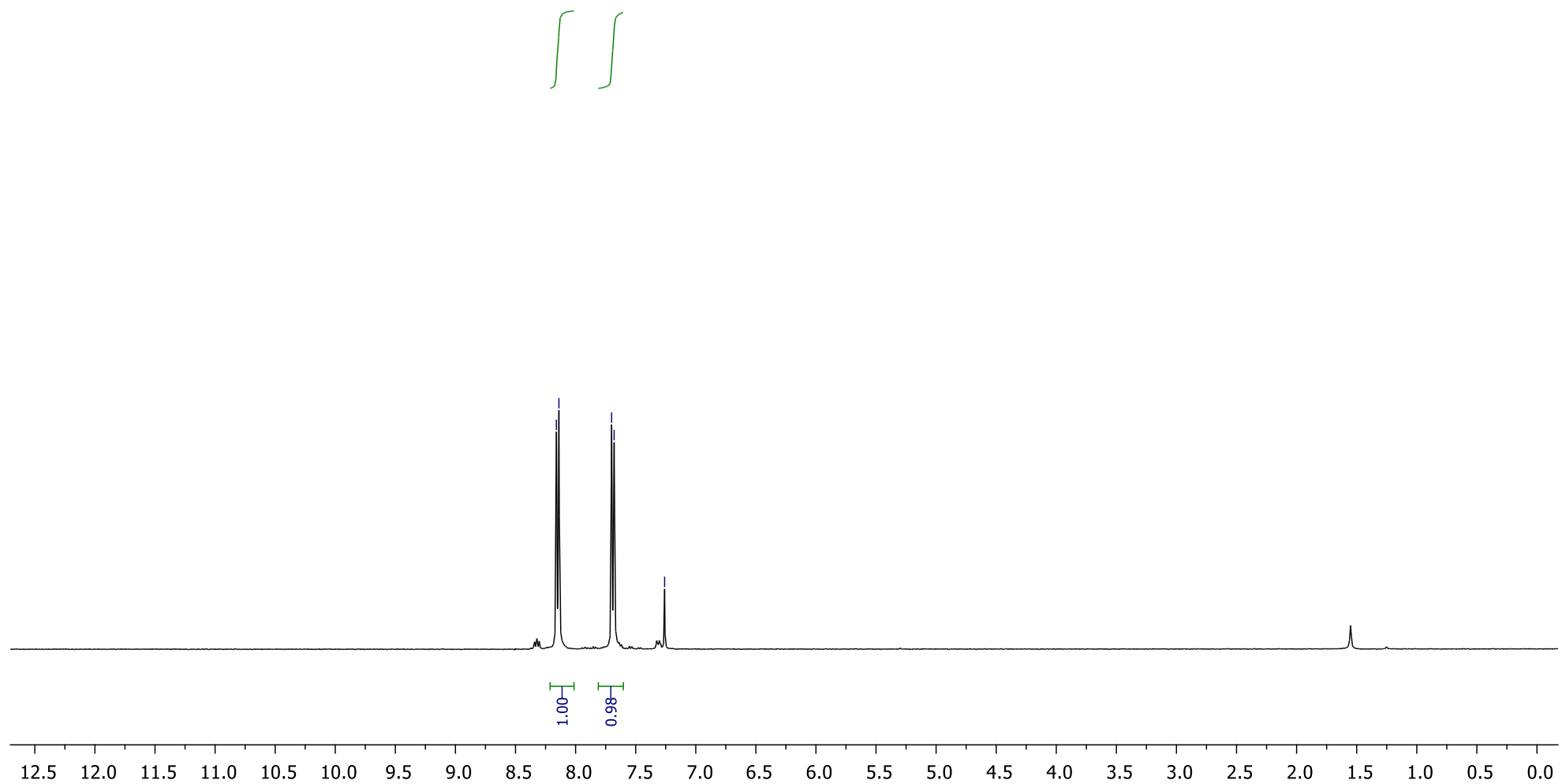
${ }^{13} \mathrm{C}\left\{{ }^{1} \mathrm{H}\right\}$ NMR $\left(151 \mathrm{MHz}, \mathrm{CDCl}_{3}\right)$

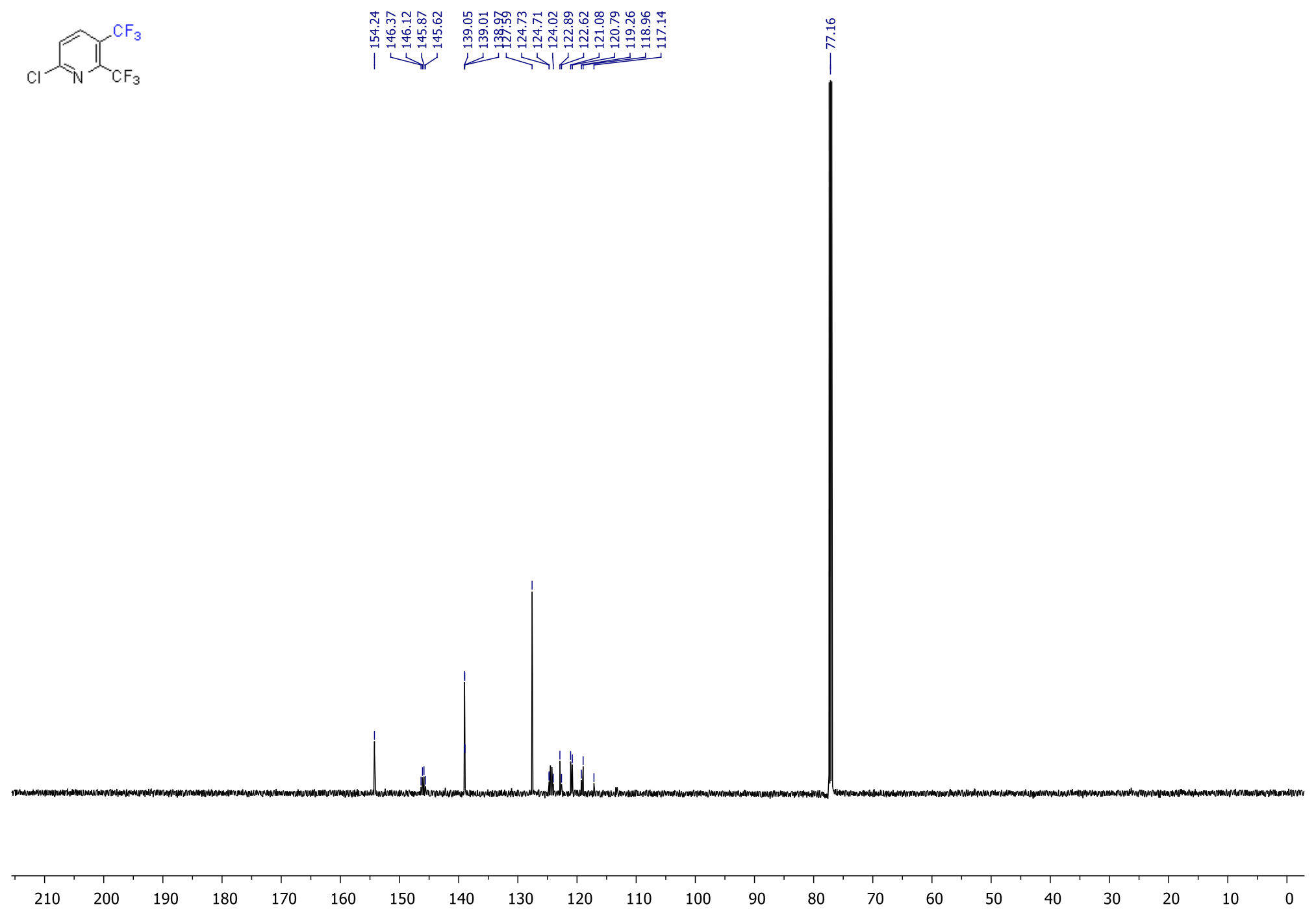


${ }^{19} \mathrm{~F}$ NMR $\left(376 \mathrm{MHz}, \mathrm{CDCl}_{3}\right)$

$\overbrace{\mathrm{N}}^{\mathrm{CF}_{3}}$

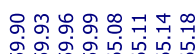

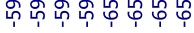

1
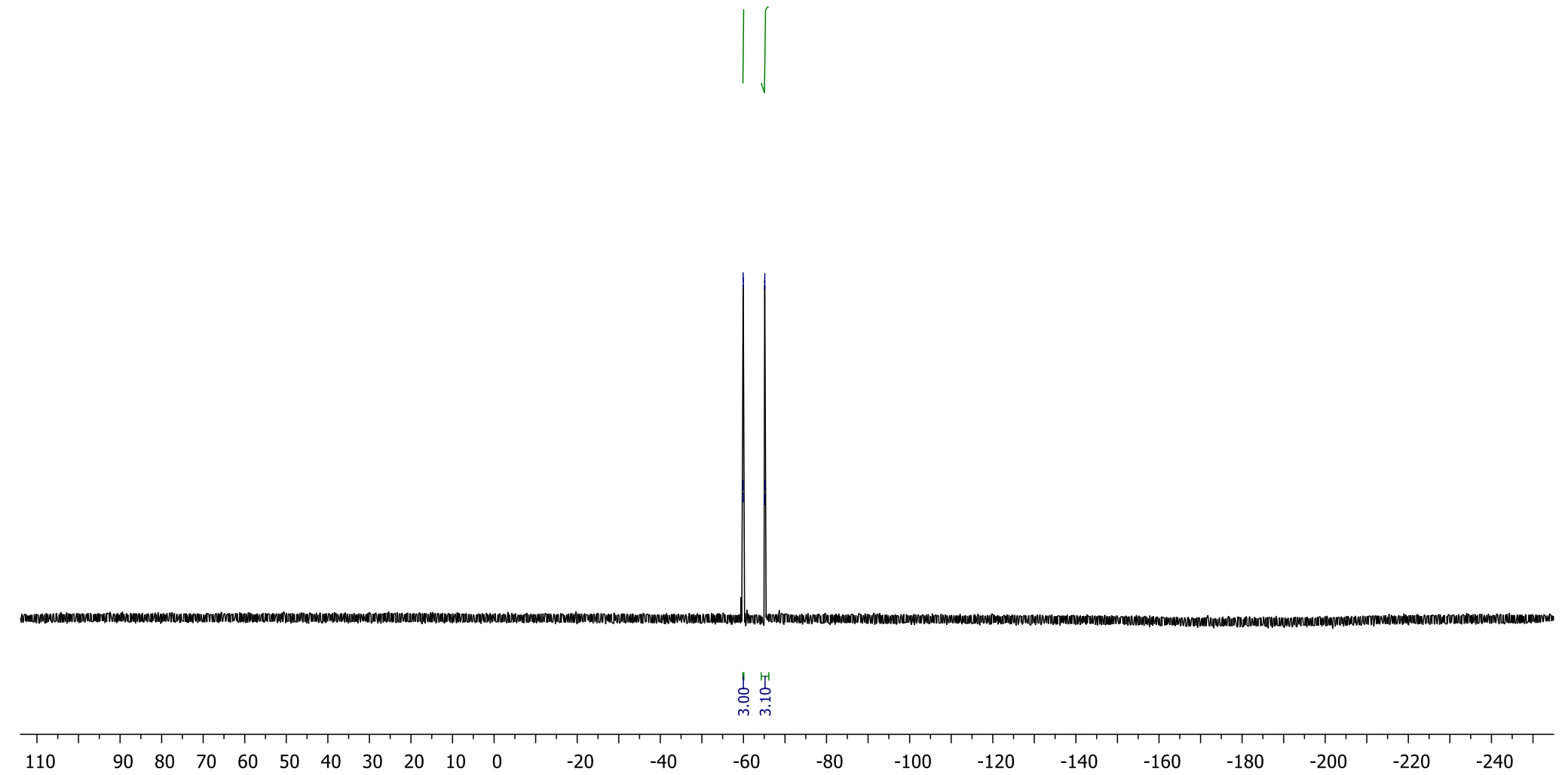
Compound 53a

${ }^{1} \mathrm{H}$ NMR (400 MHz, $\mathrm{CDCl}_{3}$ )

$\overbrace{\mathrm{N}}^{\mathrm{CO} \mathrm{F}_{3}}$

$\bigcup_{\substack{+\infty \\ \infty}}^{\substack{\infty \\ \infty}} \stackrel{\substack{\infty \\ \infty}}{i}$

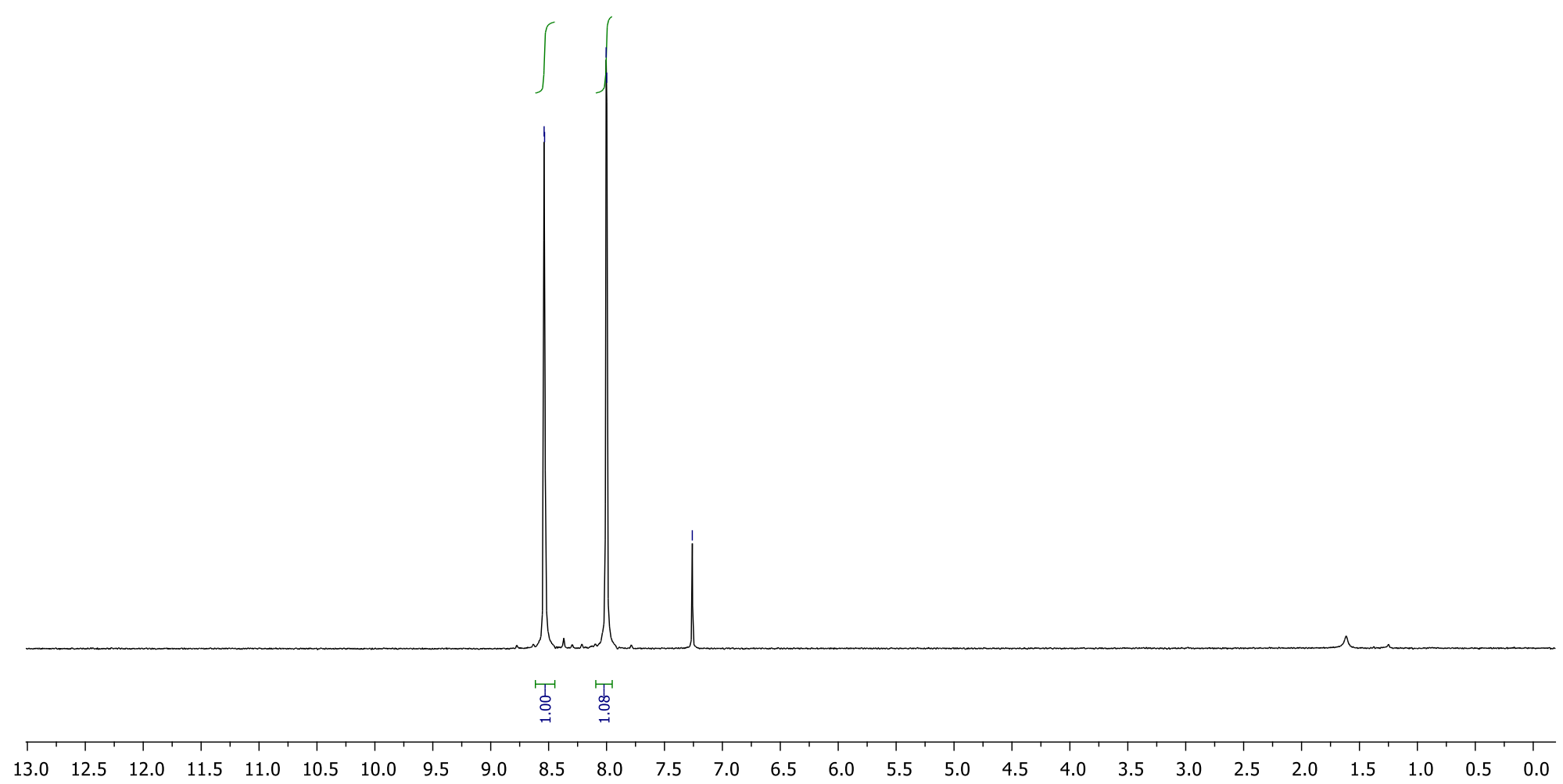


${ }^{13} \mathrm{C}\left\{{ }^{1} \mathrm{H}\right\}$ NMR $\left(126 \mathrm{MHz}, \mathrm{CDCl}_{3}\right)$

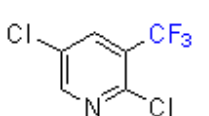

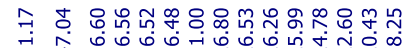

تُ

$\stackrel{\substack{i \\ i}}{i}$

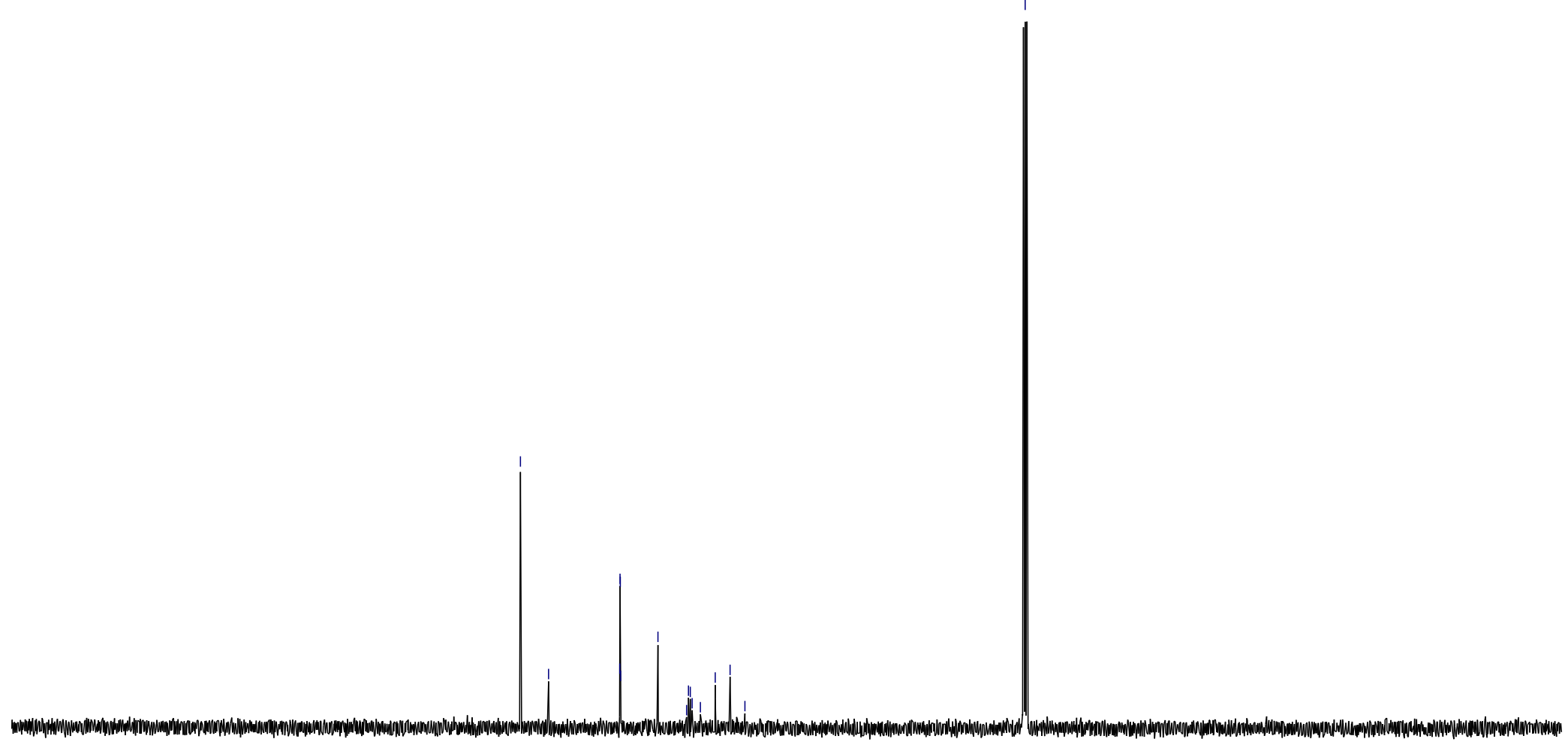


${ }^{19} \mathrm{~F}$ NMR (376 MHz, $\mathrm{CDCl}_{3}$ )

$\mathrm{C}_{\mathrm{Cl}}^{\mathrm{CF}_{3}}$

苦

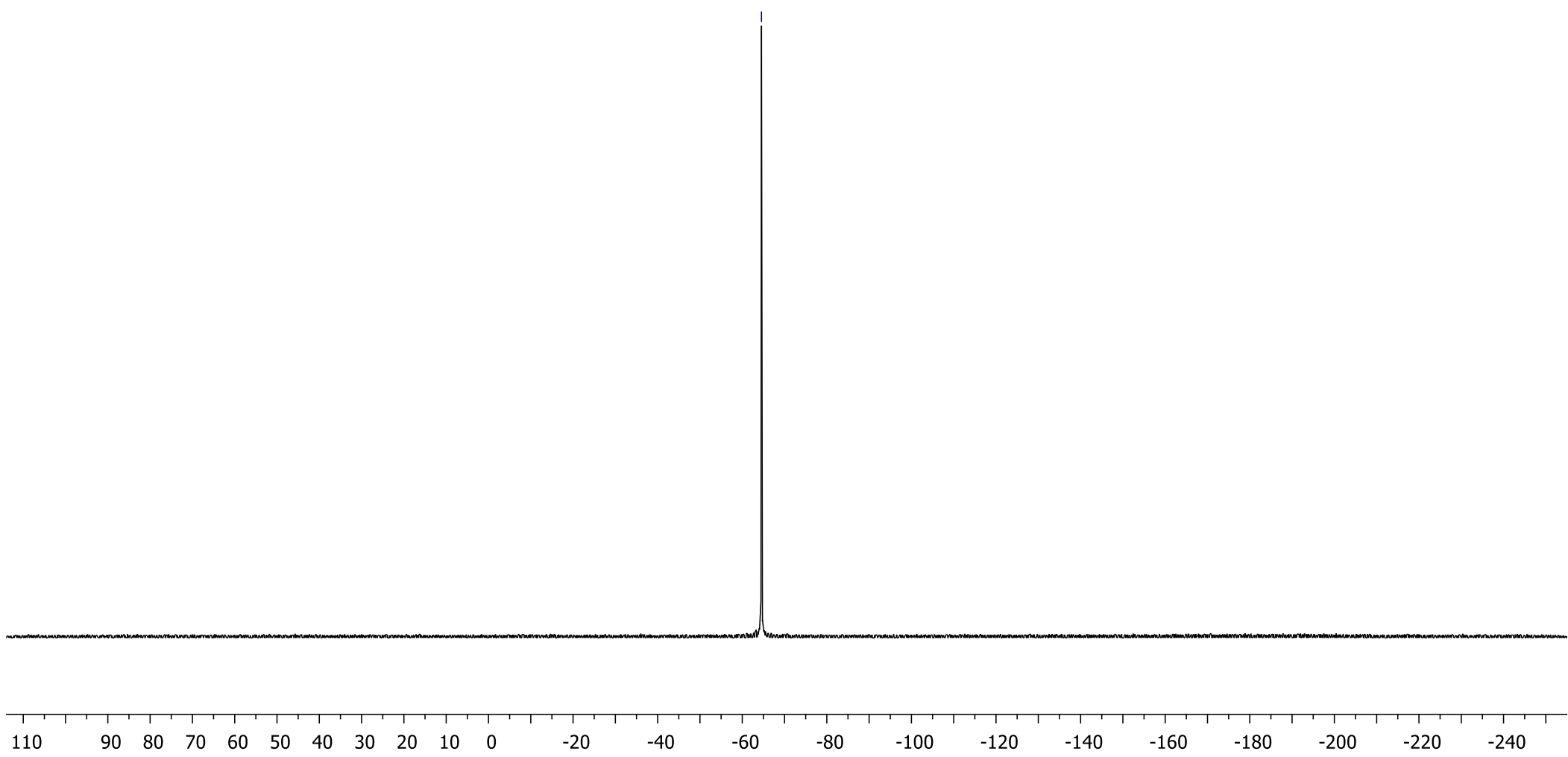


Compound 54a

${ }^{1} \mathrm{H}$ NMR (400 MHz, $\mathrm{CDCl}_{3}$ )
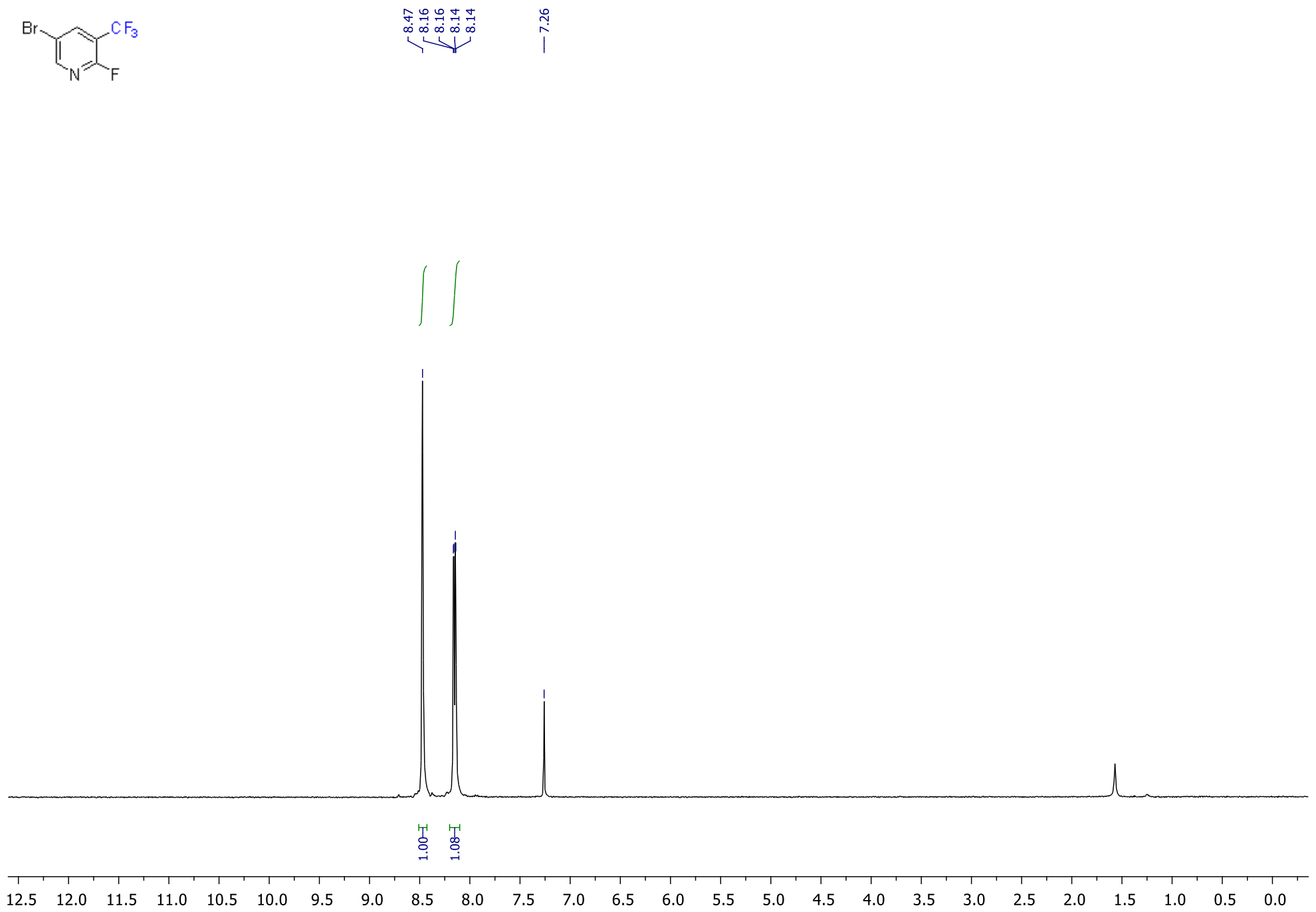
${ }^{13} \mathrm{C}\left\{{ }^{1} \mathrm{H}\right\}$ NMR $\left(126 \mathrm{MHz}, \mathrm{CDCl}_{3}\right)$

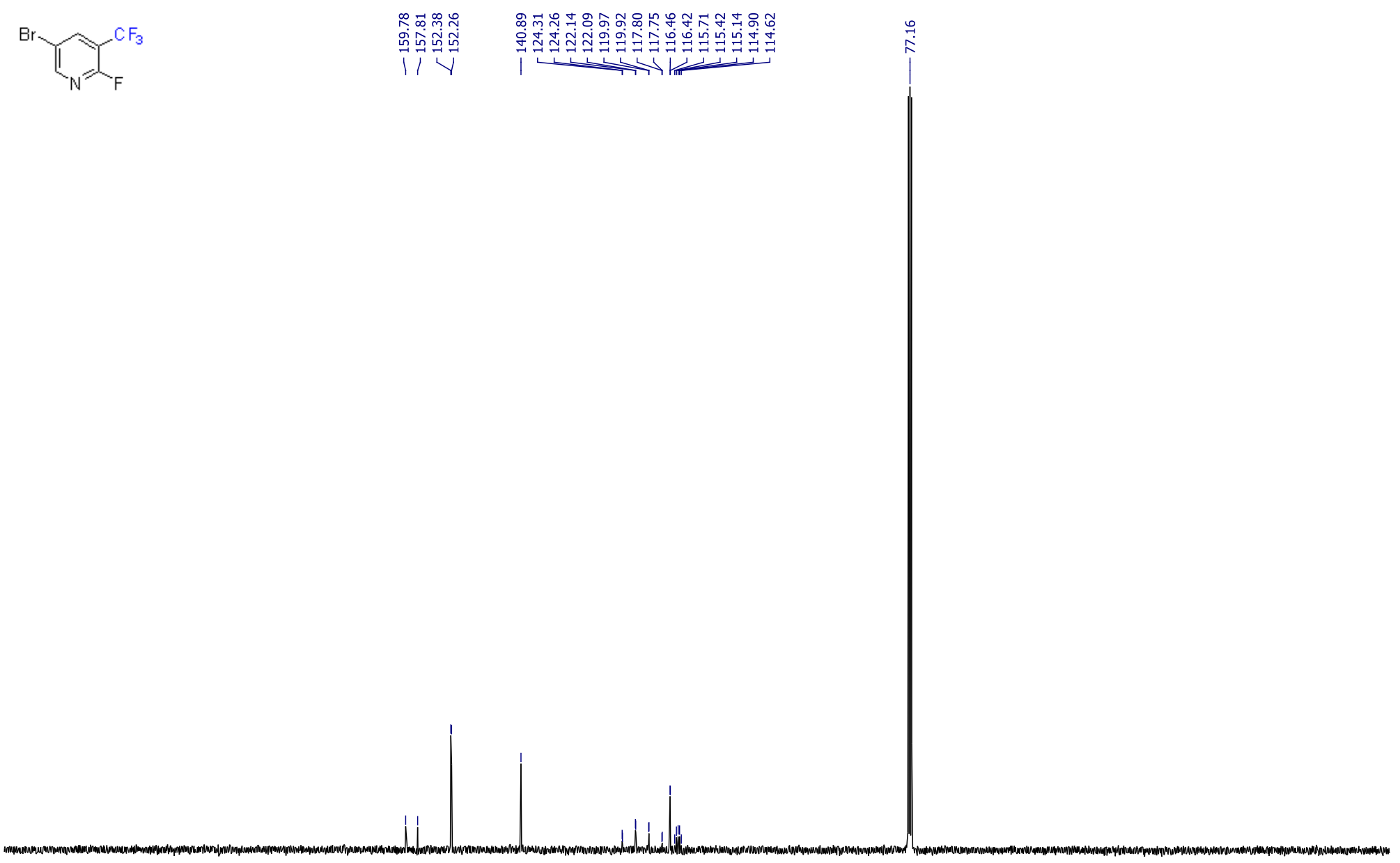

$\begin{array}{lllllllllllll}220 & 210 & 200 & 190 & 180 & 170 & 160 & 150 & 140 & 130 & 120 & 110 & 100\end{array}$ $70 \quad 60$ 
$\left.{ }^{19} \mathrm{~F} \mathrm{NMR} \mathrm{(376} \mathrm{MHz,} \mathrm{CDCl}_{3}\right)$

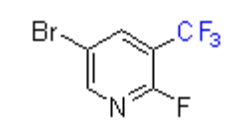

N

$\checkmark v$

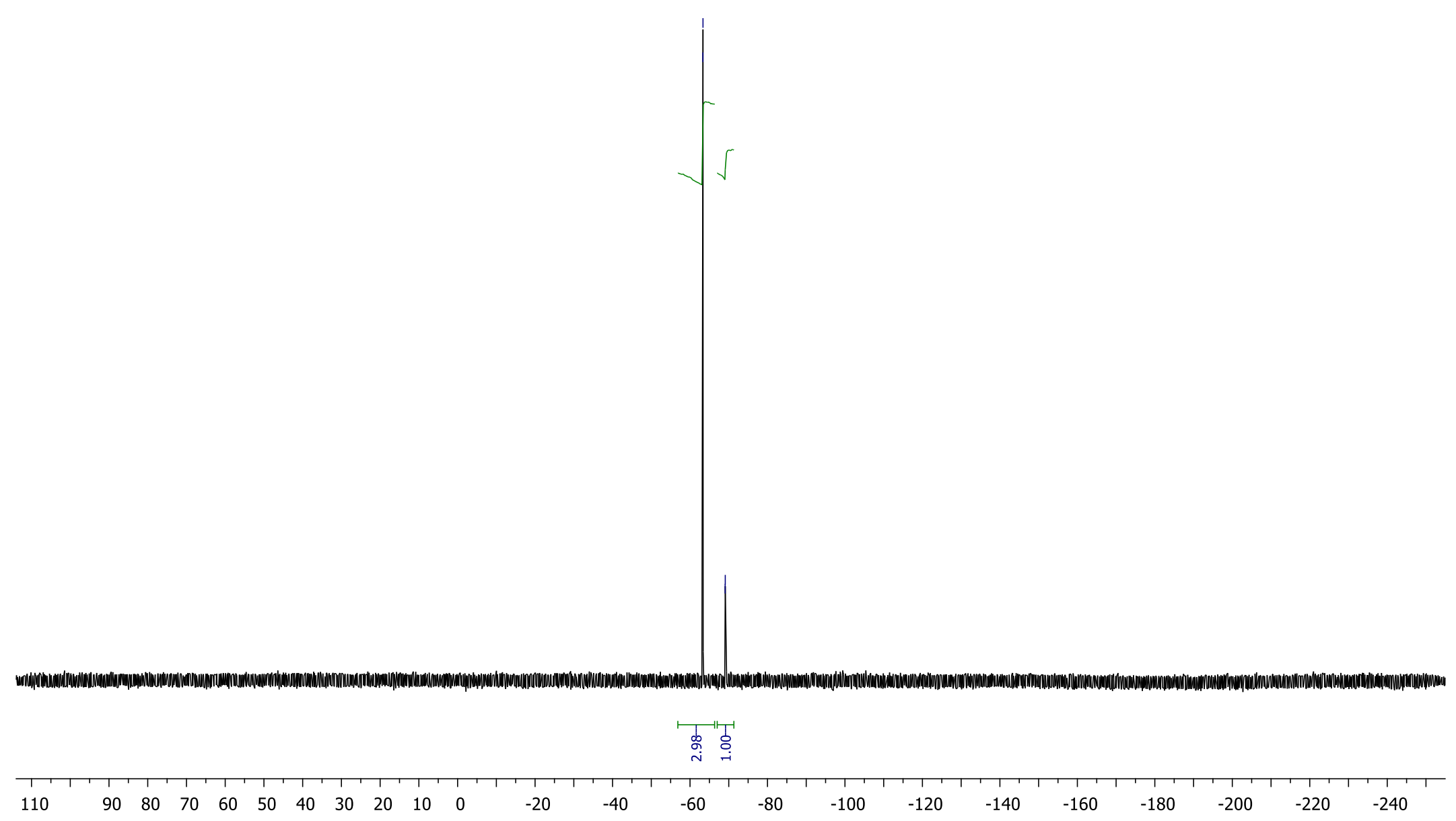


Compound 55a

${ }^{1} \mathrm{H}$ NMR (400 MHz, $\mathrm{CDCl}_{3}$ )

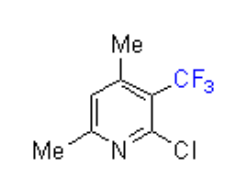

$\stackrel{\substack{1\\}}{\substack{0 \\ \hdashline}}$

$\underbrace{\stackrel{\vec{n}}{g} \mathcal{N}^{g}}$

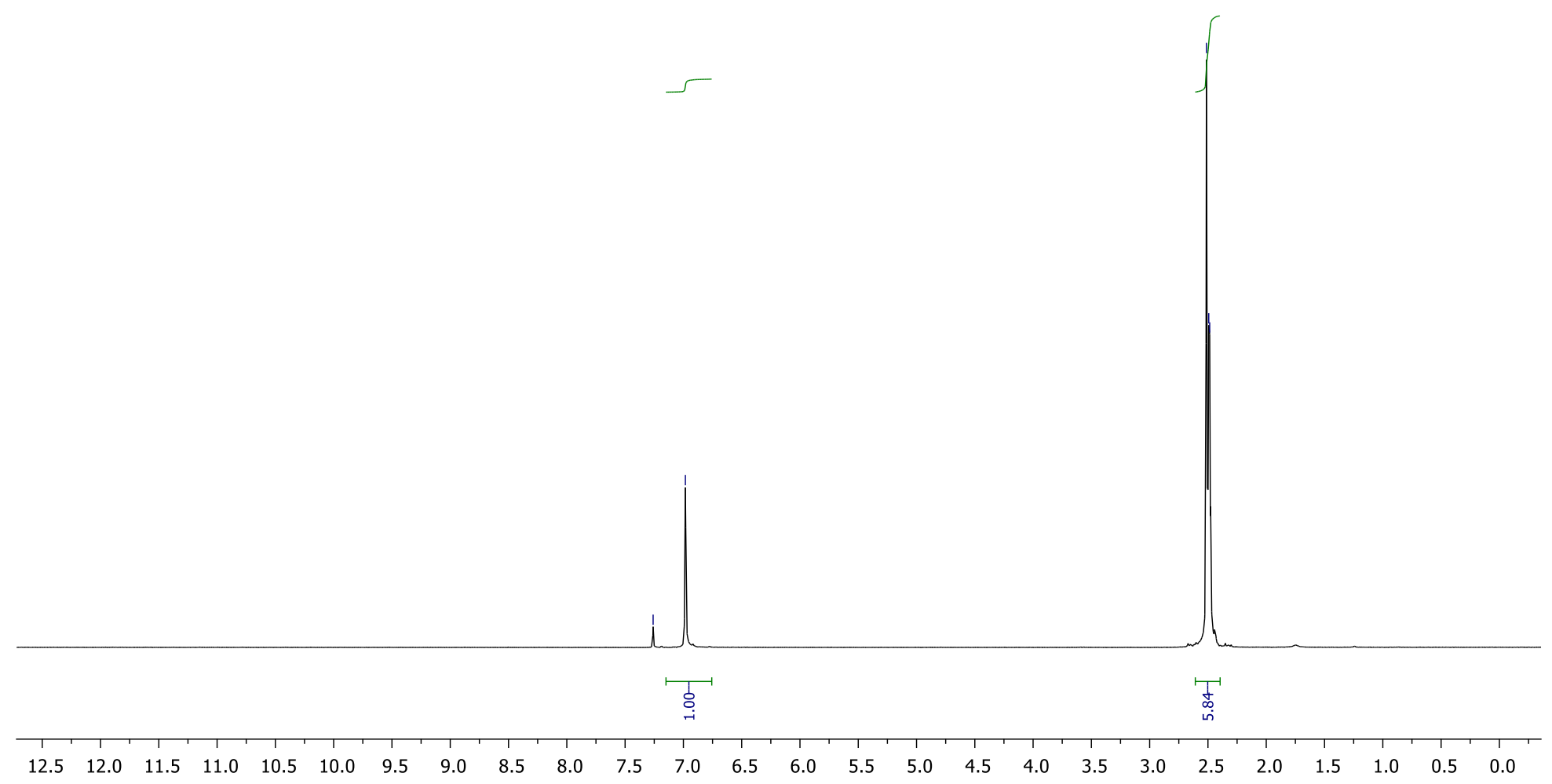


${ }^{13} \mathrm{C}\left\{{ }^{1} \mathrm{H}\right\}$ NMR $\left(126 \mathrm{MHz}, \mathrm{CDCl}_{3}\right)$
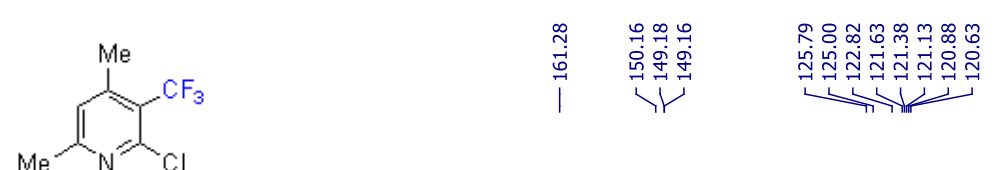

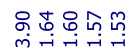

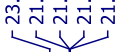

$\mathrm{Me} N \mathrm{C}$

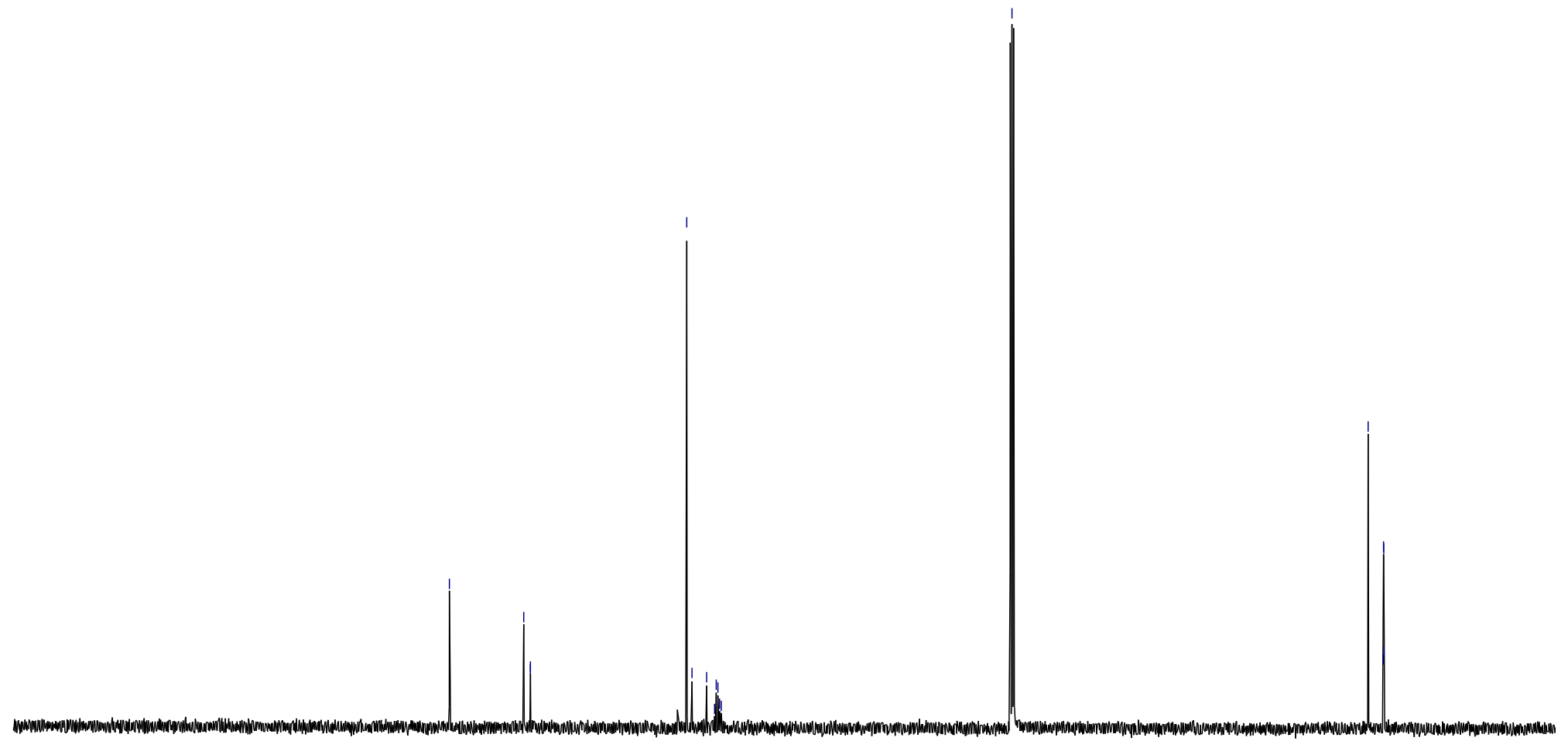

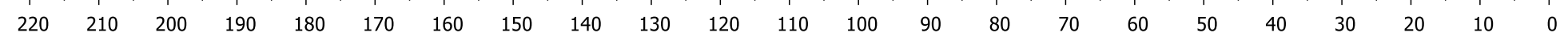


${ }^{19} \mathrm{~F}$ NMR (376 MHz, $\left.\mathrm{CDCl}_{3}\right)$
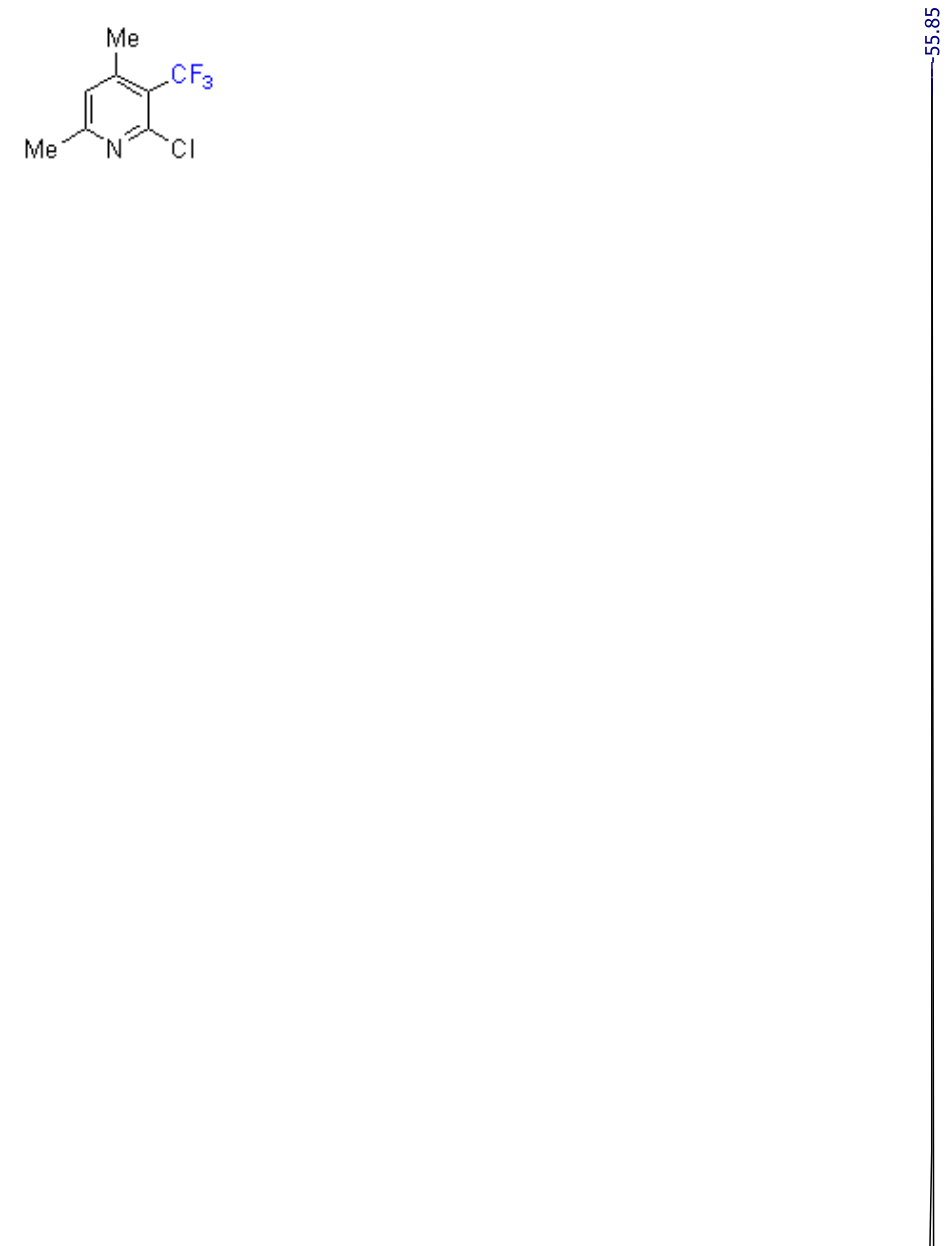


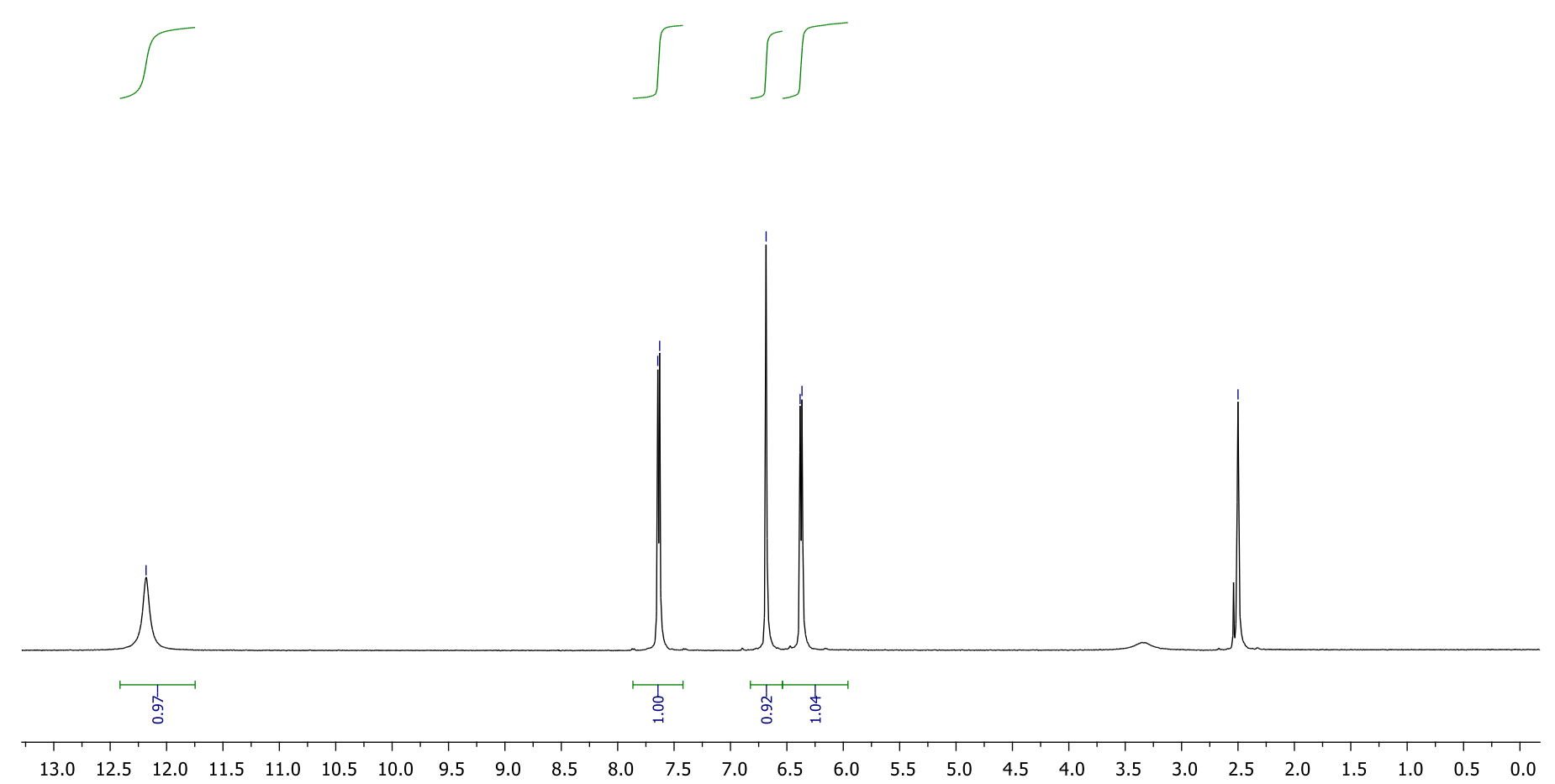

Compound 56a is also described in: a) J. Fluorine Chem. 1999, 93, 2, 153-157; b) Org. Proc. Res. Dev. 2001, 5, 5, 531-534; c) Bioorg. Med. Chem. 2001, 9, 7, 1773-1780. 


\section{${ }^{13} \mathrm{C}\left\{{ }^{1} \mathrm{H}\right\}$ NMR (151 MHz, DMSO-d 6 )}

D

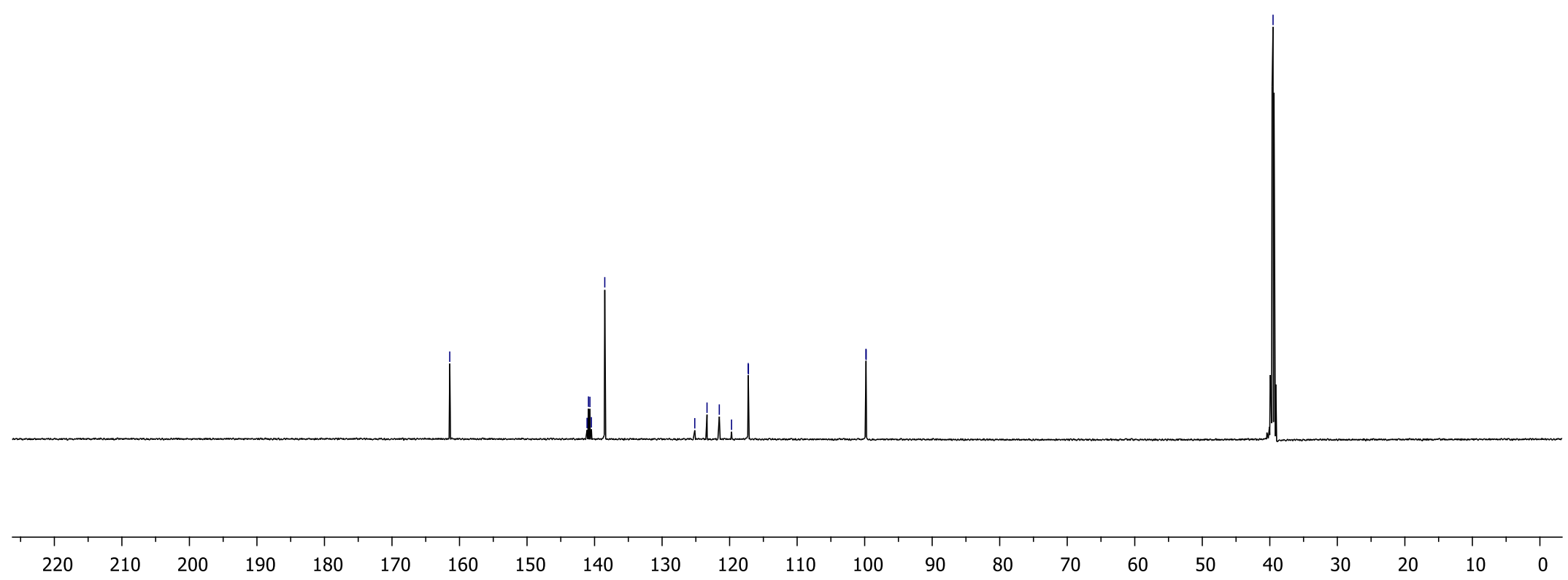


${ }^{19}$ F NMR (376 MHz, DMSO-d 6 )

$\sum_{H}^{C_{3}}$

$\overrightarrow{0}$

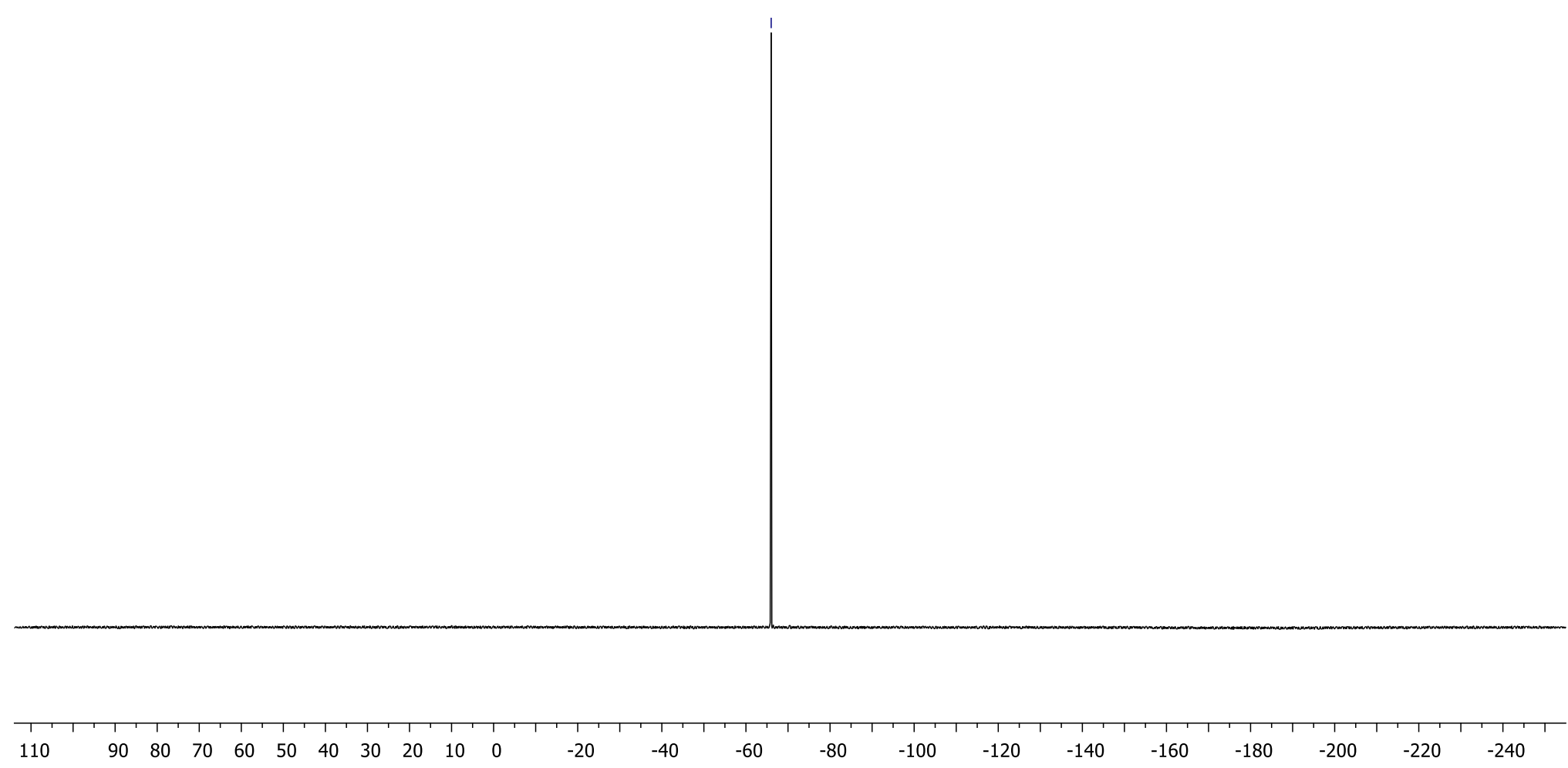




\section{Compound 57a}

${ }^{1} \mathrm{H}$ NMR (400 MHz, $\mathrm{CDCl}_{3}$ )

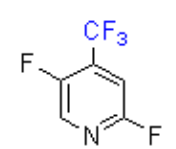

$\stackrel{\sim}{0}^{\infty}$

पr

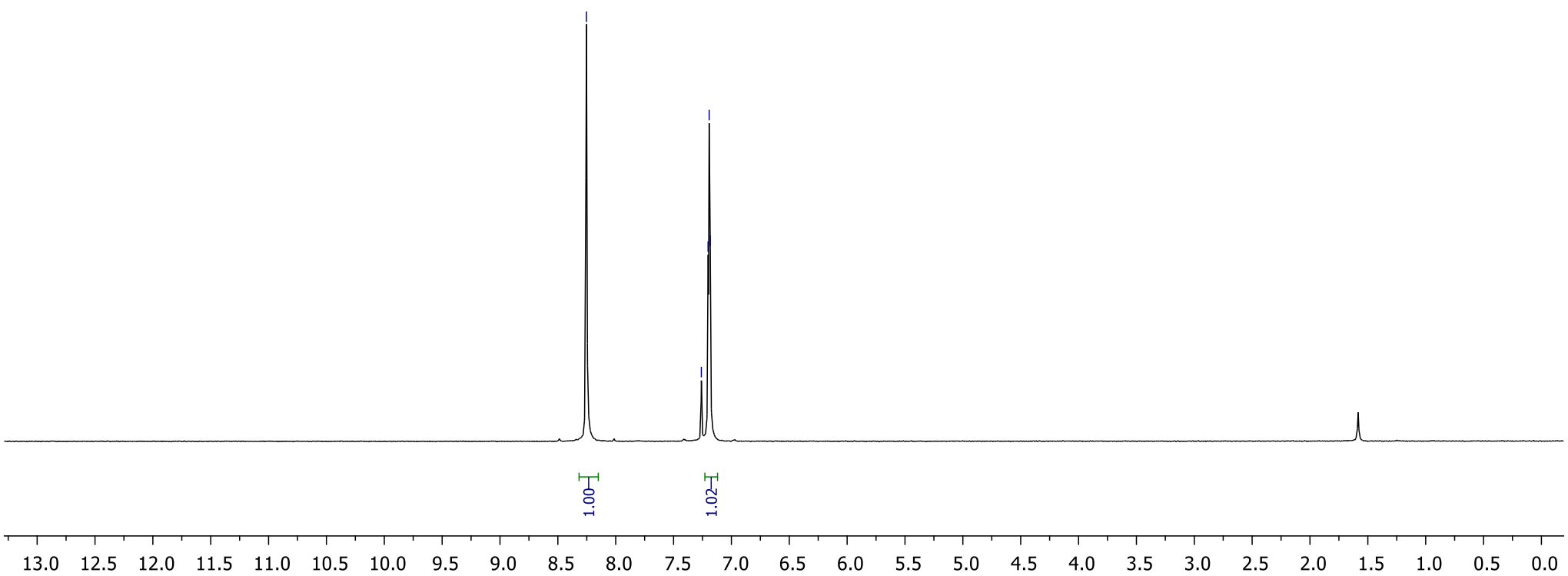


${ }^{13} \mathrm{C}\left\{{ }^{1} \mathrm{H}\right\} \mathrm{NMR}\left(151 \mathrm{MHz}, \mathrm{CDCl}_{3}\right)$

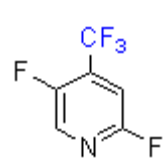

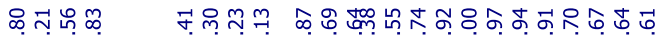

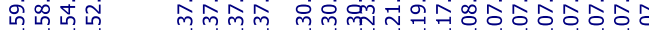

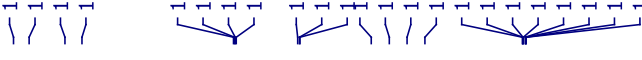

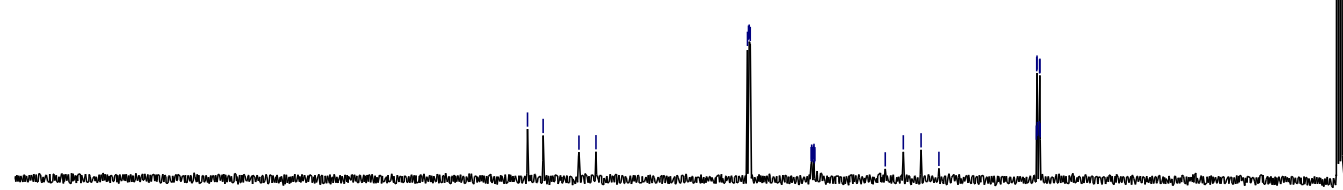

$210 \quad 200$
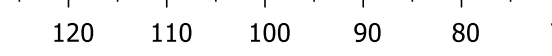
${ }^{19} \mathrm{~F}$ NMR $\left(376 \mathrm{MHz}, \mathrm{CDCl}_{3}\right)$

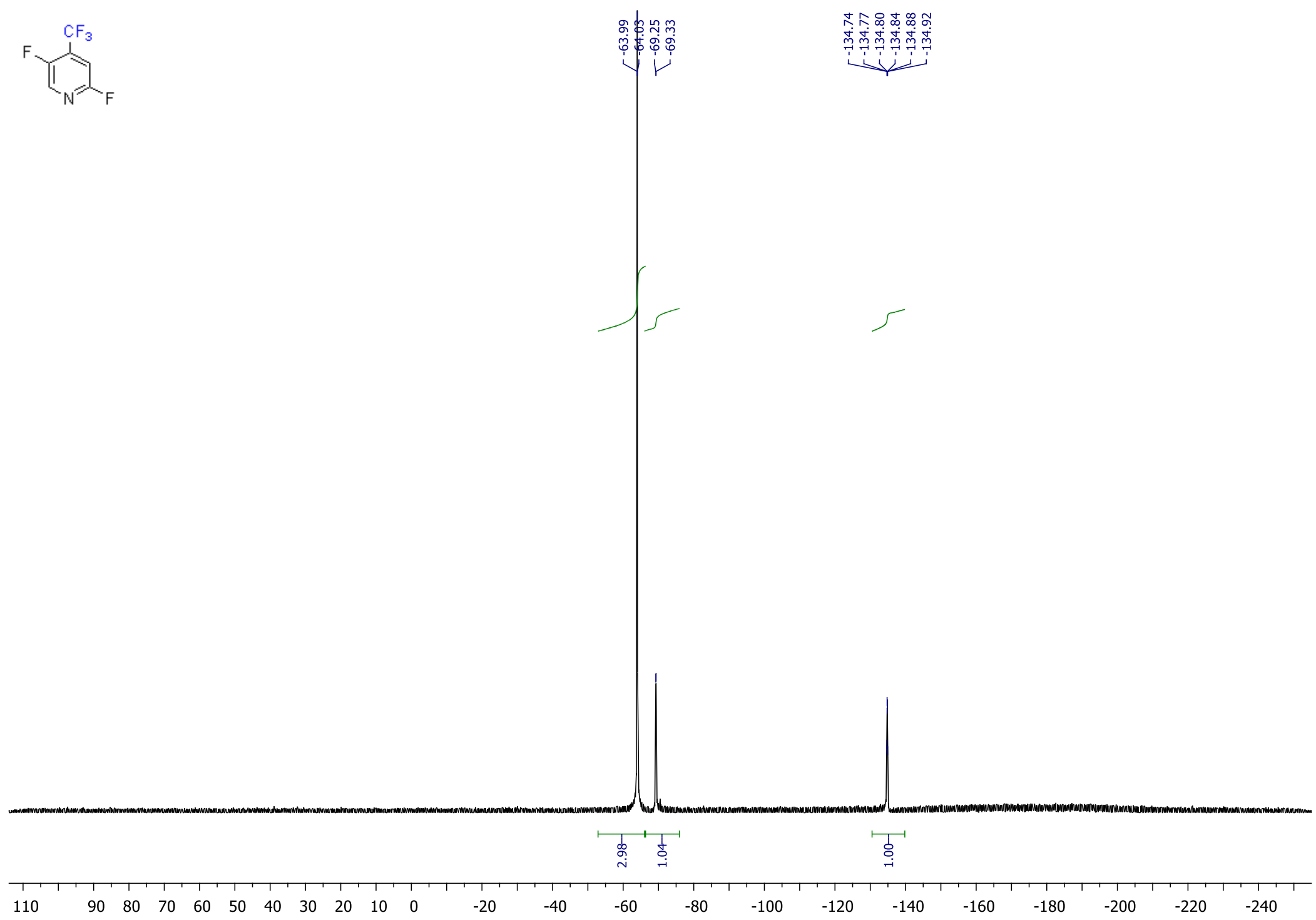


Compound 58a

${ }^{1} \mathrm{H}$ NMR (400 MHz, $\mathrm{CDCl}_{3}$ )

${ }_{\mathrm{Cl}}^{\mathrm{Cl}_{3}}$

1

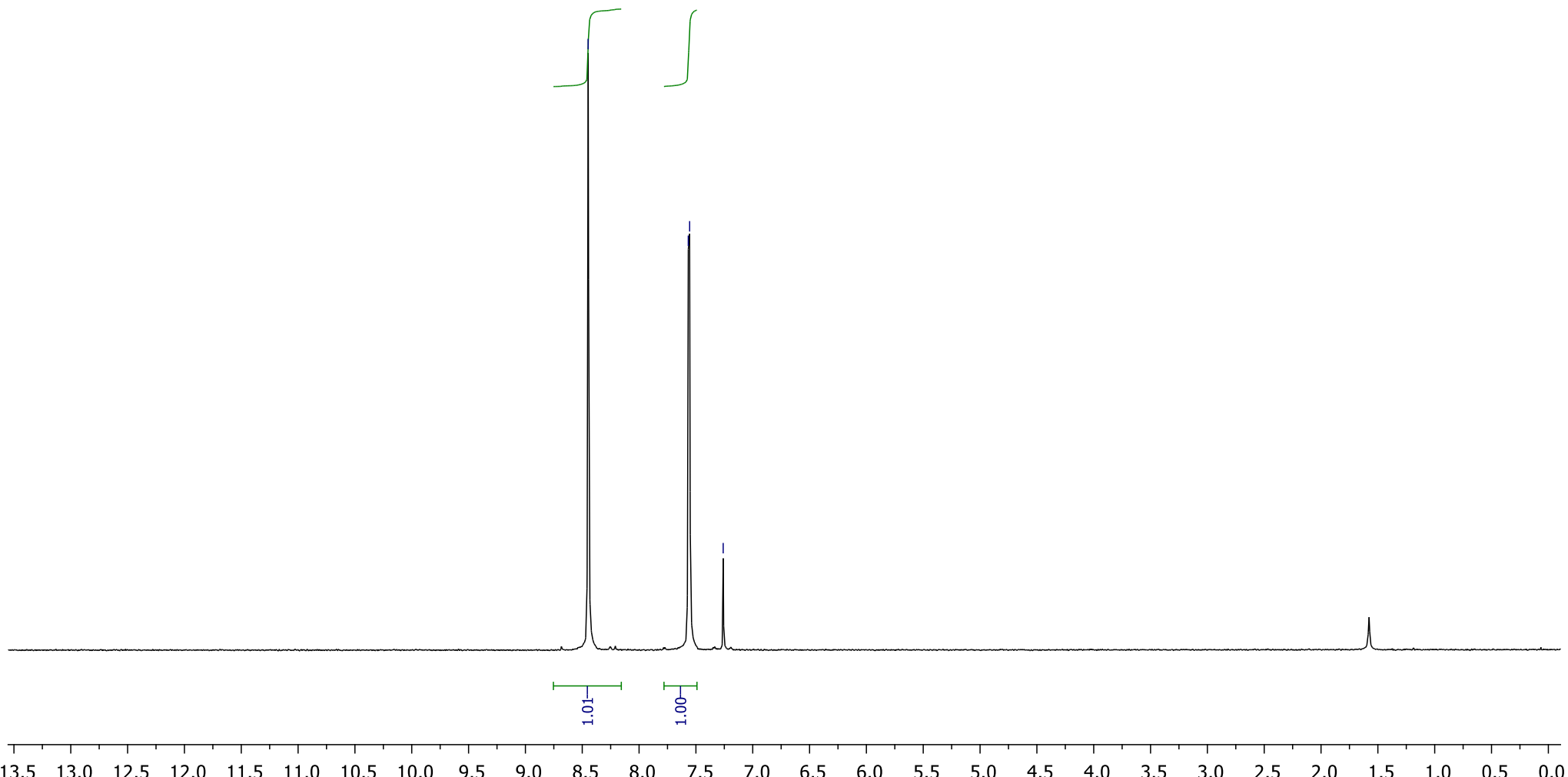




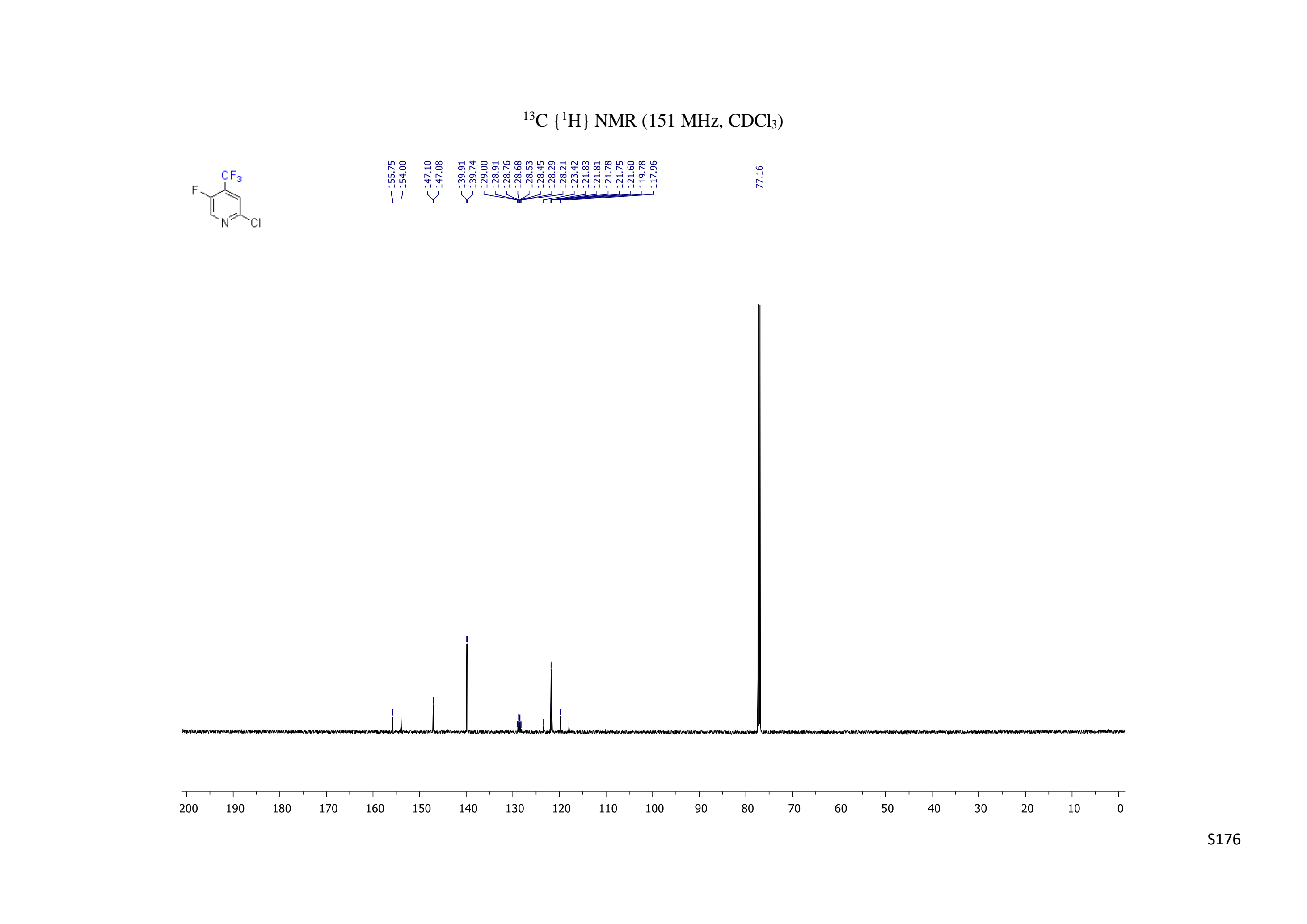


${ }^{19} \mathrm{~F}$ NMR (376 MHz, $\mathrm{CDCl}_{3}$ )
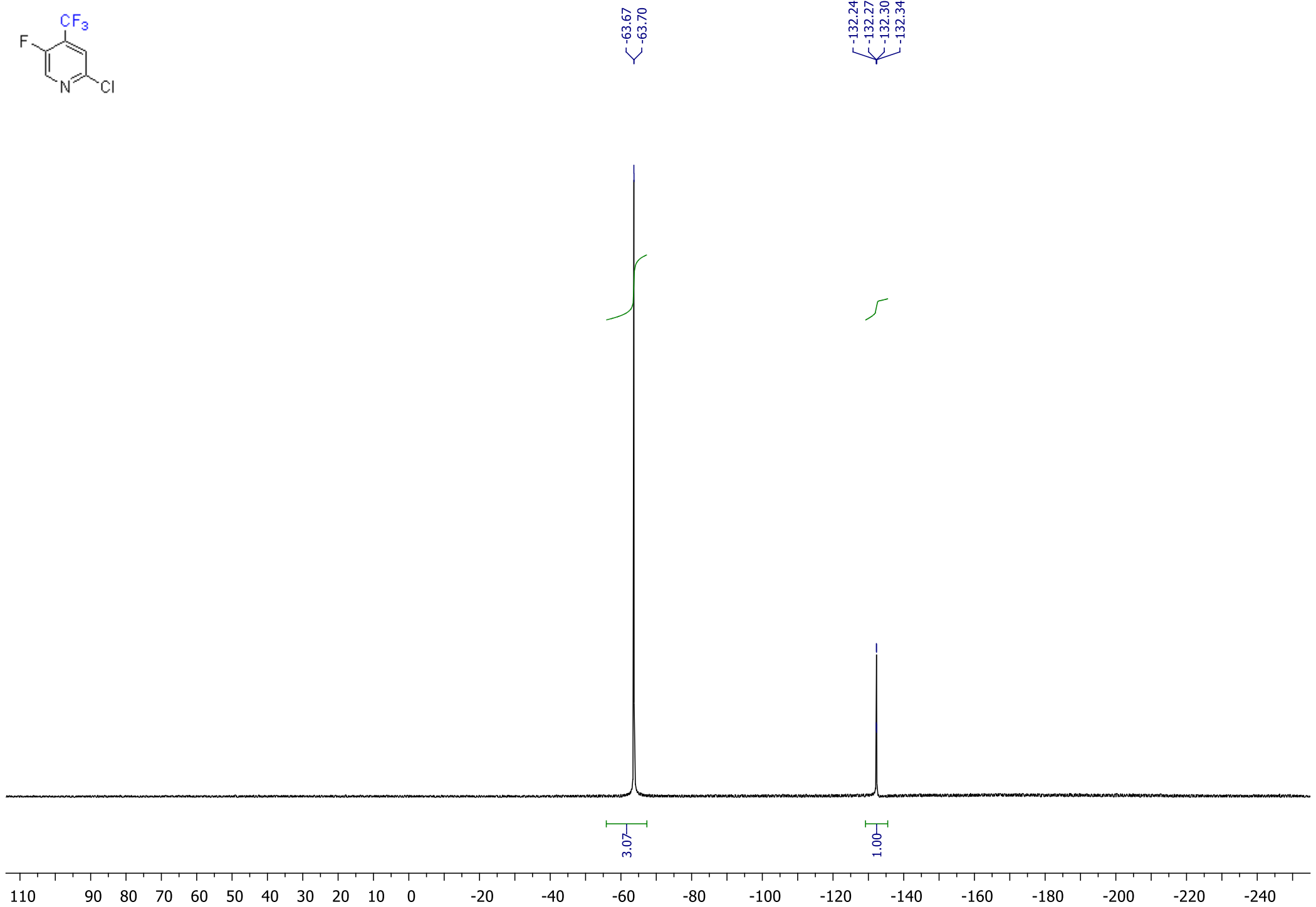
Compound 59a*

${ }^{1} \mathrm{H} \mathrm{NMR}\left(400 \mathrm{MHz}, \mathrm{CDCl}_{3}\right)$

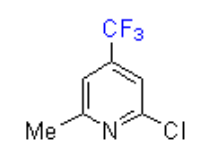

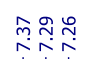

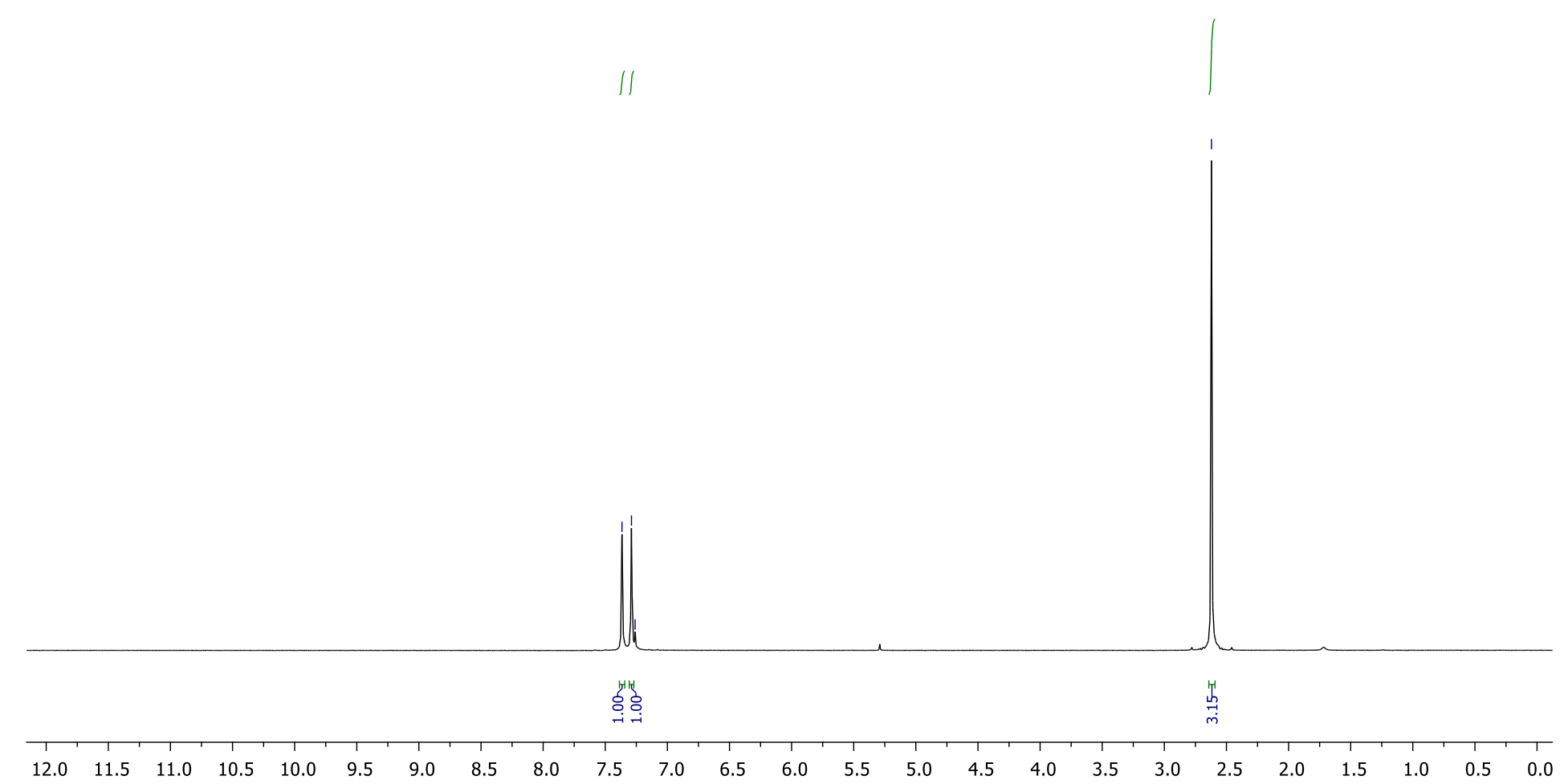

Compound 59a is also described in: Lett. Drug Des. Discov. 2015, 12, 1, 38 - 45. 
${ }^{13} \mathrm{C}\left\{{ }^{1} \mathrm{H}\right\}$ NMR $\left(126 \mathrm{MHz}, \mathrm{CDCl}_{3}\right)$

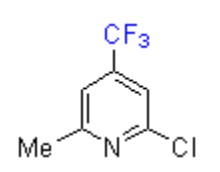

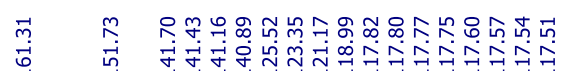

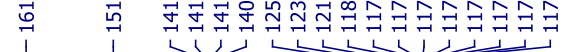
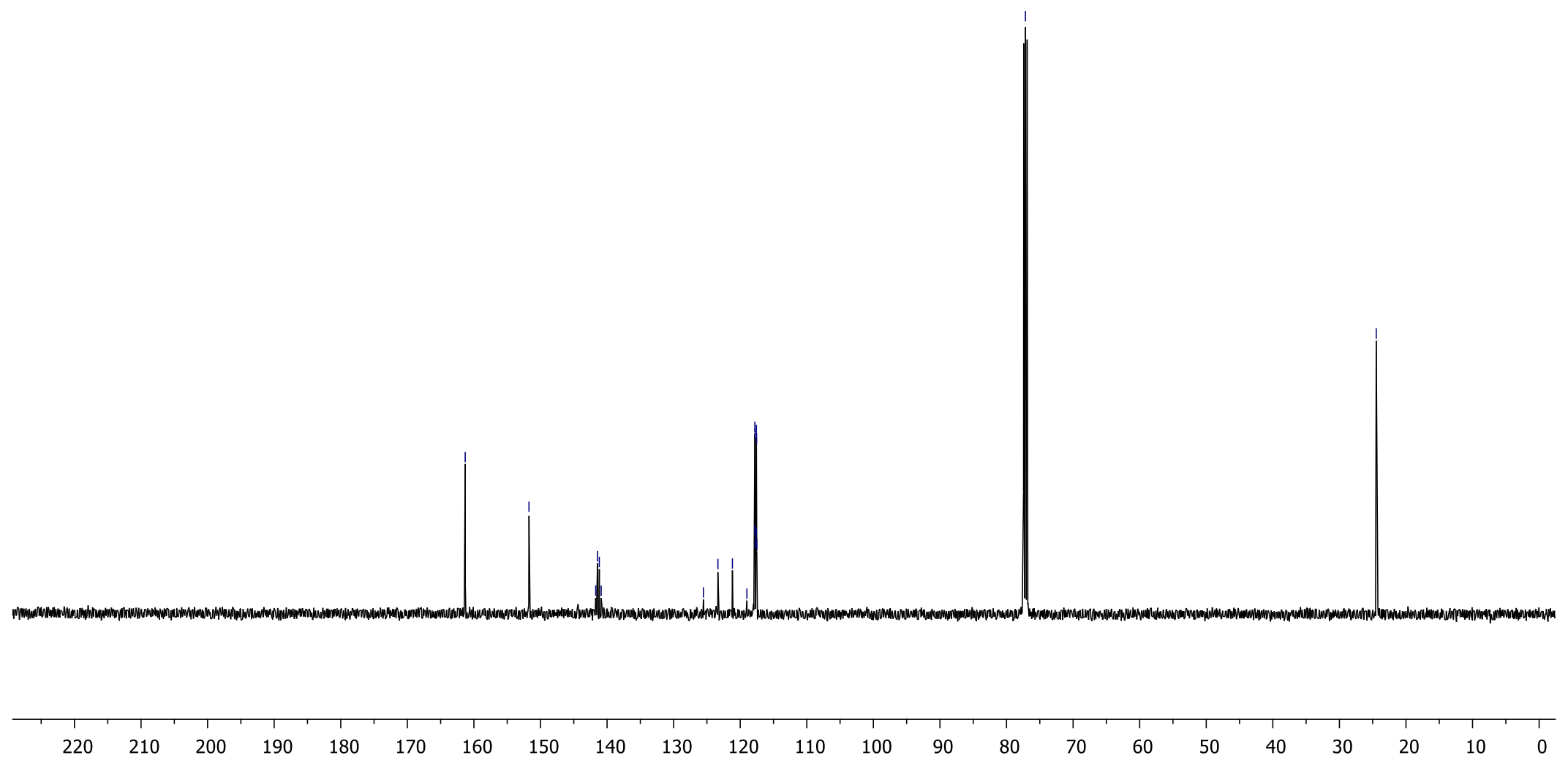
${ }^{19} \mathrm{~F}$ NMR (376 MHz, $\left.\mathrm{CDCl}_{3}\right)$



总

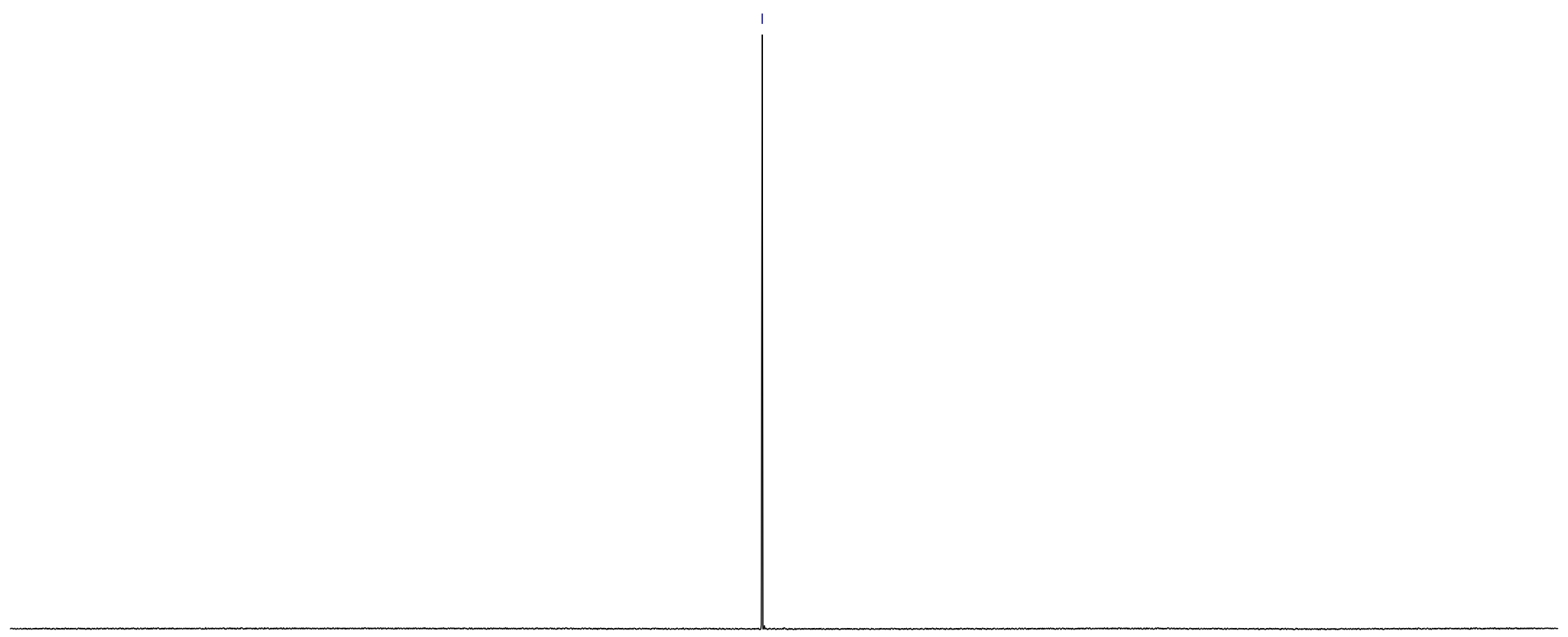


${ }^{1} \mathrm{H}$ NMR (400 MHz, DMSO-d 6 )
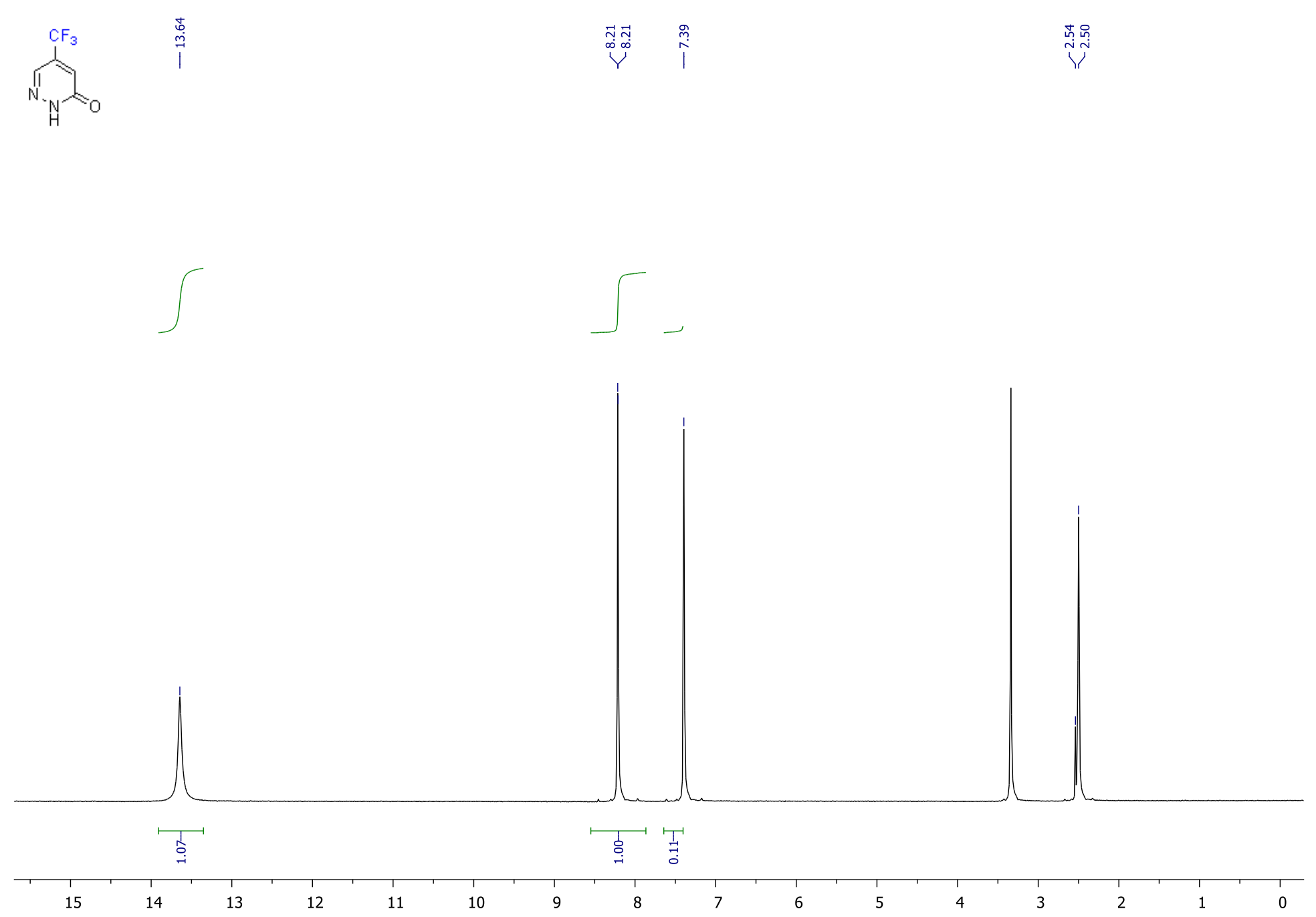
${ }^{13} \mathrm{C}\left\{{ }^{1} \mathrm{H}\right\}$ NMR (151 MHz, DMSO-d 6 )

No

m.

I $\quad$ a

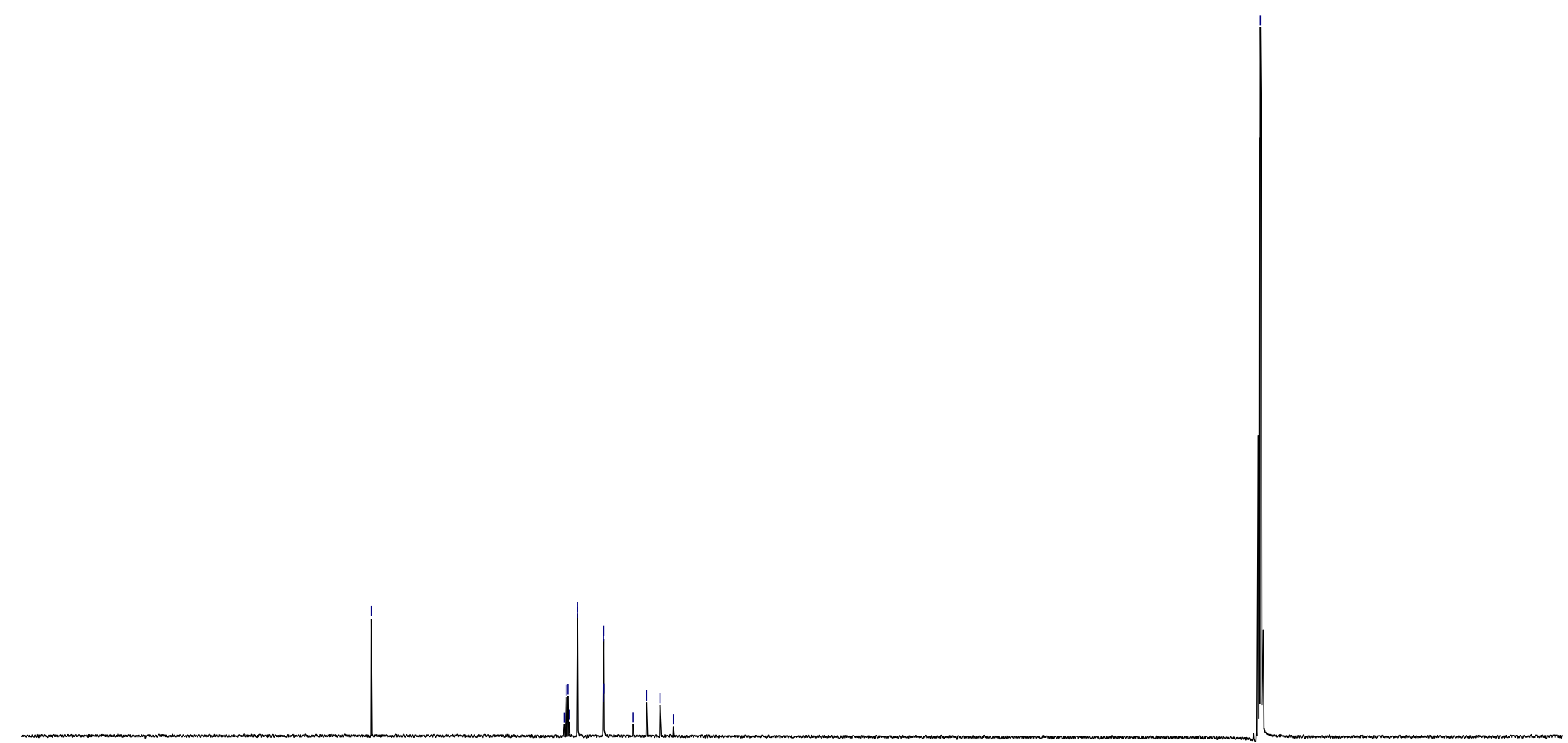


${ }^{19}$ F NMR (376 MHz, DMSO-d $)$

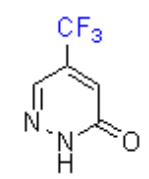

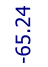

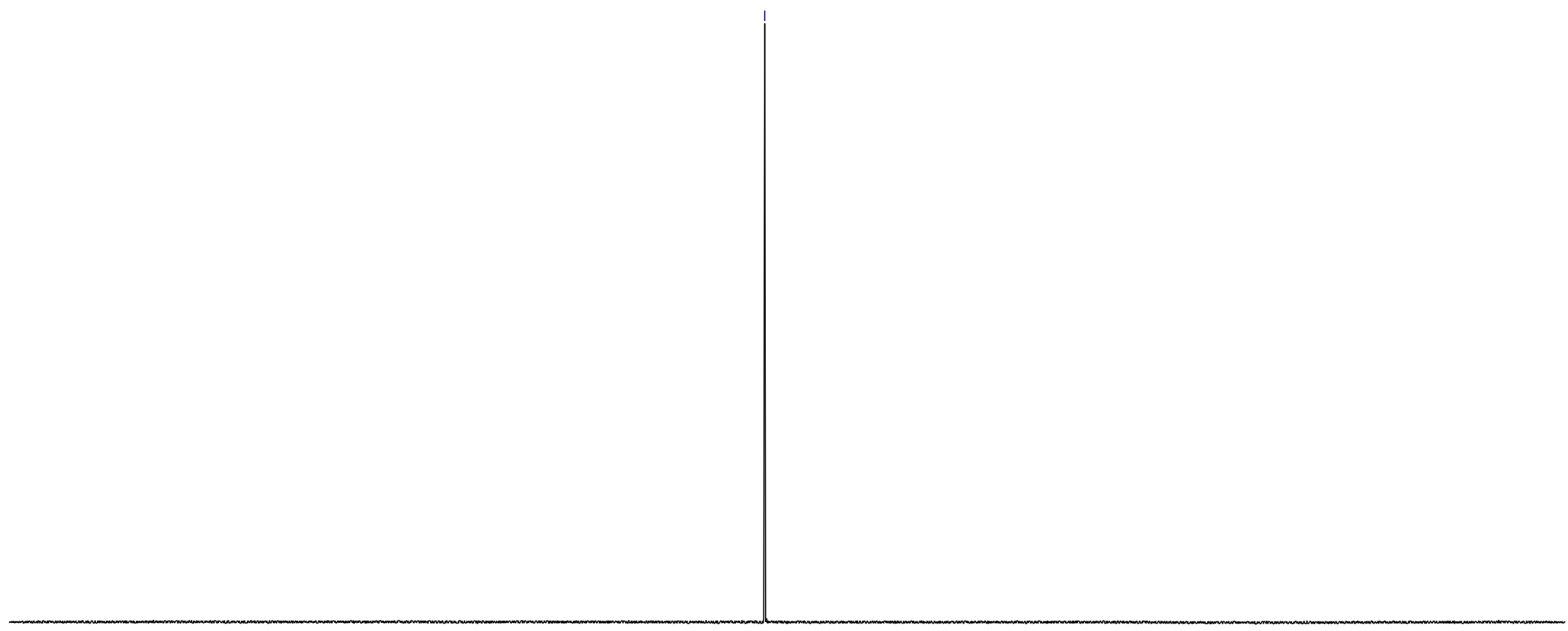

$110 \quad$\begin{tabular}{rlllllllllllllllllllllllllllllll}
\hline 0 & 80 & 70 & 60 & 50 & 40 & 30 & 20 & 10 & 0 & -20 & -40 & -60 & -80 & -100 & -120 & -140 & -160 & -180 & -200 & -220 & -240
\end{tabular} 
Compound $61 \mathrm{a}^{*}$

${ }^{1} \mathrm{H}$ NMR (400 MHz, $\left.\mathrm{CDCl}_{3}\right)$

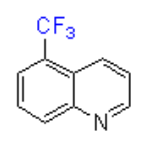

บั
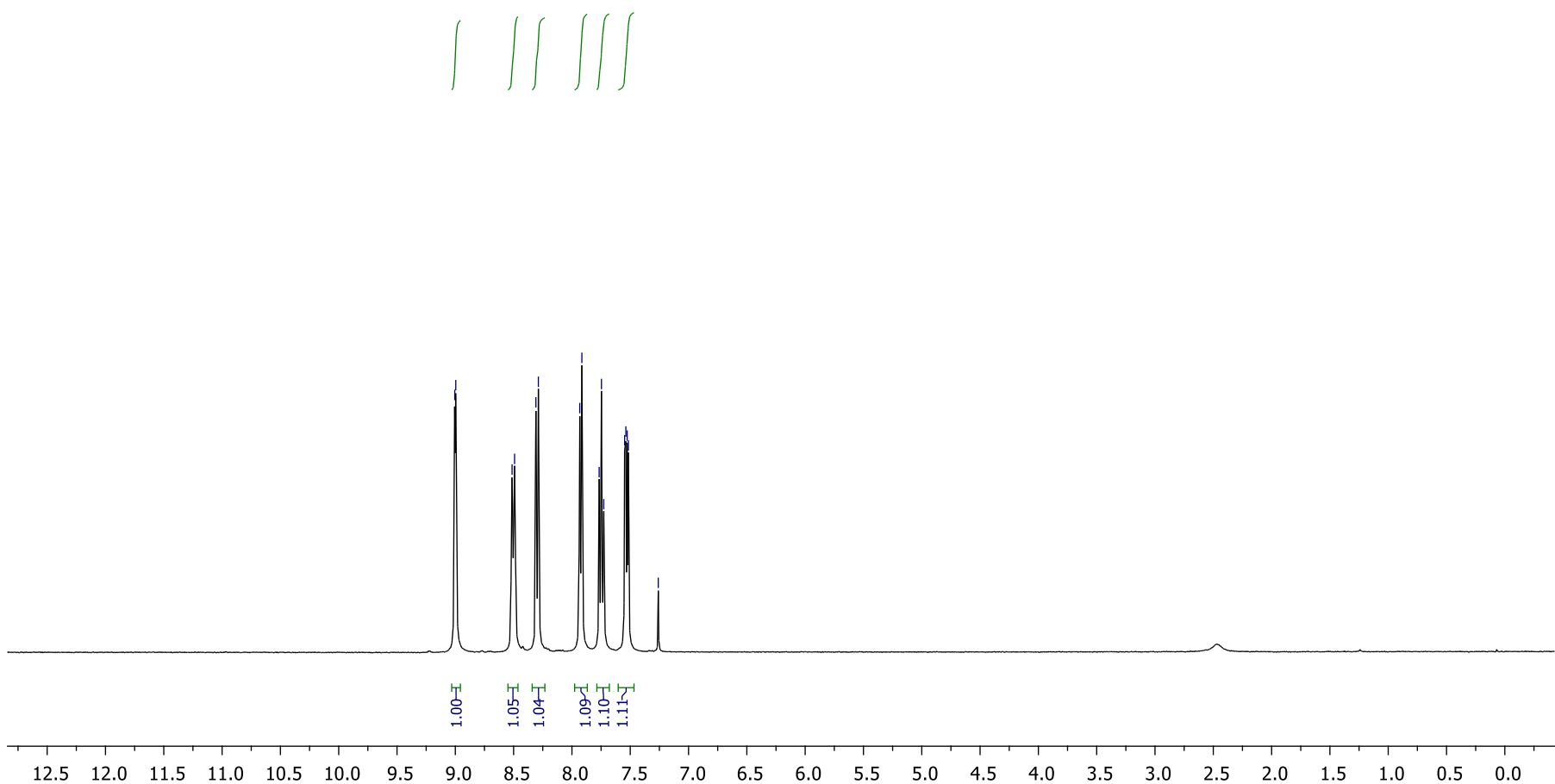

Compound 61a is also described in: a) J. Am. Chem. Soc. 1943, 65, 12, 2467-2468; b) J. Fluorine Chem. 2010, 131, 98-105; c) Org. Biomol. Chem. 2016, 14, 9088-9092; d) J. Am. Chem. Soc. 1943, 65, 12, 2467-2468. 
${ }^{13} \mathrm{C}\left\{{ }^{1} \mathrm{H}\right\}$ NMR $\left(126 \mathrm{MHz}, \mathrm{CDCl}_{3}\right)$

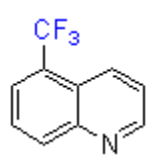

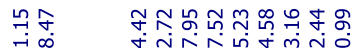

تُ

造造婇

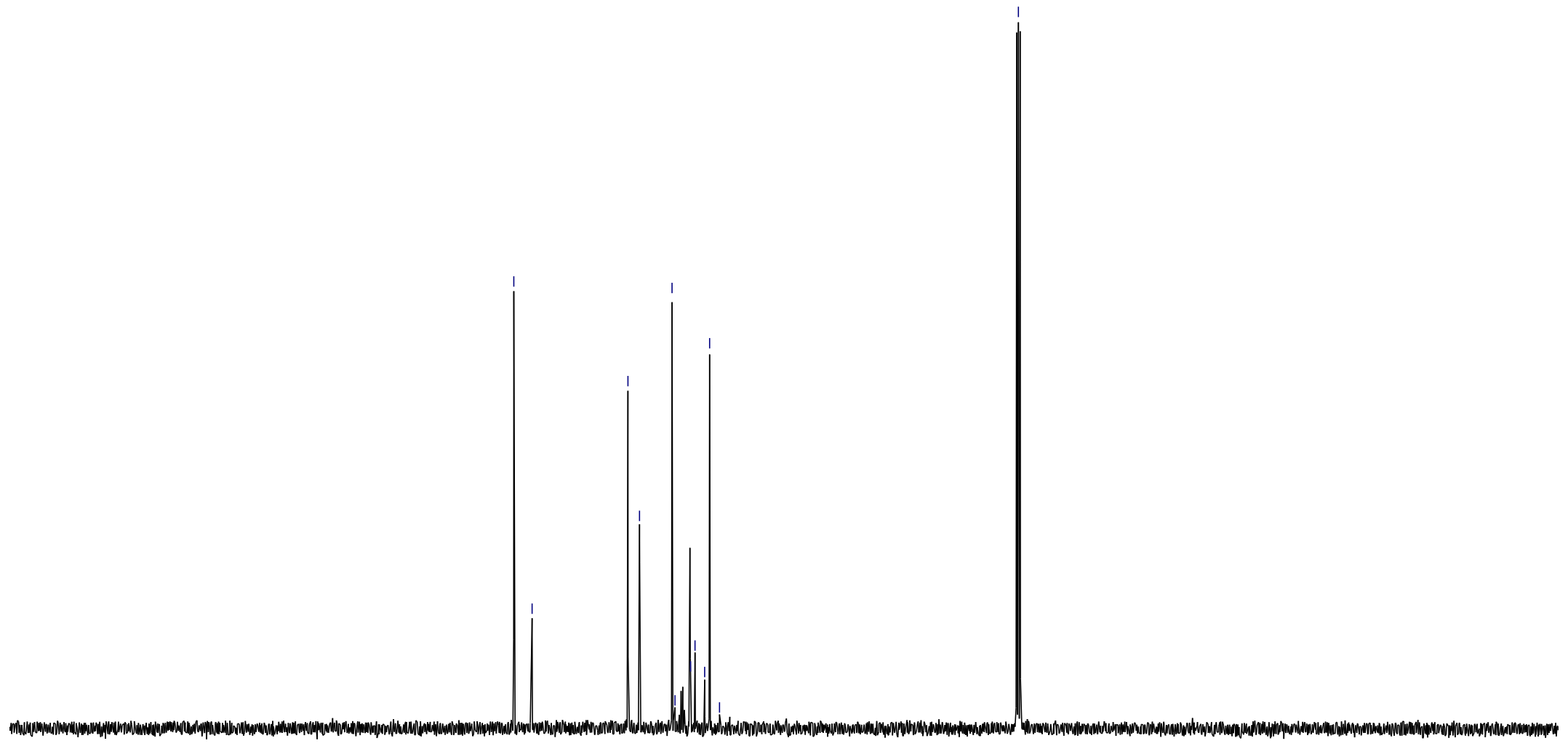

$\begin{array}{llllllllllllllllllllllll}220 & 210 & 200 & 190 & 180 & 170 & 160 & 150 & 140 & 130 & 120 & 110 & 100 & 90 & 80 & 70 & 60 & 50 & 40 & 30 & 20 & 10 & 0\end{array}$ 
$\left.{ }^{19} \mathrm{~F} \mathrm{NMR} \mathrm{(376} \mathrm{MHz,} \mathrm{CDCl}_{3}\right)$

$\overbrace{N}^{C F_{3}}$

ڤั.

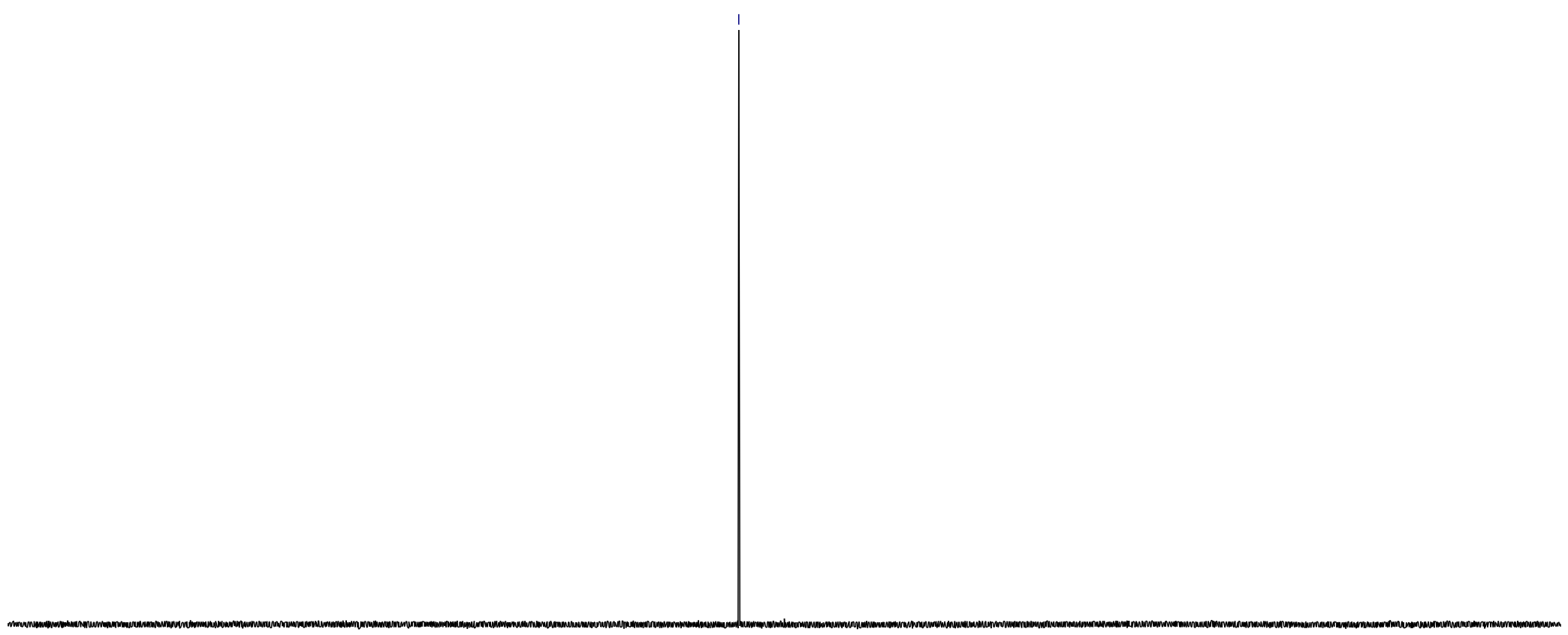


Compound $62 \mathrm{a}^{*}$

${ }^{1} \mathrm{H} \mathrm{NMR}\left(400 \mathrm{MHz}, \mathrm{CDCl}_{3}\right)$

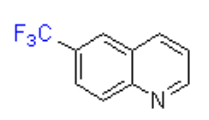

$\underbrace{\infty}$

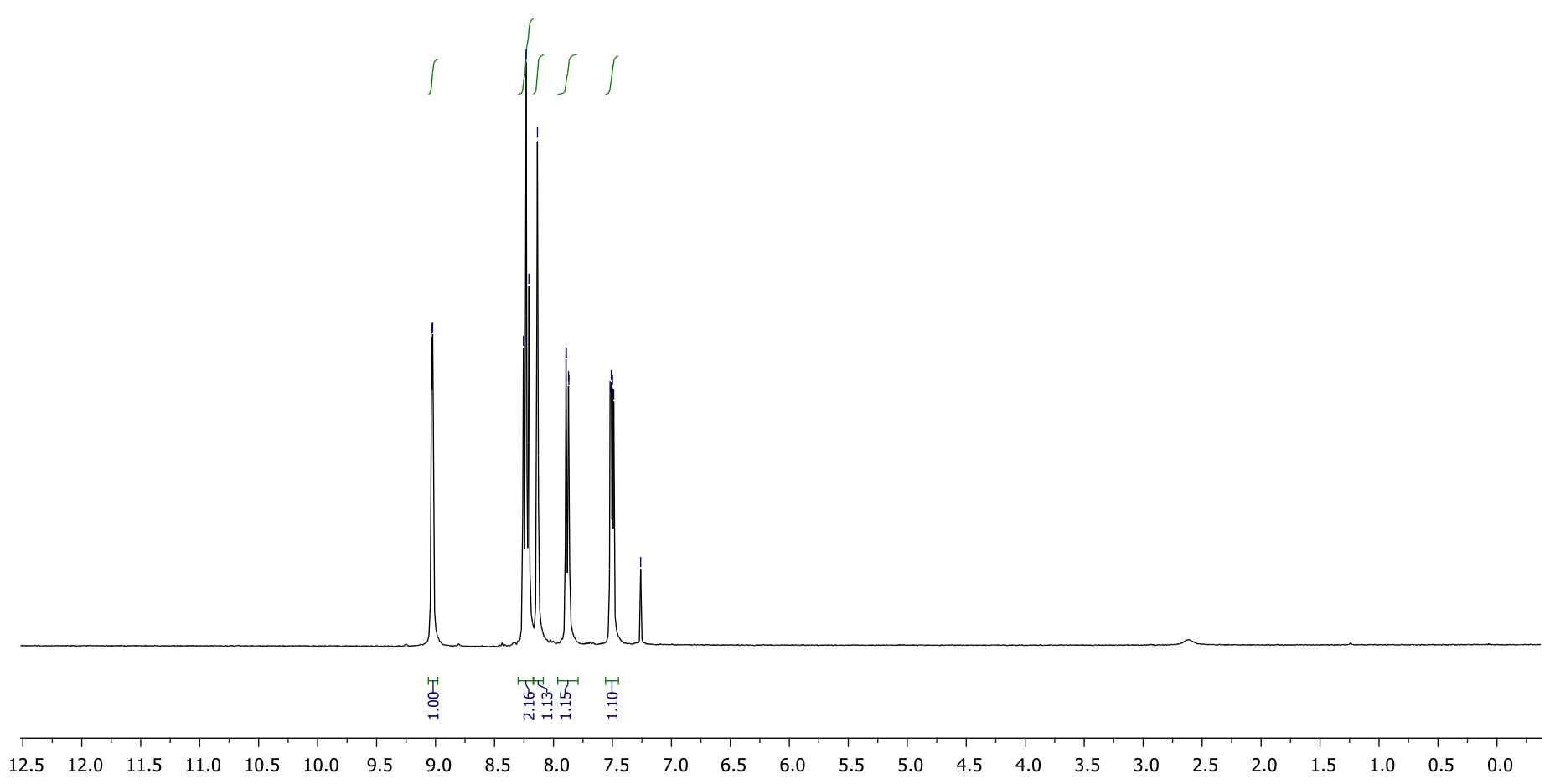

Compound 62a is also described in: a) J. Am. Chem. Soc. 2015, 137, 33, 10652-10658; b) Chem. Sci. 2019, 10, 5345-5352; c) Org. Lett. 2015, 17, 11, 27522755; d) Org. Lett. 2014, 16, 16, 4268-4271; e) RSC Adv. 2016, 6, 18525-18529. 
${ }^{13} \mathrm{C}\left\{{ }^{1} \mathrm{H}\right\}$ NMR $\left(126 \mathrm{MHz}, \mathrm{CDCl}_{3}\right)$

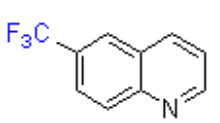

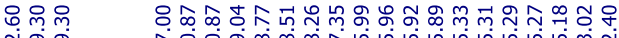

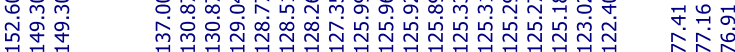

IY V V

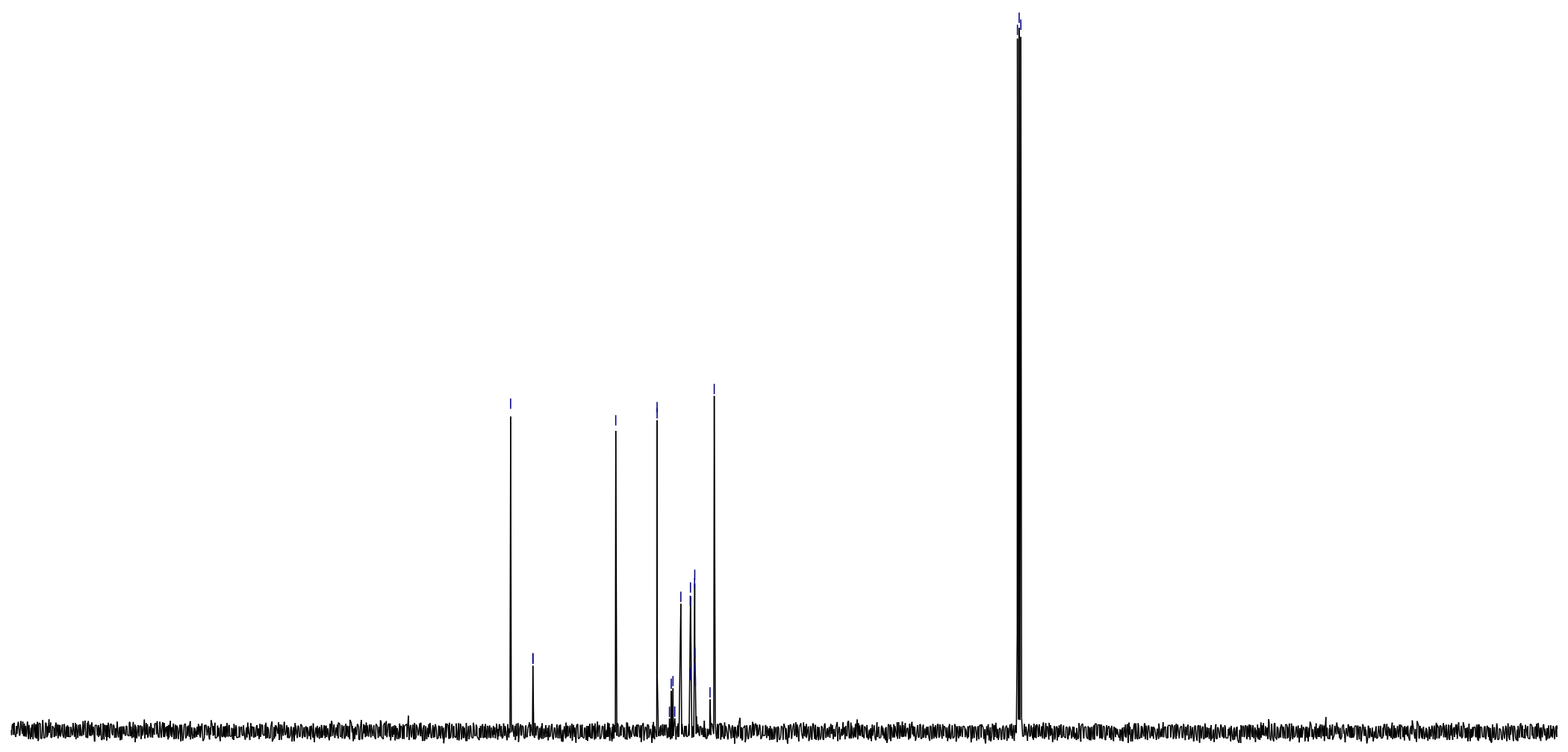

220

200

$190 \quad 180$

$170 \quad 160$

150

$130 \quad 120$

$110 \quad 100$

90

$80 \quad 70$

60

40

20

$10 \quad 0$ 
${ }^{19} \mathrm{~F}$ NMR (376 MHz, $\mathrm{CDCl}_{3}$ )

No

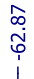

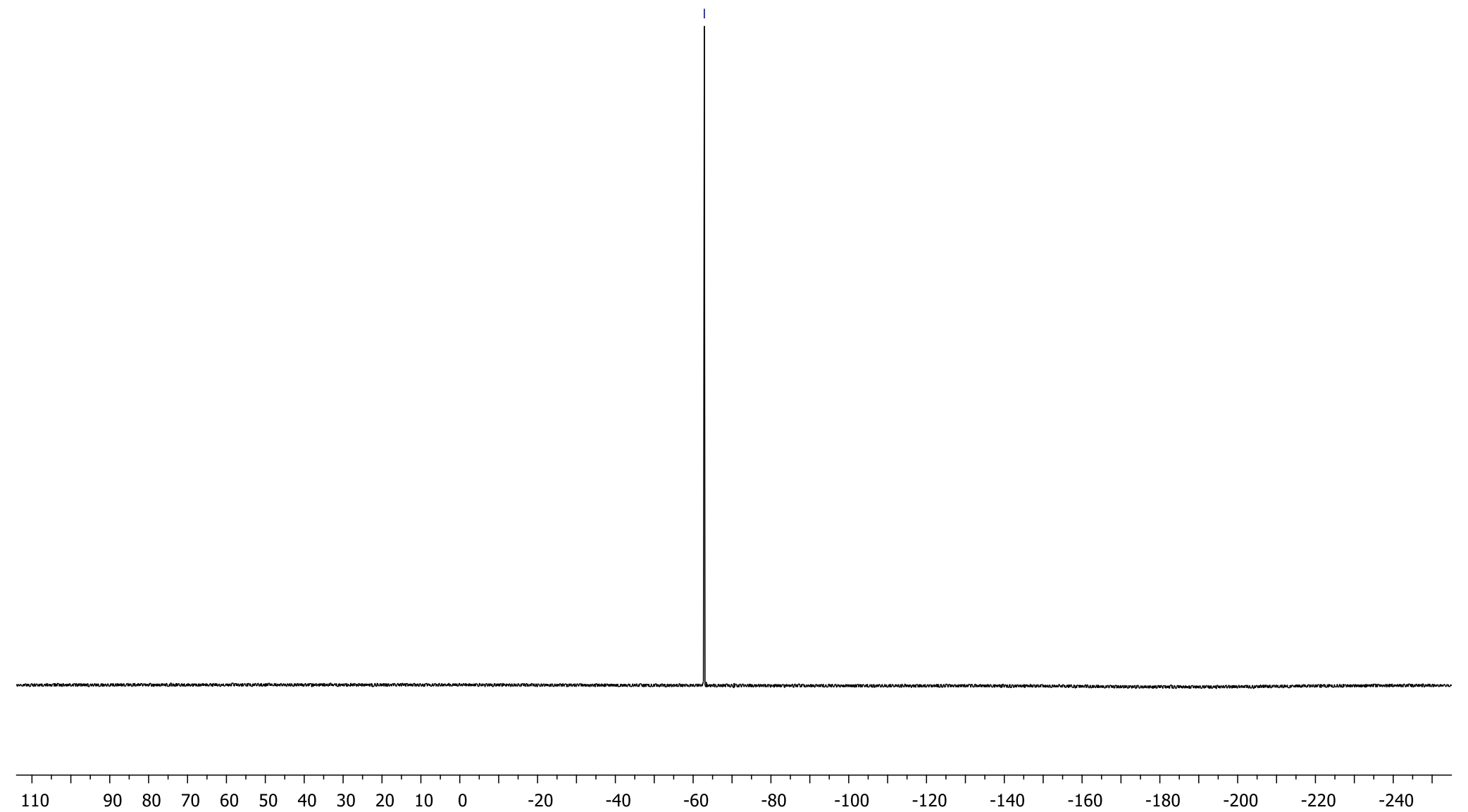


Compound $63 \mathrm{a}^{*}$

${ }^{1} \mathrm{H} \mathrm{NMR}\left(400 \mathrm{MHz}, \mathrm{CDCl}_{3}\right)$

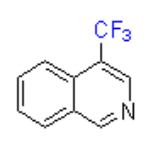

|
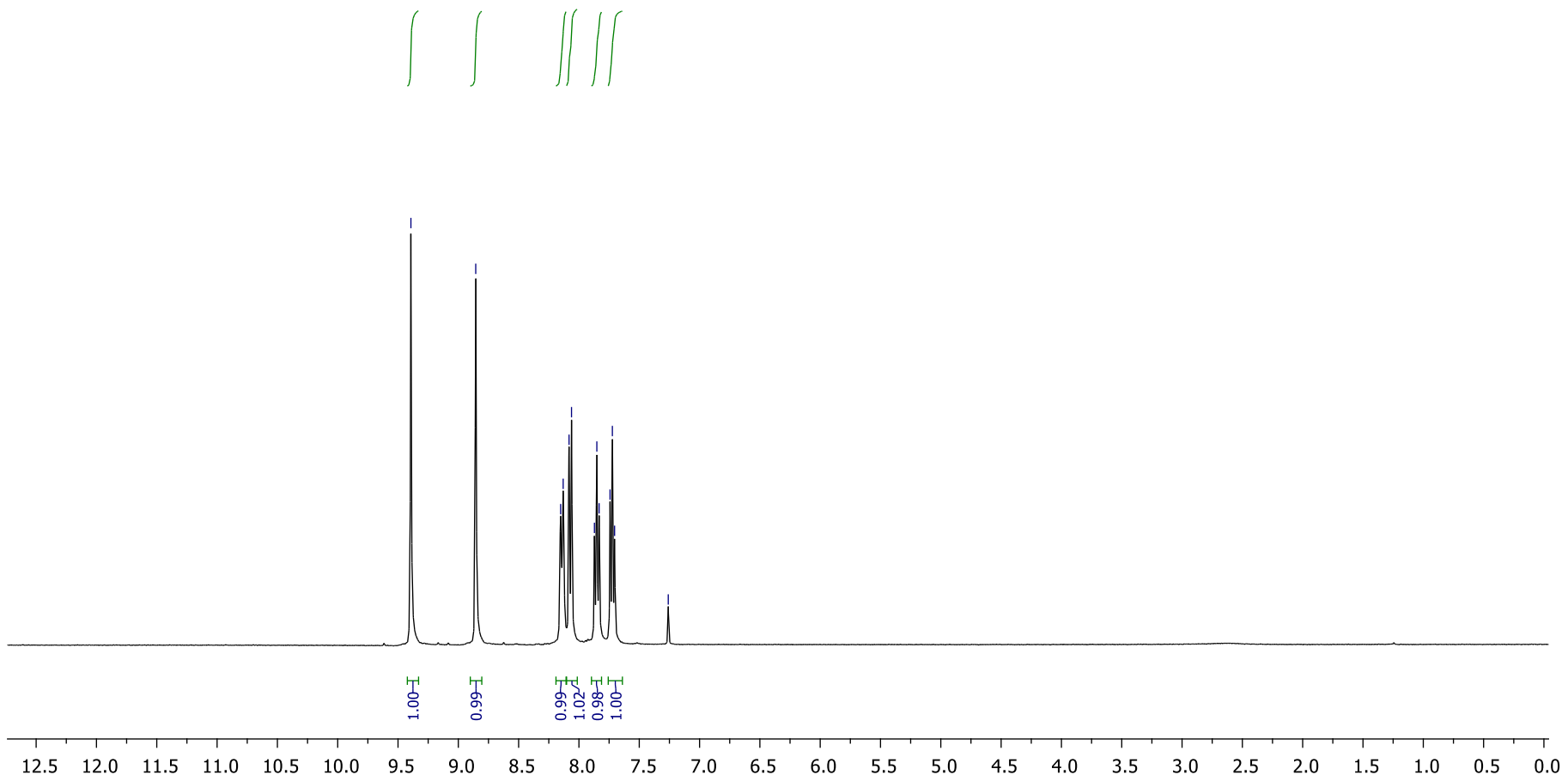

Compound 63a is also described in: a) Chem. Lett. 2005, 34, 6, 778-779; b) Org. Lett. 2014, 16, 6, 1744-1747; c) Bull. Chem. Soc. Jpn. 1988, 61, 10, 35313537. 
${ }^{13} \mathrm{C}\left\{{ }^{1} \mathrm{H}\right\}$ NMR (101 MHz, $\left.\mathrm{CDCl}_{3}\right)$
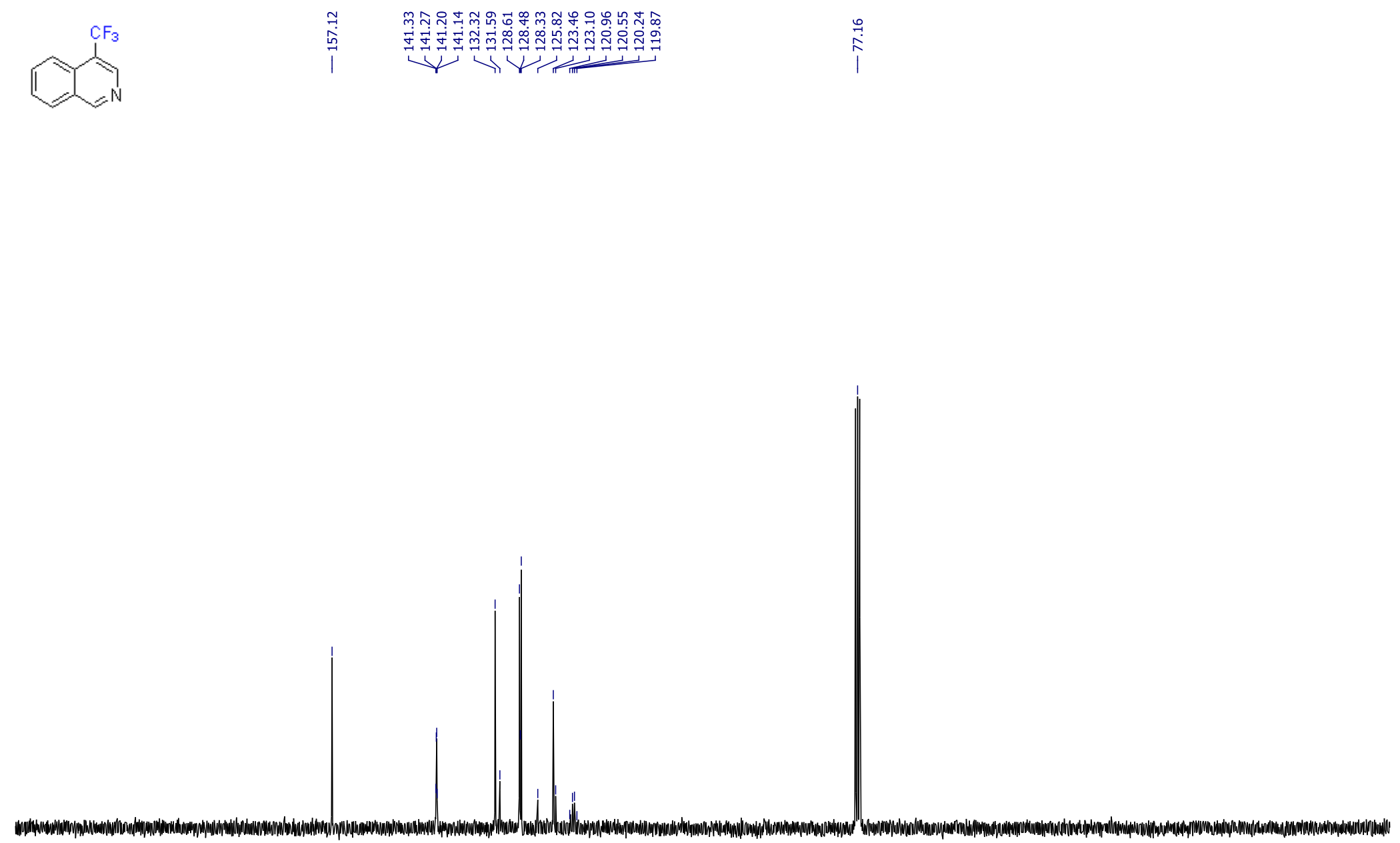
${ }^{19} \mathrm{~F}$ NMR (376 MHz, $\mathrm{CDCl}_{3}$ )

$\overbrace{N}^{C F_{3}}$

:

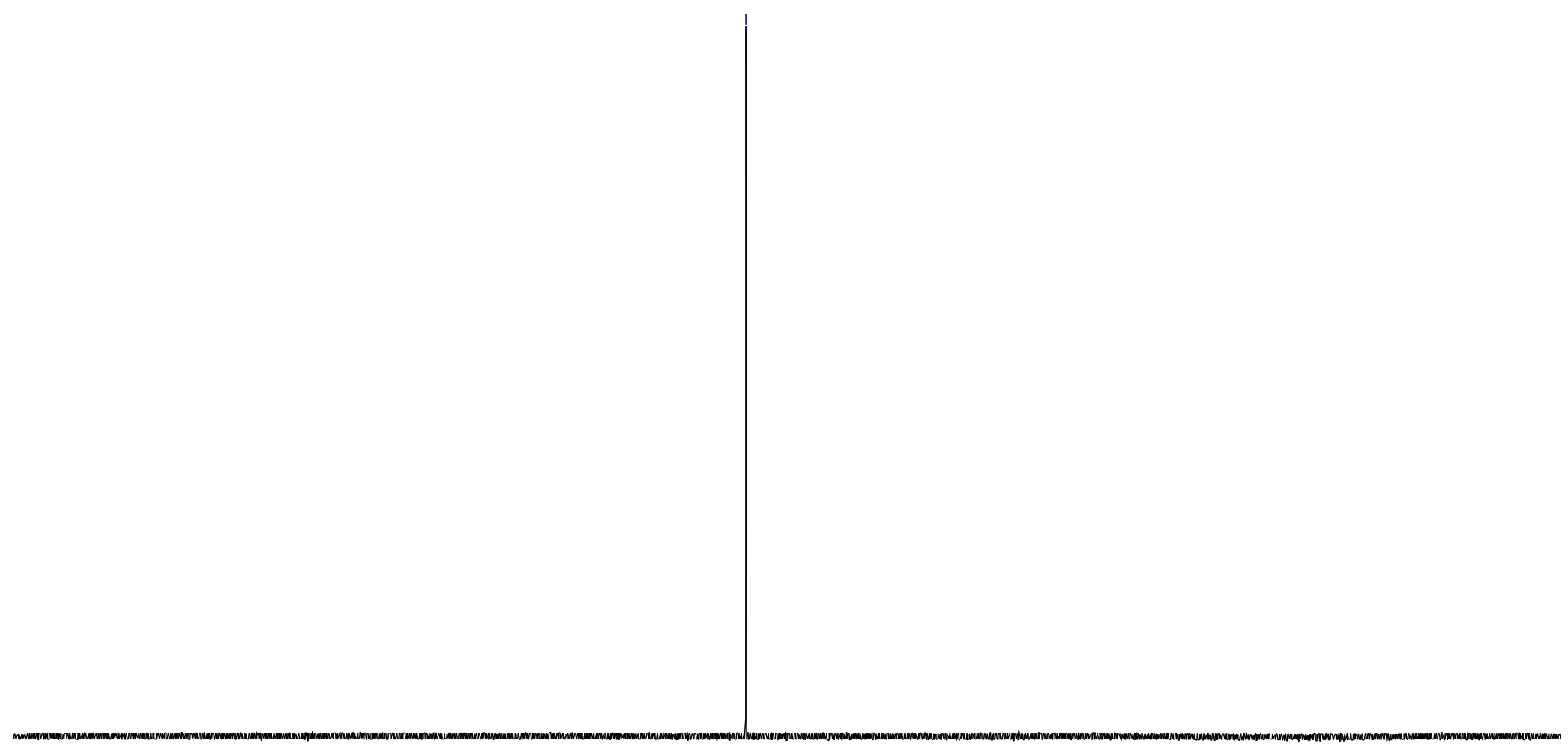

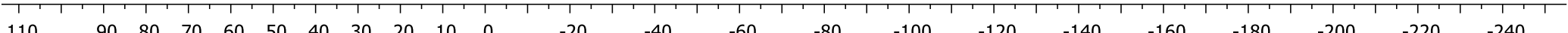


Compound $64 \mathrm{a}^{*}$

${ }^{1} \mathrm{H}$ NMR $\left(400 \mathrm{MHz}, \mathrm{CDCl}_{3}\right)$
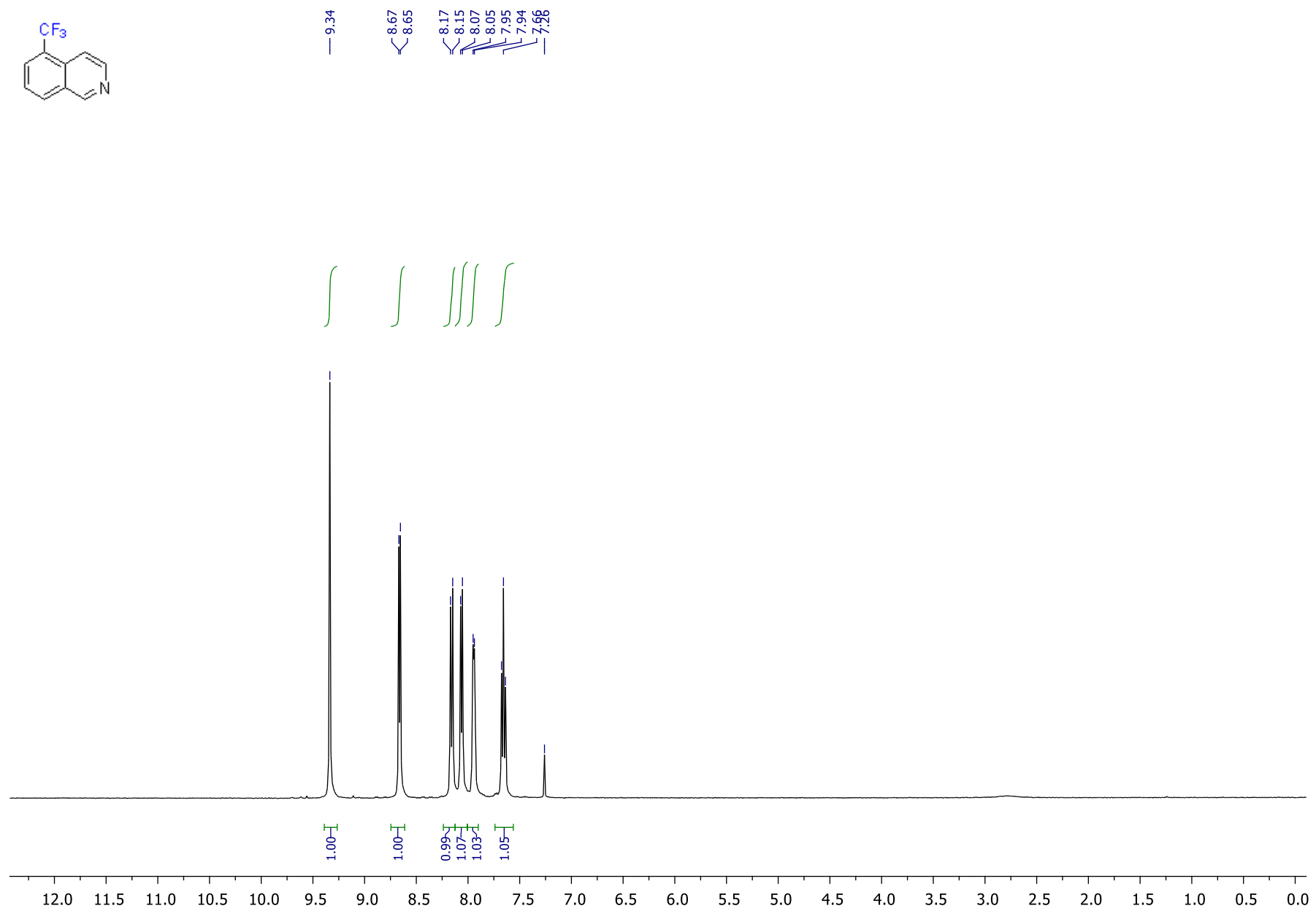

Compound 63a is also described in: a) Chem. Commun. 2014, 50, 10237-10240; b) Chem. Eur. J. 2016, 22, 6, 2075 - 2084; c) Bull. Chem. Soc. Jpn. 1988, 61, 10, 3531-3537. 
${ }^{13} \mathrm{C}\left\{{ }^{1} \mathrm{H}\right\}$ NMR $\left(126 \mathrm{MHz}, \mathrm{CDCl}_{3}\right)$

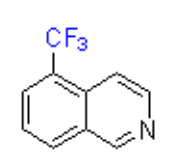

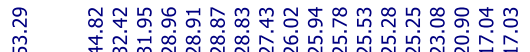

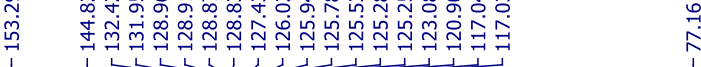

i

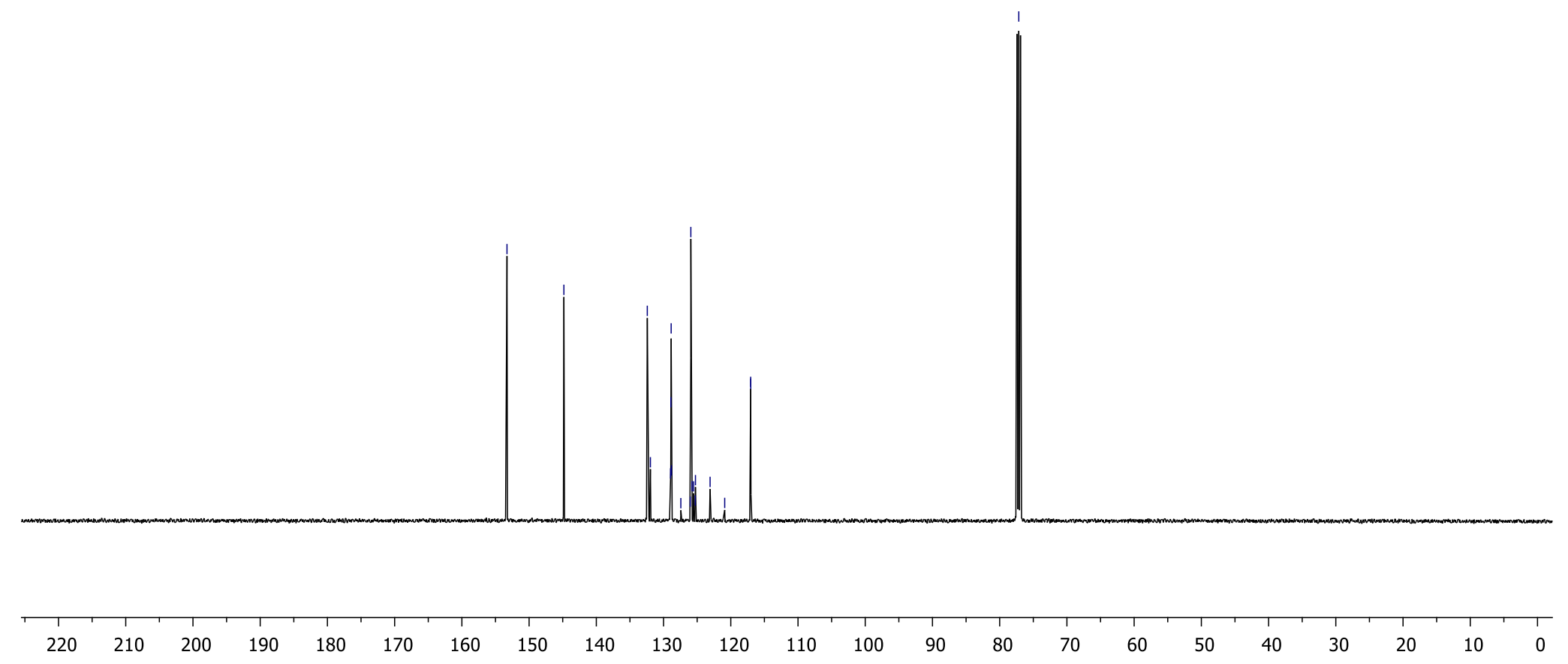


${ }^{19} \mathrm{~F}$ NMR $\left(376 \mathrm{MHz}, \mathrm{CDCl}_{3}\right)$

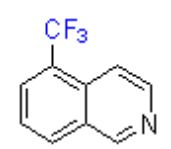

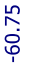

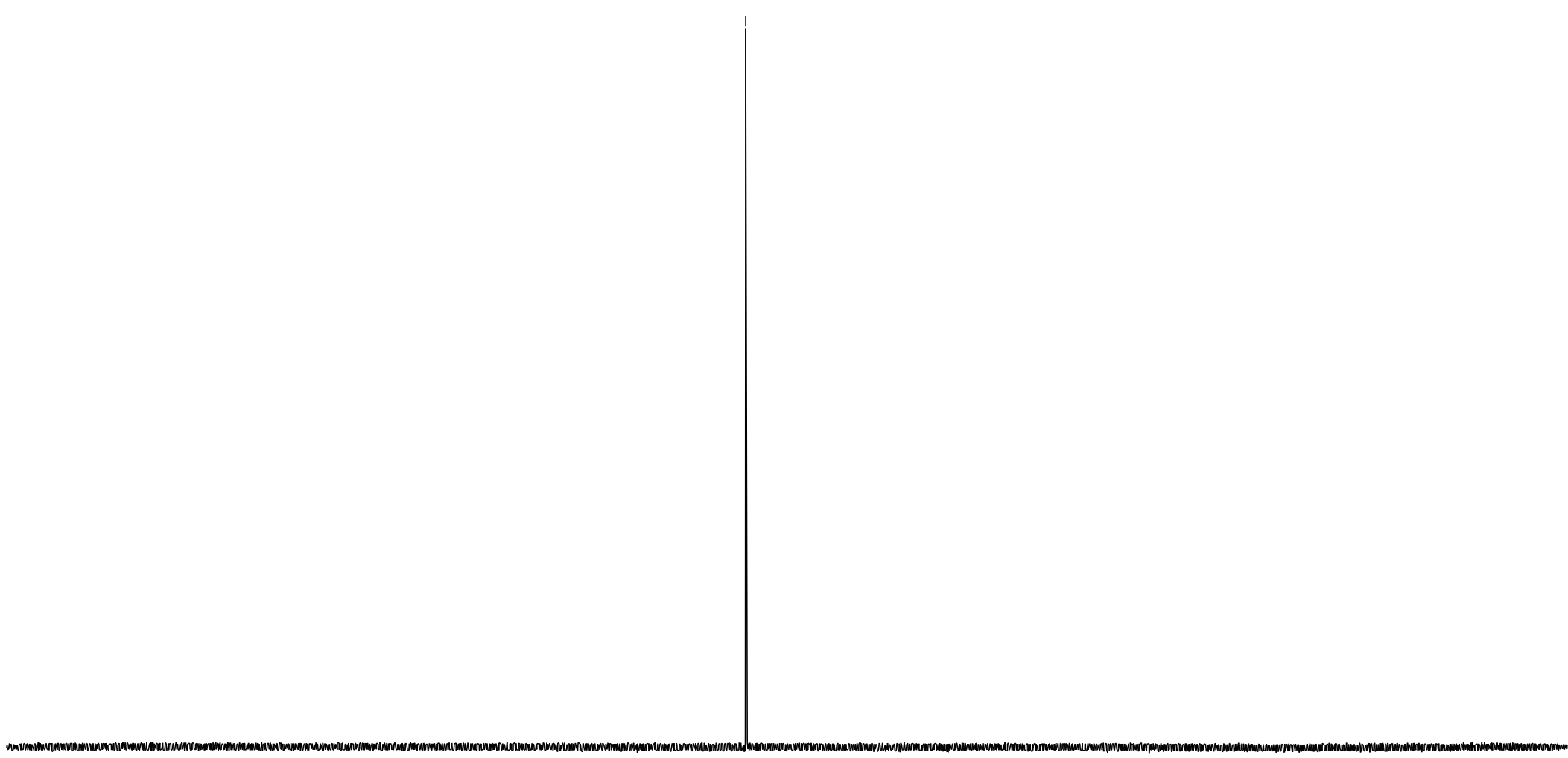

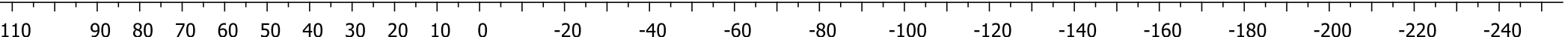




\section{Compound 65a}

${ }^{1} \mathrm{H}$ NMR $\left(400 \mathrm{MHz}, \mathrm{CDCl}_{3}\right)$
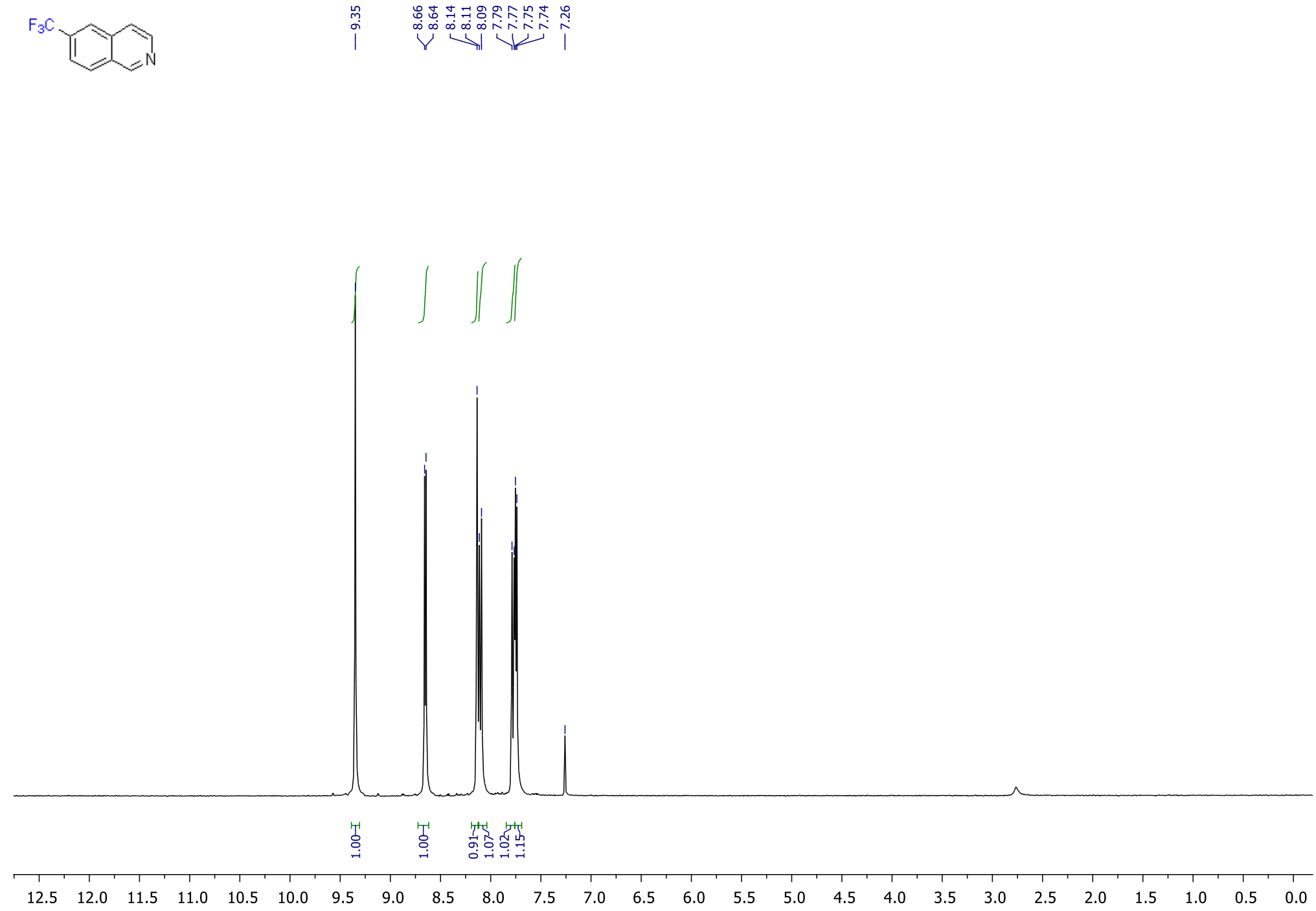
${ }^{13} \mathrm{C}\left\{{ }^{1} \mathrm{H}\right\}$ NMR $\left(126 \mathrm{MHz}, \mathrm{CDCl}_{3}\right)$

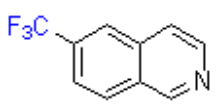

๑ัن

岱

$\stackrel{0}{\stackrel{2}{i}}$
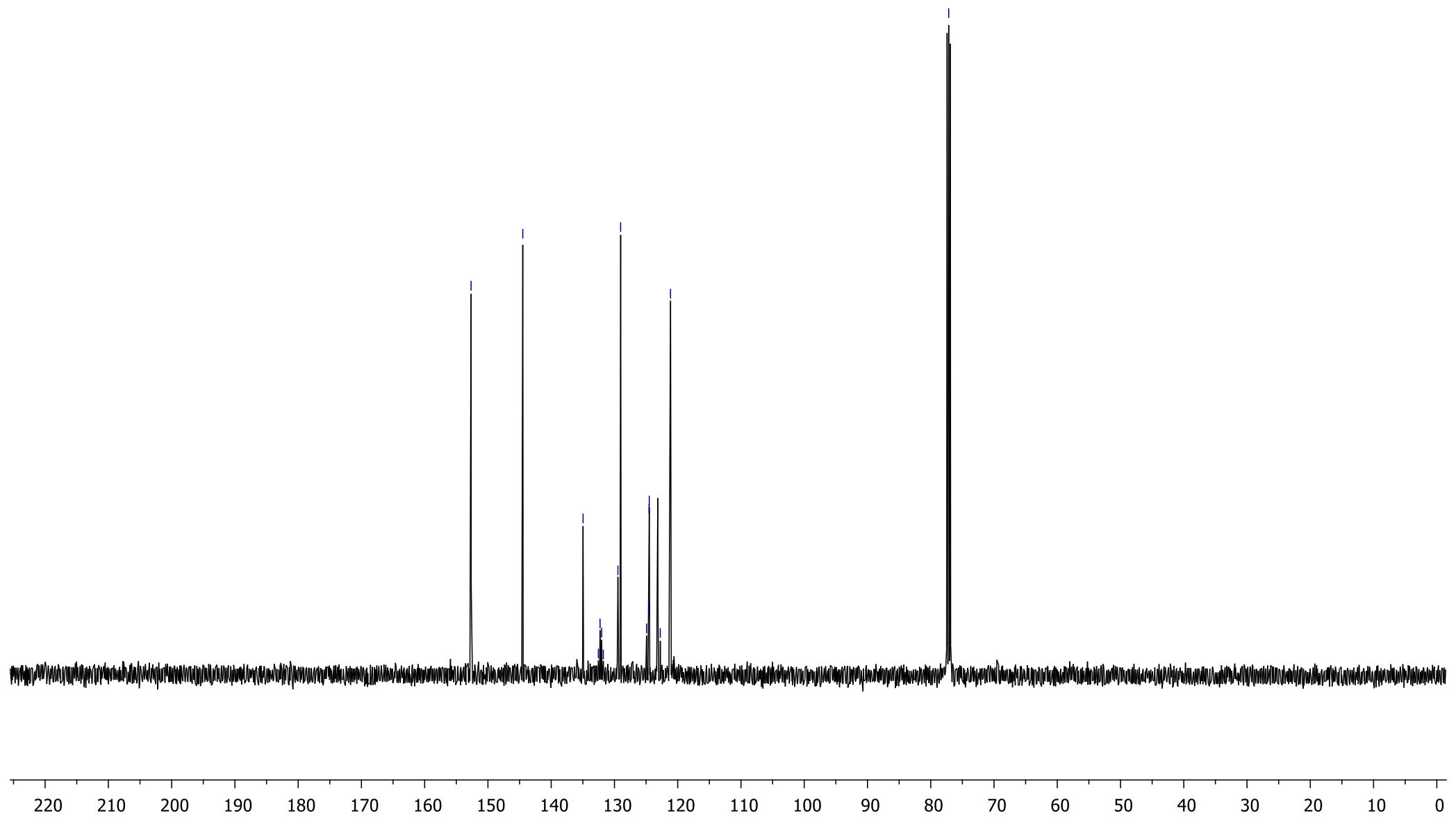
${ }^{19} \mathrm{~F}$ NMR (376 MHz, $\mathrm{CDCl}_{3}$ )
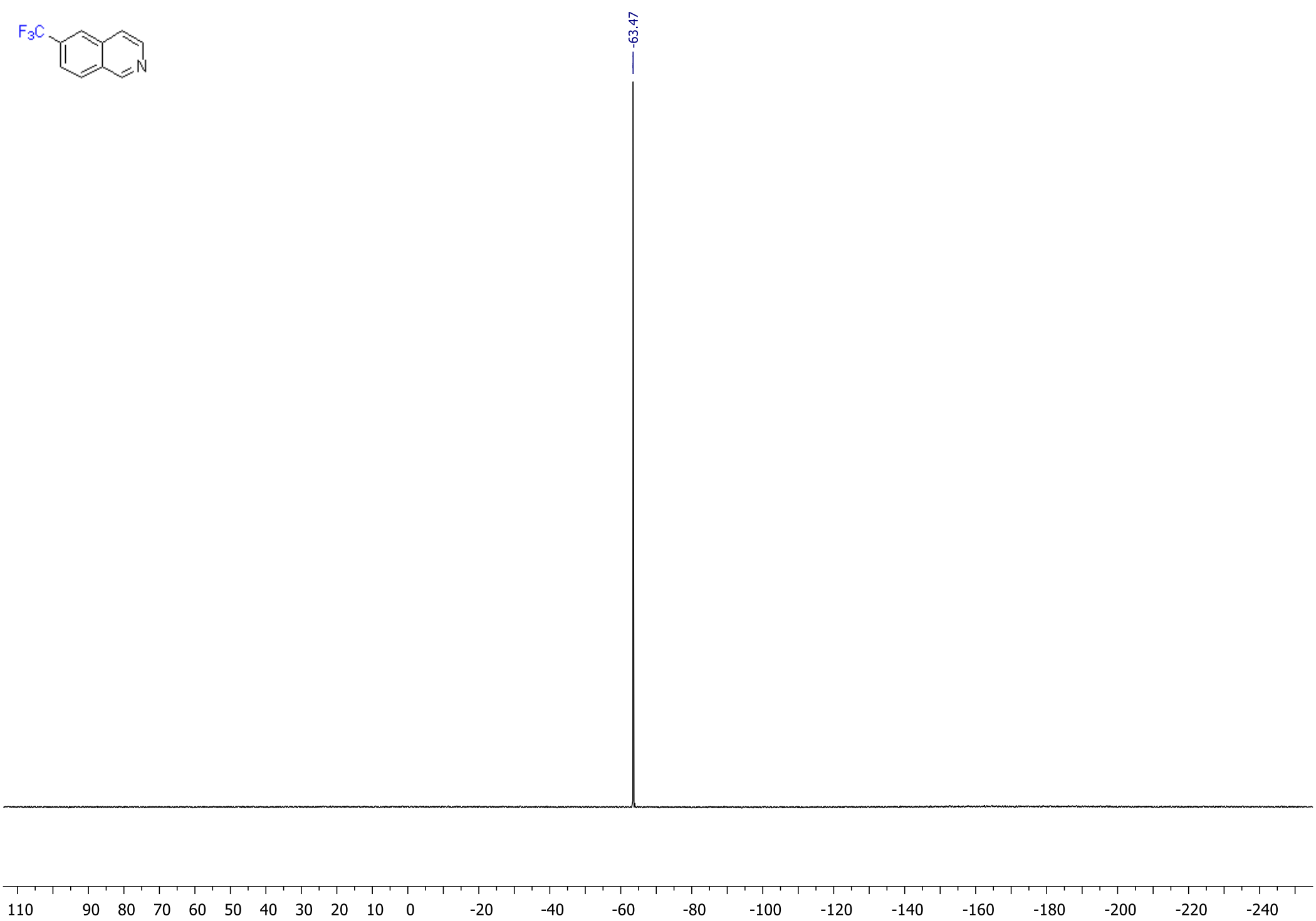


\section{Compound 66a}

${ }^{1} \mathrm{H}$ NMR (400 MHz, $\left.\mathrm{CDCl}_{3}\right)$

舟

|

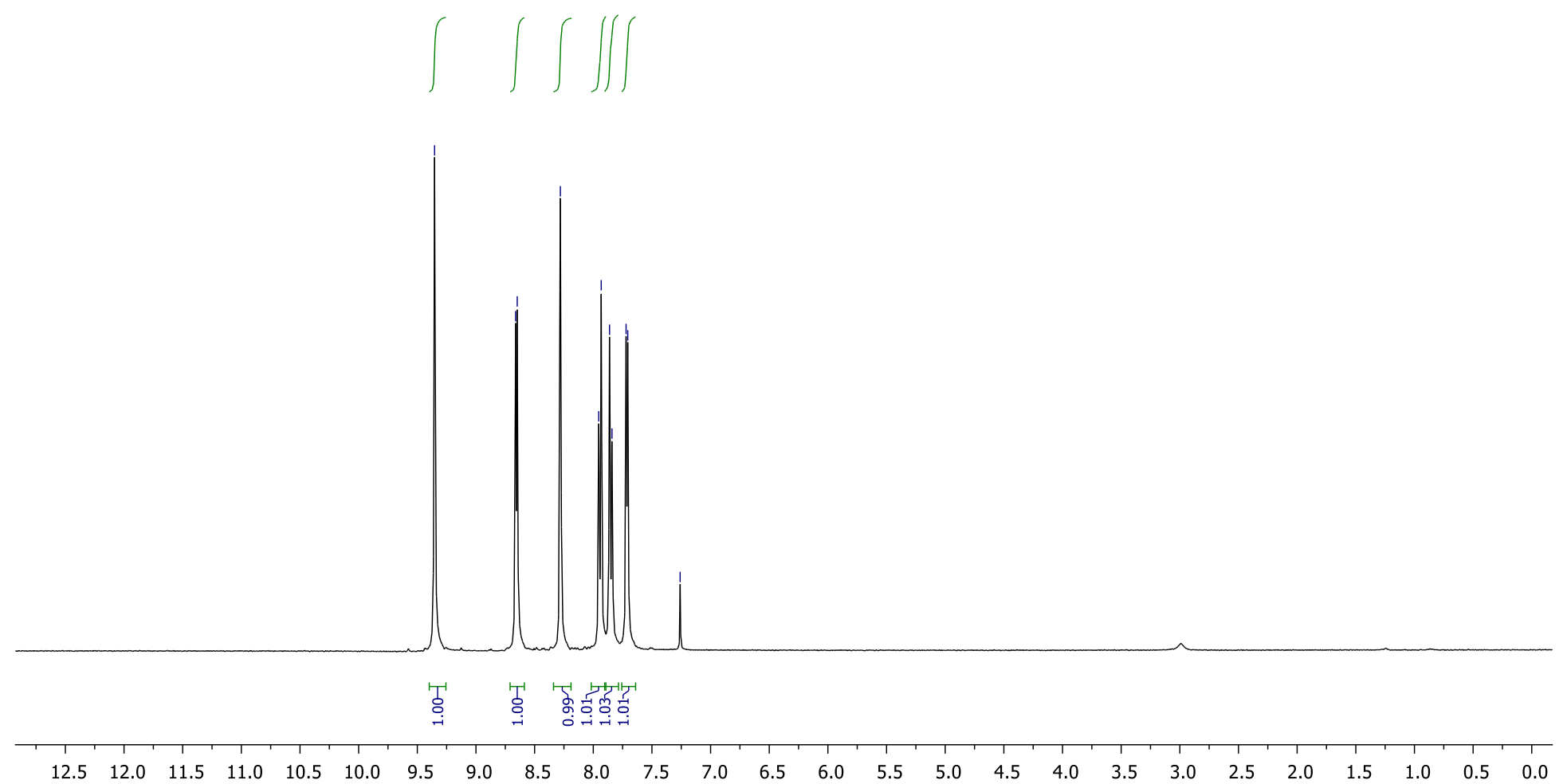


${ }^{13} \mathrm{C}\left\{{ }^{1} \mathrm{H}\right\}$ NMR $\left(126 \mathrm{MHz}, \mathrm{CDCl}_{3}\right)$

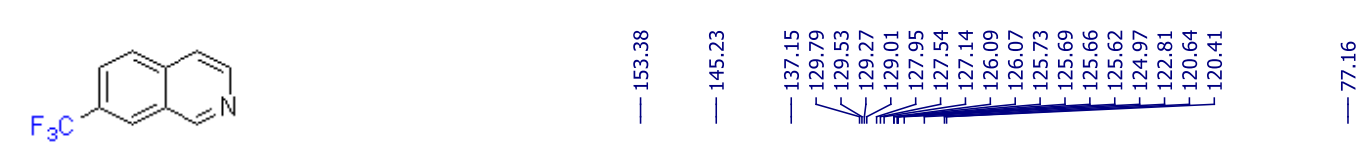

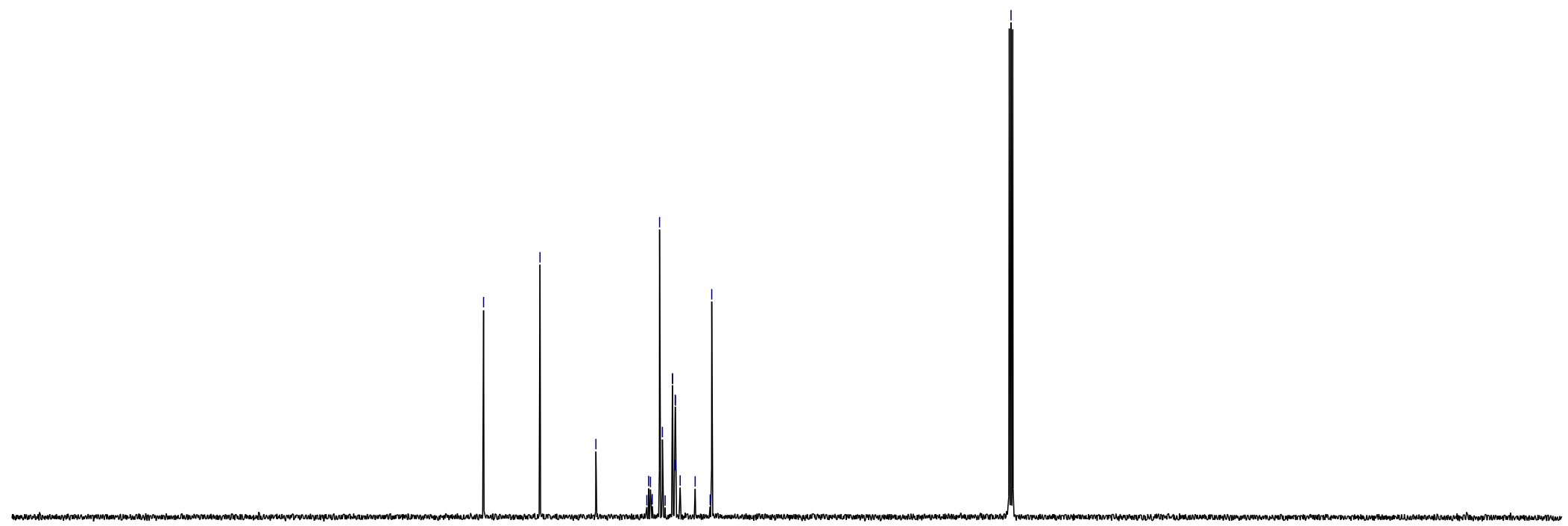

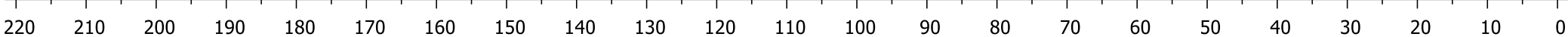


${ }^{19} \mathrm{~F}$ NMR $\left(376 \mathrm{MHz}, \mathrm{CDCl}_{3}\right)$

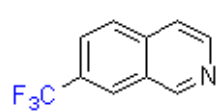

$\stackrel{\infty}{\stackrel{\infty}{0}}$

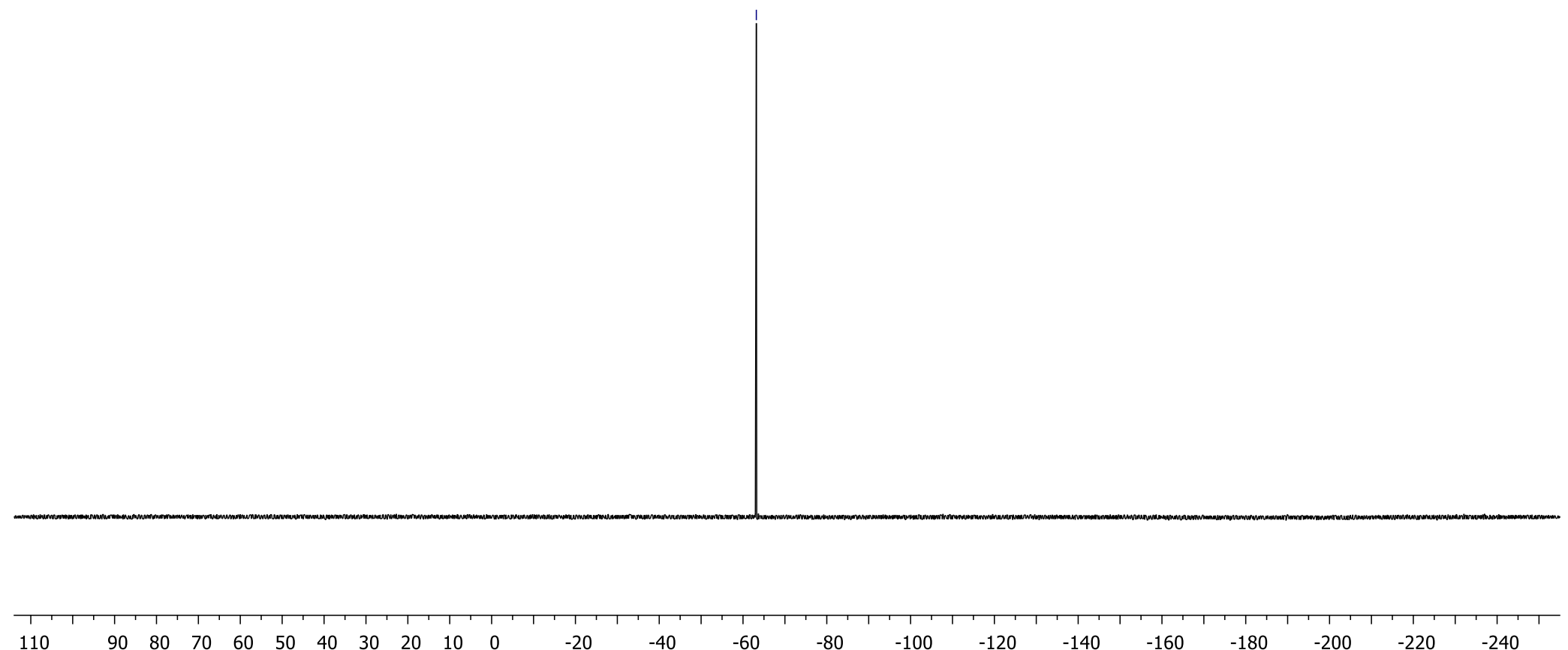


Compound $13 b^{*}$

${ }^{1} \mathrm{H} \mathrm{NMR}\left(400 \mathrm{MHz}, \mathrm{CDCl}_{3}\right)$
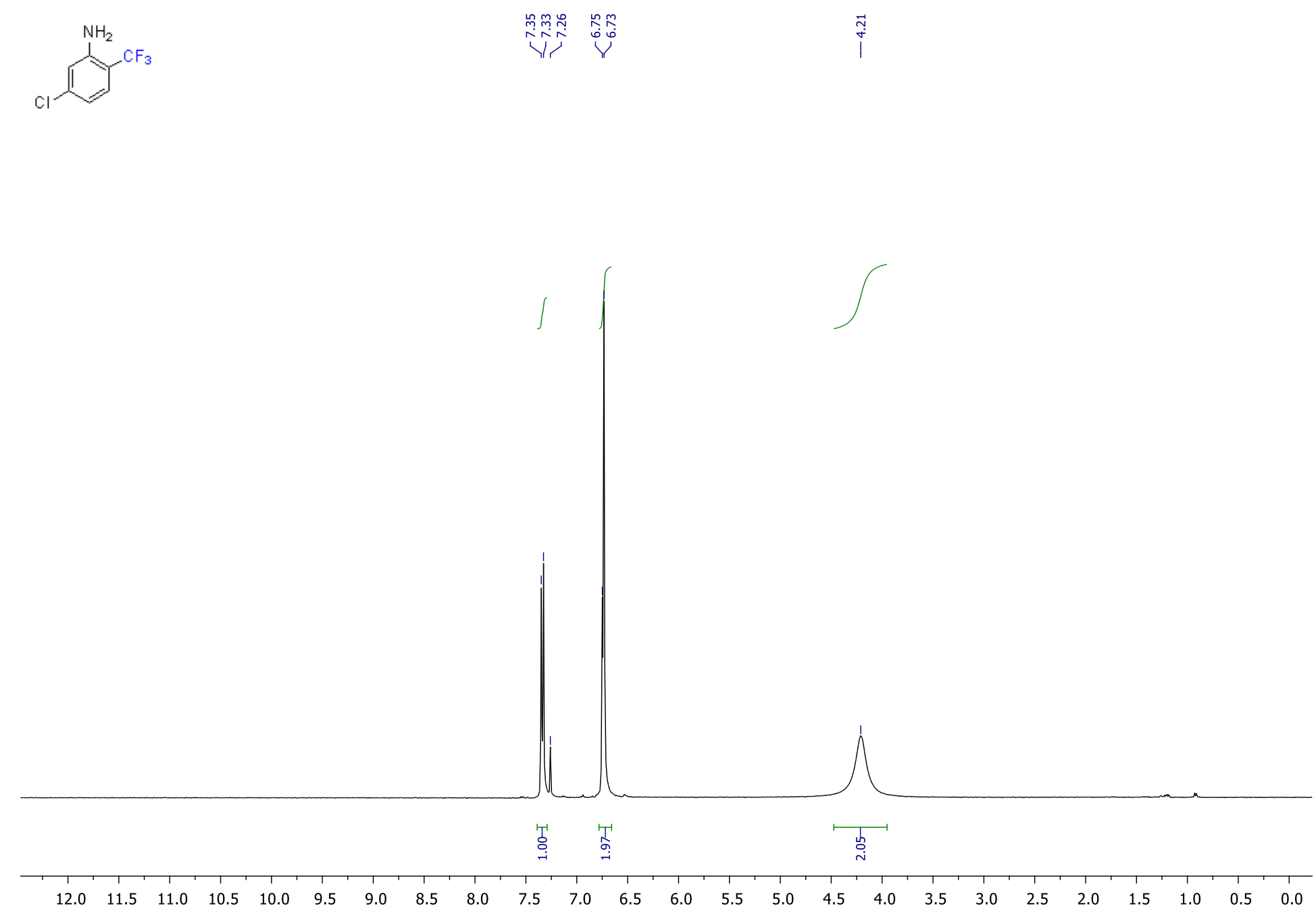

Compound 13b is also described in: a) J. Chem. Soc., Perkin Trans. 1, 1990, 2293-2299; b) ChemCatChem 2018, 10, 5, 965-970. 
${ }^{13} \mathrm{C}\left\{{ }^{1} \mathrm{H}\right\}$ NMR $\left(126 \mathrm{MHz}, \mathrm{CDCl}_{3}\right)$

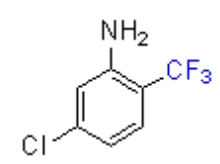

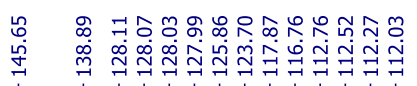

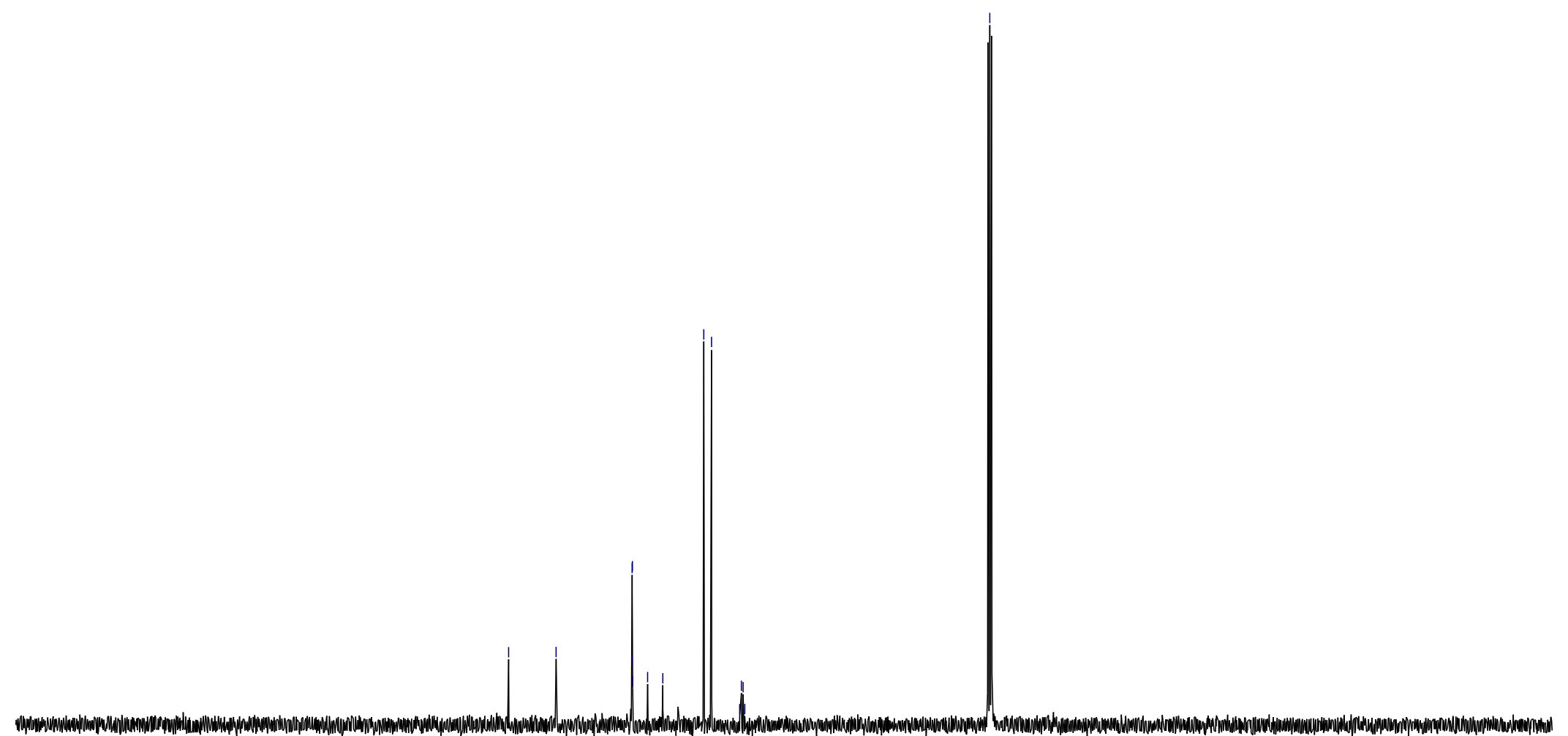


${ }^{19} \mathrm{~F}$ NMR (376 MHz, $\mathrm{CDCl}_{3}$ )

CF

$\stackrel{m}{\stackrel{m}{0}}$

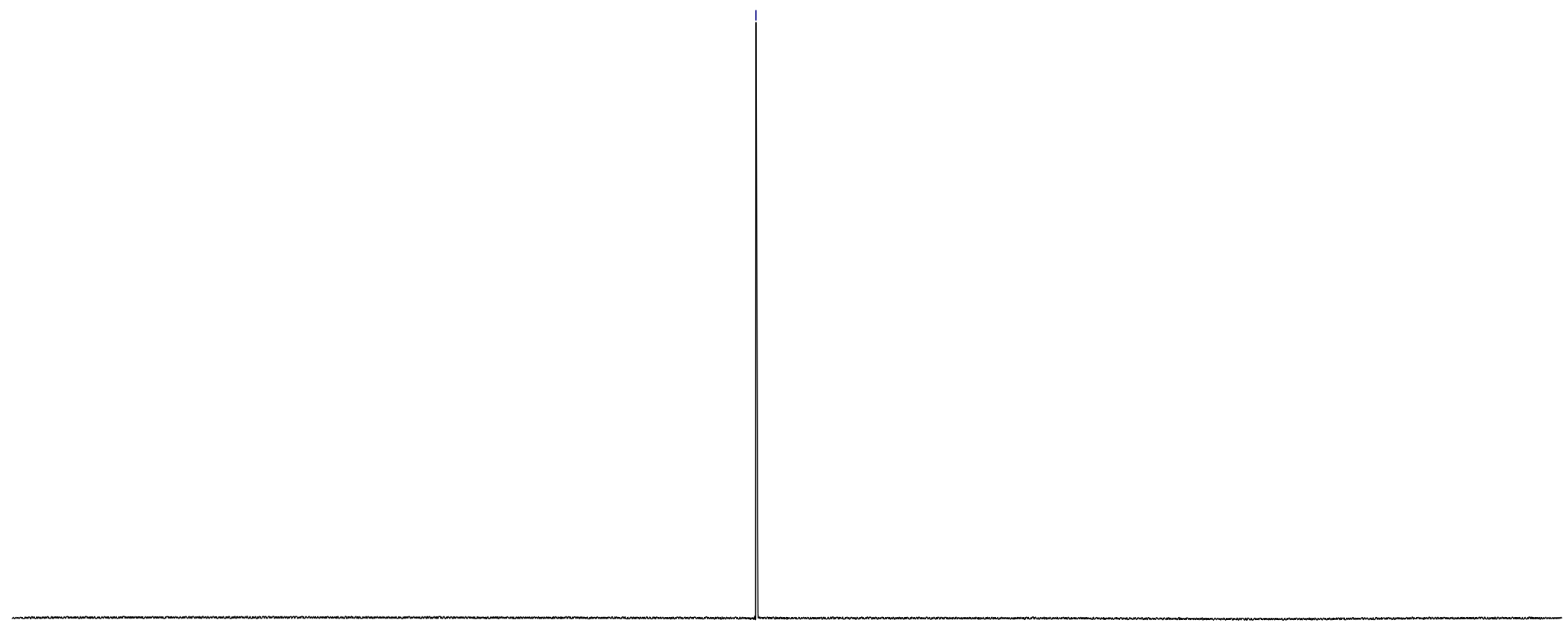

$\begin{array}{llllllllll}110 & 90 & 80 & 70 & 60 & 50 & 40 & 30 & 20 & 10\end{array}$

$-20$




\section{Compound $14 b^{*}$}

${ }^{1} \mathrm{H}$ NMR $\left(400 \mathrm{MHz}, \mathrm{CDCl}_{3}\right)$
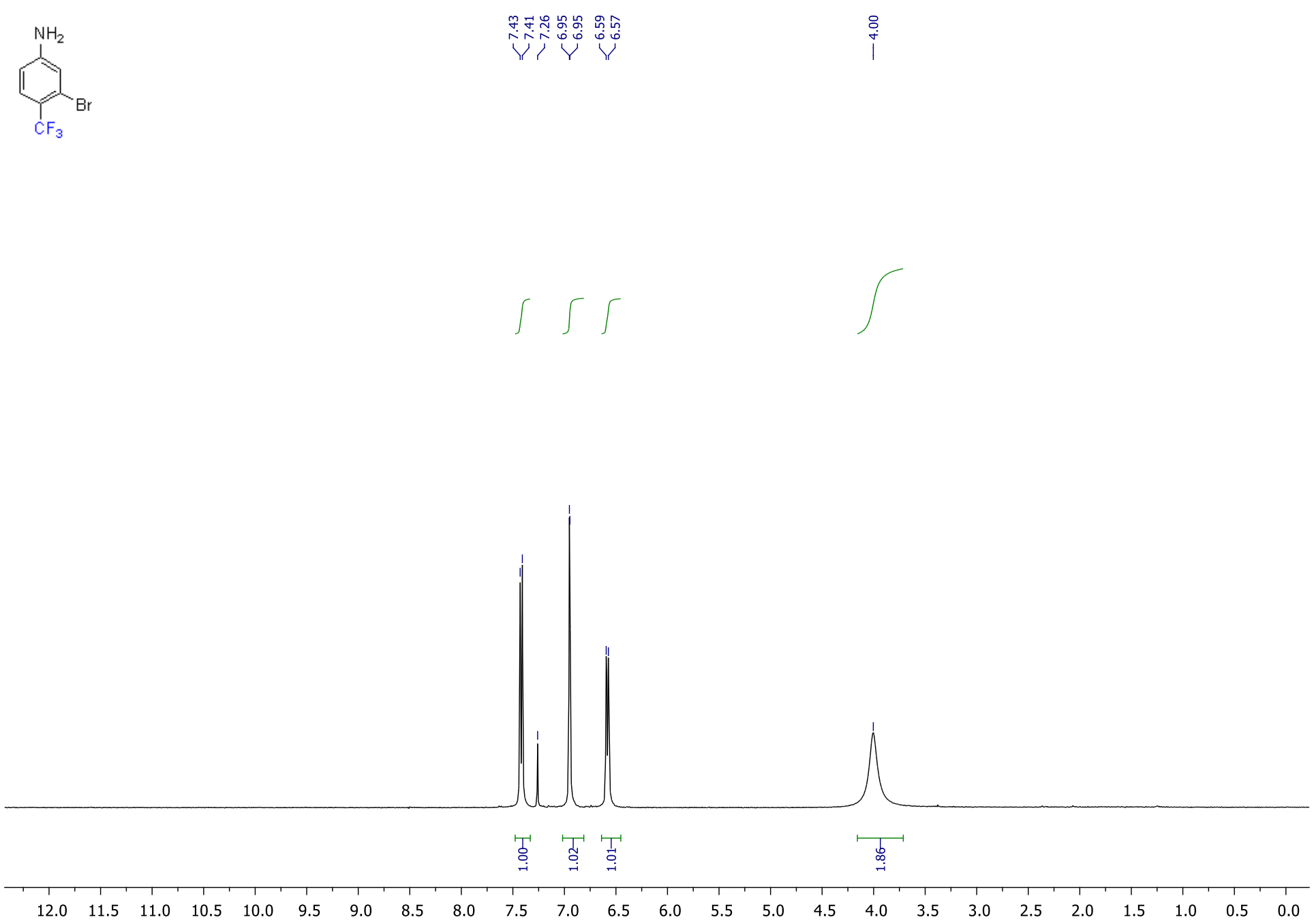

Compound 14b is also described in: a) Org. Lett. 2018, 20, 13, 3732-3735; b) Org. Lett. 2014, 16, 6, 1768-1771. 
${ }^{13} \mathrm{C}\left\{{ }^{1} \mathrm{H}\right\}$ NMR $\left(126 \mathrm{MHz}, \mathrm{CDCl}_{3}\right)$

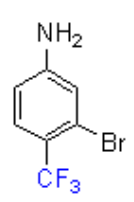

ำ 음

|

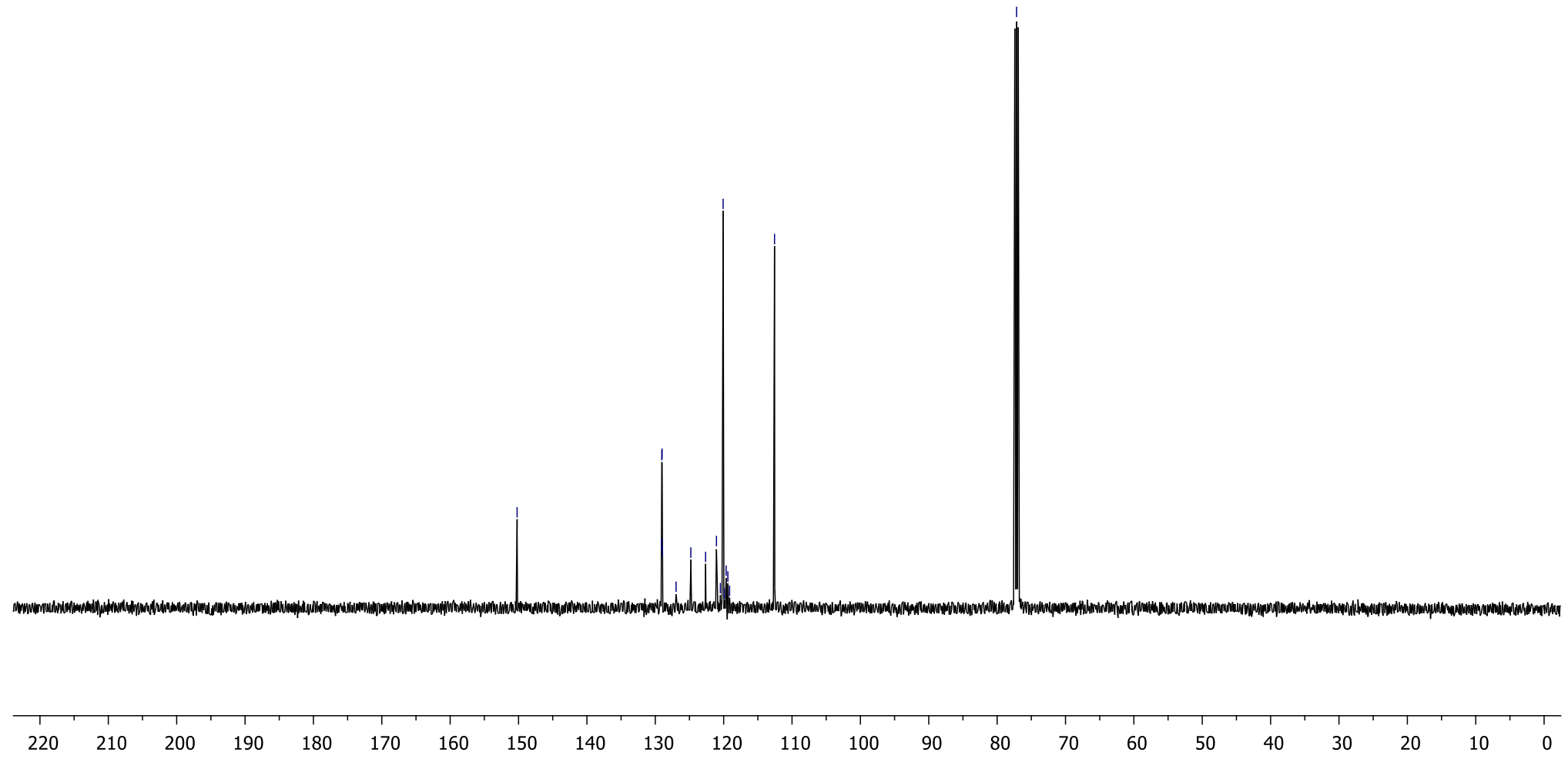


${ }^{19} \mathrm{~F}$ NMR (376 MHz, $\mathrm{CDCl}_{3}$ )

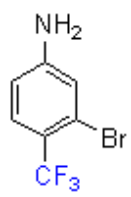

क्षें

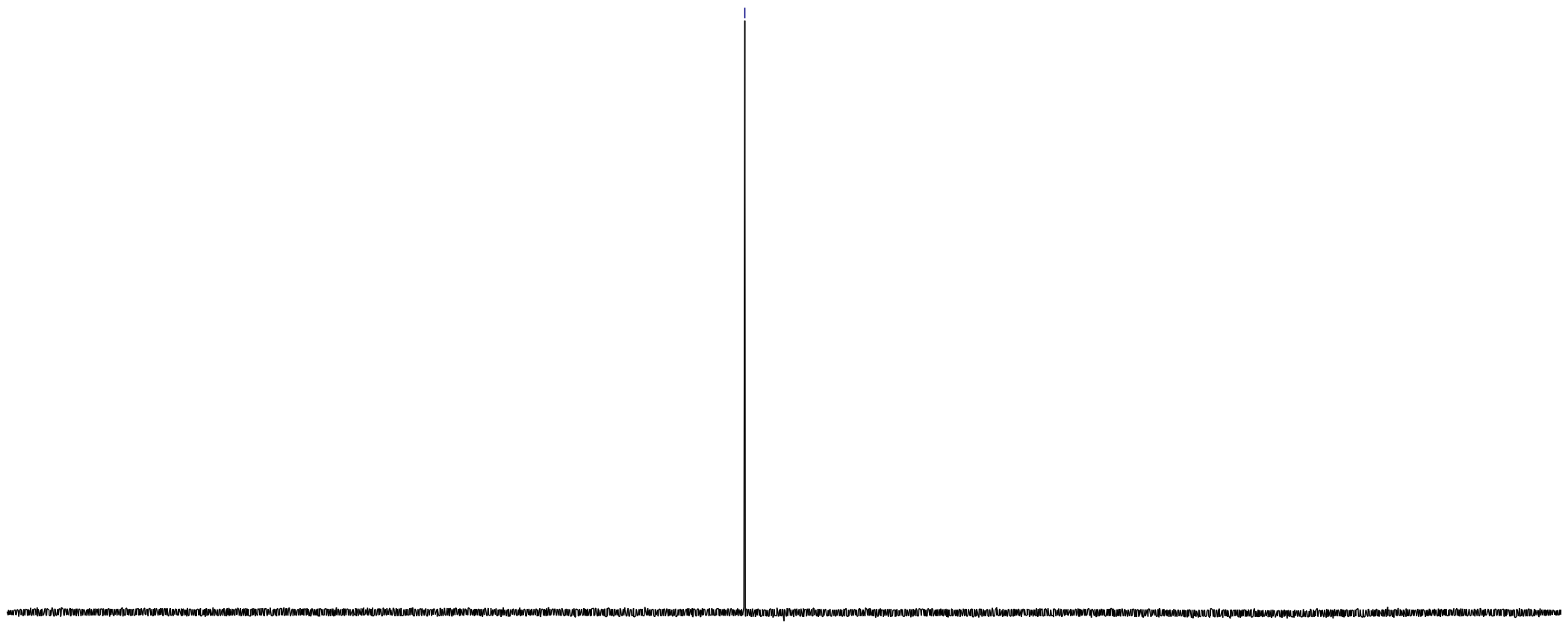

$\begin{array}{lllllllllll}110 & 90 & 80 & 70 & 60 & 50 & 40 & 30 & 20 & 10 & 0\end{array}$

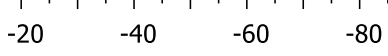

$-100 \quad-120$ 
Compound 15b

${ }^{1} \mathrm{H}$ NMR (400 MHz, $\mathrm{CDCl}_{3}$ )

(NF3
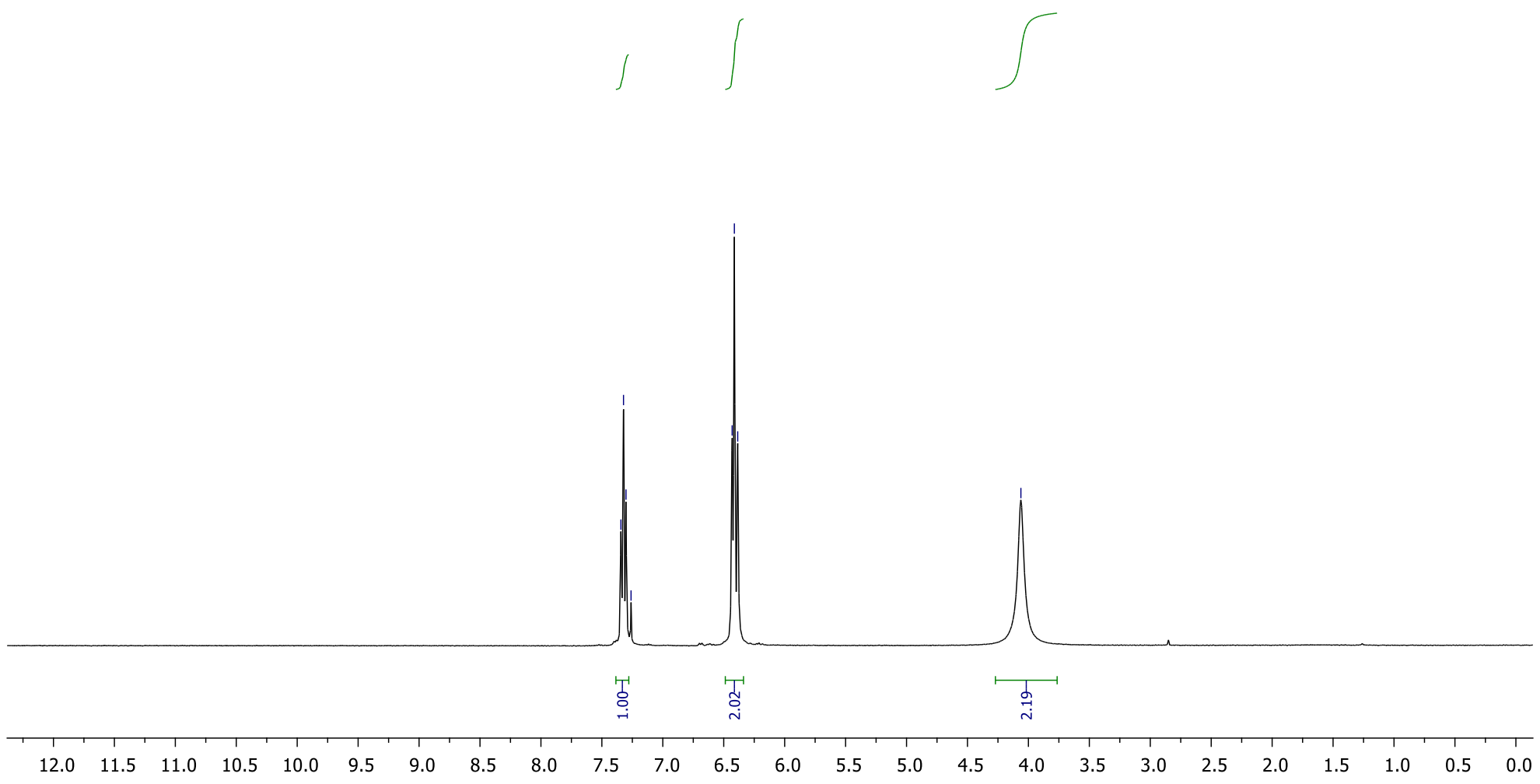
${ }^{13} \mathrm{C}\left\{{ }^{1} \mathrm{H}\right\}$ NMR $\left(126 \mathrm{MHz}, \mathrm{CDCl}_{3}\right)$

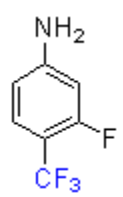

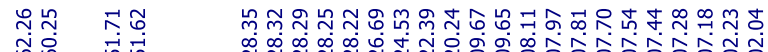

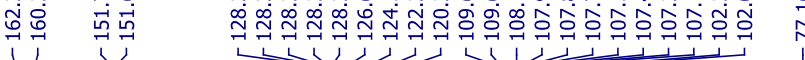

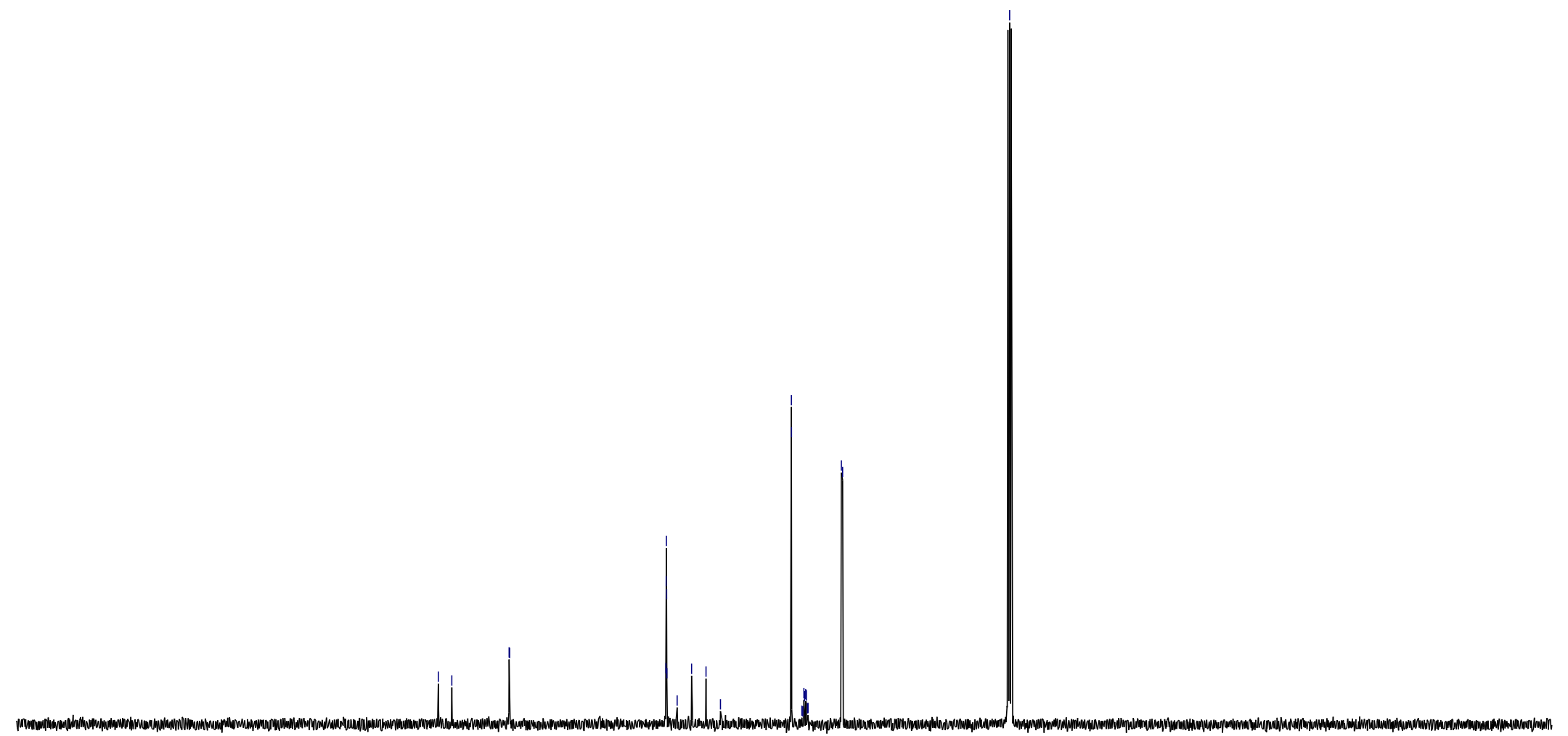

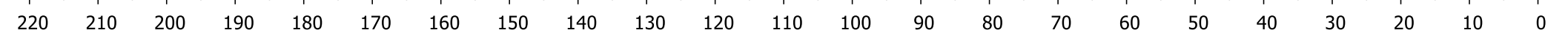


${ }^{19} \mathrm{~F}$ NMR (376 MHz, $\mathrm{CDCl}_{3}$ )

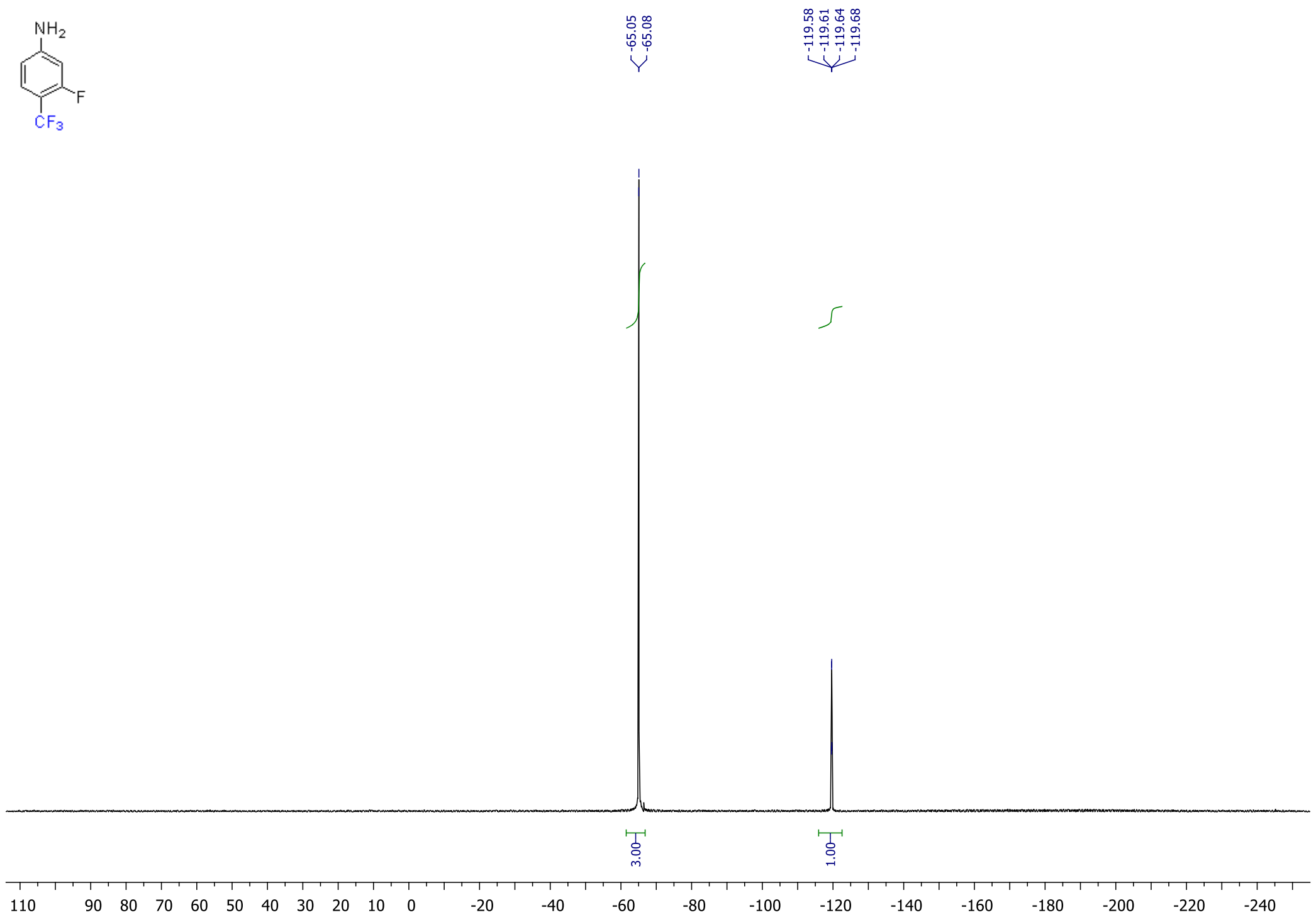




\section{Compound $16 b^{*}$}

\section{${ }^{1} \mathrm{H}$ NMR $\left(400 \mathrm{MHz}, \mathrm{CDCl}_{3}\right)$}
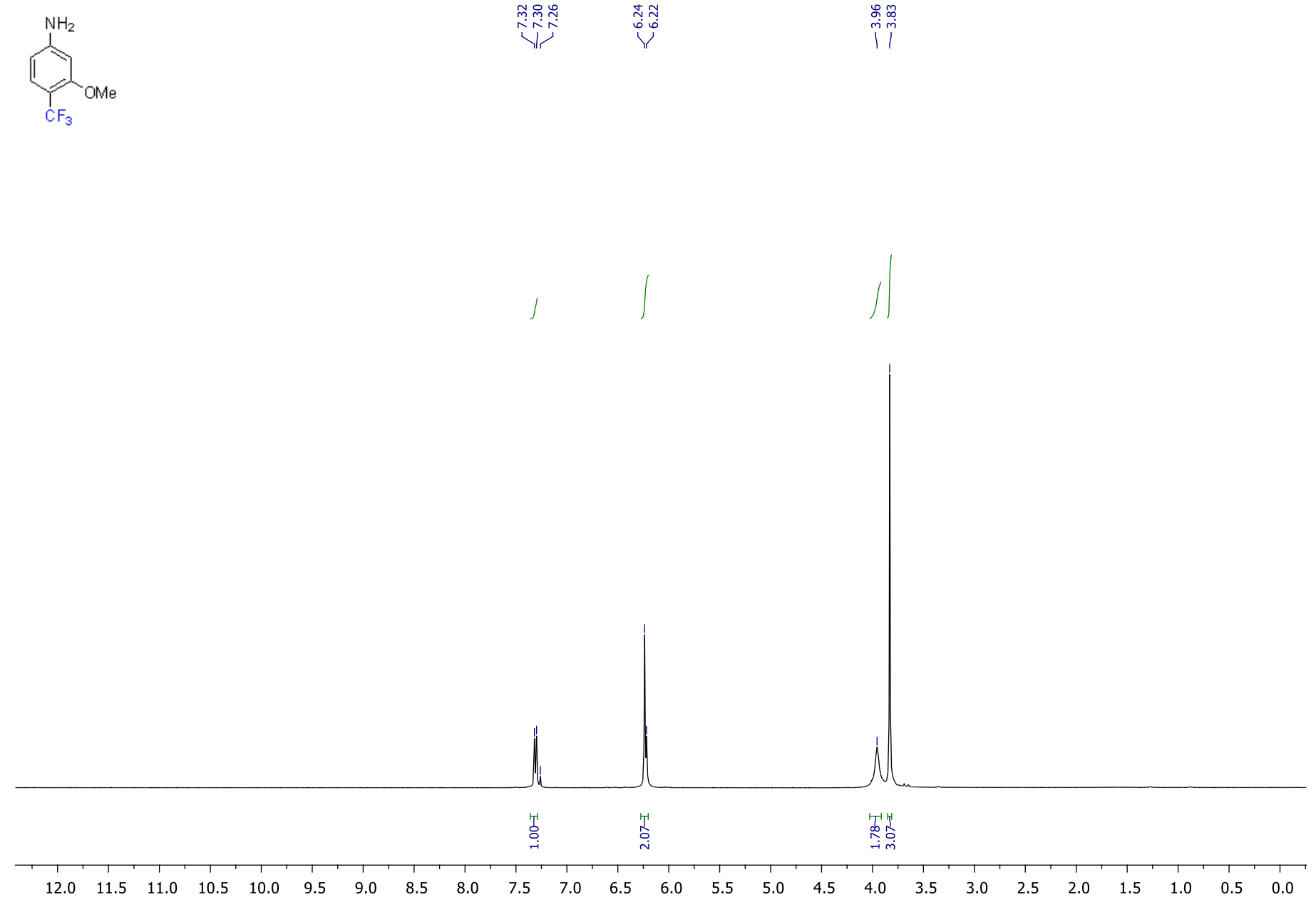

Compound 16b is also described in: a) J. Med. Chem. 2014, 57, 5, 1914-1931; b) Org. Lett. 2018, 20, 13, 3732-3735; c) J. Chem. Soc., Perkin Trans. 1, 1990, 2293-2299. 
${ }^{13} \mathrm{C}\left\{{ }^{1} \mathrm{H}\right\}$ NMR $\left(126 \mathrm{MHz}, \mathrm{CDCl}_{3}\right)$

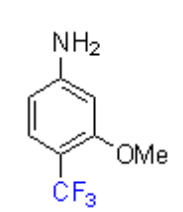

웅

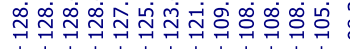

$\stackrel{0}{i}$

$\mathrm{CF}_{3}$

।
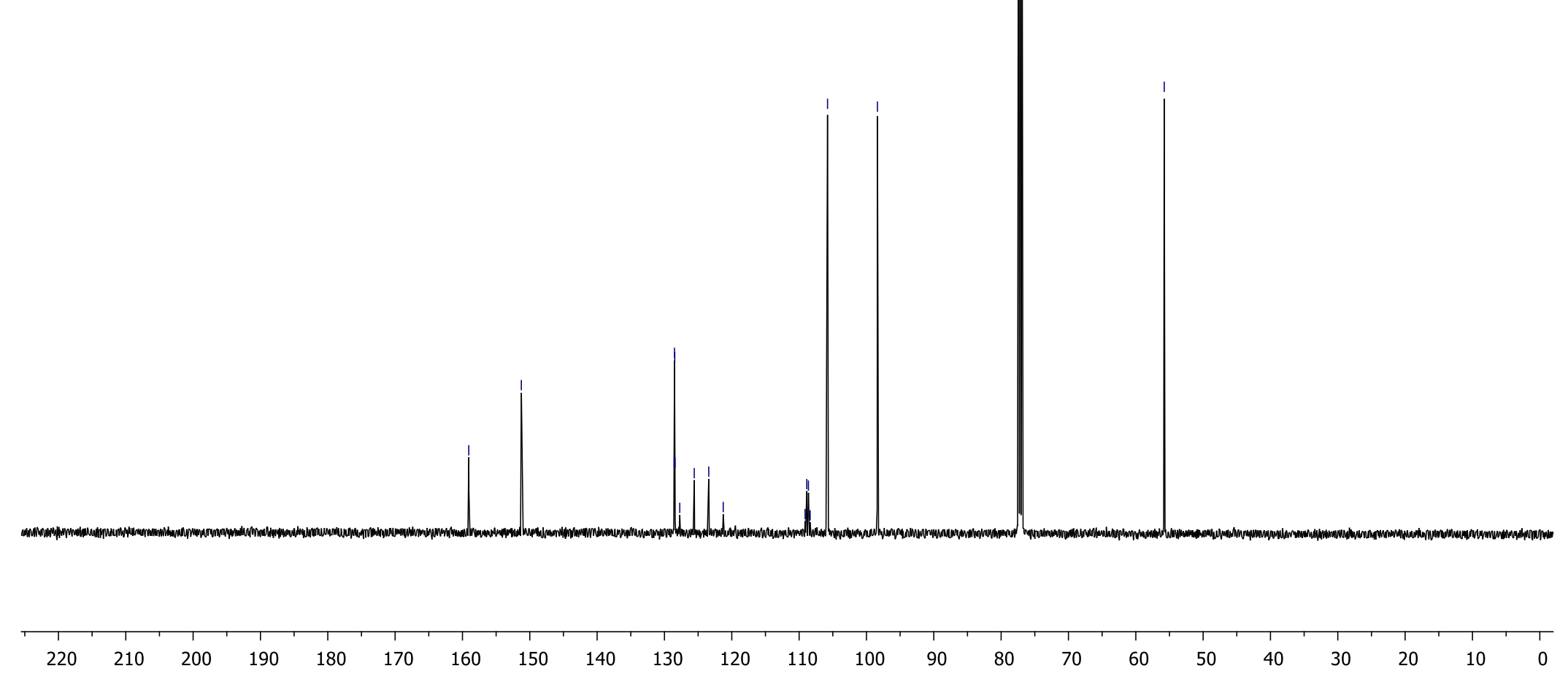
${ }^{19} \mathrm{~F}$ NMR (376 MHz, $\mathrm{CDCl}_{3}$ )

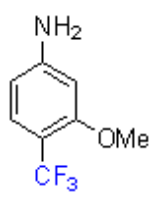

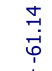

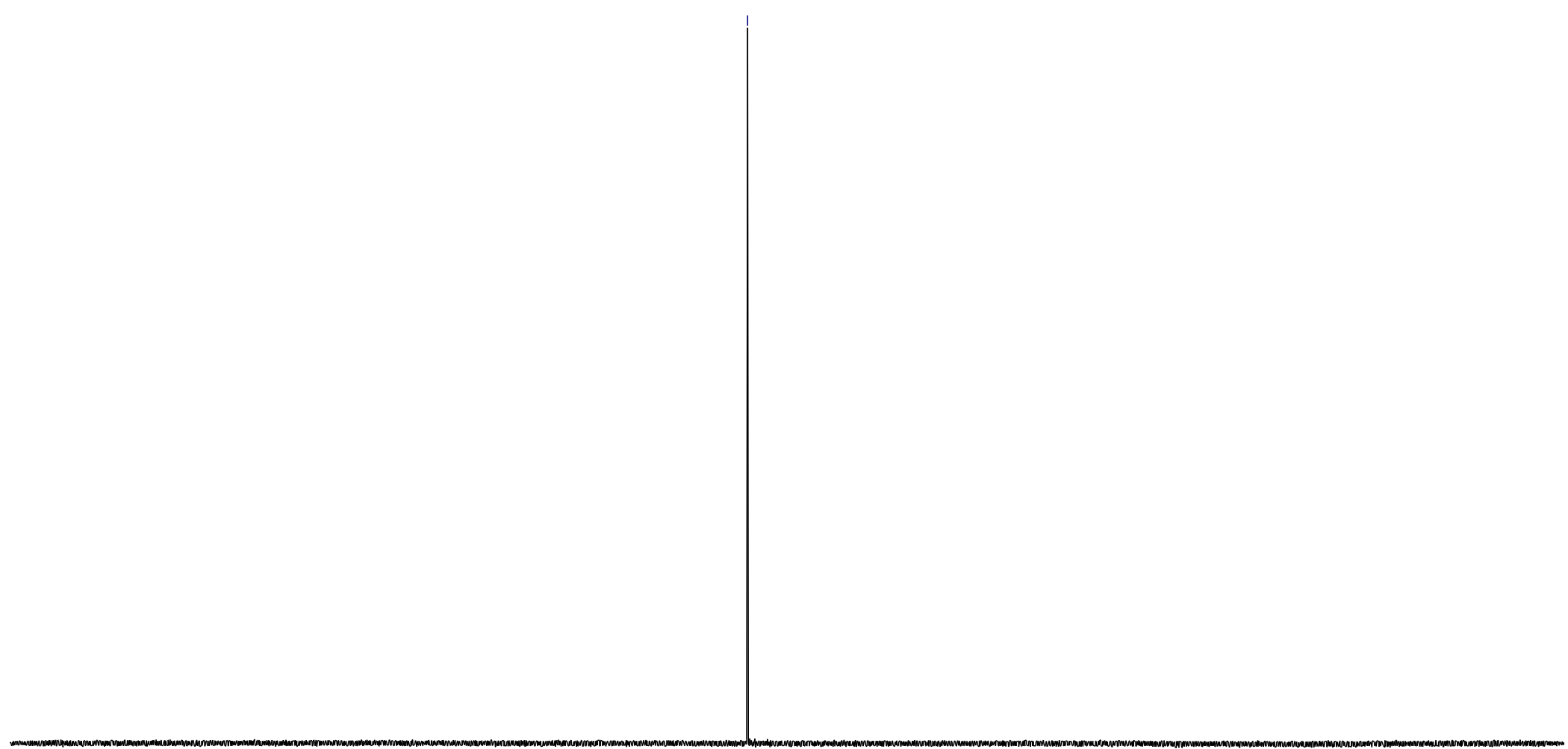

$\begin{array}{llllllllll}110 & 90 & 80 & 70 & 60 & 50 & 40 & 30 & 20 & 10\end{array}$

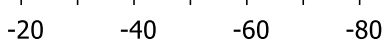

$-100 \quad-120$ 
Compound $17 \mathrm{~b}^{*}$

${ }^{1} \mathrm{H}$ NMR $\left(400 \mathrm{MHz}, \mathrm{CDCl}_{3}\right)$
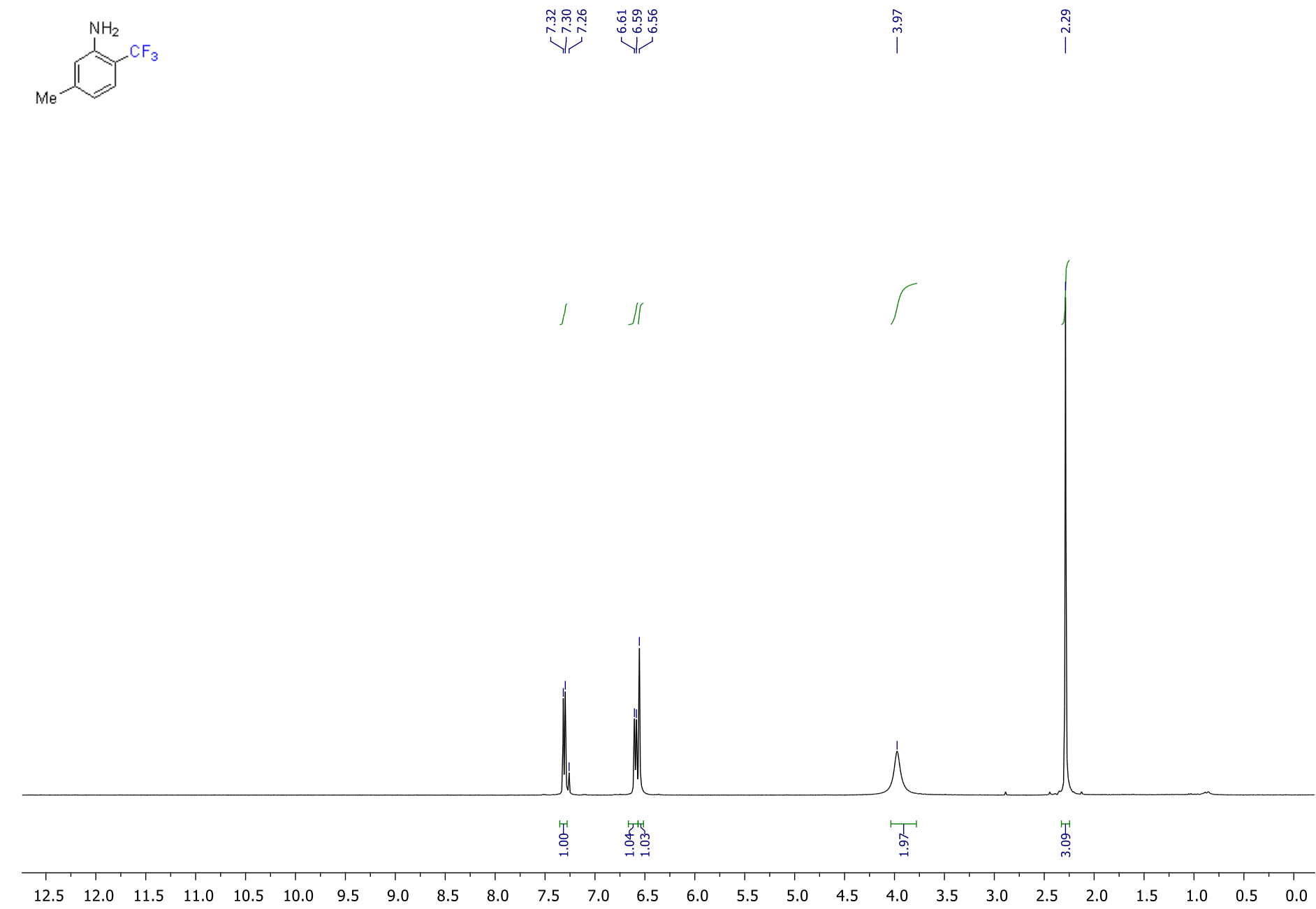

Compound 17b is also described in: a) J. Chem. Soc., Perkin Trans. 1, 1990, 2293-2299; b) ChemCatChem 2018, 10, 5, 965-970; c) Bioorg. Med. Chem. Lett. 2015, 25, 17, 3738-3743. 
${ }^{13} \mathrm{C}\left\{{ }^{1} \mathrm{H}\right\}$ NMR $\left(126 \mathrm{MHz}, \mathrm{CDCl}_{3}\right)$

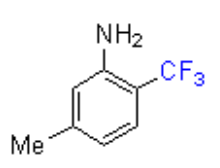

焉

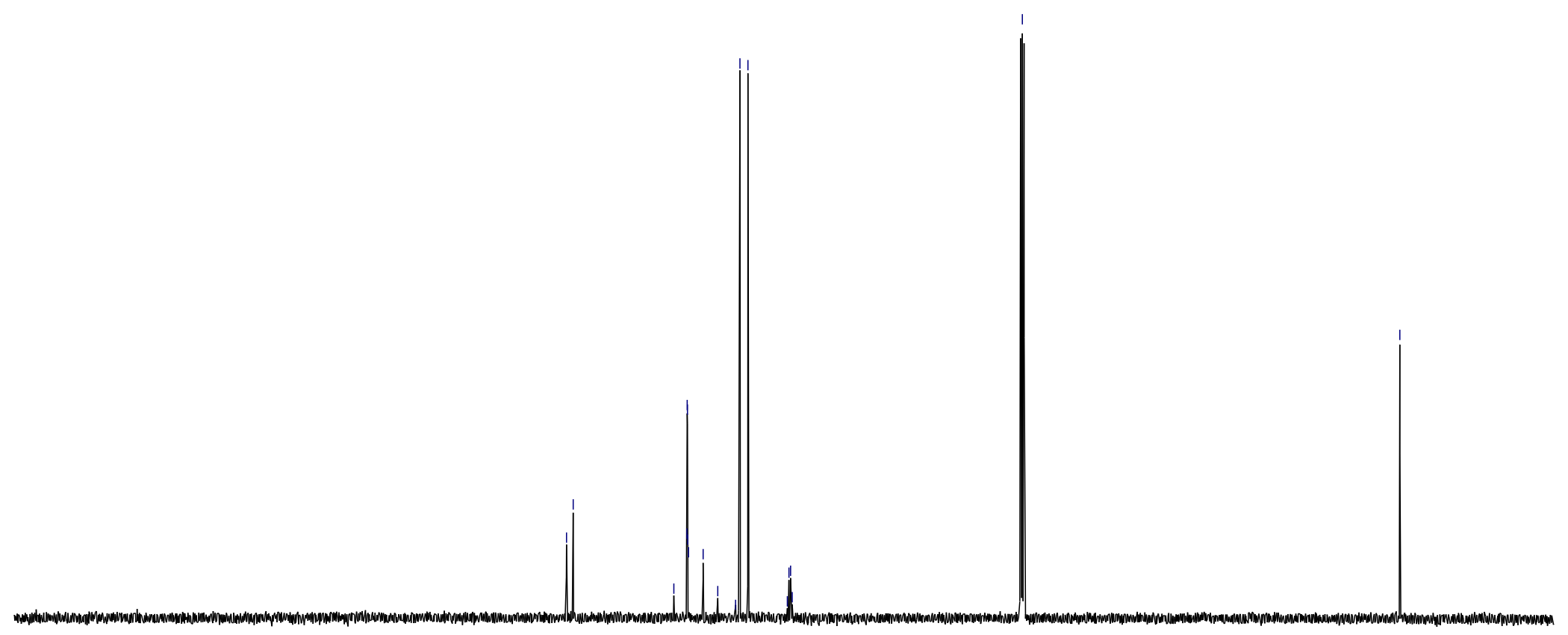


${ }^{19} \mathrm{~F}$ NMR (376 MHz, $\mathrm{CDCl}_{3}$ )

$\overbrace{}^{\mathrm{NH}_{2}}$

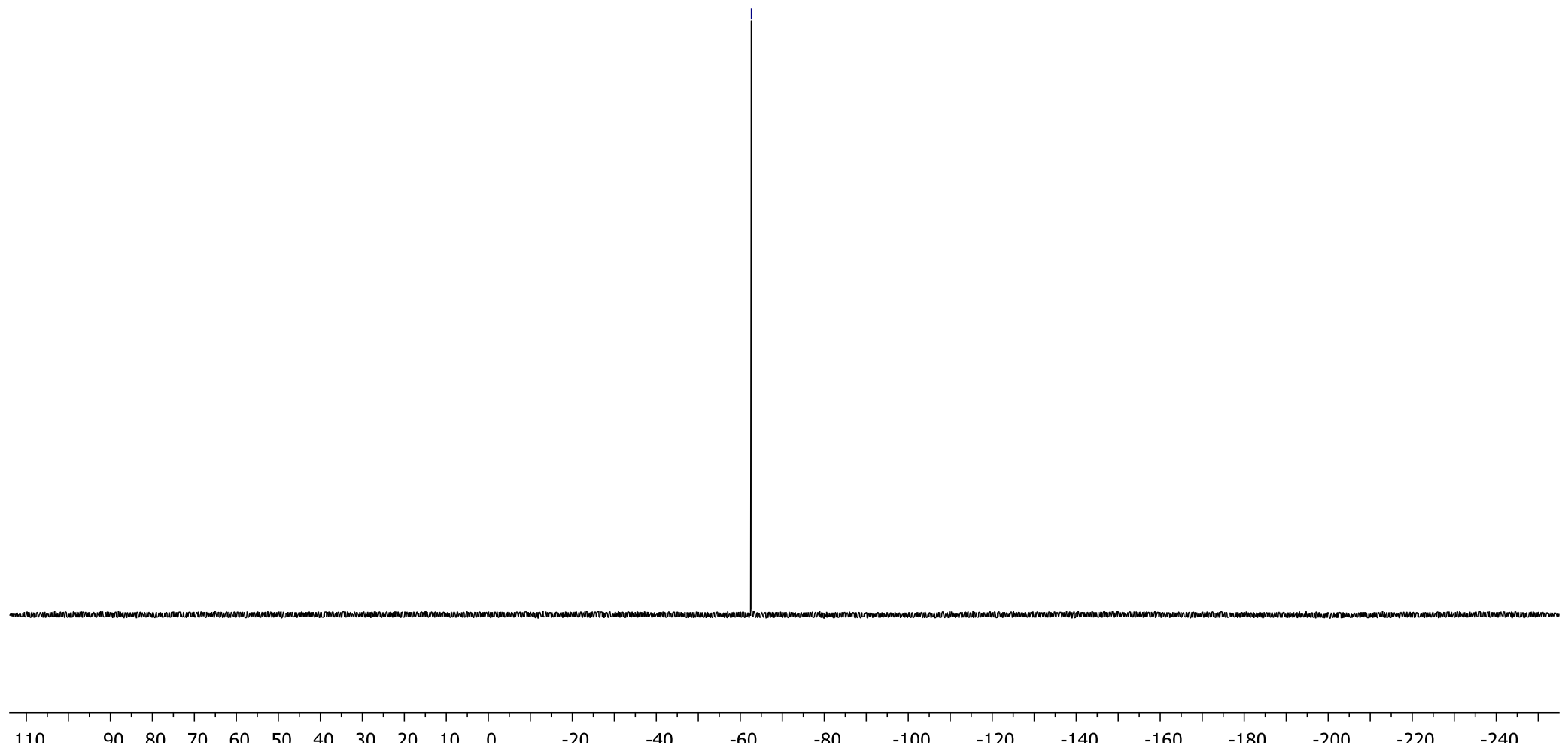


Compound 20b

${ }^{1} \mathrm{H}$ NMR (400 MHz, $\mathrm{CDCl}_{3}$ )

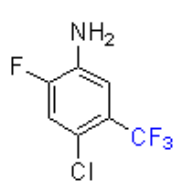

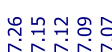

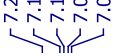

$\stackrel{\infty}{i}$

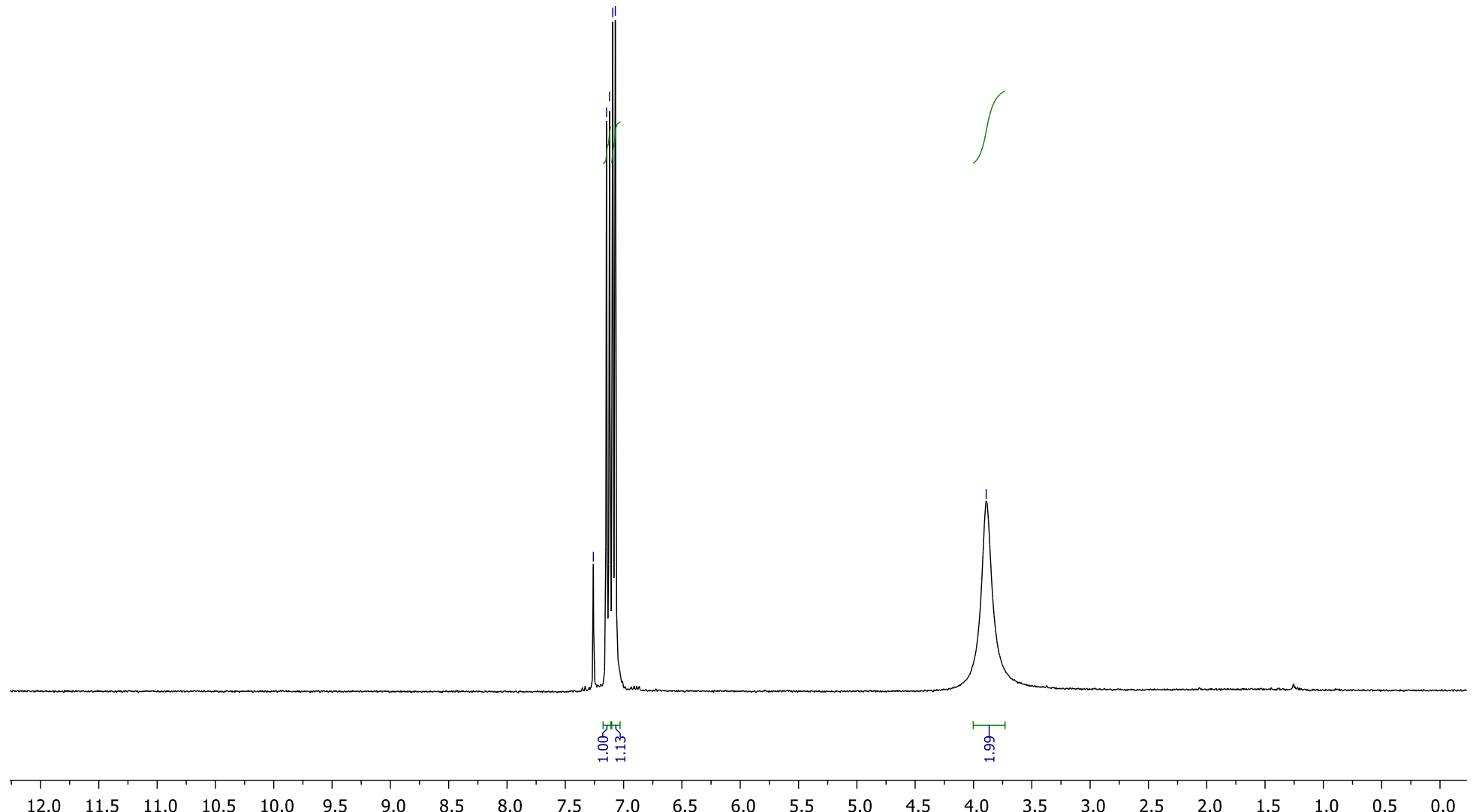


${ }^{13} \mathrm{C}\left\{{ }^{1} \mathrm{H}\right\}$ NMR $\left(126 \mathrm{MHz}, \mathrm{CDCl}_{3}\right)$

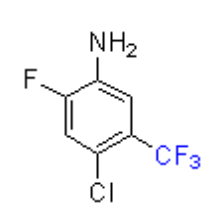

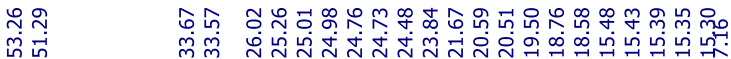

传

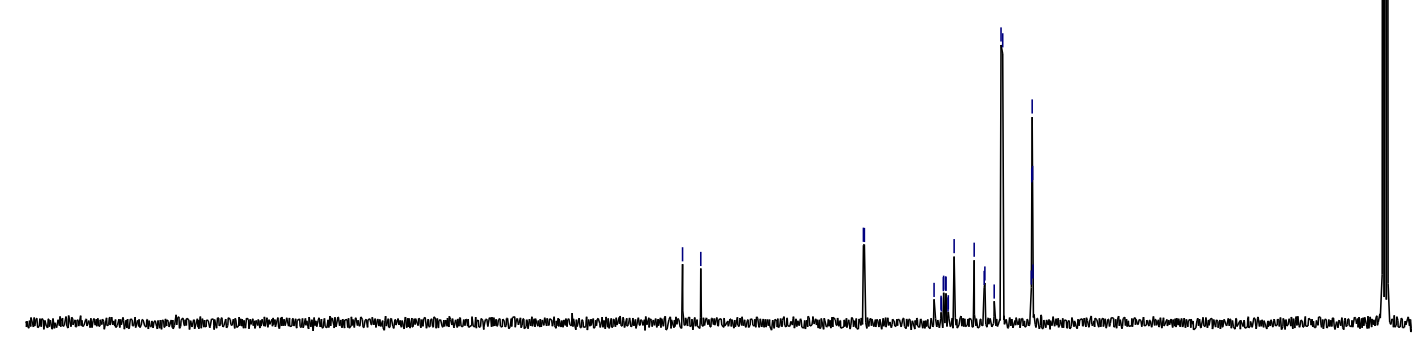

$\begin{array}{lllllll}220 & 210 & 200 & 190 & 180 & 170 & 160\end{array}$ 
${ }^{19} \mathrm{~F}$ NMR (376 MHz, $\mathrm{CDCl}_{3}$ )
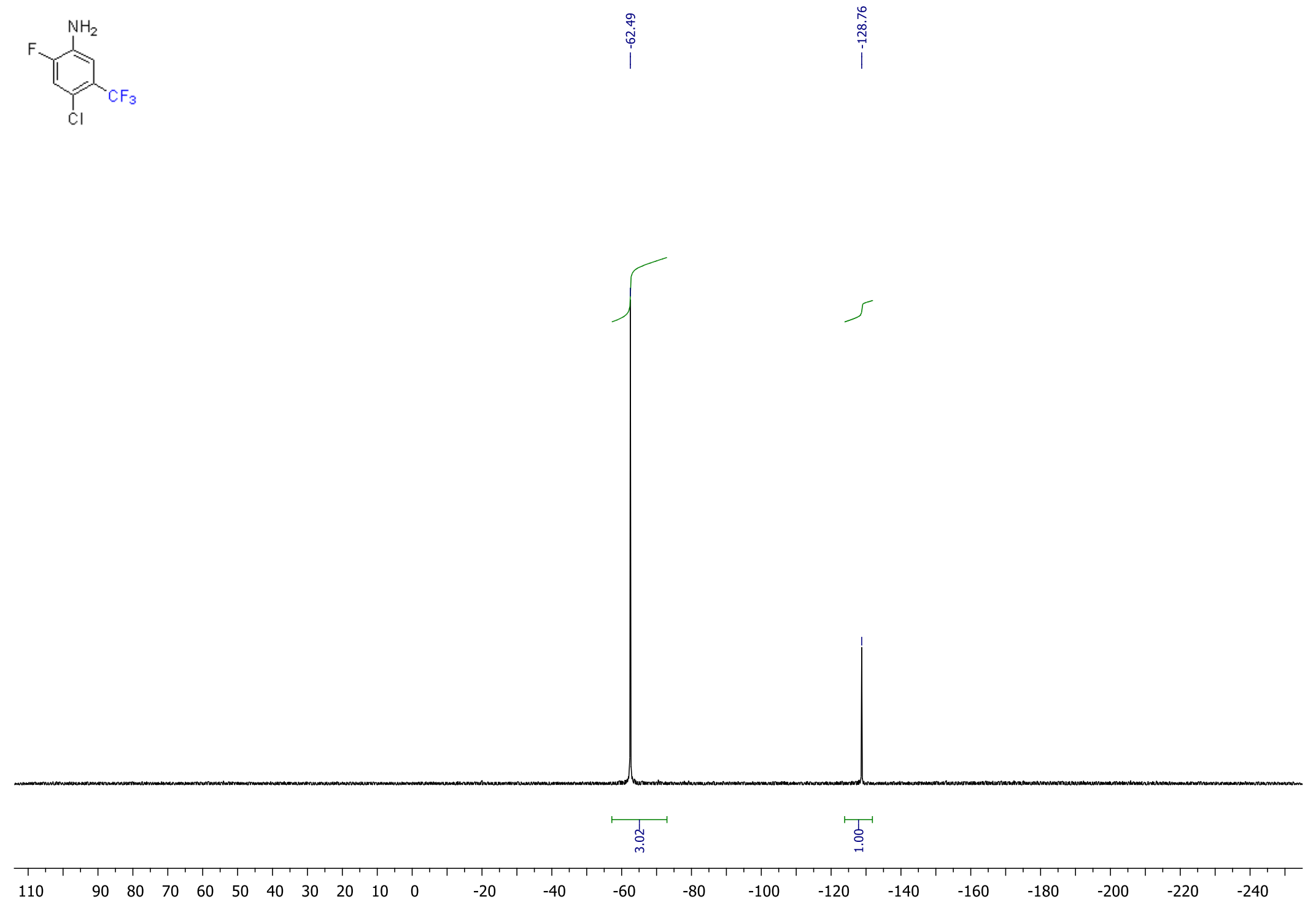
Compound 22b

${ }^{1}$ H NMR (400 MHz, DMSO-d6)

$\overbrace{\mathrm{CF}_{3}}^{{ }^{*} \mathrm{HCl}}$

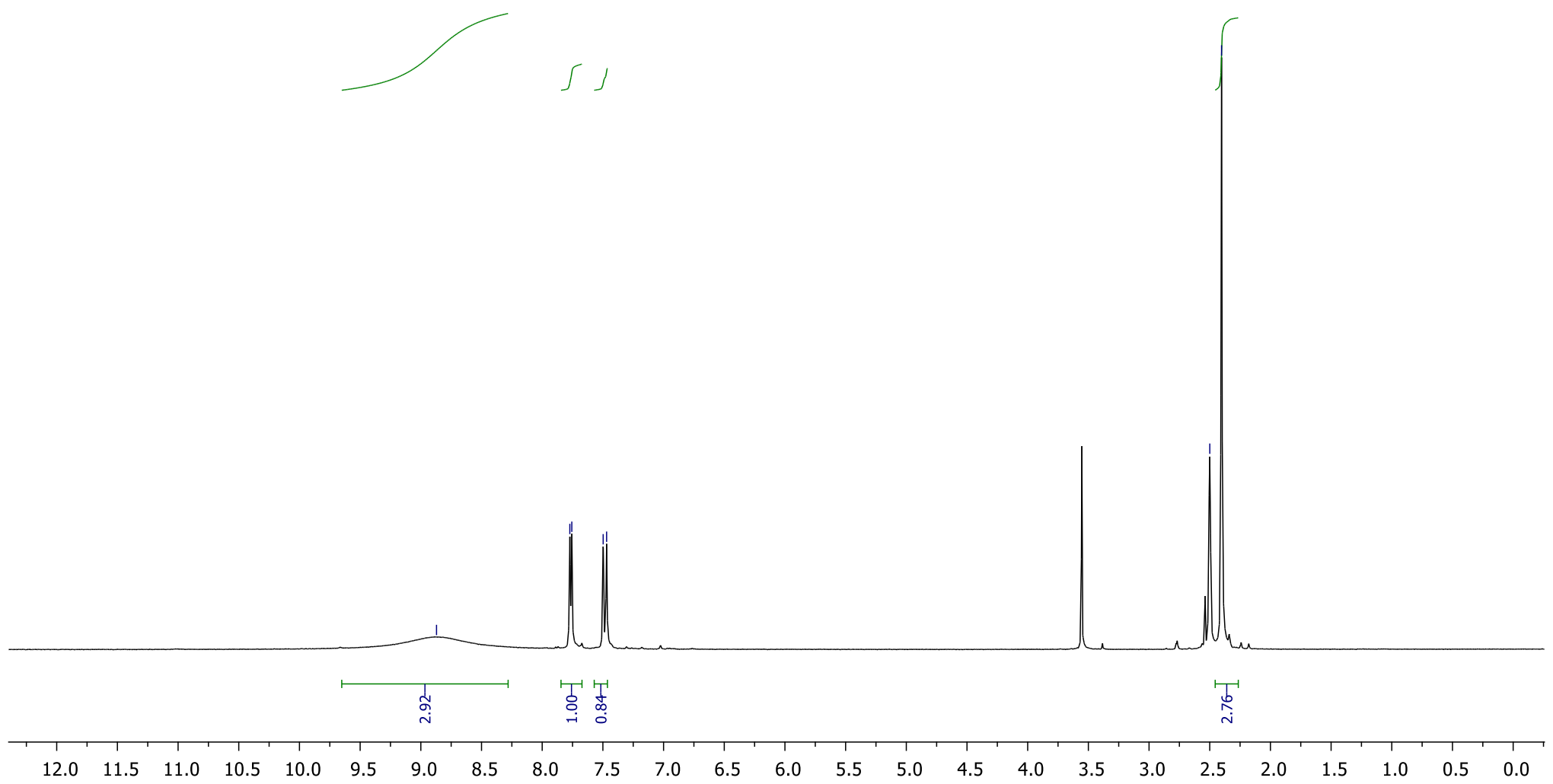


${ }^{13} \mathrm{C}\left\{{ }^{1} \mathrm{H}\right\}$ NMR (151 MHz, DMSO-d 6 )

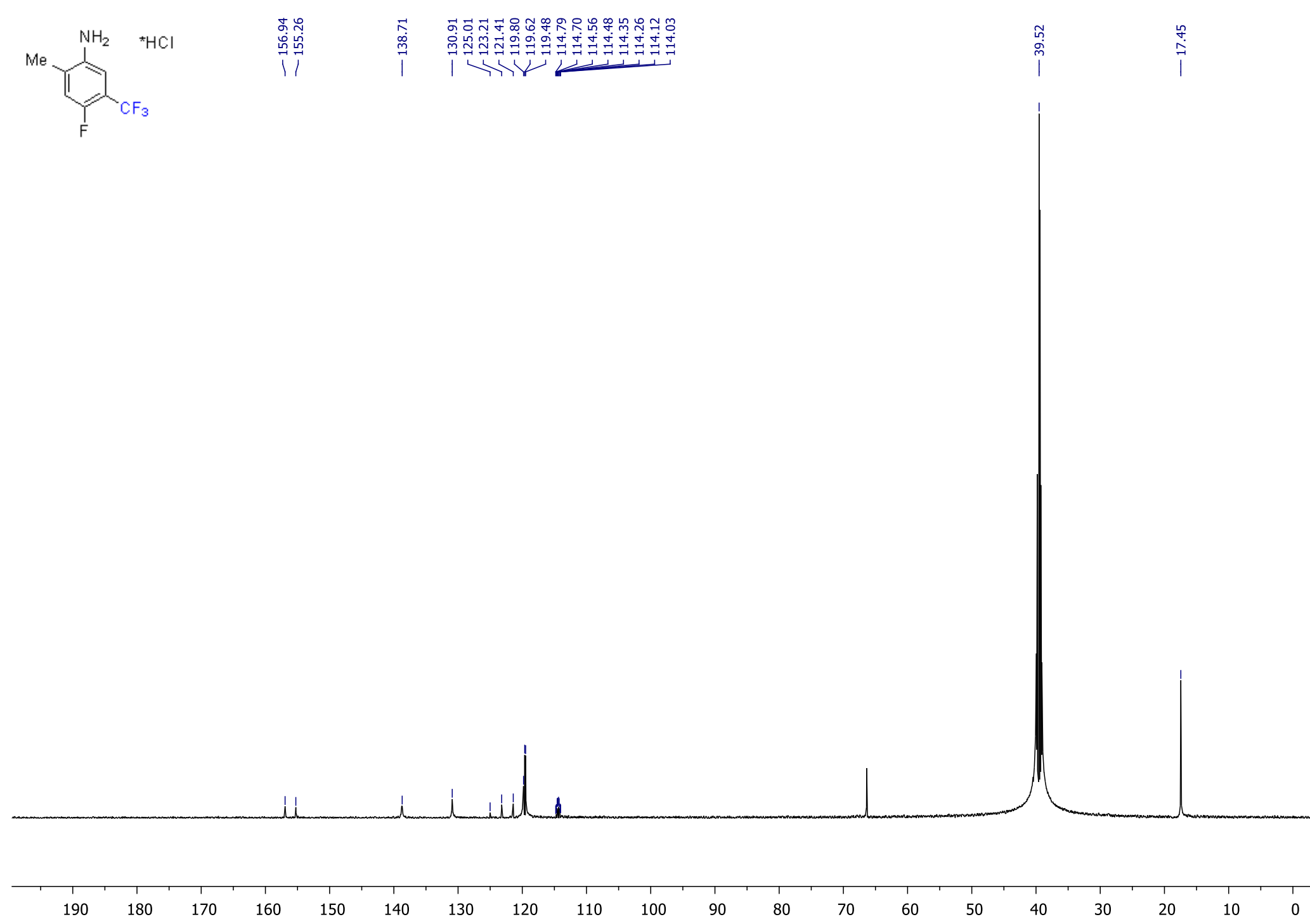


${ }^{19}$ F NMR (376 MHz, DMSO-d 6 )
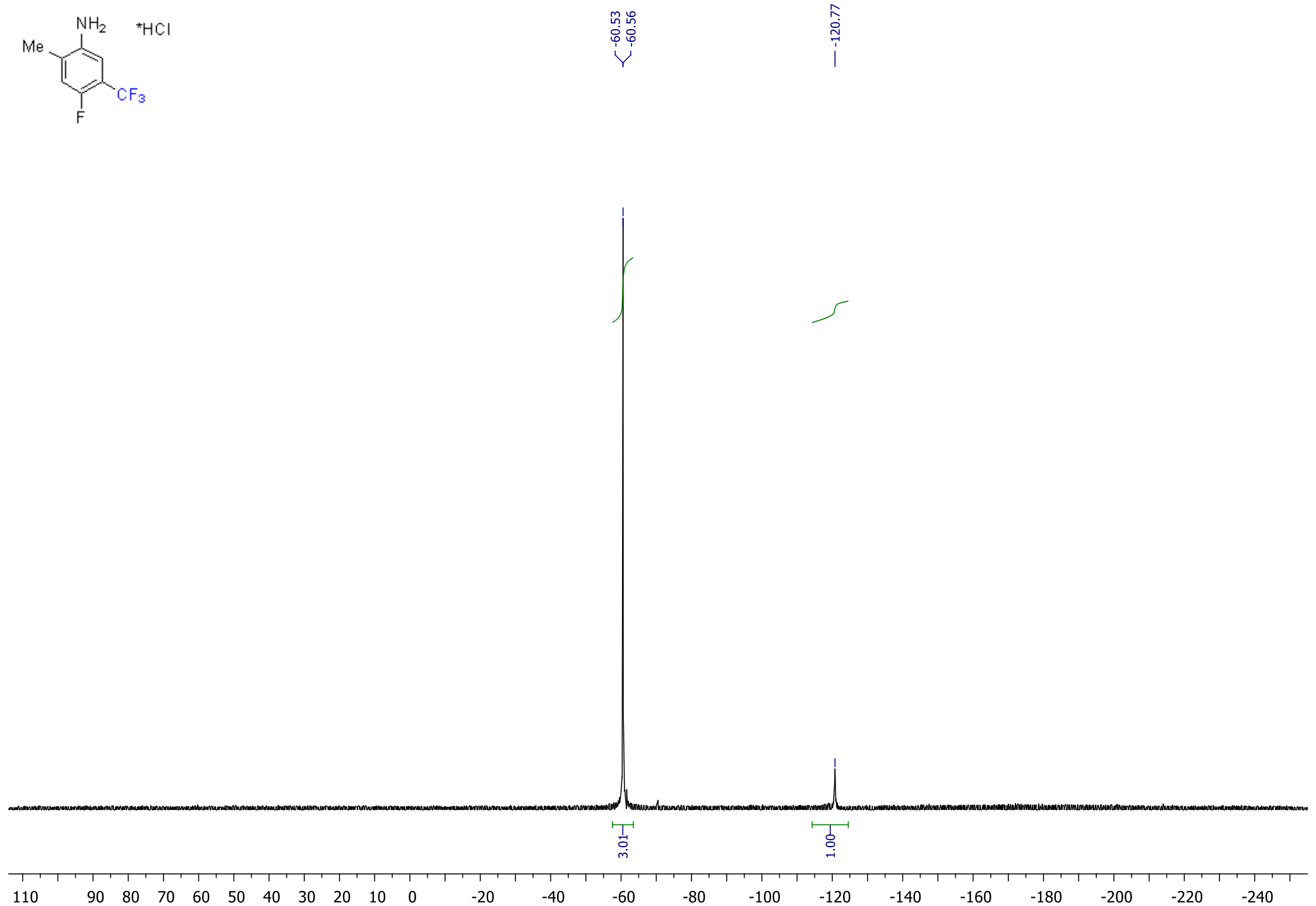

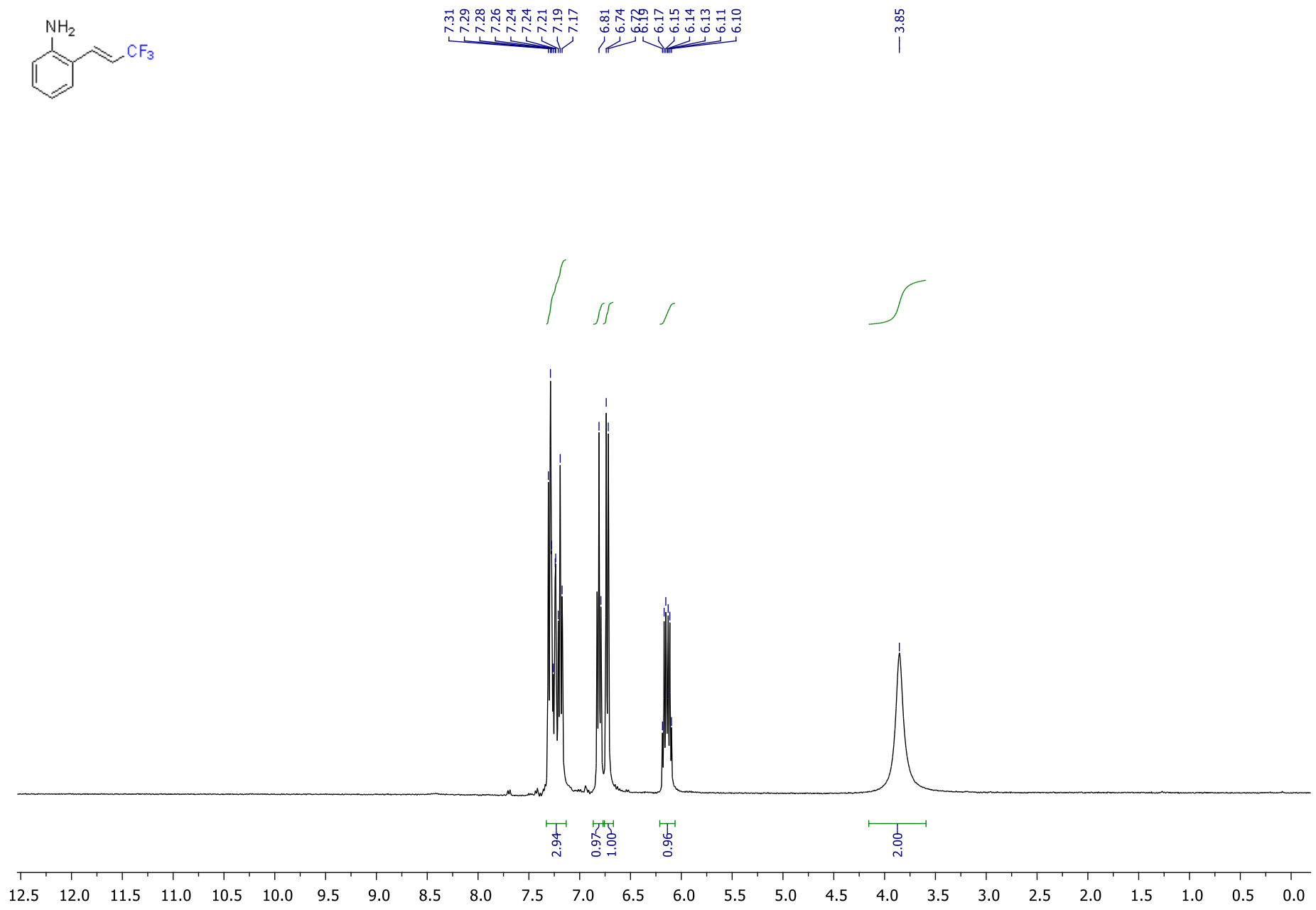

Compound 24b is also described in: a) Org. Lett. 2012, 14, 4, 1146-1149; b) J. Org. Chem. 2013, 78, 12, 6196-6201; c) J. Org. Chem. 2017, 82, 12, 61926201. 
${ }^{13} \mathrm{C}\left\{{ }^{1} \mathrm{H}\right\}$ NMR $\left(126 \mathrm{MHz}, \mathrm{CDCl}_{3}\right)$

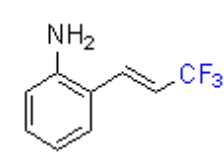

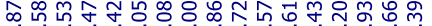
舟品品

$\stackrel{20}{1}$

11111

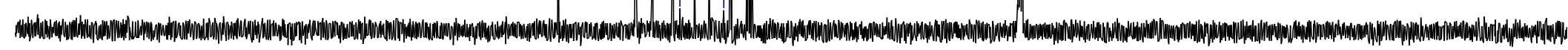

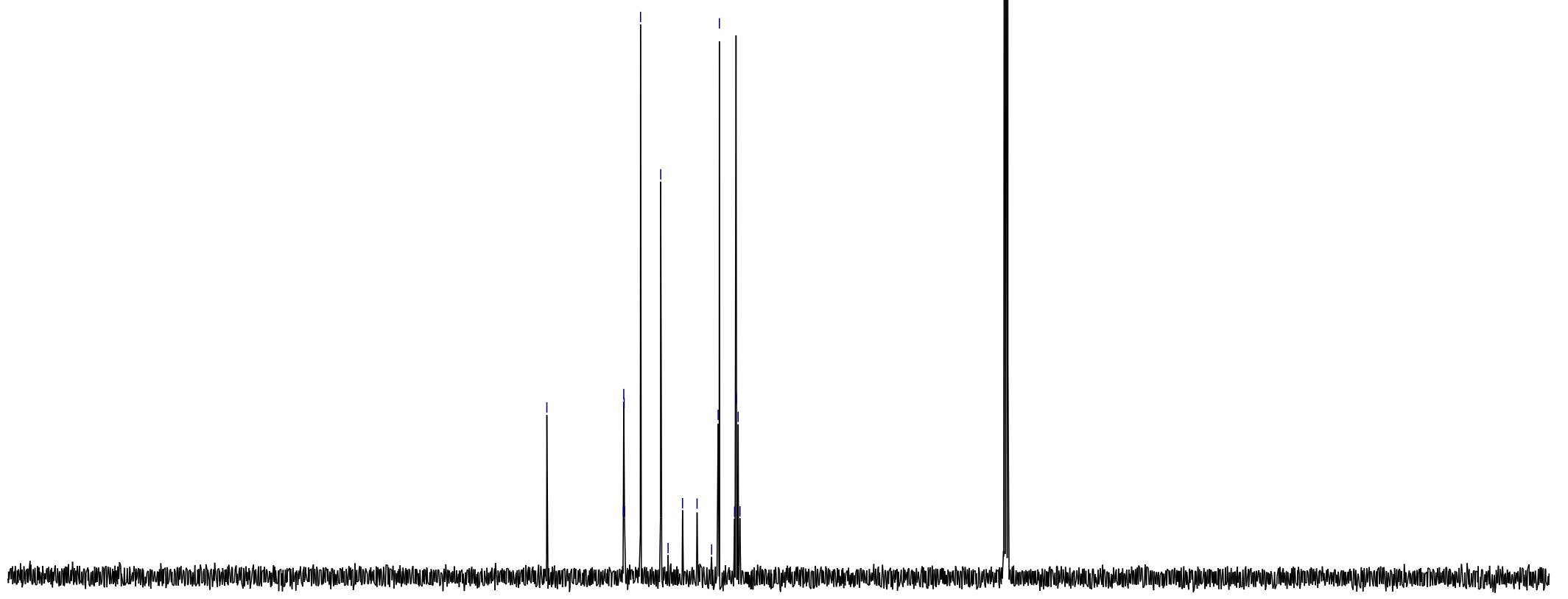

$\begin{array}{lllllllllllllllllllllll}220 & 210 & 200 & 190 & 180 & 170 & 160 & 150 & 140 & 130 & 120 & 110 & 100 & 90 & 80 & 70 & 60 & 50 & 40 & 30 & 20 & 10 & 0\end{array}$ 
${ }^{19} \mathrm{~F}$ NMR (376 MHz, $\mathrm{CDCl}_{3}$ )

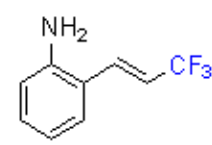

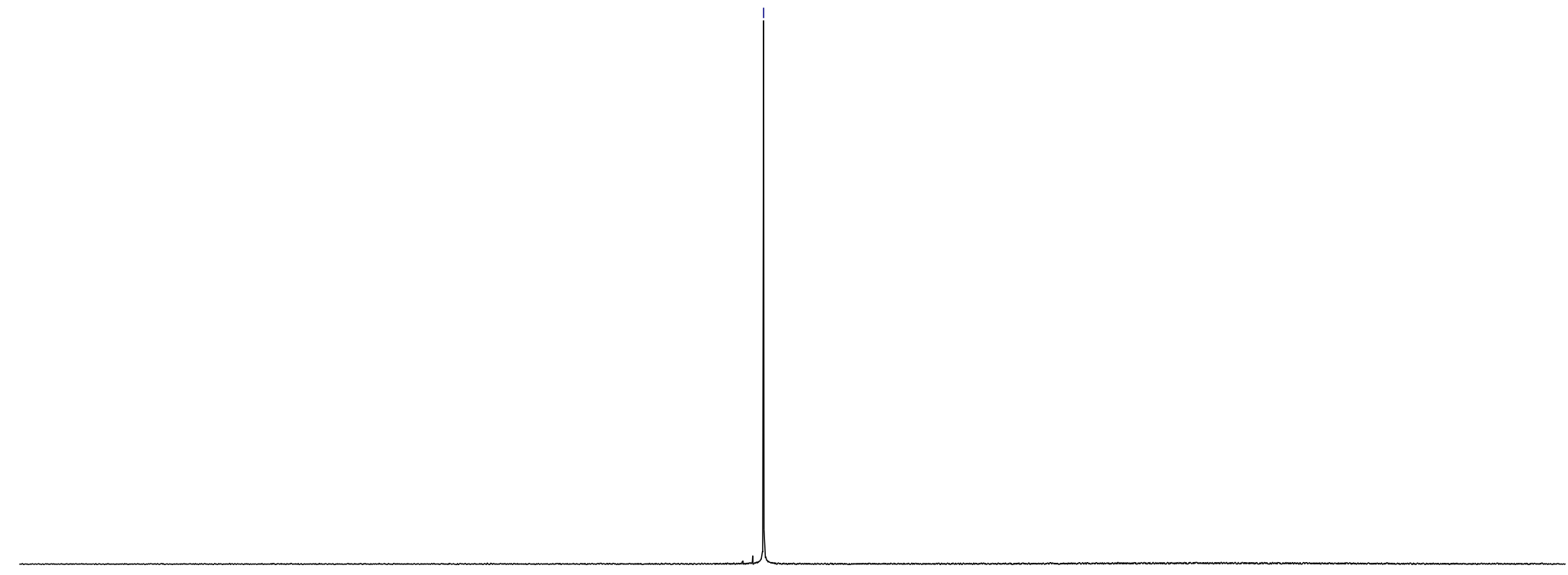



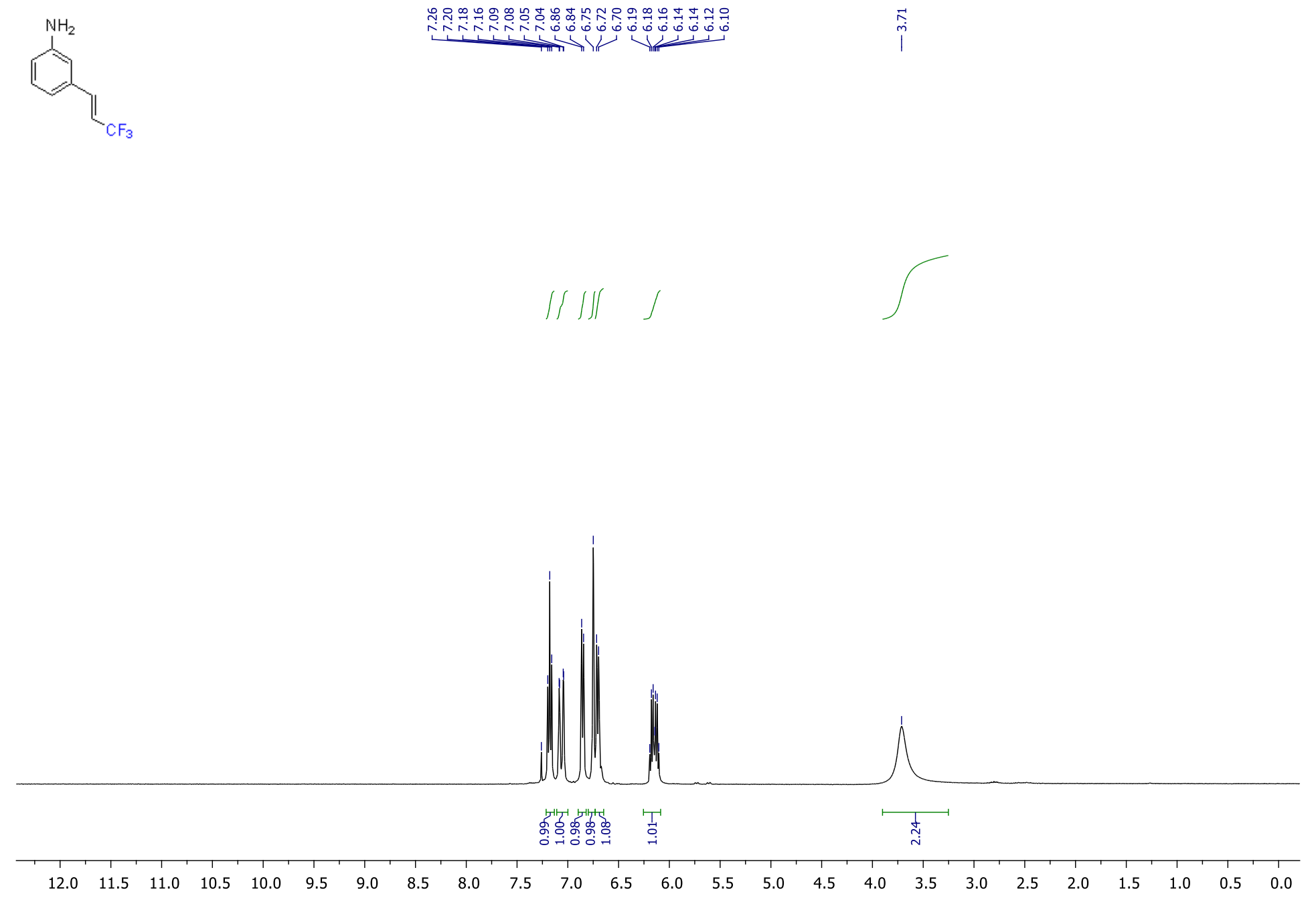

Compound 25b is also described in: J. Gen. Chem. USSR, 1961, 31, $3343-3349$. 
${ }^{13} \mathrm{C}\left\{{ }^{1} \mathrm{H}\right\}$ NMR $\left(126 \mathrm{MHz}, \mathrm{CDCl}_{3}\right)$

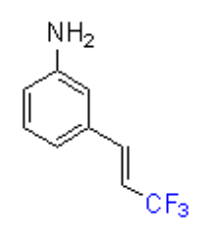

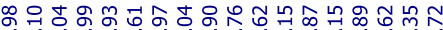

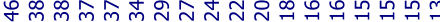

i
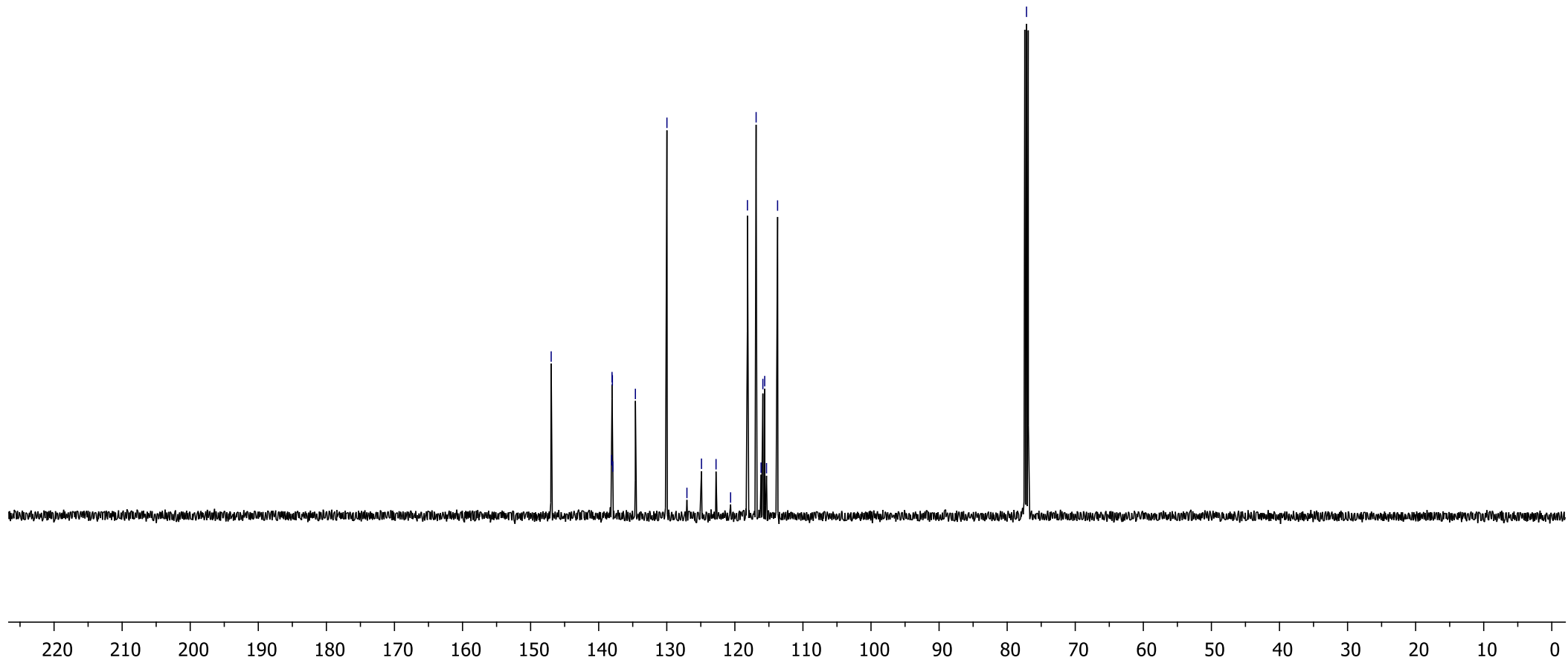
${ }^{19} \mathrm{~F}$ NMR (376 MHz, $\mathrm{CDCl}_{3}$ )

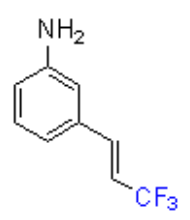

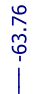

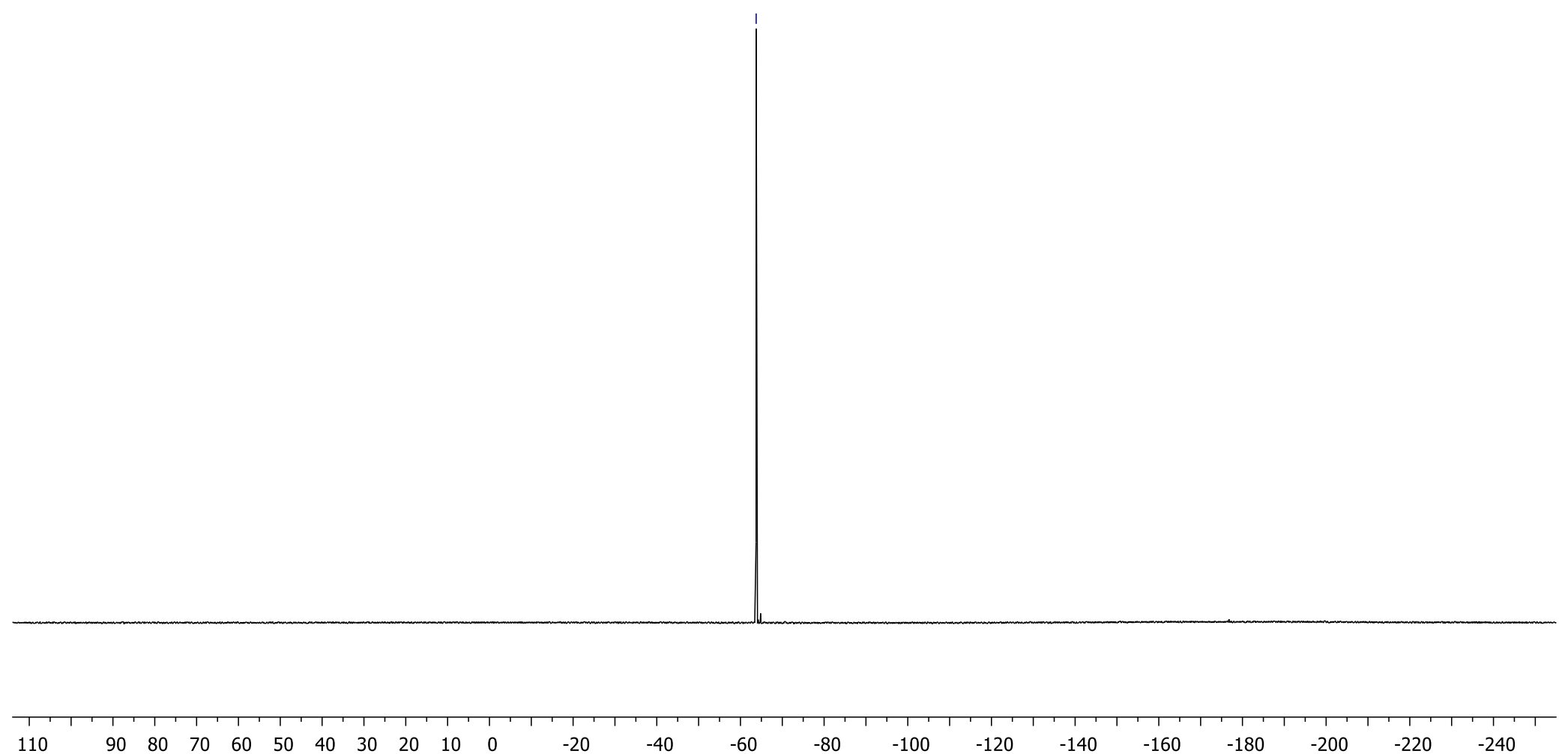


Compound 26b*
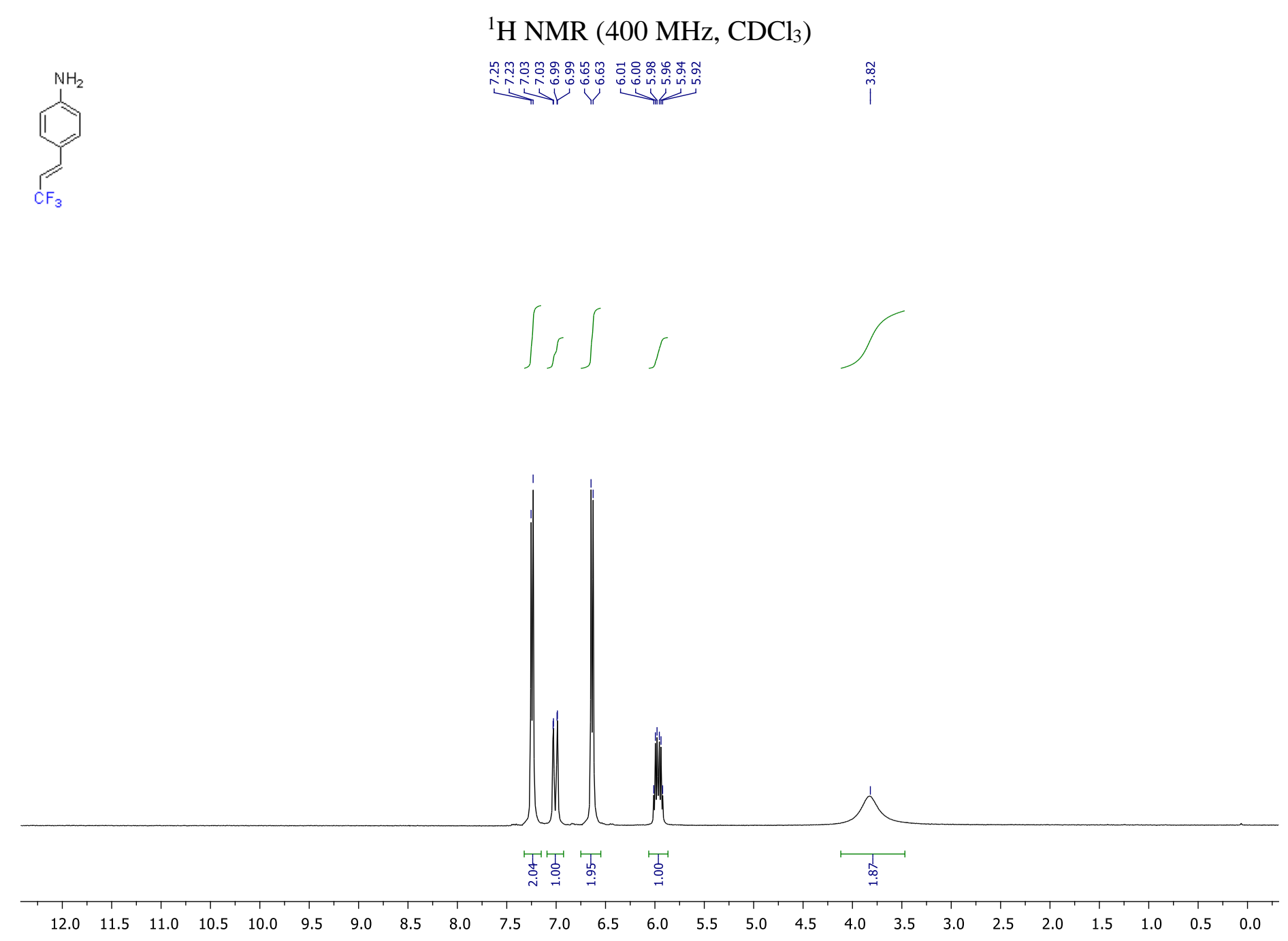

Compound 26b is also described in: a) Org. Lett. 2013, 15, 2, 406-409; b) J. Org. Chem. 2013, 78, 12, 6196-6201; c) J. Org. Chem. 2017, 82, 12, 61926201; c) J. Gen. Chem. USSR, 1961, 31, $3343-3349$. 
${ }^{13} \mathrm{C}\left\{{ }^{1} \mathrm{H}\right\}$ NMR $\left(151 \mathrm{MHz}, \mathrm{CDCl}_{3}\right)$

(1)

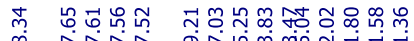

क्ष்̃

$\stackrel{1}{1}$

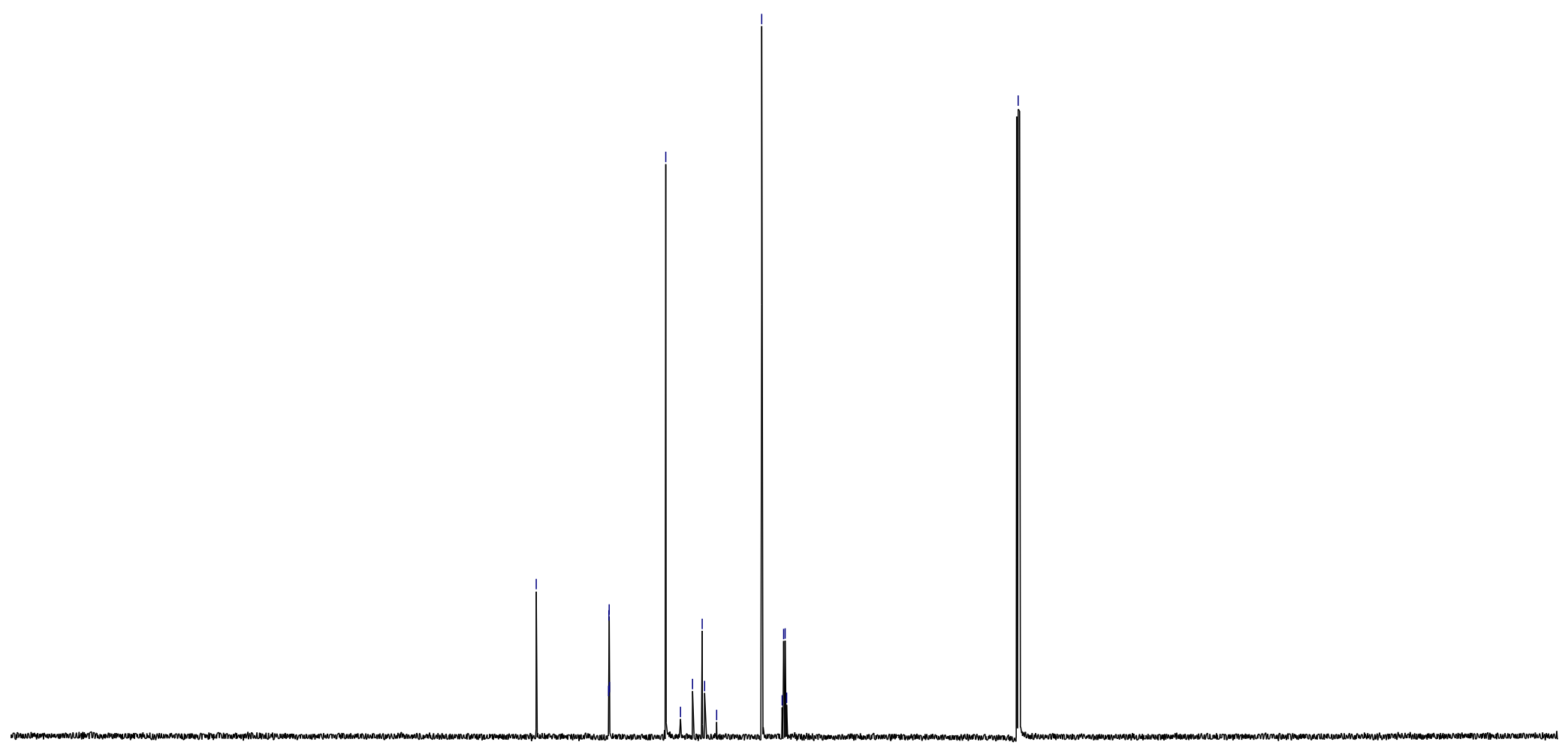


${ }^{19} \mathrm{~F}$ NMR (376 MHz, $\left.\mathrm{CDCl}_{3}\right)$

(N)

ডิ

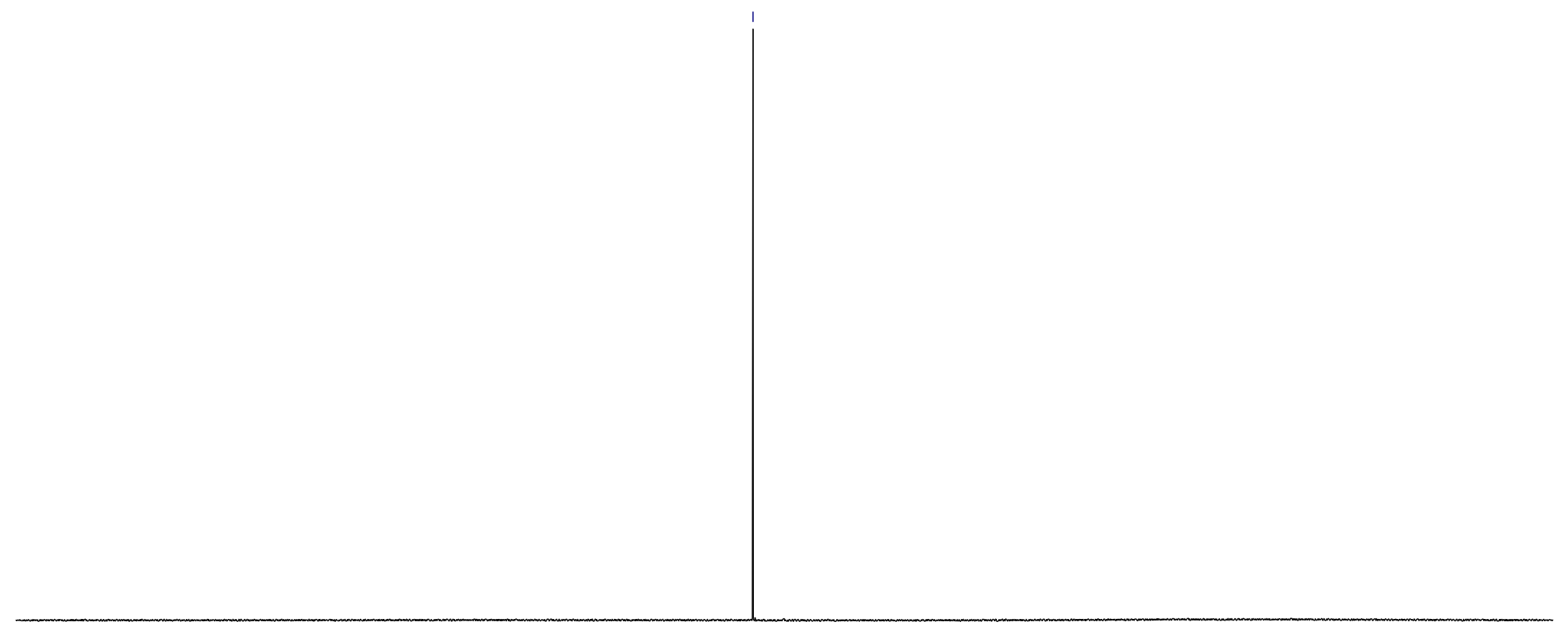

$\begin{array}{lllllllllll}110 & 90 & 80 & 70 & 60 & 50 & 40 & 30 & 20 & 10 & 0\end{array}$ 
Compound $61 b^{*}$

${ }^{1} \mathrm{H}$ NMR (400 MHz, DMSO-d 6 )

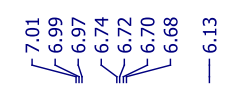

$\underbrace{m}$
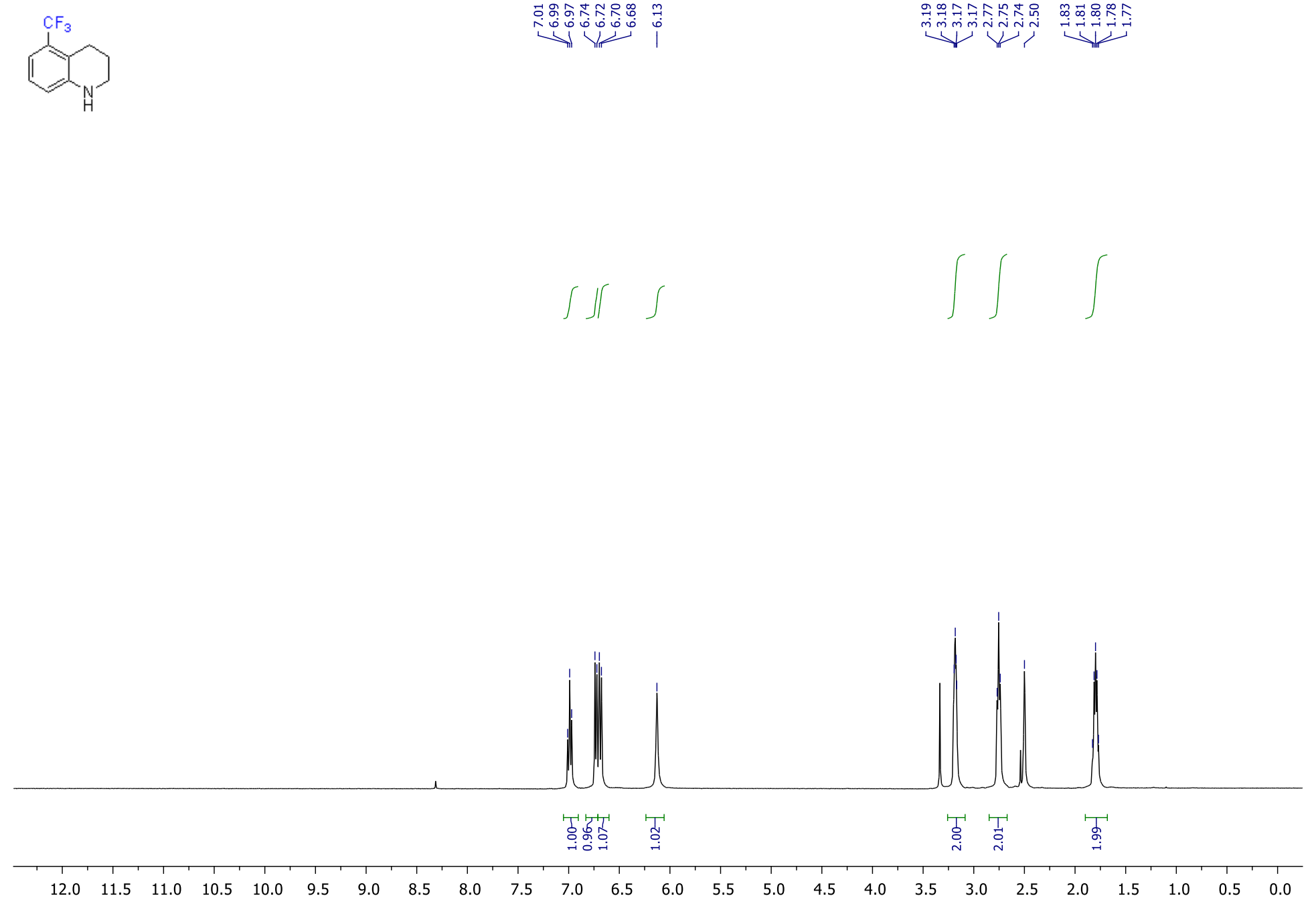

Compound 61b is also described in: Science 2016, 353, 6304, $1144-1147$. 
${ }^{13} \mathrm{C}\left\{{ }^{1} \mathrm{H}\right\}$ NMR (151 MHz, DMSO-d 6$)$

$\overbrace{\mathrm{H}}^{C F_{3}}$

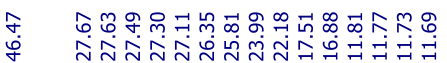

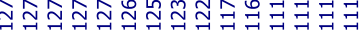

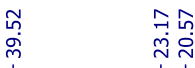

11

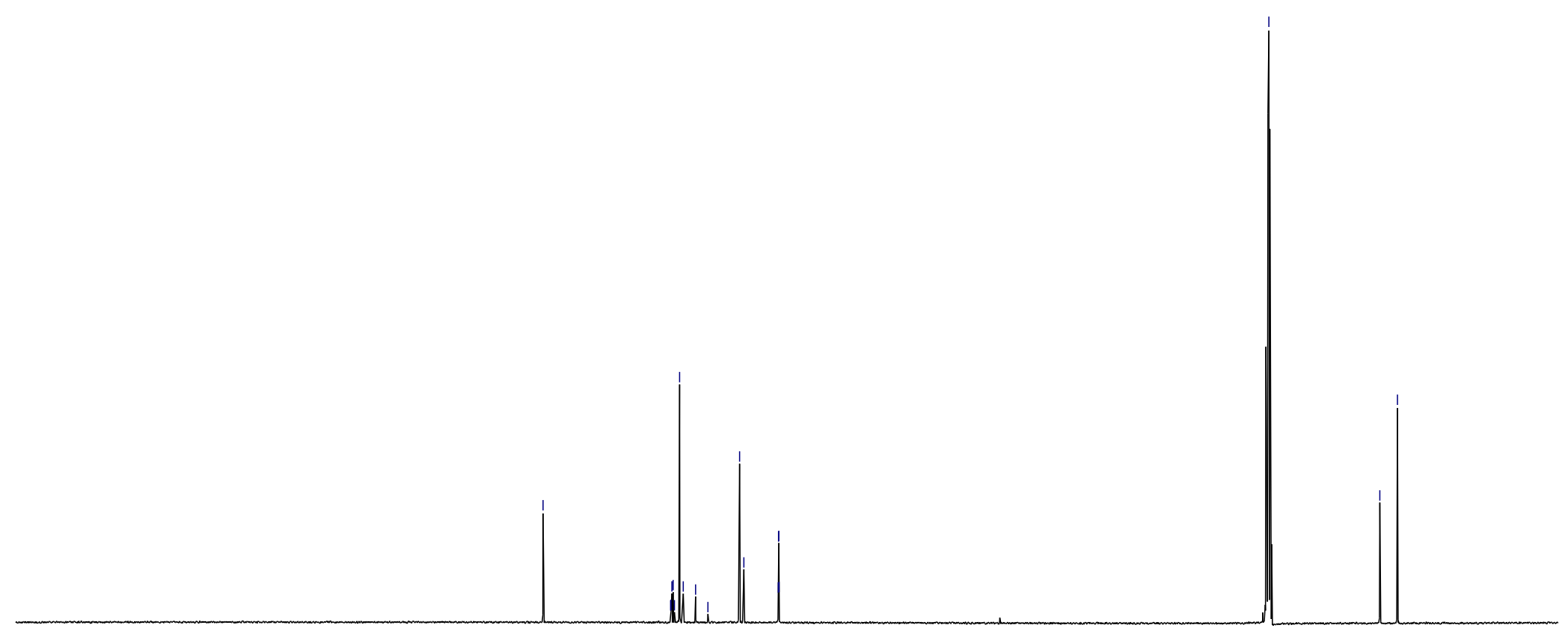

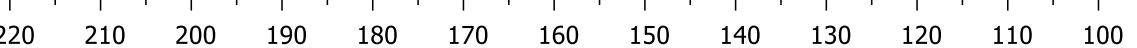

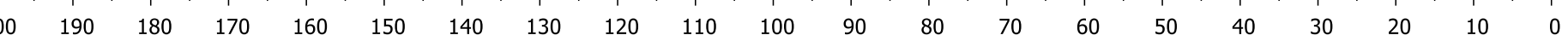




$$
+
$$


Compound $62 b^{*}$

${ }^{1} \mathrm{H}$ NMR $\left(400 \mathrm{MHz}, \mathrm{CDCl}_{3}\right)$
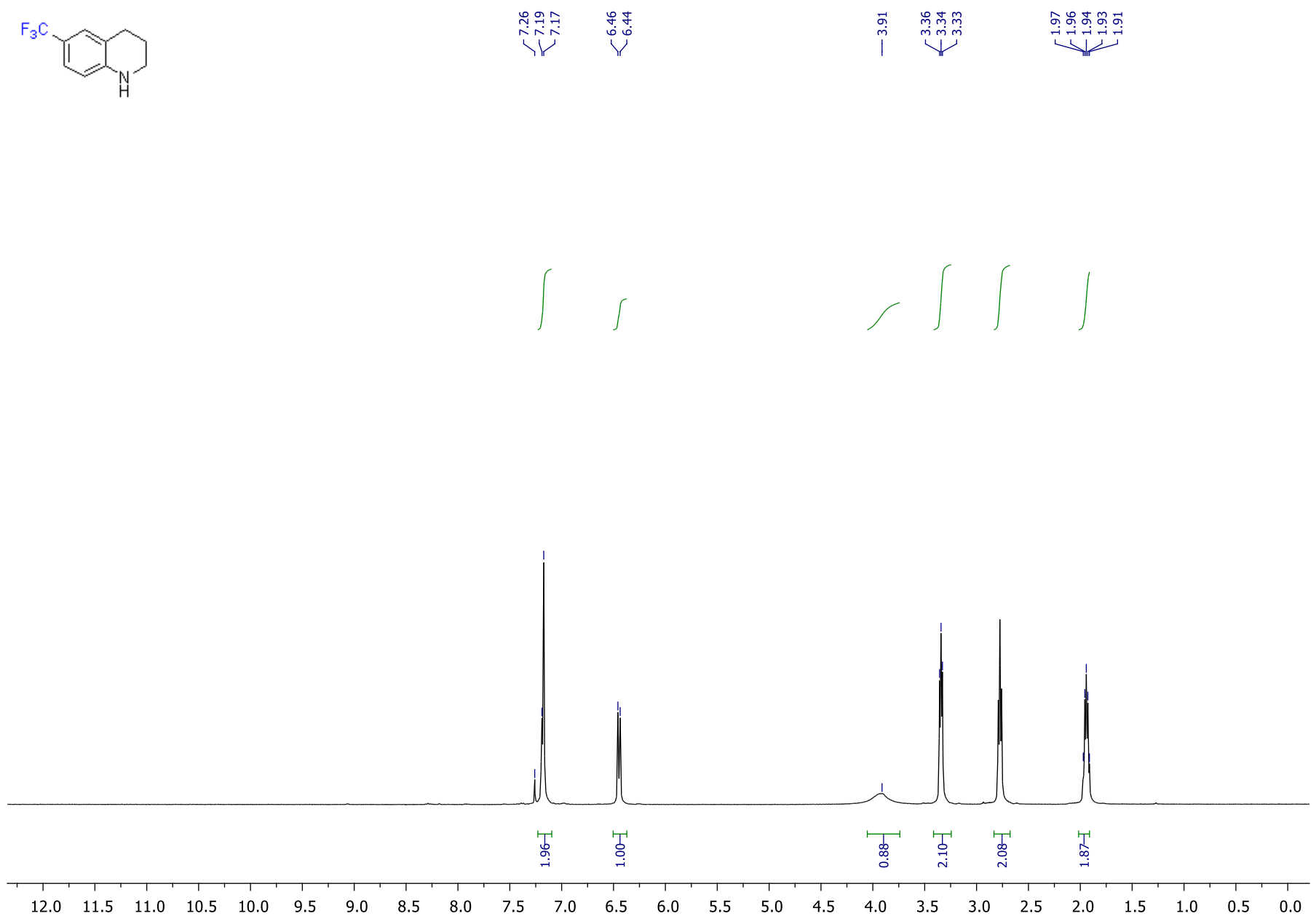

Compound 62b is also described in: a) Bioorg. Med. Chem. 2008, 16, 17, 7956-7967; b) Chem. Commun. 2012, 48, 5877-5879; c) J. Chem. Soc., Perkin Trans. 1, 1980, 1933-1939; d) J. Org. Chem. 2013, 78, 9, 4463-4472. 
${ }^{13} \mathrm{C}\left\{{ }^{1} \mathrm{H}\right\}$ NMR $\left(126 \mathrm{MHz}, \mathrm{CDCl}_{3}\right)$

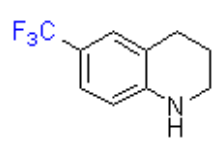

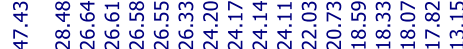

政 궈

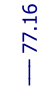

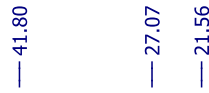

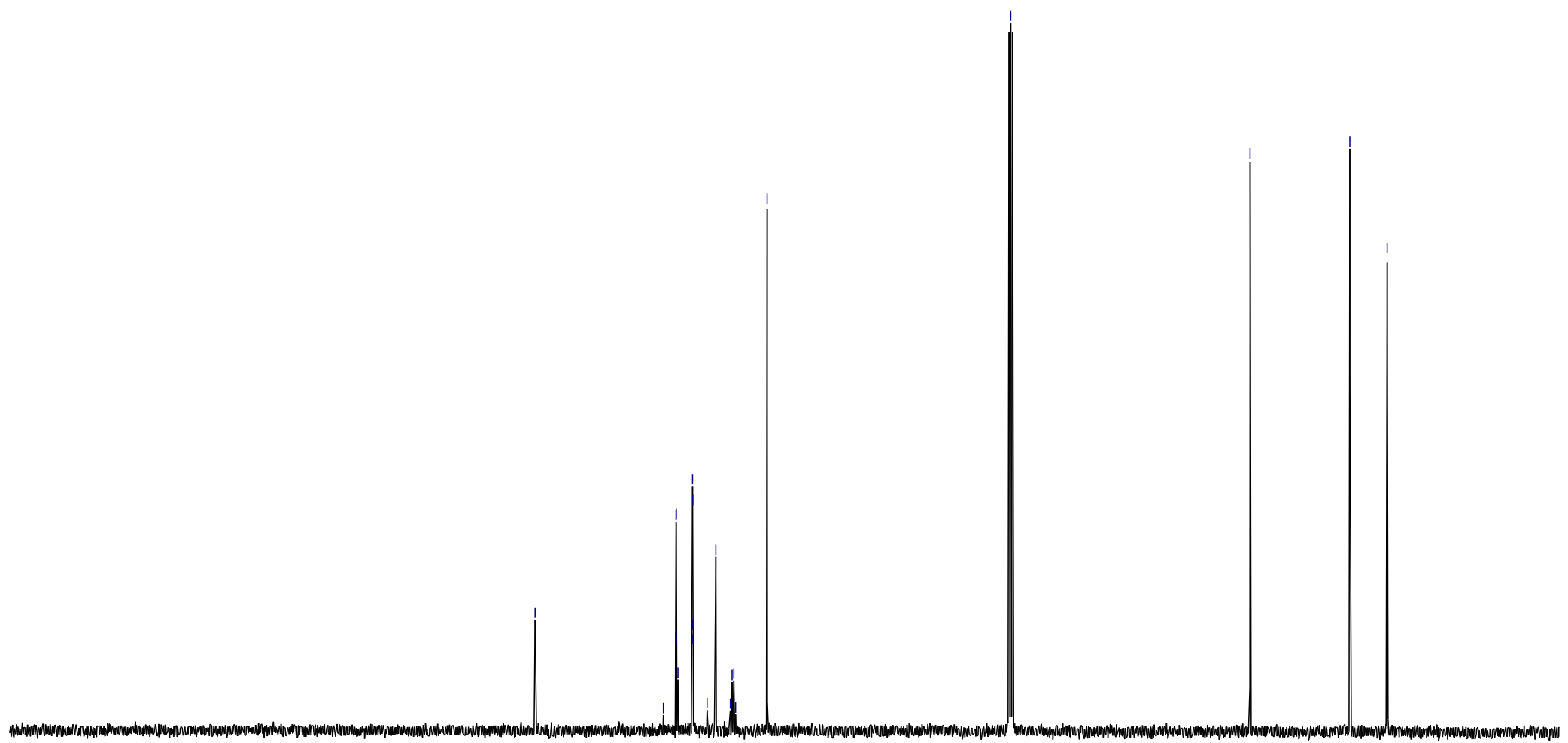

80

70

60

50

40

30

20

$10 \quad 0$ 
${ }^{19} \mathrm{~F}$ NMR (376 MHz, $\mathrm{CDCl}_{3}$ )

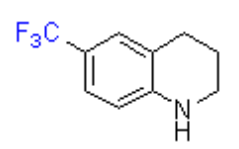

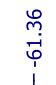
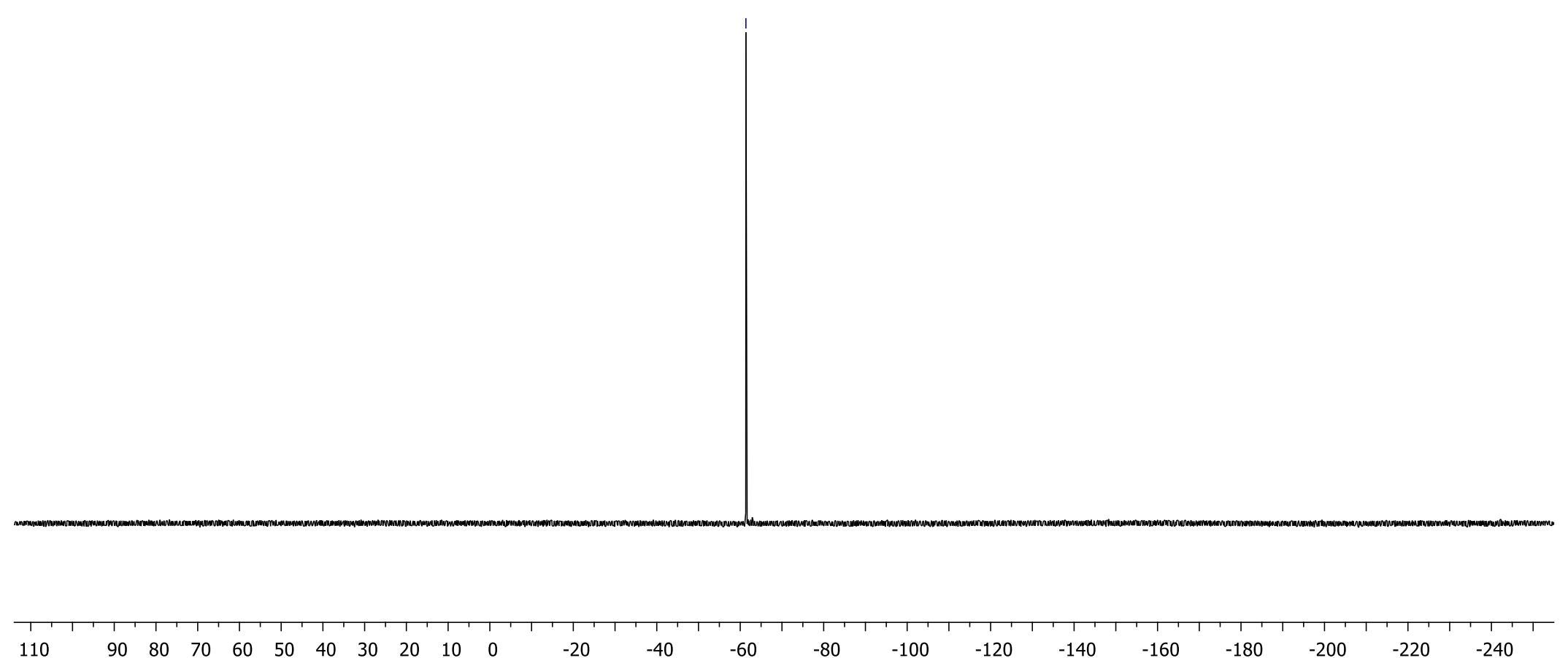


\section{Compound 63b}

${ }^{1} \mathrm{H} \mathrm{NMR}\left(400 \mathrm{MHz}, \mathrm{CDCl}_{3}\right.$

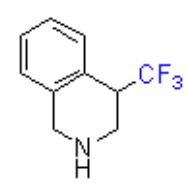

نำ

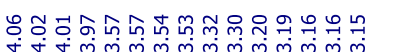

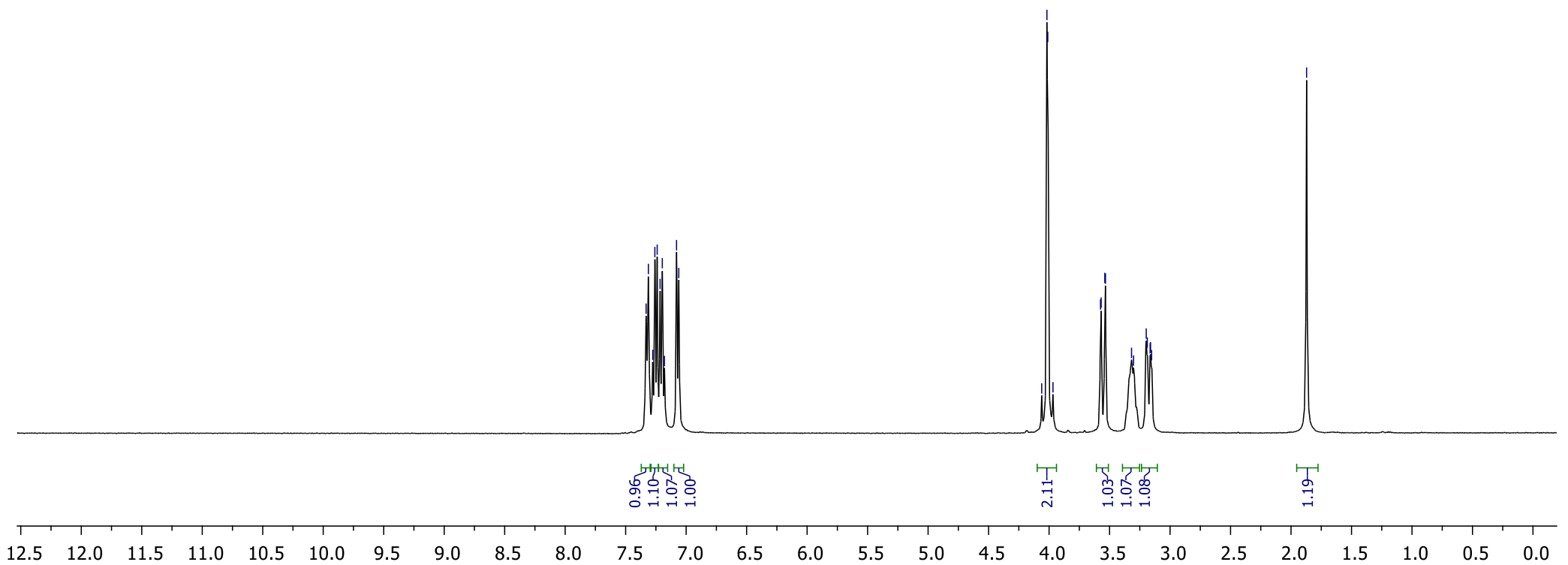


${ }^{13} \mathrm{C}\left\{{ }^{1} \mathrm{H}\right\}$ NMR $\left(126 \mathrm{MHz}, \mathrm{CDCl}_{3}\right)$

$\overbrace{\mathrm{H}} \mathrm{CF}^{3}$

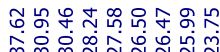

正

I Y Y

要

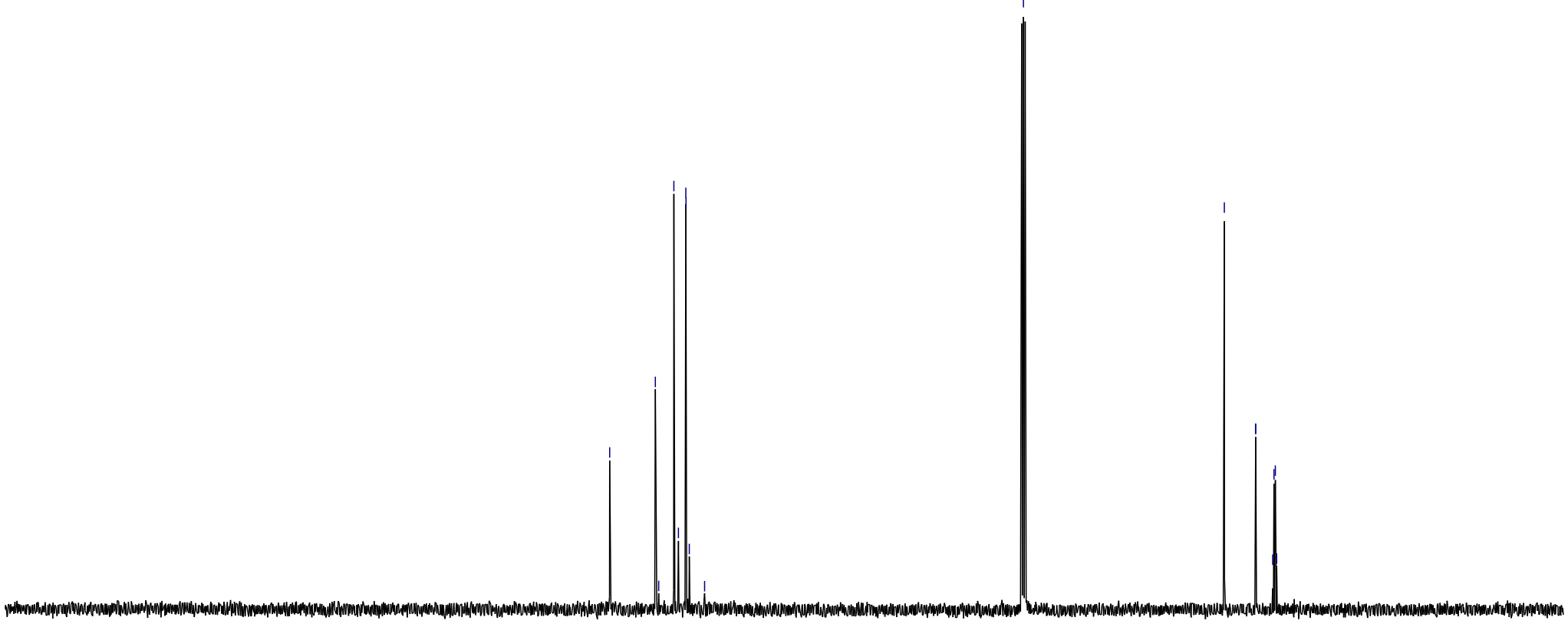

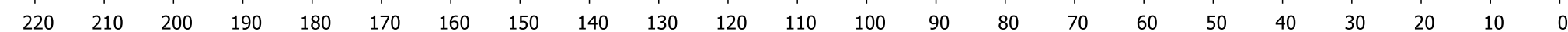


${ }^{19} \mathrm{~F}$ NMR (376 MHz, $\mathrm{CDCl}_{3}$ )

$\overbrace{n} C^{C_{3}}$

i̊ํ.

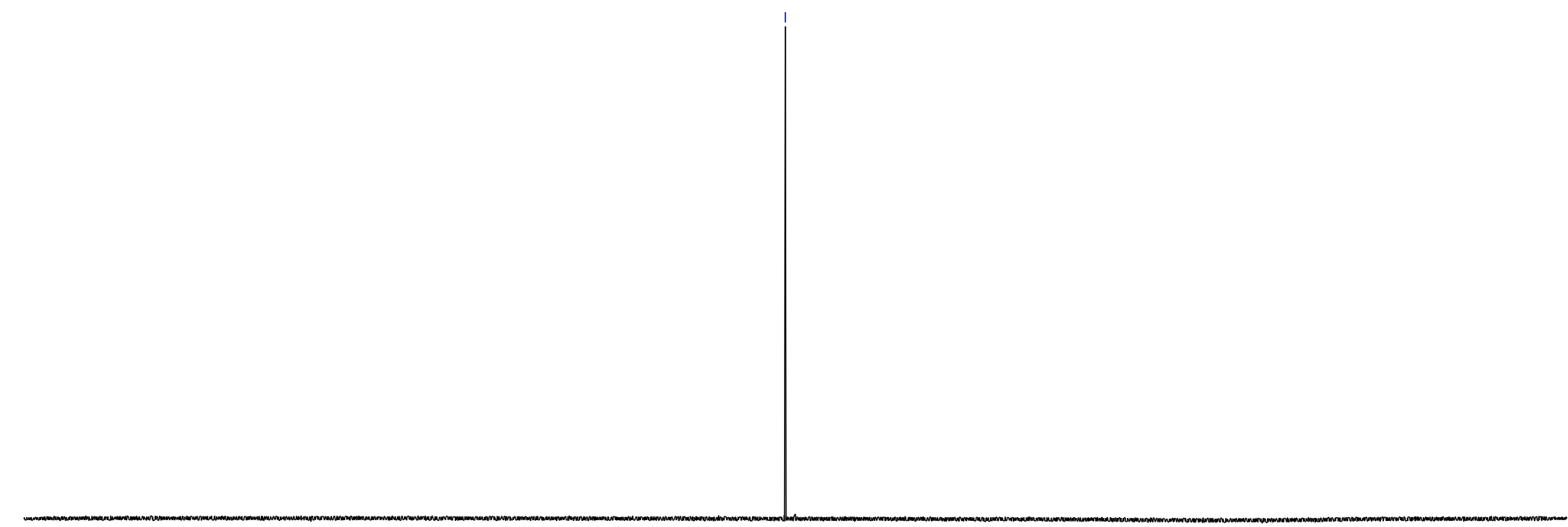

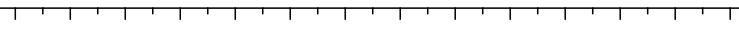

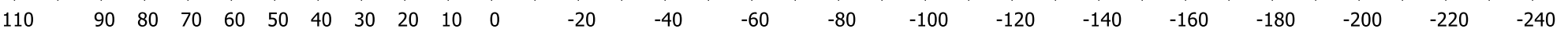




\section{Compound 64b}

${ }^{1} \mathrm{H}$ NMR (400 MHz, $\mathrm{CDCl}_{3}$ )

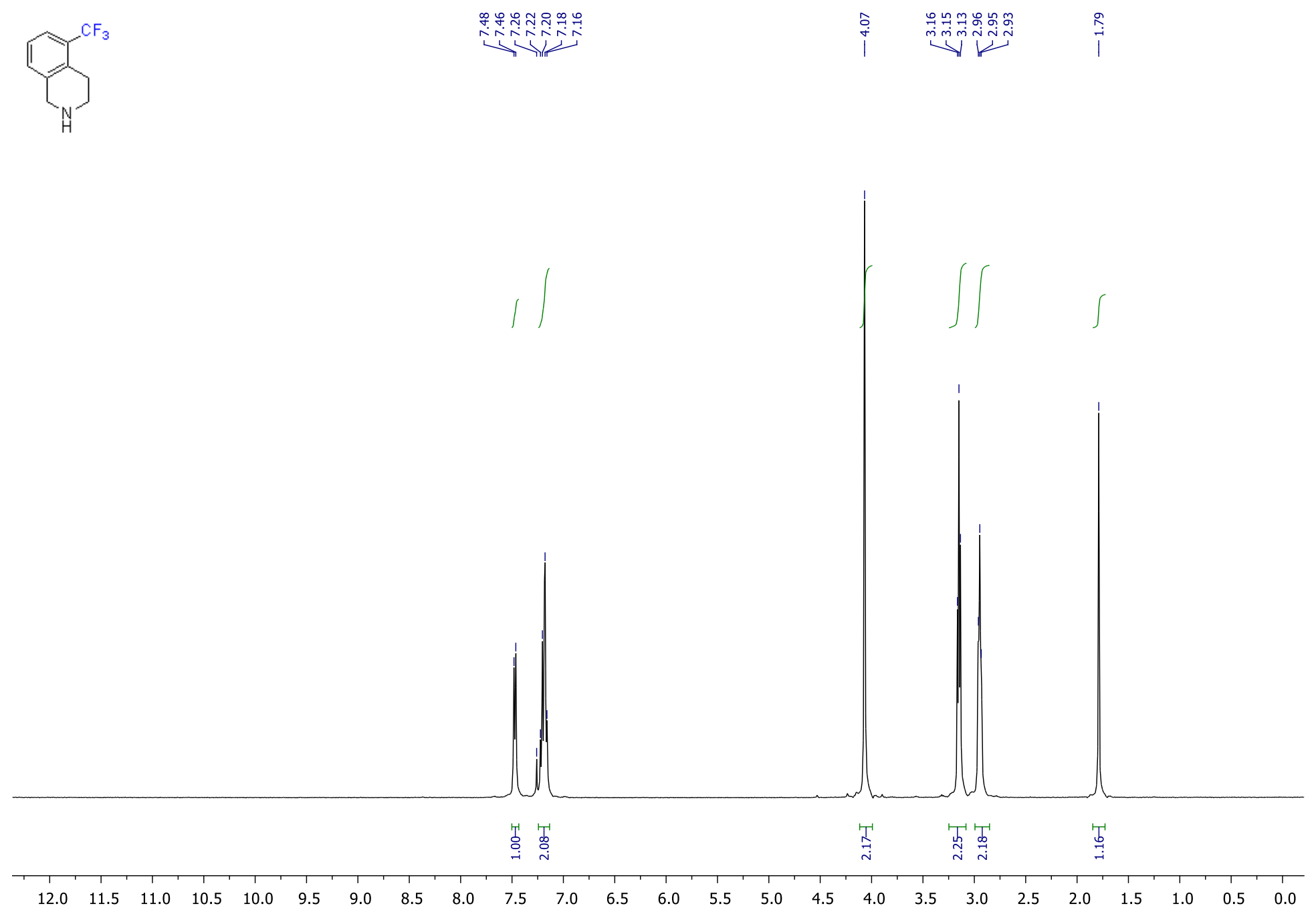


${ }^{13} \mathrm{C}\left\{{ }^{1} \mathrm{H}\right\}$ NMR $\left(126 \mathrm{MHz}, \mathrm{CDCl}_{3}\right)$
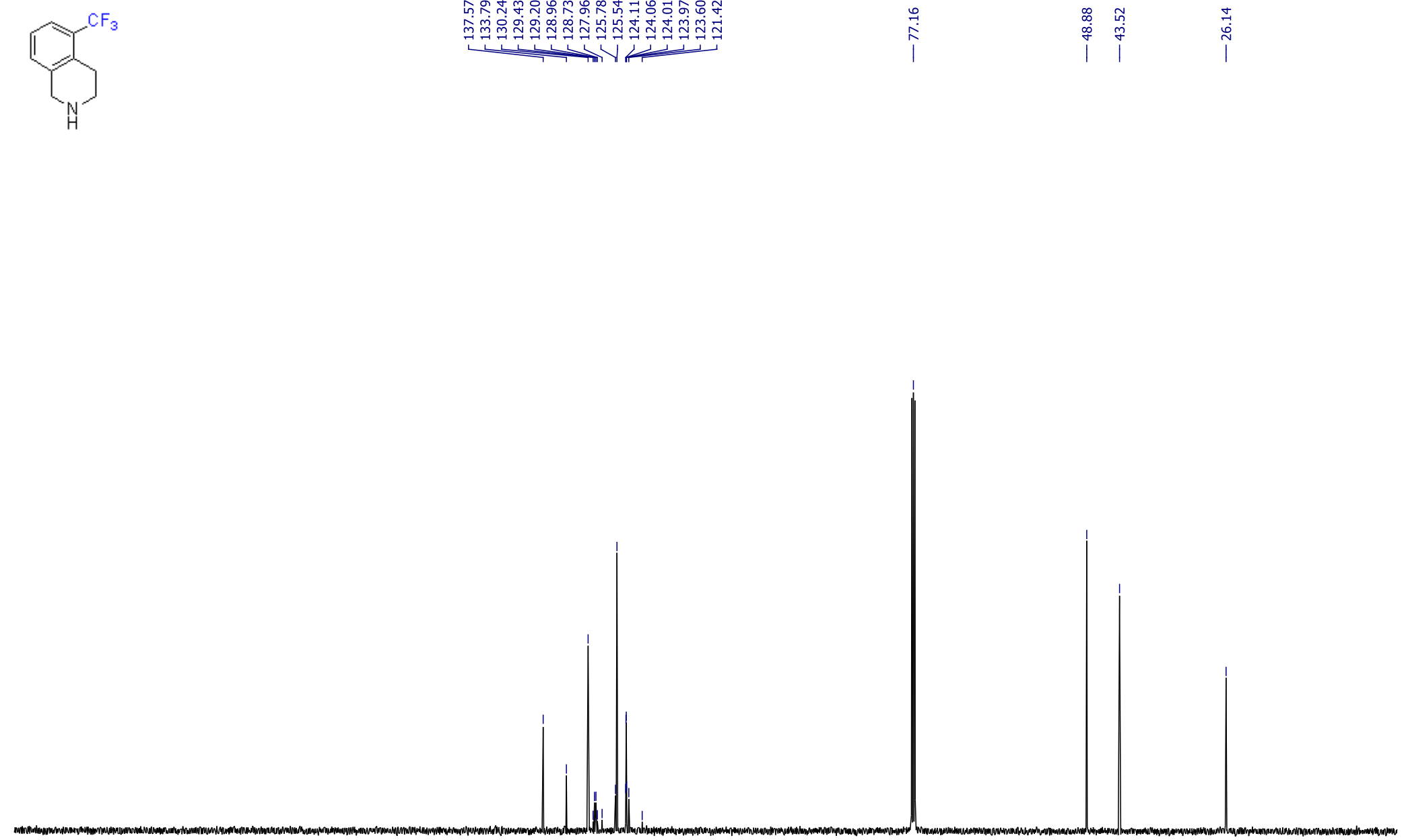

$\begin{array}{lllllllll}220 & 210 & 200 & 190 & 180 & 170 & 160 & 150 & 140\end{array}$

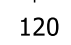

110100

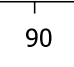


${ }^{19} \mathrm{~F}$ NMR (376 MHz, $\left.\mathrm{CDCl}_{3}\right)$
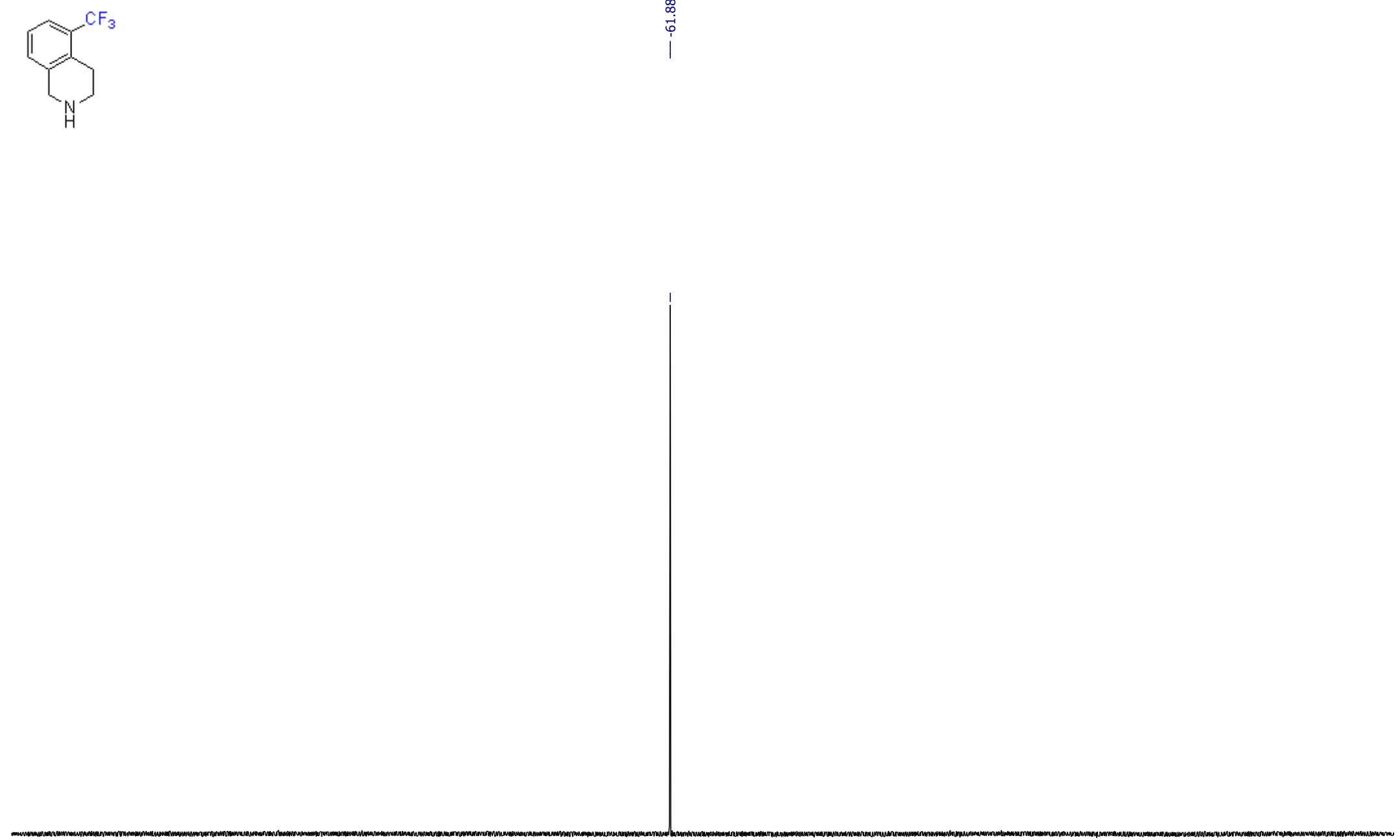

$\begin{array}{lllllllllll}110 & 90 & 80 & 70 & 60 & 50 & 40 & 30 & 20 & 10 & 0\end{array}$
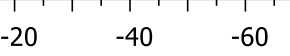


\section{Compound 65b}

${ }^{1} \mathrm{H}$ NMR (400 MHz, $\mathrm{CDCl}_{3}$ )
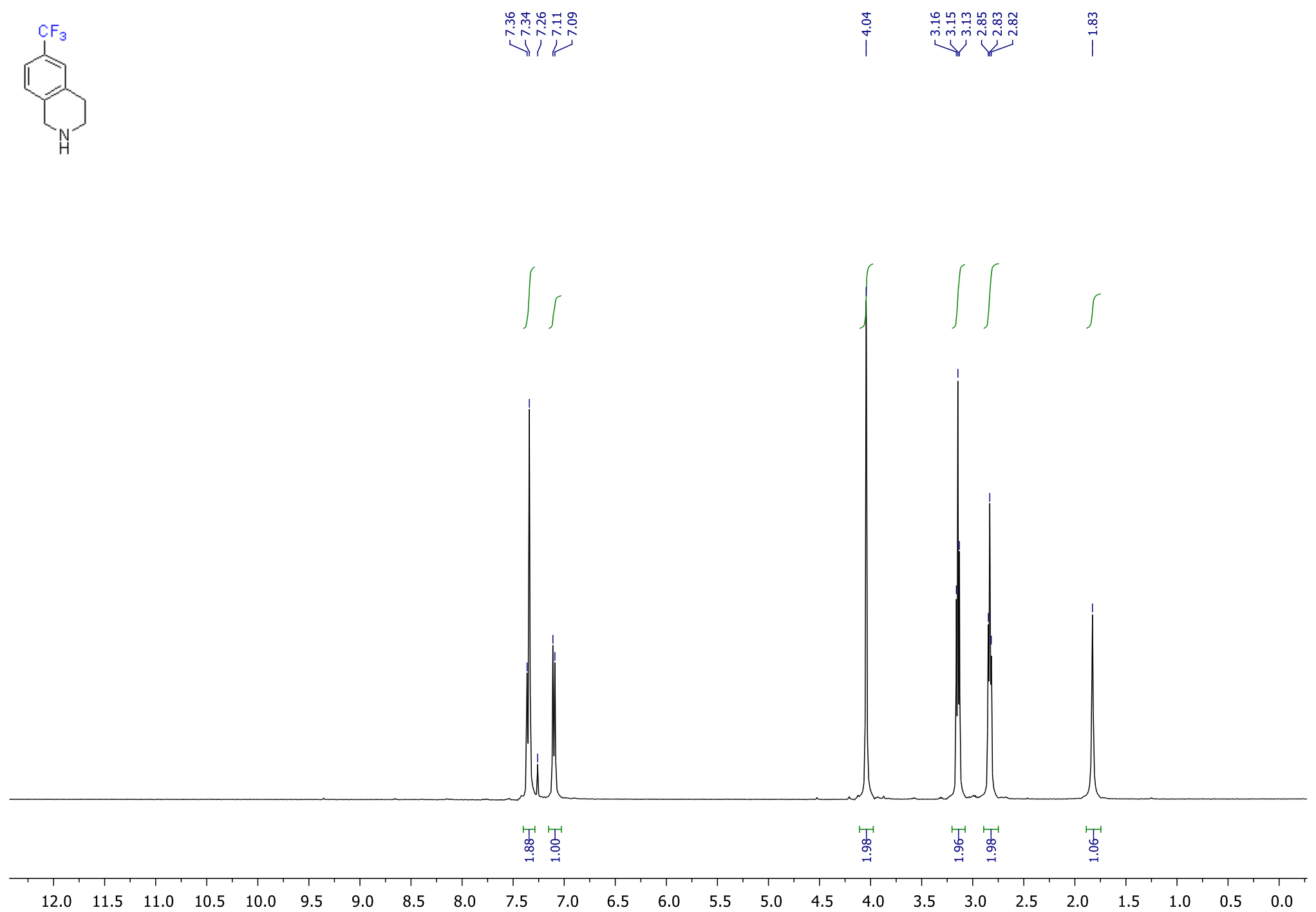
${ }^{13} \mathrm{C}\left\{{ }^{1} \mathrm{H}\right\}$ NMR $\left(126 \mathrm{MHz}, \mathrm{CDCl}_{3}\right)$

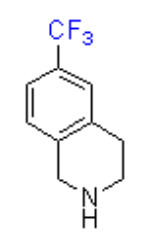

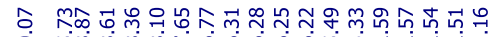

ชั

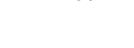

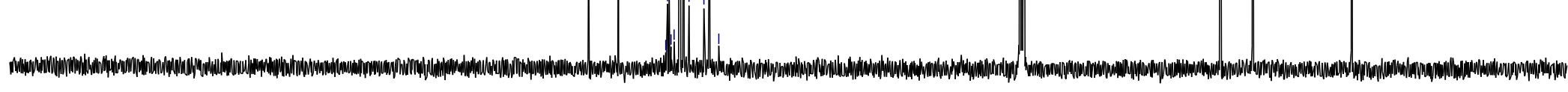

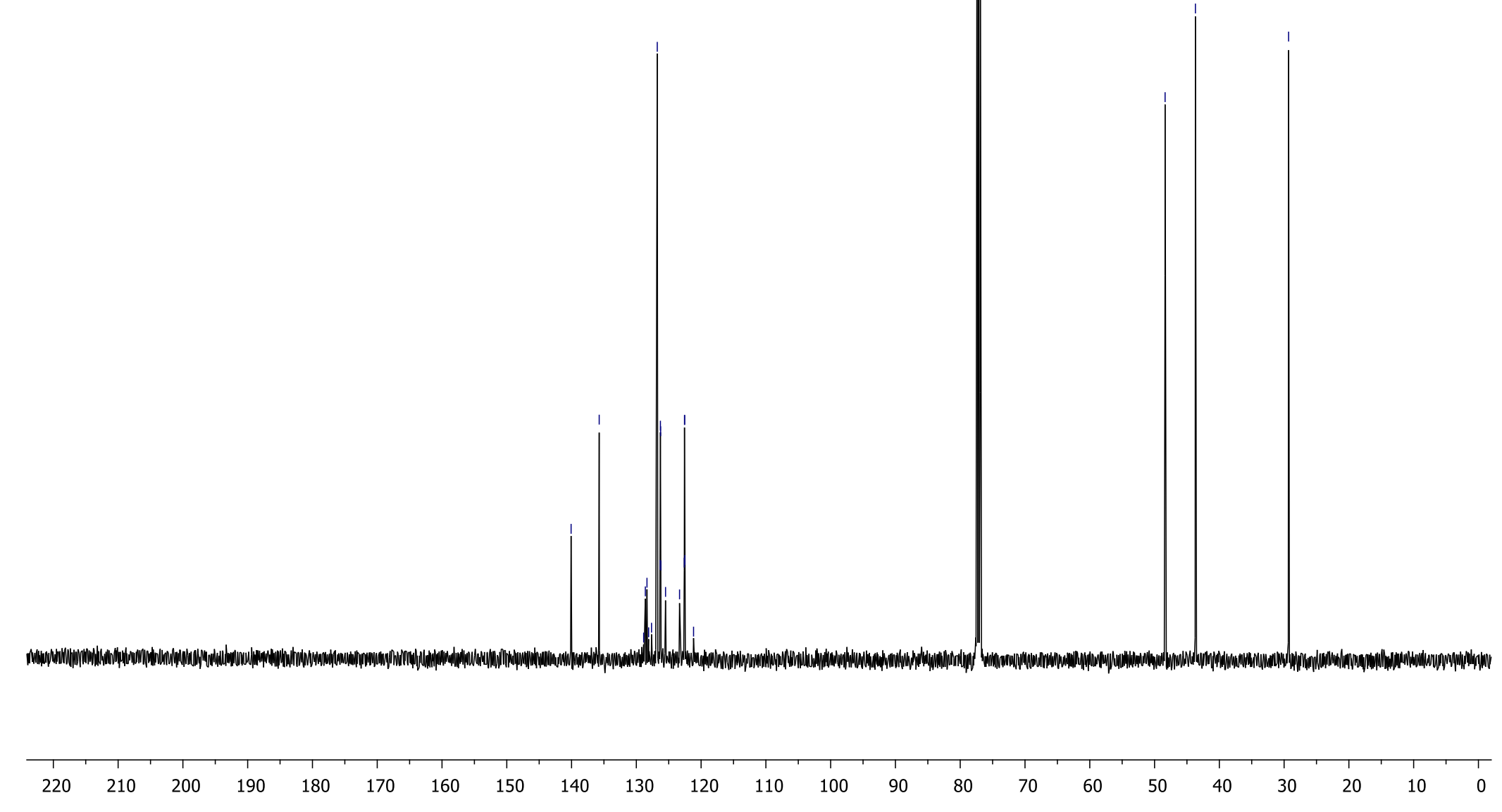


${ }^{19} \mathrm{~F}$ NMR (376 MHz, $\mathrm{CDCl}_{3}$ )
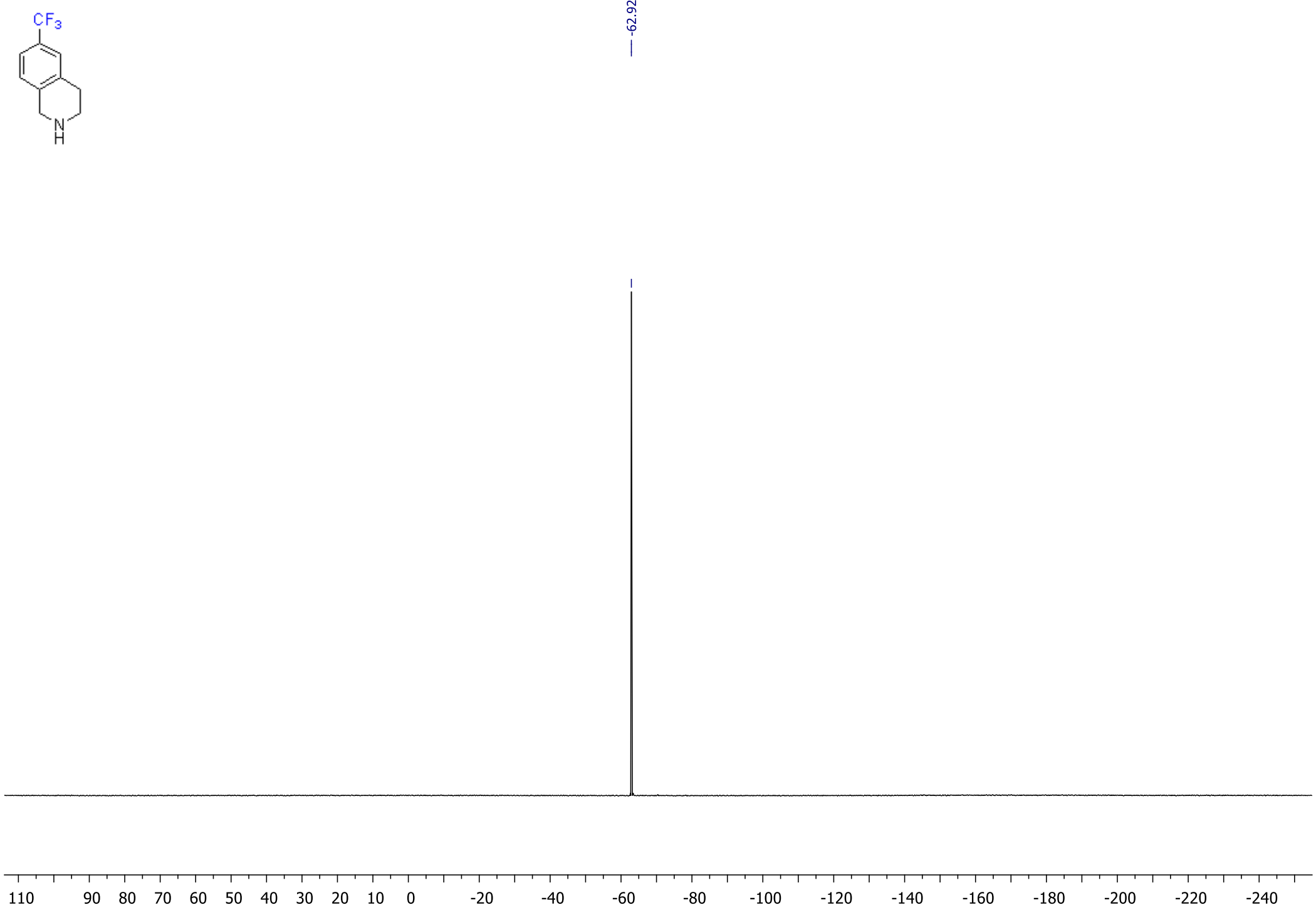
Compound 66b*

\section{${ }^{1} \mathrm{H}$ NMR $\left(400 \mathrm{MHz}, \mathrm{CDCl}_{3}\right)$}

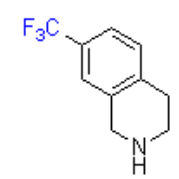

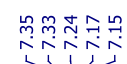

I

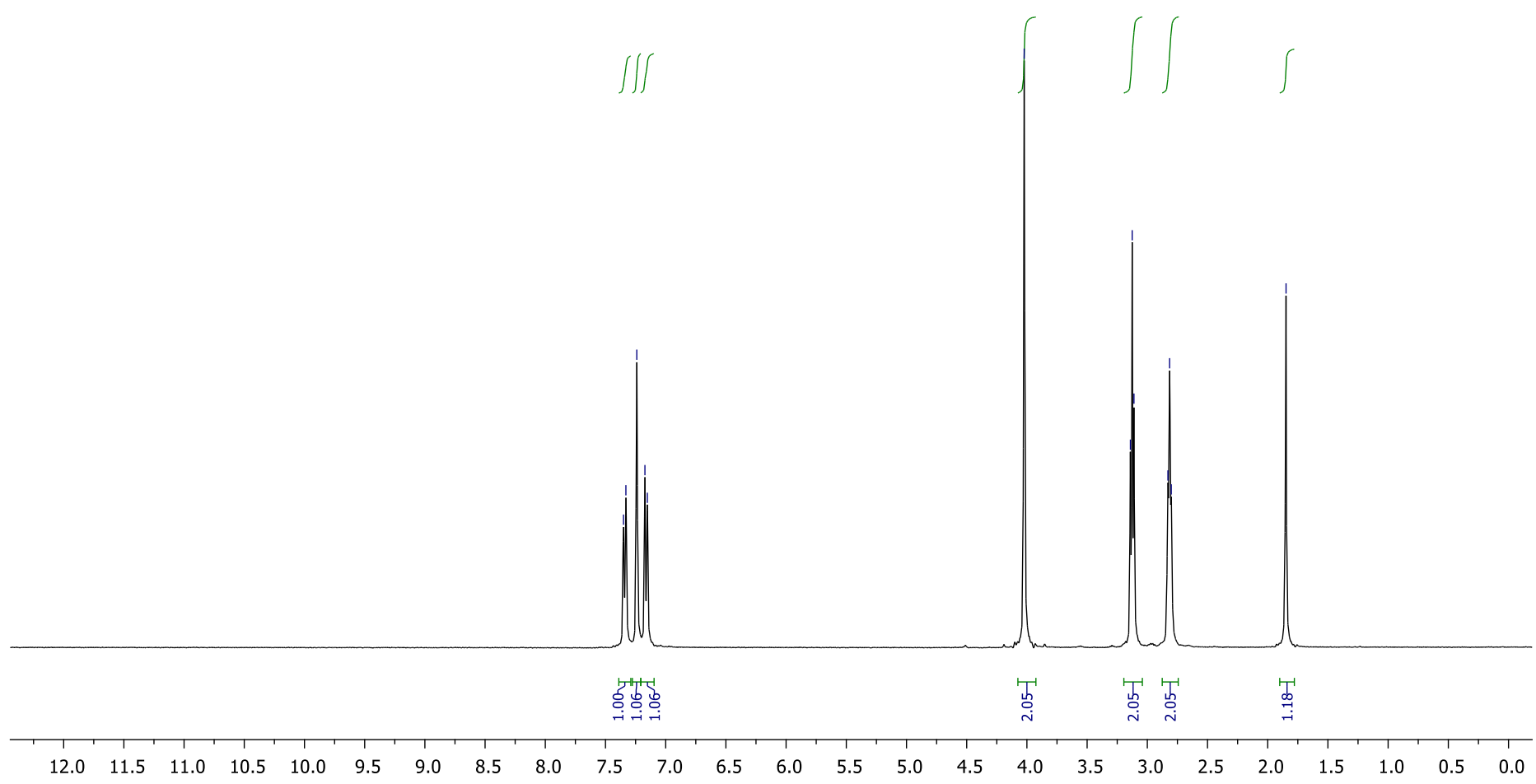

Compound 66b is also described in: a) J. Org. Chem. 1998, 63, 12, 4116-4119; b) J. Med. Chem. 1999, 42, 1, 118-134; c) J. Org. Chem. 1998, 63, 12, 41164119. 
${ }^{13} \mathrm{C}\left\{{ }^{1} \mathrm{H}\right\}$ NMR $\left(126 \mathrm{MHz}, \mathrm{CDCl}_{3}\right)$

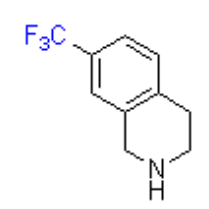

ๆ 주유ำ

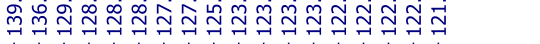

$\stackrel{1}{1}$

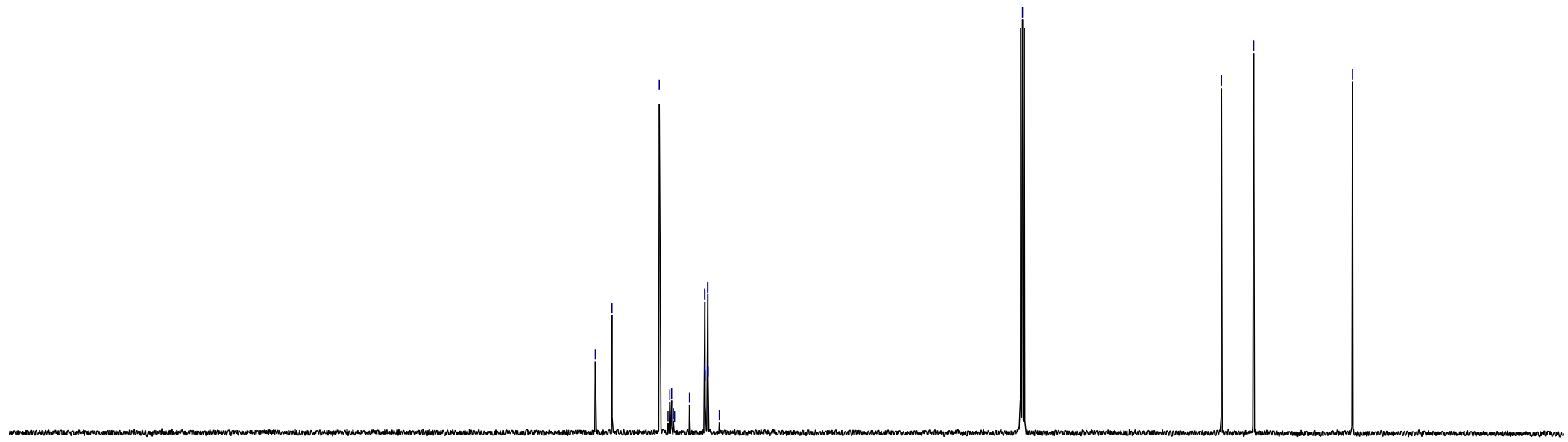

$\begin{array}{llllllll}220 & 210 & 200 & 190 & 180 & 170 & 160 & 150\end{array}$
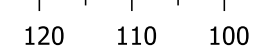

$90 \quad 80$

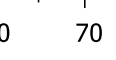

60

$50 \quad 40$ 
${ }^{19} \mathrm{~F}$ NMR $\left(376 \mathrm{MHz}, \mathrm{CDCl}_{3}\right)$
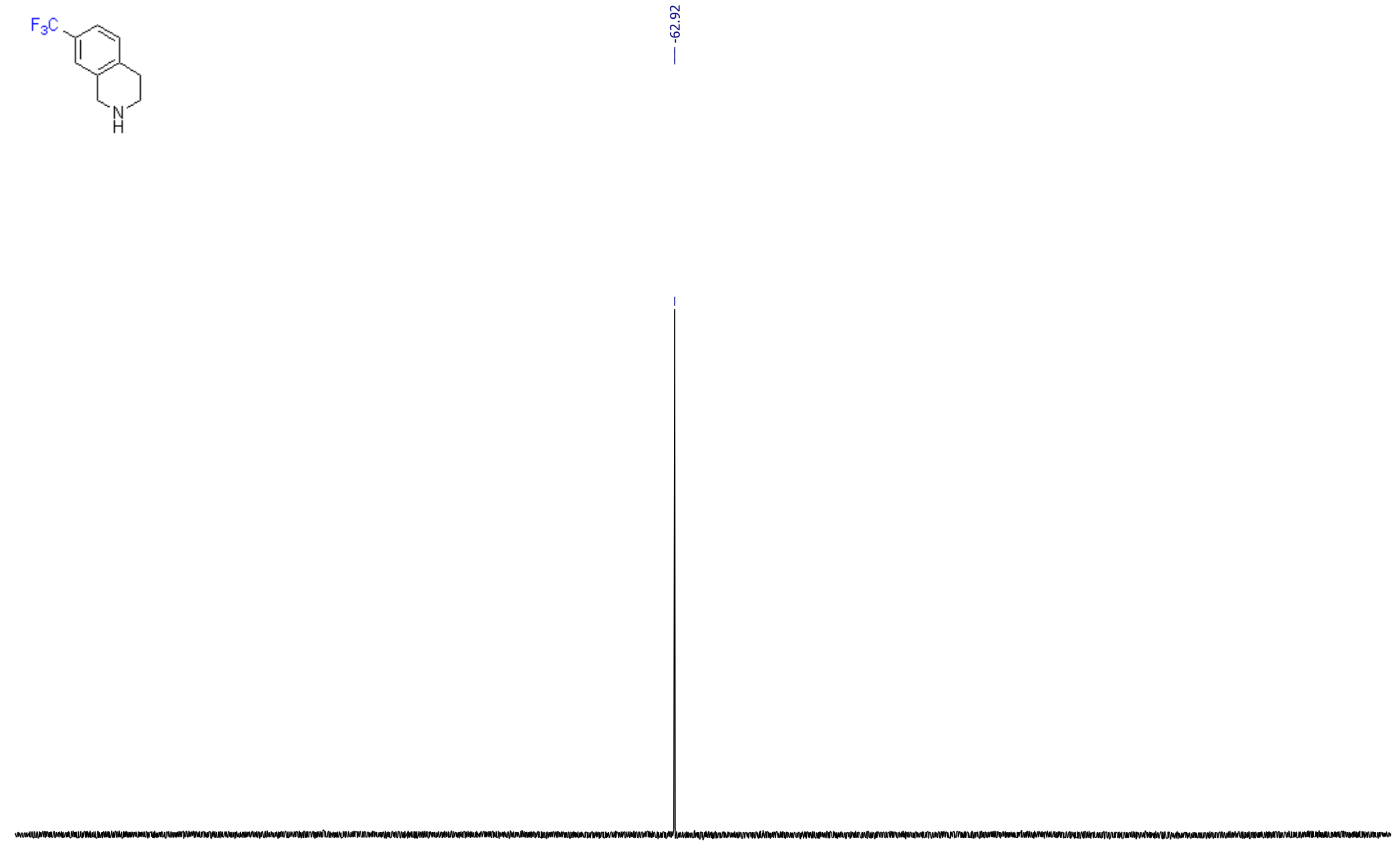

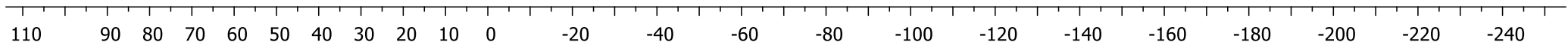




\section{Compound $45 b^{*}$}

\section{${ }^{1} \mathrm{H}$ NMR (400 MHz, DMSO-d6)}

SpinWorks 4: 6-(trifluoromethyl)piperidin-2-one

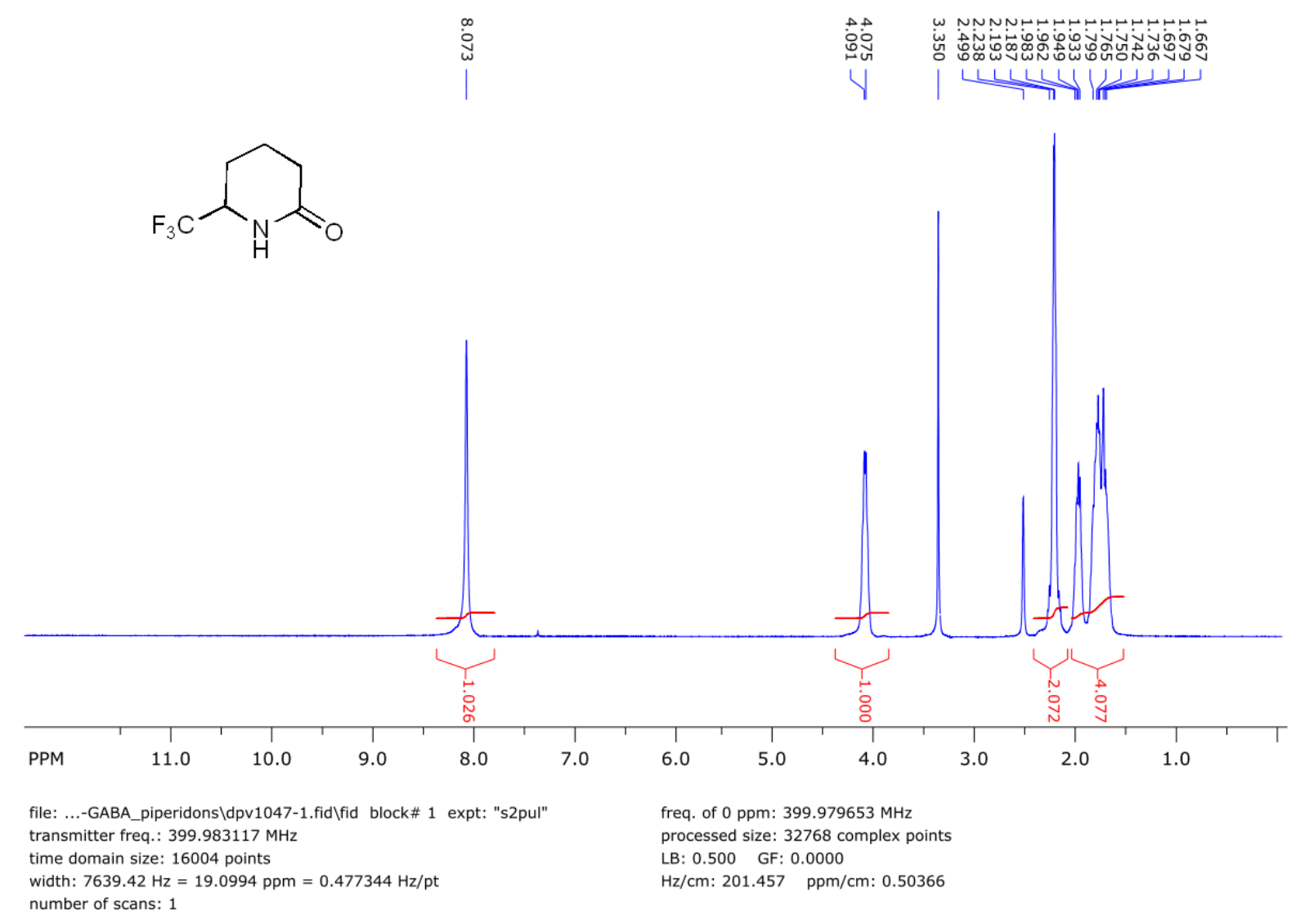

Compound 45b is also described in: Chem. Commun. 2013, 49, 7492-7494. 


\section{${ }^{13} \mathrm{C}\left\{{ }^{1} \mathrm{H}\right\}$ NMR $(100 \mathrm{MHz}$, DMSO-d 6 )}

SpinWorks 4: 6-(trifluoromethyl)piperidin-2-one-13C
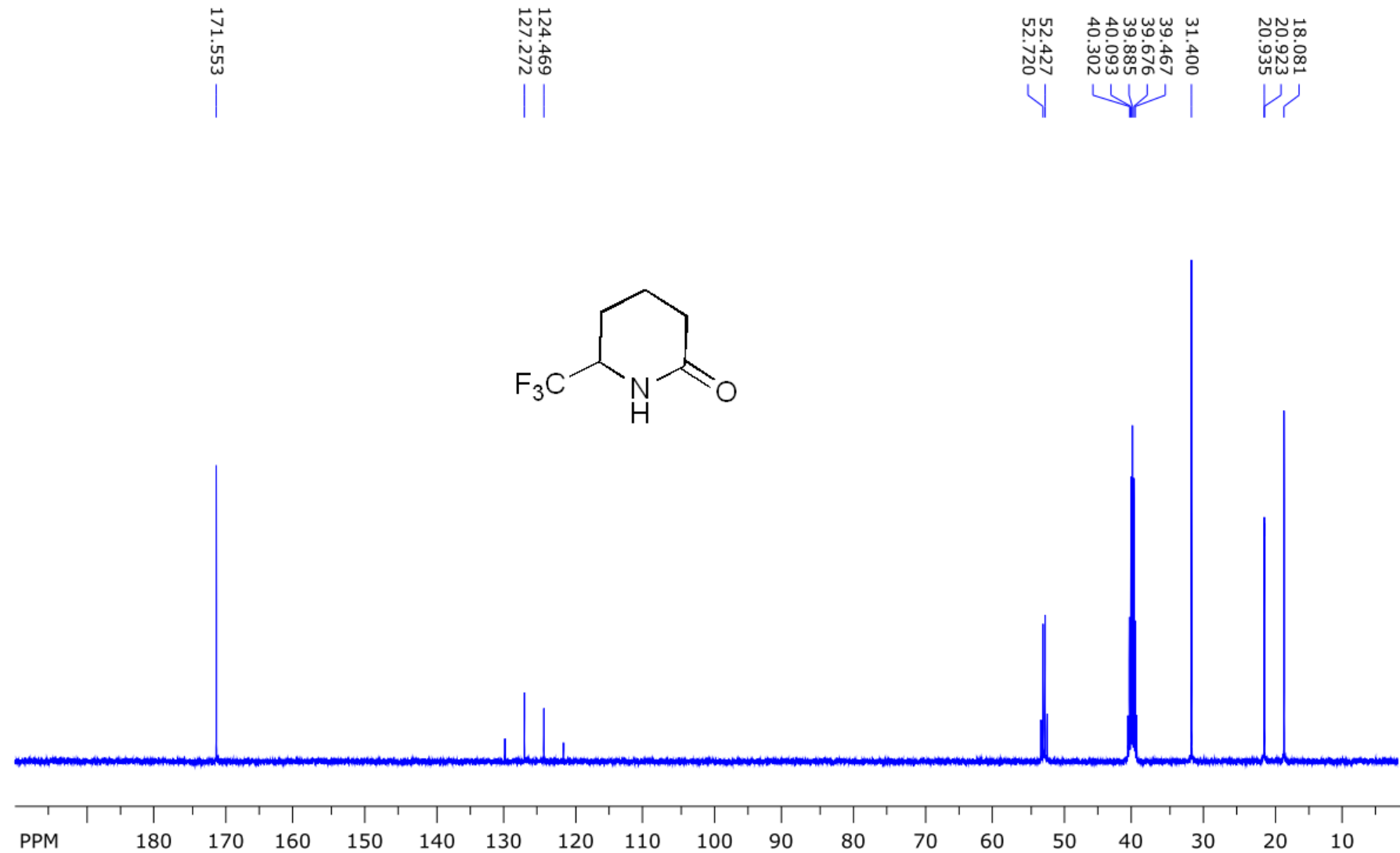

file: ...ABA_piperidons\DPV1047-7_C13\1 \fid expt: <zgpg30>

freq. of $0 \mathrm{ppm}: 100.612769 \mathrm{MHz}$

transmitter freq.: $100.622830 \mathrm{MHz}$

time domain size: 65536 points

processed size: 32768 complex points

LB: 1.000 GF: 0.0000

width: $24038.46 \mathrm{~Hz}=238.8967 \mathrm{ppm}=0.366798 \mathrm{~Hz} / \mathrm{pt}$

number of scans: 242

$\mathrm{Hz} / \mathrm{cm}: 799.859 \mathrm{ppm} / \mathrm{cm}: 7.94908$ 
${ }^{19}$ F NMR (376 MHz, DMSO-d6)

SpinWorks 4: 6-(trifluoromethyl)piperidin-2-one-19F<smiles>O=C1CCCC(C(F)(F)F)N1</smiles>

PPM

file: ...A_piperidons\dpv1047-1 F19\1|fid expt: <zg> transmitter freq: 376.472011 MHz

time domain size: 262144 points

width: $138888.89 \mathrm{~Hz}=368.9222 \mathrm{ppm}=0.529819 \mathrm{~Hz} / \mathrm{pt}$

number of scans: 4

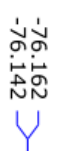


SpinWorks 4: 3-(trifluoromethyl)piperidin-2-one
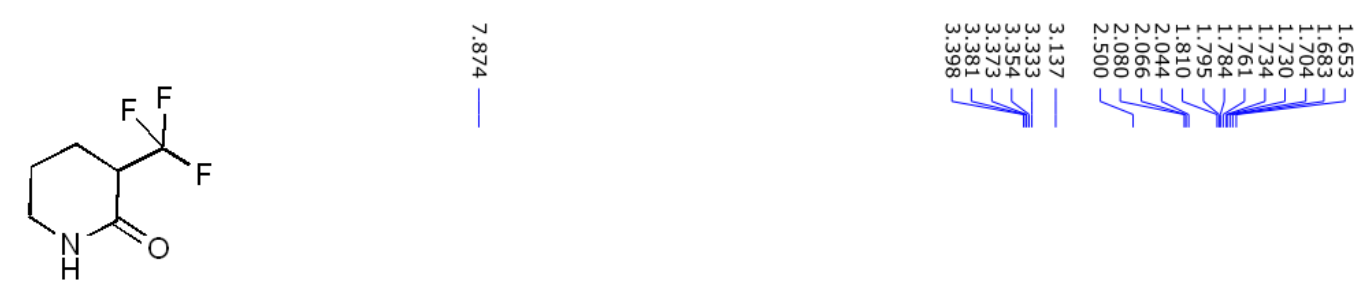

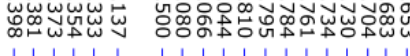
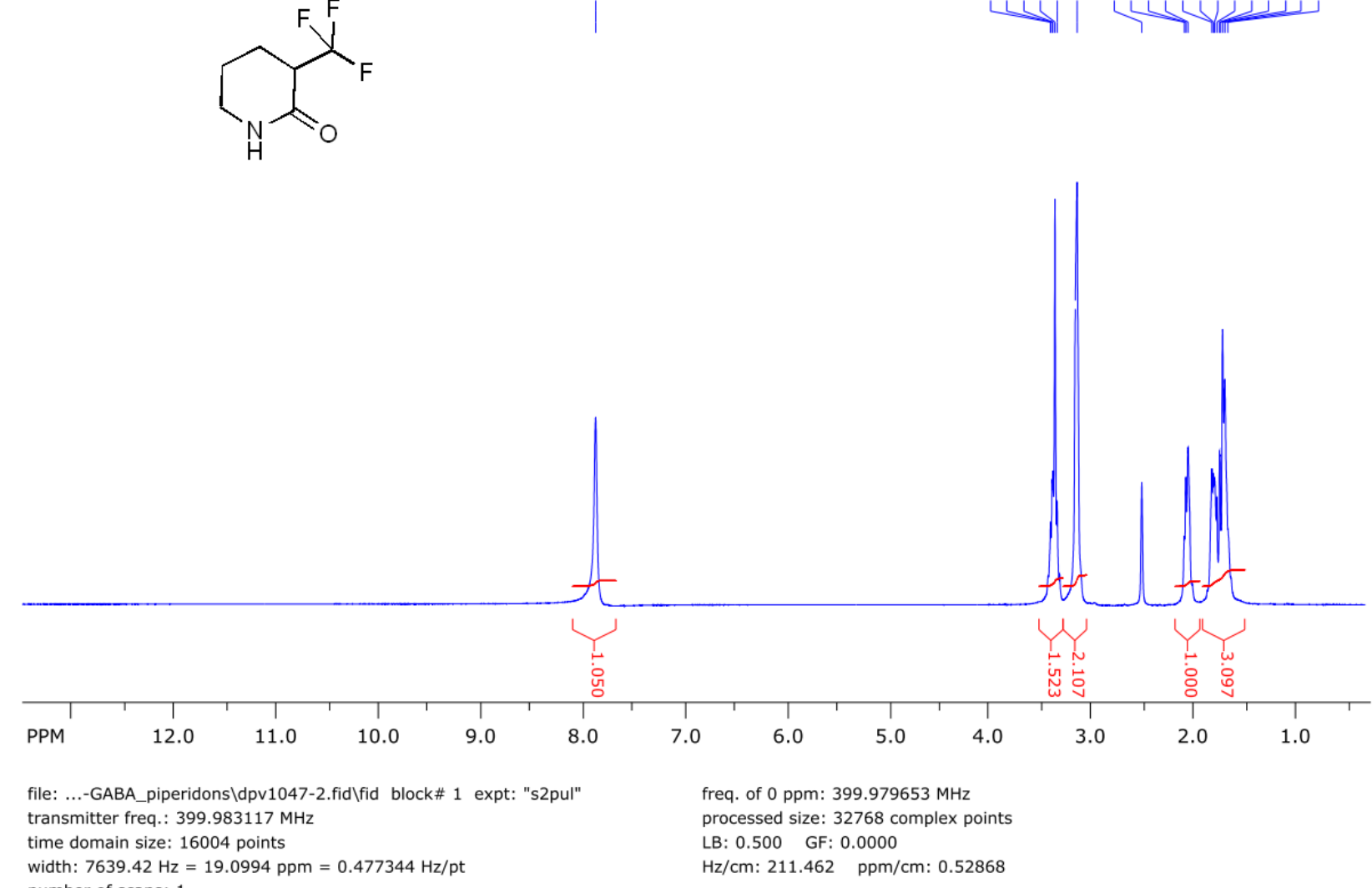

$\mathrm{Hz} / \mathrm{cm}: 211.462 \mathrm{ppm} / \mathrm{cm}: 0.52868$ 


\section{${ }^{13} \mathrm{C}\left\{{ }^{1} \mathrm{H}\right\}$ NMR $(100 \mathrm{MHz}$, DMSO-d 6 )}

SpinWorks 4: 3-(trifluoromethyl)piperidin-2-one-13C
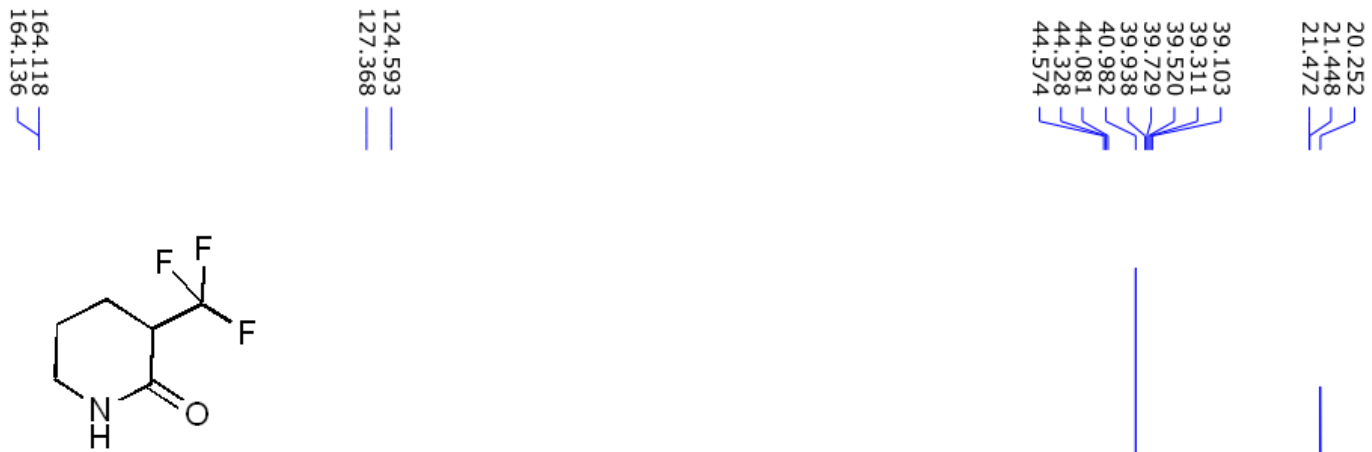

|

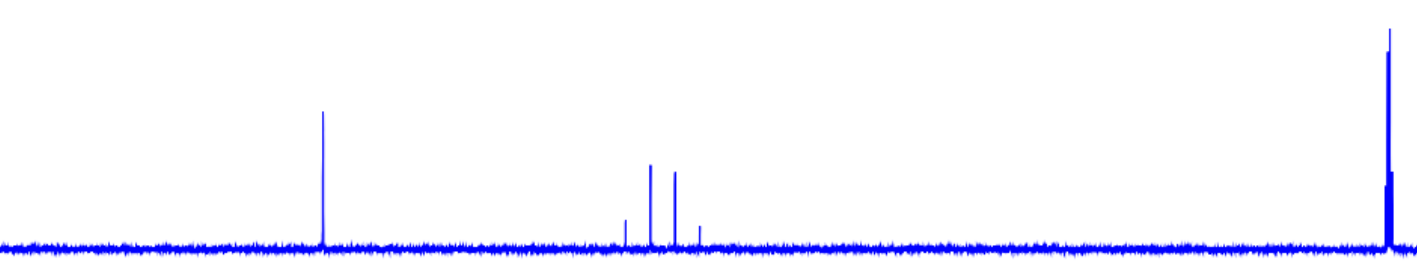

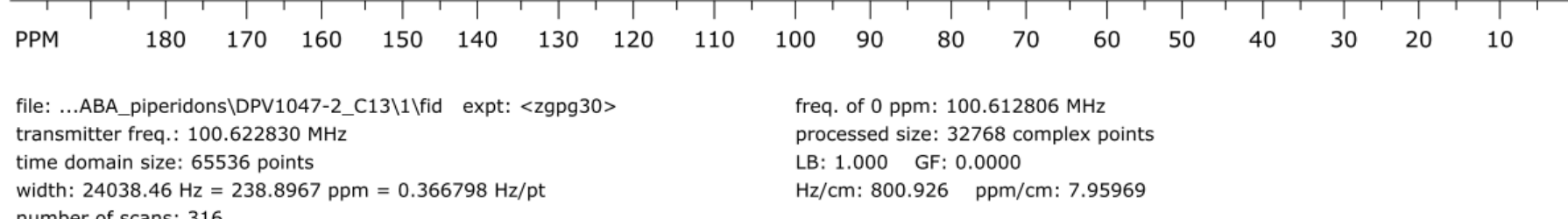

width: $24038.46 \mathrm{~Hz}=238.8967 \mathrm{ppm}=0.366798 \mathrm{~Hz} / \mathrm{pt}$

LB: $1.000 .902: 0.0000 / \mathrm{cm}: 7.95969$ 
${ }^{19}$ F NMR (376 MHz, DMSO-d6)

SpinWorks 4: 3-(trifluoromethyl)piperidin-2-one-19F
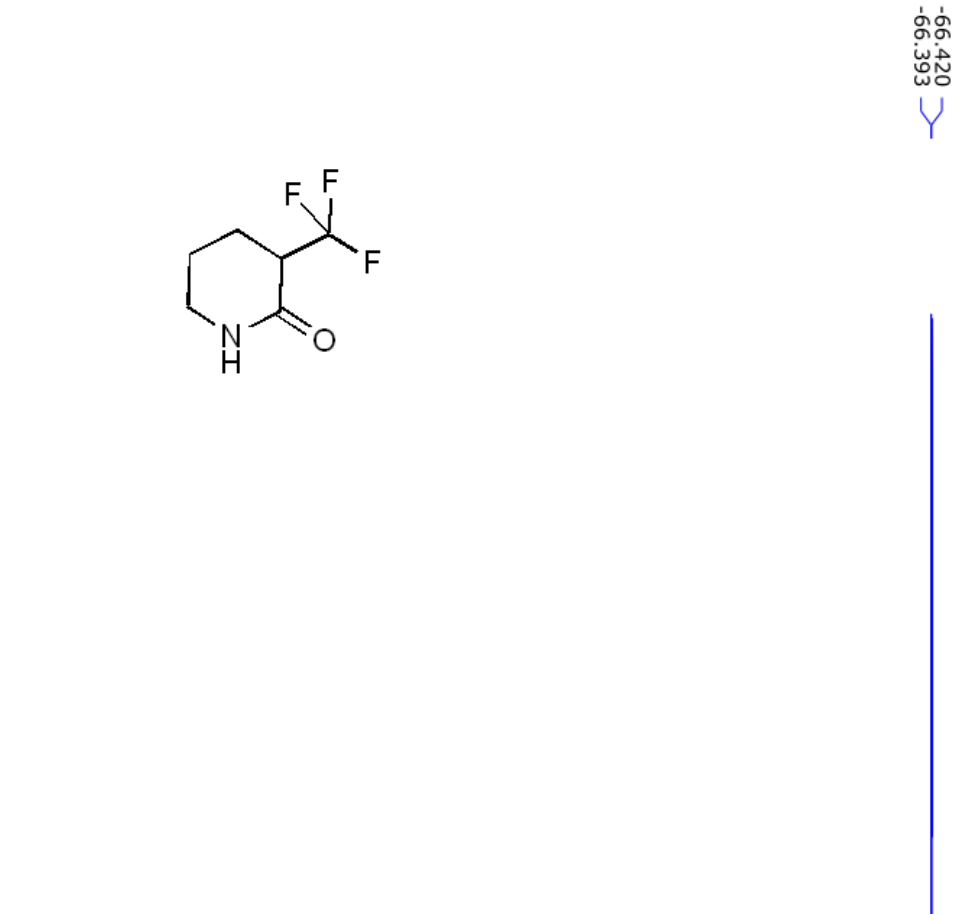

PPM

$20 \quad 10$

$0 \quad-10$

$-20 \quad-30$

$\begin{array}{cc}-40 & -50\end{array}$

$-60-70$

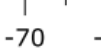

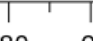

file: ...A_piperidons\dpv1047-2 F19\1\fid expt: $\langle$ zg $>$

transmitter freq.: $376.472011 \mathrm{MHz}$

freq. of $0 \mathrm{ppm}: 376.498553 \mathrm{MHz}$

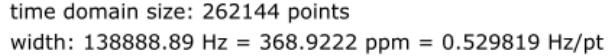

processed size: 262144 complex points

LB: 0.300 GF: 0.0000

number of scans: 4

$\mathrm{Hz} / \mathrm{cm}: 3009.002 \mathrm{ppm} / \mathrm{cm}: 7.99263$ 


\section{Compound 56b*}

\section{${ }^{1} \mathrm{H}$ NMR (400 MHz, DMSO-d 6 )}

SpinWorks 4: 4-(trifluoromethyl)piperidin-2-one

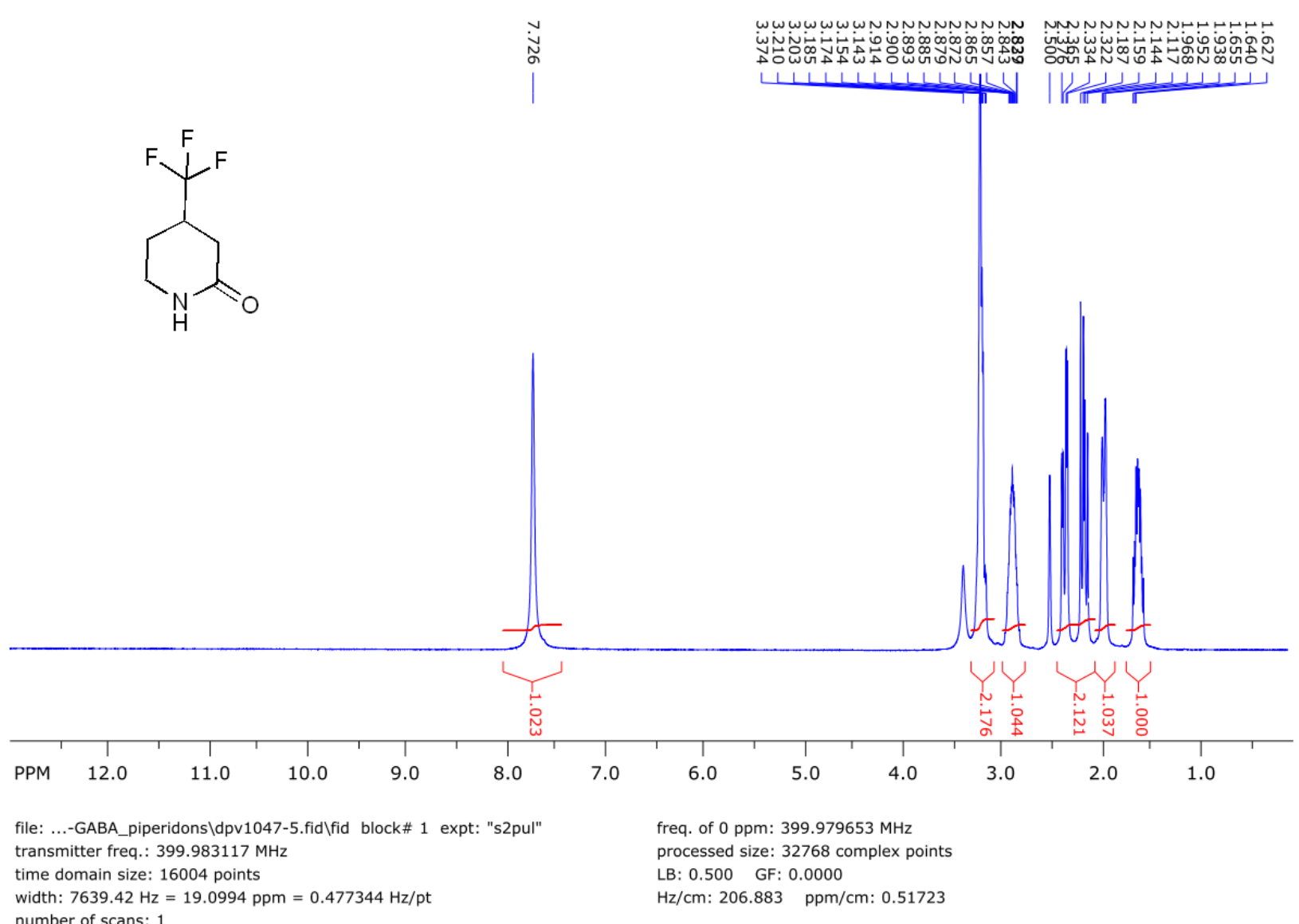

Compound 56b is also described in: Eur. J. Org. Chem. 2018, 46, 6486-6493. 


\section{${ }^{13} \mathrm{C}\left\{{ }^{1} \mathrm{H}\right\}$ NMR $(100 \mathrm{MHz}$, DMSO-d 6 )}

SpinWorks 4: 4-(trifluoromethyl)piperidin-2-one-13C
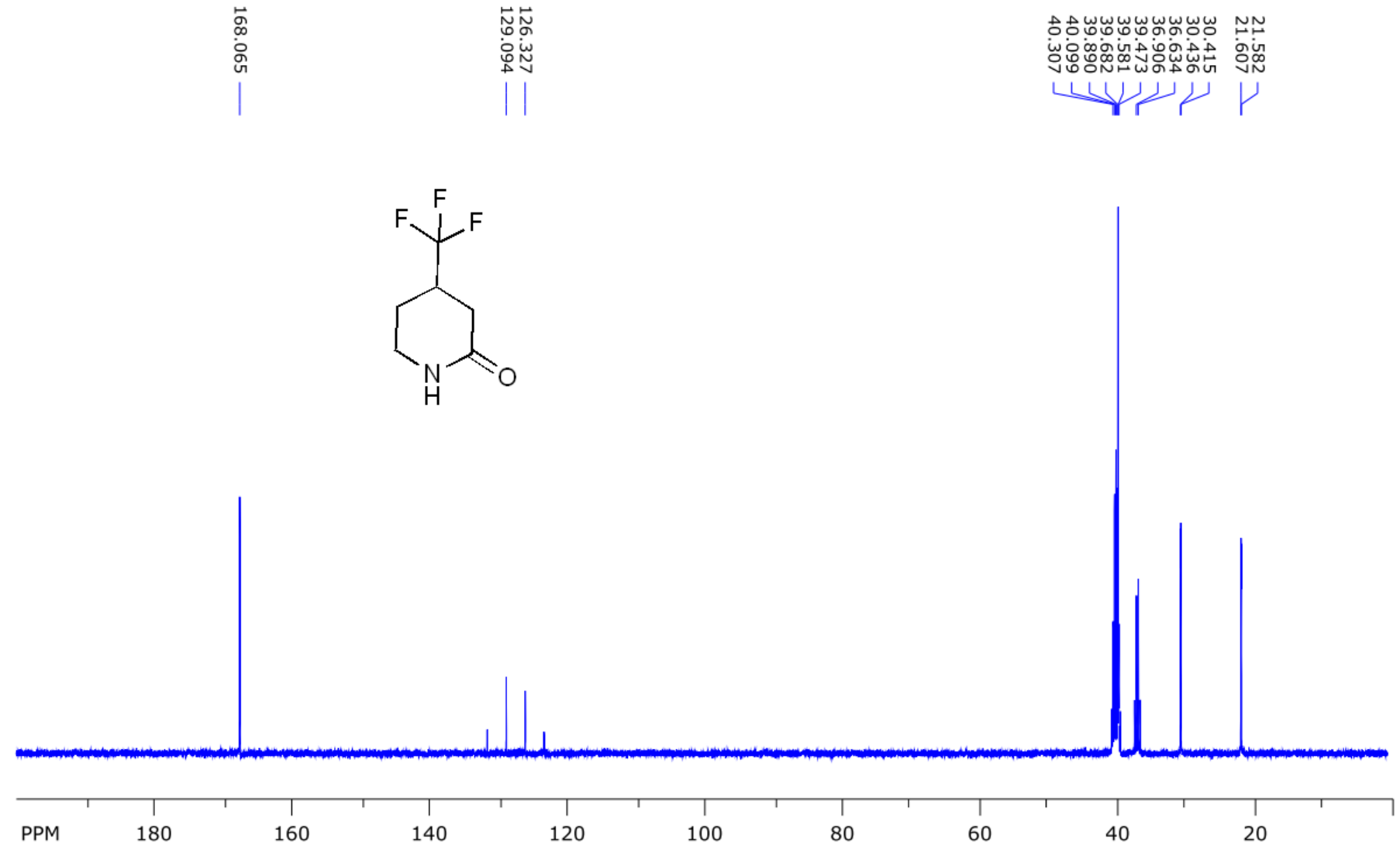

file: ...ABA piperidons\DPV1047-5 C13\1 \fid expt: <zgpg30> transmitter freq: $100.622830 \mathrm{MHz}$

freq. of $0 \mathrm{ppm}: 100.612769 \mathrm{MHz}$ time doma

width: $24038.46 \mathrm{~Hz}=238.8967 \mathrm{ppm}=0.366798 \mathrm{~Hz} / \mathrm{pt}$

processed

LB: 1.000 GF: 0.0000

$\mathrm{Hz} / \mathrm{cm}: 807.329 \mathrm{ppm} / \mathrm{cm}: 8.02332$ 
${ }^{19}$ F NMR (376 MHz, DMSO-d6)

SpinWorks 4: 4-(trifluoromethyl)piperidin-2-one-19F
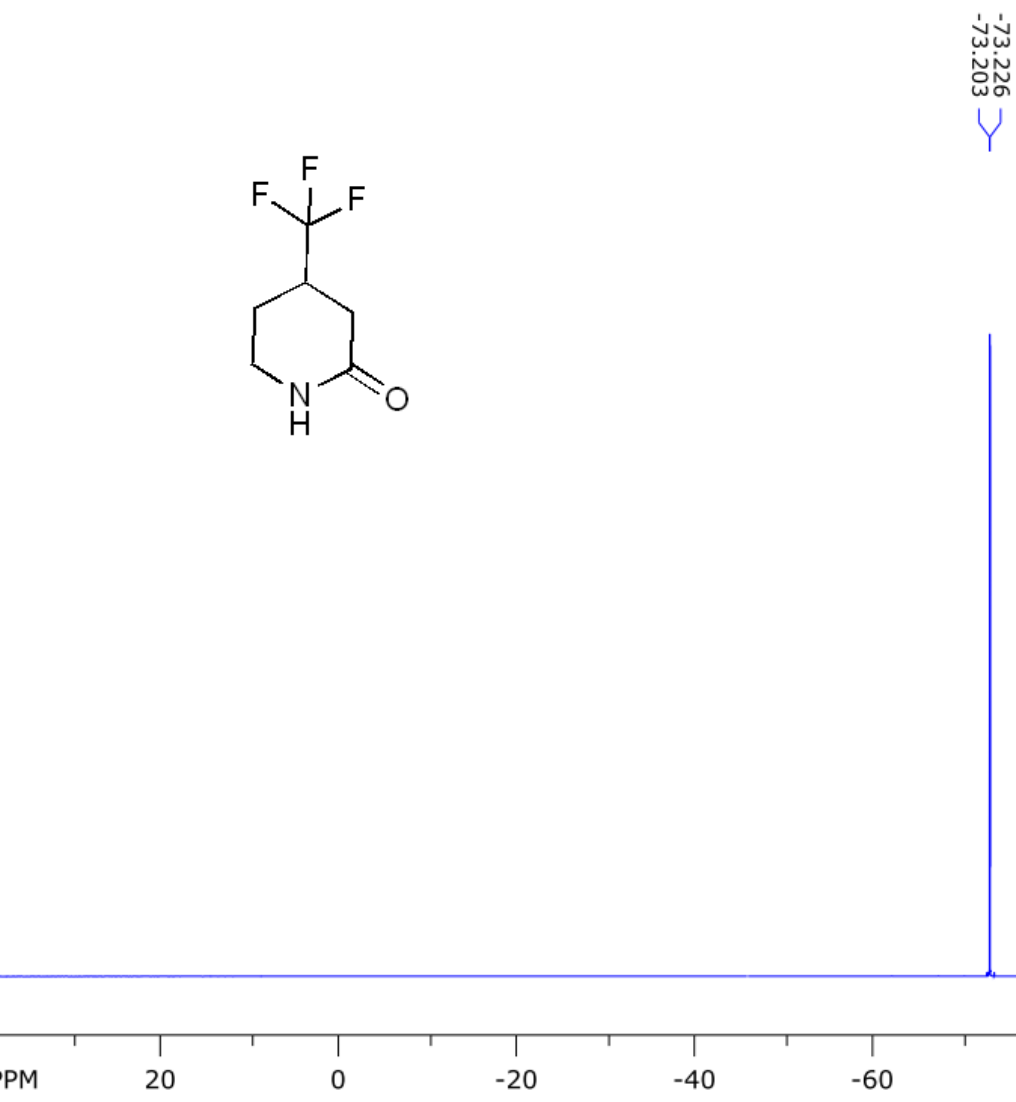

$-20$

$-40$

$-60$

$-80$

$-100$

$-120$

$-140$

file: ...A_piperidons\dpv1047-5 F19\1\fid expt: <zg>

transmitter freq.: $376.472011 \mathrm{MHz}$

time domain size: 262144 points

freq. of 0 ppm: $376.498553 \mathrm{MHz}$

width: $138888.89 \mathrm{~Hz}=368.9222 \mathrm{ppm}=0.529819 \mathrm{~Hz} / \mathrm{pt}$

number of scans: 4

LB: 0.300 GF: 0.0000

$\mathrm{Hz} / \mathrm{cm}$ : $3018.251 \mathrm{ppm} / \mathrm{cm}: 8.01720$ 
SpinWorks 4: 5-(trifluoromethyl)piperidin-2-one

ํ.<smiles>O=C1CCC(C(F)(F)F)CN1</smiles>

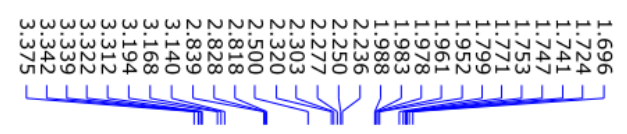

iाiा ?

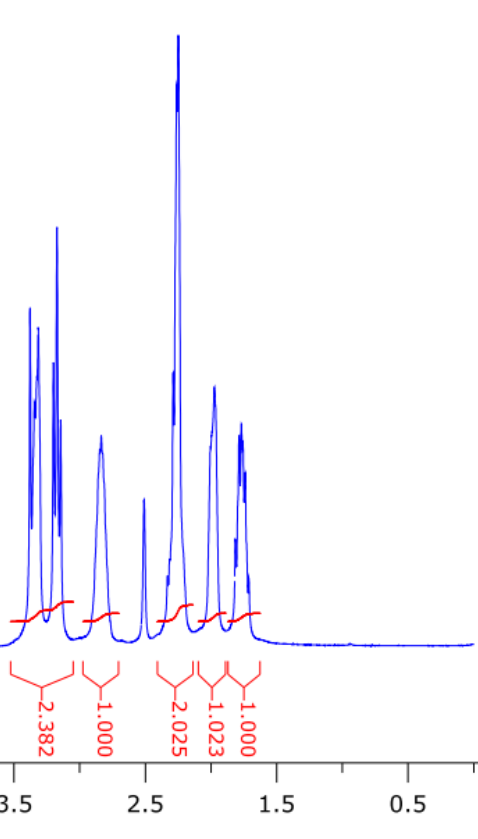

file: ...-GABA_piperidons\dpv1047-3.fid (fid block\# 1 expt: "s2pul" transmitter freq.: $399.983117 \mathrm{MHz}$

time domain size: 16004 points

width: $7639.42 \mathrm{~Hz}=19.0994 \mathrm{ppm}=0.477344 \mathrm{~Hz} / \mathrm{pt}$

freq. of o ppm: $399.979653 \mathrm{MHz}$

LB: 0.500 GF: 0.0000

number of scans: 1 


\section{${ }^{13} \mathrm{C}\left\{{ }^{1} \mathrm{H}\right\}$ NMR $(100 \mathrm{MHz}$, DMSO-d 6 )}

SpinWorks 4: 5-(trifluoromethyl)piperidin-2-one-13C

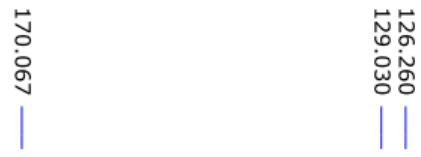

A N

|<smiles>O=C1CCC(C(F)(F)F)CN1</smiles>

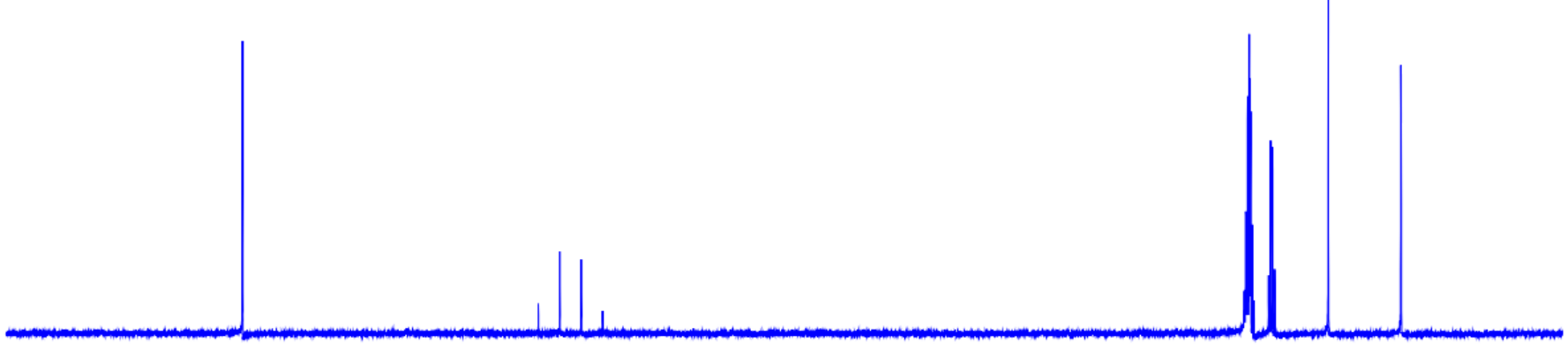

file: ...ABA_piperidons\DPV1047-3_C13\1\fid expt: <zgpg30> transmitter freq.: $100.622830 \mathrm{MHz}$

freq. of $0 \mathrm{ppm}: 100.612769 \mathrm{MHz}$

processed size: 32768 complex points

time domain size: 65536 points

complex points

width: $24038.46 \mathrm{~Hz}=238.8967 \mathrm{ppm}=0.366798 \mathrm{~Hz} / \mathrm{pt}$

1.000 GF: 0.0000

$\mathrm{Hz} / \mathrm{cm}: 809.997 \mathrm{ppm} / \mathrm{cm}: 8.04984$ 
${ }^{19}$ F NMR (376 MHz, DMSO-d6)

SpinWorks 4: 5-(trifluoromethyl)piperidin-2-one-19F

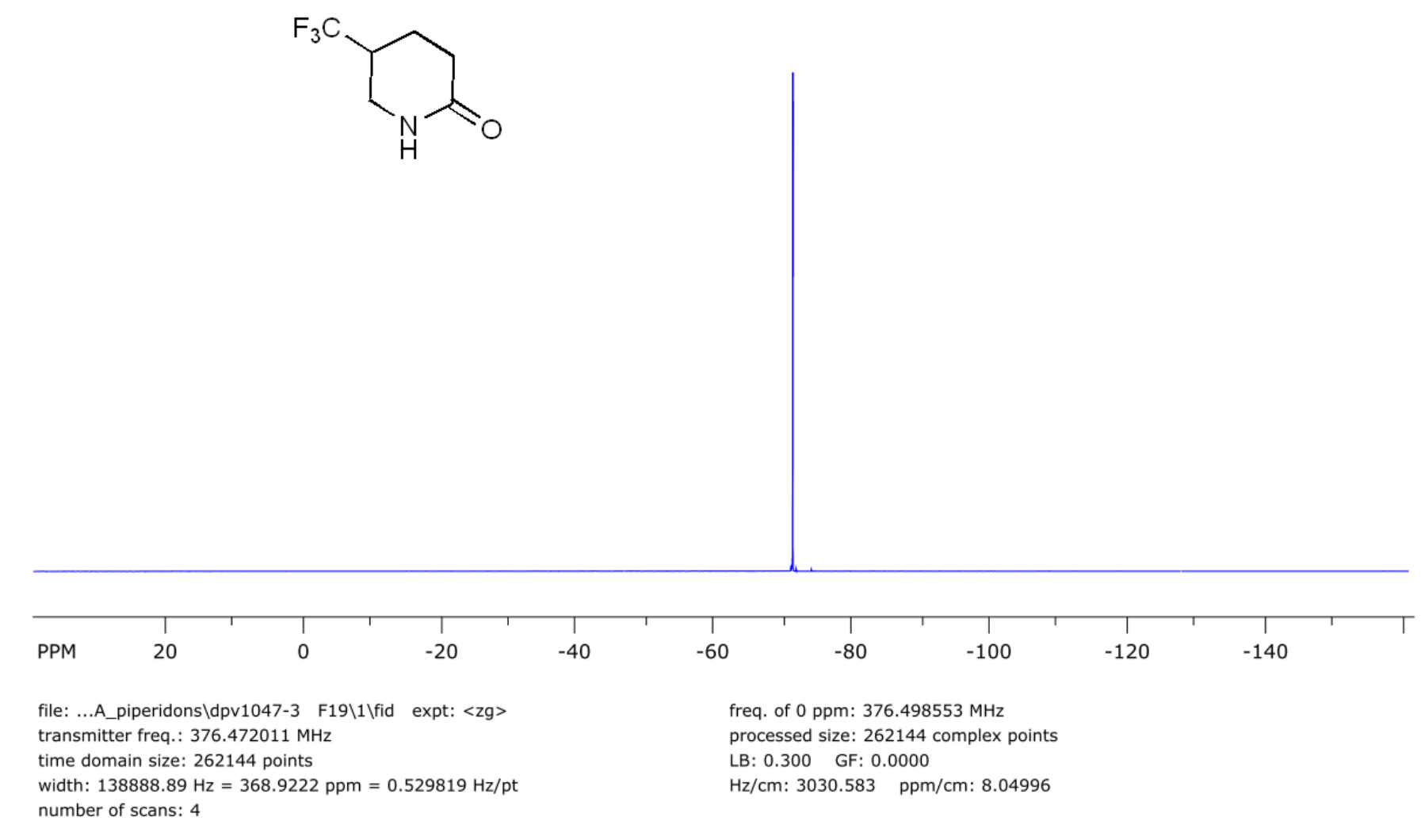


SpinWorks 4: 5-amino-2-(trifluoromethyl)pentanoic acid hydrochloride

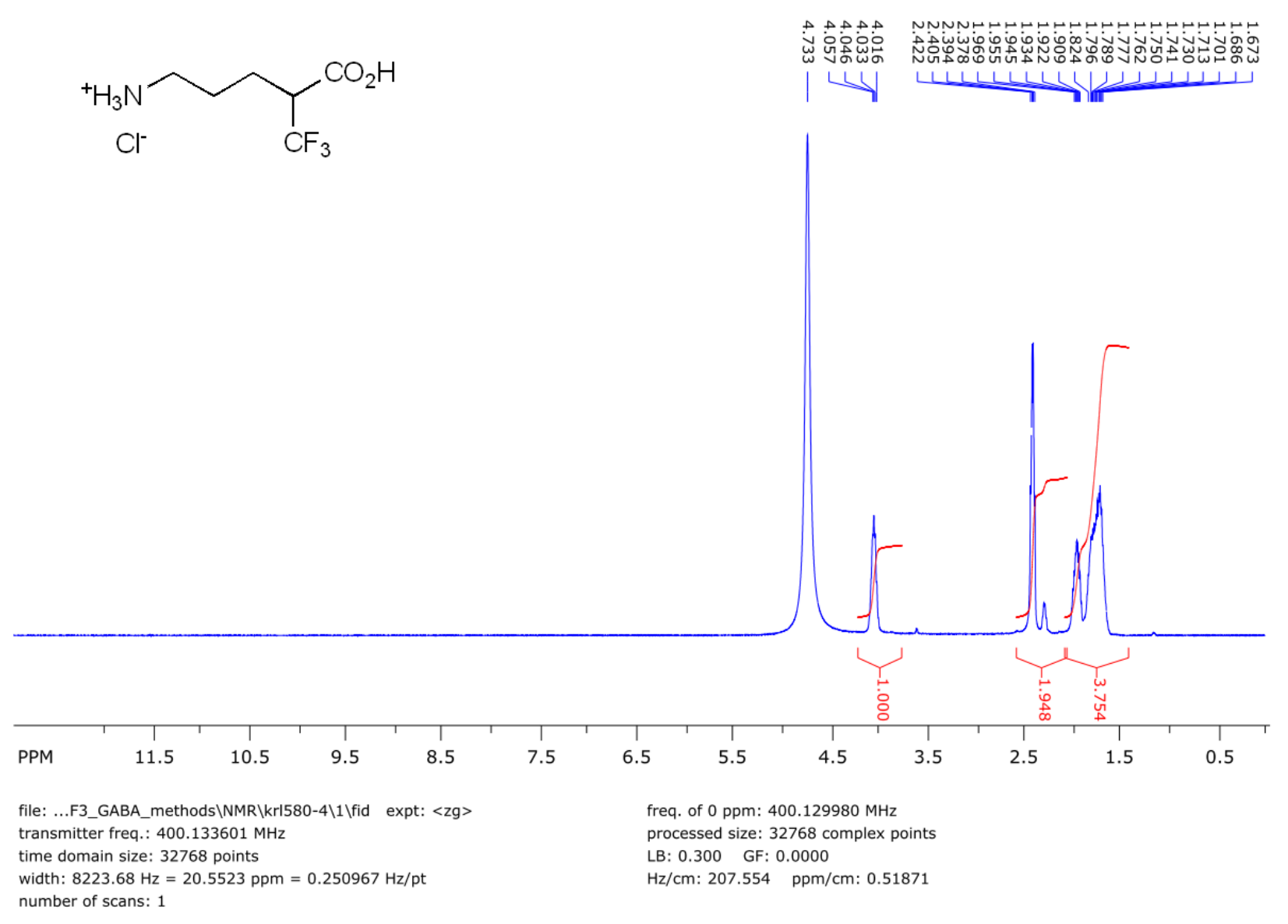




\section{${ }^{13} \mathrm{C}\left\{{ }^{1} \mathrm{H}\right\}$ NMR $\left(100 \mathrm{MHz}, \mathrm{D}_{2} \mathrm{O}\right)$}

SpinWorks 4: 5-amino-2-(trifluoromethyl)pentanoic acid hydrochloride -APT

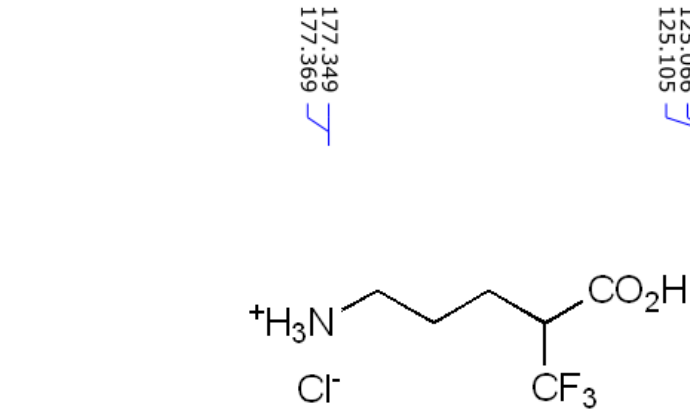

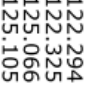

$$
\begin{aligned}
& \text { yy }
\end{aligned}
$$

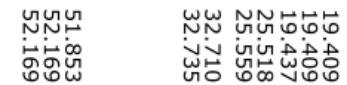$$
\text { Y Y Y H }
$$<smiles>[BH3-]</smiles>

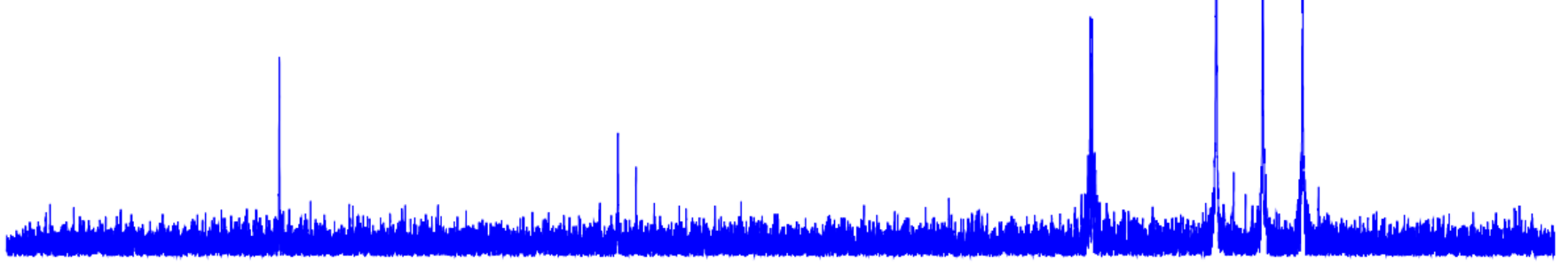

\begin{tabular}{|c|c|c|c|c|c|c|c|c|}
\hline \multirow[b]{2}{*}{ PPM } & $T$ & $T$ & $T$ & $T$ & $T$ & $T$ & 1 & $T$ \\
\hline & 200 & 180 & 160 & 140 & 120 & 100 & 80 & 60 \\
\hline \multicolumn{6}{|c|}{$\begin{array}{l}\text { file: ...A_methods } \backslash N M R \backslash k r 1580-4 \text { APT } \backslash 1 \backslash \text { fid } \text { expt: }<\text { aptjc> } \\
\text { transmitter freq.: } 100.622830 \mathrm{MHz}\end{array}$} & \multicolumn{3}{|c|}{$\begin{array}{l}\text { freq. of } 0 \mathrm{ppm}: 100.612769 \mathrm{MHz} \\
\text { processed size: } 65536 \text { complex points }\end{array}$} \\
\hline $\begin{array}{l}\text { time d } \\
\text { width: }\end{array}$ & nain siz & $\begin{array}{l}36 \text { point } \\
238.89\end{array}$ & & & & & $\begin{array}{r}\text { GF } \\
61.538\end{array}$ & $/ \mathrm{cm}: 9.55587$ \\
\hline
\end{tabular}

(3.8.867 ppm $=0.366798 \mathrm{~Hz} / \mathrm{pt}$

$\mathrm{Hz} / \mathrm{cm}: 961.538 \mathrm{ppm} / \mathrm{cm}: 9.55587$ 
${ }^{19} \mathrm{~F}$ NMR $\left(376 \mathrm{MHz}, \mathrm{D}_{2} \mathrm{O}\right)$

SpinWorks 4: 5-amino-2-(trifluoromethyl)pentanoic acid hydrochloride -19F

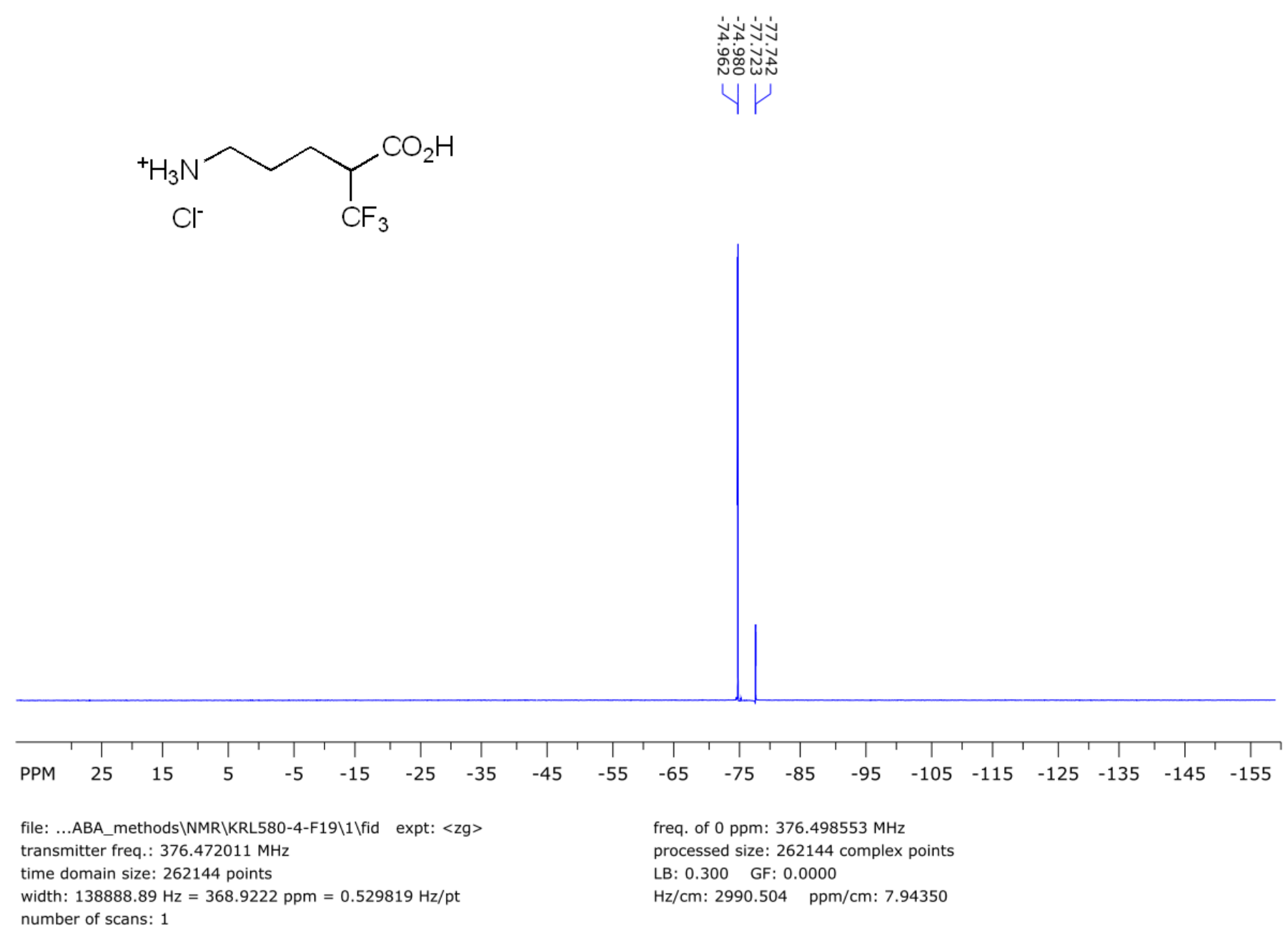


Compound 70*

${ }^{1} \mathrm{H}$ NMR (400 MHz, D $\left.2 \mathrm{O}\right)$

SpinWorks 4: 5-amino-3-(trifluoromethyl)pentanoic acid

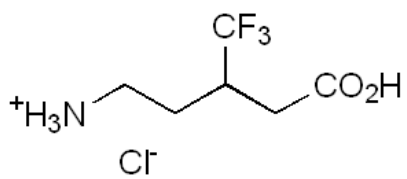

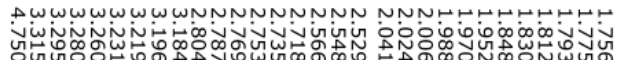
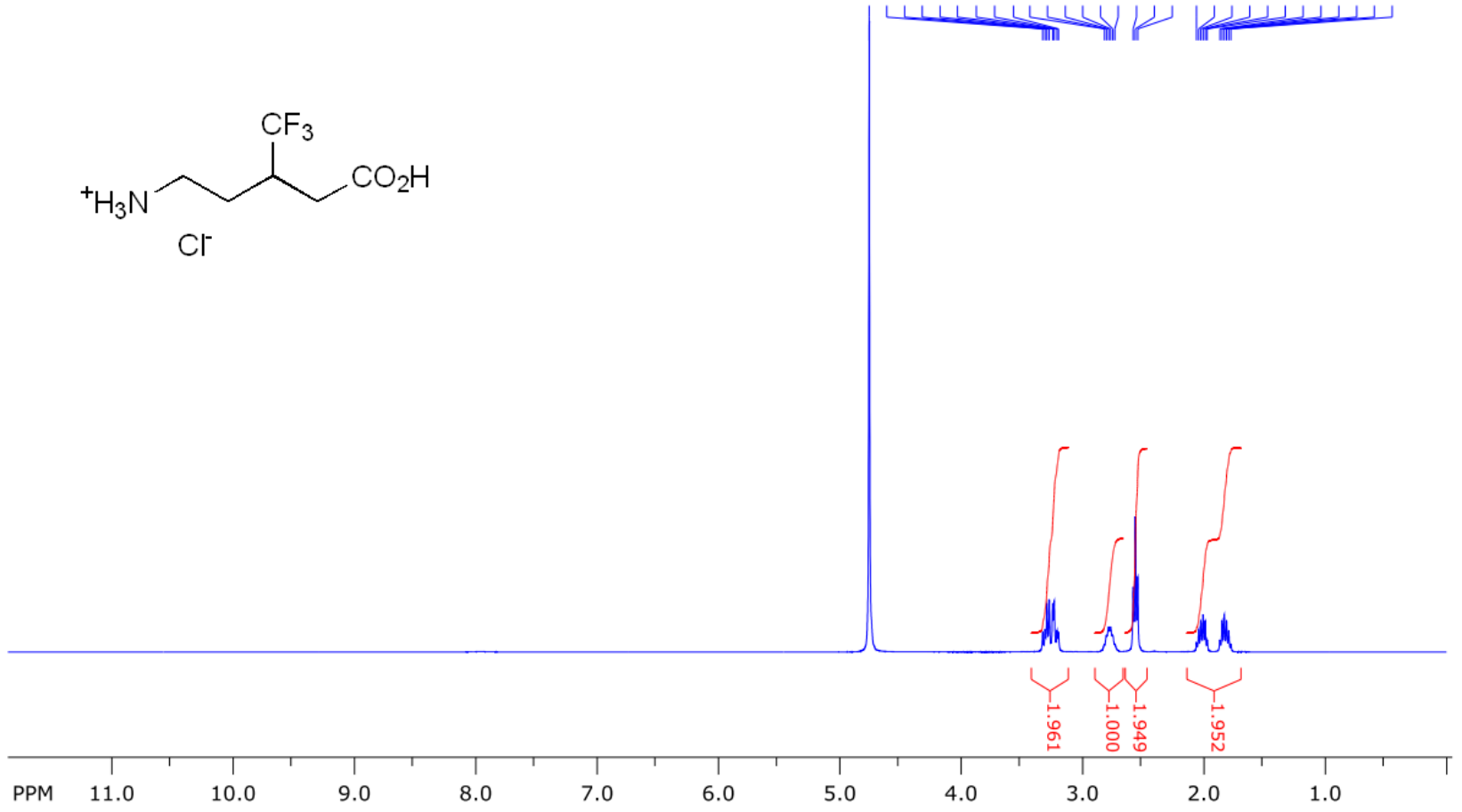

$\begin{array}{llll}\text { PPM } 11.0 & 10.0 & 9.0 & 8.0\end{array}$

file: ...F3_GABA_methods $\mid$ NMR $\backslash k r 1580-3 \backslash 1$ fid expt: <zg>

processed spm: $400.129980 \mathrm{MHz}$

transmitter freq.: $400.133601 \mathrm{MHz}$

LB: 0.300 GF: 0.0000

time domain size: 32768 points

$\mathrm{Hz} / \mathrm{cm}: 190.578 \mathrm{ppm} / \mathrm{cm}: 0.47629$

number of scans: 1

Compound 70 is also described in: Eur. J. Org. Chem. 2018, 46, 6486-6493. 


\section{${ }^{13} \mathrm{C}\left\{{ }^{1} \mathrm{H}\right\}$ NMR $\left(100 \mathrm{MHz}, \mathrm{D}_{2} \mathrm{O}\right)$}

SpinWorks 4: 5-amino-3-(trifluoromethyl)pentanoic acid -13C
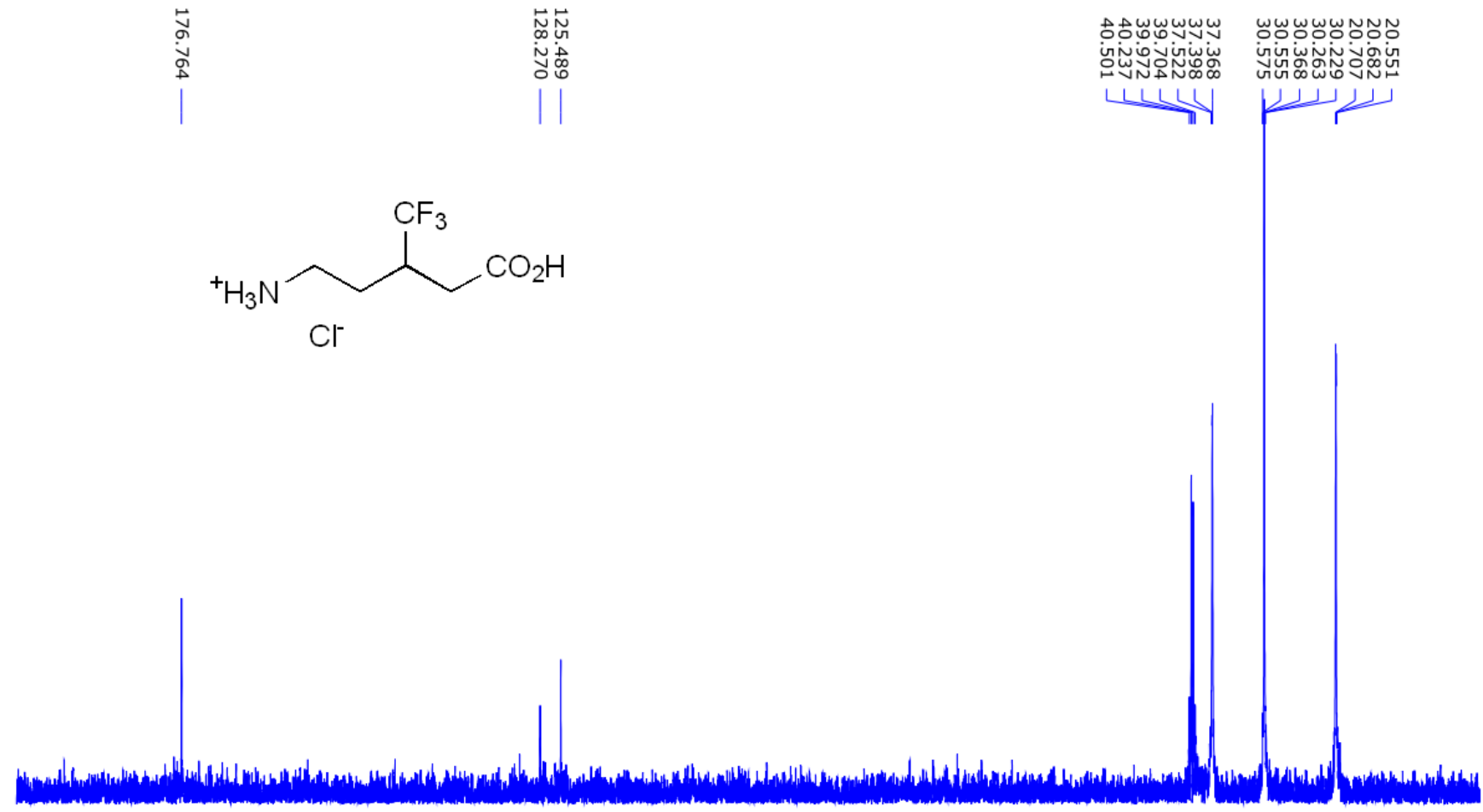

PPM $185 \quad 175$

16515

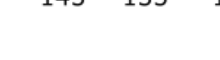

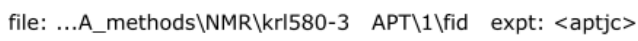
transmitter freq.: $100.622830 \mathrm{MHz}$

time domain size: 65536 points

width: $24038.46 \mathrm{~Hz}=238.8967 \mathrm{ppm}=0.366798 \mathrm{~Hz} / \mathrm{pt}$

freq. of O ppm: $100.612769 \mathrm{MHz}$

processed size: 65536 complex points

LB: 1.500 GF: 0.0000

number of scans: 273 
${ }^{19}$ F NMR (376 MHz, D $\left.2 \mathrm{O}\right)$

SpinWorks 4: 5-amino-3-(trifluoromethyl)pentanoic acid -19F

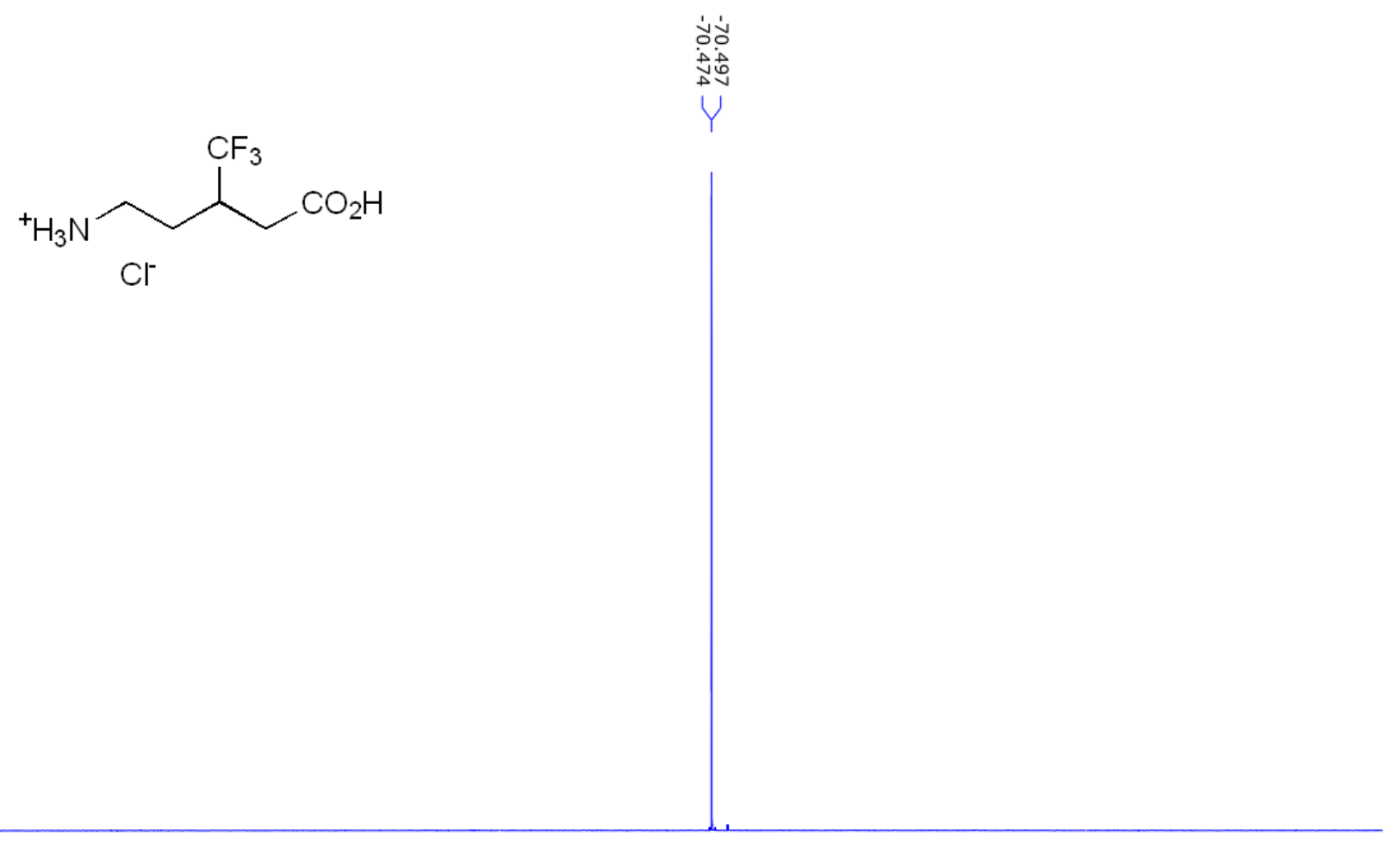

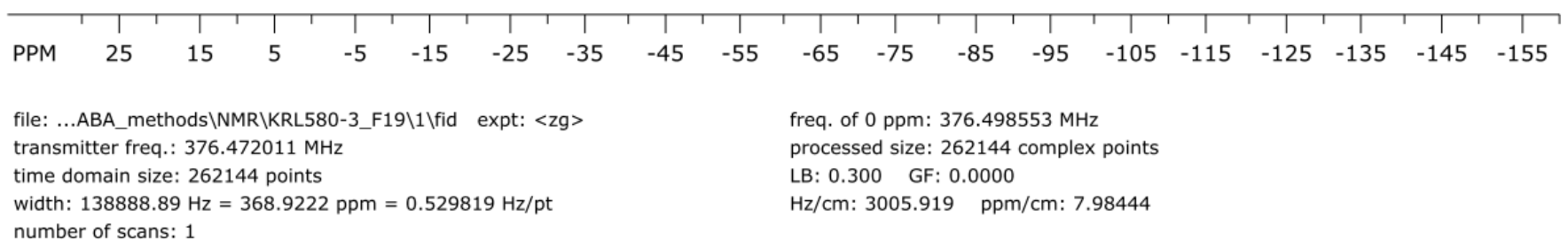




\section{Compound 71}

${ }^{1} \mathrm{H}$ NMR (400 MHz, D $2 \mathrm{O}$ )

SpinWorks 4: 5-amino-4-(trifluoromethyl)pentanoic acid

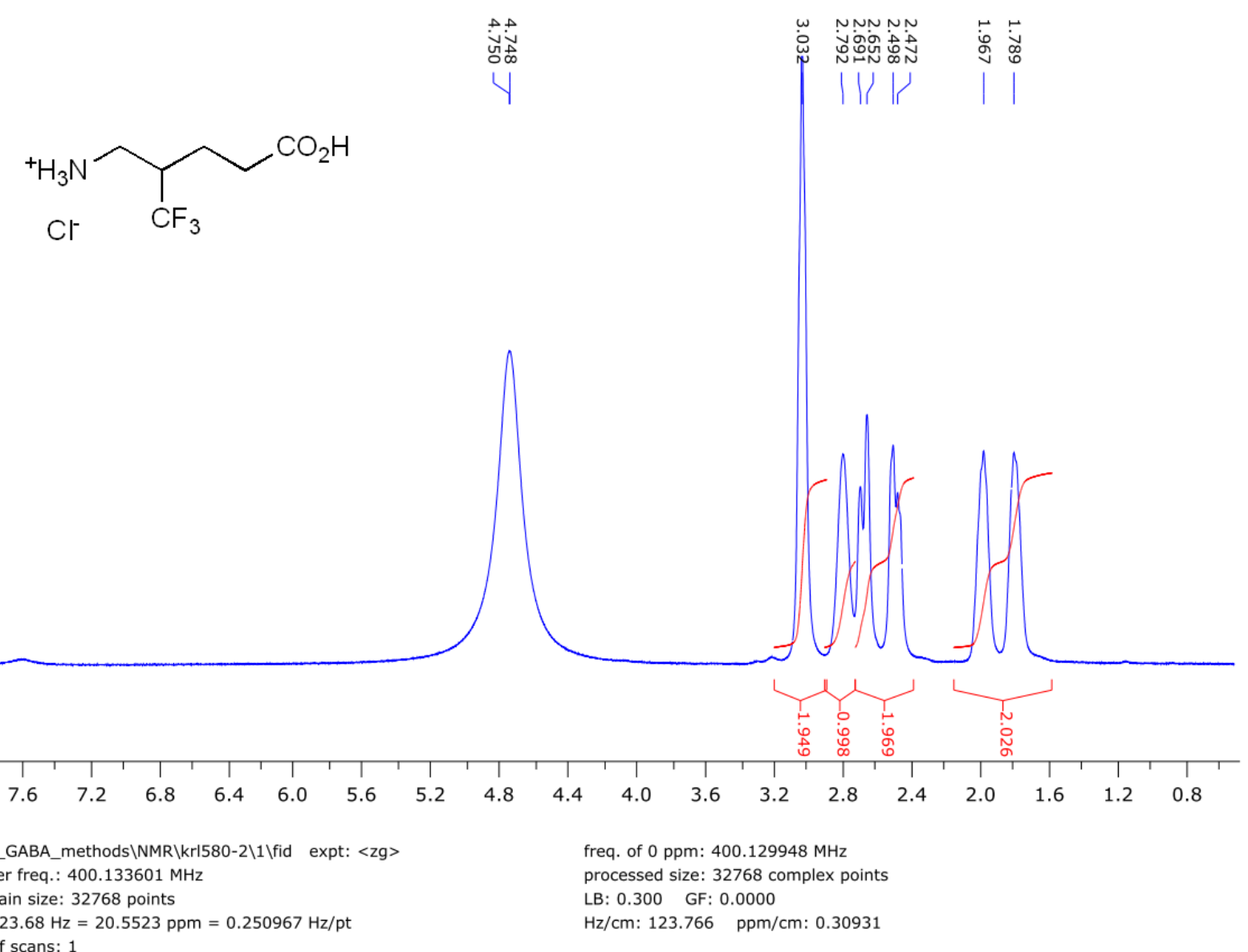




\section{${ }^{13} \mathrm{C}\left\{{ }^{1} \mathrm{H}\right\}$ NMR $\left(100 \mathrm{MHz}, \mathrm{D}_{2} \mathrm{O}\right)$}

SpinWorks 4: 5-amino-4-(trifluoromethyl)pentanoic acid 13C

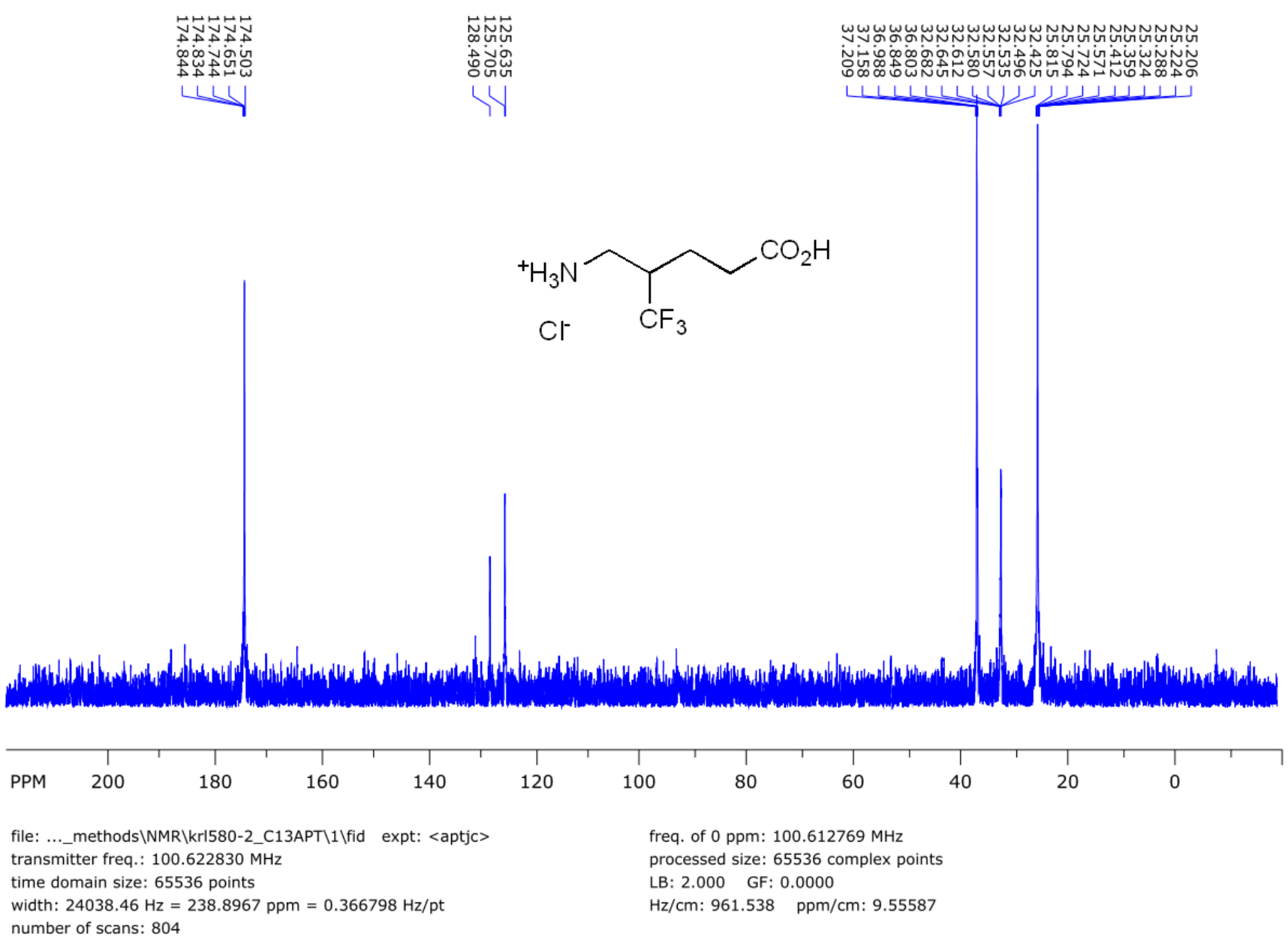


${ }^{19}$ F NMR (376 MHz, D $\left.2 \mathrm{O}\right)$

SpinWorks 4: 5-amino-4-(trifluoromethyl)pentanoic acid F19

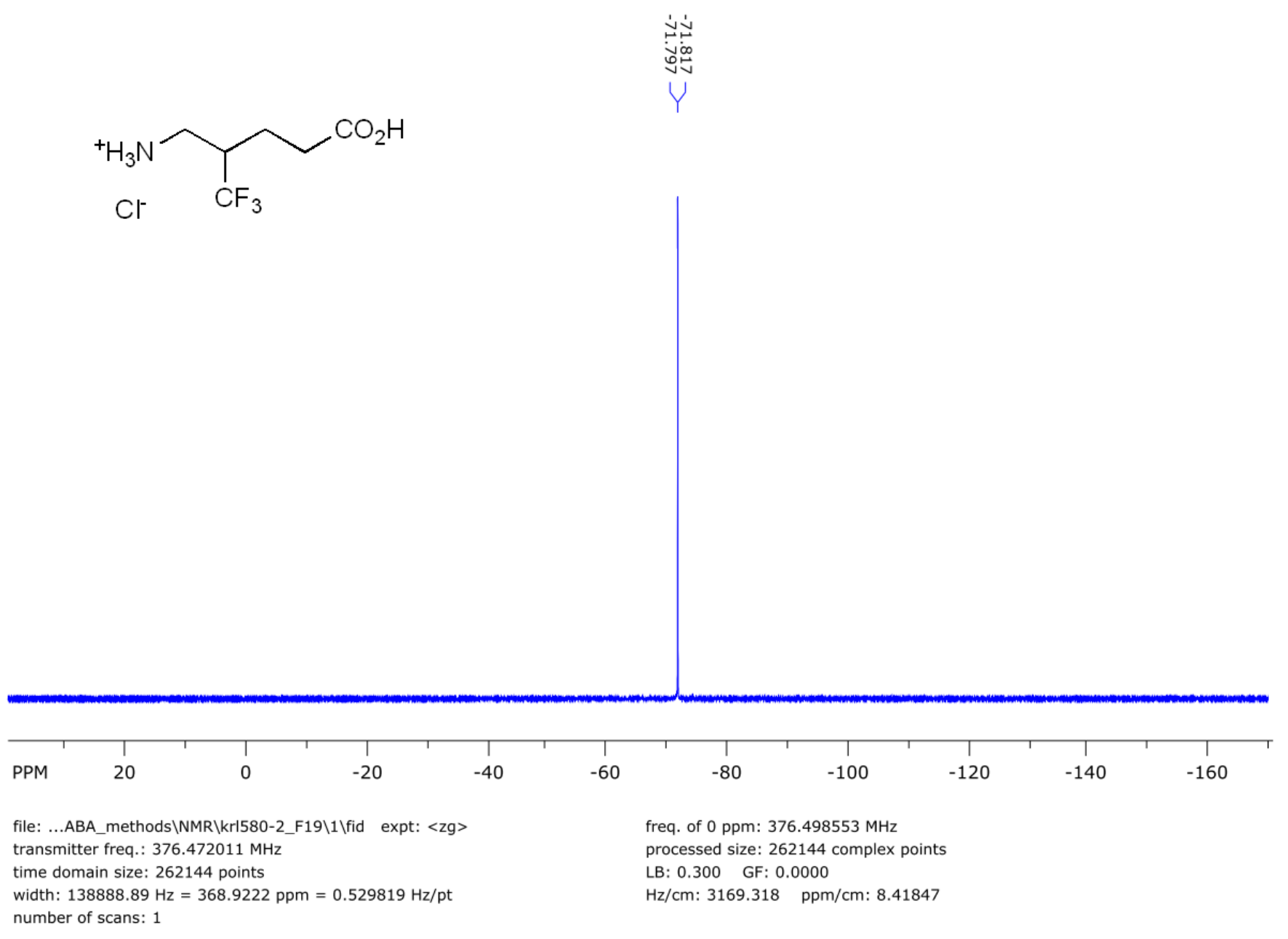


SpinWorks 4: 5-amino-5-(trifluoromethyl)pentanoic acid

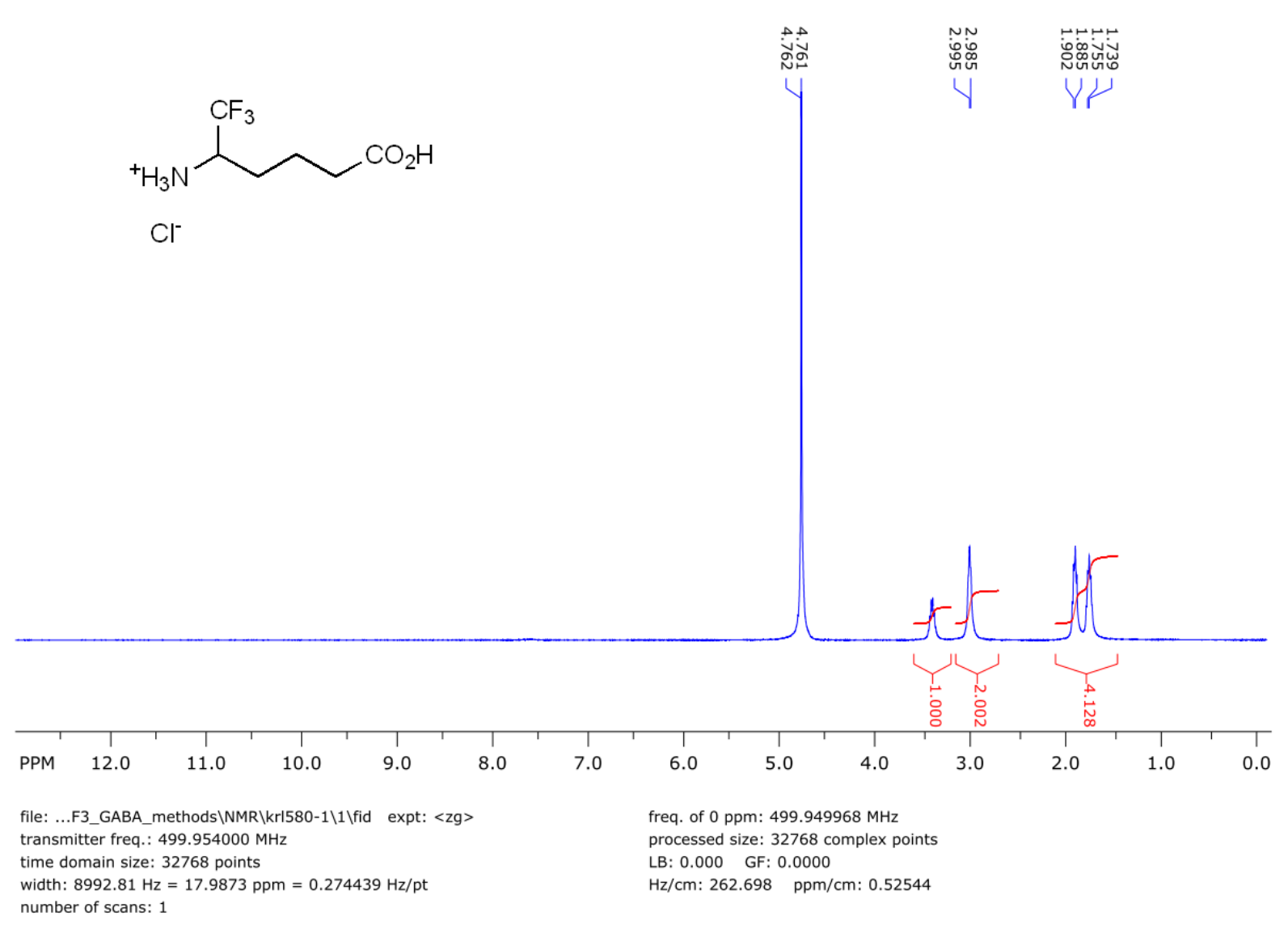




\section{${ }^{13} \mathrm{C}\left\{{ }^{1} \mathrm{H}\right\}$ NMR $\left(100 \mathrm{MHz}, \mathrm{D}_{2} \mathrm{O}\right)$}

SpinWorks 4: 5-amino-5-(trifluoromethyl)pentanoic acid 13C

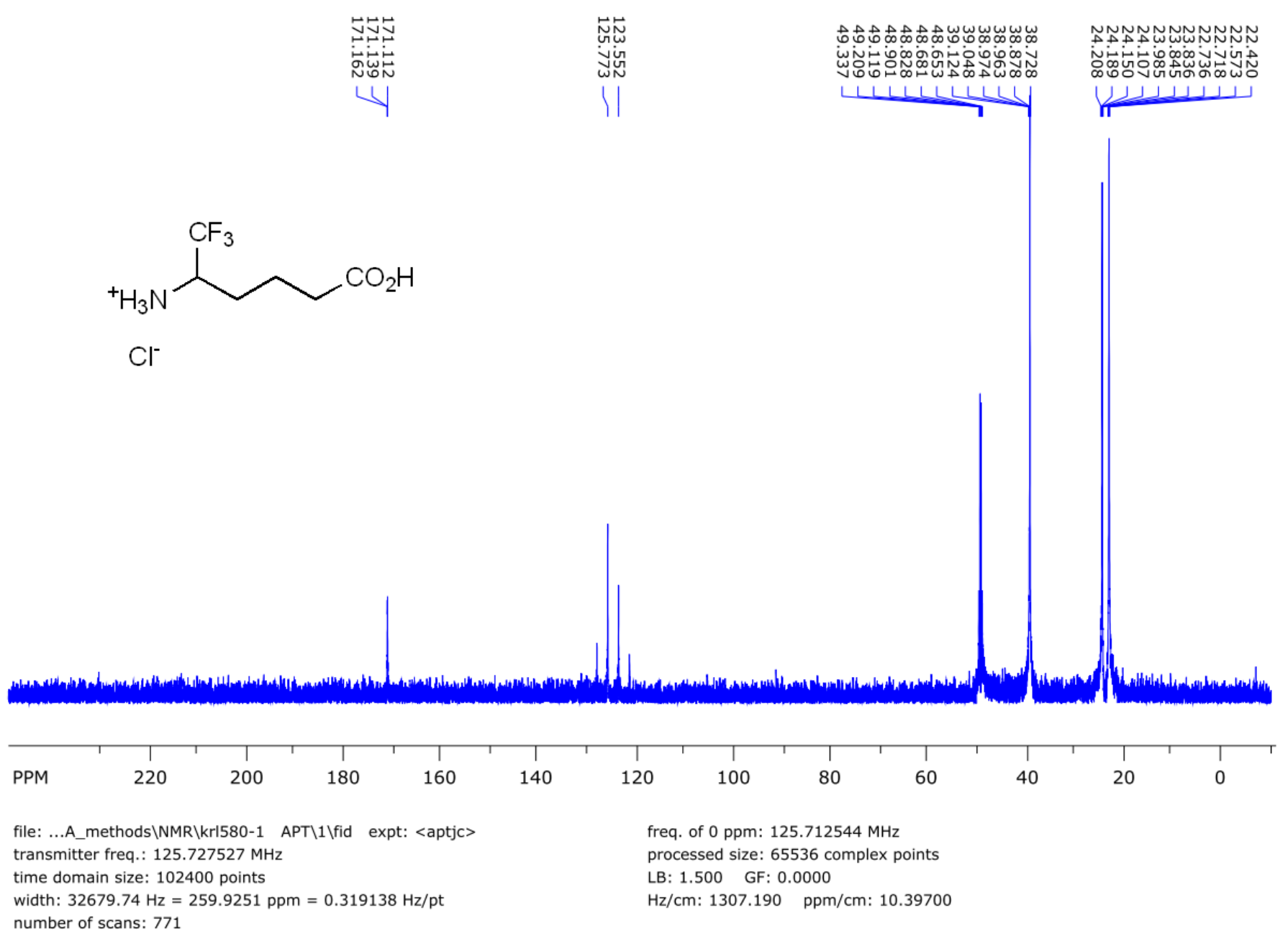


${ }^{19}$ F NMR (376 MHz, D $\left.2 \mathrm{O}\right)$

SpinWorks 4: 5-amino-5-(trifluoromethyl)pentanoic acid F19

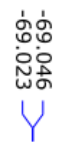<smiles>[NH3+]C(CCCC(=O)O)C(F)(F)F</smiles>

$\mathrm{Cl}^{-}$

file: ...ABA_methods\NMR\KRL580-1_F19\1\fid expt: <zg> transmitter freq.: $376.472011 \mathrm{MHz}$

freq. of $0 \mathrm{ppm}: 376.498553 \mathrm{MHz}$

time domain size: 26214

processed

width: $138888.89 \mathrm{~Hz}=368.9222 \mathrm{ppm}=0.529819 \mathrm{~Hz} / \mathrm{pt}$

LB: 0.300 GF: 0.0000

number of scans: 1

$\mathrm{Hz} / \mathrm{cm}: 5555.556 \mathrm{ppm} / \mathrm{cm}: 14.75689$ 


\section{Determination $p K_{a}$ and $p I$ for $\mathrm{CF}_{3}$-homo-GABA isomers by potentiometric titration $\mathrm{pK}_{\mathrm{a}}$ Determination of homo-GABA and its trifluoromethyl derivatives}

The $\mathrm{pK}_{\mathrm{a}}$ values of amino acids were determined by potentiometric titration method using the $\mathrm{pH}$ meter ( $\mathrm{pH}-340)$ with ESL-43-07 glass electrode (Gomel, Belarus). In the applied pH-metric method, a solution of sample is titrated over a $\mathrm{pH}$ range in which it passes from being fully protonated to fully deprotonated. The negative logarithm of dissociation constants of carboxylic $\left(\mathrm{pK}_{\mathrm{COOH}}\right)$ and amino groups $\left(\mathrm{pK}_{\mathrm{NH} 3+}\right)$ were calculated by analyzing the shape of the titration curve with using the graphical method (1. Albert A., Sergeant E. P. Ionization constants of acids and bases, Wiley, Inc., New York, 1962) and Hyperquad 2000 program. Values of isoelectric points were calculated according to the formula: $\mathrm{pI}=\left(\mathrm{pK}_{\mathrm{COOH}}+\mathrm{pK}_{\mathrm{NH} 3+)} / 2\right.$. Theoretical $\mathrm{pK}$ values were predicted with using ACD/ChemSketch program.

General titration procedure: an exact amounts of the amino acids were dissolved in aqueous solution containing $0.1 \mathrm{M} \mathrm{KCl}$ to adjust ionic strength. For the $\mathrm{pK}_{\mathrm{a}}$ determinations the hydrochloride salts of fluorinated amino acids were used. From other hand before titration of not substituted 5-aminopentanoic acid the equimolar quantities of hydrochloric acid were added in substance solution. Than the prepared solutions were titrated with standartizied base $(0.06 \mathrm{M} \mathrm{KOH})$ at constant temperature $\left(23{ }^{\circ} \mathrm{C}\right)$. The examples of obtained titration curves of fluorinated amino acids hydrochlorides are shown in Figure S1.

\begin{tabular}{|l|l|l|}
\hline \multicolumn{2}{|c|}{} & \\
\hline
\end{tabular}


(2): Compound 69;

(3): Compound70;

(4): Compound 71;

(5): Compound 72.

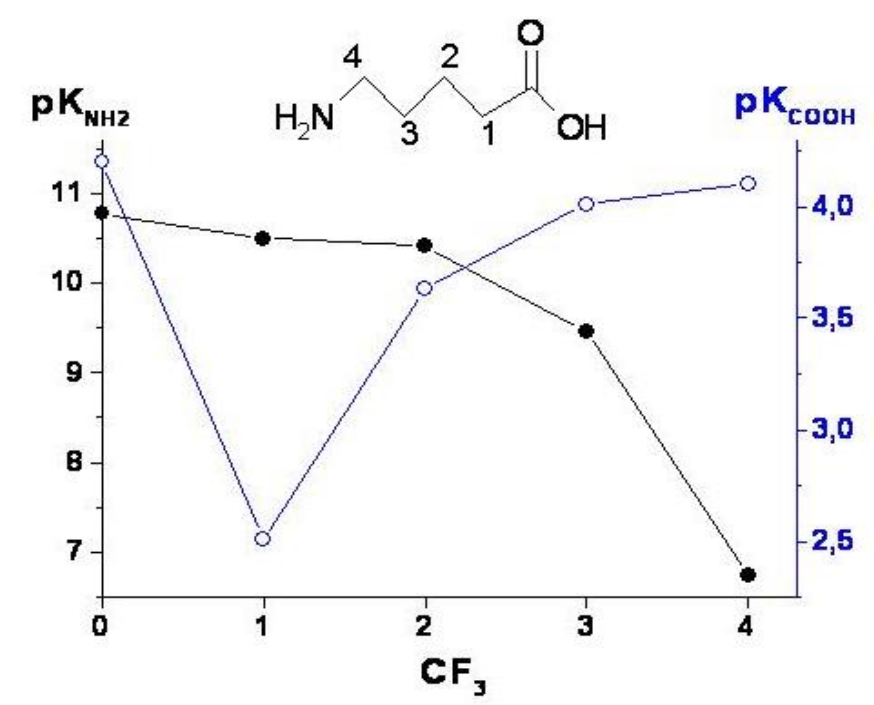

Figure S2. Dependence of $\mathrm{pK}_{\mathrm{NH} 2}$ and $\mathrm{pK}_{\mathrm{COOH}}$ values of fluorine substituted amino acids on the position of the $\mathrm{CF}_{3}$ group in the structure.

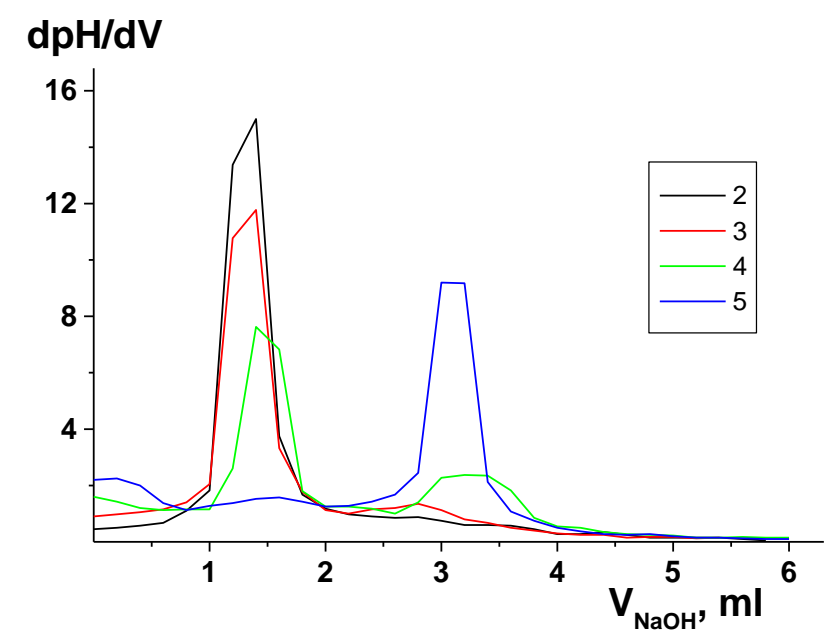

Figure S3. Differential dependence of titration curves of aqueous solutions of fluorine hydrochloride substituted aliphatic amino acids with a solution of $\mathrm{NaOH} . \mathrm{C}_{\mathrm{sub}}=0.01 \mathrm{M}, \mathrm{C}_{\mathrm{NaOH}}$ $=0.06 \mathrm{M}, \mathrm{V}_{0}=10 \mathrm{~mL}$. 
Table S1. Experimental $p K a$ values and isoelectric point $p I$ values of homo-GABA and $\mathrm{CF}_{3-}$ substituted analogs.

\begin{tabular}{|l|c|c|c|}
\hline Compound & $\mathbf{p K}\left(\mathbf{N H}_{2}\right)$ & $\mathbf{p K}\left(\mathbf{C} \mathbf{O}_{2} \mathbf{H}\right)$ & $\mathbf{p I}$ \\
\hline homo-GABA & $10.77 \pm 0.04$ & $4.20 \pm 0.03$ & 7.48 \\
\hline 2-CF - homo-GABA 69 & $10.50 \pm 0.04$ & $2.51 \pm 0.04$ & 6.51 \\
\hline 3-CF - homo-GABA 70 & $10.41 \pm 0.03$ & $3.63 \pm 0.04$ & 7.02 \\
\hline 4-CF - homo-GABA 71 & $9.46 \pm 0.02$ & $4.01 \pm 0.04$ & 6.74 \\
\hline 5- $\mathrm{CF}_{3}-$ homo-GABA 72 & $6.74 \pm 0.06$ & $4.1 \pm 0.05$ & 5.42 \\
\hline
\end{tabular}

Table S2. Theoretically calculated values of the dissociation constants of amino acids in water in the ACD lab.

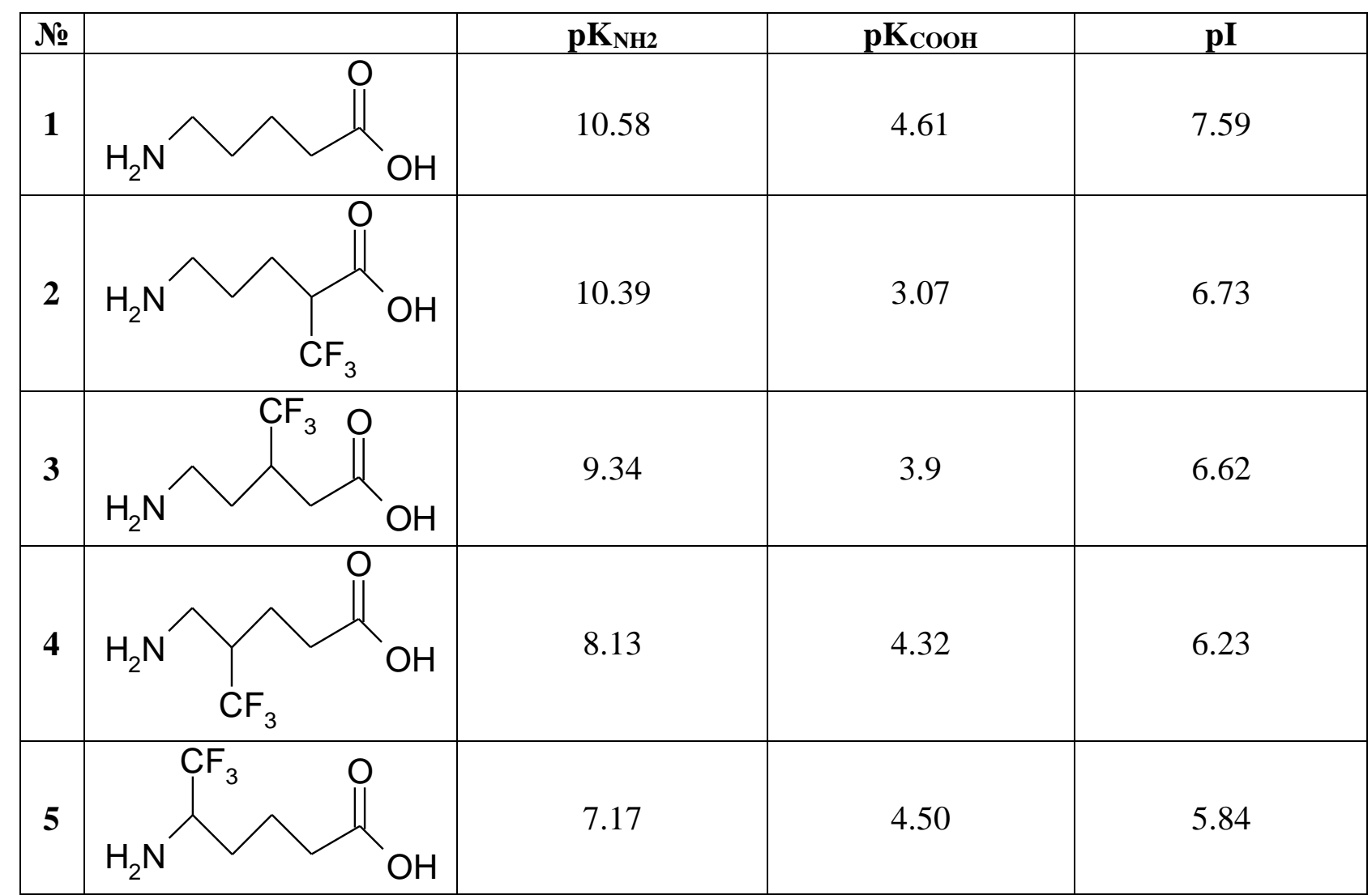




\section{Crystallographic data (X-Ray)}

Crystals of compounds 69-72 suitable for X-Ray diffractional studies were obtained by a low evaporation of a solution of listed compounds in methanol. Diffraction data were collected at room temperature on a Bruker SMART APEX II diffractometer with graphite-monochromated Mo K $\alpha$ radiation $(\lambda=0.71073 \AA)$ operating in the $\omega$ scans mode. These data were integrated using the program SAINT while the absorption correction was carried out using the program SADABS.

The structures were solved by direct methods and refined by the full-matrix least-squares technique in the anisotropic approximation for non-hydrogen atoms using the Bruker SHELXTL program package. Crystallographic data for all structures in this paper have been deposited at Cambridge Crystallographic Data Centre. Copies of the data can be obtained, free of charge, on application to CCDC, 12 Union Road, Cambridge CB21EZ, UK, (fax: +44-(0)1223-336033 or e-mail: deposit@ccdc.cam.ac.uk).

\section{Compound 69}

Crystals are monoclinic $\mathrm{C}_{6} \mathrm{H}_{11} \mathrm{NO}_{2} \mathrm{~F}_{3} \mathrm{Cl}$, at $20^{\circ} \mathrm{C}, \boldsymbol{a}=12.575$ (2), $\boldsymbol{b}=7.6962(9), \boldsymbol{c}=10.377$ (2) $\AA, \beta$ $=99.56(1)^{\circ}, \mathrm{V}=990.4(2) \AA^{3}, \mathrm{Mr}=221.61, \mathrm{Z}=4$, space group $\mathrm{P} 2_{1 / \mathrm{c}}, \mathrm{d}_{\mathrm{cal}}=1.486 \mathrm{~g} / \mathrm{cm}^{3}, \mu\left(\mathrm{MoK}_{\alpha}\right)$ $=0.401 \mathrm{~mm}^{-1}, \mathrm{~F}(000)=456$. Unit cell parameters and intensities of 9450 reflections (2878 independent, $\left.\mathrm{R}_{\text {int }}=0.043\right)$ were measured on an "Xcalibur-3" diffractometer $\left(\right.$ MoK $_{\alpha}$ radiation, CCD-detector, graphite monochromator, $\omega$-scanning, $2 \theta_{\max }=60^{\circ}$ ).

The structure was decoded by the direct method using the SHELXTL software package (Sheldrick, G. M. Acta Crystallogr., Sect. A, 2008, A64, 112-122). The positions of the hydrogen atoms were determined from the differential synthesis of electron density and refined using the "rider" model with $U_{\text {iso }}=n U_{\text {equiv }}(n=1.5$ for the hydroxyl group and protonated amino group; $n=1.2$ for the remaining hydrogen atoms) of the non-hydrogen atom associated with this hydrogen. The structure was refined by $\mathrm{F}^{2}$ by full-matrix least squares in the anisotropic approximation for non-hydrogen atoms up to $\mathrm{wR}_{2}=0.253$ by 2785 reflections $\left(\mathrm{R}_{1}=0.084\right.$ by 1686 reflections with $\mathrm{F}>4 \sigma(\mathrm{F}), \mathrm{S}=$ 1.013). 


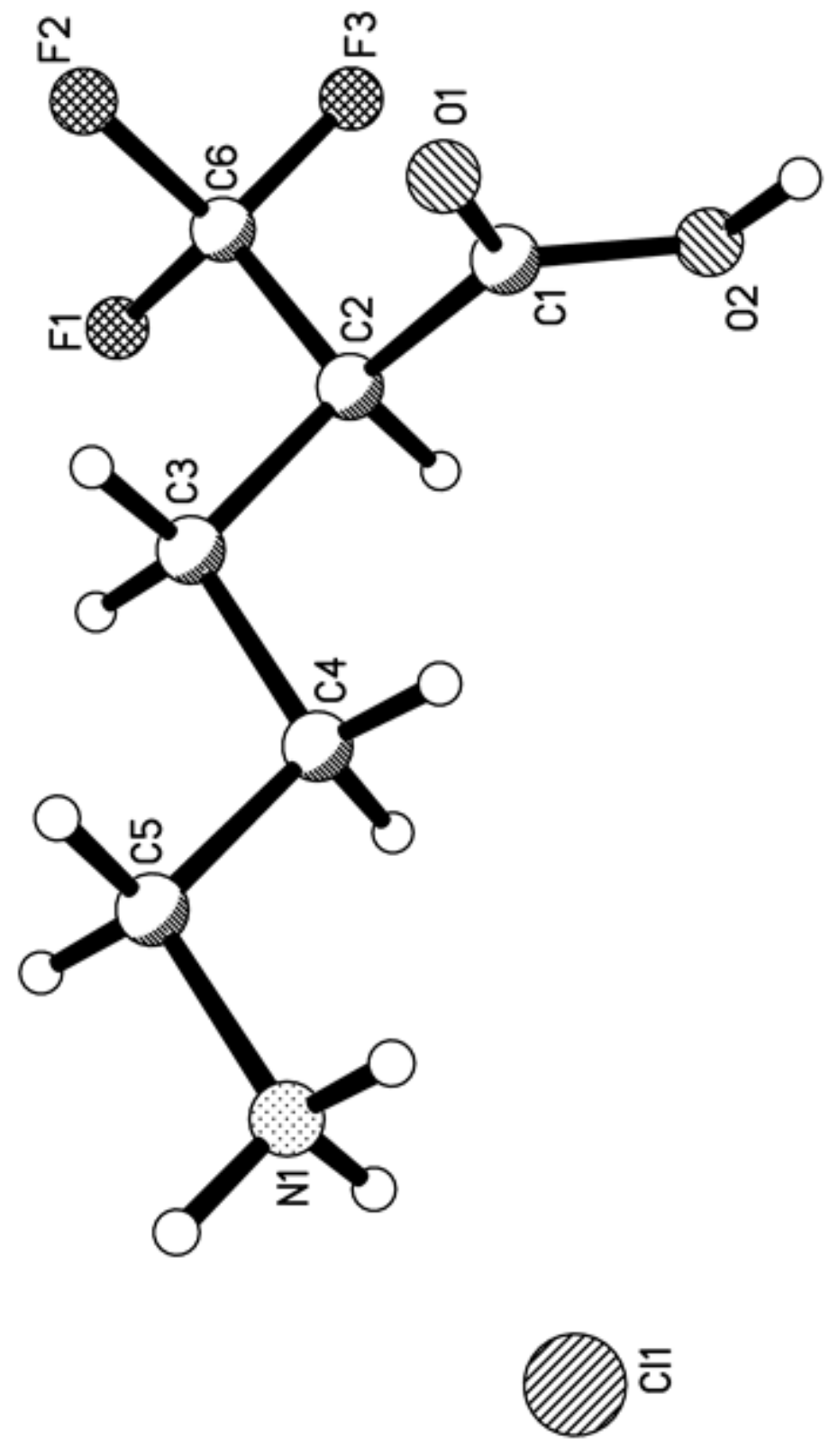

Figure S4. Structure of compound $69 * \mathbf{H C l}$ according to X-ray diffraction data. Ellipsoids are shown at $30 \%$ probability level.

\section{Compound 70}

Crystals are rhombic, $\mathrm{C}_{6} \mathrm{H}_{11} \mathrm{NO}_{2} \mathrm{~F}_{3} \mathrm{Cl} \cdot \mathrm{H}_{2} \mathrm{O}$, at $20^{\circ} \mathrm{C} \boldsymbol{a}=25.781(2), \boldsymbol{b}=6.5384(7), \boldsymbol{c}=25.212(2) \AA$, $\mathrm{V}=4250.0(6) \AA^{3}, \mathrm{M}_{\mathrm{r}}=239.62, \mathrm{Z}=16$, space group Pbcn, $\mathrm{d}_{\mathrm{cal}}=1.498 \mathrm{~g} / \mathrm{sm}^{3}, \mu\left(\mathrm{MoK}_{\alpha}\right)=0.387 \mathrm{~mm}$ ${ }^{-1}, \mathrm{~F}(000)=1984$. Unit cell parameters and intensities of 46418 reflections $\left(6194\right.$ independent, $\mathrm{R}_{\text {int }}=$ 0.077) were measured on an "Xcalibur-3" diffractometer $\left(\mathrm{MoK}_{\alpha}\right.$ radiation, CCD-detector, graphite monochromator, $\omega$-scanning, $2 \theta_{\max }=60^{\circ}$ ).

The positions of the hydrogen atoms are revealed from the difference synthesis of electron density and refined in the isotropic approximation. The structure was decoded by the direct method using 
the SHELXTL software package (Sheldrick, G. M. Acta Crystallogr., Sect. A, 2008, A64, 112-122). The structure was refined by $\mathrm{F}^{2}$ by full-matrix least squares in the anisotropic approximation for non-hydrogen atoms up to $\mathrm{wR}_{2}=0.253$ by 2785 reflections $\left(\mathrm{R}_{1}=0.127\right.$ by 6162 reflections with $\mathrm{F}>4 \sigma(\mathrm{F}), \mathrm{S}=0.922)$.

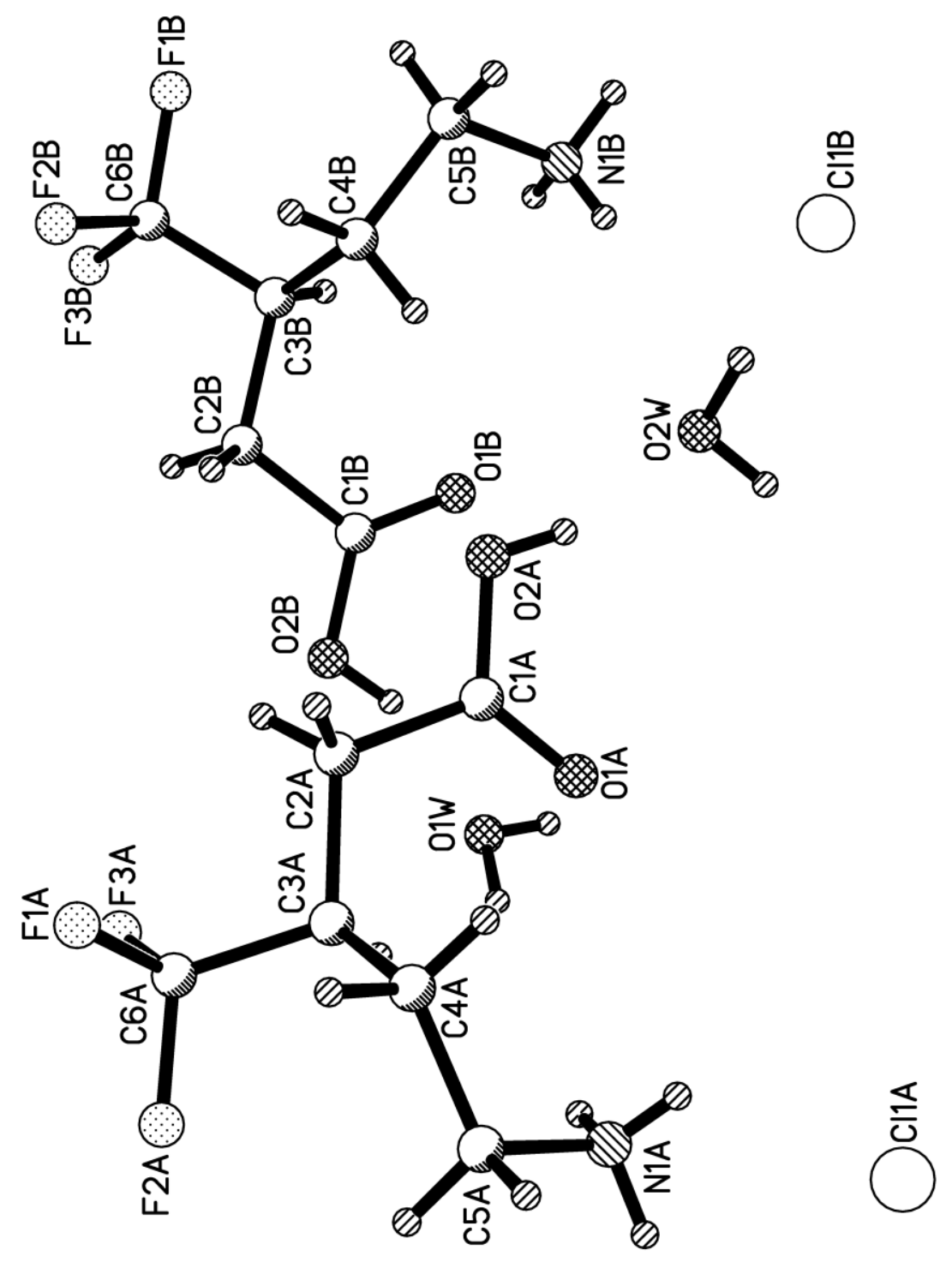

Figure S5. Structure of compound $\mathbf{7 0} * \mathbf{H C l}$ according to X-ray diffraction data. Ellipsoids are shown at $30 \%$ probability level. 


\section{Compound 71}

Crystals are monoclinic, $\mathrm{C}_{6} \mathrm{H}_{11} \mathrm{NO}_{2} \mathrm{~F}_{3} \mathrm{Cl}$, at $20^{\circ} \mathrm{C} \boldsymbol{a}=11.834(1), \boldsymbol{b}=9.7817(6), \boldsymbol{c}=8.4036(7) \AA, \beta=$ 109.386(9) $)^{\mathrm{o}}, \mathrm{V}=917.6(1) \AA^{3}, \mathrm{M}_{\mathrm{r}}=221.61, \mathrm{Z}=4$, space group $\mathrm{P} 2{ }_{1} / \mathrm{c}, \mathrm{d}_{\mathrm{cal}}=1.604 \mathrm{~g} / \mathrm{sm}^{3}, \mu\left(\mathrm{MoK}_{\alpha}\right)=$ $0.433 \mathrm{~mm}^{-1}, \mathrm{~F}(000)=456$. Unit cell parameters and intensities of 8458 reflections (4553 independent, $\left.\mathrm{R}_{\text {int }}=0.059\right)$ were measured on an "Xcalibur-3" diffractometer $\left(\right.$ MoK $_{\alpha}$ radiation, CCD-detector, graphite monochromator, $\omega$-scanning, $2 \theta_{\max }=60^{\circ}$ ).

The structure was decoded by the direct method using the SHELXTL software package (Sheldrick, G. M. Acta Crystallogr., Sect. A, 2008, A64, 112-122). The positions of the hydrogen atoms were determined from the differential synthesis of electron density and refined using the "rider" model with $\mathrm{U}_{\text {iso }}=n \mathrm{U}_{\text {equiv }}(\mathrm{n}=1.5$ for the hydroxyl group and protonated amino group; $\mathrm{n}=1.2$ for the remaining hydrogen atoms) of the non-hydrogen atom associated with this hydrogen. The structure was refined by $\mathrm{F}^{2}$ by full-matrix least squares in the anisotropic approximation for non-hydrogen atoms up to $\mathrm{wR}_{2}=0.131$ by4257 reflections $\left(\mathrm{R}_{1}=0.047\right.$ by 3545 reflections with $\mathrm{F}>4 \sigma(\mathrm{F}), \mathrm{S}=$ 1.041). 


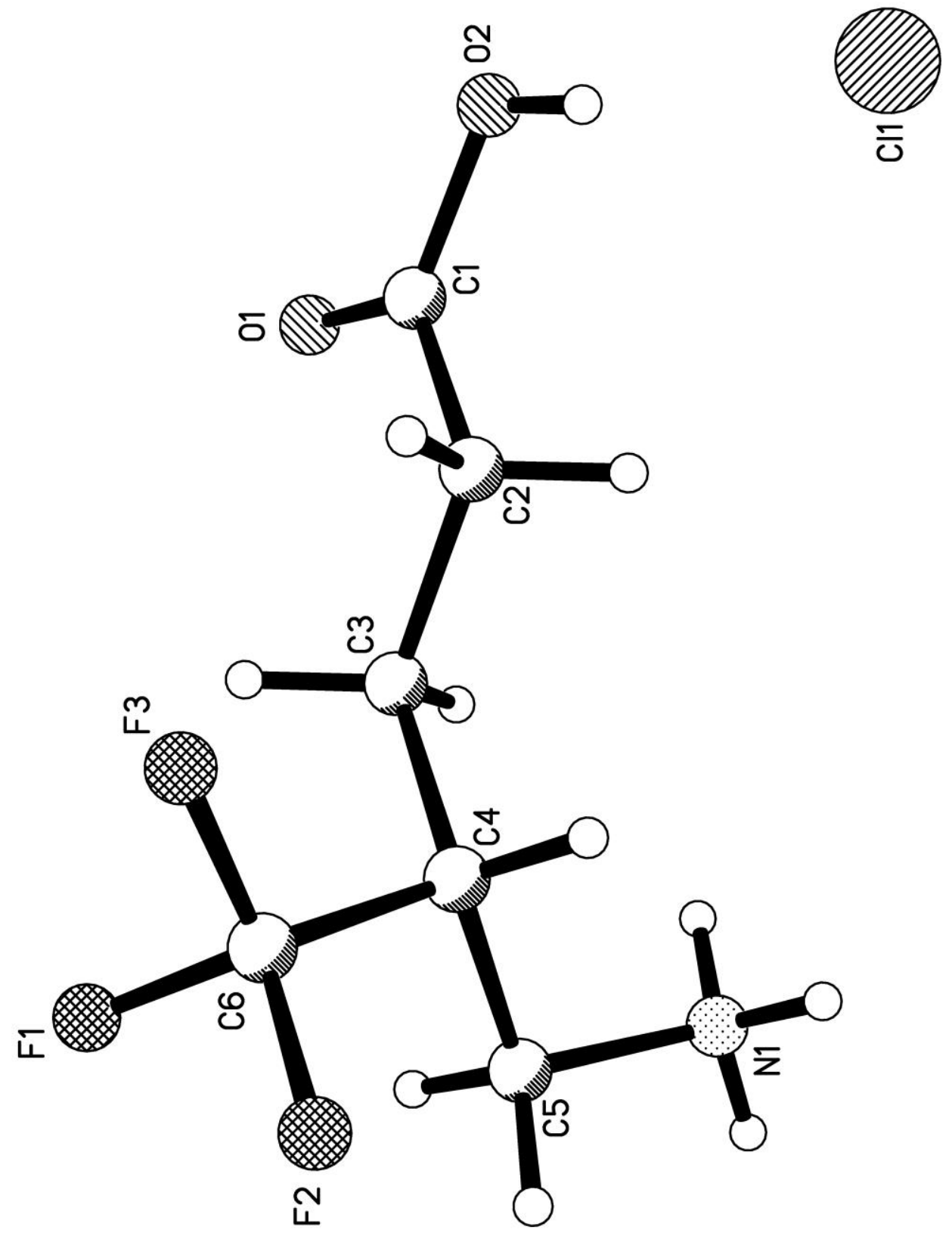

Figure S6. Structure of compound $\mathbf{7 1} * \mathbf{H C l}$ according to X-ray diffraction data. Ellipsoids are shown at $30 \%$ probability level. 


\section{Compound 72}

Crystals are rhombic, $\cdot \mathrm{C}_{6} \mathrm{H}_{11} \mathrm{~F}_{3} \mathrm{NO}_{2} \mathrm{Cl} \cdot \mathrm{H}_{2} \mathrm{O}$, at $20^{\circ} \mathrm{C} \boldsymbol{a}=11.4374(5), \boldsymbol{b}=25.6538(9), \boldsymbol{c}=7.4152(3)$ $\AA, \mathrm{V}=2175.7(2) \AA^{3}, \mathrm{M}_{\mathrm{r}}=239.62, \mathrm{Z}=8$, space group Pccn, $\mathrm{d}_{\mathrm{cal}}=1.463 \mathrm{~g} / \mathrm{sm}^{3}, \mu\left(\mathrm{MoK}_{\alpha}\right)=0.378$ $\mathrm{mm}^{-1}, \mathrm{~F}(000)=992$. Unit cell parameters and intensities of 21849 reflections (3184 independent, $\left.\mathrm{R}_{\text {int }}=0.075\right)$ were measured on an "Xcalibur-3" diffractometer $\left(\mathrm{MoK}_{\alpha}\right.$ radiation, CCD-detector, graphite monochromator, $\omega$-scanning, $2 \theta_{\max }=60^{\circ}$ ).

The structure was decoded by the direct method using the SHELXTL software package (Sheldrick, G. M. Acta Crystallogr., Sect. A, 2008, A64, 112-122). The positions of the hydrogen atoms were determined from the differential synthesis of electron density and refined using the "rider" model with $\mathrm{U}_{\text {iso }}=n \mathrm{U}_{\text {equiv }}(\mathrm{n}=1.5$ for the hydroxyl group, protonated amino group and water; $\mathrm{n}=1.2$ for the remaining hydrogen atoms) of the non-hydrogen atom associated with this hydrogen. The structure was refined by $\mathrm{F}^{2}$ by full-matrix least squares in the anisotropic approximation for nonhydrogen atoms up to $\mathrm{wR}_{2}=0.198$ by 3129 reflections $\left(\mathrm{R}_{1}=0.062\right.$ by 1800 reflections with $\mathrm{F}>4 \sigma$ $(\mathrm{F}), \mathrm{S}=0.993)$. 

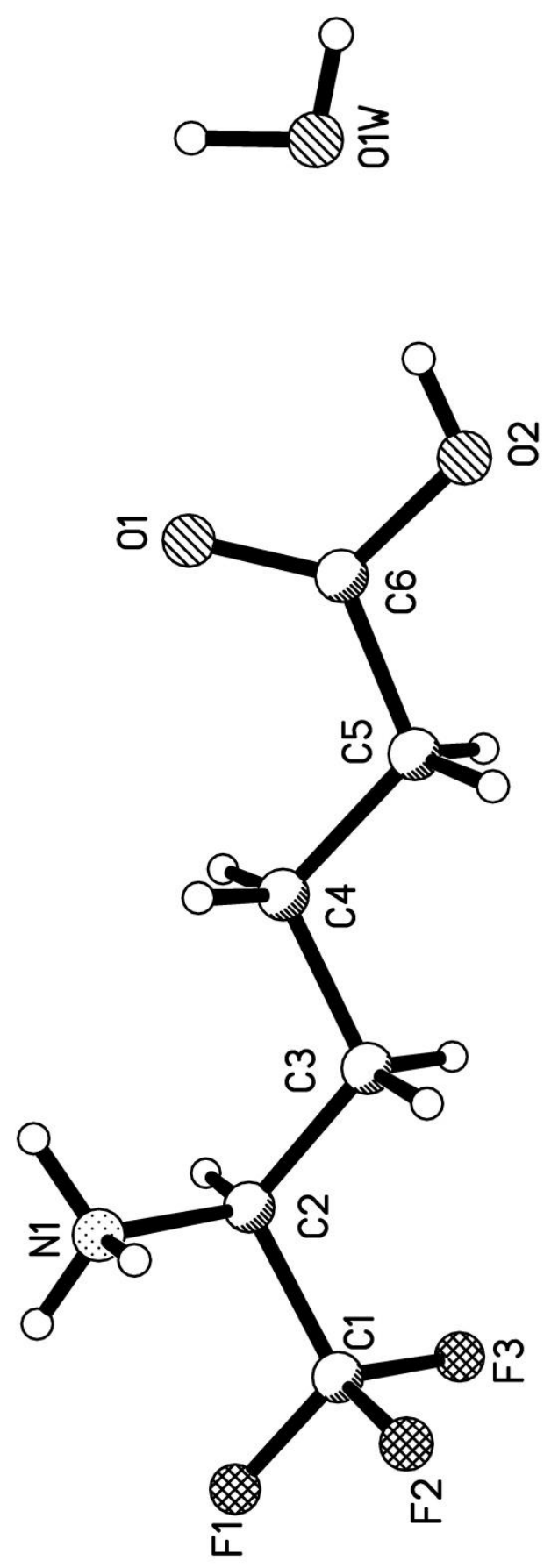

Figure S7. Structure of compound $\mathbf{7 2} * \mathbf{H C l}$ according to X-ray diffraction data. Ellipsoids are shown at $30 \%$ probability level. 


\section{Photos}

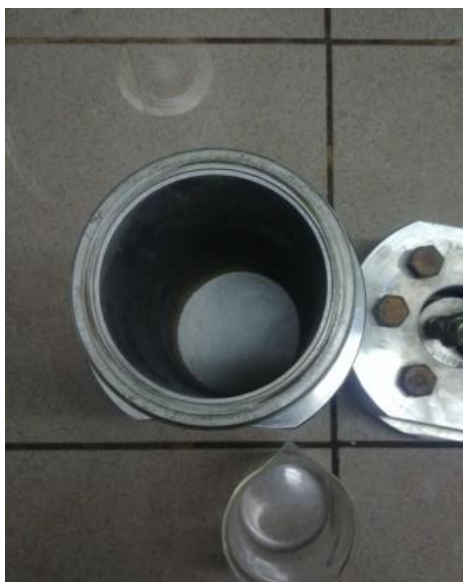

A high pressure reactor.

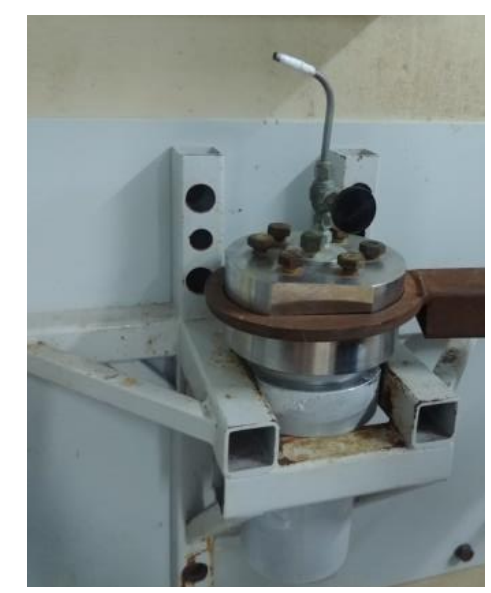

Installation of the high pressure reactor.

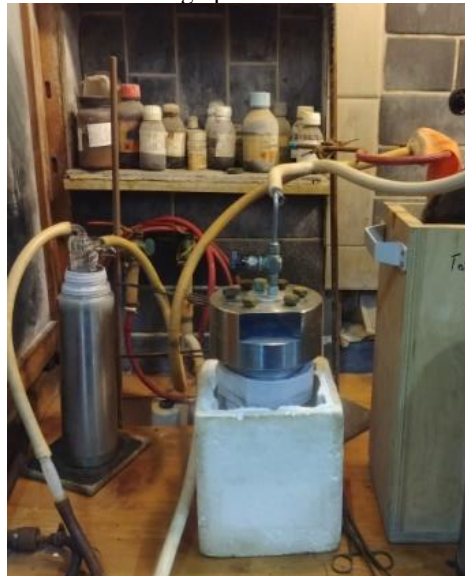

System for gas blowing.

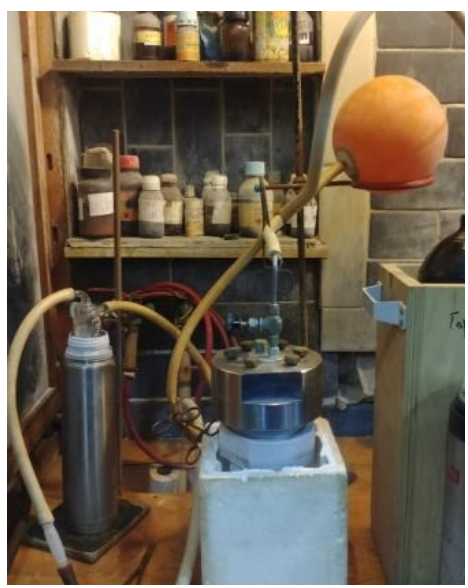

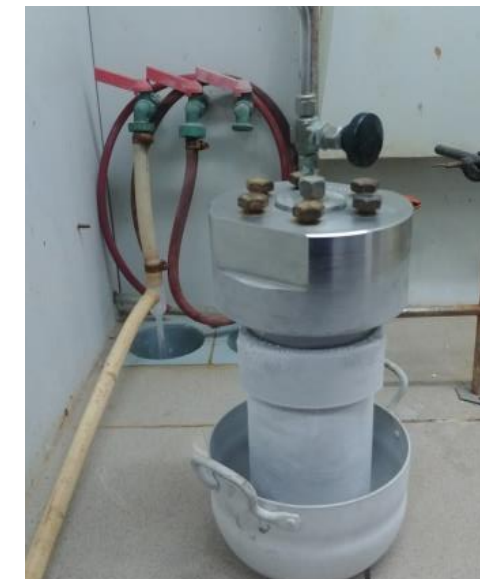

Cooling down the reaction mixture..

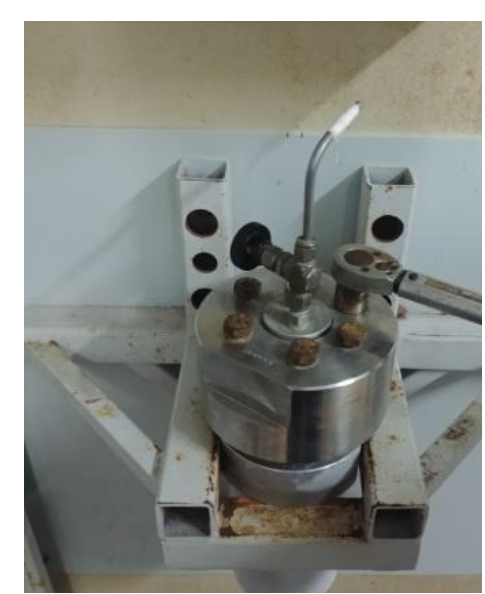

Installation of the high pressure reactor.

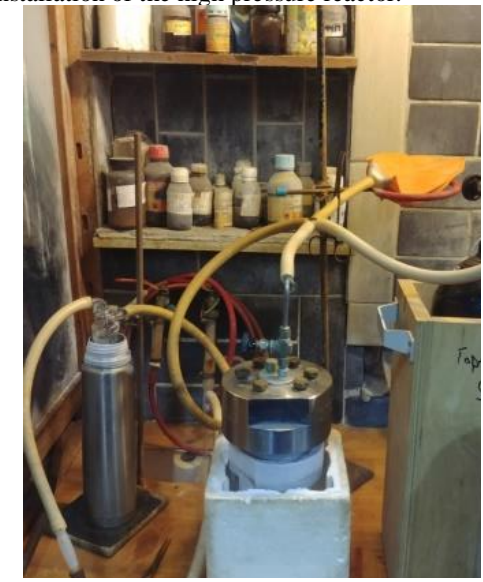

Vacuuming of the reaction system. A balloon is closed with a hemostat.

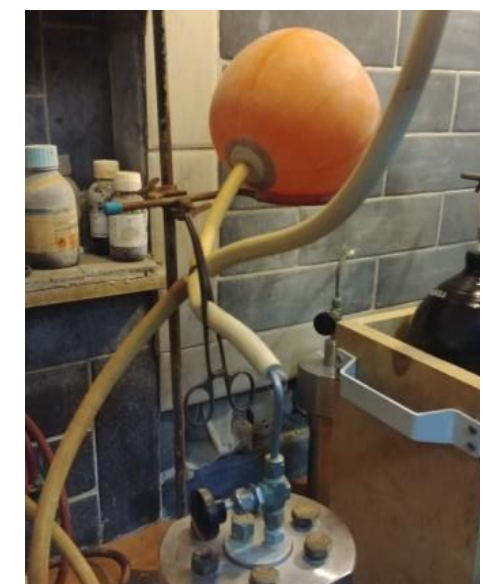

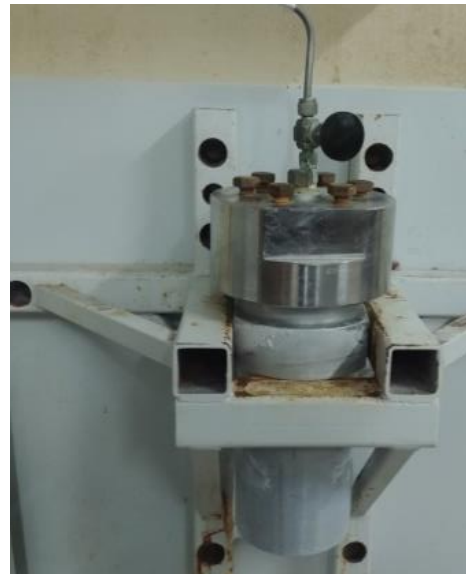

Fixing the high pressure reactor on the special

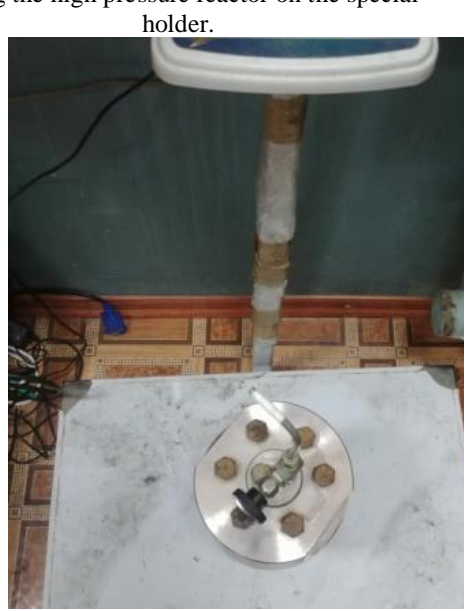

Weighing of the reaction mixture.

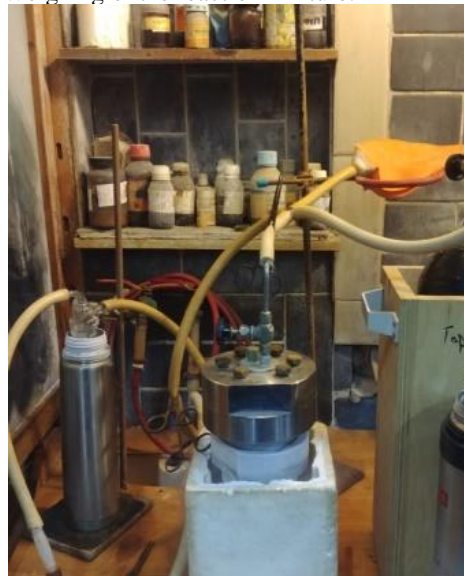

Preparation for pumping up the balloon with $\mathrm{SF}_{4}$ The high pressure reactor is closed with a hemostat

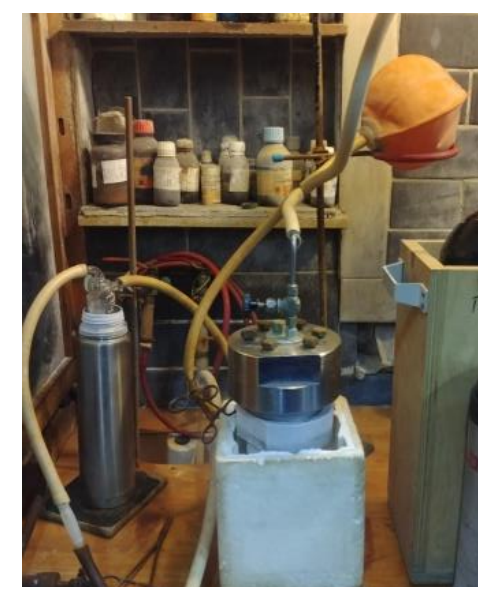


Inflating a balloon.

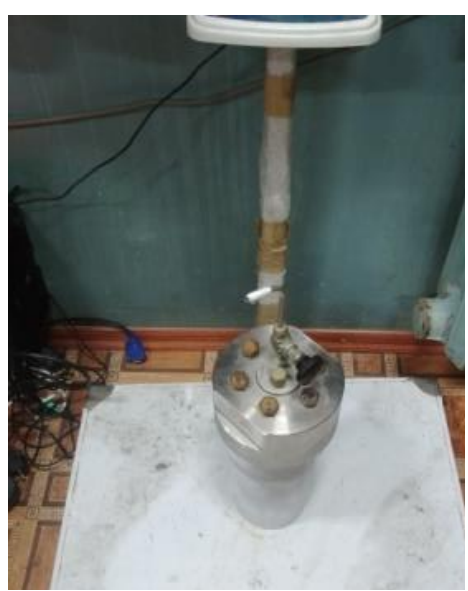

Weighing of the reaction mixture with $\mathrm{SF}_{4}$.

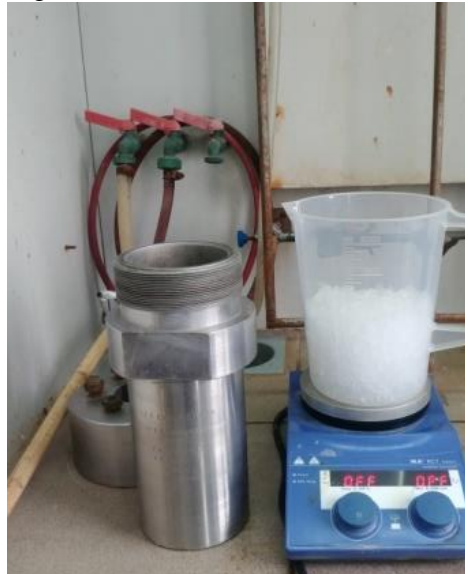

The opened high pressure reactor.
The high pressure reactor is closed with a hemostat.

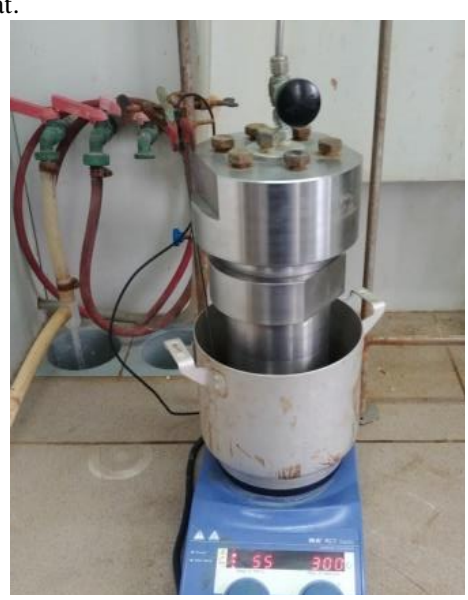

The high pressure reactor placed in oil bath.

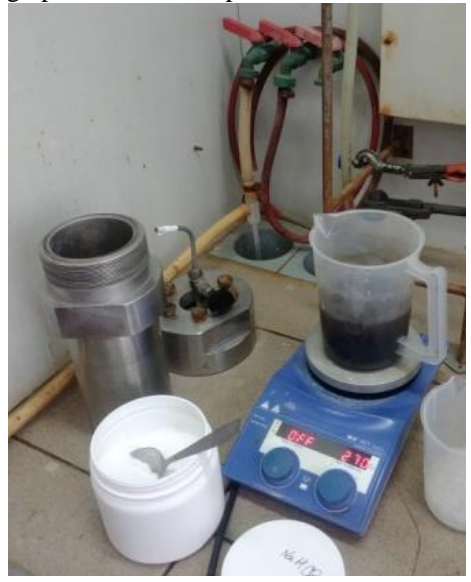

Quenching with $\mathrm{NaHCO}_{3}$
Gas injection into the reaction mixture.

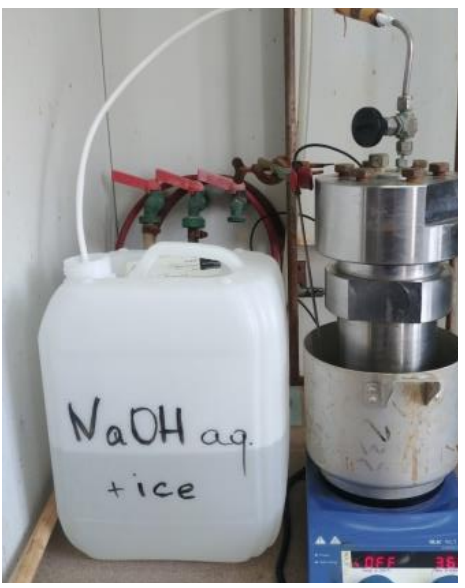

Quenching with a cold solution of $\mathrm{NaOH}$.

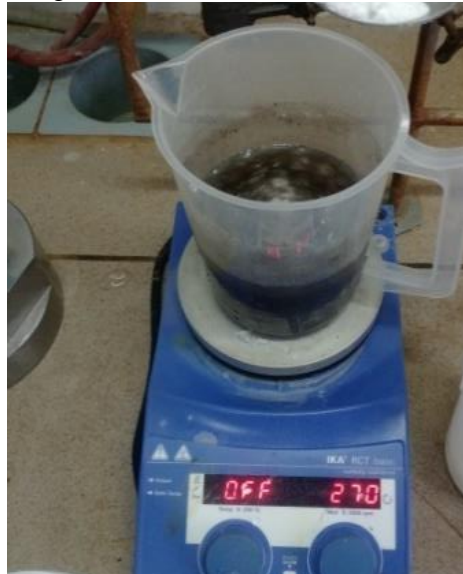

Quenching with $\mathrm{NaHCO}_{3}$ 